\title{
Physical rehabilitation approaches for the recovery of function and mobility following stroke (Review)
}

Todhunter-Brown A, Baer G, Campbell P, Choo PL, Morris J, Langhorne P, Cowie J, Sellers CE

Todhunter-Brown A, Baer G, Campbell P, Choo PL, Morris J, Langhorne P, Cowie J, Sellers CE.

Physical rehabilitation approaches for the recovery of function and mobility following stroke.

Cochrane Database of Systematic Reviews 2014, Issue 4. Art. No.: CD001920.

DOI: 10.1002/14651858.CD001920.pub3.

www.cochranelibrary.com 
TABLE OF CONTENTS

ABSTRACT

PLAIN LANGUAGE SUMMARY

Figure 1.

OBJECTIVES

METHODS

RESULTS

Figure 2.

Figure 3.

Figure 4.

DISCUSSION

AUTHORS' CONCLUSIONS

ACKNOWLEDGEMENTS

REFERENCES

CHARACTERISTICS OF STUDIES

DATA AND ANALYSES

Analysis 1.1. Comparison 1: Intervention versus no treatment: immediate outcomes, Outcome 1: Independence in ADL scales .

Analysis 1.2. Comparison 1: Intervention versus no treatment: immediate outcomes, Outcome 2: Motor function scales .........

Analysis 1.3. Comparison 1: Intervention versus no treatment: immediate outcomes, Outcome 3: Balance (Berg Balance Scale)

Analysis 1.4. Comparison 1: Intervention versus no treatment: immediate outcomes, Outcome 4: Gait velocity

Analysis 1.5. Comparison 1: Intervention versus no treatment: immediate outcomes, Outcome 5: Length of stay

Analysis 2.1. Comparison 2: Intervention versus usual care or attention control: immediate outcomes, Outcome 1: Independence in ADL scales

Analysis 2.2. Comparison 2: Intervention versus usual care or attention control: immediate outcomes, Outcome 2: Motor function scales

Analysis 2.3. Comparison 2: Intervention versus usual care or attention control: immediate outcomes, Outcome 3: Balance (Berg Balance Scale)

Analysis 2.4. Comparison 2: Intervention versus usual care or attention control: immediate outcomes, Outcome 4: Gait velocity

Analysis 2.5. Comparison 2: Intervention versus usual care or attention control: immediate outcomes, Outcome 5: Length of stay

Analysis 3.1. Comparison 3: One active intervention versus another active intervention: immediate outcomes, Outcome 1: Independence in ADL scales

Analysis 3.2. Comparison 3: One active intervention versus another active intervention: immediate outcomes, Outcome 2: Motor function scales

Analysis 3.3. Comparison 3: One active intervention versus another active intervention: immediate outcomes, Outcome 3: Balance (Berg Balance Scale)

Analysis 3.4. Comparison 3: One active intervention versus another active intervention: immediate outcomes, Outcome 4: Gait velocity

Analysis 3.5. Comparison 3: One active intervention versus another active intervention: immediate outcomes, Outcome 5: Length of stay

Analysis 4.1. Comparison 4: Intervention versus no treatment: persisting outcomes, Outcome 1: Independence in ADL scales .. Analysis 4.2. Comparison 4: Intervention versus no treatment: persisting outcomes, Outcome 2: Motor function scales ........... Analysis 4.3. Comparison 4: Intervention versus no treatment: persisting outcomes, Outcome 3: Balance (Berg Balance Scale) . Analysis 4.4. Comparison 4: Intervention versus no treatment: persisting outcomes, Outcome 4: Gait velocity ......................... Analysis 5.1. Comparison 5: Intervention versus usual care or attention control: persisting outcomes, Outcome 1: Independence in ADL scales

Analysis 5.2. Comparison 5: Intervention versus usual care or attention control: persisting outcomes, Outcome 2: Motor function scales

Analysis 5.3. Comparison 5: Intervention versus usual care or attention control: persisting outcomes, Outcome 3: Balance (Berg Balance Scale)

Analysis 5.4. Comparison 5: Intervention versus usual care or attention control: persisting outcomes, Outcome 4: Gait velocity 
Analysis 6.1. Comparison 6: One active intervention versus another active intervention: persisting outcomes, Outcome 1: Independence in ADL scales

Analysis 6.2. Comparison 6: One active intervention versus another active intervention: persisting outcomes, Outcome 2: Motor function scales

Analysis 6.3. Comparison 6: One active intervention versus another active intervention: persisting outcomes, Outcome 3: Balance (Berg Balance Scale)

Analysis 6.4. Comparison 6: One active intervention versus another active intervention: persisting outcomes, Outcome 4: Gait velocity

Analysis 7.1. Comparison 7: Subgroups. Intervention versus no treatment: immediate outcome: independence in ADL, Outcome 1: Time after stroke

Analysis 7.2. Comparison 7: Subgroups. Intervention versus no treatment: immediate outcome: independence in ADL, Outcome 2: Study geographical location

Analysis 7.3. Comparison 7: Subgroups. Intervention versus no treatment: immediate outcome: independence in ADL, Outcome 3: Dose of intervention

Analysis 7.4. Comparison 7: Subgroups. Intervention versus no treatment: immediate outcome: independence in ADL, Outcome 4: Provider of intervention

Analysis 7.5. Comparison 7: Subgroups. Intervention versus no treatment: immediate outcome: independence in ADL, Outcome 5: Treatment components included

Analysis 8.1. Comparison 8: Subgroups. Intervention versus attention control or usual care: immediate outcome: independence in ADL, Outcome 1: Time after stroke

Analysis 8.2. Comparison 8: Subgroups. Intervention versus attention control or usual care: immediate outcome: independence in ADL, Outcome 2: Study geographical location

Analysis 8.3. Comparison 8: Subgroups. Intervention versus attention control or usual care: immediate outcome: independence in ADL, Outcome 3: Dose of intervention

Analysis 8.4. Comparison 8: Subgroups. Intervention versus attention control or usual care: immediate outcome: independence in ADL, Outcome 4: Provider of intervention

Analysis 8.5. Comparison 8: Subgroups. Intervention versus attention control or usual care: immediate outcome: independence in ADL, Outcome 5: Treatment components included

Analysis 9.1. Comparison 9: Subgroups. Intervention versus no treatment: immediate outcome: motor function, Outcome 1: Time after stroke

Analysis 9.2. Comparison 9: Subgroups. Intervention versus no treatment: immediate outcome: motor function, Outcome 2: Study geographical location

Analysis 9.3. Comparison 9: Subgroups. Intervention versus no treatment: immediate outcome: motor function, Outcome 3: Dose of intervention

Analysis 9.4. Comparison 9: Subgroups. Intervention versus no treatment: immediate outcome: motor function, Outcome 4: Provider of intervention

Analysis 9.5. Comparison 9: Subgroups. Intervention versus no treatment: immediate outcome: motor function, Outcome 5: Treatment components included

Analysis 10.1. Comparison 10: Subgroups. Intervention versus attention control or usual care: immediate outcome: motor function, Outcome 1: Time after stroke

Analysis 10.2. Comparison 10: Subgroups. Intervention versus attention control or usual care: immediate outcome: motor function, Outcome 2: Study geographical location

Analysis 10.3. Comparison 10: Subgroups. Intervention versus attention control or usual care: immediate outcome: motor function, Outcome 3: Dose of intervention

Analysis 10.4. Comparison 10: Subgroups. Intervention versus attention control or usual care: immediate outcome: motor function, Outcome 4: Provider of intervention

Analysis 10.5. Comparison 10: Subgroups. Intervention versus attention control or usual care: immediate outcome: motor function, Outcome 5: Treatment components included

Analysis 11.1. Comparison 11: Subgroups. One active intervention versus another active intervention: immediate outcomes: independence in ADL, Outcome 1: Functional task training components

Analysis 11.2. Comparison 11: Subgroups. One active intervention versus another active intervention: immediate outcomes: independence in ADL, Outcome 2: Neurophysiological components

Analysis 11.3. Comparison 11: Subgroups. One active intervention versus another active intervention: immediate outcomes: independence in ADL, Outcome 3: Musculoskeletal components

Analysis 12.1. Comparison 12: Subgroups. One active intervention versus another active intervention: immediate outcomes: motor function, Outcome 1: Functional task training components 
Analysis 12.2. Comparison 12: Subgroups. One active intervention versus another active intervention: immediate outcomes: motor function, Outcome 2: Neurophysiological components

Analysis 12.3. Comparison 12: Subgroups. One active intervention versus another active intervention: immediate outcomes: motor function, Outcome 3: Musculoskeletal components

ADDITIONAL TABLES

APPENDICES

WHAT'S NEW

HISTORY 
[Intervention Review]

\section{Physical rehabilitation approaches for the recovery of function and mobility following stroke}

Alex Todhunter-Brown¹, Gillian Baer², Pauline Campbell1, Pei Ling Choo ${ }^{3}$, Jacqui Morris ${ }^{4}$, Peter Langhorne ${ }^{5}$, Julie Cowie ${ }^{6}$, Ceri E Sellers 7

1Nursing, Midwifery and Allied Health Professions Research Unit, Glasgow Caledonian University, Glasgow, UK. 2Department of Physiotherapy, Queen Margaret University, Edinburgh, UK. ${ }^{3}$ School of Health \& Life Sciences, Glasgow Caledonian University, Glasgow, UK. ${ }^{4}$ School of Nursing and Midwifery, University of Dundee, Dundee, UK. ${ }^{5}$ Academic Section of Geriatric Medicine, ICAMS, University of Glasgow, Glasgow, UK. ${ }^{6}$ NMAHP Research Unit, Glasgow Caledonian University, Glasgow, UK. ${ }^{7}$ Nursing, Midwifery and Allied Health Professions Research Unit, Glasgow Caledonian University, Glasgow, UK

Contact: Alex Todhunter-Brown, alex.todhunterbrown@gcu.ac.uk.

Editorial group: Cochrane Stroke Group.

Publication status and date: Edited (no change to conclusions), published in Issue 4, 2023.

Citation: Todhunter-Brown A, Baer G, Campbell P, Choo PL, Morris J, Langhorne P, Cowie J, Sellers CE. Physical rehabilitation approaches for the recovery of function and mobility following stroke. Cochrane Database of Systematic Reviews 2014, Issue 4. Art. No.: CD001920. DOI: 10.1002/14651858.CD001920.pub3.

Copyright ( 2023 The Cochrane Collaboration. Published by John Wiley \& Sons, Ltd.

\section{A B S T R A C T}

\section{Background}

Various approaches to physical rehabilitation may be used after stroke, and considerable controversy and debate surround the effectiveness of relative approaches. Some physiotherapists base their treatments on a single approach; others use a mixture of components from several different approaches.

\section{Objectives}

To determine whether physical rehabilitation approaches are effective in recovery of function and mobility in people with stroke, and to assess if any one physical rehabilitation approach is more effective than any other approach.

For the previous versions of this review, the objective was to explore the effect of 'physiotherapy treatment approaches' based on historical classifications of orthopaedic, neurophysiological or motor learning principles, or on a mixture of these treatment principles. For this update of the review, the objective was to explore the effects of approaches that incorporate individual treatment components, categorised as functional task training, musculoskeletal intervention (active), musculoskeletal intervention (passive), neurophysiological intervention, cardiopulmonary intervention, assistive device or modality.

In addition, we sought to explore the impact of time after stroke, geographical location of the study, dose of the intervention, provider of the intervention and treatment components included within an intervention.

\section{Search methods}

We searched the Cochrane Stroke Group Trials Register (last searched December 2012), the Cochrane Central Register of Controlled Trials (CENTRAL) (The Cochrane Library Issue 12, 2012), MEDLINE (1966 to December 2012), EMBASE (1980 to December 2012), AMED (1985 to December 2012) and CINAHL (1982 to December 2012). We searched reference lists and contacted experts and researchers who have an interest in stroke rehabilitation.

\section{Selection criteria}

Randomised controlled trials (RCTs) of physical rehabilitation approaches aimed at promoting the recovery of function or mobility in adult participants with a clinical diagnosis of stroke. Outcomes included measures of independence in activities of daily living (ADL), motor 
function, balance, gait velocity and length of stay. We included trials comparing physical rehabilitation approaches versus no treatment, usual care or attention control and those comparing different physical rehabilitation approaches.

\section{Data collection and analysis}

Two review authors independently categorised identified trials according to the selection criteria, documented their methodological quality and extracted the data.

\section{Main results}

We included a total of 96 studies (10,401 participants) in this review. More than half of the studies (50/96) were carried out in China. Generally the studies were heterogeneous, and many were poorly reported.

Physical rehabilitation was found to have a beneficial effect, as compared with no treatment, on functional recovery after stroke (27 studies, 3423 participants; standardised mean difference (SMD) 0.78 , 95\% confidence interval (CI) 0.58 to 0.97 , for Independence in ADL scales), and this effect was noted to persist beyond the length of the intervention period (nine studies, 540 participants; SMD $0.58,95 \% \mathrm{Cl} 0.11$ to 1.04). Subgroup analysis revealed a significant difference based on dose of intervention ( $P$ value $<0.0001$, for independence in $A D L$ ), indicating that a dose of 30 to 60 minutes per day delivered five to seven days per week is effective. This evidence principally arises from studies carried out in China. Subgroup analyses also suggest significant benefit associated with a shorter time since stroke (P value 0.003 , for independence in $\mathrm{ADL}$ ).

We found physical rehabilitation to be more effective than usual care or attention control in improving motor function (12 studies, 887 participants; SMD $0.37,95 \% \mathrm{Cl} 0.20$ to 0.55 ), balance (five studies, 246 participants; SMD $0.31,95 \% \mathrm{Cl} 0.05$ to 0.56 ) and gait velocity (14 studies, 1126 participants; SMD $0.46,95 \% \mathrm{Cl} 0.32$ to 0.60 ). Subgroup analysis demonstrated a significant difference based on dose of intervention ( $P$ value 0.02 for motor function), indicating that a dose of 30 to 60 minutes delivered five to seven days a week provides significant benefit. Subgroup analyses also suggest significant benefit associated with a shorter time since stroke ( $P$ value 0.05 , for independence in $A D L)$.

No one physical rehabilitation approach was more (or less) effective than any other approach in improving independence in ADL (eight studies, 491 participants; test for subgroup differences: $P$ value 0.71 ) or motor function (nine studies, 546 participants; test for subgroup differences: $P$ value 0.41 ). These findings are supported by subgroup analyses carried out for comparisons of intervention versus no treatment or usual care, which identified no significant effects of different treatment components or categories of interventions.

\section{Authors' conclusions}

Physical rehabilitation, comprising a selection of components from different approaches, is effective for recovery of function and mobility after stroke. Evidence related to dose of physical therapy is limited by substantial heterogeneity and does not support robust conclusions. No one approach to physical rehabilitation is any more (or less) effective in promoting recovery of function and mobility after stroke. Therefore, evidence indicates that physical rehabilitation should not be limited to compartmentalised, named approaches, but rather should comprise clearly defined, well-described, evidenced-based physical treatments, regardless of historical or philosophical origin.

\section{PLAIN LANGUAGE SUMMARY}

\section{Physical rehabilitation approaches for recovery of function, balance and walking after stroke}

\section{Question}

We wanted to know whether physical rehabilitation approaches are effective in recovery of function and mobility in people with stroke, and if any one physical rehabilitation approach is more effective than any other approach.

\section{Background}

Stroke can cause paralysis of some parts of the body and other difficulties with various physical functions. Physical rehabilitation is an important part of rehabilitation for people who have had a stroke. Over the years, various approaches to physical rehabilitation have been developed, according to different ideas about how people recover after a stroke. Often physiotherapists will follow one particular approach, to the exclusion of others, but this practice is generally based on personal preference rather than scientific rationale. Considerable debate continues among physiotherapists about the relative benefits of different approaches; therefore it is important to bring together the research evidence and highlight what best practice ought to be in selecting these different approaches.

\section{Study characteristics}

We identified 96 studies, up to December 2012, for inclusion in the review. These studies, involving 10,401 stroke survivors, investigated physical rehabilitation approaches aimed at promoting recovery of function or mobility in adult participants with a clinical diagnosis of stroke compared with no treatment, usual care or attention control or in comparisons of different physical rehabilitation approaches. The average number of participants in each study was 105: most studies (93\%) included fewer than 200 participants, one study had more than 1000 participants, six had between 250 and 1000 participants and 10 had 20 or fewer participants. Outcomes included measures of 
independence in activities of daily living (ADL), motor function (functional movement), balance, walking speed and length of stay. More than half of the studies (50/96) were carried out in China. These studies showed many differences in relation to the type of stroke and how severe it was, as well as differences in treatment, which varied according to both treatment type and duration.

\section{Key results}

This review brings together evidence confirming that physical rehabilitation (often delivered by a physiotherapist, physical therapist or rehabilitation therapist) can improve function, balance and walking after stroke. It appears to be most beneficial when the therapist selects a mixture of different treatments for an individual patient from a wide range of available treatments.

We were able to combine the results from 27 studies (3243 stroke survivors) that compared physical rehabilitation versus no treatment. Twenty-five of these 27 studies were carried out in China. Results showed that physical rehabilitation improves functional recovery, and that this improvement may last long-term. When we looked at studies that compared additional physical rehabilitation versus usual care or a control intervention, we found evidence to show that the additional physical treatment improved motor function (12 studies, 887 stroke survivors), standing balance (five studies, 246 stroke survivors) and walking speed (14 studies, 1126 stroke survivors). Very limited evidence suggests that, for comparisons of physical rehabilitation versus no treatment and versus usual care, treatment that appeared to be effective was given between 30 and 60 minutes per day, five to seven days per week, but further research is needed to confirm this. We also found evidence of greater benefit associated with a shorter time since stroke, but again further research is needed to confirm this.

We found evidence showing that no one physical rehabilitation approach was more effective than any other approach. This finding means that physiotherapists should choose each individual patient's treatment according to the evidence available for that specific treatment, and should not limit their practice to a single 'named' approach.

\section{Quality of the evidence}

It was difficult for us to judge the quality of evidence because we found poor, incomplete or brief reporting of information. We determined that less than $50 \%$ of the studies were of good quality, and for most studies, the quality of the evidence was unclear from the information provided. 


\section{SUMMARY OF FINDINGS}

\section{Summary of findings 1 . Summary of findings: intervention versus no treatment}

\section{Physiotherapy intervention compared with no treatment for recovery after stroke}

Patient or population: adults with stroke

Intervention: physiotherapy interventions

Comparison: no treatment

\begin{tabular}{|c|c|c|c|c|}
\hline Outcomes & $\begin{array}{l}\text { Standardised } \\
\text { mean difference } \\
(95 \% \mathrm{CI})\end{array}$ & $\begin{array}{l}\text { No. of participants } \\
\text { (studies) }\end{array}$ & $\begin{array}{l}\text { Quality of the } \\
\text { evidence } \\
\text { (GRADE) }\end{array}$ & Comments \\
\hline $\begin{array}{l}\text { Independence in ADL } \\
\text { scales } \\
\text { Immediate outcome }\end{array}$ & $\begin{array}{l}0.78(0.58 \text { to } \\
0.97)\end{array}$ & $\begin{array}{l}27 \text { studies } \\
3423 \text { participants }\end{array}$ & $\begin{array}{l}\oplus \oplus \oplus \ominus \\
\text { moderate }\end{array}$ & $\begin{array}{l}\text { Substantial heterogeneity in results. } \\
\text { Most studies are at high or unclear } \\
\text { risk of bias. Most studies are carried } \\
\text { out in China; significant subgroup } \\
\text { effect relating to geographical loca- } \\
\text { tion of the study }\end{array}$ \\
\hline $\begin{array}{l}\text { Independence in ADL } \\
\text { scales } \\
\text { Persisting outcome }\end{array}$ & $\begin{array}{l}0.58(0.11 \text { to } \\
1.04)\end{array}$ & $\begin{array}{l}9 \text { studies } \\
540 \text { participants }\end{array}$ & $\begin{array}{l}\oplus \oplus \oplus \ominus \\
\text { moderate }\end{array}$ & \\
\hline $\begin{array}{l}\text { Motor function scales } \\
\text { Immediate outcome }\end{array}$ & $\begin{array}{l}0.81(0.58 \text { to } \\
1.04)\end{array}$ & $\begin{array}{l}25 \text { studies } \\
4558 \text { participants }\end{array}$ & $\begin{array}{l}\oplus \oplus \oplus \ominus \\
\text { moderate }\end{array}$ & $\begin{array}{l}\text { Substantial heterogeneity in results. } \\
\text { Most studies are at high or unclear } \\
\text { risk of bias. Most studies are carried } \\
\text { out in China; significant subgroup } \\
\text { effect relating to geographical loca- } \\
\text { tion of the study }\end{array}$ \\
\hline $\begin{array}{l}\text { Motor function scales } \\
\text { Persisting outcome }\end{array}$ & $\begin{array}{l}1.06(0.37 \text { to } \\
1.75)\end{array}$ & $\begin{array}{l}8 \text { studies } \\
1829 \text { participants }\end{array}$ & $\begin{array}{l}\oplus \oplus \oplus \ominus \\
\text { moderate }\end{array}$ & \\
\hline $\begin{array}{l}\text { Balance (Berg Balance } \\
\text { Scale) } \\
\text { Immediate outcome }\end{array}$ & $\begin{array}{l}-0.04(-0.71 \text { to } \\
0.64)\end{array}$ & $\begin{array}{l}1 \text { study } \\
34 \text { participants }\end{array}$ & $\begin{array}{l}\oplus \odot \odot \ominus \\
\text { very low }\end{array}$ & \\
\hline $\begin{array}{l}\text { Balance (Berg Balance } \\
\text { Scale) } \\
\text { Persisting outcome }\end{array}$ & $\begin{array}{l}-0.03(-0.70 \text { to } \\
0.65)\end{array}$ & $\begin{array}{l}1 \text { study } \\
34 \text { participants }\end{array}$ & $\begin{array}{l}\oplus \odot \odot \ominus \\
\text { very low }\end{array}$ & \\
\hline $\begin{array}{l}\text { Gait velocity } \\
\text { Immediate outcome }\end{array}$ & $\begin{array}{l}0.05(-0.18 \text { to } \\
0.28)\end{array}$ & $\begin{array}{l}3 \text { studies } \\
292 \text { participants }\end{array}$ & $\begin{array}{l}\oplus \oplus \odot \odot \\
\text { low }\end{array}$ & \\
\hline $\begin{array}{l}\text { Gait velocity } \\
\text { Persisting outcome }\end{array}$ & $\begin{array}{l}-0.06(-0.29 \text { to } \\
0.18)\end{array}$ & $\begin{array}{l}3 \text { studies } \\
271 \text { participants }\end{array}$ & $\begin{array}{l}\oplus \oplus \ominus \ominus \\
\text { low }\end{array}$ & \\
\hline Length of stay & $\begin{array}{l}\text { MD }-2.85(-10.47 \\
\text { to } 4.76)\end{array}$ & $\begin{array}{l}3 \text { studies } \\
318 \text { participants }\end{array}$ & $\begin{array}{l}\oplus \oplus \ominus \ominus \\
\text { low }\end{array}$ & \\
\hline
\end{tabular}


GRADE Working Group grades of evidence.

High quality: Further research is very unlikely to change our confidence in the estimate of effect.

Moderate quality: Further research is likely to have an important impact on our confidence in the estimate of effect and may change the estimate.

Low quality: Further research is very likely to have an important impact on our confidence in the estimate of effect and is likely to

change the estimate.

Very low quality: We are very uncertain about the estimate.

\section{Summary of findings 2 . Summary of findings: intervention versus usual care or attention control}

Physiotherapy intervention compared with usual care or attention control for recovery after stroke

Patient or population: adults with stroke

Intervention: physiotherapy intervention

Comparison: usual care or attention control

\begin{tabular}{|c|c|c|c|c|}
\hline Outcomes & $\begin{array}{l}\text { Standardised } \\
\text { mean difference } \\
(95 \% \mathrm{CI})\end{array}$ & $\begin{array}{l}\text { No. of participants } \\
\text { (studies) }\end{array}$ & $\begin{array}{l}\text { Quality of the } \\
\text { evidence } \\
\text { (GRADE) }\end{array}$ & Comments \\
\hline $\begin{array}{l}\text { Independence in ADL scales } \\
\text { Immediate outcome }\end{array}$ & $\begin{array}{l}0.04(-0.27 \text { to } \\
0.35)\end{array}$ & $\begin{array}{l}6 \text { studies } \\
260 \text { participants }\end{array}$ & $\begin{array}{l}\oplus \oplus \oplus \ominus \\
\text { moderate }\end{array}$ & \\
\hline $\begin{array}{l}\text { Independence in ADL scales } \\
\text { Persisting outcome }\end{array}$ & & & & No data \\
\hline $\begin{array}{l}\text { Motor function scales } \\
\text { Immediate outcome }\end{array}$ & $\begin{array}{l}0.42(0.24 \text { to } \\
0.61)\end{array}$ & $\begin{array}{l}13 \text { studies } \\
967 \text { participants }\end{array}$ & $\begin{array}{l}\oplus \oplus \oplus \ominus \\
\text { moderate }\end{array}$ & $\begin{array}{l}\text { Removing all studies that were } \\
\text { judged as unsure or high risk of } \\
\text { bias for random sequence gener- } \\
\text { ation or allocation concealment } \\
\text { left } 7 \text { studies ( } 377 \text { participants) } \\
\text { demonstrating no significant ef- } \\
\text { fect (SMD } 0.17,95 \% \mathrm{Cl}-0.04 \text { to } \\
0.38 \text { ) }\end{array}$ \\
\hline $\begin{array}{l}\text { Motor function scales } \\
\text { Persisting outcome }\end{array}$ & $\begin{array}{l}-0.10(-0.42 \text { to } \\
0.23)\end{array}$ & $\begin{array}{l}3 \text { studies } \\
160 \text { participants }\end{array}$ & $\begin{array}{l}\oplus \oplus \Theta \ominus \\
\text { low }\end{array}$ & \\
\hline $\begin{array}{l}\text { Balance (Berg Balance Scale) } \\
\text { Immediate outcome }\end{array}$ & $\begin{array}{l}0.31(0.05 \text { to } \\
0.56)\end{array}$ & $\begin{array}{l}5 \text { studies } \\
246 \text { participants }\end{array}$ & $\begin{array}{l}\oplus \oplus \oplus \ominus \\
\text { moderate }\end{array}$ & \\
\hline $\begin{array}{l}\text { Balance (Berg Balance Scale) } \\
\text { Persisting outcome }\end{array}$ & & & & No data \\
\hline $\begin{array}{l}\text { Gait velocity } \\
\text { Immediate outcome }\end{array}$ & $\begin{array}{l}0.46(0.32 \text { to } \\
0.60)\end{array}$ & $\begin{array}{l}14 \text { studies } \\
1126 \text { participants }\end{array}$ & $\begin{array}{l}\oplus \oplus \oplus \oplus \\
\text { high }\end{array}$ & $\begin{array}{l}\text { Sensitivity analysis: studies with } \\
\text { attention control: } \\
7 \text { studies } \\
251 \text { participants }\end{array}$ \\
\hline
\end{tabular}




\begin{tabular}{|c|c|c|c|c|}
\hline & & & & SMD $0.41(0.51$ to 0.67$)$ \\
\hline $\begin{array}{l}\text { Gait velocity } \\
\text { Persisting outcome }\end{array}$ & $\begin{array}{l}0.38 \text { (0.10 to } \\
0.66)\end{array}$ & $\begin{array}{l}5 \text { studies } \\
214 \text { participants }\end{array}$ & $\begin{array}{l}\oplus \oplus \oplus \ominus \\
\text { moderate }\end{array}$ & \\
\hline Length of stay & $\begin{array}{l}\text { MD }-10.36(-48.09 \\
\text { to } 27.36)\end{array}$ & $\begin{array}{l}2 \text { studies } \\
105 \text { participants }\end{array}$ & $\begin{array}{l}\oplus \oplus \Theta \Theta \\
\text { low }\end{array}$ & \\
\hline
\end{tabular}

GRADE Working Group grades of evidence.

High quality: Further research is very unlikely to change our confidence in the estimate of effect.

Moderate quality: Further research is likely to have an important impact on our confidence in the estimate of effect and may change the estimate.

Low quality: Further research is very likely to have an important impact on our confidence in the estimate of effect and is likely to change the estimate.

Very low quality: We are very uncertain about the estimate.

\section{Summary of findings 3. Summary of findings: one active intervention versus another active intervention}

\section{One active intervention compared with another active intervention for recovery after stroke}

Patient or population: adults with stroke

Intervention: A physiotherapy intervention containing functional task training, neurophysiological or musculoskeletal components

Comparison: A physiotherapy intervention that does not contain the same category of treatment components

\begin{tabular}{|c|c|c|c|c|}
\hline Outcomes & $\begin{array}{l}\text { Standardised } \\
\text { mean difference } \\
(95 \% \mathrm{CI})\end{array}$ & $\begin{array}{l}\text { No. of partici- } \\
\text { pants } \\
\text { (studies) }\end{array}$ & $\begin{array}{l}\text { Quality of the } \\
\text { evidence } \\
\text { (GRADE) }\end{array}$ & Comments \\
\hline $\begin{array}{l}\text { 3.1.1 Includes functional training } \\
\text { versus does not include functional } \\
\text { training } \\
\text { Independence in ADL scales }\end{array}$ & $\begin{array}{l}-0.03(-0.37 \text { to } \\
0.32)\end{array}$ & $\begin{array}{l}4 \text { studies (186 } \\
\text { participants) }\end{array}$ & $\begin{array}{l}\oplus \oplus \ominus \ominus \\
\text { low }\end{array}$ & $\begin{array}{l}\text { Quality of evidence downgrad- } \\
\text { ed from "moderate" to "low" } \\
\text { because of the relatively low } \\
\text { number of studies/partici- } \\
\text { pants }\end{array}$ \\
\hline \multicolumn{5}{|l|}{ Immediate outcomes } \\
\hline $\begin{array}{l}\text { 3.1.2 Includes neurophysiological } \\
\text { versus does not include neurophys- } \\
\text { iological }\end{array}$ & $\begin{array}{l}-0.02(-0.26 \text { to } \\
0.22)\end{array}$ & $\begin{array}{l}7 \text { studies (451 } \\
\text { participants) }\end{array}$ & $\begin{array}{l}\oplus \oplus \oplus \odot \\
\text { moderate }\end{array}$ & $\begin{array}{l}\text { Evidence primarily relates to } \\
\text { interventions described as Bo- } \\
\text { bath }\end{array}$ \\
\hline \multicolumn{5}{|l|}{ Independence in ADL scales } \\
\hline \multicolumn{5}{|l|}{ Immediate outcomes } \\
\hline $\begin{array}{l}\text { 3.1.3 Includes musculoskeletal ver- } \\
\text { sus does not include musculoskele- } \\
\text { tal } \\
\text { Independence in ADL scales }\end{array}$ & $\begin{array}{l}-0.12(-0.58 \text { to } \\
0.34)\end{array}$ & $\begin{array}{l}3 \text { studies (103 } \\
\text { participants) }\end{array}$ & $\begin{array}{l}\oplus \oplus \odot \odot \\
\text { low }\end{array}$ & $\begin{array}{l}\text { Quality of evidence downgrad- } \\
\text { ed from "moderate" to "low" } \\
\text { because of the relatively low } \\
\text { number of studies/partici- } \\
\text { pants }\end{array}$ \\
\hline \multicolumn{5}{|l|}{ Immediate outcomes } \\
\hline $\begin{array}{l}\text { 3.2.1 Includes functional training } \\
\text { versus does not include functional } \\
\text { training }\end{array}$ & $\begin{array}{l}-0.16(-0.59 \text { to } \\
0.28)\end{array}$ & $\begin{array}{l}4 \text { studies (188 } \\
\text { participants) }\end{array}$ & $\begin{array}{l}\oplus \oplus \ominus \ominus \\
\text { low }\end{array}$ & $\begin{array}{l}\text { Quality of evidence downgrad- } \\
\text { ed from "moderate" to "low" } \\
\text { because of the relatively low }\end{array}$ \\
\hline
\end{tabular}




\section{Motor function scales}

Immediate outcomes number of studies/participants

\begin{tabular}{|c|c|c|c|c|}
\hline $\begin{array}{l}3.2 .2 \text { Includes neurophysiological } \\
\text { versus does not include neurophys- } \\
\text { iological }\end{array}$ & $\begin{array}{l}0.17(-0.05 \text { to } \\
0.39)\end{array}$ & $\begin{array}{l}8 \text { studies (506 } \\
\text { participants) }\end{array}$ & $\begin{array}{l}\oplus \oplus \oplus \odot \\
\text { moderate }\end{array}$ & $\begin{array}{l}\text { Evidence primarily relates to } \\
\text { interventions described as Bo- } \\
\text { bath }\end{array}$ \\
\hline \multicolumn{5}{|l|}{ Motor function scales } \\
\hline \multicolumn{5}{|l|}{ Immediate outcomes } \\
\hline $\begin{array}{l}\text { 3.2.3 Includes musculoskeletal ver- } \\
\text { sus does not include musculoskele- } \\
\text { tal } \\
\text { Motor function scales }\end{array}$ & $\begin{array}{l}-0.08(-0.53 \text { to } \\
0.36)\end{array}$ & $\begin{array}{l}4 \text { studies ( } 81 \text { par- } \\
\text { ticipants) }\end{array}$ & $\begin{array}{l}\oplus \oplus \ominus \ominus \\
\text { low }\end{array}$ & $\begin{array}{l}\text { Quality of evidence downgrad- } \\
\text { ed from "moderate" to "low" } \\
\text { because of the relatively low } \\
\text { number of studies/partici- } \\
\text { pants }\end{array}$ \\
\hline Immediate outcomes & & & & \\
\hline
\end{tabular}

GRADE Working Group grades of evidence.

High quality: Further research is very unlikely to change our confidence in the estimate of effect.

Moderate quality: Further research is likely to have an important impact on our confidence in the estimate of effect and may change the estimate.

Low quality: Further research is very likely to have an important impact on our confidence in the estimate of effect and is likely to change the estimate.

Very low quality: We are very uncertain about the estimate. 


\section{B A C K G R O U N D}

\section{Description of the condition}

Stroke is a leading cause of death and disability in many Western nations. In Australia, the UK and the USA, it is within the top 10 causes of long-term physical disability (Fisher 2013; Mathers 2006; Ovbiagele 2011). The most common and widely recognised impairment caused by stroke is motor impairment, which can be regarded as loss or limitation of function in muscle control or movement or limitation in mobility (Wade 1992a). Motor impairment after stroke typically affects the control of movement of the face, arm and leg on one side of the body (Warlow 2008) and is seen in about $80 \%$ of patients. Almost two-thirds of stroke survivors have initial mobility deficits (Jorgensen 1995; Shaughnessy 2005), and six months after a stroke, more than $30 \%$ of survivors still cannot walk independently (Jorgensen 1995; Mayo 2002; Patel 2000). Therefore, much of the focus of stroke rehabilitation, in particular, the work of physiotherapists (also known as physical therapists or rehabilitation therapists), is focused on recovery of physical independence and functional ability during activities of daily living; commonly the ultimate goal of therapy is to improve the function of walking and recovery of balance and movement (Langhorne 2009).

\section{Description of the intervention}

Various approaches to physical rehabilitation can be used after stroke, and considerable controversy and debate about the relative effectiveness of these approaches are ongoing (Carlisle 2010; Kollen 2009). Descriptions of these approaches are best considered within a historical context.

Before the 1940s, physical rehabilitation approaches primarily consisted of corrective exercises based on orthopaedic principles related to contraction and relaxation of muscles, with emphasis placed on regaining function by compensating with the unaffected limbs (Ashburn 1995; Partridge 1996). In the 1950s and 1960s, techniques based on available neurophysiological knowledge were developed to enhance recovery of the paretic side. These new approaches included the methods of Bobath (Bobath 1990; Davies 1985), Brunnström (Brunnström 1970) and Rood (Goff 1969), as well as the proprioceptive neuromuscular facilitation approach (Knott 1968; Voss 1985). In the 1980s, the potential importance of neuropsychology and motor learning was highlighted (Anderson 1986; Turnbull 1982) and the motor learning, or relearning, approach was proposed (Carr 1982). This suggested that active practice of context-specific motor tasks with appropriate feedback would promote learning and motor recovery (Carr 1980; Carr 1982; Carr 1987a; Carr 1987b; Carr 1989; Carr 1990; Carr 1998). The practical application of these approaches appeared to result in substantial differences in patient treatment. Approaches based on neurophysiological principles seemingly involved the physiotherapist moving the patient through patterns of movement, with the therapist acting as problem solver and decision maker and the patient being a relatively passive recipient (Lennon 1996). In direct contrast, motor learning approaches stressed the importance of active involvement by the patient (Carr 1982), and orthopaedic approaches emphasised muscle strengthening techniques and compensation with the non-paretic side.

Since the 1980s, the need to base neurological physiotherapy on scientific research in relevant areas such as medical science, neuroscience, exercise physiology and biomechanics, and to test the outcomes of physical interventions to develop evidencebased physiotherapy has been increasingly emphasised. However, anecdotal evidence and the results of questionnaire-based studies suggest that, traditionally, many physiotherapists continued to base their clinical practice around a 'named' treatment approach. From the 1990s, the Bobath approach, based on neurophysiological principles, came to be recognised as the most widely used method in Sweden (Nilsson 1992), Australia (Carr 1994a) and the UK (Davidson 2000; Lennon 2001; Sackley 1996). As a consequence, since this time, physiotherapists have often sought evidence related to these 'named' approaches to the physical rehabilitation of stroke patients.

In some parts of the world, clear preferences for one 'named' approach have prevailed; however in others, physical rehabilitation approaches for stroke have developed with greater eclecticism, resulting in geographical preferences for mixing particular approaches, or components from different approaches, as well as preferences for single 'named' approaches. For example, in China, where stroke rehabilitation is not yet considered standard care (Zhang 2013), standard 'approaches' to rehabilitation have been proposed, including 'standardised tertiary rehabilitation' ( $\mathrm{Hu}$ 2007 isch; Hu 2007a; Jiang 2006; Research Group 2007; Zhang 2004) and 'standardised three-phase rehabilitation' (Bai 2008; Fan 2006; Zhu 2004b). These approaches arguably appear to draw on the full range of treatment interventions available from all orthopaedic, neurophysiological and motor learning approaches described in Western literature, while incorporating traditional Chinese therapies such as acupuncture (Zhang 2013; Zhuang 2012).

More recently, calls asking physiotherapists to cease using named approaches and to stop selecting treatments based on historical perspectives have increased. Physiotherapists have been urged to refrain from using compartmentalised, named approaches and to select clearly defined and described techniques and task-specific treatments, regardless of their historical or philosophical origin (Kollen 2009; Langhammer 2012; Mayston 2000; Pomeroy 2005). Although a move away from named approaches in preference of more evidence-based approaches has been deliberately implemented in some countries, such as the Netherlands (Kollen 2009; van Peppen 2004), heated debate continues about the evidence for doing this (Carlisle 2010), and some physiotherapists around the world continue to exhibit preferences for particular named approaches (Khan 2012; Tyson 2009a; Tyson 2009b).

\section{Why it is important to do this review}

Continued controversy and debate about the relative effectiveness of physical rehabilitation approaches and evidence of clear preferences for particular named approaches in some parts of the world, despite increasing calls for this to change, justify the importance of this review.

\section{Why it is important to address limitations within previous versions of this review}

The original versions of this review classified approaches to physiotherapy on the basis of historical principles described in the literature; however we classified interventions as neurophysiological, motor learning, orthopaedic or mixed, according to the descriptor or name of the intervention provided by trialists (Pollock 2007). Table 1 displays the criteria that we used in 
classifying neurophysiological and motor learning approaches (NB: We are not using these criteria in this updated review.). However, the 2007 version of the review (Pollock 2007) identified several limitations associated with this method of classification.

1. This classification was based on Western approaches and descriptions of physiotherapy and did not incorporate physical therapy delivered across the whole world. In Pollock 2007, we identified a large number (26) of non-English language (23 Chinese) trials (and classified them as 'studies awaiting assessment'). We stated: "The information currently available from the majority of the Chinese trials awaiting assessment suggests that it is unlikely that the interventions studied in these trials will fit into the western categorisations and classifications of physiotherapy treatment approaches developed for this review. Prior to the next update of this review, the authors intend to seek advice and write additional inclusion and exclusion criteria to deal with the non-western approaches to physiotherapy for stroke".

2. The 'mixed' approach category within the review could potentially incorporate a large number of heterogeneous interventions that may not be meaningful to combine. Pollock 2007 stated: "A limitation of combining all mixed approaches is that this category potentially amalgamates any number of possible combinations of other approaches and techniques".

3. We found difficulties in determining the classifications of motor learning and mixed approaches for some studies. Pollock 2007 stated: "difficulty was experienced in distinguishing between a mixed approach (not a mixture of two different approaches, such as Stern 1970 mixing orthopaedic and neurophysiological approaches, but an unclassified mix [where the interventions were not easily classified into a 'named' approach]) and a motor learning approach. The mixed, intensive and focused approach investigated by Richards 1993 and the problem-solving approach investigated by Green 2002 and Wade 1992 had stated philosophies very similar to those of motor learning approaches. However, the described techniques and the supporting references led the reviewers to classify these interventions as mixed. This highlights a key problem with the classification of the motor learning approach. Although a motor relearning programme has been described by Carr and Shepherd (Carr 1982; Carr 1987b), these authors primarily advocate an approach based on related research in relevant areas such as medical science, neuroscience, exercise physiology and biomechanics. Such an approach is arguably one of research-based practice, rather than being based on one specific philosophy".

It was therefore essential to plan solutions and strategies to address these limitations before this update of the review was conducted.

\section{Consensus methods to inform update of this review}

To address the identified limitations within previous versions of this review, before this update we convened a stakeholder group comprising 13 purposively selected people: three stroke survivors, one carer and nine physiotherapists. Members of this group are listed and acknowledged in the Acknowledgements section. We used formal group consensus methods to reach consensus decisions around review aims and methods, while focusing on clinical relevance, as such methods are recognised to be advantageous when subjective judgements need to be organised (Nair 2011). The consensus methods were based on nominal group techniques, as this method enables the pooling of decisions and judgements from a group of informed experts, leading to votes on a range of options until ultimately group consensus is reached (Sinha 2008; Stapleton 2010). The review authors attended the stakeholder group meetings and contributed to discussions; however we did not participate in the voting process. This approach was taken to ensure that the results of the voting reflected the views of stroke survivors, carers and physiotherapists and were not biased by the opinions of the review authors. The process of stakeholder group involvement is outlined in Figure 1. 
Figure 1. The figure summarises the process undertaken by the stakeholder group, which met on three occasions (green circles). The nominal group technique was used to achieve all decisions. The blue circles represent the 'preparation phase,' which included drafting role descriptors for the SG; obtaining local University ethics and recruiting the SG and data extraction exercise of the sample of Chinese studies $(n=10)$ that had previously been identified in the 2007 version of this review. Purple circles represent the months dedicated to undertaking the systematic review.

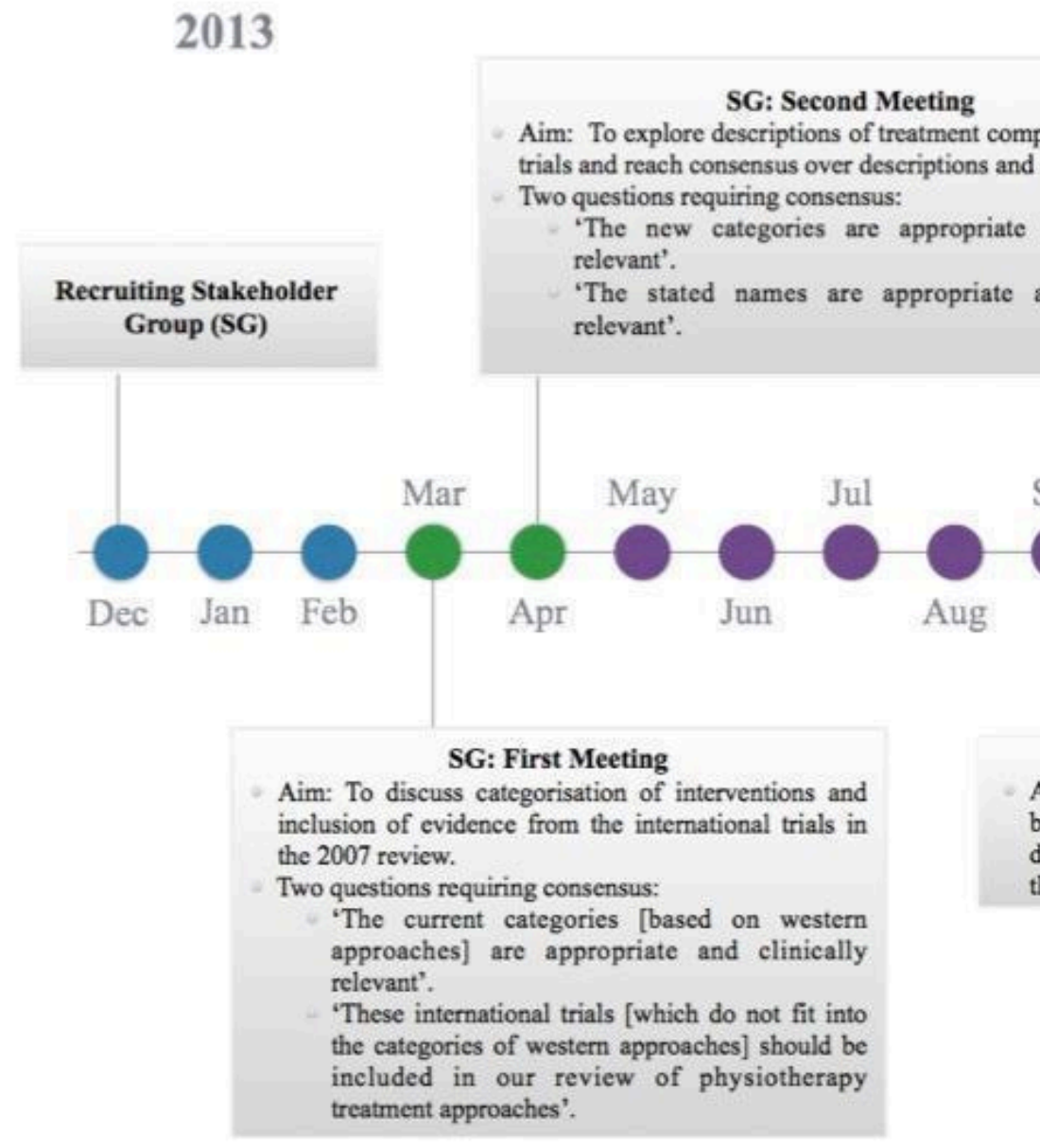

SG: Final meeting

Aim: To discuss the review, and decide how best to disseminate the findings. They discussed and agreed "clinical implications" of the results.
The stakeholder group specifically discussed the categorisation of interventions and inclusion of evidence from the international trials listed as awaiting assessment in Pollock 2007, which led to voting on two key statements.

1. "The current categories (based on western approaches) are appropriate and clinically relevant".

2. "These international trials (which do not fit into the categories of western approaches) should be included in our review of physiotherapy treatment approaches".

We determined the proportion agreeing with each statement. We audio-recorded and transcribed verbatim the consensus decision meetings. We coded and analysed qualitative data using NVivo software: $84 \%$ of group members disagreed with statement 1 , and $100 \%$ agreed with statement 2 . Two key themes and several subthemes emerged from the transcribed data. Key themes were that (1) current categories of rehabilitation approaches should be amended to enable inclusion of all international evidence and (2) current physiotherapy taxonomies have limitations and concerns that have been raised surrounding their relevance to clinical practice in the UK.

Discussion amongst stakeholder group members led to the generation of, and agreement on, a proposal that the optimal way of classifying the 'approaches' for this review consisted of using systematic categorisation of the treatment components described in relation to interventions. This discussion followed a presentation of treatment components described in a sample of 10 Chinese trials that had been listed as 'awaiting assessment' in Pollock 2007 (Chen 2004; Chu 2003; Gong 2003; Huang 2003; Pan 2004; Pang 2003; Xie 2003; Xu 2003a; Zhang 1998; Zhu 2001). On the suggestion of the stakeholder group, to further explore the range of treatment components and reach agreement on definitions of these components, we systematically extracted descriptions of physical rehabilitation approaches from the 20 trials included in Pollock 2007 (Dean 1997; Dean 2000; Duncan 1998; Duncan 2003; Gelber 1995; Green 2002; Hesse 1998; Howe 2005; Langhammer 2000; Lincoln 2003; McClellan 2004; Mudie 2002; Ozdemir 2001; Pollock 1998; Richards 1993; Salbach 2004; Stern 1970; Wade 
1992; Wang 2005; Wellmon 1997). The stakeholder group then explored the descriptions of treatment components from these 30 trials. The aim was to include a variety of types and descriptions of physical rehabilitation approaches to allow examination of whether a range of treatment components could be identified and consensus over descriptions and categorisations; this was designed as an exploration, rather than as a comprehensive aggregation. The stakeholder group debated the treatment components described within these trials of physical rehabilitation approaches, reached consensus on key components, agreed on descriptions of these components and determined categorisation for synthesis of evidence within this update of the Cochrane review.

The stakeholder group identified and defined 27 treatment components based on the interventions described within the 30 explored trials. These were grouped into seven categories: functional task training, musculoskeletal intervention (active), musculoskeletal intervention (passive), neurophysiological intervention, cardiopulmonary intervention, assistive device and modality. These categories were informed by the taxonomy described by DeJong 2004. One hundred per cent of the stakeholder group agreed with these treatment component descriptions and categories. The agreed upon categories, treatment components and definitions are listed in Table 2.

\section{O B J E C T IVES}

To determine whether physical rehabilitation approaches are effective in recovery of function and mobility in people with stroke, and to assess if any one physical rehabilitation approach is more effective than any other approach.

For the Pollock 2007 version of the review and earlier versions, the objective was to explore the effect of 'physiotherapy treatment approaches' based on historical classifications of orthopaedic, neurophysiological or motor learning principles, or on a mixture of these treatment principles. For this update of the review, the objective was to explore the effects of approaches that incorporate treatment components from each of the categories listed in Table 2, Individual treatment components were categorised as functional task training, musculoskeletal intervention (active), musculoskeletal intervention (passive), neurophysiological intervention, cardiopulmonary intervention, assistive device or modality.

In addition, we sought to explore the impact of time after stroke, geographical location of the study, dose of the intervention, provider of the intervention and treatment components included within an intervention.

\section{METHODS}

\section{Criteria for considering studies for this review}

\section{Types of studies}

We included controlled trials if the participants were randomly assigned to one of two or more treatment groups. Random assignment gives each participant entering the trial the same, predetermined, chance of receiving each of the possible treatments (e.g. by using sequentially numbered opaque sealed envelopes or computer-generated random numbers). We included trials with or without blinding of participants, physiotherapists and assessors. We excluded trials with quasi-random assignment, thereby excluding a number of trials that had been included in previous versions of this review (Hesse 1998; Ozdemir 2001; Stern 1970).

\section{Types of participants}

We included trials enrolling adult participants (over 18 years of age) with a clinical diagnosis of stroke (World Health Organization definition; Hatano 1976), which could be ischaemic or haemorrhagic in origin (confirmation of the clinical diagnosis by imaging was not compulsory).

\section{Types of interventions}

We included physical rehabilitation approaches that were aimed at promoting recovery of postural control (balance during maintenance of a posture, restoration of a posture or movement between postures) and lower limb function (including gait), as well as interventions that had a more generalised stated aim, such as improving functional ability. We excluded rehabilitation approaches that were primarily aimed at promoting recovery of upper limb movement or upper limb function.

\section{Types of outcome measures}

\section{Primary outcomes}

We defined primary outcomes as measures of disability (activity limitations; WHO 2002) and prestated relevant measures as follows.

1. Independence in activities of daily living $(A D L)^{*}$ scales. These include Barthel Activities of Daily Living Index (Mahoney 1965), Functional Independence Measure (FIM) (Keith 1987), Modified Rankin Scale (van Swieten 1988), Katz Index of Activities of Daily Living (Katz 1970) and Rehabilitation Activities Profile (van Bennekom 1995).

2. Motor function* scales. These include Motor Assessment Scale (MAS) (Carr 1985), Fugl-Meyer Assessment (lower limb section) (Fugl-Meyer 1975), Rivermead Mobility Index (Forlander 1999) and Rivermead Motor Assessment (Lincoln 1979).

\section{Secondary outcomes}

1. Balance (Berg Balance Scale) (Berg 1989; Berg 1992).

2. Gait velocity.

3. Length of stay.

We were interested in outcomes that were assessed both immediately after the end of an intervention period ('immediate outcome') and at a follow-up period ('persisting outcomes').

*See Differences between protocol and review.

\section{Search methods for identification of studies}

See the 'Specialized register' section in the Cochrane Stroke Group module. We searched for trials in all languages and arranged translation of relevant papers published in languages other than English.

\section{Electronic searches}

We searched the Cochrane Stroke Group Trials Register, which was last searched in December 2012, the Cochrane Central Register of Controlled Trials (CENTRAL) (The Cochrane Library Issue 12, 2012), MEDLINE (Ovid) (1966 to December 2012) (Appendix 1), EMBASE 
(Ovid) (1980 to December 2012) (Appendix 2), AMED (Ovid) (1985 to December 2012) (Appendix 3) and CINAHL (EBSCO) (1982 to December 2012) (Appendix 4).

With the help of the Cochrane Stroke Group Trials Search Coordinator, we developed comprehensive search strategies for MEDLINE (adapted for CENTRAL), EMBASE, AMED and CINAHL using controlled vocabulary and free text terms. We updated the search strategies for this review to incorporate new vocabulary terms.

\section{Searching other resources}

We handsearched the reference lists of all trials found using the above search methods.

For the original version of this review, we contacted relevant experts from the Physiotherapy Researchers Register, held by the Chartered Society of Physiotherapy, and asked whether they knew of any additional, unpublished or ongoing trials of rehabilitation approaches for stroke. We also placed a request on the PHYSIO email discussion list asking the list members (who originate from approximately 35 countries) if they knew of any unpublished or ongoing trials of rehabilitation approaches for stroke. We identified no relevant additional, unpublished or ongoing trials through contact with experts from the Physiotherapy Researchers Register and received no relevant responses from the PHYSIO email discussion list.

For future updates of this review, we plan to expand search resources to include the REHABDATA Database (www.naric.com/? $\mathrm{q}=\mathrm{en} / \mathrm{REHABDATA}$ ), Wangfangdata, a database of Chinese studies (www.wanfangdata.com/) and the major ongoing trials and research registers.

\section{Data collection and analysis}

\section{Selection of studies}

One review author (AP or PC or PLC) read the titles of the identified references and eliminated obviously irrelevant studies. We obtained the abstracts for the remaining studies and then, based on the inclusion criteria (types of studies, types of participants, aims of interventions, outcome measures), two review authors (AP, PC, PLC or GB) independently ranked these as relevant, irrelevant or unsure. We discussed abstracts written in Chinese, with one review author (PLC) translating relevant sections and verbally providing information to other review authors in English (AP, PC). We excluded studies ranked as irrelevant by all review authors and obtained the full text of all remaining studies.

We considered the full texts of studies ranked as relevant or unsure and resolved disagreements through discussion between review authors. We included all trials that were assessed to investigate different physical rehabilitation approaches and excluded all trials of single specific treatments. Single specific treatments included biofeedback, functional electrical stimulation, treadmill walking, acupuncture, ankle-foot orthoses, continuous passive movement and transcutaneous electrical nerve stimulation. Some of these single specific treatments have been the subject of other Cochrane reviews (e.g. Moseley 2005; Pomeroy 2006).

\section{Data extraction and management}

Two review authors independently performed the data extraction, and we contacted study authors to request missing data when possible (AP, GB, PC, PLC). The data extracted included the following (when possible): trial setting (e.g. hospital, community); details of participants (e.g. age, gender, side of hemiplegia, stroke classification, co-morbid conditions, premorbid disability); inclusion and exclusion criteria; and all assessed outcomes. The review authors resolved disagreements by discussion and contacted study authors for clarification when necessary. For papers published in Chinese, one review author (PLC) performed data extraction and translated relevant sections of text, which a second review author (AP, PC) checked.

Two review authors (AP, PLC) independently scrutinised the descriptions of interventions provided in each included trial and determined the treatment components included within each trial, based on the agreed upon definitions of treatment components (Table 2). Descriptions of interventions that were available only in Chinese were translated (and components classified) by one review author (PLC) and the translated descriptions used for the classification of components by a second review author (AP). We resolved disagreements through discussion and obtained further information from trialists when necessary (and possible).

\section{Assessment of risk of bias in included studies}

Two review authors independently documented the methodological quality of the studies, recording the following quality criteria: randomisation (allocation concealment); baseline comparison of groups; blinding of recipients and providers of care to treatment group/study aims; blinding of outcome assessor; possibility of contamination/co-intervention by the therapists providing the intervention; completeness of follow-up and other potential confounders (AP, GB, PC, PLC). The review authors resolved disagreements by discussion and contacted study authors for clarification when necessary.

One review author (PLC) translated relevant extracts related to the methodological quality of studies written in Chinese and assessed their methodological quality. A second review author (AP) checked the documentation of methodological quality, based on the translated extracts.

\section{Measures of treatment effect}

We presented all analysed outcome measures as continuous data. We calculated standardised mean differences (SMDs) and 95\% confidence intervals $(\mathrm{Cls})$, using a random-effects model for all outcomes analysed, with the exception of length of stay, for which we calculated mean differences (MDs) and $95 \% \mathrm{Cls}$, as length of stay was reported in number of days by all studies.

\section{Unit of analysis issues}

\section{Dealing with missing data}

\section{Assessment of heterogeneity}

\section{Assessment of reporting biases}

\section{Data synthesis}

We changed the comparisons included in the review for this update, based on consensus decisions reached by the expert stakeholder group (see Background). In earlier versions of this review, the comparisons were structured around 'named' rehabilitation approaches, as reported in the included studies (e.g. Bobath, Motor Relearning Programme). For this 
update, we planned to carry out comparisons of physical rehabilitation approaches that included treatment components within the categories of functional task training, musculoskeletal intervention (active), musculoskeletal intervention (passive), neurophysiological intervention, cardiopulmonary intervention, assistive device and modality (see Table 2). Categories were based on the treatment components described within each included study. We planned to compare active interventions with (1) no treatment, (2) usual care or control and (3) another active intervention.

\section{Subgroup analysis and investigation of heterogeneity}

We planned to carry out subgroup analysis to explore the effects of time post stroke of participants, geographical location of the study, dose of the intervention and the profession of the person who delivered the intervention (i.e. physiotherapist, nurse, therapy assistant). We also planned to explore the effects of including different individual treatment components.

\section{Sensitivity analysis}

We planned to carry out sensitivity analyses to explore the effects of methodological quality, based on assessment of risk of bias.

\section{RE S U L T S}

\section{Description of studies}

Results of the search

Results of the search are displayed in Figure 2. 
Figure 2. Study flow diagram.

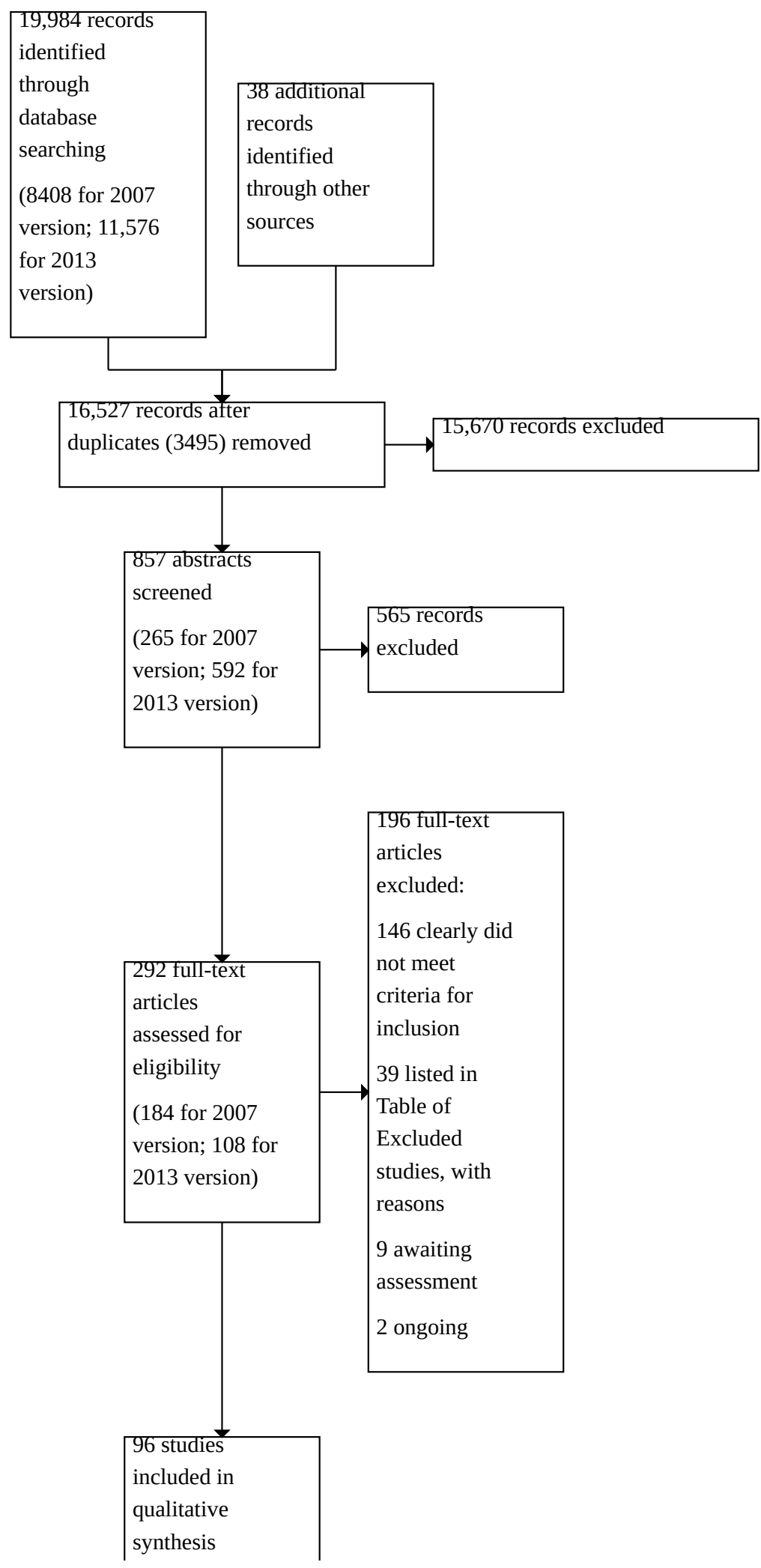


Figure 2. (Continued)

\begin{tabular}{|l|}
$\mid \begin{array}{l}\text { 4uantaue } \\
\text { synthesis }\end{array}$ \\
\hline $\begin{array}{l}\text { 41 studies } \\
\text { included in } \\
\text { meta-analysis } \\
\text { of intervention } \\
\text { versus no } \\
\text { treatment } \\
\text { 22 studies } \\
\text { included in } \\
\text { meta-analysis } \\
\text { of intervention } \\
\text { versus usual } \\
\text { care or } \\
\text { attention control } \\
13 \text { studies } \\
\text { included in } \\
\text { meta-analysis } \\
\text { of one active } \\
\text { intervention } \\
\text { versus another } \\
\text { active } \\
\text { intervention }\end{array}$ \\
\hline
\end{tabular}

\section{7 version}

For the 2007 version of this review, we identified 8408 potentially relevant trials by electronic searching; we considered 184 full papers and included 20 trials (1087 participants) (Dean 1997; Dean 2000; Duncan 1998; Duncan 2003; Gelber 1995; Green 2002; Hesse 1998; Howe 2005; Langhammer 2000; Lincoln 2003; McClellan 2004; Mudie 2002; Ozdemir 2001; Pollock 1998; Richards 1993; Salbach 2004; Stern 1970; Wade 1992; Wang 2005; Wellmon 1997).

\section{3 update}

For this update of this review, we identified 11,576 (8120 with duplicates removed) potentially relevant studies. We considered 108 full papers and included 96 trials (10,401 participants) (including the 20 within the 2007 version).

We identified two relevant ongoing studies (see Characteristics of ongoing studies), and we had insufficient information to reach decisions on nine studies (see Characteristics of studies awaiting classification). We excluded three studies that had been included in the 2007 version, as they used quasi-random assignment (Hesse 1998; Ozdemir 2001; Stern 1970) (see Figure 2).

\section{Included studies}

We included a total of 96 studies (10,401 participants) in this review. Two studies divided participants according to type of stroke (ischaemic or haemorrhagic) before randomisation and presented results within these two groups: These have been entered as four separate studies: Hu 2007 haem and Hu 2007 isch, and Zhu 2007 isch and Zhu 2007 haem. The data for Fang 2004 were presented in two groups, according to the age of participants, so these data have also been presented separately (Fang 2004 old and Fang 2004 young). Thus a total of 99 studies are referenced as included studies in this review. Details of these 99 studies are provided in Characteristics of included studies.

The mean number of participants was 105 (SD 151). Ninety-two of the 99 studies included fewer than 200 participants. One study had more than 1000 participants (Zhang 2004; 1078 participants); and six had between 250 and 1000 participants (Bai 2008, 364; Behrman 2011, 408; Hu 2007 haem, 352; Hu 2007 isch, 965; Kwakkel 2008, 250; Zhao 2003, 300). Ten studies included 20 or fewer participants (Aksu 2001, 20; Allison 2007, 17; Bale 2008, 18; Carlson 2006, 11; Dean 1997, 20; Dean 2000, 12; Dean 2007, 12; Duncan 1998, 20; Kim 2012, 20; Stephenson 2004, 18). 


\section{Intervention categories}

Details of the categories and treatment components of the active interventions are provided in Table 3. Of the 99 studies, 23 investigated two active interventions (19 of which directly compared two active interventions; and four of which had three intervention groups, of which two were active interventions). Thus a total of 122 active interventions were studied (99 included studies plus 23 studies with a second active intervention).

The most common intervention category was functional task training, with 101 of the 122 active interventions categorised as including treatment components from functional task training.

1. Of these 101 interventions, 20 included only functional task training components.

2. Of these 101 interventions, 26 included functional task training plus one other category. The second category was neurophysiological for six interventions; modality for two interventions; musculoskeletal (passive) for nine interventions; musculoskeletal (active) for eight interventions and cardiopulmonary for one intervention.

3. Of these 101 interventions, 32 included functional task training plus two other categories. The other categories included neurophysiological for 11 interventions; musculoskeletal (active) and musculoskeletal (passive) for 13 interventions; musculoskeletal (active or passive) plus other categories for seven interventions; and cardiopulmonary and assistive devices for one intervention.

4. Of these 101 interventions, 19 included functional task training plus three other categories. The other categories were neurophysiological plus musculoskeletal (active) plus musculoskeletal (passive) for nine interventions; neurophysiological plus other categories for eight interventions; and musculoskeletal (active) plus musculoskeletal (passive) plus another category for two interventions.

5. Of these 101 interventions, four included functional task training plus four other categories. The other categories were modalities, musculoskeletal (passive), musculoskeletal (active) and neurophysiological for three interventions; assistive devices, musculoskeletal (passive), musculoskeletal (active) and neurophysiological for one intervention; and modalities, musculoskeletal (passive), musculoskeletal (active) and assistive devices for one intervention.

Of the remaining 21 of the 122 interventions, most (17 interventions) included components from the neurophysiological category.

1. Of these 17 interventions, only 12 implemented neurophysiological treatment components.

2. Of these 17 interventions, five implemented neurophysiological plus a combination of musculoskeletal (active), musculoskeletal (passive) and/or modalities.

Of the remaining four interventions:

1. one included musculoskeletal (active) and musculoskeletal (passive) components;

2. two included musculoskeletal (passive) components only; and

3. one included a modality only (this modality was acupuncture; Zhuang 2012).

\section{Comparison groups}

The studies included in this review compare an active intervention with:

1. no treatment (55 studies: see Table 4 for further details);

2. usual care (19 studies) or attention control (11 studies) (see Table 5 for further details); or

3. another active intervention (23 studies: see Table 6 for further details).

A total of 108 comparisons were performed, as five of the 99 studies contributed data on more than one comparison. Four studies contributed data on three comparisons: Cooke 2006, Mudie 2002 and Richards 1993 each compared two active treatments with usual care, and Baer 2007 compared two active treatments with no treatment. Of the 99 studies, one contributed data on two comparisons: Kwakkel 2002 compared an active intervention with both an attention control group and a no treatment group.

\section{Study location}

Table 7 lists the geographical locations of the included studies. Of the 99 included studies, 97 recruited participants from one country or continent, and two studies recruited participants from two countries or continents (Brock 2005: Australia and Europe; Thaut 2007: North America and Europe). A total of 54 studies were carried out in China; 17 in Europe; 10 in North America and Canada; seven in Australia and New Zealand; eight in Asia, excluding China and one in South America.

The mean study size was greater in studies carried out in China (mean 138, SD 189 participants) than in other parts of the world (Europe: mean 76, SD 60; North America and Canada: mean 74, SD 122; Australia and New Zealand: mean 48, SD 48; Asia, excluding China: mean 46, SD 30).

The settings for recruitment of participants and for administration of the intervention are summarised in Table 7.

Table 8 illustrates the types of control interventions included in studies in different geographical locations. Of the 54 studies, 44 including a no-treatment comparison were carried out in China.

\section{Study participants}

Table 9 displays details of the participants included in the studies.

In 38 of the 99 studies, the time since stroke was 30 days or less (Allison 2007; Bai 2008; Chen 2004; Chen 2010; Dean 2007; Deng 2011; Fan 2006; Gelber 1995; Hou 2006; Howe 2005; Hu 2007 haem; Hu 2007 isch; Huang 2003; Jiang 2006; Jing 2006; Li 2005; Liao 2006; Lincoln 2003; Liu 2003; Ni 1997; Qian 2004; Qian 2005; Richards 1993; Thaut 2007; Torres-Arreola 2009; Wang 2005; Wang 2006; Wu 2006; Xiao 2003; Xie 2003; Xu 2003a; Xu 2003b; Xu 2004; Yan 2002; Zhu 2001; Zhu 2004b; Zhu 2007 isch; Zhu 2007 haem).

In 12 of the 99 studies, the time since stroke was 90 days or less (Bale 2008; Cooke 2006; Duncan 1998; Ge 2003; Mudie 2002; Pollock 1998; Verheyden 2006; Verma 2011; Wang 2004b; Wei 1998; Zhu 2006; Zhuang 2012).

In eight of the 99 studies, the time since stroke was six months or less (Blennerhassett 2004; Brock 2005; Chan 2006; Duncan 2003; Holmgren 2006; Kwakkel 2002; Kwakkel 2008; Wellmon 1997). 
In three of the 99 studies, the time since stroke was 12 months or less (McClellan 2004; Salbach 2004; Yelnik 2008).

In 10 of the 99 studies, the time since stroke was longer than 12 months (Baer 2007; Chen 2006; Dean 1997; Dean 2000; Dean 2006; Hui-Chan 2009; Kim 2011; Kim 2012; Mudge 2009; Wade 1992).

The time since stroke was not stated in 28 of the 99 studies (Aksu 2001; Behrman 2011; Carlson 2006; Chu 2003; Fang 2003; Fang 2004 old; Fang 2004 young; Green 2002; Langhammer 2000; Langhammer 2007; Lennon 2006; Li 1999; Li 2003; Pan 2004; Pang 2003; Pang 2006; Shin 2011; Stephenson 2004; Tang 2009; Wang 2004a; Xie 2005; Xu 1999; Xue 2006; Yin 2003a; Zhang 1998; Zhang 2004; Zhao 2002; Zhao 2003).

\section{Dose of intervention}

The duration of the intervention period was 28 days or less in 35 studies (Allison 2007; Baer 2007; Bale 2008; Blennerhassett 2004; Brock 2005; Carlson 2006; Chen 2010; Dean 1997; Dean 2000; Dean 2007; Fang 2003; Fang 2004 old; Fang 2004 young; Howe 2005; HuiChan 2009; Kim 2012; Lennon 2006; Liao 2006; Liu 2003; Mudge 2009; Pang 2003; Pollock 1998; Shin 2011; Stephenson 2004; Thaut 2007; Verma 2011; Wang 2004b; Wang 2005; Wellmon 1997; Xiao 2003; Xu 2003a; Xu 2003b; Yelnik 2008; Zhao 2003; Zhuang 2012); 12 weeks or less in 24 studies (Chan 2006; Chen 2004; Chen 2006; Cooke 2006; Deng 2011; Duncan 1998; Holmgren 2006; Huang 2003; Kim 2011; Kwakkel 2008; Li 1999; McClellan 2004; Mudie 2002; Ni 1997; Salbach 2004; Tang 2009; Verheyden 2006; Wang 2004a; Wei 1998; Xu 1999; Xu 2004; Xue 2006; Yan 2002; Zhao 2002); between 12 weeks and six months in 16 studies (Bai 2008; Behrman 2011; Duncan 2003; Green 2002; Hou 2006; Hu 2007 haem; Hu 2007 isch; Jiang 2006; Kwakkel 2002; Pang 2006; Torres-Arreola 2009; Wang 2006; Wu 2006; Xie 2005; Zhang 2004; Zhu 2004b) and over six months in three studies (Chu 2003; Dean 2006; Langhammer 2007). The intervention period was unclear in 21 of the 99 studies (Aksu 2001; Fan 2006; Ge 2003; Gelber 1995; Jing 2006; Langhammer 2000; Li 2003; Li 2005; Lincoln 2003; Pan 2004; Qian 2004; Qian 2005; Richards 1993; Wade 1992; Xie 2003; Yin 2003a; Zhang 1998; Zhu 2001; Zhu 2006; Zhu 2007 isch; Zhu 2007 haem).

The frequency of intervention was more than once per day in 22 studies (Blennerhassett 2004; Carlson 2006; Fan 2006; Hou 2006; Jiang 2006; Kim 2012; Li 1999; Liu 2003; McClellan 2004; Ni 1997; Pan 2004; Pang 2003; Wang 2004a; Wellmon 1997; Xie 2003; Xie 2005; Xu 1999; Xue 2006; Yan 2002; Zhu 2004b; Zhu 2007 isch; Zhu 2007 haem); once per day for five to seven days per week for 30 to 60 minutes in 33 studies (Allison 2007; Bai 2008; Bale 2008; Chu 2003; Dean 1997; Dean 2007; Deng 2011; Fang 2003; Fang 2004 old; Fang 2004 young; Holmgren 2006; Hui-Chan 2009; Kim 2011; Kwakkel 2002; Langhammer 2000; Lennon 2006; Li 2005; Liao 2006; Mudie 2002; Pang 2006; Pollock 1998; Shin 2011; Thaut 2007; Verma 2011; Wang 2004b; Wang 2005; Wei 1998; Xu 2004; Yelnik 2008; Zhao 2002; Zhu 2001; Zhu 2006; Zhuang 2012); three to four times per week in 12 studies (Brock 2005; Chan 2006; Cooke 2006; Dean 2000; Dean 2006; Duncan 1998; Duncan 2003; Mudge 2009; Salbach 2004; Stephenson 2004; Verheyden 2006; Xiao 2003); one to two times per week in two studies (Chen 2006; Kwakkel 2008) and less frequent than once per week in one study (Baer 2007). The frequency was unclear in 29 of the 99 studies (Aksu 2001; Behrman 2011; Chen 2004; Chen 2010; Ge 2003; Gelber 1995; Green 2002; Howe 2005; Hu 2007 haem; Hu 2007 isch; Huang 2003; Jing 2006; Langhammer 2007; Li 2003; Lincoln 2003; Qian 2004; Qian 2005; Richards 1993;
Tang 2009; Torres-Arreola 2009; Wade 1992; Wang 2006; Wu 2006; Xu 2003a; Xu 2003b; Yin 2003a; Zhang 1998; Zhang 2004; Zhao 2003).

Table 10 displays the length and dose of intervention for those studies with Independence in ADL or motor function data in comparisons with no treatment, and Table 11 displays this information for studies with comparisons with usual care or attention control.

\section{Definition of dose}

We preplanned subgroup analyses to explore the effect of dose of intervention. We defined dose as including the components of (1) length of a single treatment session, (2) frequency of treatment sessions and (3) duration of the intervention period. However, because of the availability of data and the complexities associated with variations in these three components, our subgroup analyses of dose explored only the combination of (1) length of a single treatment session and (2) frequency of treatment sessions. We also performed additional sensitivity analyses to explore the impact of (3) duration of intervention period on subgroup analysis results.

\section{Outcome measures for analysis}

The included trials used a large number of heterogeneous outcome measures. The many diverse outcome measures recorded in the included studies made it impossible for review authors to analyse all of the documented data. Based on the prestated groupings of relevant outcomes and the availability of data from specific measures in the included trials, the review authors for the previous version of the review made the decision to concentrate data analysis on independence in ADL, motor function,, balance, muscle strength, gait velocity and length of rehabilitation stay. For this update, we decided to remove the muscle strength outcome, as this outcome was rarely reported in the included studies.

*Independence in ADL was called 'global dependency' and motor function was called 'functional independence in mobility' in previous versions of this review. See Differences between protocol and review.

Outcome measures were recorded at several different time points during and after the intervention period. For the analyses in the review, we classed 'immediate outcomes' as data that were recorded at the end of the treatment period or at the time point nearest to the end of the treatment period. If the intervention comprised a change in treatment throughout the whole of a participant's rehabilitation period, then data were recorded from the outcome measures noted at the time of discharge from rehabilitation or at the time point nearest to discharge. When studies also reported follow-up data, we included these as analysis of 'persisting outcome'. The time points at which data were recorded are clearly documented and stated for each trial in the Characteristics of included studies table and are summarised in Table 4, Table 5 and Table 6.

Of the 99 studies, 79 included outcome measures suitable for inclusion in an analysis of immediate outcomes, and 27 provided a follow-up outcome measure. Details of these outcome measures are provided below. 


\section{Studies included in meta-analysis}

\section{Independence in ADL scales}

Forty-nine studies reported 'immediate outcome' data for a measure of independence in ADL. This was the Barthel Index (or modified Barthel Index) for 45 studies (Chen 2004; Chen 2006; Chen 2010; Chu 2003; Duncan 1998; Fang 2003; Fang 2004 old; Fang 2004 young; Green 2002; Holmgren 2006; Hou 2006; Huang 2003; Jing 2006; Langhammer 2007; Langhammer 2000; Lennon 2006; Li 1999; Li 2005; Lincoln 2003; Liu 2003; Mudie 2002; Pan 2004; Pang 2003; Pang 2006; Pollock 1998; Richards 1993; Thaut 2007; Torres-Arreola 2009; Wade 1992; Wu 2006; Xie 2003; Xu 1999; Xu 2003a; Xu 2003b; Xu 2004; Xue 2006; Yan 2002; Zhang 1998; Zhang 2004; Zhao 2002; Zhao 2003; Zhu 2006; Zhu 2007 isch; Zhu 2007 haem; Zhuang 2012) and the Functional Independence Measure (FIM) for four studies (Chan 2006; Gelber 1995; Ni 1997; Yelnik 2008).

Sixteen studies reported 'persisting outcome' data for a measure of independence in ADL. This was the Barthel Index (or modified Barthel Index) for 14 studies (Chen 2004; Fang 2003; Fang 2004 old; Fang 2004 young; Green 2002; Holmgren 2006; Hou 2006; Jing 2006; Lincoln 2003; Mudie 2002; Torres-Arreola 2009; Verma 2011; Wade 1992; Zhao 2003) and the FIM for two studies (Gelber 1995; Yelnik 2008). Verma 2011 reported a follow-up measurement but not an immediate measurement for the Barthel Index.

Standard deviations for Zhu 2007 isch and Zhu 2007 haem were estimated from the reported range, and data for Chen 2010 and Zhao 2003 were estimated from categorical data; it was preplanned to explore the effect of including these studies.

\section{Motor function scales}

Fifty studies reported 'immediate outcome' data for a measure of motor function. This was the Rivermead Motor Assessment for six studies (Cooke 2006; Green 2002; Kwakkel 2008; Lincoln 2003; Mudge 2009; Wade 1992); the Motor Assessment Scale for six studies (Bale 2008; Langhammer 2007; Langhammer 2000; Lennon 2006; McClellan 2004; Wang 2005) and the Fugl-Meyer Assessment for 38 studies (Chen 2010; Chu 2003; Deng 2011; Duncan 1998; Duncan 2003; Fang 2003; Fang 2004 old; Fang 2004 young; Hu 2007 haem; Hu 2007 isch; Huang 2003; Jing 2006; Li 1999; Liao 2006; Liu 2003; Ni 1997; Pan 2004; Qian 2005; Richards 1993; Tang 2009; Thaut 2007; Wang 2004a; Wang 2004b; Wei 1998; Wu 2006; Xu 2003a; Xu 2003b; Xu 2004; Xue 2006; Yin 2003a; Zhang 1998; Zhang 2004; Zhao 2002; Zhu 2001; Zhu 2006; Zhu 2007 isch; Zhu 2007 haem; Zhuang 2012).

Twelve studies reported 'persisting outcome' data for a measure of motor function. This was the Rivermead Motor Assessment for six studies (Cooke 2006; Green 2002; Kwakkel 2008; Lincoln 2003; Mudge 2009; Wade 1992); the Motor Assessment Scale for one study (McClellan 2004); the Rivermead Mobility Index for one study (Cooke 2006) and the Fugl-Meyer Assessment for five studies (Fang 2003; Fang 2004 old; Fang 2004 young; Jing 2006; Zhao 2002).

Richards 1993 and Yin 2003a included two active treatment groups so are entered twice into analyses, with the control group data 'shared' as the comparison group for the two active interventions. Standard deviations for Green 2002, Mudge 2009, Zhu 2007 isch and Zhu 2007 haem were estimated from the reported range, it was preplanned to explore the effect of including these studies. Data from Jing 2006 were not included in analyses of one approach versus another, as both of the two treatment groups were assessed as including similar treatment components.

\section{Balance (Berg Balance Scale)}

Eleven studies reported 'immediate outcome' data for measures of balance (Brock 2005; Chan 2006; Duncan 1998; Duncan 2003; Holmgren 2006; Kim 2012; Richards 1993; Salbach 2004; Shin 2011; Wang 2005; Yelnik 2008). Holmgren 2006 and Yelnik 2008 also reported 'persisting outcome' data.

Richards 1993 included two active treatment groups so is entered twice into analyses, with the control group data 'shared' as the comparison group for the two active interventions. The data for Holmgren 2006 standard deviations were calculated from the reported confidence intervals.

\section{Gait velocity}

Twenty-three studies reported 'immediate outcome' data for measures of gait velocity (Bale 2008; Blennerhassett 2004; Brock 2005; Cooke 2006; Dean 1997; Dean 2000; Dean 2006; Dean 2007; Duncan 1998; Duncan 2003; Gelber 1995; Green 2002; Hui-Chan 2009; Kim 2012; Kwakkel 2008; Lincoln 2003; Richards 1993; Salbach 2004; Stephenson 2004; Thaut 2007; Verma 2011; Wade 1992; Yelnik 2008), and 13 studies reported 'persisting outcome' data (Blennerhassett 2004; Cooke 2006; Dean 2000; Dean 2007; Gelber 1995; Green 2002; Hui-Chan 2009; Kwakkel 2008; Lincoln 2003; Mudge 2009; Verma 2011; Wade 1992; Yelnik 2008).

Cooke 2006 and Richards 1993 included two active treatment groups so are entered twice into analyses, with the control group data 'shared' as the comparison group for the two active interventions. Standard deviations for Green 2002 and Mudge 2009 were estimated from the reported range, and data for Bale 2008 were estimated from categorical data; it was preplanned to explore the effect of including these studies.

\section{Length of stay}

Eight studies reported data relating to length of stay (Blennerhassett 2004; Gelber 1995; Holmgren 2006; Langhammer 2000; Langhammer 2007; Li 2003; Li 2005; Torres-Arreola 2009).

\section{Studies included in meta-analysis comparisons}

\section{Intervention versus no treatment}

Of the 54 studies included in this review that compared an active intervention with no treatment, 41 included data suitable for inclusion in meta-analysis. These were 'immediate outcome' data relating to Independence in ADL for 28 studies; motor function for 28 studies; balance for one study and gait velocity for three studies. Three of these studies reported length of stay. 'Persisting outcome' data were available relating to independence in ADL for 10 studies; motor function for 10 studies; balance for one study and gait velocity for three studies. (See Table 4 for further details.)

\section{Intervention versus attention control or usual care}

Of the 27 studies included in this review that compared an active intervention with usual care (17) or attention control (10), 22 included data suitable for inclusion in meta-analysis. These were 'immediate outcome' data relating to independence in ADL for eight studies; motor function for 13 studies; balance for six studies and gait velocity for 16 studies. Two of these studies reported 
length of stay. 'Persisting outcome' data were available relating to independence in ADL for no studies; motor function for four studies; balance for no studies and gait velocity for six studies. (See Table 5 for further details.)

\section{One active intervention versus another active intervention}

Of the 23 studies included in this review that compared two different active interventions, 13 included data suitable for inclusion in meta-analysis. These were 'immediate outcome' data relating to independence in ADL for seven studies; motor function for eight studies; balance for four studies and gait velocity for seven studies. Four of these studies reported length of stay. 'Persisting outcome' data were available relating to independence in ADL for two studies; motor function for no studies; balance for no studies and gait velocity for two studies. (See Table 6 for further details.)

Data from three studies comparing one active intervention with another active intervention (Chen 2006; Cooke 2006; Jing 2006) were available but were not included in meta-analyses, as the two active treatment groups were classified as including similar treatment components.

\section{Excluded studies}

Studies listed in the Characteristics of excluded studies table were limited to those for which discussions were required between review authors to reach consensus. Thirty-nine studies are listed; we considered a further 147 as full papers but excluded them, as we agreed that they clearly did not meet the inclusion criteria. We needed to look at full papers because insufficient details were provided in the abstracts; the main reasons for excluding studies at this stage were that they were not randomised controlled trials (RCTs) or that they investigated a single specific treatment (such as electrical stimulation or treadmill training).

\section{Risk of bias in included studies}

Details of the methodological quality of the studies are provided in Characteristics of included studies, and risk of bias is summarised in Figure 3 and Figure 4. We assessed only 40 of the 99 studies to have low risk of bias for sequence generation; 29 of 99 for allocation concealment and 51 of 99 for blinding of outcome assessor. Poor reporting led to our assigning 'unclear' risk of bias in most cases, with 56 of 99,59 of 99 and 39 of 99 studies having unclear risk of bias for sequence generation, allocation concealment and blinding of outcome assessor, respectively. We assessed a larger proportion (72 of 99) to have low risk of bias for being 'free of systematic differences in baseline characteristics of groups,' as this information could generally be determined from tables of characteristics of participants. When no systematic differences in baseline characteristics of groups were noted, there was no need for study authors to adjust for baseline characteristics; this was also therefore assessed to show low risk of bias for a similar number of studies.

\section{Figure 3. Risk of bias graph: review authors' judgements about each risk of bias item presented as percentages} across all included studies.

Random sequence generation (selection bias)

Allocation concealment (selection bias)

Blinding of outcome assessment (detection bias): All outcomes Incomplete outcome data (attrition bias): All outcomes

Free of systematic differences in baseline characteristics of groups compared?

Did authors adjust for baseline differences in their analyses?

Other bias

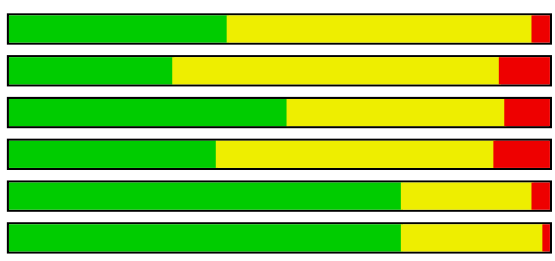

-

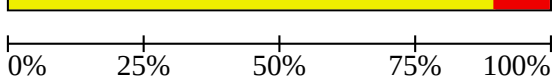


Figure 4. Risk of bias summary: review authors' judgements about each risk of bias item for each included study.

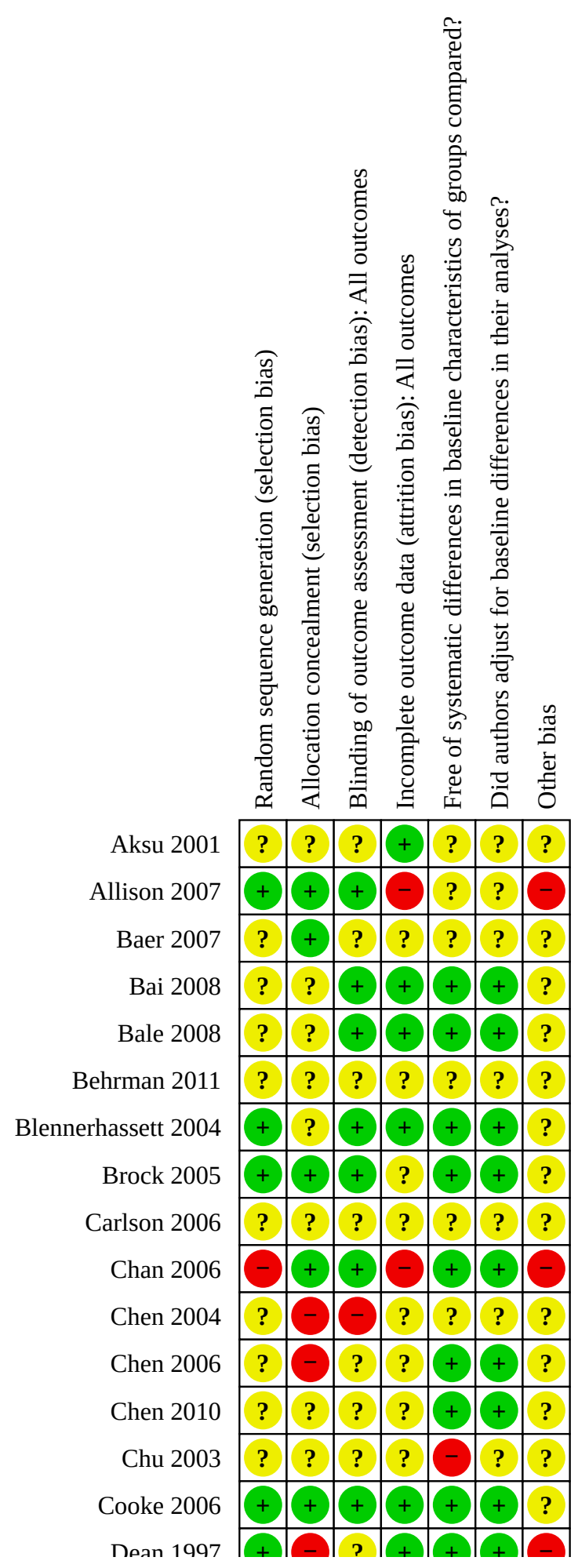


Figure 4. (Continued)

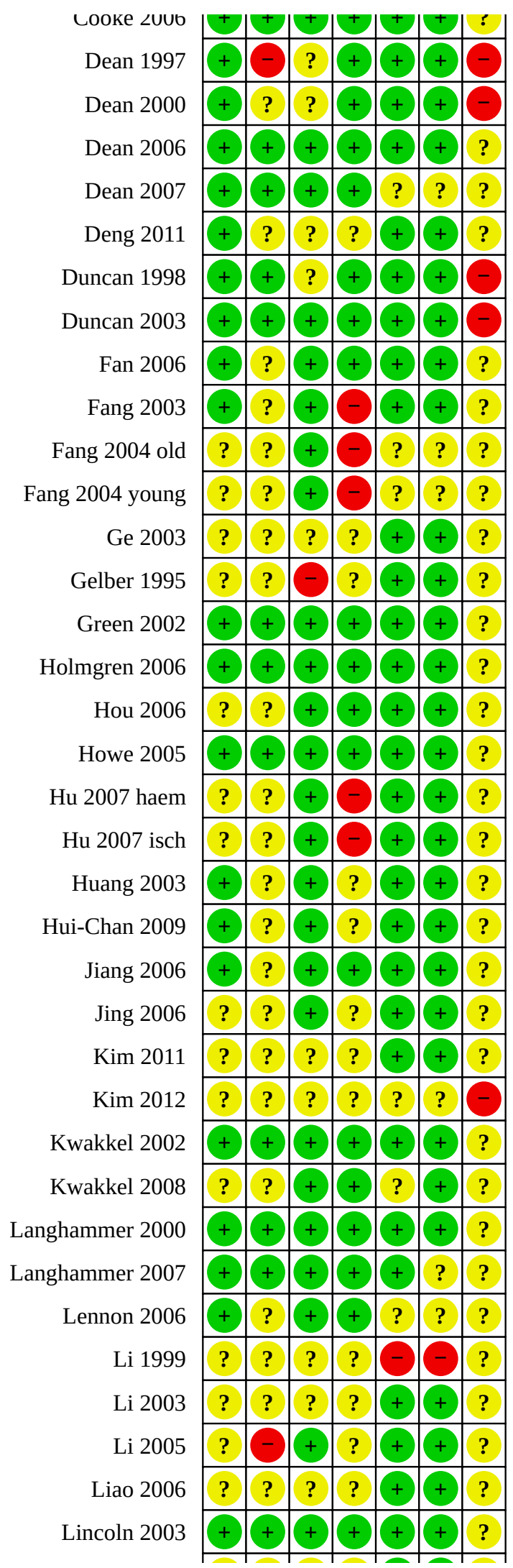


Figure 4. (Continued)

\begin{tabular}{|c|c|c|c|c|c|c|c|}
\hline Lincoln 2003 & + & + & + & + & \pm & + & $?$ \\
\hline Liu 2003 & $?$ & $?$ & $?$ & $?$ & + & + & $?$ \\
\hline McClellan 2004 & + & + & + & + & + & + & $?$ \\
\hline Mudge 2009 & + & + & + & + & + & + & $?$ \\
\hline Mudie 2002 & + & + & + & + & + & + & $?$ \\
\hline Ni 1997 & $?$ & $?$ & $?$ & $?$ & $?$ & $?$ & $?$ \\
\hline Pan 2004 & $?$ & $?$ & $?$ & $?$ & + & + & $?$ \\
\hline Pang 2003 & $?$ & $?$ & $?$ & $?$ & + & + & $?$ \\
\hline Pang 2006 & $?$ & $(-$ & - & + & $?$ & $?$ & - \\
\hline Pollock 1998 & + & + & - & - & $?$ & $?$ & - \\
\hline Qian 2004 & $?$ & - & + & $?$ & + & + & $?$ \\
\hline Qian 2005 & $?$ & - & + & $?$ & + & + & $?$ \\
\hline Richards 1993 & + & + & + & $?$ & $?$ & $?$ & $?$ \\
\hline Salbach 2004 & + & + & - & $?$ & $?$ & $?$ & $?$ \\
\hline Shin 2011 & $?$ & $?$ & $?$ & $?$ & + & + & $?$ \\
\hline Stephenson 2004 & $?$ & $?$ & $?$ & $?$ & $?$ & $?$ & $?$ \\
\hline Tang 2009 & $?$ & $?$ & $?$ & $?$ & + & + & $?$ \\
\hline Thaut 2007 & + & + & + & - & + & + & $?$ \\
\hline Torres-Arreola 2009 & + & + & + & - & - & $?$ & $?$ \\
\hline Verheyden 2006 & $?$ & + & + & + & + & + & $?$ \\
\hline Verma 2011 & + & + & + & + & + & + & $?$ \\
\hline Wade 1992 & + & + & + & $?$ & $?$ & $?$ & $?$ \\
\hline Wang 2004a & $?$ & $?$ & + & + & + & + & $?$ \\
\hline Wang 2004b & $?$ & - & - & + & + & + & $?$ \\
\hline Wang 2005 & + & + & + & + & $?$ & $?$ & $?$ \\
\hline Wang 2006 & $?$ & $?$ & $?$ & $?$ & + & + & $?$ \\
\hline Wei 1998 & $?$ & $?$ & $?$ & + & + & + & $?$ \\
\hline Wellmon 1997 & $?$ & $?$ & - & + & $?$ & $?$ & C \\
\hline Wu 2006 & $?$ & $?$ & $?$ & + & + & + & $?$ \\
\hline Xiao 2003 & $?$ & $?$ & $?$ & $?$ & + & + & $?$ \\
\hline Xie 2003 & $?$ & $?$ & $?$ & $?$ & + & + & $?$ \\
\hline Xie 2005 & $?$ & $?$ & + & $?$ & + & + & $?$ \\
\hline Xu 1999 & $?$ & $?$ & $?$ & $?$ & $?$ & $?$ & $?$ \\
\hline Xu 2003a & $?$ & $?$ & $?$ & $?$ & + & + & $?$ \\
\hline Xu 2003b & $?$ & $?$ & ? & $?$ & + & + & $?$ \\
\hline Xu 2004 & ? & $?$ & $?$ & $?$ & + & + & $?$ \\
\hline
\end{tabular}


Figure 4. (Continued)

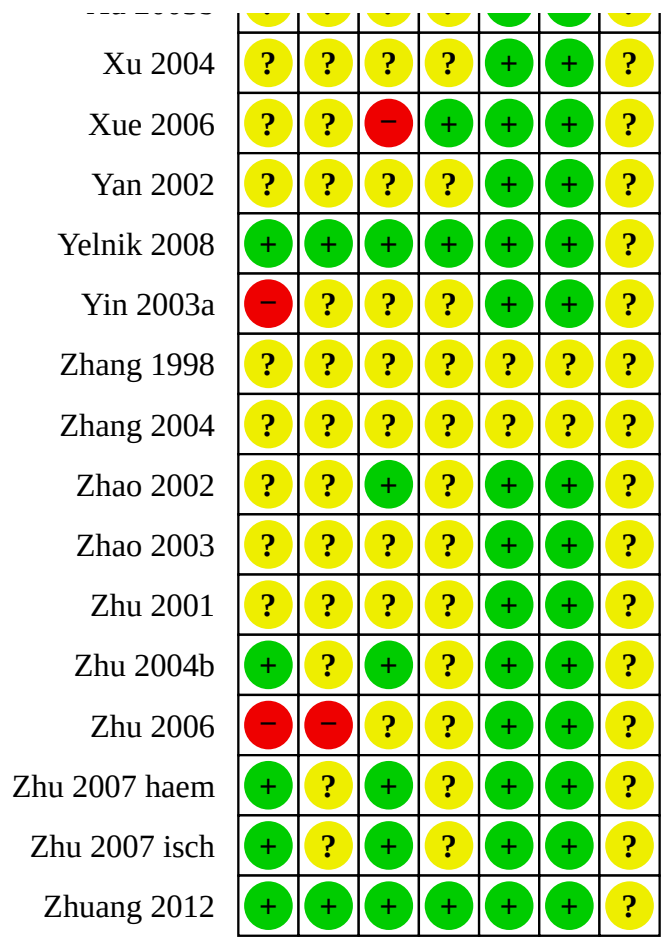

\section{Effects of interventions}

See: Summary of findings 1 Summary of findings: intervention versus no treatment; Summary of findings 2 Summary of findings: intervention versus usual care or attention control; Summary of findings 3 Summary of findings: one active intervention versus another active intervention

The results are described below under the comparisons carried out for each of the explored outcomes (1. Independence in ADL scale; 2. Motor function scale; 3. Balance; 4 . Gait velocity and 5. Length of stay) for both immediate and persisting outcomes. Table 12 provides a summary of the analyses performed, stating the numbering of analyses; Table 13 provides a summary of the subgroup analyses performed, along with the numbering of subgroup analyses.

(Section numbering corresponds to numbering of relevant analyses. Four 'empty' forest plots are provided, for which no data were available. These include the following: Analysis 5.1-Intervention versus usual care; persisting outcomesglobal dependency; Analysis 5.3-Intervention versus usual care; persisting outcomes-balance; Analysis 6.2-One intervention versus another; persisting outcomes-functional independence and Analysis 6.3-One intervention versus another; persisting outcomes-balance. Despite the absence of data, these forest plots have been left, as this maintains the consistency of numbering of the section headings and analyses, which we believe aids the accessibility of this section.)

\section{Comparison 1. Intervention versus no treatment, immediate outcomes}

\subsection{Independence in ADL scale}

We pooled data from 27 studies (3423 participants) in the analysis, demonstrating that the intervention had a significantly beneficial effect compared with no intervention (SMD $0.78,95 \% \mathrm{Cl} 0.58$ to $0.97)$. However, substantial heterogeneity was found $\left(I^{2}=85 \%\right)$.

Significant differences were noted between the subgroups of different categories of treatment components ( $P$ value $<0.00001)$.

For the subgroup of studies that combined functional task training and musculoskeletal components (Analysis 1.1.2; nine studies, 967 participants) and for studies that combined functional task training and neurophysiological and musculoskeletal components (Analysis 1.1.6; 12 studies, 1838 participants), a significant effect of the intervention compared with no intervention was seen (SMD $0.97,95 \% \mathrm{Cl} 0.67$ to $1.27, \mathrm{I}^{2}=76 \%$; and SMD $0.96,95 \% \mathrm{Cl} 0.66$ to 1.27 , $1^{2}=86 \%$, respectively). See Analysis 1.1 .

\section{Sensitivity analysis: risk of bias}

Removing studies judged to be at high risk of bias for at least one assessed quality of component led to our removing Chu 2003, Fang 2003, Fang 2004 old, Fang 2004 young, Hu 2007 haem, Hu 2007 isch, Li 1999, Wu 2006, Xue 2006, Yin 2003a and Zhu 2006. Data from the remaining 17 studies (2655 participants) demonstrated a similar direction of effect (SMD $0.98,95 \% \mathrm{Cl} 0.63$ to $1.34, \mathrm{I}^{2}$ = 94\%); additionally, removing the studies for which data had been estimated from reported ranges (Green 2002; Zhu 2007 haem; Zhu 2007 isch) left data from 15 studies (2346 participants), demonstrating a similar direction of effect (SMD 1.07, 95\% CI 0.66 to $\left.1.47,\left.\right|^{2}=95 \%\right)$. 
Removing all studies judged as having unclear or high risk of bias for random sequence generation or allocation concealment left only two studies (Green 2002; Wade 1992; 150 participants), which showed no significant benefit of intervention versus no treatment (SMD $-0.06,95 \% \mathrm{Cl}-0.30$ to $0.19, \mathrm{~L}=0 \%$ ). (These sensitivity analyses are not illustrated within forest plots.)

\section{Subgroup analysis: time after stroke}

A significant difference between subgroups was noted according to time post stroke (P value 0.003 ), with a suggestion of greater effect in studies with participants who were within 30 days post stroke. See Analysis 7.1.

\section{Subgroup analysis: study geographical location}

Twenty-five (3173 participants) of the 27 studies were carried out in China, and only two studies (250 participants) were carried out in Europe (Green 2002; Wade 1992). A significant difference between these subgroups was noted ( $P$ value $<0.00001$ ). See Analysis 7.2.

\section{Subgroup analysis: dose of intervention}

Eight studies (711 participants) provided the intervention more than once per day; 11 studies (1027 participants) provided daily intervention five to seven days per week for between 30 and 60 minutes; four studies provided a less frequent intervention than this and the dose was not stated in another four studies (see Table 10). When the studies in which the dose was not stated were excluded (as it was not appropriate to include this group), a significant difference between subgroups was noted ( $P$ value $<$ 0.00001 ) (analysis not shown). A significant difference was also seen between the subgroup of more than one intervention per day and the subgroup receiving daily intervention ( $P$ value 0.02 ) (analysis not shown). The effect size was greater in studies with a greater dose of intervention, with an indication that a dose of between 30 and 60 minutes once per day for five to seven days a week was beneficial, but that more than once-daily intervention may provide even greater benefit. See Analysis 7.3.

\section{Sensitivity analysis: dose and duration of the intervention period}

We explored the effect of the length of the intervention period for studies that provided the intervention once per day or provided daily interventions five to seven days per week for between 30 and 60 minutes (19 studies). The order of these studies within Analysis 7.3.1 and 7.3.2 is from least to most intervention (with studies for which length of intervention is not stated at the 'most' end). Six studies did not state the length of intervention and were removed from the analysis (Pan 2004; Xie 2003; Zhang 1998; Zhu 2006; Zhu 2007 haem; Zhu 2007 isch). Four studies (298 participants) (Fang 2004 old; Fang 2004 young; Liu 2003; Pang 2006) had a length of intervention of 15 days or less: including only these studies leads to a non-significant effect (SMD 0.54, 95\% Cl -0.01 to 1.09$)$, but with very substantial heterogeneity $(12=80 \%)$. Eight studies (938 participants) (Huang 2003; Li 1999; Xu 1999; Xu 2003b; Xu 2004; Xue 2006; Yan 2002; Zhao 2003) had a length of intervention of approximately one month; including only these studies demonstrated a significant effect of the intervention (SMD $1.06,95 \% \mathrm{Cl} 0.63$ to 1.48 ), with very substantial heterogeneity (I2 $=88 \%$ ). (These sensitivity analyses are not illustrated within forest plots.)

\section{Subgroup analysis: provider of the intervention}

Five studies (1158 participants) stated that the intervention was provided by a 'therapist'; six studies (429 participants) stated that it was provided by a therapist with help from family members (although in three of these studies, the role of the 'therapist' is not explicit; Xu 1999; Xu 2004; Zhang 1998). Two of the studies stated that it was a 'physiotherapist' and two stated 'nurse' or 'rehabilitation nurse.' In some cases it was stated that another professional, such as an occupational therapist (Wade 1992) or a doctor (Zhang 2004), also contributed. The provider was not stated in 12 studies. When studies in which the provider was not stated were excluded (as it was not appropriate to include this group), a significant difference between subgroups was noted ( $P$ value 0.0001 ) (analysis not shown), with an indication of greater effect when the provider was 'therapist' or 'therapist plus family.' However, each of the other subgroups contained only two studies. See Analysis 7.4.

\section{Subgroup analysis: treatment components included}

Twenty-three studies (3055 participants) included functional task training components; 15 studies (2106 participants) included neurophysiological training components and 23 studies (3033 participants) included musculoskeletal components. No significant difference between these subgroups was observed ( $P$ value 0.99). See Analysis 7.5.

\subsection{Motor function scale}

We pooled data from 25 studies (4558 participants) in the analysis, demonstrating that the intervention had a significantly beneficial effect compared with no intervention (SMD $0.81,95 \% \mathrm{Cl} 0.58$ to 1.04). However, considerable heterogeneity was present $\left(I^{2}=\right.$ $92 \%)$. Significant differences between the subgroups of different categories of treatment components were noted ( $P$ value $<0.0001)$. See Analysis 1.2.

\section{Sensitivity analysis: risk of bias}

Removing studies judged to be at high risk of bias for at least one assessed quality of component led us to remove Chu 2003, Fang 2003, Fang 2004 old, Hu 2007 isch, Li 1999, Xue 2006, Yin 2003a and Zhu 2006. Data from the remaining 18 studies (2655 participants) demonstrated a similar direction of effect (SMD 0.95, 95\% Cl 0.60 to $1.29,12=93 \%$ ); additionally removing the studies for which data had been estimated from reported ranges (Green 2002; Zhu 2007 isch; Zhu 2007 haem) left data from 15 studies (2416 participants), also demonstrating a similar direction of effect (SMD 1.02, 95\% Cl 0.63 to $1.14,1^{2}=94 \%$ ). Removing the one study that was visually a clear outlier (Zhao 2002) left 14 studies (2236 participants) and still demonstrated a similar direction of effect (SMD $0.79,95 \% \mathrm{Cl} 0.54$ to $\left.1.03,\left.\right|^{2}=84 \%\right)$.

Removing all studies judged as having unclear or high risk of bias for random sequence generation or allocation concealment left only two studies (Green 2002; Wade 1992; 250 participants), which showed no significant benefit of intervention versus no treatment ( $\mathrm{SMD} 0.14,95 \% \mathrm{Cl}-0.31$ to $0.58,12=67 \%$ ). (These sensitivity analyses are not illustrated within forest plots.)

\section{Subgroup analysis: time after stroke}

A significant difference between subgroups was noted according to time post stroke (P value 0.02). However, when studies in which 
the time after stroke was not stated were excluded, no significant difference between subgroups was noted ( $P$ value 0.06 ) (analysis not shown). See Analysis 9.1.

\section{Subgroup analysis: study geographical location}

Twenty-three (4308 participants) of the 25 studies were carried out in China, and only two studies (250 participants) were carried out in Europe (Green 2002; Wade 1992). A significant difference between these subgroups was reported (P value 0.005). See Analysis 9.2.

\section{Subgroup analysis: dose of intervention}

Four studies (434 participants) provided the intervention more than once per day; 11 studies (1080 participants) provided daily intervention five to seven days per week for between 30 and 60 minutes; five studies provided less frequent interventions than this and the dose was not stated in another four studies (see Table 10). When studies in which the dose was not stated were excluded (as it was not appropriate to include this group), a significant difference between subgroups was noted ( $P$ value 0.0007 ) (analysis not shown). However, no significant differences between the subgroup of more than one intervention per day and the subgroup receiving daily intervention was noted ( $P$ value 0.20 ) (analysis not shown). The effect size was greater with studies with a greater dose of intervention, with an indication that a dose of at least 30 to 60 minutes once per day for five to seven days a week was beneficial. See Analysis 9.3.

\section{Sensitivity analysis: dose and duration of the intervention period}

We explored the effect of the length of the intervention period for studies that provided the intervention once per day or provided daily intervention five to seven days per week for between 30 and 60 minutes (17 studies). The order of these studies within Analysis 9.3.1 and 9.3.2 is from least to most intervention (with studies for which length of intervention is not stated at the 'most' end). Seven studies did not state the length of intervention, and we removed them from the analysis (Pan 2004; Yin 2003a; Zhang 1998; Zhu 2001; Zhu 2006; Zhu 2007 haem; Zhu 2007 isch). Three studies (220 participants) (Fang 2004 old; Fang 2004 young; Liu 2003) had a length of intervention of 15 days or less; including only these studies leads to a non-significant effect (SMD $0.71,95 \% \mathrm{Cl}-0.36$ to $1.79)$, but with very substantial heterogeneity $(12=92 \%)$. Six studies (716 participants) (Chu 2003; Huang 2003; Wang 2004a; Xu 2003b; Xue 2006; Zhao 2002) had a length of intervention of approximately one month; including only these studies demonstrates a significant effect of intervention (SMD $1.45,95 \% \mathrm{Cl} 0.39$ to 2.51 ), with very considerable heterogeneity $\left(I^{2}=97 \%\right)$. (These sensitivity analyses are not illustrated within forest plots.)

\section{Subgroup analysis: provider of the intervention}

Seven studies (1356 participants) stated that the intervention was provided by a 'therapist'; two studies (152 participants) stated that it was provided by a therapist with help from family members (although the role of the 'therapist' was not explicit in Zhang 1998). Two of the studies stated that it was a 'physiotherapist' and three stated 'nurse' or 'rehabilitation nurse.' In some cases it was stated that another professional, such as an occupational therapist (Wade 1992) or a doctor (Zhang 2004), also contributed. The provider was not stated in 11 studies. When studies in which the provider was not stated were excluded (as it was not appropriate to include this group), a significant difference between subgroups was noted ( $P$ value 0.004 ) (analysis not shown). However, excluding the group stating 'physiotherapist' demonstrated no significant difference between the remaining groups ( $P$ value 0.15 ) (analysis not shown). See Analysis 9.4.

\section{Subgroup analysis: treatment components included}

Twenty-three studies (4330 participants) included functional task training components; 13 studies (2033 participants) included neurophysiological training components and 22 studies (4240 participants) included musculoskeletal components. No significant differences between these subgroups were reported ( $P$ value 0.74 ). See Analysis 9.5.

\subsection{Balance (Berg Balance Scale)}

Only one study (34 participants) reported a Berg Balance Scale score for a comparison of intervention versus no treatment (SMD $-0.04,95 \% \mathrm{Cl}-0.71$ to 0.64$)$.

\subsection{Gait velocity}

Three studies (292 participants), all investigating functional task training interventions, reported gait velocity, demonstrating no significant benefit of intervention compared with no treatment (SMD $0.05,95 \% \mathrm{Cl}-0.18$ to 0.28 ), with no statistical heterogeneity (12 $=0 \%)$.

\subsection{Length of stay}

Three studies (318 participants), all investigating functional task training plus musculoskeletal interventions, reported length of stay. Analysis demonstrated no statistically significant effect of the intervention on length of stay (MD $-2.85,95 \% \mathrm{Cl}-10.47$ to 4.76$)$, with very considerable heterogeneity $(12=96 \%)$.

\section{Comparison 2. Intervention versus attention control or usual care, immediate outcomes}

\subsection{Independence in ADL scale}

We pooled data from six studies (260 participants) in this analysis. For all six studies, the control intervention was categorised as 'usual care' and the intervention was given in addition to this usual care. Two studies each compared two active intervention groups with usual care; both active intervention groups were included in the analysis, with the control group participants 'shared' between these comparisons (Mudie 2002; Richards 1993). The meta-analysis found no evidence that the addition of the active intervention had any significant effect compared with usual care only (SMD 0.04, 95\% $\mathrm{Cl}-0.27$ to 0.35$)$, with low heterogeneity $(12=21 \%)$. See Analysis 2.1 .

\section{Sensitivity analysis: comparison group}

The comparison group was classed as usual care for five studies and as attention control for one study (Chen 2010). In two studies the usual care was categorised as comprising neurophysiological components (Pollock 1998; Richards 1993); in one study it was categorised as comprising functional task training and neurophysiological and musculoskeletal components (Duncan 1998). No details of the usual care were provided for Mudie 2002 or for Langhammer 2007. The attention control intervention in another study was Traditional Chinese Medicine (TCM) massage therapy (Chen 2010). Sensitivity analyses to explore the effects of these different types of 'usual care' or attention control found no effect on the direction of these results, although it was noted that the study with the TCM massage therapy comparison intervention 
(Chen 2010; 106 participants) did find a significant benefit of active intervention as compared with TCM massage therapy (SMD 0.53, $95 \% \mathrm{Cl} 0.14$ to 0.92 ). (These sensitivity analyses are not illustrated within forest plots.)

\section{Sensitivity analysis: risk of bias}

Removing studies judged to be at high risk of bias for at least one assessed quality of component led to the removal of Pollock 1998 but did not alter the direction of the results (SMD 0.03, 95\% $\mathrm{Cl}-0.32$ to 0.38 ); additionally removing Chen 2010, as these data were estimated from categorical data, did not alter the direction of the results (SMD $-0.19,95 \% \mathrm{Cl}-0.53$ to 0.15 ). (These sensitivity analyses are not illustrated within forest plots.) No further studies were removed by excluding those judged as unsure or high risk of bias for random sequence generation or allocation concealment.

\section{Subgroup analysis: time after stroke}

A significant difference between subgroups was noted according to time post stroke ( $P$ value 0.05 ), suggesting that a greater effect may occur with a smaller time after stroke. See Analysis 8.1.

\section{Subgroup analysis: study geographical location}

A significant difference between subgroups was noted based on study geographical location ( $P$ value 0.04 ). The only study with a positive effect was carried out in China. See Analysis 8.2.

\section{Subgroup analysis: dose of intervention}

Two studies (46 participants) provided daily intervention five to seven days per week for between 30 and 60 minutes; two studies (85 participants) provided two to three interventions per week and the dose was not stated in another three studies (see Table 11). When studies in which the dose was not stated were excluded (as it was not appropriate to include this group), no significant difference between subgroups was noted (P value 0.58 ) (analysis not shown). See Analysis 8.3.

Data were insufficient to enable sensitivity analyses to explore length of intervention. The length of the intervention period in the four studies that stated this information was four weeks (Pollock 1998), six weeks (Mudie 2002), eight weeks (Duncan 1998) and four three-month sessions (Langhammer 2007).

\section{Subgroup analysis: provider of intervention}

Four studies (124 participants) stated that the intervention was provided by a 'physiotherapist'; one study stated that it was a 'Bobath-trained physiotherapist.' In Duncan 1998 an occupational therapist also contributed. The provider was not stated in one study. When studies in which the provider was not stated were excluded (as it was not appropriate to include this group), no significant difference between subgroups was noted ( $P$ value 0.43 ) (analysis not shown). See Analysis 8.4.

\section{Subgroup analysis: treatment components included}

Six studies (244 participants) included functional task training components; three studies (54 participants) included neurophysiological training components and four studies (208 participants) included musculoskeletal components. No significant difference between these subgroups was reported ( $P$ value 0.58 ). See Analysis 8.5.

\subsection{Motor function scale}

We pooled data from 13 studies (967 participants) in this analysis. Each of two studies compared two active intervention groups with usual care; both active intervention groups were included in the analysis, and the control group participants were 'shared' between these comparisons (Cooke 2006; Richards 1993). Meta-analysis demonstrated a significant effect of intervention compared with usual care (SMD $0.42,95 \% \mathrm{Cl} 0.24$ to 0.61 ), with moderate heterogeneity $(12=42 \%)$.

Significant differences between the subgroups of different categories of treatment components were noted ( $P$ value 0.02), with some indication of greater effect when neurophysiological components were included.

For the subgroup of studies that combined functional task training and neurophysiological and musculoskeletal components (Analysis 2.2.6; four studies, 281 participants), a significant effect of intervention compared with usual care was reported (SMD 0.46, $95 \% \mathrm{Cl} 0.21$ to 0.70$)$, with no statistical heterogeneity $(12=0 \%)$. See Analysis 2.2.

\section{Sensitivity analysis: comparison group}

The comparison group was classed as usual care for 11 studies and as attention control for two studies. Usual care included both functional task training and neurophysiological components (plus other components) for four studies (Cooke 2006; Duncan 1998; Qian 2005; Tang 2009); functional task training (plus other components) for two studies (Duncan 2003; Kwakkel 2008); neurophysiological (with or without other components) for one study (Richards 1993); musculoskeletal (passive) for one study (Wang 2004b) and was not described for three studies (Langhammer 2007; McClellan 2004; Wei 1998). The attention control was TCM massage therapy for one study (Chen 2010) and a social intervention for another study (Mudge 2009). Sensitivity analyses to explore the effects of these different types of 'usual care' or attention control found no effect on the direction of these results. (These sensitivity analyses are not illustrated within forest plots.)

\section{Sensitivity analysis: risk of bias}

Removing studies judged to be at high risk of bias for at least one assessed quality of component led to the removal of Duncan 1998, Duncan 2003 and Qian 2005, and left data from nine studies (733 participants), which did not alter the direction of the results (SMD 0.31, 95\% Cl 0.13 to 0.49); additionally removing Mudge 2009 (as these data were estimated from the range) and Chen 2010 (as these data were estimated from categorical data) did not alter the direction of the results (seven studies, 569 participants; SMD 0.31, $95 \% \mathrm{Cl} 0.10$ to 0.53$)$.

However, removing all studies judged as unclear or high risk of bias for random sequence generation or allocation concealment led to the removal of Chen 2010, Kwakkel 2008, Qian 2005, Tang 2009, Wang 2004b and Wei 1998, leaving seven studies (377 participants) and demonstrating no significant effect of intervention compared with usual care or control (SMD $0.17,95 \% \mathrm{Cl}-0.04$ to 0.38 ), with no statistical heterogeneity $\left(I^{2}=0 \%\right)$. (These sensitivity analyses are not illustrated within forest plots.) 


\section{Subgroup analysis: time after stroke}

No statistically significant difference between subgroups was noted according to time post stroke (P value 0.34 ). See Analysis 10.1.

\section{Subgroup analysis: study geographical location}

Five studies (348 participants) were carried out in China; three (405 participants) in Europe; three (75 participants) in North America and Canada and two (79 participants) in Austalia and New Zealand. A significant difference between these subgroups was reported $(P$ value 0.002 ), with studies carried out in China having a greater effect size. See Analysis 10.2.

\section{Subgroup analysis: dose of intervention}

No studies provided the intervention more than once per day; four studies (242 participants) provided daily intervention five to seven days per week for between 30 and 60 minutes; four studies (269 participants) provided intervention three or four times per week and three studies (327 participants) provided intervention twice weekly (see Table 11). The dose was not stated in another two studies. When studies in which the dose was not stated were excluded (as it was not appropriate to include this group), a significant difference between subgroups was noted ( $P$ value 0.002 ) (analysis not shown), with a greater effect size in studies with a greater dose of intervention. No significant difference between the subgroup of three to four interventions per week and the subgroup with one to two interventions per week was reported ( $P$ value 0.39 ) (analysis not shown). See Analysis 10.3.

\section{Sensitivity analysis: dose and duration of intervention period}

The effect of the length of the intervention period was explored by ordering studies within Analysis 9.3 from least to most intervention (with studies in which length of intervention is not stated at the 'most' end). One study did not state the length of intervention (Qian 2005) and was removed from the analyses. Four studies (228 participants) (Cooke 2006; McClellan 2004; Mudge 2009; Wang 2004b) had a length of intervention of four to six weeks; including only these studies leads to a non-significant effect (SMD 0.22, $95 \% \mathrm{Cl}-0.08$ to 0.51$)$, with low heterogeneity $\left({ }^{2}=14 \%\right)$. Five studies (504 participants) (Duncan 1998; Duncan 2003; Kwakkel 2008; Tang 2009; Wei 1998) had a length of intervention of eight to 14 weeks; including only these studies demonstrates a significant effect of intervention (SMD $0.57,95 \% \mathrm{Cl} 0.34$ to 0.80 ), with moderate heterogeneity $(12=31 \%)$. (These sensitivity analyses are not illustrated within forest plots.)

\section{Subgroup analysis: provider of intervention}

Eight studies (619 participants) stated that the intervention was provided by a 'physiotherapist'; two stated the provider was a 'therapist' and one a 'nurse.' In Duncan 1998 and Duncan 2003, an occupational therapist also contributed; in Kwakkel 2008 a sports therapist contributed and in Mudge 2009, physiotherapy students contributed. In Cooke 2006 the provider was described as a 'research physiotherapist.' The provider was not stated in two studies. When studies in which the provider was not stated were excluded (as it was not appropriate to include this group), a significant difference between subgroups was reported ( $P$ value 0.02) (analysis not shown), but the low number of studies in some groups makes it difficult to determine the direction of effect. See Analysis 10.4.

\section{Subgroup analysis: treatment components included}

Eleven studies (827 participants) included functional task training components; eight studies (467 participants) included neurophysiological training components and 10 studies (818 participants) included musculoskeletal components. No significant difference between these subgroups was noted (P value 0.12 ). See Analysis 10.5.

\subsection{Balance (Berg Balance Scale)}

We pooled the data from five studies (246 participants) in this analysis. One study compared two active intervention groups with usual care; both active intervention groups were included in the analysis, with control group participants 'shared' between these comparisons (Richards 1993).

Meta-analysis demonstrated a significant effect of intervention compared with usual care (SMD $0.31,95 \% \mathrm{Cl} 0.05$ to 0.56 ), with no statistical heterogeneity $\left(I^{2}=0 \%\right)$.

No significant differences between the subgroups of different categories of treatment components were reported ( $P$ value 0.90$)$. See Analysis 2.3.

\section{Sensitivity analysis: comparison group}

The comparison group was classed as usual care for four studies and as attention control for one study. The usual care included functional task training and neurophysiological components in one study (Duncan 1998), functional task training (plus other components) in two studies (Duncan 2003; Kim 2012) and only neurophysiological components in one study (Richards 1993). The attention control comprised upper limb training (Salbach 2004). Sensitivity analyses to explore the effects of these different types of 'usual care' or attention control found no effect on the direction of these results. (These sensitivity analyses are not illustrated within forest plots.)

\section{Sensitivity analysis: risk of bias}

Removing studies judged to be at high risk of bias for at least one assessed quality of component led to the removal of all studies apart from Richards 1993 and did not demonstrate a significant effect (SMD 0.40, 95\% Cl -0.48 to 1.28). However, removing all studies judged as unclear or high risk of bias for random sequence generation or allocation concealment led to the removal of only Kim 2012 and had no effect on the direction of the results. (These sensitivity analyses are not illustrated within forest plots.)

\subsection{Gait velocity}

We pooled data from 14 studies (1126 participants) in this analysis. Two studies compared two active intervention groups with usual care; both active intervention groups were included in the analysis, with control group participants 'shared' between these comparisons (Cooke 2006; Richards 1993).

Meta-analysis demonstrated a significant effect of intervention compared with usual care (SMD $0.46,95 \% \mathrm{Cl} 0.32$ to 0.60 ), with little heterogeneity $\left(1^{2}=14 \%\right)$.

No significant differences between the subgroups of different categories of treatment components were reported ( $P$ value 0.86 ). See Analysis 2.4. 


\section{Sensitivity analysis: comparison group}

The comparison group was classed as usual care for seven studies and as attention control for seven studies. The usual care included functional task training and musculoskeletal and neurophysiological components in two studies (Cooke 2006; Duncan 1998), functional task training and musculoskeletal components for three studies (Behrman 2011; Duncan 2003; Kim 2012); functional task training and musculoskeletal and cardiopulmonary components for one study (Kwakkel 2008) and only neurophysiological components for one study (Richards 1993). The attention control comprised upper limb training for three studies (Blennerhassett 2004; Dean 2000; Dean 2006); cognitive training for two studies (Dean 1997; Dean 2007) and a social intervention for one study (Mudge 2009). Sensitivity analyses to explore the effects of these different types of 'usual care' or attention control found no effect on the direction of these results. When only the studies with attention control comparisons were included, seven studies (251 participants) found a significant effect in favour of the intervention compared with attention control (SMD $0.41,95 \% \mathrm{Cl} 0.15$ to 0.67$)$, with low heterogeneity $(12=20 \%)$. When only the studies with usual care comparisons were included, seven studies (775 participants) also found a significant effect in favour of the intervention compared with usual care (SMD 0.50, 95\% Cl 0.34 to 0.67$)$, with low heterogeneity $(12=9 \%)$. (These sensitivity analyses are not illustrated within forest plots.)

\section{Sensitivity analysis: risk of bias}

Removing studies judged to be at high risk of bias for at least one assessed quality of component led to the removal of Dean 1997, Dean 2000, Duncan 1998, Duncan 2003 and Kim 2012, leaving data from eight studies (876 participants), which did not alter the direction of the results (SMD $0.53 .95 \% \mathrm{CI} 0.39$ to 0.67 ); additionally removing Mudge 2009 (as these data were estimated from the range) did not alter the direction of the results (seven studies, 818 participants; SMD 0.52, $95 \% \mathrm{Cl} 0.35$ to 0.68 ).

Removing all studies judged as unclear or high risk of bias for random sequence generation or allocation concealment led to the removal of Behrman 2011, Dean 1997, Dean 2000, Kim 2012 and Kwakkel 2008, leaving nine studies (568 participants), and did not alter the direction of the results (SMD $0.42,95 \% \mathrm{Cl} 0.25$ to 0.59 ). (These sensitivity analyses are not illustrated within forest plots.)

\subsection{Length of stay}

Two studies (105 participants), both investigating functional task training plus musculoskeletal interventions, reported length of stay. This analysis demonstrated no statistically significant effect of intervention on reported length of stay (MD $-10.36,95 \% \mathrm{Cl}-48.09$ to 27.36), with substantial heterogeneity $\left(I^{2}=83 \%\right)$. See Analysis 2.5 .

\section{Comparison 3. One active intervention versus another active intervention, immediate outcomes}

\subsection{Independence in ADL scale}

\subsubsection{Includes functional task training versus does not include functional task training}

Four studies (186 participants) compared a group receiving an intervention that contained functional task training components with a group receiving an alternative intervention. In all four studies, the alternative intervention comprised neurophysiological components (Langhammer 2000; Lincoln 2003; Mudie 2002;
Richards 1993). Three of the studies investigated only functional task training components (Langhammer 2000; Lincoln 2003; Mudie 2002), and one investigated functional task training plus musculoskeletal components and modalities (Richards 1993). This analysis demonstrated no significant differences between interventions comprising the different types of components (SMD $-0.03,95 \% \mathrm{Cl}-0.37$ to 0.32$)$, with low heterogeneity $(\mathrm{I}=19 \%)$. Sensitivity analysis to remove the one study with additional components (Richards 1993) did not change the direction of the results. See Analysis 3.1.

\section{Subgroup analysis: functional task training components}

Analysis 11.1 explores the effect of different functional task training components. No significant differences between these subgroups were reported (P value 0.59).

\subsubsection{Includes neurophysiological versus does not include neurophysiological}

Seven studies (451 participants) compared a group receiving an intervention that contained neurophysiological components with a group that received an alternative intervention. Five of the studies investigated the effect of neurophysiological components only (Langhammer 2000; Lincoln 2003; Mudie 2002; Richards 1993; Zhuang 2012); one of the studies investigated neurophysiological components combined with functional task training, modalities and musculoskeletal (passive) (Li 2005) and one investigated neurophysiological plus functional task training (Gelber 1995). In all seven studies, the neurophysiological component included components described as 'Bobath.' In six of the studies, the alternative intervention included functional task training: functional task training only in three studies (Langhammer 2000; Lincoln 2003; Mudie 2002); functional task training plus musculoskeletal components in two studies (Gelber 1995; Richards 1993) and functional task training plus modalities in one study ( $\mathrm{Li}$ 2005). In one study the alternative intervention was a modality (acupuncture) (Zhuang 2012). This analysis demonstrated no significant differences between interventions, which did or did not include neurophysiological/Bobath treatment (SMD -0.02, 95\% CI -0.26 to 0.22$)$, with low heterogeneity $(12=28 \%)$. Sensitivity analyses to explore the effects of different comparison components did not change the direction of the results. We preplanned sensitivity analysis to explore the effects of including Zhuang 2012, as the alternative intervention group did not receive active physical rehabilitation in addition to acupuncture; removing this study did not lead to significant differences in the direction of the results. See Analysis 3.1.

\section{Subgroup analysis: neurophysiological components}

Analysis 11.2 explores the effects of different neurophysiological components. No significant differences between these subgroups were noted ( $P$ value 0.45$)$.

\subsubsection{Includes musculoskeletal versus does not include musculoskeletal}

Three studies (103 participants) compared a group that received an intervention containing musculoskeletal components with a group that received an alternative intervention. All three studies combined musculoskeletal components with components from other categories. Gelber 1995 and Richards 1993 combined both active and passive musculoskeletal components with functional task training and modalities or assistive devices. In both of 
these studies, the musculoskeletal components included muscle strengthening. Li 2005 implemented only passive musculoskeletal components (passive movement and body positioning) combined with functional task training, neurophysiological and modalities. The alternative intervention comprised only neurophysiological components in one study (Richards 1993); functional task training and neurophysiological in one study (Gelber 1995) and functional task training and modality in one study (Li 2005). This analysis demonstrated no significant differences between interventions that did or did not include musculoskeletal components (SMD -0.12, $95 \% \mathrm{Cl}-0.58$ to 0.34$)$, with low heterogeneity $(12=21 \%)$. Sensitivity analyses to explore the effects of different components did not change the direction of the results. See Analysis 3.1.

\section{Subgroup analysis: musculoskeletal components}

Analysis 11.3 explores the effects of different musculoskeletal components. No significant differences between these subgroups were reported (P value 0.11 ). However, this finding is based on a low number of studies.

\section{Sensitivity analysis: risk of bias}

Removing studies judged to be at high risk of bias for at least one assessed quality of component led to the removal of Gelber 1995 and Li 2005 and did not alter the direction of the results for any of the subgroups.

Removing studies judged as unclear or high risk of bias for random sequence generation or allocation concealment led to the removal of Li 2005 and did not alter the direction of the results. (These sensitivity analyses are not illustrated within forest plots.)

\subsection{Motor function scale}

\subsubsection{Includes functional task training versus does not include} functional task training

Four studies (188 participants) compared a group receiving an intervention that contained functional task training components with a group that received an alternative intervention. In all four studies, the alternative intervention comprised neurophysiological components (Langhammer 2000; Lincoln 2003; Richards 1993; Wang 2005). Two of the studies investigated only functional task training components (Langhammer 2000; Lincoln 2003); one investigated functional task training plus musculoskeletal components (Wang 2005) and one investigated functional task training plus musculoskeletal components and modalities (Richards 1993). This analysis demonstrated no significant differences between interventions comprising the different types of components (SMD $-0.16,95 \% \mathrm{Cl}-0.59$ to 0.28 ), with moderate heterogeneity $(12=45 \%)$. Sensitivity analyses to explore the effects of different components did not change the direction of the results. See Analysis 3.2.

\section{Subgroup analysis: functional task training components}

Analysis 12.1 explores the effects of different functional task training components. No significant differences between these subgroups were reported ( $P$ value 0.48 ).

\subsubsection{Includes neurophysiological versus does not include neurophysiological}

Eight studies (506 participants) compared a group that received an intervention containing neurophysiological components with a group that received an alternative intervention. Five of the studies investigated the effect of neurophysiological components only (Langhammer 2000; Lincoln 2003; Richards 1993; Wang 2005; Zhuang 2012); two of the studies investigated neurophysiological components combined with functional task training (Bale 2008; Gelber 1995) and one investigated neurophysiological components combined with functional task training and passive musculoskeletal components (Liao 2006). In all eight studies, the neurophysiological component included components described as 'Bobath.' In seven of the studies, the alternative intervention included functional task training: functional task training only in two studies (Langhammer 2000; Lincoln 2003); functional task training plus musculoskeletal components in three studies (Bale 2008; Liao 2006; Wang 2005) and functional task training plus musculoskeletal components and modalities or assistive devices in two studies (Gelber 1995; Richards 1993). In one study the alternative intervention was a modality (acupuncture) (Zhuang 2012). This analysis demonstrated no significant differences between interventions that did or did not include neurophysiological or Bobath treatment (SMD 0.17, 95\% Cl -0.05 to 0.39$)$, with low heterogeneity $\left(I^{2}=24 \%\right)$. Sensitivity analyses to explore the effects of different comparison components did not change the direction of the results. We preplanned a sensitivity analysis to explore the effect of including Zhuang 2012, as the alternative intervention group did not receive active physical rehabilitation in addition to acupuncture; removing this study did not result in significant differences in the direction of the results. See Analysis 3.2.

\section{Subgroup analysis: neurophysiological components}

Analysis 12.2 explores the effects of different neurophysiological components. No significant differences between these subgroups were reported ( $P$ value 0.76$)$.

\subsubsection{Includes musculoskeletal versus does not include musculoskeletal}

Four studies (81 participants) compared a group that received an intervention containing musculoskeletal components with a group that received an alternative intervention. All four studies combined musculoskeletal components with components from other categories. Bale 2008 combined active musculoskeletal components with functional task training, and Gelber 1995, Richards 1993 and Wang 2005 combined both active and passive musculoskeletal components with functional task training (with or without modalities or assistive devices). In all four studies, the musculoskeletal components included muscle strengthening. The alternative intervention comprised only neurophysiological in two studies (Richards 1993; Wang 2005) and functional task training and neurophysiological in two studies (Bale 2008; Gelber 1995). This analysis demonstrated no significant differences between interventions that did or did not include musculoskeletal components (SMD $-0.08,95 \% \mathrm{Cl}-0.53$ to 0.36 ), with no statistical heterogeneity $(12=0 \%)$. Sensitivity analyses to explore the effects of different components did not change the direction of the results. See Analysis 3.2.

\section{Subgroup analysis: musculoskeletal components}

Analysis 12.3 explores the effects of different musculoskeletal components. No significant differences between these subgroups were reported (P value 0.15 ). However, this finding is based on a low number of studies. 


\section{Sensitivity analysis: risk of bias}

No studies were judged to be at high risk of bias for at least one assessed quality component. Removing Bale 2008, as these data were estimated from categorical data, did not alter the direction of the results.

Removing studies judged as unclear or high risk of bias for random sequence generation or allocation concealment led to the removal of Bale 2008, Gelber 1995 and Liao 2006 from the analyses but did not alter the direction of the results. (These sensitivity analyses are not illustrated within forest plots.)

\subsection{Balance (Berg Balance Scale)}

Four studies (83 participants) compared one active intervention with another active intervention and reported a measure of balance (Brock 2005; Richards 1993; Shin 2011; Wang 2005). No significant differences were found for comparisons of interventions containing different categories of treatment components (see Analysis 3.3).

\subsection{Gait velocity}

\subsubsection{Includes functional task training versus does not include} functional task training

Three studies (73 participants) compared a group receiving an intervention that contained functional task training components with a group that received an alternative intervention. In all three studies, the alternative intervention comprised neurophysiological components (Lincoln 2003; Richards 1993; Verma 2011). Two of the studies investigated only functional task training components (Lincoln 2003; Verma 2011), and one investigated functional task training plus musculoskeletal components and modalities (Richards 1993). This analysis demonstrated no significant differences between interventions comprising the different types of components (SMD $0.43,95 \% \mathrm{Cl}-0.37$ to 1.22 ), with substantial heterogeneity $(12=73 \%)$. Sensitivity analyses to explore the effects of different components did not change the direction of the results. See Analysis 3.4.

\subsubsection{Includes neurophysiological versus does not include neurophysiological}

Seven studies (278 participants) compared a group receiving an intervention that contained neurophysiological components with a group that received an alternative intervention. Four of the studies investigated the effect of neurophysiological components only (Lincoln 2003; Richards 1993; Thaut 2007; Verma 2011); two studies investigated neurophysiological components combined with functional task training (Bale 2008; Gelber 1995) and one investigated neurophysiological components combined with functional task training and cardiovascular training components (Brock 2005). In all seven studies, the neurophysiological component included components described as 'Bobath.' In all of the studies, the alternative intervention included functional task training: functional task training only in three studies (Lincoln 2003; Thaut 2007; Verma 2011); functional task training plus musculoskeletal components in one study (Bale 2008); functional task training plus musculoskeletal components and modalities or assistive devices in two studies (Gelber 1995; Richards 1993) and functional task training plus cardiovascular training components in one study (Brock 2005). Analysis demonstrated no significant differences between interventions that did or did not include neurophysiological or Bobath treatments (SMD $-0.12,95 \% \mathrm{Cl}-0.95$ to 0.70$)$, with substantial heterogeneity $\left({ }^{2}=89 \%\right)$. Sensitivity analyses to explore the effects of different comparison components did not change the direction of the results. See Analysis 3.4.

\subsubsection{Includes musculoskeletal versus does not include musculoskeletal}

Three studies (45 participants) compared a group receiving an intervention that contained musculoskeletal components with a group that received an alternative intervention (Bale 2008; Gelber 1995; Richards 1993). Analysis demonstrated no significant differences between interventions that did or did not include musculoskeletal components (SMD $-0.47,95 \% \mathrm{Cl}-1.67$ to 0.74 ), with substantial heterogeneity $\left(I^{2}=71 \%\right)$. Sensitivity analyses to explore the effects of different components did not change the direction of the results. See Analysis 3.4.

\subsection{Length of stay}

3.5.1. Includes functional task training versus does not include functional task training

One study (53 participants) compared a group receiving an intervention containing functional task training components with a group that received an alternative intervention. This study demonstrated that the functional task training intervention resulted in a reduced length of stay. See Analysis 3.5.

\subsubsection{Includes neurophysiological versus does not include neurophysiological}

Three studies (141 participants) compared a group receiving an intervention that contained neurophysiological components with a group that received an alternative intervention. This analysis demonstrated a significantly reduced length of stay (MD 11.36, $95 \% \mathrm{Cl} 1.52$ to 21.19 ) for the groups that did not receive the neurophysiological components, with substantial heterogeneity $(12$ $=74 \%)$. See Analysis 3.5 .

\subsubsection{Includes musculoskeletal versus does not include musculoskeletal}

Two studies (88 participants) compared a group receiving an intervention that contained musculoskeletal components with a group that received an alternative intervention. This analysis demonstrated no significant differences between interventions that did or did not include musculoskeletal components (MD 8.71, $95 \% \mathrm{Cl}-12.92$ to 30.34 ), with considerable heterogeneity ( $12=91 \%)$. It should be noted that both of these studies are also included in comparison 3.5.2, but that Gelber 1995 compared musculoskeletal components with neurophysiological components, whilst Li 2005 combined musculoskeletal and neurophysiological components. See Analysis 3.5.

\section{Comparison 4. Intervention versus no treatment, persisting outcomes}

\subsection{Independence in $A D L$ scale}

We pooled data from nine studies (540 participants) in this analysis, which demonstrated that intervention had a significantly beneficial effect compared with no intervention (SMD $0.58,95 \% \mathrm{Cl} 0.11$ to 1.04). However, substantial heterogeneity was found $(12=83 \%)$. See Analysis 4.1.

Significant differences between the subgroups of different categories of treatment components were found ( $P$ value 0.0002 ). 
These results are similar to the results for immediate Independence in ADL outcomes (Analysis 1.1).

Sensitivity analysis to explore the effects of studies with high or uncertain risk of bias did not alter the direction of the results.

\subsection{Motor function scale}

We pooled data from eight studies (1829 participants) in this analysis, which demonstrated that intervention had a significantly beneficial effect compared with no treatment (SMD 1.06, 95\% $\mathrm{Cl} 0.37$ to 1.75$)$, with very considerable heterogeneity $(12=$ $97 \%)$. Significant differences between the subgroups of different categories of treatment components were reported ( $P$ value 0.002 ). See Analysis 4.2.

Sensitivity analysis to explore the effects of studies with high or uncertain risk of bias demonstrated that the significant effect was not maintained if studies with high or uncertain risk of bias were removed (SMD 1.67, 95\% $\mathrm{Cl}-0.25$ to 3.59 ).

\subsection{Balance (Berg Balance Scale)}

Only one study (Holmgren 2006) reported follow-up data for balance outcomes (see Analysis 4.3).

\subsection{Gait velocity}

The three studies that reported immediate outcomes for gait velocity also reported follow-up outcomes. Similar to the analysis of immediate outcomes, no statistically significant effect was found for intervention versus no treatment (SMD $-0.06,95 \% \mathrm{Cl}-0.29$ to 0.18). See Analysis 4.4.

\section{Comparison 5. Intervention versus attention control or usual care, persisting outcomes}

\subsection{Independence in ADL scale}

No studies comparing intervention with control or usual care reported a follow-up outcome for an independence in ADL scale.

\subsection{Motor function scale}

We pooled data from three studies (160 participants) in this analysis, which demonstrated no significant differences between intervention and control (SMD $-0.10,95 \% \mathrm{Cl}-0.42$ to 0.23 ), with no statistical heterogeneity $\left(I^{2}=0 \%\right)$. See Analysis 5.2 .

\subsection{Balance (Berg Balance Scale)}

No studies comparing intervention with control or usual care reported a follow-up outcome for the Berg Balance Scale.

\subsection{Gait velocity}

We pooled data from five studies (214 participants) in this analysis, which demonstrates that intervention had a significantly beneficial effect compared with usual care or control (SMD 0.38, 95\% CI 0.10 to 0.66$)$, with no statistical heterogeneity $\left(I^{2}=0 \%\right)$. See Analysis 5.4.

This result is similar to the results for the immediate gait velocity outcomes (Analysis 1.4).

\section{Comparison 6. One active intervention versus another} active intervention, persisting outcomes

\subsection{Independence in ADL scale}

\subsubsection{Includes functional task training versus does not include} functional task training

One study (Verma 2011; 30 participants) compared a group receiving an intervention that contained functional task training components with a group that received an alternative intervention. This study did not contribute data to the analysis of immediate outcomes, as only follow-up data were provided. Follow-up was at six weeks, after a two-week intervention period. The data suggest a significant benefit of functional task training (SMD 1.33, 95\% Cl 0.52 to 2.13). See Analysis 6.1.

\subsubsection{Includes neurophysiological versus does not include neurophysiological}

Two studies (57 participants) compared a group receiving an intervention that contained neurophysiological components with a group that received an alternative intervention. This analysis demonstrated a significant detrimental effect of the intervention that included neurophysiological or Bobath treatments (SMD -0.95, $95 \% \mathrm{Cl}-1.67$ to -0.22$)$.See Analysis 6.1.

\subsubsection{Includes musculoskeletal versus does not include musculoskeletal}

One study (27 participants) compared a group receiving an intervention that contained musculoskeletal components with a group that received an alternative intervention. The data showed no significant differences between groups that did and did not receive musculoskeletal components (SMD $0.58,95 \% \mathrm{Cl}-0.19$ to 1.36). See Analysis 6.1.

\section{Subgroup analysis}

A statistically significant difference was reported between subgroups including different types of components ( $P$ value 0.0001 ), with an indication of greater beneficial effect of interventions that included functional task training or musculoskeletal components.

\subsection{Motor function scale}

No studies comparing two different active interventions reported a follow-up outcome for a motor function scale.

\subsection{Balance (Berg Balance Scale)}

No studies comparing two different active interventions reported a follow-up outcome for the Berg Balance Scale.

\subsection{Gait velocity}

6.4.1. Includes functional task training versus does not include functional task training

One study (Verma 2011; 30 participants) compared a group receiving an intervention that contained functional task training components with a group that received an alternative intervention containing neurophysiological components. This study did not contribute data to the analysis of immediate outcomes, as only follow-up data were provided. Follow-up was at six weeks, after a two-week intervention period. The data suggest significant benefits of functional task training (SMD $1.14,95 \% \mathrm{Cl} 0.36$ to 1.92). See Analysis 6.4. 


\subsubsection{Includes neurophysiological versus does not include} neurophysiological

Two studies (43 participants) compared a group receiving an intervention that contained neurophysiological components with a group that received an alternative intervention (containing functional task training for Verma 2011 and functional task training plus musculoskeletal components for Gelber 1995). This analysis demonstrated a significant detrimental effect of the intervention that included neurophysiological or Bobath treatments (SMD -0.82, $95 \% \mathrm{Cl}-1.60$ to -0.05$)$. See Analysis 6.4 .

\subsubsection{Includes musculoskeletal versus does not include musculoskeletal}

One study (14 participants) compared a group that received an intervention containing musculoskeletal components with a group that received an alternative intervention, which contained neurophysiological components. The data show no significant differences between groups that did and did not receive musculoskeletal components (SMD 0.33, 95\% Cl -0.74 to 1.40 ). See Analysis 6.4.

\section{Subgroup analysis}

A statistically significant difference was noted between the subgroups including different types of components (P value 0.002), with an indication of greater beneficial effect of interventions that included functional task training or musculoskeletal components.

\section{DISCUSSION}

\section{Key findings}

This review included 96 studies (10,401 participants) that explored the effects of different physical rehabilitation approaches. More than half of the studies (50/99) were carried out in China. Fifty-one studies compared a physiotherapy intervention with no treatment; 42 of these studies were carried out in China. Twenty-seven studies compared a physiotherapy intervention with usual care or attention control. Twenty-four studies compared two different active physical rehabilitation approaches. Data were available for meta-analysis from 34 studies comparing intervention with no treatment; 16 studies comparing intervention with usual care or attention control and 14 studies comparing two different active interventions. Key findings arising from meta-analyses were as follows.

\section{Intervention versus no treatment}

1. Moderate-quality evidence showed a beneficial effect of physical rehabilitation on measures of independence in ADL and motor function. This finding was sustained at follow-up assessments, although the size of the benefit was reduced. Quality of reporting of studies within this comparison was generally poor, and risk of bias was frequently unclear for key methodological criteria.

2. There was insufficient evidence to support conclusions relating to the effect of physical rehabilitation on balance, gait velocity or length of stay.

3. A significant difference between subgroups based on time since stroke was noted, with an indication of benefit associated with shorter time since stroke.

4. A significant difference between subgroups based on geographical location was reported, but most studies were carried out in China with participants who were within 30 days post stroke. All studies carried out in China were assessed at high or unclear risk of bias.

5. A significant difference between subgroups based on dose of intervention was noted, with an indication that a dose of between 30 and 60 minutes once per day for five to seven days a week was beneficial, but that more than once-daily intervention may provide even greater benefit. It was not possible to draw conclusions relating to duration of the intervention period, with substantial heterogeneity within analyses.

6. Significant differences between subgroups based on provider of intervention were noted, but it is difficult to reach generalised conclusions from these subgroup analyses.

7. Results of the subgroup analyses must be interpreted with caution, as a complex interrelationship between some of the subgroups is likely. For example, studies with the least time since stroke were carried out in China, meaning that reported effects attributed to geographical location may be related to time since stroke (and vice versa).

8. No significant differences were noted between studies that investigated different components or categories of intervention.

In summary, moderate-quality evidence indicates that physical rehabilitation has a beneficial effect on independence in ADL and motor function after stroke, and that this effect persists beyond the end of the intervention period, when compared with no treatment. Evidence shows greater benefit associated with a shorter time since stroke. Evidence also suggests that a dose of 30 to 60 minutes per day delivered five to seven days per week is effective, and that more frequent or increased doses may provide even greater benefit. Substantial heterogeneity was observed between the studies included in these analyses, and most of the studies were at high or uncertain risk of bias.

This evidence principally arises from China, where a particular healthcare system and cultures and beliefs are associated with health and disease. In China, physiotherapy or rehabilitation traditionally has not been routinely provided within acute hospital settings; therefore this evidence is highly relevant to stroke care settings in China. Arguably this evidence does not have any direct implications for settings in which no treatment would not be considered to be an ethical alternative for hospitalised patients with stroke, but the indirect implications of this evidence base may have universal relevance. Evidence suggests that 30 to 60 minutes of physical rehabilitation per day, delivered five to seven days per week, is beneficial for recovery of function, but that no one individual approach to physical rehabilitation is better than any other approach.

\section{Intervention versus usual care or attention control}

1. Moderate- to high-quality evidence shows a beneficial effect of physical rehabilitation on measures of motor function, balance and gait velocity. Moderate-quality evidence also shows that this beneficial effect was maintained at follow-up for gait velocity, but insufficient data were available at follow-up to permit conclusions for other outcomes.

2. No evidence was found of any benefit of intervention on measures of independence in ADL, but relatively few studies included data for this outcome.

3. A significant difference between subgroups was noted based on time since stroke, with an indication of benefit associated with 
a shorter time since stroke. No evidence of this effect was seen in follow-up data.

4. For measures of motor function, a significant difference between subgroups based on dose of intervention was observed, with an indication that a dose of 30 to 60 minutes five to seven days per week was significantly more beneficial than an intervention delivered three to four times per week. No difference between subgroups was described for measures of independence in ADL. It was not possible to draw conclusions relating to duration of the intervention period, with substantial heterogeneity within analyses.

5. No significant differences were reported between studies that investigated different components or categories of intervention.

In summary, moderate- to high-quality evidence shows that physical rehabilitation is more effective than usual care or attention control in improving motor function, balance and gait velocity. Evidence suggests greater benefit associated with a shorter time since stroke. Evidence also suggests that a dose of 30 to 60 minutes delivered five to seven days a week provides significant benefit. In particular, high-quality evidence indicates that physical rehabilitation has an impact on gait, with significant increases in gait velocity maintained at follow-up assessments. Some evidence suggests that benefit may be greater if rehabilitation is carried out earlier after stroke, but these findings should be interpreted cautiously. As with the comparison of intervention versus no treatment, this evidence suggests that no one individual approach to physical rehabilitation is better than any other approach for recovery of function or mobility.

\section{One active intervention versus another active intervention}

1. Moderate-quality evidence shows no difference between interventions that include neurophysiological components and interventions that do not include neurophysiological components. This evidence primarily arose from interventions that were described as 'Bobath.' Very limited evidence indicates that interventions including neurophysiological components resulted in a longer hospital stay.

2. Low-quality evidence shows no differences between interventions that include components of functional task training and interventions that do not include components of functional task training. No evidence suggests that any specific functional task training components are more effective than other interventions.

3. Low-quality evidence shows no differences between interventions that include musculoskeletal components and interventions that do not include musculoskeletal components. No evidence suggests that any specific musculoskeletal components are more effective than other interventions.

In summary, evidence suggests that no one physical rehabilitation approach is more effective in promoting recovery of function or mobility after stroke than any other approach. These findings are supported by the subgroup analyses carried out for the comparisons of intervention versus no treatment or usual care, which found no significant effects of different treatment components or categories of intervention.

\section{Physical rehabilitation approaches and components synthesised within this review}

This review synthesises evidence relating to the effectiveness of different physical rehabilitation approaches. The original focus of the comparisons within this review (2007 and earlier versions) was the effectiveness of different named approaches to physiotherapy, based on a historical perspective. The original review was carried out in direct response to a consultation exercise conducted in Scotland that aimed to identify the 'burning questions' of Scottish stroke rehabilitation workers, and that identified 'different (named) treatment approaches' to be amongst the most important questions posed by physiotherapists (Legg 2000). Following consultation with key stakeholders (physiotherapists, stroke survivors and carers), the focus for this update of the review was determined to be the individual treatment components that constitute physical rehabilitation approaches. This is an important, albeit arguably subtle, shift, enabling synthesis of evidence based on different philosophies and from different cultures, with systematic categorisation of individual treatment components, regardless of their philosophical or theoretical origin. This change in focus is in line with recommendations made within the 2007 version of this review.

The studies within this review included 121 active interventions; most of these (99 interventions) included treatment components categorised as functional task training. Most of the interventions incorporated treatment components from at least two different categories of intervention, with only 33 interventions focusing on just one category of intervention (20 interventions focused on functional task training only; 13 focused on neurophysiological interventions only). The finding that most studies include a combination of different treatment components, generally arising from at least two of the different categories defined for this review, highlights this fact: Physiotherapists appear to be basing their interventions not on one single historical or philosophical approach, but rather on a pragmatic eclectic approach that utilises a range of different treatment components, often regardless of their historical or philosophical origins. This pragmatic approach, which adopts a mix of components from different approaches, was supported by the previous version of this review, which concluded that a mix of components from different approaches was significantly more effective than no treatment or placebo in recovery of functional independence following stroke, and provides justification for the decision to change the focus of the review for this update.

\section{Identification of relevant trials}

The identification of all relevant trials was confounded by several factors.

1. Inconsistent and poorly defined terminology: Electronic searching was difficult because the names given to different physiotherapy rehabilitation approaches are poorly documented, often have several derivations and have varied over time. Furthermore, the interventions were not always described as 'physiotherapy' or 'physical therapy,' but sometimes were described as 'rehabilitation,' 'training' or 'exercise.' This was particularly true for studies emerging from China, which frequently described interventions as 'early rehabilitation.' Studies investigating circuit training or exercise classes sometimes met the inclusion criteria for this review; 
again, identification of these was made difficult by lack of use of the term 'physiotherapy' or 'physical therapy.'

2. Change in focus of the review: As described above, for this update of the review, a subtle change in focus was applied-from 'named' rehabilitation approaches to the individual treatment components that constitute physical rehabilitation approaches. No change to the search strategy or to selection criteria was implemented, and we do not believe that any changes would be justified. It could be argued that decisions made relating to the exclusion of studies from the search results for previous versions of this review may be different in light of the changed focus. However, selection of relevant trials for this review has always been challenging, and we do not believe that the change in focus of the review has affected study selection in one direction or another.

3. Lack of detail within the abstracts: Lack of information on study methods, participants and interventions potentially increases the chance that a relevant trial may be excluded. However, when uncertainty arose, we obtained full papers.

4. Material published in journals not included in electronic databases, and unpublished material: Although substantial effort was made to identify unpublished material and material in journals not cited in the databases searched, relevant trials may not have been identified.

5. Material published in Chinese: A substantial number of the included studies were carried out in China and were published in Chinese. Our electronic searching successfully identified studies for which an abstract was available in Chinese, as well as a number of studies based on English titles. However, we believe it is likely that we will not have identified all relevant Chinese trials, in particular those for which only English titles were available and those not published in journals included in the electronic databases that we searched.

6. Different cultures and healthcare systems: Decisions were made to include some studies in which the provider of the intervention was not clearly a physiotherapist or a physical therapist; these decisions were often related to the fact that physiotherapists may not be routinely found in all healthcare settings around the world. This provided additional challenges in relation to determining whether a study was investigating a 'physical rehabilitation' approach. In particular, many studies in China simply referred to a 'therapist,' but in some instances, the provider was a doctor or a nurse. We carried out subgroup analyses to explore the effect of the stated intervention provider on outcome. However, many studies did not explicitly state this, which limited conclusions that could be made from this subgroup analysis.

\section{Completeness of published studies}

Many of the relevant trials that we included were published only as abstracts or as brief reports. This was frequently the case for studies published in Chinese, for which published versions were often less than two pages long. Although we contacted study authors, when possible, to confirm study eligibility, we did not have the time or resources to contact all study authors for further information on trial design or study results. Thus, in general the completeness of study information is low, resulting in a high number of studies for which risk of bias is classed as 'unclear' and a high number of studies that do not contribute data to the analyses.
Relatively few studies followed up with participants after the intervention had ended: Data were available immediately at the end of intervention for 49 studies for the independence in ADL outcome and for 50 studies for the motor function outcome, but only for 16 and 12 studies, respectively, for a longer-term follow-up outcome. Follow-up data from studies comparing intervention with no treatment demonstrate that significant benefit of intervention is maintained, but the size of the benefit was observed to lessen. In the comparison of intervention versus usual care or control, lack of follow-up data limits the ability of review authors to draw any generalisable conclusions relating to whether observed benefits are maintained.

\section{Descriptions of interventions}

Clear, concise documentation of complex physical interventions is exceptionally difficult to achieve. The written information provided by study authors regarding interventions administered in the included trials is included in the Characteristics of included studies table. Although many of the included studies attempt to describe all administered interventions, the available documentation is often insufficient to allow confident and accurate repetition of the applied rehabilitation approach. Problems with documentation of interventions generally are not the fault of researchers or therapists, but rather are due to the fundamental problem of recording methods of physical handling skills and techniques, and the nature of the often intimate relationship between stroke survivor and physiotherapist. Documentation of this process would generally be complex and 'wordy'; therefore often it is not possible to present within research papers with limitations on length. These problems are confounded by the fact that treatments applied are often ultimately the decision of a single physiotherapist, based on an individual assessment of a unique stroke survivor's movement disorders.

Furthermore, the common basis of physical rehabilitation 'approaches' is that they are holistic. All body parts and movements can be assessed and treated based on the selected approach; however, a physiotherapist may select to concentrate on the treatment of one particular body part or movement during a treatment session. Subsequently, treatments given to specific stroke survivors by individual therapists may vary enormously. This review attempted to limit this variation slightly by excluding trials that had provided interventions only to the upper limb. Nevertheless, although we grouped together studies that included treatment components within similar categories, it is conceivable that substantial differences exist between the physical interventions given to participants within the same treatment group.

\section{Categorisation of treatment components within interventions}

The comparisons carried out within the review relied on categorisation of treatment components that were described within the published papers. Two independent review authors categorised the described treatments using agreed definitions of individual treatment components. This process relied on adequate descriptions within published papers. Papers that published only very brief descriptions of interventions therefore may have resulted in categorisations that were not truly reflective of the intervention delivered. 
Furthermore, this process of categorisation was highly dependent on the language and terminology provided within a written description. For example, an author may state "activities aimed at improved gait." This description would result in categorisation only within the functional task training component of "walking." However, in practice, this intervention could have included components such as active or active-assisted movement, sensorimotor facilitation and muscle strengthening. Therefore our method of categorisation is likely to have underestimated rather than overestimated the numbers of treatment components and intervention categories. Hence, if any inaccuracy exists, the interventions are likely to be more "mixed" and eclectic than has been captured by our method of categorisation.

A number of difficulties were encountered in distinguishing between interventions that included only functional task training components and those that also included musculoskeletal (active) components. In particular, the review authors encountered difficulties in determining whether an intervention focused on a functional task might also include active or active-assisted movement. This reality was due to the fact that all functional task training necessitates active movement, and overlap between practice of an active-movement and practice of a functional task can be inevitable. This is an area that we recommend for further exploration in relation to the descriptions and definitions of treatment components proposed for this review.

Discussion also focused on whether the categories of 'assistive devices' and 'modalities' would be better combined into one joint category. The separate categories were agreed and defined by the stakeholder group participants, which is why they have been used within this review. However, we recommend that merging of these categories be explored before future updates of this review are prepared.

\section{Treatment components within named approaches}

We were aware when developing the definitions for categorisation of described interventions that a number of studies have stated a named approach (e.g. 'Bobath,' 'Motor-relearning programme') without providing any description of the treatment components included within the approach. We therefore wrote definitions such that these studies could be captured by our system of categorisation of individual treatment components. However, including studies that have provided only the name of an approach without providing any descriptions potentially introduces a number of biases. These biases occur as a result of the fact that the content of named approaches potentially changes over time and in keeping with geographical or personal preferences and biases. In particular, several studies reported that the intervention was 'Bobath,' and much debate has surrounded the content of physiotherapy interventions based on the Bobath concept. This debate arises largely from the fact that the content of the Bobath approach has changed over time, published descriptions are limited, and the content of current therapy is variable (Carr 1994a; DeJong 2004; Langhammer 2012; Mayston 2008; Nilsson 1992; Pomeroy 2001b; Sackley 1996; Tyson 2009b; Turner 1995). A summary of the philosophy or theory of some of the key named approaches was drawn up for the first version of this review and is provided in Table 1.

\section{Translation of descriptions of interventions}

Thirty-eight of the included full papers were published in languages other than English; all were published in Chinese. For these papers, we sought translation of the intervention description into English. In addition, several included studies were carried out in China and the papers published in English, but by authors for whom English clearly was not the first language. These translations provided a number of challenges in relation to interpretation of meanings and subsequent classification of treatment components. For example, in several papers, it was unclear whether 'standing up training' referred to activities carried out in standing (i.e. training to promote standing balance) or to sit-to-stand training. In these cases, decisions were made based on discussion between two review authors (one of whom was a Chinese-speaking physiotherapist (PLC)).

\section{Geographical location of studies}

Subgroup analyses found evidence of an association between effect size and geographical location, with an indication that studies carried out in China may have a greater effect size. This finding may be due to reporting biases and may reflect biases associated with publication, location, citation and language. This finding may also reflect a true difference in the effects of interventions carried out in different geographical locations, which may be a result of differences in culture, traditions, training and implementation of interventions. However, this difference may also be related to the comparison interventions, especially if they consist of no treatment or usual care. All studies that compared intervention against no treatment in the acute phase of stroke were carried out in China; this is a reflection of the fact that 'usual care' can comprise no rehabilitation within these geographical settings. Consequently, the finding that intervention is more effective than no treatment in improving independence in ADL and in motor function may, arguably, be applicable only to settings in China. Evidence shows that usual care can vary considerably, both regionally and nationally as well as internationally. Hence, geographical location could be a confounding variable in the comparison of intervention against usual care.

\section{Risk of bias of included studies}

Judgement of quality of evidence was very difficult because of poor, incomplete or brief reporting of information. Less than $50 \%$ of the studies were judged to be at low risk of bias for selection, detection and attrition bias; however, for most of the studies, this information was unclear, and risk of bias was judged to be high in less than $10 \%$ of the studies. Sensitivity analyses were carried out to explore the effect of including studies with high or unclear risk of bias. These sensitivity analyses generally found that removal of studies with high or unclear risk of bias did not alter the direction (or significance) of the results. Thus, although the quality of most of the evidence included in this review remains uncertain, the fact that inclusion of these studies does not affect the direction of results gives us greater confidence in our findings. The main message arising from this review in relation to quality of the evidence is that it is essential that reporting of methodological features of RCTs of physiotherapy interventions is improved, and that studies are reported using the CONSORT guidelines for reporting (Schulz 2010).

Studies that used quasi-random assignment were excluded from this update of the review (previous versions had included quasi- 
randomised trials). This led to exclusion of three studies that had been included in the 2007 version (Hesse 1998; Ozdemir 2001; Stern 1970). However, we found information about the method of randomisation particularly difficult to judge in a number of studies included in this review, particularly studies published in Chinese, in which use of the term 'random' in English abstracts did not always reflect the descriptions provided in Chinese versions of the study. There is an urgent need for trialists to address the issue of adequate reporting of methods of randomisation. It is possible that we have inadvertently included in this review trials that used quasi-random assignment, rather than true random assignment.

In most studies, it was unclear whether participants were blinded to the study group and aims. The nature of rehabilitation interventions and the ethical requirement to obtain informed consent often make it difficult, if not impossible, to blind participants. If the aims and objectives of the study were apparent to the participants, this could confound the study results. It is generally impossible to blind the treating therapist because treating therapists have to be familiar with the intervention they are administering. Therapists who strongly favoured one approach over another could introduce performance bias. In several studies, the same therapist administered treatment to participants in both study groups; this potentially introduced considerable contamination between groups. The 'beliefs' of stroke survivors and therapists may further contribute to biases within many of these studies, and the large number of different geographical locations in which studies were carried out means that the studies were carried out with participants living in a wide variety of cultures, which could potentially impact the response to physical rehabilitation. Many of the included trials did not state that they used a blinded assessor. Lack of blinding of assessors potentially introduces considerable bias into the study results. This is particularly important in studies in which therapists often have strong beliefs in support of a particular approach.

\section{Heterogeneity of included trials}

In addition to limitations of the study methods, the trials included in the review had considerable heterogeneity. The key areas of heterogeneity were related to interventions and to participants.

1. Interventions: Although attempts have been made to categorise the interventions using a systematic, rigorous and valid method, considerable variation may still exist between studies that have used similar types of treatment components. Furthermore, substantial variations in dose and intensity and in length of the treatment period were noted. Also different is the fact that some interventions were carried out only when a therapist is present, whilst in other studies, independent practice of activities outside therapy sessions was encouraged.

2. Participants: The participant populations in the different included studies were heterogeneous. They varied from limited populations (e.g. pure motor stroke only) to those inclusive of all stroke survivors. Considerable variation in the time since stroke was also noted. The validity of combining results from such heterogeneous samples is debatable. We recommend that future updates consider subgroup analysis to explore the initial impairment of included participants.

Although we have carried out subgroup analyses to explore issues relating to the heterogeneity of both the interventions (i.e. dose, components) and the participants (time since stroke) and other issues (geographical location, provider of intervention), it is likely that a complex interrelationship exists between some of the subgroups that we have been unable to explore. For example, studies carried out in China tended to be those with the least time since stroke, meaning that effects found that have been attributed to geographical location could be due equally to time since stroke (and vice versa). We believe that this will be true for a number of other variables. We recommend that future updates of this review plan to explore issues associated with the interrelationship of these variables.

\section{Potential biases in the review process}

\section{Publication bias}

As has been discussed above, the identification of all relevant trials was confounded by a number of factors, and, despite a rigorous search strategy, we are not fully confident that we will have successfully identified all studies. Consequently, this review may be biased towards particular types of studies and publications. For example, we are not confident that we will have successfully identified all relevant studies published in China, or in Chinese. Similarly, we may be missing other non-English studies or studies published in journals that are not included in the electronic databases that we searched.

\section{Treatment components and categorisation of interventions}

We introduced a method of categorisation of interventions that was agreed by a stakeholder group of physiotherapists, stroke survivors and carers. This method of categorisation has not been tested or explored further, and such testing is necessary to confirm the relevance and validity of the identified categories. In the absence of any other suitable method of categorisation of treatment components, we believe that we have adopted a robust, justifiable method-based on consensus between physiotherapists, stroke survivors and carers. We argue that the involvement of this expert stakeholder group has considerably enhanced our review and is substantially advantageous compared with the alternative of having researchers make decisions over the categorisation of interventions and the structure of comparisons. Feedback from the stakeholder group members confirms that the group perceived that their input benefited the format of the review and made the review more clinically relevant. However, because of the limited nature of our resources, most members of the stakeholder group were based in Scotland. Given known differences in physiotherapy practice in different parts of the world, we recommend that the proposed categorises are explored and amended to reflect international practices in relation to stroke rehabilitation.

We recognise that the terminology used, particularly in the titles of the categories, may not be universally accepted or understood. We acknowledge that the appropriateness of terms such as 'functional,' 'neurophysiological' and 'musculoskeletal' can be debated when used in the way we have used them within this review. However, these terms were selected by the stakeholder group to have clinical meaning and were informed by the terminology used by DeJong 2004.

\section{Appraisal of quality in non-English language papers}

Non-English (Chinese) papers included in the review were appraised and assessed for risk of bias by one review author with the language skills to translate relevant sections of the 
papers. This review author also possessed the necessary quality appraisal skills and had detailed expertise of physiotherapy and stroke rehabilitation. A second review author then considered the assessment of risk of bias by the first review author, based on the translations of relevant extracts provided by the first review author. Thus, although two review authors did consider the quality of these non-English language papers, the assessment of the second review author was based entirely on the translation provided by the first review author. This method means that if the first review author made any errors in translation, or missed information provided in the non-English text, the second review author will not have identified this. Thus, although having two review authors for these papers offers advantages, it does not provide the same level of 'independence' as the process of having two independent review authors for the English language papers. However, given the volume of non-English papers that we have included, and the available resources for this review, we believe that we have taken all steps available to us to minimise potential biases in this process.

\section{Conclusions arising from this review}

Following completion of the analyses and results of this review, this information was presented to our stakeholder group, which comprised physiotherapists, stroke survivors and carers. For each of the three main comparisons and associated subgroup analyses, group members discussed the clinical implications and key messages arising from the results. The points discussed have been incorporated within the Discussion and Authors' conclusions sections of this review. In particular, the stakeholder group members highlighted the need to specifically draw out information pertaining to the dose of interventions delivered within the studies, as this was believed to have important implications for clinical practice. We believe that this process of consultation considerably removes potential biases from the process of reaching conclusions from this review, as the conclusions reflect the views of expert clinicians, stroke survivors and carers, rather than the potentially biased viewpoints of researchers and academics.

\section{Previous versions of this review}

The previous (2007) version of this review concluded that "no one physiotherapy approach has been shown to be more advantageous to the promotion of recovery of lower limb function or postural control" and that "physiotherapy using a mix of components from different approaches is more beneficial than no treatment or placebo control for the recovery of functional independence after stroke." These findings supported the conclusion that "this evidence provides a sound scientific rationale for physiotherapists to use a selection of treatments, regardless of their philosophical or theoretical origin." This updated review agrees with, and adds considerable evidence to, these previous conclusions. Although the 2007 version concluded that evidence was insufficient to suggest that one approach was superior to another, this review can now conclude that moderate-quality evidence indicates that there is no difference between approaches.

\section{Other reviews}

A number of other published reviews agree with the conclusion that physiotherapists should not use compartmentalised, named approaches, but should select clearly defined and described techniques and task-specific treatments, regardless of their historical or philosophical origin (Kollen 2009; Langhammer 2012; Mayston 2008; Pomeroy 2005).
This review is in agreement with a review of evidence relating specifically to the Bobath approach (Kollen 2009), which concluded that there was "no evidence for the superiority of Bobath therapy or any other approach on sensorimotor control of the upper and lower limb, dexterity, mobility, ADL, HRQOL, and cost-effectiveness."

Zhang 2013 recently completed a review that has similarities to our comparison of physiotherapy treatment versus no treatment. Zhang 2013 aimed specifically to review RCTs that compared rehabilitation versus standard care after stroke in China. This review pooled evidence from 31 trials (5220 participants) that reported independence in ADL (Barthel Index) and 27 trials (4501 participants) that reported motor function (Fugl-Meyer Assessment). In contrast, we identified and pooled the results of 27 studies (3423 participants) and 25 studies (4558 participants), respectively. (NB: Our figures also include non-Chinese studies.) We are unclear whether the differences in included trials reflected a more effective strategy for identification of Chinese studies by Zhang 2013; differences in selection criteria between reviews or a combination of these. Before future updates of our review, we plan to speak to the authors of Zhang 2013 to discuss differences in identification and selection of trials between the reviews. Despite differences in the numbers of trials included, the results and conclusions of our review were in agreement with those of Zhang 2013, which concluded that "there is some evidence that rehabilitation post stroke is more effective than no rehabilitation, improving activities of daily living and reducing disability." Both Zhang 2013 and our review highlight the limitations relating to low reporting quality and study heterogeneity. A key difference between our review and Zhang 2013 is that Zhang 2013 did not attempt to explore the specific treatment components investigated by the studies.

\section{AUTHORS' CONCLUSIONS}

\section{Implications for practice}

Physical rehabilitation, using a mix of components from different approaches, is effective for recovery of function and mobility after stroke. Evidence relating to dose of physical rehabilitation is limited by substantial heterogeneity and does not support robust conclusions. However, there is some suggestion that treatment sessions of 30 to 60 minutes five to seven days a week may provide a beneficial effect, and more frequent sessions may provide added benefit. Evidence also suggests greater benefit associated with a shorter time since stroke. These hypotheses require robust evaluation before clinical recommendations can be made.

Current evidence indicates that no one approach to physical rehabilitation is more (or less) effective in promoting recovery of function and mobility after stroke. Therefore, clinical selection of the most appropriate physical treatments for individual stroke survivors should be undertaken using evidenced-based interventions and critical clinical reasoning. Members of the stakeholder group for this review agreed that key implications for practice arising from this evidence related to meeting the need for personnel involved in delivering stroke rehabilitation and educating therapists to:

1. select treatment components based on assessment of the individual stroke survivor, with consideration of the full range of treatment techniques that they have the skills and expertise to administer; and 
2. implement evidence-based rehabilitation after stroke, with critical evaluation and awareness that the current evidence shows that no one approach is superior to any other.

In conclusion, this review provides evidence indicating that physical rehabilitation should not be limited to compartmentalised, named rehabilitation approaches, but should comprise clearly defined, well-described, evidence-based physical treatments regardless of historical or philosophical origin.

\section{Implications for research}

Moderate-quality evidence now shows that physiotherapy using a mix of components from different approaches is more beneficial than no treatment, usual care or attention control, and that no single approach is more (or less) effective than any other. Researchers should add to this body of evidence by determining which individual treatment components contribute towards the beneficial effects. High-quality randomised trials and systematic reviews are needed to determine the effectiveness of clearly described individual techniques and task-specific treatments, regardless of their historical or philosophical origin. Many Cochrane reviews have already been published that include a large body of trials exploring the effectiveness of specific single treatments, and it is important that researchers are familiar with this evidence and plan future research according to relevant recommendations within these reviews. Researchers should identify whether there are any gaps in this evidence base so that these can be addressed. It is important that the current Cochrane reviews are kept upto-date. With a high volume of research being published in the Chinese literature, systematic reviewers must ensure that they have adequate resources to support review updates and to adequately address and explore the potential variations in clinical practice and trial design in studies arising from different geographical locations.

In addition to research evaluating single specific treatments, we recommend that researchers adopt pragmatic research designs to investigate the effectiveness of skilled physiotherapists in providing patient-centred interventions, for which treatment components are selected following individual patient assessment. Valid, reliable methods of systematic documentation and description of patientcentred physical rehabilitation must be explored, such that robust RCTs and systematic reviews are supported. We recommend evaluation and exploration of the classifications and definitions of treatment components used within this review, before future updates of this review are undertaken. Furthermore, we recommend that future research explores physical rehabilitation in relation to, and in combination with, other evidence-based nonphysical interventions, such as medical and drug interventions (e.g. thrombolysis, Traditional Chinese Medicine, transcranial magnetic stimulation (TMS)). Studies emerging from China have demonstrated the ability to include relatively large numbers of participants, and lessons should be learnt from these large clinical trials.

This review synthesises the evidence in relation to function and mobility after stroke. Research is needed to consider the full range of outcomes that may be associated with improved function and mobility. These outcomes include the clinical and cost benefits potentially associated with a reduction in falls or emergency hospital admissions, and the impact of community and social care teams and services. All benefits in relation to stroke survivor-perceived quality of life, psychological mood, social participation, return to work and carer strain and wellbeing should be considered. This review found that many RCTs did not assess long-term follow-up, and it is essential that future RCTs plan follow-up assessments as a key feature of their design. Adequate resources should be sought to ensure that follow-up assessments are possible. The observed reduction in effect size in the available follow-up data supports research that explores additional or longer-term physiotherapy or both. Furthermore, future research should consider the long-term benefits of physical rehabilitation interventions and should explore the effects of follow-up physiotherapy assessment, self-management and treatment in maintaining benefits and preventing deterioration. Self-referral systems that will enable stroke survivors to gain followup physiotherapy when they believe it is necessary should also be explored.

Improvement in the reporting of RCTs of physical rehabilitation interventions is urgently needed, and we urge researchers to follow the CONSORT guidelines for reporting of clinical trials (Schulz 2010). A wide variety of outcome measures are used to assess the effects of physical rehabilitation, and we recommend research that leads to consensus and standardisation of some core outcome measures for use within future RCTs. We urge researchers to follow the guidance offered by the COMET Initiative relating to the development and reporting of core outcomes.

A stakeholder group was central to this review update, and this update has demonstrated that user involvement in Cochrane reviews is feasible and valued and can significantly impact the review structure and methods. We recommend similar models of user involvement within other Cochrane reviews and evidence syntheses.

\section{ACKNOWLEDGEMENTS}

\section{Current versions of this review}

1. Scottish Government's Chief Scientist Office, for providing funding to support this review update.

2. Hazel Fraser, Cochrane Stroke Group Managing Editor, for continued support.

3. Brenda Thomas, Cochrane Stroke Group Trials Search Coordinator, for continued support in running searches and obtaining some of the trials.

4. Trialists who responded to emails and provided various additional details about their studies: Ayse Karaduman.

5. Ashma Krishan, Cochrane Stroke Group Statistician, for responding to queries and providing help relating to metaanalyses.

6. Tom Crocker and Jaime Smith, Academic Unit of Elderly Care and Rehabilitation, University of Leeds, for assisting with the process of identification of treatment components, and providing us with feedback on their experiences of similar projects.

7. Charles Lai and Philip Chan, Hong Kong Hospital Authority, for providing advice relating to physiotherapy practice in Hong Kong and China.

8. Ming Liu for supplying us with a copy of the Chinese Stroke Rehabiliation Guidelines. 
9. Julie Bernhardt for replying to queries in relation to her systematic review relating to stroke rehabilitation in China (Zhang 2013).

10.Sandra Paget, Consumer Reviewer, for providing comments on the review.

\section{Stakeholder group}

The stakeholder group was formed specifically to support the current update of this review. The broad aims of the group were to (1) ensure that the review was clinically relevant; (2) reach consensus on decisions surrounding the incorporation of Chinese studies into the previous Western classification of rehabilitation approaches and (3) guide dissemination to ensure that the updated review impacts on practice. The stakeholder group met for three half-days throughout the period of this review update and responded to email requests in addition to this. The input of the stakeholder group was highly valuable and had considerable impact on this review update, in particular, regarding (1) decisions around updating the way of classifying rehabilitation approaches, and incorporating non-Western approaches, within the review, (2) development of the classification system and definitions of individual treatment components, (3) selection of items for subgroup analysis and (4) interpretation of the results and assessment of implications for clinical practice and future research. All 13 members of the stakeholder group made enthusiastic and effective contributions to this review. These members included:

1. Carolyn Agnew, Lead Physiotherapist, Fife Rehabilitation Service;

2. Mrs Caroline Arhard, BSc (Hons) Physiotherapy, Stroke Specialist Physiotherapist in acute stroke, The Rehabilitation and Assessment Directorate, NHS Greater Glasgow and Clyde;

3. Jacqueline Boyle, BSc (Hons), Physiotherapist;
4. Audrey Campbell, Stroke Rehabilitation Physiotherapist, Douglas Grant Rehabilitation Centre, NHS Ayrshire and Arran;

5. Mrs Heather Goodare, MA (Oxon) Dip Couns (Brighton), consumer peer reviewer for The Cochrane Collaboration, carer for a stroke survivor;

6. Wendy Juner, Team Lead Physiotherapist, NHS Lothian;

7. Jill Skelly, Senior Physiotherapist, NHS Tayside;

8. Mark Smith, Grad Dip Phys, BSc (Hons), MPhil, MCSP; Consultant Physiotherapist NHS Lothian; visiting lecturer at Queen Margaret University, University of Glasgow and Robert Gordon's University; member of the National Advisory Committee for Stroke and the Scotland Committee of the Stroke Association; and

9. A further five members who have chosen to remain anonymous.

\section{Previous versions of this review}

1. Chest Heart and Stroke Scotland, for funding the STEP project, which made the first version of this review possible.

2. The Big Lottery Fund for current funding of the STEP project.

3. Brenda Thomas, Cochrane Stroke Group Trials Search Coordinator, for help in writing the search strategy, running the searches and obtaining some of the trials.

4. Lynsey Smyth, STEP, for help with obtaining trials and tracking down references and authors.

5. The trialists who responded to emails and provided various additional details about their studies: Cath Dean, John Green, Stefan Hesse, Susan Hillier, Tracey Howe, Ayse Karaduman, Nadina Lincoln, Nancy Mayo, Carol Richards, Nancy Salbach, Judith Salter, Joni Stoker-Yates, Paulette van Vliet, Bob Wellmon, Guo-rong Wei and Sharon Wood-Dauphinee.

6. Dr You Hong, who translated sections of Chinese trials.

7. Janet Carr and Roberta Shepherd, who sent us detailed comments on the published protocol. 


\section{RE F E R E N C E S}

\section{References to studies included in this review}

Aksu 2001 \{published data only\}

Aksu S, Armutlu K, Cetisli N, Guclu A, Atay S, Guniken Y, et al. Numbers of exercises in acute stroke rehabilitation: is it important? Neurorehabilitation and Neural Repair 2001;15(4):348.

\section{Allison 2007 \{published data only\}}

Allison R, Dennett R. Pilot randomized controlled trial to assess the impact of additional supported standing practice on functional ability post stroke. Clinical Rehabilitation 2007;21(7):614-9.

\section{Baer 2007 \{published data only\}}

Baer G, Durward B, Weller D. An investigation into a homebased mobility rehabilitation programme for late-stage stroke. A pilot randomised controlled trial. Physiotherapy 2007;93 Suppl 1:S546.

\section{Bai 2008 \{published data only\}}

Bai Y, Hu Y, Chen W, Wang X, Cheng A, Jiang C, et al. Study of effects of standardized three stage rehabilitation treatment on motor function in ischemic stroke patients. Journal of Rehabilitation Medicine 2008;Suppl 46:109.

* Bai Y, Hu Y, Wu Y, Zhu Y, He Q, Jiang C, et al. A prospective, randomized, single-blinded trial on the effect of early rehabilitation on daily activities and motor function of patients with hemorrhagic stroke. Journal of Clinical Neuroscience 2012;19:1376-9.

\section{Bale 2008 \{published data only\}}

Bale M, Strand LI. Does functional strength training of the leg in subacute stroke improve physical performance? A pilot randomized controlled trial. Clinical Rehabilitation 2008;22:911-21.

\section{Behrman 2011 \{published data only\}}

Behrman A, Sullivan KJ, Wu SS, Nadeau SE, Dobkin BH, Azen SP, et al. Functional walking ability 2 to 6 months after stroke: effects of progressive task-specific and impairment-based exercise rehabilitation programs compared to usual care. Stroke 2011;42(11):e610.

\section{Blennerhassett 2004 \{published data only\}}

Blennerhassett J, Dite W. Additional task-related practice improves mobility and upper limb function early after stroke: a randomised controlled trial. Australian Journal of Physiotherapy 2004;50(4):219-24.

\section{Brock 2005 \{published data only\}}

Brock K, Haase G, Rotacher G, Cotton S. Does physiotherapy based on the Bobath concept achieve greater improvement in walking ability compared to structured task practice? A pilot randomised controlled trial. Neurorehabilitation and Neural Repair 2012;26(6):727-8.

* Brock K, Haase G, Rothacher G, Cotton S. Does physiotherapy based on the Bobath concept, in conjunction with a task practice, achieve greater improvement in walking ability in people with stroke compared to physiotherapy focused on structured task practice alone? A pilot randomized controlled trial. Clinical Rehabilitation 2011;25(10):903-12.

Brock K, Haase G Cotton S, Thompson M. Effects of physiotherapy interventions based on the Bobath concept versus structured task practice for improving the ability to walk outdoors following stroke. International Journal of Stroke 2010;5 Suppl 1:11.

Brock K. Regaining the ability to walk outdoors following stroke: the efficacy of the Bobath concept compared to structural task practice. Australian New Zealand Clinical Trials Registry (ANZCTR), 2005. http://www.anzctr.org.au/.

Carlson 2006 \{published data only\}

Carlson TD, Cahn L, Leloup B. The effects of intense massed practice on balance and ambulation post-stroke: a pilot study. Platforms, thematic posters, and posters for CSM 2007. Journal of Neurologic Physical Therapy 2006;30(4):211.

Chan 2006 \{published data only\}

Chan DY, Chan CC, Au DK. Motor relearning programme for stroke patients: a randomized controlled trial. Clinical Rehabilitation 2006;20(3):191-200.

Chen 2004 \{published data only\}

Chen Q, Wang H-X, Wang T, Shan C-L. Effects of early rehabilitation on the activities of daily living in hemiplegic patients. Zhongguo Linchuang Kangfu 2004;8(22):4418-9.

\section{Chen 2006 \{published data only\}}

Chen XF. Effect of community-based-rehabilitation on activities of daily life and cognitive function in stroke patients. Zhongguo Linchuang Kangfu 2006;10(32):4-6.

\section{Chen 2010 \{published data only\}}

Chen Q, Li L. Observation on curative effect of rehabilitation exercise therapy to treat patients with post-stroke hemiplegia and muscles spasm. Zhongguo Huli Zazhì 2010;24(1A):14-6.

\section{Chu 2003 \{published data only\}}

Chu P. Effect of early facilitation techniques on motor function of stroke patients. Zhongguo Linchuang Kangfu 2003;7(7):1189.

\section{Cooke 2006 \{published data only\}}

Cooke E, Tallis R, Miller S, Pomeroy V. The effects of type and intensity of physiotherapy on lower limb strength and function after stroke. UK Stroke Forum Conference 2007:25-6.

* Cooke EV, Tallis RC, Clark A, Pomeroy VM. Efficacy of functional strength training on restoration of lower-limb motor function early after stroke: phase I randomized controlled trial. Neurorehabilitation and Neural Repair 2010;24(1):88-96.

Cooke EV, Tallis RC, Miller S, Pomeroy VM. The effects of functional strength training on lower limb strength and function after stroke. In: UK Stroke Forum Conference. 2006:95. 


\section{Dean 1997 \{published and unpublished data\}}

Dean CM, Shepherd RB, Adams R. The effect of specific balance training in sitting on support and balance through the lower limbs following stroke. In: Proceedings of the 1996 National Physiotherapy Congress. 1996:148-9.

* Dean CM, Shepherd RB. Task-related training improves performance of seated reaching tasks after stroke. A randomized controlled trial. Stroke 1997;28:722-8.

Dean CM. Personal correspondence. May 2000.

\section{Dean 2000 \{published data only\}}

Dean CM, Richards CL, Malouin F. Task-related circuit training improves performance of locomotor tasks in chronic stroke: a randomised, controlled pilot trial. Archives of Physical Medicine and Rehabilitation 2000;81:409-17.

\section{Dean 2006 \{published data only\}}

Dean C, Rissel C, Sharkey M, Sherrington C, Cumming R, Lord S, et al. Ongoing exercise opportunities to prevent falls and enhance mobility in community dwellers after stroke: the stroke club trial. International Journal of Stroke 2010;5:12.

Dean C. Exercise intervention to prevent falls, enhance mobility and increase physical activity in community dwellers after stroke: a randomised controlled trial. Australian New Zealand Clinical Trials Registry (ANZCTR), 2006. http:// www.anzctr.org.au/.

Dean CM, Rissel C, Sharkey M, Sherrington C, Cumming RG, Barker RN, et al. Exercise intervention to prevent falls and enhance mobility in community dwellers after stroke: a protocol for a randomised controlled trial. BMC Neurology 2009;9:38.

* Dean CM, Rissel C, Sherrington C, Sharkey M, Cumming RG, Lord SR, et al. Exercise to enhance mobility and prevent falls after stroke: the community stroke club randomized trial. Neurorehabilitation and Neural Repair 2012;26(9):1046-57.

\section{Dean 2007 \{published data only\}}

Dean CM, Channon EF, Hall JM. Sitting training early after stroke improves sitting ability and quality and carries over to standing up but not to walking: a randomised controlled trial. Australian Journal of Physiotherapy 2007;53:97-102.

\section{Deng 2011 \{published data only\}}

Deng RY, Xing FM, Wang L, Zhao HY. Influence of ADL system intervention on movement function and quality of life of stroke patients. Journal of Jilin University Medicine Edition 2011;37(6):1129-32.

\section{Duncan 1998 \{published and unpublished data\}}

Duncan P, Richards L, Wallace D, Stoker-Yates J, Pohl P, Luchies $C$, et al. A randomized, controlled pilot study of a homebased exercise program for individuals with mild and moderate stroke. Stroke 1998;29:2055-60.

\section{Duncan 2003 \{published data only\}}

Duncan P, Studenski S, Richards L, Gollub S, Lai SM, Reker D, et al. Randomized clinical trial of therapeutic exercise in subacute stroke. Stroke 2003;34:2173-80.

\section{Fan 2006 \{published data only\}}

Fan W, Hu Y, Wu Y. Clinical study of standardized three stages' rehabilitation program in promoting motor function in stroke patients with hemiplegia. Zhongguo Linchuang Kangfu 2006;21(6):484-7.

\section{Fang 2003 \{published data only\}}

Fang Y, Chen X, Li H, Huang R, Zeng J. A study on additional early physiotherapy after stroke and factors affecting functional recovery. Clinical Rehabilitation 2003;17:608-17.

\section{Fang 2004 old \{published data only\}}

Fang Y-N, Li H, Zeng J-S, Chen X-H, Ma M-M, Lin J-W, et al. Analysis of the factors affecting the functional rehabilitation of senile stroke patients. Zhongguo Linchuang Kangfu 2004;8(10):1818-20.

\section{Fang 2004 young \{published data only\}}

Fang Y-N, Li H, Zeng J-S, Chen X-H, Ma M-M, Lin J-W, et al. Analysis of the factors affecting the functional rehabilitation of senile stroke patients. Zhongguo Linchuang Kangfu 2004;8(10):1818-20.

\section{Ge 2003 \{published data only\}}

Ge W, Hu J. Impact of early rehabilitation therapy on ADL in stroke patients with paralysis. Zhongguo Linchuang Kangfu 2003;7(5):853.

\section{Gelber 1995 \{published data only\}}

Gelber DA, Good DC, Herrmann D. Comparison of two physical therapy approaches in the treatment of the pure motor hemiparetic patient. Neurology 1993;43:A234.

* Gelber DA, Josefczyk PB, Herrman D, Good DC, Verhulst SJ. Comparison of two therapy approaches in the rehabilitation of the pure motor hemiparetic stroke patient. Journal of Neurologic Rehabilitation 1995;9:191-6.

Josefczyk PB, Gelber D. Stroke rehabilitation outcome: a prospective comparison of traditional functional rehabilitation $v$ neurodevelopmental technique in the moderately impaired stroke patient. Journal of Neurologic Rehabilitation 1995;9(2):127-8.

\section{Green 2002 \{published data only\}}

* Green J, Forster A, Bogle S, Young J. Physiotherapy for patients with mobility problems more than 1 year after stroke: a randomised controlled trial. Lancet 2002;359:199-203.

Green J, Young J, Forster A, Collen F, Wade D. Combined analysis of two randomized trials of community physiotherapy for patients more than one year post stroke. Clinical Rehabilitation 2004;18(3):249-52.

\section{Holmgren 2006 \{published data only\}}

* Holmgren E, Lindstrom B, Gosman-Hedstrom G, Nyberg L, Wester $\mathrm{P}$. What is the benefit of a high intensive exercise program? A randomized controlled trial. Advances in Physiotherapy 2010;12(3):115-24. 
Holmgren E. Evaluation of an intervention program targeted at improving balance and functional skills after stroke. ClinicalTrials.gov 2006.

Holmgren ES, Wester P. Evaluation of an intervention program targeted at improving balance and functional skills after stroke. Cerebrovascular Diseases 2009;27:238.

\section{Hou 2006 \{published data only\}}

Hou H, Wang T, Wang H. Effects of 3-phase rehabilitation on functional outcome in hemiplegia after stroke. Zhongguo Linchuang Kangfu 2006;21(1):61-3.

\section{Howe 2005 \{published data only\}}

Howe TE, Taylor I, Finn P, Jones H. Lateral weight transference exercises following acute stroke: a preliminary study of clinical effectiveness. Clinical Rehabilitation 2005;19:45-53.

\section{Hu 2007 haem \{published data only\}}

Hu YS. Clinical study of standardized tertiary rehabilitation program in promoting upper and lower limbs motor function in stroke patients. National Medical Journal of China 2007;87(33):2358-60.

\section{Hu 2007 isch \{published data only\}}

Hu YS. Clinical study of standardized tertiary rehabilitation program in promoting upper and lower limbs motor function in stroke patients. National Medical Journal of China 2007;87(33):2358-60.

\section{Huang 2003 \{published data only\}}

Huang W-M, Gao Z, Ran C-F, Li Q, Li H-M. Evaluation of early movement intervention for functional recovery of patients with stroke. Zhongguo Linchuang Kangfu 2003;7(25):3484-5.

\section{Hui-Chan 2009 \{published data only\}}

Hui-Chan CW, Ng SS, Mak MK. Effectiveness of a home-based rehabilitation programme on lower limb functions after stroke. Xianggang Yi Xue Za Zhi 2009;15(3 Suppl 4):42-6.

\section{Jiang 2006 \{published data only\}}

Jiang C, Hu Y, Wu Y. Cost-effectiveness evaluation in stroke patients received standard tertiary rehabilitation for 6 months. Zhongguo Linchuang Kangfu 2006;21(2):114-7.

* Jiang C, Hu Y, Wu Y. The cost-effectiveness analysis of early rehabilitation of cerebral vascular accident patients. Zhongguo Linchuang Kangfu 2006;21(11):973-6.

\section{Jing 2006 \{published data only\}}

Jing ZW, Han QY, Wang Z, Zhang, JW, Zhang ZQ, Han CH, et al. Effect of early occupational therapy on the activities of daily life in stroke patients. Zhongguo Linchuang Kangfu 2006;10(4):54-6.

\section{Kim 2011 \{published data only\}}

Kim Y, Kim E, Gong W. The effects of trunk stability exercise using PNF on the functional reach test and muscle activities of stroke patients. Journal of Physical Therapy Science 2011;23(5):699-702.

\section{Kim 2012 \{published data only\}}

Kim B, Lee S, Bae Y, Yu J, Kim T. The effect of a task-oriented training on trunk control ability, balance and gait of stroke patients. Journal of Physical Therapy Science 2012;24(6):519-22.

\section{Kwakkel 2002 \{published data only\}}

Kwakkel G, Wagenaar RC. Effect of duration of upper- and lower-extremity rehabilitation sessions and walking speed on recovery of interlimb coordination in hemiplegic gait. Physical Therapy 2002;82(5):432-48.

\section{Kwakkel 2008 \{published data only\}}

Kwakkel G. Effects of a task-oriented circuit training to enhance walking competency after stroke. Nederlands Trial Register, 2008. http://www.trialregister.nl.

* van de Port I, Wevers LE, Lindeman E, Kwakkel G. Effects of circuit training as alternative to usual physiotherapy after stroke: randomised controlled trial. BMJ 2012;344:e2672.

\section{Langhammer 2000 \{published data only\}}

Langhammer B, Stanghelle J. Can physiotherapy after stroke based on the Bobath Concept result in improved quality of movement compared to the Motor Relearning Programme. Physiotherapy Research International 2011;16(2):69-80.

Langhammer B, Stanghelle JK, Sykehus S. Motor learning in the rehabilitation of stroke patients: short term or for life? A followup 1 and 4 years poststroke. Journal of Aging and Physical Activity 2004;12(3):412.

Langhammer B, Stanghelle JK. Bobath or motor relearning programme? A follow-up one and four years post stroke. Clinical Rehabilitation 2003;17(7):731-4.

* Langhammer B, Stranghelle JK. Bobath or motor relearning programme? A comparison of two different approaches of physiotherapy in stroke rehabilitation: a randomized controlled study. Clinical Rehabilitation 2000;14:361-9.

Langhammer B. Motor learning theories in the acute stroke rehabilitation. Practical consequences of a randomised controlled study. International Journal of Rehabilitation Research 2004;27(Suppl 1):116.

\section{Langhammer 2007 \{published data only\}}

Langhammer B, Lindmark B, Stanghelle JK. Stroke patients and long-term training: is it worthwhile? A randomized comparison of two different training strategies after rehabilitation. Clinical Rehabilitation 2007;21(6):495-510.

\section{Lennon 2006 \{published data only\}}

Lennon S, Harpur W, Fullerton K, Power M, Wiggam I, MacSherry A. The effects of gait-specific training within Bobath therapy in people after acute stroke: a pilot randomized controlled trial. Neurorehabilitation and Neural Repair 2006;20(1):166.

* Lennon SM, Wheatley-Smith L, Harpur WM, Fullerton K, Power M, Wiggam I. Gait-specific training within Bobath therapy in acute stroke: a randomised controlled trial. Cerebrovascular Diseases 2009;27:240-1. 
Lennon SM, Wheatley-Smith L. The content of gait re-education for acute stroke within a randomised controlled trial of Bobath therapy. Cerebrovascular Diseases 2009;27:241.

\section{Li 1999 \{published data only\}}

Li M, Chen Y, Jiang J. Early rehabilitation of acute hemiplegic stroke: experience of 30 cases. Zhongguo Shi Yong Nei Ke Za Zhi 1999;19(6):352-3.

\section{Li 2003 \{published data only\}}

Li F. Impact of early rehabilitation nursing on paralyzed limbs in patients with cerebral hemorrhage. Zhongguo Linchuang Kangfu 2003;7(5):841.

\section{Li 2005 \{published data only\}}

Li HF, Wang JH, Feng JC, Gao F. Application of motor relearning therapy in the early rehabilitation of stroke: a randomized controlled comparison. Zhongguo Linchuang Kangfu 2005;9(29):1-3.

\section{Liao 2006 \{published data only\}}

Liao L, Luo W, Chen S. The effect of trunk control training on balance and lower limb function in patients with hemiplegia. Zhongguo Linchuang Kangfu 2006;21(7):608.

\section{Lincoln 2003 \{unpublished data only\}}

Lincoln N, Van Vliet P. A comparison of a UK approach to stroke rehabilitation with a movement science based approach. Personal communication 2000.

* van Vliet PM, Lincoln NB, Foxall A. Comparison of Bobath based and movement science based treatment for stroke: a randomised controlled trial. Journal of Neurology Neurosurgery and Psychiatry 2005;76:503-8.

\section{Liu 2003 \{published data only\}}

Liu G, Zhang H. Early rehabilitation and prognosis of post-stroke hemiplegic patients. Zhongguo Linchuang Kangfu 2003;7(3):506.

\section{McClellan 2004 \{published data only\}}

McClellan R, Ada L. A six-week, resource-efficient mobility program after discharge from rehabilitation improves standing in people affected by stroke: placebo-controlled, randomised trial. Australian Journal of Physiotherapy 2004;50:163-7.

\section{Mudge 2009 \{published data only\}}

Mudge S, Barber PA, Stott NS. Circuit-based rehabilitation improves gait endurance but not usual walking activity in chronic stroke: a randomized controlled trial. Archives of Physical Medicine and Rehabilitation 2009;90(12):1989-96.

\section{Mudie 2002 \{published data only\}}

Mudie MH, Winzeler-Mercay U, Radwan S, Lee L. Training symmetry of weight distribution after stroke: a randomised controlled pilot study comparing task-related reach, Bobath and feedback training approaches. Clinical Rehabilitation 2002;16:582-92.

\section{Ni 1997 \{published data only\}}

$\mathrm{Ni}$ CM, Fu J, Liu CY. Influence of early rehabilitation on walking function of the patients with acute stroke. Zhongguo Linchuang Kangfu 1997;12(4):164-5.

\section{Pan 2004 \{published data only\}}

Pan C-H, He J-Q, Pu S-X, Wan X-L, Gao C. Effects of early rehabilitation therapy on the motor function of limbs and activities of daily living in patients with hemiplegia after stroke. Zhongguo Linchuang Kangfu 2004;8(13):2404-5.

\section{Pang 2003 \{published data only\}}

Pang G. Early rehabilitation care effect on activities of daily life of stroke patients. Zhongguo Linchuang Kangfu 2003; 7(7):1230-1.

\section{Pang 2006 \{published data only\}}

Pang AL, Ren H, Chang LH, Gong SX, Ma SP. Effects of cocktail treatment on motor function of patients with ischemic stroke. Zhongguo Linchuang Kangfu 2006;10(32):6-8.

Pollock 1998 \{published and unpublished data\} Pollock AS, Durward BR, Rowe PJ, Paul JP. The effect of independent practice of motor tasks by stroke patients: a pilot randomized controlled trial. Clinical Rehabilitation 2002:16:473-80.

* Pollock AS. An Investigation into Independent Practice as an Addition to Physiotherapy Intervention for Patients with Recently Acquired Stroke [Dissertation]. Edinburgh: Queen Margaret College, 1998.

\section{Qian 2004 \{published data only\}}

Qian K-L, Wang T. Effect of early rehabilitation therapy on short and long term functional assessment in hemiplegic patients after stroke. Zhongguo Linchuang Kangfu 2004;8(25):5210-1.

\section{Qian 2005 \{published data only\}}

Qian KL, Wang T. Application of flexor reflex therapy in rehabilitation of lower extremity of hemiplegic patients after brain injury. Zhongguo Linchuang Kangfu 2005;9(5):24-5.

\section{Richards 1993 \{published and unpublished data\}}

Malouin F, Potvin M, Prevost J, Richards CL, Wood-Dauphinee S. Use of intensive task-oriented gait training program in a series of patients with acute cerebrovascular accidents. Physical Therapy 1992;72(11):781-93.

Malouin F, Richards CL, Wood-Dauphinee S, Williams JI. A randomized controlled trial comparing early and intensive task-specific physical therapy to conventional therapy in acute stroke patients. Canadian Journal of Rehabilitation 1993;7:27-8.

* Richards CL, Malouin F, Wood-Dauphinee S, Williams JI, Bouchard JP, Brunet D. Task-specific physical therapy for optimization of gait recovery in acute stroke patients. Archives of Physical Medicine and Rehabilitation 1993;74:612-20.

\section{Salbach 2004 \{published data only\}}

Salbach NM, Mayo NE, Wood-Dauphinee S, Hanley JA, Richards $\mathrm{CL}$, Cote R. A task-orientated intervention enhances walking distance and speed in the first year post stroke: 
a randomised controlled trial. Clinical Rehabilitation 2004;18:509-19.

\section{Shin 2011 \{published data only\}}

Shin WS, Lee SW, Lee YW, Choi SB, Song CH. Effects of combined exercise training on balance of hemiplegic stroke patients. Journal of Physical Therapy Science 2011;23(4):639-43.

\section{Stephenson 2004 \{published data only\}}

Stephenson J, Maitland M, Beckstead J. Body weight support treadmill training compared with PNF training in persons with chronic stroke. Journal of Neurologic Physical Therapy 2004;28(4):186.

\section{Tang 2009 \{published data only\}}

Tang W, Yang B. Influence of sensory function training on motor function of cerebral apoplexy patients complicated with hemiplegia. Zhongguo Huli Zazhì 2009;23(7C):1905-6.

\section{Thaut 2007 \{published data only\}}

Thaut MH, Leins AK, Rice RR, Argstatter H, Kenyon GP, McIntosh GC, et al. Rhythmic auditory stimulation improves gait more than NDT/Bobath training in near-ambulatory patients early poststroke: a single-blind, randomized trial. Neurorehabilitation and Neural Repair 2007;21(5):455-9.

\section{Torres-Arreola 2009 \{published data only\}}

Torres-Arreola L del P, Doubova Dubova SV, Hernandez SF, Torres-Valdez LE, Constantino-Casas NP, Garcia-Contreras F, et al. Effectiveness of two rehabilitation strategies provided by nurses for stroke patients in Mexico. Journal of Clinical Nursing 2009;18:2993-3002.

\section{Verheyden 2006 \{published data only\}}

Verheyden G, Vereeck L, Truijen S, De Weerdt W. Additional exercises improve trunk performance after stroke: an assessorblinded randomized controlled trial. In: UK Stroke Forum Conference. 2007:31.

Verheyden G, Vereeck L, Truijen S, Nieuwboer A, De Weerdt W. Trunk rehabilitation after stroke: a pilot randomized controlled trial. Neurorehabilitation and Neural Repair 2006;20(1):118-9.

* Verheyden G, Vereeck L, Truijen S, Troch M, Lafosse C, Saeys W, et al. Additional exercises improve trunk performance after stroke: a pilot randomized controlled trial. Neurorehabilitation and Neural Repair 2009;23(3):281-6.

\section{Verma 2011 \{published data only\}}

Verma R, Arya K, Garg RK, Singh T. Task-oriented circuit class training program with motor imagery for gait rehabilitation in poststroke patients: a randomized controlled trial. Topics in Stroke Rehabilitation 2011;18(1):620-32.

\section{Wade 1992 \{published data only\}}

Wade DT, Collen FM, Robb GF, Warlow CP. Physiotherapy intervention late after stroke and mobility. $B M J$ 1992;304:609-13.

\section{Wang 2004a \{published data only\}}

Wang Q-R, Gan Z-R, Lu H, Hu X, Liu H-L, Cai C-Q, et al. Effect of early exercise therapy on the recovery of motor function in patients with cerebral infarction and the changes of somatosensory evoked potentials. Zhongguo Linchuang Kangfu 2004;8(28):6023-5.

\section{Wang 2004b \{published data only\}}

Wang WM. Treatment of limb spasticity in hemiplegic patients within 4 months after stroke: curative effect of neural facilitation combined with low frequency pulse current. Zhongguo Linchuang Kangfu 2004;8(25):5224-5.

\section{Wang 2005 \{published data only\}}

Wang RY, Chen HI, Chen CY, Yang YR. Efficacy of Bobath versus orthopaedic approach on impairment and function at different motor recovery stages after stroke: a randomized controlled study. Clinical Rehabilitation 2005;19:155-64.

\section{Wang 2006 \{published data only\}}

Wang H, Wang T, Chen Q. The dynamic analysis of effects of 3-phase rehabilitation intervention on muscle tone and function in hemiplegia after stroke. Zhongguo Linchuang Kangfu 2006;21(9):807-10.

\section{Wei 1998 \{published data only\}}

* Wei G, Song L, Huang M et al. Effects of selective trunk activities training on the recovery of hemiplegic patients. Zhongguo Linchuang Kangfu 1998;13(3):108-10.

Wei G, Song L, Huang M. Effects of selective trunk activities training on the recovery of hemiplegic patients (Abstract PORR-392-27). In: Proceedings of the World Confederation of Physical Therapy. Yokohama, Japan: Science Council of Japan and the Japanese Physical Therapy Association, May 23-28, 1999.

\section{Wellmon 1997 \{published and unpublished data\}}

Wellmon R, Newton RA. An examination of changes in gait and standing symmetry associated with the practice of a weight shifting task. Neurology Report 1997;21(2):52-3.

\section{Wu 2006 \{published data only\}}

Wu X, Ni C, Han R. Effect of early rehabilitation on spasticity, activities of daily living and motor function in patients with hemiplegia after stroke. Zhongguo Linchuang Kangfu 2006;21(6):494-6.

\section{Xiao 2003 \{published data only\}}

Xiao W, Wang J, Luo Z, Xie R. The economic health evaluation to the early intensive rehabilitation on patients with stroke. Zhongguo Linchuang Kangfu 2003;7(3):372-3.

Xie 2003 \{published data only\}

Xie S, Zhu M, Zhang X. Effect of early rehabilitation nursing on activities of daily living in patients with stroke. Zhongguo Linchuang Kangfu 2003;7(1):143.

\section{Xie 2005 \{published data only\}}

Xie XH, Chen WH, Yang R, Wu WQ, Zhang W, Qi Q, et al. Effects of rehabilitation training on quality of life in stroke patients at different stages after the episode. Zhongguo Linchuang Kangfu 2005;9(32):47-9.

Physical rehabilitation approaches for the recovery of function and mobility following stroke (Review) 


\section{Xu 1999 \{published data only\}}

Xu L, Shong Q, Zuo H. Effects of rehabilitation treatment on the hemiplegic patients following stroke. Jiangxi Medical Journal 1999;34(3):149-50.

\section{Xu 2003a \{published data only\}}

Xu Y, Liu S. Effects of early rehabilitation on patients with paralysis after cerebral infarction. Zhongguo Linchuang Kangfu 2003;7(1):127.

\section{Xu 2003b $\{$ published data only\}}

$\mathrm{Xu} \mathrm{H}$, Sun S, Wang M. The effect of early rehabilitation on hemiplegic limbs of patients with stroke. Zhongguo Linchuang Kangfu 2003;7(3):503.

\section{Xu 2004 \{published data only\}}

Xu BH, Yu RQ, Yu W, Xie B, Huang YX. Effects of early rehabilitation on activities of daily living and complications in acute stroke patients. Beijing Da Xue Xue Bao 2004;36(1):75-8.

\section{Xue 2006 \{published data only\}}

Xue J, Bai L, Guo QR, Yang CR, Lu J. Efficacy of early intervention of motor relearning program on post-stroke hemiplegia: a randomized controlled observation. Neural Regeneration Research 2006;1(3):277-9.

\section{Yan 2002 \{published data only\}}

Yan YL. Effects of early rehabilitation on lower extremity motor function in stroke hemiplegia. Zhongguo Linchuang Kangfu 2002;6(3):331-2.

\section{Yelnik 2008 \{published data only\}}

Yelnik AP, Le Breton F, Colle FM, Bonan IV, Hugeron C, Egal V, et al. Rehabilitation of balance after stroke with multisensorial training: a single-blind randomized controlled study. Neurorehabilitation and Neural Repair 2008;22(5):468-76.

\section{Yin 2003a \{published data only\}}

Yin H, Tian Y. Early rehabilitation of hemiplegic extremities after stroke and effect observation of therapy with intermediate frequency. Zhongguo Linchuang Kangfu 2003;7(7):1198.

\section{Zhang 1998 \{published data only\}}

Zhang X. Observation of the effects of early rehabilitation intervention for cerebral apoplexy and hemiplegia. Heilongjiang Nursing Journal 1998;4(11):1-2.

\section{Zhang 2004 \{published data only\}}

Zhang T, Li LL, Bi S, Mei YW, Xie RM, Luo ZM, et al. Effects of three-stage rehabilitation treatment on acute cerebrovascular diseases: a prospective, randomized, controlled, multicenter study. Zhonghua Yi Xue Za Zhi 2004;84(23):1948-54.

\section{Zhao 2002 \{published data only\}}

Zhao F, Ji X. Early stage rehabilitation and nursing care in cerebral apoplexy patients with hemiparalysis. Zhongguo Huli Zazhì 2002;16(4):194-6.
Zhao 2003 \{published data only\}

Zhao F, Wang L, Tian G, Zhou J, Han J. Early rehabilitation intervention promoting ability of daily living in acute stroke patients. Zhongguo Linchuang Kangfu 2003;7(5):851.

\section{Zhu 2001 \{published data only\}}

Zhu Y-L, Hu Y-S, Yang P-J. Effects of three months' rehabilitation on motor function in hemiplegics after stroke. Chinese Journal of Rehabilitation Theory and Practice 2001;7(4):155-6.

Zhu 2004b \{published data only\}

Zhu GX, Hu YS, Wu Y, Zhu YL, Han X, Sun LM, et al. Effects of standardized three-stage rehabilitation on recovery of neurological function in stroke patients with hemiplegia. Zhonghua Yi Xue Za Zhi 2004;84(23):1955-8.

Zhu 2006 \{published data only\}

Zhu J, Liao L, Chen S. Influence of rehabilitative nursing intervention on activities of daily living of senile cerebral apoplexy patients. Zhongguo Huli Zazhì 2006;20(4A):890-2.

\section{Zhu 2007 haem \{published data only\}}

Zhu XJ, Wang T, Chen Q, Wang X, Hou H, Wang HX. The effects of standardised rehabilitation treatment on the outcome of activities of daily living in patients with hemiplegia after stroke. Chinese Journal of Cerebrovascular Diseases 2007;4(6):254-9.

Zhu 2007 isch \{published data only\}

Zhu X, Wang T, Chen Q, Wang X, Hou H, Wang H. The effects of early rehabilitation on quality of life for elderly patients with stroke. Journal of Rehabilitation Medicine 2008;Suppl 46:118.

* Zhu XJ, Wang T, Chen Q, Wang X, Hou H, Wang HX. The effects of standardized rehabilitation treatment on the outcome of activities of daily living in patients with hemiplegia after stroke. Chinese Journal of Cerebrovascular Diseases 2007;4(6):254-9.

\section{Zhuang 2012 \{published data only\}}

Zhuang LX, Xu SF, D'Amado CR, Jia C, He J, Han DX, et al. An effectiveness study comparing acupuncture, physiotherapy, and their combination in poststroke rehabilitation: a multicentered, randomized, controlled clinical trial. Alternative Therapies in Health and Medicine 2012;18(3):8-14.

\section{References to studies excluded from this review}

Acalha 2010 \{published data only\}

Acalha T, MG, Suzuki SO, Berg TL. Effects of the task oriented and auditory cues for chronic stroke patients. Revista Terapia Manual 2010;8(39):441-7.

Dean 2000a \{unpublished data only\}

Dean CM, Malouin F, Richards CL. Locomotor task-related training for chronic stroke: multiple single case design. [Unpublished].

de Paula Oliveira 2007 \{published data only\} de Paula Oliveira T, Voos M, Korim W, Piemonte M. Homeexercise program for stroke patients: improvement in functional performance. Physiotherapy 2007;93 Suppl 1:S563-4. 
Dickstein 1986 \{published data only\}

Dickstein R, Hocherman S, Pillar T, Shaham R. Stroke rehabilitation: three exercise therapy approaches. Physical Therapy 1986;66(8):1233-8.

\section{Eng 2003 \{published data only\}}

Eng JJ, Chu KS, Kim CM, Dawson AS, Carswell A, Hepburn K. A community-based group exercise program for persons with chronic stroke. Medicine and Science in Sports and Exercise 2003;35:1271-8.

\section{English 2003 \{published data only\}}

English C, Warden-Flood A, Stiller K, Hillier S. Is task-related circuit training an effective means of providing rehabilitation to acute stroke patients? Australian Journal of Physiotherapy 2003;49 Suppl 4:s9-s10.

\section{Gong 2003 \{published data only\}}

Gong S, Zhang J, Yu K. Effect of early rehabilitation training on daily life activity of patients with hemiplegia after stroke. Zhongguo Linchuang Kangfu 2003;7(5):848-9.

\section{Gregson 2003 \{published data only\}}

Gregson J. Exercise training early after stroke: a feasibility study. Current Controlled Trials, 2003. http://www.controlledtrials.com/.

\section{Hesse 1998 \{published data only\}}

Hesse S, Jahnke MT, Schaffrin A, Lucke D, Reiter F, Konrad M. Immediate effects of therapeutic facilitation on the gait of hemiparetic patients as compared with walking with and without a cane. Electroencephalography and Clinical Neurophysiology 1998;109:515-22.

\section{Inaba 1973 \{published data only\}}

Inaba M, Edberg E, Montgomery J, Gillis MK. Effectiveness of functional training, active exercise, and resistive exercise for patients with hemiplegia. Physical Therapy 1973;53(1):28-35.

\section{Karaduman 2001 \{published data only\}}

Karaduman A, Armutlu K, Aksu A, Aras O, Arikan H. The comparison of different exercise-treatment methods in the rehabilitation of early stage hemiplegic patients. Neurorehabilitation and Neural Repair 2001;15(4):348.

\section{Khanna 2003 \{published data only\}}

Khanna PB. A randomised controlled study of the immediate and long term benefits of conventional stroke rehabilitation with task related group therapy in chronic stroke patients. www.controlled-trials.com (accessed July 2005).

\section{Kim 2001 \{published data only\}}

Kim CM, Eng JJ, MacIntyre DL, Dawson AS. Effects of isokinetic strength training on walking in persons with stroke: a double-blind controlled pilot study. Journal of Stroke and Cerebrovascular Diseases 2001;10:265-73.

\section{Krutulyte 2003 \{published data only\}}

Krutulyte G, Kimtys A, Krisciunas A. The effectiveness of physical therapy methods (Bobath and motor relearning program) in rehabilitation of stroke patients. Medicina 2003;39(9):889-95.

\section{Li 2004 \{published data only\}}

Li K, Dou Z-L, Zhu H-X, Zheng J-L, Lan Y. Effect of supported balance training on balance and activities of daily living in convalescent stroke patients. Zhongguo Linchuang Kangfu 2004;8(34):7620-1.

\section{Lin 2004 \{published data only\}}

Lin JH, Hsieh CL, Lo SK, Chai HM, Liao LR. Preliminary study of the effect of low-intensity home-based physical therapy in chronic stroke patients. Kaohsiung Journal of Medical Science 2004;20(1):18-23.

\section{Liu 2008 \{published data only\}}

Liu L, Zhu L. Efficacy observation of modified comprehensive rehabilitation therapy in patients with moderate to severe hemiplegia in acute stage of stroke. Chinese Journal of Cerebrovascular Diseases 2008;5(3):117-20.

\section{Meng 2005 \{published data only\}}

Meng ZX, Ni CM, Li JB, Han R. Effects of early rehabilitation on the functional outcomes of stroke patients estimated with functional comprehensive assessment. Chinese Journal of Clinical Rehabilitation 2005;9(5):1-3.

\section{Ng 2005 \{published data only\}}

$\mathrm{Ng}$ SS. Effectiveness of an Innovative Home-Based Rehabilitation Program on Lower Limb Functions in Subjects with Chronic Stroke: A Randomized, Controlled Trial. Hong Kong Polytechnic University (People's Republic of China), 2005.

Nissan-Lavi 2009 \{published data only\}

Nissan-Lavi L, Carmeli E. Stroke patients in the community: comparison of the effect of physical therapy group training and walking training. Journal of the Israeli Physical Therapy Society (JIPTS) 2009;11(1):28.

Ozdemir 2001 \{published data only\}

Ozdemir F, Birtane M, Tabatabaei R, Kokino S, Ekuklu G. Comparing stroke rehabilitation outcomes between acute inpatient and nonintense home settings. Archives of Physical Medicine and Rehabilitation 2001;82:1375-9.

Pomeroy 2001a \{published data only\}

Pomeroy VM. The immediate effects of neurofacilitatory physiotherapy on gait parameters early post-stroke. The National Research Register 2001;1:Publication ID: NO217063260.

\section{Pyoria 2007 \{published data only\}}

Pyoria O, Talvitie U, Nyrkko H, Kautiainen H, Pohjolainen T, Kasper V. The effect of two physiotherapy approaches on physical and cognitive functions and independent coping at home in stroke rehabilitation. A preliminary follow-up study. Disability and Rehabilitation 2007;29(6):503-11.

\section{Qiu 2004 \{published data only\}}

Qui W-H, Hu X-Q, Zheng J-L, Li K, Lan Y, Chen X-M. Effect of balance function on limb function and activities of daily living in hemiplegic patients. Zhongguo Linchuang Kangfu 2004;8(31):6872-3. 
Richards 2004 \{published data only\}

Richards CL, Malouin F, Bravo G, Dumas F, Wood-Dauphinee S. The role of technology in task-oriented training in persons with subacute stroke: a randomised controlled trial. Neurorehabilitation and Neural Repair 2004;18:199-211.

Salter 1991 \{published data only\}

Salter J, Camp Y, Pierce LL, Mion LC. Rehabilitation nursing approaches to cerebrovascular accident: a comparison of two approaches. Rehabilitation Nursing 1991;16(2):62-6.

\section{Stern 1970 \{published data only\}}

Stern PH, McDowell F, Miller JM, Robinson M. Effects of facilitation exercise techniques in stroke rehabilitation. Archives of Physical Medicine and Rehabilitation 1970;51:526-31.

\section{Stuart 2008 \{published data only\}}

Macko RF, Benvenuti F, Stanhope S, Macellari V, Taviani, A, Nesi B, et al. Adaptive physical activity improves mobility function and quality of life in chronic hemiparesis. Journal of Rehabilitation Research and Development 2008;45(2):323-8.

Stuart M, Benvenuti F, Macko R, Taviani A, Segenni L, Mayer F, et al. Community-based adaptive physical activity program for chronic stroke: feasibility, safety, and efficacy of the Empoli model. Neurorehabilitation and Neural Repair 2009;23(7):726-34

Stuart M, Chard S, Benvenuti F, Steinwachs S. Community exercise: a vital component to healthy aging. Healthcare Papers 2009;10(1):23-8.

Stuart M, Chard S, Roettger S. Exercise for chronic stroke survivors: a policy perspective. Journal of Rehabilitation Research and Development 2008;45(2):329-35.

* Stuart M. Adaptive physical activity for chronic stroke (APAStroke). ClinicalTrials.gov 2008.

\section{Thielman 2004 \{published data only\}}

Thielman GT, Dean CD, Gentile AM. Rehabilitation of reaching after stroke: task-relating training versus progressive resistive exercise. Archives of Physical Medicine and Rehabilitation 2004;85:1613-8.

\section{Wagenaar 1990 \{published data only\}}

Wagenaar RC, Meijer OG, van Wieringen PCW, Kuik DJ, Hazenberg GJ, Lindeboom J, et al. The functional recovery of stroke: a comparison between neuro-developmental treatment and the Brunnström method. Scandinavian Journal of Rehabilitation Medicine 1990;22:1-8.

\section{Wang 2005b \{published data only\}}

Wang A, Wang Y, Hu P. Influence of comprehensive nursing intervention on extremities function in patients with cerebrovascular accidents. Chinese Nursing Research 2005;19(1A):37-39.

\section{Wolny 2003 \{published data only\}}

Wolny T, Saulicz E, Gnat R, Bacik B. The effectiveness of PNF method in gait reeducation in hemiplegic patients - a preliminary study. In: Proceedings of the 14th International
Congress of the World Confederation for Physical Therapy. Barcelona, Spain, 2003:Abst. RR-PO-0326.

Wood 1994 \{published data only\}

Wood-Dauphinee S. The impact of task-oriented physical therapy on locomotion recovery of stroke patients. [Unpublished].

\section{Xu 2008 \{published data only\}}

$\mathrm{Xu} Y$. The effect of early intensive exercise on patients with hemiplegia. Journal of Rehabilitation Medicine 2008;Suppl 46:75.

\section{Yin 2003b \{published data only\}}

Yin S. The recovery comparison of comprehensive rehabilitation and simple pharmacotherapy on the patients after stroke during the period of sequela. Chinese Journal of Clinical Rehabilitation 2003;7(1):140.

\section{Yu 2008 \{published data only\}}

Yu J, Hu Y, Wu Y, Chen W, Cui X, Lu W, et al. An analysis about the effects of standardized community-based rehabilitation (CBR) therapy on ADL for patients after stroke in China. Journal of Rehabilitation Medicine 2008;Suppl 46:110.

\section{Zhong 2006 \{published data only\}}

Zhong M, Mo H, Liu H. Evidence-based nursing on early rehabilitation of cerebral infarction patients with hemiplegia. Chinese Nursing Research 2006;20(2B):393-5.

\section{Zhou 2003 \{published data only\}}

Zhou J, Wang J. The effect of earlier functional training improving ability of living self care of patients with stroke. Zhongguo Linchuang Kangfu 2003;7(3):508.

\section{Zhu 2004a \{published data only\}}

Zhu Y-L, Hu Y-S, Wu Y, Xu Y-M. Possibility of functional recovery with early rehabilitation training during the first 3 months in hemiplegic patients with stroke. Zhongguo Linchuang Kangfu 2004;8(1):18-9.

\section{References to studies awaiting assessment}

Giraux 2008 \{published data only\}

Giraux P. Impact of prolonged physical therapy on gait performance of chronic phase stroke patients (HEMIMARCHE). ClinicalTrials.gov 2008.

\section{Li 2000 \{published data only\}}

* Li H, Jia Z, Song L. Early rehabilitation effect on stroke hemiplegia. Chinese Journal of Physical Medicine 1998;20:1-2.

Li H. Long term effect of early rehabilitation on stroke hemiplegic patients. Chinese Journal of Physical Medicine and Rehabilitation 2000;22(1):15-7.

\section{Matsumoto 2010 \{published data only\}}

Matsumoto $\mathrm{S}$. The effect of trunk facilitation in post-stroke patients. UMIN Clinical Trials Registry (UMIN-CTR), 2010. http:// www.umin.ac.jp/ctr/. 
Richardson 2011 \{published data only\}

Richardson J, Fleck R, Hladysh G, McBay C, MacKay E, Thorlakson R, et al. Fit for function: a community wellness program for persons with stroke. Stroke 2011;42(11):e596.

\section{Sanchez-Sanchez 2011 \{published data only\}}

Sanchez-Sanchez ML, Igual-Camacho C, Villaplana-Alcazar M, Rubio-Moreno MC, Viosca-Herrero E. Gait recovery after stroke: a physiotherapy protocol based on clinical and functional criteria. Cerebrovascular Diseases 2011;31 Suppl 2:202.

\section{Wang 2005a \{published data only\}}

Wang W. Effect of systematic rehabilitative training on motor function and activity of daily life in post stroke hemiplegic patients. Chinese Journal of Clinical Rehabilitation 2005;9(21):14-5.

\section{Wang 2012 \{published data only\}}

Wang W, Ru X, Dai H, Wu S, Jiang B. A new model of rehabilitation study at community level for stroke survivors in five urban populations in China. Neuroepidemiology 2012;39(3-4):191.

\section{Yau 2010 \{published data only\}}

Yau WWM, Wong WL. The feasibility and efficacy of an augmented exercise program for acute stroke patients. International Journal of Stroke 2010;5:346-7.

\section{Zhang 2008 \{published data only\}}

Zhang J, Wu S, Huang Y, Long Y, Chen T, Feng L, et al. The effects of standardized three stages rehabilitation program in promoting active function in stroke patients with hemiplegia. Journal of Rehabilitation Medicine 2008;Suppl 46:111 (Abst PP002-060).

\section{References to ongoing studies}

\section{Cross 2009 \{published data only\}}

Cross J. FeST1vAIS. Functional strength training to improve walking and upper limb function in people at least 1 year after stroke. UK Clinical Research Network Portfolio Database, 2009. http://public.ukcrn.org.uk/search/:last accessed 27th June 2013.

Cross J. Functional strength training to improve walking and upper limb function in people at least one year after stroke: a phase II trial. Current Controlled Trials, 2009. http:// www.controlled-trials.com/:last accessed 27th June 2013.

* Mares K, Cross J, Clark A, Barton GR, Poland F, O'Driscoll ML, et al. The FeSTivaLS trial protocol: a randomized evaluation of the efficacy of functional strength training on enhancing walking and upper limb function later post stroke. International Journal of Stroke 2013;5:374-82.

\section{Kumaran 2010 \{published data only\}}

Kumaran S. Effectiveness and feasibility of a task and context based exercise program in stroke patients: a randomized controlled trial. Clinical Trials Registry - India (CTRI), 2010. http://www.ctri.in:last accessed 19th June 2013.

\section{Additional references}

\section{Affolter 1980}

Affolter F. Perceptual processes as prerequisites for complex human behaviours. International Rehabilitation Medicine 1980;3:3-9.

\section{Anderson 1986}

Anderson M, Lough S. A psychological framework for neurorehabilitation. Physiotherapy Practice 1986;2:74-82.

\section{Ashburn 1995}

Ashburn A. A review of current physiotherapy in the management of stroke. In: Harrison MA, editors(s). Physiotherapy in Stroke Management. London: Churchill Livingstone, 1995:3-22.

\section{Ayres 1972}

Ayres AJ. Sensory Integration and Learning Disabilities. Los Angeles: Western Psychological Services, 1972.

\section{Berg 1989}

Berg K, Wood-Dauphinee S, Williams JI, Gayton D. Measuring balance in the elderly: preliminary development of an instrument. Physiotherapy Canada 1989;41:304-11.

\section{Berg 1992}

Berg K, Wood-Dauphinee SL, Williams JI, Maki BE. Measuring balance in the elderly: validation of an instrument. Canadian Journal of Public Health 1992;83(S2):S7-S11.

\section{Bobath 1959}

Bobath B. Observations on adult hemiplegia and suggestions for treatment. Physiotherapy 1959;45:279-89.

\section{Bobath 1966}

Bobath K. The Motor Deficit in Patients with Cerebral Palsy. London: Heinemann, 1966.

\section{Bobath 1970}

Bobath B. Adult Hemiplegia: Evaluation and Treatment. London: Heinemann Medical, 1970.

\section{Bobath 1978}

Bobath B. Adult Hemiplegia: Evaluation and Treatment. 2nd edition. London: Heinemann Medical, 1978.

\section{Bobath 1990}

Bobath B. Adult Hemiplegia: Evaluation and Treatment. 3rd edition. London: Butterworth-Heinemann, 1990.

\section{Bower 1993}

Bower E. Physiotherapy for cerebral palsy: a historical review. Baillières Clinical Neurology 1993;2(1):29-54.

\section{Brunnström 1956}

Brunnström S. Associated reactions of the upper extremity in adult hemiplegia: an approach to training. Physical Therapy Review 1956;36:225-36. 


\section{Brunnström 1961}

Brunnström S. Motor behaviours of adult hemiplegia patients. American Journal of Occupational Therapy 1961;15(1):6-12.

\section{Brunnström 1970}

Brunnström S. Movement Therapy in Hemiplegia. London: Harper and Row, 1970.

\section{Carlisle 2010}

Carlisle D. Bobath under fire. http://www.csp.org.uk/frontline/ article/bobath-under-fire 2010;16(1).

\section{Carr 1980}

Carr JH, Shepherd RB. Physiotherapy in Disorders of the Brain. London: Heinemann Medical, 1980.

\section{Carr 1982}

Carr JH, Shepherd RB. A Motor Relearning Programme for Stroke. London: Heinemann Medical, 1982.

\section{Carr 1985}

Carr JH, Shepherd RB, Nordholm L, Lynne, D. Investigation of a new motor assessment scale for stroke patients. Physical Therapy 1985;65:175-80.

\section{Carr 1987a}

Carr JH, Shepherd RB. Movement Science: Foundations for Physical Therapy in Rehabilitation. Rockville, MD: Aspen, 1987.

\section{Carr 1987b}

Carr JH, Shepherd RB. A Motor Relearning Programme for Stroke. 2nd edition. London: Heinemann Medical, 1987.

\section{Carr 1989}

Carr JH, Shepherd RB. A motor learning model for stroke rehabilitation. Physiotherapy 1989;89:372-80.

\section{Carr 1990}

Carr JH, Shepherd RB. A motor learning model for rehabilitation of the movement disabled. In: Ada L, Canning C, editors(s). Key Issues in Neurological Physiotherapy. Oxford: Heinemann Medical, 1990:1-24.

\section{Carr 1994a}

Carr JH, Mungovan SF, Shepherd RB, Dean CM, Nordholm LA. Physiotherapy in stroke rehabilitation: bases for Australian physiotherapists' choice of treatment. Physiotherapy Theory and Practice 1994;10:201-9.

\section{Carr 1998}

Carr JH, Shepherd RB. Neurological Rehabilitation. Optimising Motor Performance. Oxford: Butterworth-Heinemann, 1998.

\section{Carr 2002}

Carr JH, Shepherd RB. Stroke Rehabilitation: Guidelines for Exercise and Training to Optimize Motor Skill. Oxford: Butterworth-Heinemann, 2002.

\section{COMET Initiative}

COMET (Core Outcome Measures in Effectiveness Trials) Initiative. http://www.comet-initiative.org/ (Last accessed 6 February 2014).

\section{Cotton 1983}

Cotton E, Kinsman R. Conductive Education and Adult Hemiplegia. Edinburgh: Churchill Livingstone, 1983.

\section{Davidson 2000}

Davidson I, Waters K. Physiotherapists working with stroke patients: a national survey. Physiotherapy 2000;86(2):69-80.

\section{Davies 1985}

Davies PM. Steps to Follow. A Guide to the Treatment of Adult Hemiplegia. Berlin: Springer-Verlag, 1985.

\section{Davies 1990}

Davies PM. Right in the Middle. Selective Trunk Activity in the Treatment of Adult Hemiplegia. Berlin: Springer-Verlag, 1990.

\section{DeJong 2004}

DeJong G, Horn S, Gassaway J, Slavin M, Dijkers M. Toward a taxonomy of rehabilitation interventions: using an inductive approach to examine the 'Black Box' of rehabilitation. Archives of Physical Medicine and Rehabilitation 2004;85:678-86.

\section{Fisher 2013}

Fisher A, Martin J, Srikusalanukul W, Davis M. Trends in stroke survival incidence rates in older Australians in the new millennium and forecasts into the future. Journal of Stroke and Cerebrovascular Diseases 2013;5:1-12.

\section{Forlander 1999}

Forlander DA, Bohannon RW. Rivermead Mobility Index: a brief review of research to date. Clinical Rehabilitation 1999;13:97-100.

\section{Fugl-Meyer 1975}

Fugl-Meyer AR, Jaasko L, Leyman I, Olsson S, Steglind S. The post-stroke hemiplegic patient: I. A method for evaluation of physical performance. Scandinavian Journal of Rehabilitation Medicine 1975;975(7):13-31.

\section{Goff 1969}

Goff B. Appropriate afferent stimulation. Physiotherapy 1969;55:9-17.

\section{Hatano 1976}

Hatano S. Experience from a multicentre stroke register: a preliminary report. Bulletin of the World Health Organization 1976;54:541-53.

\section{Hu 2007a}

Hu Y, Wu Y, Liu S et al. Standardized tertiary rehabilitation on the comprehensive functions of cerebral strokes patients with hemiplegia. Chinese Journal of Rehabilitation Medicine 2007;22(1):3-8. 


\section{Johnstone 1980}

Johnstone M. Home Care for the Stroke Patient: Living in a Pattern. Edinburgh: Churchill Livingstone, 1980.

\section{Johnstone 1989}

Johnstone M. Current advances in the use of pressure splints in the management of adult hemiplegia. Physiotherapy 1989;75(7):381-4.

\section{Jorgensen 1995}

Jorgensen HS, Nakayama H, Raaschou HO, Olsen TS. Recovery of walking function in stroke patients - the Copenhagen stroke study. Archives of Physical Medicine and Rehabilitation 1995;76(1):27-32.

\section{Kabat 1953}

Kabat H, Knott M. Proprioceptive facilitation technics for treatment of paralysis. Physical Therapy Review 1953;33:53-64.

\section{Katz 1970}

Katz S, Down TD, Cash HR, Grotz RC. Progress in the development of the index of ADL. The Gerontologist 1970;10(1):20-30.

\section{Keith 1987}

Keith RA, Granger CV, Hamilton BB, Sherwin FS. The functional independence measure: a new tool for rehabilitation. Advances Clinical Rehabilitation 1987;1:6-18.

\section{Khan 2012}

Khan FR, Vijesh PV, Rahool S, Radha AA, Sukumaran S, Kurupath R. Physiotherapy practice in stroke rehabilitation: a cross-sectional survey of physiotherapists in the state of Kerala, India. Topics Stroke Rehabilitation 2012;19(5):405-10.

\section{Kinsman 1988}

Kinsman R, Verity R, Waller J. A conductive education approach for adults with neurological dysfunction. Physiotherapy 1988;74(5):227-30.

\section{Knott 1968}

Knott M, Voss DE. Proprioceptive Neuromuscular Facilitation. 2nd edition. New York: Harper and Row, 1968.

\section{Kollen 2009}

Kollen BJ, Lennon S, Lyons B, Wheatley-Smith L, Scheper M, Buurke JH, et al. The effectiveness of the Bobath concept in stroke rehabilitation: what is the evidence? Stroke 2009;40:e89-97.

\section{Langhammer 2012}

Langhammer B, Stanghelle JK. Concerns about standards of reporting clinical trials: an RCT comparing the Bobath concept and motor relearning interventions for rehabilitation of stroke patients as an exemplar. Physiotherapy Research International 2012;17(4):244-7.

\section{Langhorne 2009}

Langhorne P, Coupar F, Pollock A. Motor recovery after stroke: a systematic review. Lancet Neurology 2009;8(8):741-54.

\section{Legg 2000}

Legg L, Pollock A, Langhorne, Sellars C. A multidisciplinary research agenda for stroke rehabilitation. British Journal of Therapy and Rehabilitation 2000;7(7):319-24.

\section{Lennon 1996}

Lennon S. The Bobath concept: a critical review of the theoretical assumptions that guide physiotherapy practice in stroke rehabilitation. Physical Therapy Reviews 1996;1:35-45.

\section{Lennon 2001}

Lennon S, Baxter D, Ashburn A. Physiotherapy based on the Bobath concept in stroke rehabilitation: a survey within the UK. Disability and Rehabilitation 2001;23(6):254-62.

\section{Lincoln 1979}

Lincoln NB, Leadbitter D. Assessment of motor function in stroke patients. Physiotherapy 1979;65:48-51.

\section{Mahoney 1965}

Mahoney FI, Barthel DW. Functional evaluation: the Barthel Index. Md State Medical Journal 1965;14:61-65.

\section{Mathers 2006}

Mathers CD, Loncar D. Projections of global mortality and burden of disease from 2002 to 2030. PLoS Medicine 2006;3:e442.

\section{Mayo 2002}

Mayo NE, Wood-Dauphinee S, Cote R, Durcan L, Carlton J. Activity, participation, and quality of life 6 months poststroke. Archives of Physical Medicine and Rehabilitation 2002;83(8):1035-42.

\section{Mayston 2000}

Mayston MJ. The Bobath concept today. Talk given at the Chartered Society of Physiotherapy Congress 2000. www.bobath.org.uk/BobathConceptToday.html (last accessed October 2006)

\section{Mayston 2008}

Mayston M. Editorial: Bobath Concept: Bobath@50: midlife crisis - What of the future? Physiotherapy Research International 2008;13(3):131-6.

\section{Moseley 2005}

Moseley AM, Stark A, Cameron ID, Pollock A. Treadmill training and body weight support for walking after stroke. Cochrane Database of Systematic Reviews 2005, Issue 4. Art. No: CD002840. [DOI: 10.1002/14651858.CD002840]

\section{Nair 2011}

Nair R, Aggarwal R, Khanna D. Methods of formal consensus in classification/diagnostic criteria and guideline development. Seminars in Arthritis and Rheumatism 2011;41(2):95-105.

\section{Nilsson 1992}

Nilsson L, Nordholm L. Physical therapy in stroke rehabilitation: bases for Swedish physiotherapists' choice of treatment. Physiotherapy Theory and Practice 1992;8:49-55. 


\section{Ovbiagele 2011}

Ovbiagele B, Nguyen-Huynh MN. Stroke epidemiology: advancing our understanding of disease mechanism and therapy. Neurotherapeutics 2011;8:319-29.

\section{Partridge 1996}

Partridge CJ. Physiotherapy approaches to the treatment of neurological conditions - an historical perspective. In: Edwards S, editors(s). Neurological Physiotherapy. A Problem-Solving Approach. London: Churchill Livingstone, 1996:3-14.

\section{Patel 2000}

Patel AT, Duncan PW, Lai S-M, Studenski S. The relation between impairments and functional outcomes poststroke. Archives of Physical Medicine and Rehabilitation 2000;81(10):1357-63.

\section{Perry 1967}

Perry CE. Principles and techniques of the Brunnström approach to the treatment of hemiplegia. American Journal of Physical Medicine 1967;46(1):789-815.

\section{Pfeiffer 1975}

Pfeiffer E. A short portable mental status questionnaire for the assessment of organic brain deficit in elderly patients. Journal of American Geriatrics Society 1975;23:433-41.

\section{Pomeroy 2001b}

Pomeroy V, Niven D, Barrow S, Faragher E, Tallis R. Unpacking the black box of nursing and therapy practice for the post-stroke shoulder pain: a precursor to evaluation. Clinical Rehabilitation 2001;15:67-83.

\section{Pomeroy 2005}

Pomeroy VM, Cooke E, Hamilton S, Whittet A, Tallis RC. Development of a schedule of current physiotherapy treatment used to improve movement control and functional use of the lower limb after stroke: a precursor to a clinical trial. Neurorehabilitation and Neural Repair 2005;19:350-9.

\section{Pomeroy 2006}

Pomeroy VM, King L, Pollock A, Baily-Hallam A, Langhorne P. Electrostimulation for the recovery of movement or functional ability in patients after stroke. Cochrane Database of Systematic Reviews 2006, Issue 2. Art. No: CD003241. [DOI: 10.1002/14651858.CD003241]

\section{Research Group 2007}

Research Group A of Standardized Tertiary Rehabilitation Program. A multicenter, randomized and controlled clinical trial of standardized tertiary rehabilitation program in promoting cognitive function of stroke patients with hemiplegia. Fudan University Journal of Medical Sciences 2007;34(1):6-11.

\section{Rood 1954}

Rood M. Neurophysiological reactions as a basis for physical therapy. Physical Therapy Review 1954;34(9):444-9.

\section{Sackley 1996}

Sackley CM, Lincoln NB. Physiotherapy treatment for stroke patients: a survey of current practice. Physiotherapy Theory and Practice 1996;12:87-96.

\section{Sawner 1992}

Sawner K, LaVigne J. Brunnström's Movement Therapy in Hemiplegia: A Neurophysiological Approach. 2nd edition. Philadelphia: Lippincott, 1992.

\section{Schulz 2010}

Schulz KF, Altman DG, Moher D. CONSORT 2010 Statement: updated guidelines for reporting parallel group randomised trials. BMJ 2010;340:c332.

\section{Shaughnessy 2005}

Shaughnessy M, Michael KM, Sorkin JD, Macko RF. Steps after stroke - capturing ambulatory recovery. Stroke 2005;36:1305-7.

\section{Sinha 2008}

Sinha I, Jones L, Smyth RL, Williamson PR. A systematic review of studies that aim to determine which outcomes to measure in clinical trials in children. PLoS Medicine 2008;5(4):e96.

\section{Stapleton 2010}

Stapleton T, Connelly D. Occupational therapy practice in predriving assessment post stroke in the Irish context: findings from a nominal group technique meeting. Topics Stroke Rehabilitation 2010;17(1):58-68.

\section{Stockmeyer 1967}

Stockmeyer SA. An interpretation of the approach of Rood to the treatment of neuromuscular dysfunction. American Journal of Physical Medicine 1967;46(1):900-67.

\section{Turnbull 1982}

Turnbull GI. Some learning theory implications in neurological physiotherapy. Physiotherapy 1982;68:38-41.

\section{Turner 1995}

Turner PA, Whitfield TA. Physiotherapists' reasons for selection of treatment techniques: a cross-national survey. Physiotherapy Theory Practice 1999;15:235-46.

\section{Tyson 2009a}

Tyson SF, Connell LA, Lennon S, Busse ME. What treatment packages do UK physiotherapists use to treat postural control and mobility problems after stroke? Disability and Rehabilitation 2009;31(18):1494-500.

\section{Tyson 2009b}

Tyson SF, Connell LA, Busse ME, Lennon S. What is Bobath? A survey of UK stroke physiotherapists' perceptions of the content of the Bobath concept to treat postural control and mobility problems after stroke. Disability and Rehabilitation 2009;31(6):448-57.

\section{van Bennekom 1995}

van Bennekom CA, Jelles F, Lankhorst GJ, Bouter LM. The Rehabilitation Activities Profile: a validation study of its use as a disability index with stroke patients. Archives of Physical Medicine and Rehabilitation 1995;76(6):501-7.

\section{van Peppen 2004}

Van Peppen R, Kwakkel G, Wood-Dauphinee S, Hendriks H, Van der Wees P, Dekker J. The impact of physical therapy on 
functional outcomes after stroke: what's the evidence? Journal of Clinical Rehabilitation 2004;18:833-62.

\section{van Swieten 1988}

van Swieten J, Koudstaal P, Visser M, Schouten $\mathrm{H}$, et al. Interobserver agreement for the assessment of handicap in stroke patients. Stroke 1988;19(5):604-7.

\section{Voss 1967}

Voss DE. Proprioceptive neuromuscular facilitation. American Journal of Physical Medicine 1967;46(1):838-99.

\section{Voss 1985}

Voss DE, Ionta MK, Myers BJ. Proprioceptive Neuromuscular Facilitation Patterns and Techniques. 3rd edition. Philadelphia: Harper \& Row, 1985.

\section{Wade 1992a}

Wade DT. Measurement in Neurological Rehabilitation. 1st edition. Oxford: Oxford University Press, 1992.

\section{Warlow 2008}

Warlow C, van Gijn J, Dennis M, et al. Stroke: Practical Management. 3rd edition. Oxford: Blackwell Publishing, 2008.

\section{WHO 2002}

The International Classification of Functioning, Disability and Health (ICF). Towards a common language for functioning, disability and health. World Health Organization, Geneva 2002.

\section{Wilson 1987}

Wilson B, Cockburn J, Halligan P. Development of a behavioral test of visuospatial neglect. Archives of Physical Medicine Rehabilitation 1987;68:98-102.

\section{Zhang 2013}

Zhang WW, Speare S, Churilov L, Thuy M, Donnan G, Bernhardt J. Stroke rehabilitation in China: a systematic review and meta-analysis. International Journal of Stroke 2013;29:1-9.

\section{References to other published versions of this review Pollock 2007}

Pollock A, Baer G, Pomeroy VM, Langhorne P. Physiotherapy treatment approaches for the recovery of postural control and lower limb function following stroke. Cochrane Database of Systematic Reviews 2007, Issue 1. Art. No: CD001920. [DOI: 10.1002/14651858.CD001920.pub2]

* Indicates the major publication for the study

\section{CHARACTERISTICS OF STUDIES}

Characteristics of included studies [ordered by study ID]

Aksu 2001

\section{Study characteristics}

Study design: RCT with three treatment groups
Method of randomisation: not stated ("Patients were randomly divided into three groups")

Participants Number of participants: $n=20$

Inclusion criteria: "patients, whose functional levels were similar, were included"

Interventions (1) Group $1(\mathrm{n}=9)$

"Four exercises ... were chosen from Bobath's neurodevelopmental approach"

The individual components delivered are listed in Table 3. Based on the individual components, this intervention is categorised as a neurophysiological intervention

Length of intervention period: not stated

Number of sessions and length of individual sessions: not stated

Intervention provider: not stated

(2) Group $2(n=7)$

"Six exercises ... were chosen from Bobath's neurodevelopmental approach"

The individual components delivered are listed in Table 3. Based on the individual components, this intervention is categorised as a neurophysiological intervention 
Aksu 2001 (Continued)

Length of intervention period: not stated

Number of sessions and length of individual sessions: not stated

Intervention provider: not stated

(3) Group $3(n=4)$

"Eight exercises ... were chosen from Bobath's neurodevelopmental approach"

The individual components delivered are listed in Table 3. Based on the individual components, this intervention is categorised as a neurophysiological intervention

Length of intervention period: not stated

Number of sessions and length of individual sessions: not stated

Intervention provider: not stated

This study is classified as active intervention one (neurophysiological) versus active intervention two (neurophysiological) versus active intervention three (neurophysiological) (Table 6)

\begin{tabular}{ll}
\hline Outcomes & Other secondary outcome measures: Stroke Rehabilitation Assessment of Movement (STREAM) \\
& $\begin{array}{l}\text { Time points when outcomes were assessed: "after the stroke in the first week, motor assessment was } \\
\text { performed" }\end{array}$ \\
\hline Notes & Abstract only \\
& No data available for analysis \\
\hline
\end{tabular}

\section{Risk of bias}

\begin{tabular}{|c|c|c|}
\hline Bias & Authors' judgement & Support for judgement \\
\hline $\begin{array}{l}\text { Random sequence genera- } \\
\text { tion (selection bias) }\end{array}$ & Unclear risk & No information provided \\
\hline $\begin{array}{l}\text { Allocation concealment } \\
\text { (selection bias) }\end{array}$ & Unclear risk & No information provided \\
\hline $\begin{array}{l}\text { Blinding of outcome as- } \\
\text { sessment (detection bias) } \\
\text { All outcomes }\end{array}$ & Unclear risk & No information provided \\
\hline $\begin{array}{l}\text { Incomplete outcome data } \\
\text { (attrition bias) } \\
\text { All outcomes }\end{array}$ & Low risk & No dropouts \\
\hline $\begin{array}{l}\text { Free of systematic differ- } \\
\text { ences in baseline charac- } \\
\text { teristics of groups com- } \\
\text { pared? }\end{array}$ & Unclear risk & No information provided \\
\hline $\begin{array}{l}\text { Did authors adjust for } \\
\text { baseline differences in } \\
\text { their analyses? }\end{array}$ & Unclear risk & No information provided \\
\hline Other bias & Unclear risk & Limited information: abstract only \\
\hline
\end{tabular}




\section{Study characteristics}

Study design: RCT
Methods of randomisation: "Randomization was achieved via the use of 20 sealed envelopes, 10 of each
containing a specific group allocation. A staff member who was blind to the study selected a sealed en-
velope for each participant, indicating the group allocation"

Participants

Number of participants: $\mathrm{n}=17$

Inclusion criteria: "Patients with a confirmed diagnosis of recent stroke were eligible for inclusion"

Exclusion criteria: terminally ill, suffering from an unstable co-morbidity and unable to participate safely (physically or mentally) in additional sessions of standing

Interventions

(1) Intervention group $(n=7)$

"The second treatment group (intervention group) received the conventional treatment session, and in addition had a further session of 45 minutes standing practice on each working day. This was provided by a physiotherapy assistant and typically involved the use of either standing frames, tilt tables or standing at tables to provide support while enabling standing to occur"

"Participants progressed to standing by a table for support or free standing during rehabilitation as able. Participants were encouraged to be active whilst standing-practising reaching tasks, sit-to-stand movements and so on, and were given rest periods as necessary throughout the 45 -minute session. It was not possible for the physiotherapists providing the conventional treatment to be blind to the extra intervention occurring, due to the organization of therapy on the ward. This treatment regime was continued throughout the participant's stay in the rehabilitation unit. After discharge from the unit the participant was referred for outpatient or community-based physiotherapy. Intensity of follow-up offered was based on community assessment and was typically one or two sessions of treatment per week"

The individual components delivered are listed in Table 3. Based on the individual components, this intervention is categorised as comprising functional task training and musculoskeletal intervention (active)

Length of intervention period: ranged from 14 to 28 days dependent upon length of stay on the unit

Number of sessions and length of individual sessions: "received the conventional treatment session, and in addition had a further session of 45 minutes standing practice on each working day"

Intervention provider: "conventional physiotherapy sessions were provided [by] one of three physiotherapists on the ward; the additional session of standing practice was provided by a physiotherapy assistant"

(2) Control group $(n=10)$

"Control group received conventional physiotherapy treatment from one of the three physiotherapists working on the ward. This was typically a session of 45 minutes treatment on each working day, including work on strengthening, improving movement, mobility, and upper limb function"

The individual components delivered are listed in Table 3. Based on the individual components, this intervention is categorised as comprising functional task training and musculoskeletal intervention (active)

Length of intervention period: ranged from 14 to 28 days dependent upon length of stay on the unit Number of sessions and length of individual sessions: "session of 45 minutes treatment on each working day"

Intervention provider: "conventional physiotherapy sessions were provided [by] one of 3 physiotherapists on the ward" 
Allison 2007 (Continued)

This study is classified as active Intervention one (functional task training, musculoskeletal (active)) versus active intervention two (functional task training, musculoskeletal (active)) (Table 6)

\begin{tabular}{|c|c|c|}
\hline \multirow[t]{4}{*}{ Outcomes } & \multirow{2}{*}{\multicolumn{2}{|c|}{$\begin{array}{l}\text { Measures of motor function: gross functional tool section of Rivermead Motor Assessment } \\
\text { Measures of postural control and balance: Berg Balance Scale }\end{array}$}} \\
\hline & & \\
\hline & \multicolumn{2}{|c|}{ Other secondary outcome measures: trunk control test } \\
\hline & \multicolumn{2}{|c|}{ Time points when outcomes were assessed: week one, week two and week 12} \\
\hline Notes & \multicolumn{2}{|c|}{ No outcomes included in analysis (data reported as median and IQR) } \\
\hline \multicolumn{3}{|l|}{ Risk of bias } \\
\hline Bias & Authors' judgement & Support for judgement \\
\hline $\begin{array}{l}\text { Random sequence genera- } \\
\text { tion (selection bias) }\end{array}$ & Low risk & $\begin{array}{l}\text { "Randomization was achieved via the use of } 20 \text { sealed envelopes, } 10 \text { of each } \\
\text { containing a specific group allocation" }\end{array}$ \\
\hline $\begin{array}{l}\text { Allocation concealment } \\
\text { (selection bias) }\end{array}$ & Low risk & $\begin{array}{l}\text { "A staff member who was blind to the study selected a sealed envelope for } \\
\text { each participant, indicating the group allocation" }\end{array}$ \\
\hline $\begin{array}{l}\text { Blinding of outcome as- } \\
\text { sessment (detection bias) } \\
\text { All outcomes }\end{array}$ & Low risk & $\begin{array}{l}\text { "The measurements were conducted by a staff member who did not work on } \\
\text { the unit, and who was blind to the treatment allocation" }\end{array}$ \\
\hline $\begin{array}{l}\text { Incomplete outcome data } \\
\text { (attrition bias) } \\
\text { All outcomes }\end{array}$ & High risk & $\begin{array}{l}\text { "Three of the participants allocated to the additional practice group withdrew } \\
\text { from the additional treatment within the first week of the study, all of them cit- } \\
\text { ing fatigue as the reason they would not continue. One of these consented to } \\
\text { further measures being taken but two withdrew totally from the study. Results } \\
\text { were analysed on an intention to treat basis, but the two participants who only } \\
\text { completed the first week of measures were excluded" }\end{array}$ \\
\hline
\end{tabular}

\begin{tabular}{|c|c|c|}
\hline $\begin{array}{l}\text { Free of systematic differ- } \\
\text { ences in baseline charac- } \\
\text { teristics of groups com- } \\
\text { pared? }\end{array}$ & Unclear risk & Limited demographic data available at baseline \\
\hline $\begin{array}{l}\text { Did authors adjust for } \\
\text { baseline differences in } \\
\text { their analyses? }\end{array}$ & Unclear risk & No information provided \\
\hline Other bias & High risk & $\begin{array}{l}\text { "It was not possible for the physiotherapists providing the conventional treat- } \\
\text { ment to be blind to the extra intervention occurring, due to the organization } \\
\text { of therapy on the ward." "Fatigue may be a significant barrier to participate in } \\
\text { more intensive programmes" }\end{array}$ \\
\hline
\end{tabular}

Baer 2007

\section{Study characteristics}

\begin{tabular}{ll}
\hline Methods & Study design: single-blind RCT \\
& Method of randomisation: not stated ("subjects were randomly allocated to one of three groups") \\
\hline Participants & Number of participants: $\mathrm{n}=64$
\end{tabular}


Baer 2007 (Continued)

Inclusion criteria: not stated

Interventions

(1) Part practice group $(\mathrm{n}=$ not stated)

Participants in the part practice group were taught "part practice exercises targeted at mobility and lower limb function"

The individual components delivered are listed in Table 3. Based on the individual components, this intervention is categorised as comprising functional task training

Length of intervention period: four weeks

Number of sessions and length of individual sessions: total number of sessions: three, but the length of individual sessions was not stated

Intervention provider: research physiotherapist

(2) Whole practice group $(\mathrm{n}=$ not stated)

Participants in the whole practice group were taught "whole practice exercises targeted at mobility and lower limb function"

The individual components delivered are listed in Table 3. Based on the individual components, this intervention is categorised as comprising functional task training

Length of intervention period: four weeks

Number of sessions and length of individual sessions: total number of sessions: three, but the length of individual sessions was not stated

Intervention provider: research physiotherapist

(3) Control group $(\mathrm{n}=$ not stated)

Participants in the control group were "given information about stroke"

The individual components delivered are listed in Table 3. Based on the individual components, this intervention is categorised as no intervention

Length of intervention period: four weeks

Number of sessions and length of individual sessions: total number of sessions: three, but the length of individual sessions was not stated

Intervention provider: research physiotherapist

This study is classified as active intervention one (functional task training) versus active intervention two (functional task training) (Table 6) versus no treatment (Table 4)

Time points when outcomes were assessed: "Baseline outcome measures were taken on two occasions prior to the intervention phase and meaned (base). The baseline was compared to outcomes taken at the end of intervention (OM3) and a 48 hour retention test (OM4)"

No suitable data available for analysis

\section{Risk of bias}


Baer 2007 (Continued)

\section{Bias Authors' judgement Support for judgement}

Random sequence genera- Unclear risk No information provided

tion (selection bias)

\begin{tabular}{ll}
\hline $\begin{array}{l}\text { Allocation concealment } \\
\text { (selection bias) }\end{array}$ & Low risk
\end{tabular}

(selection bias)

\begin{tabular}{lll}
\hline $\begin{array}{l}\text { Blinding of outcome as- } \\
\text { sessment (detection bias) } \\
\text { All outcomes }\end{array}$ & Unclear risk & No information provided \\
\hline $\begin{array}{l}\text { Incomplete outcome data } \\
\text { (attrition bias) }\end{array}$ & Unclear risk & No information provided
\end{tabular}

(attrition bias)

All outcomes

Free of systematic differ-
ences in baseline charac- $\quad$ Unclear risk information provided about the individual groups

teristics of groups compared?

\begin{tabular}{lll}
\hline $\begin{array}{l}\text { Did authors adjust for } \\
\text { baseline differences in } \\
\text { their analyses? }\end{array}$ & Unclear risk & No information provided about the individual groups \\
\hline Other bias & Unclear risk & Limited information-abstract only \\
\hline
\end{tabular}

\section{Bai 2008}

\section{Study characteristics}

\begin{tabular}{ll} 
Methods & Study design: RCT \\
& Method of randomisation: not stated ("patients were randomized into two treatment groups") \\
\hline Participants & Number of participants: $\mathrm{n}=364$ \\
& Inclusion criteria: "admission[s] were stabilized within one week of symptoms, Glasgow Coma Scale \\
& (GCS) score $>8$, limb disability, aged between 40 years and 80 years" \\
& Exclusion criteria: "cerebrovascular disease history with residual symptoms, onset of ICH more than \\
three weeks prior, tetraplegia, history of dementia and not resident locally"
\end{tabular}

Interventions (1) Early rehabilitation group $(\mathrm{n}=183)$

"All patients underwent the same routine internal medical intervention, but those in the rehabilitation group underwent a standardized three-stage rehabilitation program. Patients from both groups could receive help from their relatives, if necessary, patients from both groups had access to daily training for unilateral spatial neglect, speech deficit, and for swallowing, bowel, and bladder dysfunction"

"The standardized three-stage rehabilitation program included physical and occupational therapies and emphasized $A D L$ training immediately after enrolment. These training programs were carried out by therapists in emergency hospitals, rehabilitation centers, and communities and were divided into primary, secondary, and third rehabilitation. The primary rehabilitation, which was aimed at practicing the basic ADL, was conducted at the Emergency Department or Neurology Department during the first month after stroke. The secondary rehabilitation was conducted at the Physical Department during the 
Bai 2008 (Continued)

second and third month after stroke to help develop balance and walking. The third rehabilitation was conducted to enhance ADL and motor functions during the fourth to sixth month"

The individual components delivered are listed in Table 3. Based on the individual components, this intervention is categorised as functional task training

Length of intervention period: six months

Number of sessions and length of individual sessions: "During primary rehabilitation, individual physical therapy began within 24 hours of admission and was performed for 45 minutes per day, five days per week"

Intervention provider: "Their relatives or nurses were trained to rehabilitate the patients at home. If patients were transferred to community centers instead, they were visited by therapists who directed their training every two weeks..treatment was conducted by one therapist per patient"

(2) Control group $(n=181)$ "Routine internal medical intervention"

The individual components delivered are listed in Table 3. Based on the individual components, this intervention is categorised as no intervention

Length of intervention period: no intervention

Number of sessions and length of individual sessions: no intervention

Intervention provider: no intervention

This study is classified as intervention (functional task training) versus no treatment (Table 4)

$\begin{array}{ll}\text { Outcomes } & \text { Measures of independence in ADL: modified Barthel Index } \\ & \text { Measures of motor function: Fugl-Meyer Assessment } \\ & \text { Time points when outcomes were assessed: "were administered at various times, including at admis- } \\ \text { sion and at one, three, and six months after the stroke" } & \begin{array}{l}\text { No outcomes included in analysis (data shown in graphical representation only and SD not reported). } \\ \text { Clarification of data sought but not obtained }\end{array}\end{array}$

\section{Risk of bias}

\begin{tabular}{lll}
\hline Bias & Authors' judgement & Support for judgement \\
\hline $\begin{array}{l}\text { Random sequence genera- } \\
\text { tion (selection bias) }\end{array}$ & Unclear risk & No information provided \\
\hline $\begin{array}{l}\text { Allocation concealment } \\
\text { (selection bias) }\end{array}$ & Unclear risk & No information provided \\
\hline $\begin{array}{l}\text { Blinding of outcome as- } \\
\text { sessment (detection bias) } \\
\text { All outcomes }\end{array}$ & Low risk & $\begin{array}{l}\text { "All measurements were recorded by an assessor who was blinded to the study } \\
\text { design and details" }\end{array}$ \\
& $\begin{array}{l}\text { "If the assessments took place when the patients were still hospitalized, trial } \\
\text { staff were informed of an impending assessor visit so that the assessor, who } \\
\text { was blinded to study design, would not inadvertently view a rehabilitation ses- } \\
\text { sion" }\end{array}$ \\
\hline
\end{tabular}

$\begin{array}{ll}\begin{array}{l}\text { Incomplete outcome data } \\ \text { (attrition bias) }\end{array} & \begin{array}{l}\text { "Six months after the stroke, } 12 \text { patients (four in the rehabilitation group and } \\ \text { eight in the control group) were lost to follow-up, and seven patients (two in } \\ \text { All outcomes }\end{array} \\ & \begin{array}{l}\text { the rehabilitation group and five in the control group) died" (relatively low } \\ \text { numbers lost to follow-up in relation to participant size of } 364 \text { ) }\end{array}\end{array}$


Bai 2008 (Continued)

Free of systematic differences in baseline charac-

Low risk teristics of groups compared?

Did authors adjust for $\quad$ Low risk information provided
baseline differences in
their analyses?

"On admission, the rehabilitation group and the control group were comparable on all baseline measures"

Other bias Unclear risk No information provided

Bale 2008

\section{Study characteristics}

\begin{tabular}{ll}
\hline Methods & $\begin{array}{l}\text { Study design: RCT } \\
\text { Method of randomisation: "Patients who volunteered and gave written informed consent were ran- } \\
\text { domly allocated to two different training groups, either a functional strength training group or a train- } \\
\text { ing-as-usual group by drawing lots. From a total number of 20, } 10 \text { were allotted to each training group" }\end{array}$ \\
\hline Participants & $\begin{array}{l}\text { Number of participants: } \mathrm{n}=18 \\
\text { Inclusion criteria: first onset of stroke with reduced muscle strength in the affected leg, ability to under- } \\
\text { Exclusion criteria: significant sensory or cognitive sequelae, arrhythmia, uncontrolled angina pectoris } \\
\text { or hypertension or co-morbidities that could mask the sequelae from the stroke and patients with no } \\
\text { motor control of the affected leg }\end{array}$
\end{tabular}

Interventions

(1) Functional strength training (intervention) group $(n=8)$

"Had training to improve the muscle strength of the lower extremities three days a week, and trained arm functions and activities of daily living the remaining two days. The functional strength training programme was designed to facilitate appropriate power in the weak muscles of the affected leg in graded activities or sequences of activities. Most of the exercises were weight-bearing and also challenged standing balance"

The exercises are well described in the appendix-including strength training exercises such as stepups, standing, sit-to-stand-to-sit, heel and toe rises and bridging

"Each strengthening exercise was performed according to the principle of 10 to 15 repetitions maximum to achieve moderate fatigue in one set"

"The patients trained in ADLs such as walking, sitting-to-standing, stair climbing, etc. if time permitted after the strengthening exercises"

The individual components delivered are listed in Table 3. Based on the individual components, this intervention is categorised as comprising functional task training and musculoskeletal intervention (active)

Length of intervention period: four weeks

Number of sessions and length of individual sessions: 50 minutes/d five days a week

Intervention provider: different physiotherapists trained participants in the two intervention groups

(2) Training as usual (comparison) group $(n=10)$ 
Bale 2008 (Continued)

"The patients in the training-as-usual group had traditional training influenced by the Bobath Concept, with a central focus on normalizing muscle tone and movements on the affected side, symmetrical use of the body and relearning activities of daily living, often using manual guiding and facilitation techniques. Use of excessive muscle power was avoided to prevent associated reactions during training"

"As part of their basic rehabilitation, all patients participated in multidisciplinary training programmes. Questionnaires were filled in by nurses and occupational therapists at week 3 of each patient's training period to obtain information about attendance and quantity of training in the wards, and in sessions with occupational therapists"

The individual components delivered are listed in Table 3. Based on the individual components, this intervention is categorised as comprising functional task training and neurophysiological intervention

Length of intervention period: four weeks

Number of sessions and length of individual sessions: 50 minutes/d five days a week

Intervention provider: Different physiotherapists trained participants in the two intervention groups

This study is classified as active intervention one (functional task training, musculoskeletal (active)) versus active intervention two (functional task training, neurophysiological) (Table 6)

Measures of motor function: Motor Assessment Scale
Measures of voluntary movement: gait speed over 12 metres
Measures of muscle strength: dynamometer measures of knee extension and flexion
Other secondary outcome measures: maximum weight bearing, Patient Global Impression of Change
Time points when outcomes were assessed: at inclusion and after four weeks

Notes

\section{Risk of bias}

\begin{tabular}{lll} 
Bias & Authors' judgement & Support for judgement \\
\hline $\begin{array}{l}\text { Random sequence genera- } \\
\text { tion (selection bias) }\end{array}$ & Unclear risk & $\begin{array}{l}\text { "Randomly allocated to two different training groups, either a functional } \\
\text { strength training group or a training-as-usual group by drawing lots" }\end{array}$ \\
\hline $\begin{array}{l}\text { Allocation concealment } \\
\text { (selection bias) }\end{array}$ & Unclear risk & Not enough information provided \\
\hline $\begin{array}{l}\text { Blinding of outcome as- } \\
\text { sessment (detection bias) } \\
\text { All outcomes }\end{array}$ & Low risk & $\begin{array}{l}\text { "Two physiotherapists performed the physical measurements, and were blind- } \\
\text { ed to the patients' group assignment. Before the study started, the testers } \\
\text { were trained to perform the measurements based on a test protocol. To im- } \\
\text { prove reliability they tested four patients independently at the same time, and } \\
\text { discussed their scores afterwards" }\end{array}$
\end{tabular}

\begin{tabular}{lll}
\hline $\begin{array}{l}\text { Incomplete outcome data } \\
\text { (attrition bias) } \\
\text { All outcomes }\end{array}$ & Low risk & No dropouts \\
\hline $\begin{array}{l}\text { Free of systematic differ- } \\
\text { ences in baseline charac- } \\
\begin{array}{l}\text { teristics of groups com- } \\
\text { pared? }\end{array}\end{array}$ & Low risk & $\begin{array}{l}\text { "At inclusion there were no statistical significant differences between the } \\
\text { groups, neither in demographic variables nor in physical performance mea- } \\
\text { sures" }\end{array}$ \\
\hline
\end{tabular}


Bale 2008 (Continued)

Did authors adjust for baseline differences in their analyses?
Low risk No information provided

Other bias Unclear risk No information provided

Behrman 2011

\section{Study characteristics}

\begin{tabular}{ll}
\hline Methods & $\begin{array}{l}\text { Study design: single-blind RCT } \\
\text { Method of randomisation: not stated }\end{array}$ \\
\hline Participants & Number of participants: $\mathrm{n}=408$ \\
& $\begin{array}{l}\text { Inclusion criteria: "We compared the effect of two interventions to usual care initiated at two months } \\
\text { and assessed at } 6 \text { months post-stroke in } 408 \text { adults stratified by walking impairment }(\text { severe, }<0.4 \mathrm{~m} / \mathrm{s} ; \\
\text { moderate, } 0.4-0.79 \mathrm{~m} / \mathrm{s}) "\end{array}$
\end{tabular}

Interventions

(1) Locomotor training programme group $(n=139)$

Task-specific walking training using body-weight-support-treadmill and over-ground practice provided in clinics plus usual care

The individual components delivered are listed in Table 3. Based on the individual components, this intervention is categorised as comprising functional task training

Length of intervention period: 12 to 16 weeks

Number of sessions and length of individual sessions: 36 sessions, length of individual sessions not stated

Intervention provider: physical therapists

(2) Home exercise programme group $(n=126)$

"Impairment-based, progressive strength and balance exercise at home plus usual care"

The individual components delivered are listed in Table 3. Based on the individual components, this intervention is categorised as comprising functional task training and musculoskeletal intervention (active)

Length of intervention period: 12 to 16 weeks

Number of sessions and length of individual sessions: 36 sessions, length of individual sessions not stated

Intervention provider: physical therapists

(3) Usual care group $(n=143)$

The individual components delivered are listed in Table 3. Based on the individual components, this intervention is categorised as comprising functional task training and musculoskeletal intervention (active)

Length of intervention period: 12 to 16 weeks

Number of sessions and length of individual sessions: 36 sessions, length of individual sessions not stated 
Behrman 2011 (Continued)

Intervention provider: physical therapists

This study is classified as intervention (functional task training, musculoskeletal (active)) versus usual care (functional task training, musculoskeletal (active)) (Table 5)

\section{Outcomes}

Measures of voluntary movements: the proportion of participants that transitioned to a higher functional walking level six months post stroke, walking speed and distance

Measures of postural control and balance: daily step number

Measures of participation: functional status (specific scale not stated)

Measures of quality of life and social isolation: quality of life (specific scale not stated)

Time points when outcomes were assessed: six months post stroke

\begin{tabular}{ll}
\hline Notes & Abstract only \\
& Locomotor training programme (group one) intervention includes treadmill training as key interven- \\
tion and therefore is not relevant to this study
\end{tabular}

\section{Risk of bias}

\begin{tabular}{|c|c|c|}
\hline Bias & Authors' judgement & Support for judgement \\
\hline $\begin{array}{l}\text { Random sequence genera- } \\
\text { tion (selection bias) }\end{array}$ & Unclear risk & No information provided \\
\hline $\begin{array}{l}\text { Allocation concealment } \\
\text { (selection bias) }\end{array}$ & Unclear risk & No information provided \\
\hline $\begin{array}{l}\text { Blinding of outcome as- } \\
\text { sessment (detection bias) } \\
\text { All outcomes }\end{array}$ & Unclear risk & No information provided \\
\hline $\begin{array}{l}\text { Incomplete outcome data } \\
\text { (attrition bias) } \\
\text { All outcomes }\end{array}$ & Unclear risk & Insufficent information provided \\
\hline $\begin{array}{l}\text { Free of systematic differ- } \\
\text { ences in baseline charac- } \\
\text { teristics of groups com- } \\
\text { pared? }\end{array}$ & Unclear risk & Baseline characteristics not reported \\
\hline $\begin{array}{l}\text { Did authors adjust for } \\
\text { baseline differences in } \\
\text { their analyses? }\end{array}$ & Unclear risk & Not applicable \\
\hline Other bias & Unclear risk & Limited information available-abstract only \\
\hline
\end{tabular}

\section{Blennerhassett 2004}

\section{Study characteristics}

Methods Study design: prospective single-blind RCT


Blennerhassett 2004 (Continued)

Method of randomisation: "Subjects were assigned randomly to either an Upper Limb or Mobility training group. Randomisation was performed by a person independent from the study drawing a presealed opaque envelope that specified group allocation"

Pumber of participants: $\mathrm{n}=30$
Inclusion criteria: "The selection criteria were the ability to walk 10 metres with close supervision (with
or without walking aids) and ability to provide informed consent"
Exclusion criteria: "Deteriorating medical condition, or if they were independent community ambula-
tors"

"In addition to the study intervention, all subjects received their usual interdisciplinary rehabilitation, which included one hour of physiotherapy, five days a week. This physiotherapy was based predominantly upon the Movement Science approach (Carr 2002). The duration of interdisciplinary therapy was recorded"

"Both the Mobility and Upper Limb Groups received additional task-related practice for one hour a day, five days per week for four weeks. After the four weeks training, participants ceased the additional practice and continued with their interdisciplinary rehabilitation program. Each session consisted of a circuit of 10 five-minute workstations, with up to four subjects in each session. A physiotherapy department staff member supervised all sessions closely, and all activities were customised and progressed to suit individual subjects"

Mobility circuit classes were conducted separately from the upper limb sessions

"Mobility Group activities included warm-up and endurance tasks using stationary bikes and treadmills, followed by functional tasks such as sit to stand, step-ups, obstacle course walking, standing balance, stretching as required, and strengthening using traditional gymnasium equipment"

The individual components delivered are listed in Table 3. Based on the individual components, this intervention is categorised as comprising cardiopulmonary intervention, functional task training and musculoskeletal intervention (active)

Length of intervention period: four weeks

Number of sessions and length of individual sessions: "Usual interdisciplinary rehabilitation, which included one hour of physiotherapy, five days a week. Additional task-related practice for one hour a day, five days per week"

Intervention provider: physiotherapists

(2) Upper limb group $(n=15)$

"Upper Limb Group activities commenced with a warm-up (arm ergometer) followed by functional tasks to improve reach and grasp, hand-eye coordination activities, stretching as required, and strengthening using traditional gymnasium equipment. Therapist-assisted exercises were incorporated for subjects with limited control of arm or hand movement"

The individual components delivered are listed in Table 3. Based on the individual components, this intervention is categorised as comprising upper limb training

Length of intervention period: four weeks

Number of sessions and length of individual sessions: "Usual interdisciplinary rehabilitation, which included one hour of physiotherapy, five days a week. Additional task-related practice for one hour a day, five days per week"

Intervention provider: physiotherapists 
Blennerhassett 2004 (Continued)

This study is classified as intervention (functional task training and musculoskeletal intervention (active)) versus attention control (upper limb training) (Table 5). The intervention group also received cardiopulmonary intervention

Measures of motor function: Motor Assessment Scale (upper arm and hand)
Measures of postural control and balance: Timed Up and Go test, Step test
Measures of voluntary movements: Six-Minute Walk test
Other secondary outcome measures: Jebsen Taylor Hand Function Test
Time points when outcomes were assessed: "Measures of both mobility and upper limb function were
performed on three occasions: (1) prior to commencement in the trial, (2) immediately after the 4-week
additional training, and (3) at follow-up six months after completing the additional training"

Notes

\section{Risk of bias}

\begin{tabular}{lll}
\hline Bias & Authors' judgement & Support for judgement \\
\hline $\begin{array}{l}\text { Random sequence genera- } \\
\text { tion (selection bias) }\end{array}$ & Low risk & $\begin{array}{l}\text { "Randomisation was performed by a person independent from the study } \\
\text { drawing a pre-sealed opaque envelope that specified group allocation" }\end{array}$ \\
\hline $\begin{array}{l}\text { Allocation concealment } \\
\text { (selection bias) }\end{array}$ & Unclear risk & $\begin{array}{l}\text { "Subjects were not blinded to the research procedure although they were not } \\
\text { told of the study hypotheses. Treating physiotherapists were not told of group } \\
\text { allocations although they may have found out through interaction with sub- } \\
\text { jects during physiotherapy treatments" }\end{array}$ \\
\hline
\end{tabular}

\begin{tabular}{|c|c|c|}
\hline $\begin{array}{l}\text { Blinding of outcome as- } \\
\text { sessment (detection bias) } \\
\text { All outcomes }\end{array}$ & Low risk & $\begin{array}{l}\text { "An independent assessor who was blinded to group allocation and previous } \\
\text { test results, and was not involved in the treatment of the subject, performed } \\
\text { all tests" }\end{array}$ \\
\hline
\end{tabular}

All outcomes all tests"

Incomplete outcome data Low risk
(attrition bias)

(attrition bias)

Dropouts accounted for: "All subjects completed four weeks of additional training and follow-up was $100 \%$ at four weeks and $97 \%$ at six months. All subjects completed the mobility and MAS measurements on the initial and four week test. There were data missing from the JTHFT. At the four week assessment only 12 subjects from each group were able to perform the JTHFT. Of these subjects, the Mobility Group had six left and six right side affected, and the Upper Limb Group had seven left and five right side affected. One subject from the Upper Limb Group was not tested at the six month follow-up as he sustained a hip fracture secondary to a fall after discharge"

\begin{tabular}{|c|c|c|}
\hline $\begin{array}{l}\text { Free of systematic differ- } \\
\text { ences in baseline charac- } \\
\text { teristics of groups com- } \\
\text { pared? }\end{array}$ & Low risk & $\begin{array}{l}\text { "The groups were comparable at commencement of the study for factors such } \\
\text { as age, gender, type of stroke, side affected, time from stroke onset to rehabili- } \\
\text { tation admission, or time between onset and commencing the study ( } p=0.52 \\
\text { to 1.00) (see Tables } 1 \text { and } 2 \text { )" }\end{array}$ \\
\hline $\begin{array}{l}\text { Did authors adjust for } \\
\text { baseline differences in } \\
\text { their analyses? }\end{array}$ & Low risk & No information provided \\
\hline Other bias & Unclear risk & $\begin{array}{l}\text { "The number of additional practice sessions attended and total duration of in- } \\
\text { terdisciplinary therapy were similar for the two groups ( } p=0.57 \text { to } 0.87 \text { ). In ad- } \\
\text { dition, no difference was found between groups for the duration of mobility } \\
\text { and upper limb related practice delivered within the usual physiotherapy ses- } \\
\text { sions }(p=0.35 \text { to } 0.60) \text { " }\end{array}$ \\
\hline
\end{tabular}




\section{Study characteristics}

Methods

Study design: multi-centre RCT

Method of randomisation: "Randomization was done through a computer-generated, stratified, blocked randomization procedure. Patients were stratified according to time period from date of stroke to date of commencement in the trial. The two strata were four weeks to eight weeks post-stroke at commencement of the trial and more than eight weeks post-stroke. This stratification aimed to improve the likelihood of the two groups being similar in terms of initial severity and speed of recovery post-stroke, as those with milder stroke and a quicker rate of recovery are likely to improve more rapidly during the time period of the study. Separate computer-generated randomizations were used for each site. Opaque envelopes were used to conceal group allocation. Participants were randomized and assigned to the intervention groups after the baseline assessments were carried out"

Participants Number of participants: $\mathrm{n}=26$

Inclusion criteria: "first or recurrent stroke, haemorrhage or infarct, between four weeks and 20 weeks post-stroke at commencement of trial, currently participating in an inpatient or outpatient rehabilitation programme and able to walk 15 metres indoors on a level surface, with or without an aid, with supervision"

Exclusion criteria: "independent mobility indoors achieved within four weeks post-stroke, premorbid mobility limited to walking indoors only, unable to follow single stage verbal commands with visual prompts and mobility disability due primarily to pathologies other than stroke"

Interventions

(1) Bobath intervention $(n=12)$

"Intervention A was based on the Bobath concept. In this intervention, participants received individual treatment prescription based on the Bobath concept towards the goal of improving walking ability in different environmental contexts. This intervention included detailed assessment of the individual's movement strategies and the neurological and neuromuscular deficits underlying motor dysfunction. Treatment strategies were individualized and aimed at both reducing the severity of impairments where they impacted on function, and optimizing postural and movement strategies to improve efficiency and maximize function"

"The specific goal of therapy in this study was to improve the ability of the participant to walk safely in different environments, including components of endurance, walking on slopes, going up and down a single step and walking over rough ground. The session incorporated structured task practice (as described in intervention B) for $1 / 6$ of the treatment time allocated"

The individual components delivered are listed in Table 3. Based on the individual components, this intervention is categorised as comprising cardiopulmonary intervention, functional task training and neurophysiological intervention

Length of intervention period: two weeks

Number of sessions and length of individual sessions: "Participants in both groups received six one hour physiotherapy sessions over a two-week period. During the intervention period, participants did not receive any other physiotherapy aimed at improving mobility, posture, balance or lower limb function. Instead, the intervention sessions replaced the usual physiotherapy treatment for mobility. Additional physiotherapy was provided in sitting or lying for other rehabilitation goals, such as independence in bed mobility and recovery of upper limb function"

Intervention provider: "Both interventions were performed by physiotherapists with at least five years' postgraduate experience and at least two years' experience in the fields of rehabilitation or neurology. In addition, therapists providing Intervention $A$ had to have also completed a Basic Bobath Course and at least two Advanced Bobath Courses (a minimum of 180 hours of formal training acquired over a minimum of three years)"

(2) Task practice intervention $(\mathrm{n}=14)$ 
Brock 2005 (Continued)

"Participants receiving Intervention B undertook physiotherapy based on structured task practice. The supervised exercise programme aimed to provide repeated task specific practice of environmental contexts frequently encountered in walking outdoors. The tasks practised focused on increasing endurance, walking on slopes, going up and down a single step and walking over rough ground. Based on principles of motor learning, the therapist provided instruction in how to perform the task, including demonstration, verbal cueing to correct ineffective adaptive motor patterns and feedback on the performance of the task as specified by the study protocol, as well as supervision for safety. The therapist did not provide hands-on assistance or guidance during tasks. Half of the task practice component was conducted as repetitive practice using standardized equipment in the gymnasium, reflecting the closed skill stage of learning. The other half of the time was spent in environments outside the gymnasium, walking on varying surfaces indoors and outdoors, bringing in other environmental contexts, such as differing attentional demands and changes in direction"

The individual components delivered are listed in Table 3. Based on the individual components, this intervention is categorised as comprising cardiopulmonary intervention and functional task training

Length of intervention period: two weeks

Number of sessions and length of individual sessions: "Participants in both groups received six one hour physiotherapy sessions over a two-week period"

Intervention provider: "Physiotherapists with at least five years' postgraduate experience and at least two years' experience in the fields of rehabilitation or neurology"

This study was classified as: active intervention one (functional task training, neurophysiological) versus active intervention two (functional task training) (Table 6). Both intervention groups also received cardiopulmonary intervention

Outcomes Measures of postural control and balance: Berg Balance Scale

Measures of voluntary movements: adapted Six-Minute Walk test, gait velocity

Time points when outcomes were assessed: "Measures were taken at baseline, and following treatment...Post-intervention assessment measures were recorded between one and five days following the intervention"

Notes

\section{Risk of bias}

\section{Bias}

Authors' judgement Support for judgement

Random sequence genera- Low risk tion (selection bias)

"Randomization was done through a computer-generated, stratified, blocked randomization procedure. Separate computer-generated randomizations were used for each site. Opaque envelopes were used to conceal group allocation. Participants were randomized and assigned to the intervention groups after the baseline assessments were carried out"

\section{Allocation concealment Low risk}

(selection bias)

"Patients were stratified according to time period from date of stroke to date of commencement in the trial. The two strata were four weeks to eight weeks post-stroke at commencement of the trial and more than eight weeks poststroke. This stratification aimed to improve the likelihood of the two groups being similar in terms of initial severity and speed of recovery post-stroke, as those with milder stroke and a quicker rate of recovery are likely to improve more rapidly during the time period of the study"

Blinding of outcome as- Low risk sessment (detection bias) All outcomes
"Measures were taken at baseline, and following treatment, by a physiotherapist who was blind to group assignment" 
Brock 2005 (Continued)

Incomplete outcome data Unclear risk (attrition bias)

All outcomes
"Twenty-nine participants were recruited to the study. Note that two participants did not complete the study because they were discharged from inpatient rehabilitation earlier than anticipated and could not complete the study as outpatients. One participant was withdrawn due to ill health"

Both of the early discharges were in the same (Bobath) group. Intention-totreat analysis not completed-unclear whether these dropouts could contribute to attrition bias
Free of systematic differ- Low risk ences in baseline characteristics of groups compared?
"No significant differences between groups at baseline for the six minute walk test $(P=0.79)$, gait velocity $(P=0.27)$ and Berg Balance Scale $(P=0.77) "$

\begin{tabular}{|c|c|c|}
\hline $\begin{array}{l}\text { Did authors adjust for } \\
\text { baseline differences in } \\
\text { their analyses? }\end{array}$ & Low risk & No information provided \\
\hline Other bias & Unclear risk & $\begin{array}{l}\text { "No data are available regarding the number of patients eligible to participate } \\
\text { or reasons for not participating. However, for both centres, the main limiting } \\
\text { factor to recruitment was planned discharge to a distant locality shortly after } \\
\text { achieving walking with supervision, preventing participation" }\end{array}$ \\
\hline
\end{tabular}

Carlson 2006

\section{Study characteristics}

\begin{tabular}{ll}
\hline Methods & Study design: RCT \\
& Method of randomisation: not stated ("Subjects were randomised into treatment $(n=6)$ and control $(n$ \\
$=5)$ groups")
\end{tabular}

\section{Participants}

Number of participants: $\mathrm{n}=11$

Inclusion criteria: not stated ("Eleven subjects with hemiparesis..participated in this study")

(1) Treatment group $(n=6)$

"received intense massed practice ... with interventions focused on forcing use of the affected lower extremity"

The individual components delivered are listed in Table 3. Based on the individual components, this intervention is categorised as functional task training

Length of intervention period: two weeks

Number of sessions and length of individual sessions: three hours/d

Intervention provider: not stated

(2) Control group $(n=5)$

"Control subjects did not receive any intervention"

The individual components delivered are listed in Table 3. Based on the individual components, this intervention is categorised as no intervention

Length of intervention period: no intervention

Intervention provider: no intervention 
Number of sessions and length of individual sessions: no intervention

This study is classified as intervention (functional task training) versus no treatment (Table 4)

\begin{tabular}{ll}
\hline Outcomes & Measures of postural control and balance: Berg Balance Scale \\
& Measures of voluntary movements: self-selected gait speed \\
Other secondary outcome measures: weight-bearing ratios in quiet standing and weight-bearing ratios \\
during ambulation \\
Time points when outcomes were assessed: "testing was performed at pre-test, post-test, and again at \\
3-month follow-up" \\
Abstract only \\
Notes data suitable for analysis
\end{tabular}

\section{Risk of bias}

\begin{tabular}{|c|c|c|}
\hline Bias & Authors' judgement & Support for judgement \\
\hline $\begin{array}{l}\text { Random sequence genera- } \\
\text { tion (selection bias) }\end{array}$ & Unclear risk & No information provided \\
\hline $\begin{array}{l}\text { Allocation concealment } \\
\text { (selection bias) }\end{array}$ & Unclear risk & No information provided \\
\hline $\begin{array}{l}\text { Blinding of outcome as- } \\
\text { sessment (detection bias) } \\
\text { All outcomes }\end{array}$ & Unclear risk & No information provided \\
\hline $\begin{array}{l}\text { Incomplete outcome data } \\
\text { (attrition bias) } \\
\text { All outcomes }\end{array}$ & Unclear risk & Insufficient information available \\
\hline $\begin{array}{l}\text { Free of systematic differ- } \\
\text { ences in baseline charac- } \\
\text { teristics of groups com- } \\
\text { pared? }\end{array}$ & Unclear risk & No information provided \\
\hline $\begin{array}{l}\text { Did authors adjust for } \\
\text { baseline differences in } \\
\text { their analyses? }\end{array}$ & Unclear risk & No information provided \\
\hline Other bias & Unclear risk & Limited information available \\
\hline
\end{tabular}

\section{Chan 2006}

\section{Study characteristics}

Study design: matched-pair RCT
Methods of randomisation: "Random assignment of patients into the sequential function-based motor
relearning group and the conventional therapy (control) group was conducted in two stages. The first
stage involved arranging patients into a block of six participants and then forming the six into pairs by
matching their age and gender; then, if possible, they were matched according to the level of inten-
sity of physiotherapy and speech therapy received in the hospital. Patients who did not form a best-


Chan 2006 (Continued)

matched pair were automatically entered into the next block for further matching and randomization. The patients were excluded from the study if their characteristics were not matched by the end of the second round. The second stage involved randomly assigning the two patients in each of the bestmatched pairs to either the motor relearning or the control groups by drawing one of two sealed envelopes designating the group membership"

Participants Number of participants: $n=66$

Inclusion criteria: "patients had to be between 21 and 65 years of age (eligible admission to outpatient setting), diagnosed as having suffered a first stroke, the stroke must have occurred within the previous 12 months, and patients had to be able to follow simple instructions as screened by the Chinese version of the Cognistat"

Exclusion criteria: "conditions were complicated by other musculoskeletal injuries and/or visual deficits"

Interventions
(1) Motor relearning group $(n=33)$

"Both programmes were conducted in the same treatment area located in the occupational therapy department of the hospital. The patients in both programmes also received physical therapy in the form of lower limb strengthening and trunk balance exercises. The patients attended physical therapy from one to three times a week. Their attendance was monitored throughout the study, and the number of sessions was incorporated into the analysis of the results"

"In the motor relearning programme, the intervention technique followed four sequential steps: identification of the missing performance components (step 1), training using remedial exercises (step 2), training using functional task components (step 3), and transfer of skills to functional task performance (step 4). A total of 24 remedial tasks (used in step 2) and 10 functional tasks (used in step 4) were designed to cover deficits in static and dynamic sitting balance, and static and dynamic standing balance. Throughout the training session, the therapist stressed the importance of relating the training processes taking place in steps 2 and 3 to practices in step 4 . The training progressed by advancing from a sitting to a standing position, and from static to dynamic balancing. The criteria established for progression were clearly defined, and those patients who demonstrated the desirable skills were upgraded to another stage of training"

The individual components delivered are listed in Table 3. Based on the individual components, this intervention is categorised as functional task training

Length of intervention period: six weeks

Number of sessions and length of individual sessions: three two-hour sessions each week (18 sessions total)

Intervention provider: occupational therapist: "The researcher conducted the motor relearning programme"

(2) Conventional therapy group $(n=33)$

"The skill training method was adopted for use with patients receiving the conventional therapy programme. The same number of remedial and functional tasks was covered as in the motor relearning programme. The selection of remedial tasks followed the principle of progressing from a sitting to a standing position, and from static to dynamic balance. The training of functional tasks began with simple self-care and bedside tasks, such as bed mobility, and progressed to more complicated tasks, such as use of community facilities. Unlike the motor relearning programme, the patients were not involved in identifying their own missing performance components (Table 2). The relationships between the practices of the remedial tasks and the entire functional task performance were not reinforced"

The individual components delivered are listed in Table 3. Based on the individual components, this intervention is categorised as functional task training

Length of intervention period: six weeks 
Chan 2006 (Continued)

Number of sessions and length of individual sessions: three two-hour sessions each week (18 sessions total)

Intervention provider: "another occupational therapist conducted the conventional therapy programme. The therapist responsible for the conventional therapy programme was trained in the standardized procedure for conducting the control programme"

This study is classified as active intervention one (functional task training) versus active intervention two (functional task training) (Table 6)

Outcomes
Measures of independence in ADL: Functional Independence Measure
Measures of postural control and balance: Berg Balance Scale, Timed Up and Go test
Measures of participation: instrumental ADL
Measures of quality of life and social isolation: Community Integration Questionnaire
Time points when outcomes were assessed: "The assessment schedule was laid out at baseline (after
randomization), two weeks, four weeks, and six weeks"
The two active interventions appear fairly similar. As the two active treatment groups were classified as
including similar treatment components, data from this study have not been included within the com-
parisons of one active intervention versus another active intervention

\section{Risk of bias}

\section{Bias}

Random sequence genera- High risk tion (selection bias)

\section{Authors' judgement Support for judgement}

"Random assignment of patients into the sequential function-based motor relearning group and the conventional therapy (control) group was conducted in two stages. The first stage involved arranging patients into a block of six participants and then forming the six into pairs by matching their age and gender; then, if possible, they were matched according to the level of intensity of physiotherapy and speech therapy received in the hospital. Patients who did not form a best-matched pair were automatically entered into the next block for further matching and randomization. The patients were excluded from the study if their characteristics were not matched by the end of the second round. The second stage involved randomly assigning the two patients in each of the best-matched pairs to either the motor relearning or the control groups by drawing one of two sealed envelopes designating the group membership"

Judged as high risk, as if participants were not matched, then they could be excluded-thus participants did not all have an equal chance of being entered into the trial. The study authors state: "The best matched process used in the randomization might introduce biases into the selection of patients to participate in the study"

$\begin{aligned} & \text { Allocation concealment } \quad \text { Low risk } \\ & \text { (selection bias) }\end{aligned} \quad \begin{aligned} & \text { "The randomization process was carried out by a registration clerk who was } \\ & \text { not involved in any part of the study" }\end{aligned}$

Blinding of outcome as- Low risk

All outcomes sessment (detection bias)

\begin{abstract}
"All the clinical outcome assessments were conducted by two occupational therapists who were working in the orthopaedic unit of the same outpatient centre. The assessors were blind to the group membership of the patients they assessed. Training sessions were provided to all raters on administering and scoring each clinical instrument in order to minimize any biases associated with the assessment process"
\end{abstract}

In the motor relearning group, seven participants discontinued the intervention for the following reasons: "suspended treatment due to travelling for more than two weeks $(n=2)$; re-admitted to hospital due to medical problem
High risk (attrition bias)

All outcomes 
Chan 2006 (Continued)

$(n=1)$; excluded from analysis due to the drop-out of matched counterpart in conventional therapy group $(n=4) "$

In the conventional therapy group, seven participants discontinued the intervention for the following reasons: "suspended due to irregular attendance ( $\mathrm{n}$ $=1)$; re-admitted to hospital due to medical problem $(n=1)$; defaulted treatment $(n=2)$; excluded from analysis due [to] drop-out of matched counterpart in motor relearning group $(n=3) "$

Judged at high risk as the result of exclusion of participants because their "matched counterpart" dropped out

Free of systematic differ- Low risk ences in baseline characteristics of groups compared?
"There were no significant differences in the demographic and medical characteristics of the patients between the motor relearning and control groups. Also, no significant differences were found in their scores on the five outcome measures at the baseline"

\begin{tabular}{|c|c|c|}
\hline $\begin{array}{l}\text { Did authors adjust for } \\
\text { baseline differences in } \\
\text { their analyses? }\end{array}$ & Low risk & No information provided \\
\hline \multirow[t]{2}{*}{ Other bias } & High risk & $\begin{array}{l}\text { Judged at high risk, as study authors state: "Although the assessors were } \\
\text { blind to the study, the motor relearning programme was conducted by the re- } \\
\text { searcher, who might have provided more enthusiastic interventions than the } \\
\text { therapist who conducted the control programme" }\end{array}$ \\
\hline & & $\begin{array}{l}\text { Additionally, "The patients received other treatment interventions in addi- } \\
\text { tion to the motor relearning or control programme, such as physiotherapy and } \\
\text { speech therapy, that could contaminate the treatment effects. The patients at- } \\
\text { tended the treatment sessions as day-patients, and their engagement in activ- } \\
\text { ities other than those conducted during treatment possibly further contami- } \\
\text { nated these effects" }\end{array}$ \\
\hline
\end{tabular}

\section{Chen 2004}

\section{Study characteristics}

\begin{tabular}{ll}
\hline Methods & Study design: RCT \\
& Method of randomisation: not stated \\
\hline Participants & Number of participants: $\mathrm{n}=78$ \\
& Inclusion criteria: "Met the 1995 National stroke diagnosis guidelines, first stroke as confirmed by CT or \\
& MRI scan, persistent deficits in motor function, within three weeks after stroke, no severe conditions of \\
the heart, liver, kidney and medically stable" & \\
Age stated in paper as "between $40-48 . "$ However, review authors have assumed that this is a typo, as \\
mean age reported in the study is 60.95 years for the rehabilitation group and 62.36 years for the con- \\
trol group
\end{tabular}

Interventions (1) Rehabilitation group $(\mathrm{n}=39)$

"Patients in the rehabilitation group were given physical training in addition to routine treatment. The treatment group commenced daily therapy after they were medically stable. Each training session began with the therapist guiding and delivering the therapy lasting $45 \mathrm{~min} /$ day. Participant's family learned the exercises alongside each training session, using approximately 0.5 hour/day to consolidate and reinforce the exercises taught" 
(a) Correct positioning of limbs in bed (supine position, lateral position with affected limbs at lower side and healthy limbs at upper side and lateral position with affected limbs at upper side and healthy limbs at lower side)

(b) Passive ranging exercises of all joints of the affected limbs (therapist placed one hand on the proximal joint and another hand on the distal joint to deliver gentle, slow and rhythmic movements to the joints)

(c) Neuromuscular facilitation techniques (combined joint repositioning and compression while performing ranging exercises on the limbs; tapping, brushing and striking the skin)

(d) Active ranging exercises of the affected limbs (used a band to assist the affected limbs in carrying out active-assisted range of motion exercises at the shoulder, elbow and wrist, as well as active ranging exercises of all joints)

(e) Training in sitting balance, standing and gait re-education, commencing up stair and down stair practice when possible

(f) ADL training (participants actively worked on completing eating, washing, combing, dressing activities); learned all possible techniques to achieve the above actions, participants with severe impairment to learn single-hand and single-leg techniques of manoeuvring the wheelchair

The individual components delivered are listed in Table 3. Based on the individual components, this intervention is categorised as comprising functional task training, musculoskeletal intervention (active), musculoskeletal intervention (passive) and neurophysiological intervention

Length of intervention period: not stated, but final outcome assessment was done after three months of therapy

Number of sessions and length of individual sessions: 45 minutes per day. No further details stated Intervention provider: therapist during training session. Participants' family provided reinforcement of exercises taught during training session

(2) Control group $(n=39)$

"Patients in the control group received routine treatment"

The individual components delivered are listed in Table 3. Based on the individual components, this intervention is categorised as comprising no intervention

Length of intervention period: not stated

Number of sessions and length of individual sessions: not stated

Intervention provider: not stated

This study is classified as intervention (functional task training, musculoskeletal intervention (active), musculoskeletal intervention (passive), neurophysiological intervention) versus no treatment (Table 4)

Measures of independence in ADL: Barthel Index
Time points when outcomes were assessed: "before therapy (not stated when specifically), after 1
month of therapy, after 3 months of therapy"

Notes Original study translated from Chinese to English

\section{Risk of bias}


Chen 2004 (Continued)

Random sequence genera- Unclear risk Not stated
tion (selection bias)

\begin{tabular}{ll}
\hline $\begin{array}{l}\text { Allocation concealment } \\
\text { (selection bias) }\end{array}$ & High risk \\
& $\begin{array}{l}\text { "Study design was done by first author, data collection was done by all au- } \\
\text { thors, intervention was delivered by therapist, outcome assessment done by } \\
\text { all authors, training given" }\end{array}$
\end{tabular}

\begin{tabular}{lll}
\hline Blinding of outcome as- & High risk & As above \\
sessment (detection bias) & \\
All outcomes &
\end{tabular}

\begin{tabular}{|c|c|c|}
\hline $\begin{array}{l}\text { Incomplete outcome data } \\
\text { (attrition bias) } \\
\text { All outcomes }\end{array}$ & Unclear risk & No dropouts reported \\
\hline $\begin{array}{l}\text { Free of systematic differ- } \\
\text { ences in baseline charac- } \\
\text { teristics of groups com- } \\
\text { pared? }\end{array}$ & Unclear risk & $\begin{array}{l}\text { Experimental group has a slightly higher score in Barthel Index than control } \\
\text { group at baseline }\end{array}$ \\
\hline $\begin{array}{l}\text { Did authors adjust for } \\
\text { baseline differences in } \\
\text { their analyses? }\end{array}$ & Unclear risk & No information provided \\
\hline Other bias & Unclear risk & No information provided \\
\hline
\end{tabular}

\section{Chen 2006}

\section{Study characteristics}

\begin{tabular}{ll}
\hline Methods & Study design: RCT \\
& Method of randomisation: not stated \\
\hline Participants & Number of participants: $\mathrm{n}=45$ \\
& $\begin{array}{l}\text { Inclusion criteria: "stroke patients diagnosed according to the evaluative standard revised by the } \\
\text { Fourth [National] Academic Conference of Cerebral Vascular Disease and lived in communities around } \\
\text { the Second Hospital of Zhengzhou University from } 2003 \text { to } 2005 \text { " }\end{array}$
\end{tabular}

Interventions (1) Rehabilitation group $(\mathrm{n}=25)$

"All patients underwent the same routine medical treatment, patients from the rehabilitation group [were] additionally treated with community-based rehabilitation for 3 months. Appropriate therapies were given after detailed examination and rehabilitation assessment, giving community-based rehabilitation training at home, giving the patient and family members comprehensive guidance, with regular follow up assessment, with all questions encountered during the rehabilitation process answered timely, and continuously adjusting the rehabilitation treatment according to the patient's response"

Rehabilitation measures: "Treatment during the flaccid stage was on preventing joint contractures and deformity, preventing secondary complications; treatment during the spasticity stage was on controlling muscle spasticity and abnormal movements to encourage normal movement patterns to emerge. Main content included: passive ranging exercises of all joints, rolling from affected and non-affected sides practice, balance ability training (including sitting and standing balance practice), transfers (bed to chair transfer, sit to stand transfer) training, gait training (ambulation, up and down stairs) and stretching in wrist extension and ankle dorsiflexion. ADL training included feeding, donning, personal hygiene management etc. At the same time, psychological recovery and social adaptation training 
Chen 2006 (Continued)

were given, mainly on fostering good patient-doctor relationships to help them build confidence, release negative emotions, guide and encourage them to express their feelings. Training was given in a way that adapted to the training environment, overcame limitation in resources through simplification, adapted to the situation and presenting condition, focused on involving the family members' participation, alterations to the home environment, maximising the resources at home"

The individual components delivered are listed in Table 3. Based on the individual components, this intervention is categorised as comprising functional task training and musculoskeletal intervention (passive)

Length of intervention period: three months

Number of sessions and length of individual sessions: two/wk, no other details given Intervention provider: not stated

(2) Control group $(n=20)$

"Routine medical treatment"

The individual components delivered are listed in Table 3. Based on the individual components, this intervention is categorised as comprising no intervention

Length of intervention period: not stated

Number of sessions and length of individual sessions: not stated

Intervention provider: not stated

This study is classified as intervention (functional task training, musculoskeletal (passive)) versus no treatment (Table 4)

Measures of independence in ADL: Barthel Index
Other secondary outcomes: Mini Mental State Examination (MMSE)
Time points when outcomes were assessed: before intervention and after three months of communi-
ty-based rehabilitation

Notes Original study translated from Chinese to English

\section{Risk of bias}

\begin{tabular}{lll}
\hline Bias & Authors' judgement & Support for judgement \\
\hline $\begin{array}{l}\text { Random sequence genera- } \\
\text { tion (selection bias) }\end{array}$ & Unclear risk & No information provided \\
\hline
\end{tabular}

\begin{tabular}{ll}
\hline $\begin{array}{l}\text { Allocation concealment } \\
\text { (selection bias) }\end{array}$ & High risk \\
& "Design, intervention and outcome assessment were completed by the au- \\
thors and the relevant medical personnel in the same discipline"
\end{tabular}

Blinding of outcome as- $\quad$ Unclear risk $\quad$ No blinding of outcome assessors reported
sessment (detection bias)
All outcomes

\begin{tabular}{l}
\hline Incomplete outcome data Unclear risk $\quad$ No dropouts reported \\
(attrition bias) \\
All outcomes
\end{tabular}

Free of systematic differ- Low risk $\quad$ No significant baseline differences
ences in baseline charac-


Chen 2006 (Continued) teristics of groups compared?

\begin{tabular}{lll}
\hline $\begin{array}{l}\text { Did authors adjust for } \\
\text { baseline differences in } \\
\text { their analyses? }\end{array}$ & Low risk & \\
\hline Other bias & Unclear risk & No information provided \\
\hline
\end{tabular}

Chen 2010

\section{Study characteristics}

\begin{tabular}{ll}
\hline Methods & Study design: RCT \\
& Method of randomisation: not stated \\
\hline Participants & Number of participants: $\mathrm{n}=106$ \\
& $\begin{array}{l}\text { Inclusion criteria: first ever stroke between February } 2006 \text { and December } 2008, \text { no obvious sign of psy- } \\
\text { chological or memory deficits, fulfilled neurological examination standards and confirmed by CT scan } \\
\text { or MRI }\end{array}$
\end{tabular}

"Test group used rehabilitation exercise therapy

(a) Passive ranging exercises (rehabilitation method during flaccid period): while patient was in supine, therapist provided exercise therapy to each of the patient's joints according to movable range of each joint (see Table 1), with the following exercise principles: (i) Exercise progressed from proximal joints to distant joints; (ii) Exercise single joints first -> gradually progressing to combined movement of several joints; (iii) Exercise upper and lower limbs on non-affected side first, until patient became used to it, before exercising affected limbs; (iv) Each exercise done slowly $3 s-5 s$, repeated 5 times -10 times, at beginning using slow and gentle motion, avoiding overly fast flexing and extending, paying attention to patient's pain level, avoiding straining. Only if exercise direction was correct, would a safe and effective rehabilitation goal be realised

(b) Active ranging exercises (rehabilitation exercise during recovery period): patient independently chose the exercise position and exercise method, with emphasis on hand exercises, assisted by some equipment, we gave appropriate guidance and monitoring, with exercise speed, repetition number and interval being determined by patient's specific condition. Active ranging exercises had to obey: (i) Active ranging exercises performed on the foundation of passive ranging exercises, in order not to induce tiredness and pain; (ii) Among the exercises, more practice done for relaxation of tensed muscles; (iii) First simple movements, then complicated movements; (iv) During practice, same actions done for non-affected limbs, to aid in recovery of function of paralysed limbs. During the process of active ranging exercise, coordination practice must be emphasised, to gradually improve level of coordination through a long period of training. From individual joints, and uni-directional simple exercises to complex coordinated movements, movement complexity and precision [were] gradually increased; starting from exercises symmetrical to both sides of body; during gait training, initial requirement was for gait to be stable, accurate and natural, and afterwards practised walking forwards in a straight line and crossing obstacles etc. Repeated practice of a single movement, in order to develop the biological foundation of a habit, and form a new neural pathway"

The individual components delivered are listed in Table 3. Based on the individual components, this intervention is categorised as comprising functional task training, musculoskeletal intervention (active) and musculoskeletal intervention (passive)

Length of intervention period: four weeks 
Chen 2010 (Continued)

Number of sessions and length of individual sessions: not stated

Intervention provider: not stated

(2) Control group $(n=53)$

Used Traditional Chinese Tui Na

The individual components delivered are listed in Table 3. Based on the individual components, this intervention is categorised as comprising massage

Length of intervention period: four weeks

Number of sessions and length of individual sessions: not stated

Intervention provider: Traditional Chinese Tui Na practitioner

This study is classified as intervention (functional task training, musculoskeletal (active), musculoskeletal (passive)) versus attention control (massage) (Table 5)

\begin{tabular}{|c|c|}
\hline \multirow[t]{4}{*}{ Outcomes } & Measures of independence in ADL: Barthel Index \\
\hline & Measure of motor function: Fugl-Meyer Assessment (FMA) \\
\hline & Measures of tone/spasticity: "Modified Ashworth Spasticity Rating Scale" \\
\hline & Time points when outcomes were assessed: before intervention and after four weeks of intervention \\
\hline \multirow[t]{3}{*}{ Notes } & $\begin{array}{l}\text { Both groups were given the same conventional medicine to reduce intracranial pressure, nourish } \\
\text { nerves, prevent and cure symptoms, maintain electrolyte balance and provide symptomatic and sup- } \\
\text { portive treatment; on admission, while lying on non-affected side and supine, all used orthopaedic de- } \\
\text { vices on affected side. Four weeks equals one treatment cycle }\end{array}$ \\
\hline & Original study translated from Chinese to English \\
\hline & Mean and SD computed from categorical data \\
\hline
\end{tabular}

\section{Risk of bias}

\begin{tabular}{|c|c|c|}
\hline Bias & Authors' judgement & Support for judgement \\
\hline $\begin{array}{l}\text { Random sequence genera- } \\
\text { tion (selection bias) }\end{array}$ & Unclear risk & No information provided \\
\hline $\begin{array}{l}\text { Allocation concealment } \\
\text { (selection bias) }\end{array}$ & Unclear risk & No information provided \\
\hline $\begin{array}{l}\text { Blinding of outcome as- } \\
\text { sessment (detection bias) } \\
\text { All outcomes }\end{array}$ & Unclear risk & No information provided \\
\hline $\begin{array}{l}\text { Incomplete outcome data } \\
\text { (attrition bias) } \\
\text { All outcomes }\end{array}$ & Unclear risk & No dropouts reported \\
\hline $\begin{array}{l}\text { Free of systematic differ- } \\
\text { ences in baseline charac- } \\
\text { teristics of groups com- } \\
\text { pared? }\end{array}$ & Low risk & No significant baseline differences \\
\hline
\end{tabular}


Chen 2010 (Continued)
Did authors adjust for
Low risk
No information provided baseline differences in

Noinformation provided
their analyses?

Other bias Unclear risk No information provided

Chu 2003

\section{Study characteristics}

$\begin{array}{ll}\text { Methods } & \text { Study design: RCT } \\ \text { Method of randomisation: not stated } \\ \text { "All the cases were divided into rehabilitation group (30 cases) and control group (28 cases) randomly" }\end{array}$

\begin{tabular}{ll}
\hline Participants & Number of participants: $\mathrm{n}=58$ \\
& Inclusion criteria: "58 stroke cases who lived in our hospital from March 1999 to October 2001 accord- \\
& ed with the diagnosis criteria instituted on the fourth national cerebrovascular disease conference in \\
& $\begin{array}{l}\text { 1995, all the cases were confirmed by CT and MRI and all the cases didn't suffer severe dysnoesia [cog- } \\
\text { nitive deficits]" }\end{array}$
\end{tabular}

Interventions $\quad$ (1) Rehabilitation group $(\mathrm{n}=30)$

"Rehabilitation group received unobstruction exercises after receiving first evaluation. Unobstruction techniques: (a) position of limbs, (b) active and passive exercises of joint of upper and lower limbs including flexion, extension, internal rotation, abduction; actions in each direction repeated 10 to 20 times, (c) bridge movement on bed: patient lied on the back, flex knee and hip, clamped a small pillow between knees, played breech elevation and hip extension and repeat these actions, (d) wiping or striking suffered limbs up to down with brush or little hammer in order to promote recovery of sensory, (e) transferring and balance exercises

Exercise of sitting balance ability: patient's healthy hand was bound on the tail of bed by a special belt. Head of bed was elevated to 30 degrees. Nurse put patient's upper limb on trunk, pushed healthy upper limb. This method could enhance strength of extensor muscle of upper limbs and trunk muscle. We increased sitting angle everyday, elongated sitting time and control sitting balance. Exercises from sitting to standing position: nurse stood in front of patient, patient embrace nurse's waist with healthy hand. Nurse fixed patient's suffered hand on waist. The other hand was put in the subaxillary position of the suffered side, which delivered sufficient weight loading to suffered side. When patients stood up, nurse called patient attention to look forward to the direction of nurse's face. Exercise of standing balance: patients received bedside bridge movement and exercise of lower limb swing in order to practice standing balance ability. Exercise of plain walking: patients walked with supporter or step at first, then feet move by turn. Nurse also could bind suffered foot and help elevation if needed. Before walk, below part of suffered limb was hanged in front of chest in order to keep functional position of upper limb and should joint. We should try to avoid tiredness and try to ensure exercise quality. Exercise of going upstairs and downstairs: according to the rule of 'healthy leg up first, suffered leg down first,' training flexor and extensor muscles and joints of lower limbs. Exercise of functions of upper limb and hand: Bobath shaking hands, suffered side upper limb takes assisted active movement with help of healthy side upper limb; inducing upper limb muscle movement and hand performance exercise; patients took flexion and extension of should, elbow, twist and finger joint, grasp, hold and pinch movement. Exercises were performed from easy to difficult, from tough to delicate"

The individual components delivered are listed in Table 3. Based on the individual components, this intervention is categorised as comprising functional task training, musculoskeletal intervention (active), musculoskeletal intervention (passive) and neurophysiological intervention

Length of intervention period: varied from "20 days to 14 months with a mean rehabilitation time of 41.3 days" 
Chu 2003 (Continued)

Number of sessions and length of individual sessions: "above exercises were taken 1 per day, 40 to 60 min/time"

Intervention provider: nurses

(2) Control group $(n=28)$

"Two group received routine neurologic treatment and nursing. Control group didn't receive rehabilitation exercise"

The individual components delivered are listed in Table 3. Based on the individual components, this intervention is categorised as no intervention

Length of intervention period: no intervention

Number of sessions and length of individual sessions: no intervention

Intervention provider: no intervention

This study is classified as intervention (functional task training, musculoskeletal (active), musculoskeletal (passive), neurophysiological) versus no treatment (Table 4)

Measures of independence in ADL: Barthel Index
Measures of motor function: Fugl-Meyer Assessment
Time points when outcomes were assessed: baseline "was taken when patients' signs and symptoms of
nerve system were stable. The same doctor evaluated patients again when treatment was over"

Notes Short paper only with limited detail regarding randomisation.

This paper was written in English, and the extracts above are direct quotes.

\section{Risk of bias}

\begin{tabular}{lll}
\hline Bias & Authors' judgement & Support for judgement \\
\hline $\begin{array}{l}\text { Random sequence genera- } \\
\text { tion (selection bias) }\end{array}$ & Unclear risk & No information provided \\
\hline $\begin{array}{l}\text { Allocation concealment } \\
\text { (selection bias) }\end{array}$ & Unclear risk & No information provided \\
\hline $\begin{array}{l}\text { Blinding of outcome as- } \\
\text { sessment (detection bias) } \\
\text { All outcomes }\end{array}$ & Unclear risk & No information provided \\
\hline $\begin{array}{l}\text { Incomplete outcome data } \\
\text { (attrition bias) } \\
\text { All outcomes }\end{array}$ & Unclear risk & No dropouts described \\
\hline
\end{tabular}

\begin{tabular}{lll}
$\begin{array}{l}\text { Free of systematic differ- } \\
\text { ences in baseline charac- } \\
\text { teristics of groups com- } \\
\text { pared? }\end{array}$ & High risk & $\begin{array}{l}\text { "Significant difference in age, gender, lesion characteristics and side of paraly- } \\
\text { sis between two groups" }\end{array}$ \\
\hline $\begin{array}{l}\text { Did authors adjust for } \\
\text { baseline differences in } \\
\text { their analyses? }\end{array}$ & Unclear risk & No information provided \\
\hline
\end{tabular}

Other bias Unclear risk No information provided




\section{Study characteristics}

Methods
Study design: observer-blind, phase one RCT

Method of randomisation: "An independent statistician produced a pretrial computer generated randomized group allocation order in blocks of 9 per trial center. Allocation was stratified by baseline scores for unilateral visual spatial neglect (Star Cancellation Test, 50-54 no spatial neglect and 0-49 unilateral spatial neglect present). Allocation was concealed in sequentially numbered sealed opaque envelopes held by an independent administrator. Envelopes were opened in response to a telephone request from the research physiotherapist (blinded to measures) after the assessor (blinded to group allocation) had completed baseline measures"

\section{Participants}

\section{Number of participants: $\mathrm{n}=109$}

Inclusion criteria: "inpatients older than 18 years, between 1 and 13 weeks after anterior circulatory stroke (hemorrhage or infarction), some voluntary muscle contraction in the paretic lower limb (a score of at least $28 / 100$ on the lower limb section of the motricity index), with potential for clinically important improvement was present, able to follow a 1-stage command, independently mobile (with or without aids), prior to the index stroke, no orthopedic surgery, no trauma affecting the lower limb in the last 8 weeks, and there was no previous history of neurological disease other than stroke"
Interventions
(1) Additional conventional therapy (CPT + CPT) group $(n=35)$

"Focused on those interventions in the treatment schedule that emphasized control/quality of movement and gave prominence to sensory stimulation and preparation of joint and muscle alignment prior to activating muscle or a functional task. Additional CPT was therefore strongly therapist hands-on, with provision of passive movements, active assisted exercise, and/or hands-on intervention to facilitate muscle activity or functional ability. Some active exercise and repetitive practice of functional tasks [were] included but without systematic progression in resistance or repetition"

The individual components delivered are listed in Table 3. Based on the individual components, this intervention is categorised as functional task training, musculoskeletal intervention (active), musculoskeletal intervention (passive) and neurophysiological intervention

Length of intervention period: six weeks

Number of sessions and length of individual sessions: standardised treatment schedules for up to one hour, four days a week for six weeks (total $=24$ hours)

Intervention provider: research physiotherapists (independent of the clinical team)

(2) Functional strength training (FST + CPT) group $(n=36)$

"Delivery of FST directed participants' attention to the exercise/activity being performed, appropriate verbal feedback on performance, and repetition (therapist hands-off). Content of FST focused on repetitive, progressive resistive exercise during goal-directed functional activity. The emphasis was on producing appropriate muscle force for the functional activity being practiced. Treatment progressed systematically using repetition and increase in resistance by, for example, changing the limb's relationship to gravity, increasing the range of movement or distance over which bodyweight was transported, and changing the weight of external objects used to provide resistance. Treatment activities progressed systematically from light to heavy loads and from few to many repetitions. Participants performed repetitive exercise of functional tasks such as sit-to-stand-to-sit, stair climbing/step ups, inside and outside walking, transfer training, bed mobility, and treadmill training with and without the use of a bodyweight support system"

The individual components delivered are listed in Table 3. Based on the individual components, this intervention is categorised as functional task training, musculoskeletal intervention (active) and neurophysiological intervention

Length of intervention period: six weeks 
Number of sessions and length of individual sessions: standardised treatment schedules for up to one hour, four days a week for six weeks (total $=24$ hours)

Intervention provider: research physiotherapists (independent of the clinical team)

(3) Conventional physiotherapy (CPT) group $(n=38)$

"Routine CPT included soft tissue mobilization, facilitation of muscle activity, facilitation of coordinated multijoint movement, tactile and proprioceptive input, resistive exercise, and functional retraining"

The individual components delivered are listed in Table 3. Based on the individual components, this intervention is categorised as usual care (functional task training, musculoskeletal intervention (active), musculoskeletal intervention (passive) and neurophysiological intervention)

Length of intervention period: six weeks

Number of sessions and length of individual sessions: standardised treatment schedules for up to one hour, four days a week for six weeks (total $=24$ hours)

Intervention provider: research physiotherapists (independent of the clinical team)

This study is classified as active Intervention one (functional task training, musculoskeletal (active), musculoskeletal (passive), neurophysiological) versus active intervention two (functional task training, musculoskeletal (active), neurophysiological) (Table 6) versus usual care (functional task training, musculoskeletal (active), musculoskeletal (passive), neurophysiological) (Table 5)

\section{Outcomes Measures of motor function: modified Rivermead Motor Assessment}

Measures of voluntary movements: 10 -Metre Walk test, ability to walk at $0.8 \mathrm{~m} / \mathrm{s}$ or more, temporal-spatial gait parameters (symmetry of step length and step time)

Measure of muscle strength: torque around the paretic knee during concentric isokinetic extension, followed immediately by flexion (using the CYBEX NORM isokinetic dynamometer)

Measures of quality of life and social isolation: EuroQol

Time points when outcomes were assessed: "Participants provided written informed consent and then completed baseline measures. Intervention began on the following day for 6 weeks. Participants repeated the measurement battery on completion of the intervention phase (outcome) and 12 weeks later (follow-up)"

Notes

As the two active treatment groups were classified as including similar treatment components, data from this study have not been included within the comparisons of one active intervention versus another active intervention

\section{Risk of bias}

Bias Authors' judgement Support for judgement

Random sequence genera- Low risk tion (selection bias)

Allocation concealment Low risk
(selection bias)

"An independent statistician produced a pretrial computer generated randomized group allocation order in blocks of 9 per trial center. Allocation was stratified by baseline scores for unilateral visual spatial neglect (Star Cancellation Test, 50-54 no spatial neglect and 0-49 unilateral spatial neglect present)"

"Allocation was concealed in sequentially numbered sealed opaque envelopes held by an independent administrator. Envelopes were opened in response to a telephone request from the research physiotherapist (blinded to measures) after the assessor (blinded to group allocation) had completed baseline measures"

\begin{tabular}{|c|c|c|}
\hline $\begin{array}{l}\text { Blinding of outcome as- } \\
\text { sessment (detection bias) }\end{array}$ & Low risk & $\begin{array}{l}\text { "research physiotherapist (blinded to measures)" } \\
\text { "assessor (blinded to group allocation)" }\end{array}$ \\
\hline
\end{tabular}

Physical rehabilitation approaches for the recovery of function and mobility following stroke (Review) Copyright $\odot 2023$ The Cochrane Collaboration. Published by John Wiley \& Sons, Ltd. 
Cooke 2006 (Continued)

All outcomes

$\begin{array}{ll}\begin{array}{l}\text { Incomplete outcome data } \\ \text { (attrition bias) }\end{array} & \text { Low risk } \\ \text { All outcomes } & \text { "At outcome, } 10(9 \%) \text { participants had withdrawn. At follow-up, a further } 18 \\ & \begin{array}{l}\text { participants had withdrawn. Every effort was made to measure participants } \\ \text { at outcome and follow-up even if they had withdrawn from therapy (inten- } \\ \text { tion-to-treat principle)" }\end{array}\end{array}$

\begin{tabular}{|c|c|c|}
\hline $\begin{array}{l}\text { Free of systematic differ- } \\
\text { ences in baseline charac- }\end{array}$ & Low risk & $\begin{array}{l}\text { "All measures were balanced at baseline with the exception of hemiplegic side } \\
\text { and number able to walk at } 0.8 \mathrm{~m} / \mathrm{s} \text { or more" }\end{array}$ \\
\hline
\end{tabular}
teristics of groups compared?

\begin{tabular}{lll}
\hline $\begin{array}{l}\text { Did authors adjust for } \\
\text { baseline differences in } \\
\text { their analyses? }\end{array}$ & Low risk & \\
\hline Other bias & Unclear risk & No information provided \\
\hline
\end{tabular}

Dean 1997

\section{Study characteristics}

\begin{tabular}{ll}
\hline Methods & Study design: RCT \\
& Method of randomisation: blocked randomisation ("subjects drew a card from a box that was originally \\
& filled with 10 control and 10 experimental cards")
\end{tabular}

Participants

Number of participants: $\mathrm{n}=20$

Inclusion criteria: diagnosed with stroke more than one year previously, discharged from rehabilitation, able to understand instructions, able to give informed consent, no orthopaedic problems that could interfere with ability to perform seated reaching tasks and able to sit unsupported for 20 minutes

Interventions (1) Motor learning group $(\mathrm{n}=10)$

Standardised training programme designed to improve sitting balance through reaching with the unaffected hand

"The training for the experimental group was designed to improve sitting balance and involved emphasis on appropriate loading of the affected leg while practicing reaching tasks using the unaffected hand to grasp objects located beyond arm's length. The reaching tasks were performed under systematically varied conditions. Distance and direction were varied by changing the location of the object. Seat height, movement speed, object weight, and extent of thigh support on the seat were also varied. The training was advanced by increasing the number of repetitions and complexity of the tasks"

The individual components delivered are listed in Table 3. Based on the individual components, this intervention is categorised as comprising functional task training

Length of intervention period: two weeks

Number of sessions and length of individual sessions: 10 sessions over two weeks, average of 30 minutes

Intervention provider: "the training programs were carried out by the first author in the subject's home"

(2) Placebo group $(\mathrm{n}=10)$

Cognitive manipulative tasks, involving reaching the unaffected hand over very small distances 
Dean 1997 (Continued)

"Control group had sham training that incorporated the performance of cognitive-manipulative tasks while seated at a table. Sham training was performed so that subjects would consider themselves involved in a training program and to eliminate any effect due to placebo. They performed manipulative tasks using the unaffected hand over small distances (less than $50 \%$ of arm length). Training was advanced over sessions by increasing the repetitions and cognitive difficulty of the tasks. The subjects in the control group performed an equal number of reaching movements as the subjects assigned to the experimental group; however, the nature of the tasks ensured that only a minimum balance perturbation occurred"

The individual components delivered are listed in Table 3. Based on the individual components, this intervention is categorised as comprising an attention control

Length of intervention period: two weeks

Number of sessions and length of individual sessions: 10 sessions over two weeks, average of 30 minutes

Intervention provider: "the training programs were carried out by the first author in the subject's home"

This study is classified as intervention (functional task training) versus attention control (cognitive) (Table 5)

Measures of postural control and balance: ground reaction force during reaching; EMG during reaching; maximum distance reached; ground reaction force during rising to stand

Measures of voluntary movement: timed 10-Metre Walk

Other measures: time to complete cognitive task

Time points when outcomes were assessed: "pretest and posttest group design was used"

\section{Notes}

\section{Risk of bias}

\section{Bias \\ Authors' judgement Support for judgement}

Random sequence genera- Low risk tion (selection bias)
"Subjects were randomly assigned to either the experimental or control group. Randomization was blocked to ensure equal numbers in the groups. The procedure involved random sampling without replacement; subjects drew a card from a box that was originally filled with 10 control and 10 experimental cards"

\begin{tabular}{|c|c|c|}
\hline $\begin{array}{l}\text { Allocation concealment } \\
\text { (selection bias) }\end{array}$ & High risk & $\begin{array}{l}\text { "Subjects drew a card from a box that was originally filled with } 10 \text { control and } \\
10 \text { experimental cards" }\end{array}$ \\
\hline & & $\begin{array}{l}\text { Judged at high risk, as concealment would have been broken once } 10 \text { were in } \\
\text { one group }\end{array}$ \\
\hline
\end{tabular}

Blinding of outcome as- $\quad$ Unclear risk
sessment (detection bias)

All outcomes

\section{The assessor was blinded for some assessments. The outcome measures for} which there was no blinded assessor were recorded by computer

However, the assessor could have encouraged some participants more than others

\begin{tabular}{ll}
\hline $\begin{array}{l}\text { Incomplete outcome data } \\
\text { (attrition bias) }\end{array}$ & Low risk \\
All outcomes & $\begin{array}{l}\text { Dropouts accounted for: "One subject from the control group dropped out of } \\
\text { the study because of an acute neurological episode that required hospitaliza- } \\
\text { tion" }\end{array}$
\end{tabular}

Free of systematic differ- Low risk ences in baseline charac- "no significant differences between the groups in terms of age, time since stroke, or walking velocity" 
Dean 1997 (Continued) teristics of groups compared?

\begin{tabular}{|c|c|c|}
\hline $\begin{array}{l}\text { Did authors adjust for } \\
\text { baseline differences in } \\
\text { their analyses? }\end{array}$ & Low risk & No information provided \\
\hline Other bias & High risk & $\begin{array}{l}\text { One therapist (principal investigator) carried out all treatments. The use of on- } \\
\text { ly one therapist provides a potential source of contamination between groups } \\
\text { or the introduction of performance bias }\end{array}$ \\
\hline
\end{tabular}

\section{Dean 2000}

\section{Study characteristics}

Study design: RCT
Methods of randomisation: After baseline measurement, participants were grouped into matched pairs
according to their average gait speed. Participants in each pair were randomly assigned to experimen-
tal or control group, using an independent person to draw cards from boxes

Participants Number of participants: $\mathrm{n}=12$

Inclusion criteria: first stroke, more than three months post stroke, discharged from rehabilitation, able to attend rehabilitation centre three times a week for four weeks and able to walk $10 \mathrm{~m}$ independently

Exclusion criteria: any medical condition that would prevent participation in a training programme

Interventions (1) Motor learning group $(n=6)$

Standardised circuit programme designed to strengthen the muscles in the affected leg in a functionally relevant way and provide for practice of locomotor-related tasks

"For the experimental group, the exercise class was designed as a circuit program, with subjects completing practice at a series of work stations as well as participating in walking races and relays with other members of the group. The workstations were designed to strengthen the muscles in the affected leg in a functionally relevant way and provide for practice of locomotor related tasks. The 10 workstations incorporated into the circuit were: (1) sitting at a table and reaching in different directions for objects located beyond arm's length to promote loading of the affected leg and activation of affected leg muscles; (2) sit-to-stand from various chair heights to strengthen the affected leg extensor muscles and practice this task; (3) stepping forward, backward, and sideways onto blocks of various heights to strengthen the affected leg muscles; (4) heel lifts in standing to strengthen the affected plantarflexor muscles; (5) standing with the base of support constrained, with feet in parallel and tandem conditions reaching for objects, including down to the floor, to improve standing balance; (6) reciprocal leg flexion and extension using the Kinetron in standing to strengthen leg muscles; (7) standing up from a chair, walking a short distance, and returning to the chair to promote a smooth transition between the two tasks; the remaining stations (8) walking on a treadmill; (9) walking over various surfaces and obstacles and (10) walking over slopes and stairs provided the opportunity for practice of walking under variant conditions"

The individual components delivered are listed in Table 3. Based on the individual components, this intervention is categorised as comprising functional task training and musculoskeletal intervention (active)

Length of intervention period: four weeks

Number of sessions and length of individual sessions: one-hour programme, three days/wk

Intervention provider: "All training sessions were organized into a group exercise class, conducted by the one of the investigators who was assisted by another physiotherapist" 
Dean 2000 (Continued)

(2) Placebo group $(n=6)$

Standardised circuit programme designed to improve function of the upper limb

The individual components delivered are listed in Table 3. Based on the individual components, this intervention is categorised as comprising attention control (upper limb)

Length of intervention period: four weeks

Number of sessions and length of individual sessions: one-hour programme, three days/wk

Intervention provider: "All training sessions were organized into a group exercise class, conducted by the one of the investigators who was assisted by another physiotherapist"

This study was classified as intervention (functional task training, musculoskeletal (active)) versus attention control (upper limb) (Table 5)

Outcomes

Measures of voluntary movement: timed 10-Metre Walk; Six-Minute Walk test; Step test; Timed Up and Go test; laboratory gait assessment

Other measures: strength and dexterity of the upper limb

Time points when outcomes were assessed: "Subjects were evaluated three times: before the training (pretraining), at the end of the training (posttraining), and 2 months later (follow-up)"

\section{Notes}

\section{Risk of bias}

\begin{tabular}{lll}
\hline Bias & Authors' judgement & Support for judgement \\
\hline $\begin{array}{l}\text { Random sequence genera- } \\
\text { tion (selection bias) }\end{array}$ & Low risk & $\begin{array}{l}\text { After baseline measurement, participants were grouped into matched pairs } \\
\text { according to their average gait speed. Participants in each pair were random- } \\
\text { ly assigned to experimental or control group, using an independent person to } \\
\text { draw cards from boxes }\end{array}$ \\
\hline $\begin{array}{l}\text { Allocation concealment } \\
\text { (selection bias) }\end{array}$ & Unclear risk & $\begin{array}{l}\text { Although participants were paired according to average gait speed, it is not } \\
\text { clear how this matching was performed; if the person doing the matching was } \\
\text { not blind to the other characteristics of the participants, there is the potential } \\
\text { for selection bias }\end{array}$ \\
\hline
\end{tabular}

Blinding of outcome as- Unclear risk The assessor was blinded for all assessments except one (Six-Minute Walk test)
sessment (detection bias) All outcomes

\begin{tabular}{lll}
$\begin{array}{l}\text { Incomplete outcome data } \\
\text { (attrition bias) } \\
\text { All outcomes }\end{array}$ & Low risk & Dropouts accounted for \\
& $\begin{array}{l}\text { Nine of } 12 \text { completed training and pretraining and post-training assessments } \\
\text { Eight of } 12 \text { completed follow-up (two-month) assessment }\end{array}$ \\
\hline $\begin{array}{l}\text { Free of systematic differ- } \\
\begin{array}{l}\text { ences in baseline charac- } \\
\text { teristics of groups com- } \\
\text { pared? }\end{array}\end{array} \quad$ Low risk & $\begin{array}{l}\text { "Subjects completed the pretraining evaluation and were then grouped into } \\
\text { matched pairs according to the average walking speed at the pretraining eval- } \\
\text { uation" }\end{array}$
\end{tabular}
pared?

Did authors adjust for Low risk No information provided
baseline differences in
their analyses?


Dean 2000 (Continued)

Other bias High risk
The same therapist conducted the training sessions for both groups and was responsible for progression of treatment, etc; this may potentially contaminate the groups

The study included only participants who were able to travel to the rehabilitation centre and prepared to meet the costs of this. The results of this study therefore can be applied only to equally motivated participants

\title{
Study characteristics
}

Methods Study design: prospective, multi-centre, parallel RCT

Method of randomisation: "eligible participants were randomly allocated within each stroke club to an experimental group (EG) or a control group (CG), using random permuted blocks of 2 to 6 participants. The allocation sequence was computer generated before commencement of the study, and a set of consecutively numbered, sealed opaque envelopes containing the allocation was centrally generated for each stroke club"

\begin{abstract}
Participants $\quad$ Number of participants: $\mathrm{n}=151$
Inclusion criteria: "participants were invited to participate if they had suffered 1 or more strokes, were able to walk $10 \mathrm{~m}$ independently with or without a mobility aid, gained medical clearance, were willing to join the NSW Stroke Recovery Association and commit to a weekly exercise class and home program for 12 months, and were able to give informed consent"

Exclusion criteria: "Folstein Mini-Mental State Examination score of less than 20, insufficient language skills to participate in assessment and intervention, and a medical condition precluding exercise, such as unstable cardiovascular disease or other uncontrolled chronic conditions that would interfere with training and testing protocols"
\end{abstract}

Interventions

(1) Experimental group (EG) $(n=76)$

The $E G$ received an exercise intervention designed to enhance mobility, prevent falls and increase physical activity-the WEBB programme. The programme involved "task-related" training with progressive balance and strengthening exercises, as well as walking and stair climbing. Typical exercises included calf raisers while standing, sit-stand, step-ups, standing with reduced base of support, graded reaching activities in standing and forward, backward and sideways stepping and walking. The intervention was delivered in a weekly circuit-style group exercise class and a home exercise programme, and advice to increase walking was given

The individual components delivered are listed in Table 3. Based on the individual components, this intervention is categorised as comprising functional task training and musculoskeletal intervention (active)

Length of intervention period: "Classes planned to be delivered weekly for 40 weeks over a 1-year period and a home exercise program to be completed at least 3 times per week"

Number of sessions and length of individual sessions: "Each class and home program session was designed to take 45 to 60 minutes"

Intervention provider: "exercise classes were delivered by a physiotherapist who also designed individual home programs, which were reviewed and modified monthly"

(2) Control group (CG) $(n=75)$

"The CG exercise class was designed to improve upper-limb function, manage upper-limb contracture with task-related strength and coordination training, and improve cognition with matching, sorting and 
Dean 2006 (Continued)

sequencing tasks. The CG was also prescribed a home program aimed to make them use their affected arm and keep their mind occupied with cognitive leisure tasks such as word and number puzzles"

The individual components delivered are listed in Table 3. Based on the individual components, this intervention is categorised as comprising upper limb training

Length of intervention period: "Classes planned to be delivered weekly for 40 weeks over a 1-year period and a home exercise program to be completed at least 3 times per week"

Number of sessions and length of individual sessions: "Each class and home program session was designed to take 45 to 60 minutes"

Intervention provider: "Exercise classes were delivered by a physiotherapist who also designed individual home programs, which were reviewed and modified monthly"

This study was classified as intervention (functional task training and musculoskeletal (active)) versus attention control (upper limb and cognition) (Table 5)

Outcomes

Measures of postural control and balance: Timed Up and Go test, Step test, Timed 5 STS, maximum balance range

Measures of voluntary movements: Six-Minute Walk test, 10-Metre Walk test

Measures of muscle strength: knee strength (affected and intact)

Measures of participation: Adelaide Activities Profile

Measures of quality of life and social isolation: Health-Related Quality of Life SF (Short Form)-12, version two

Other secondary outcome measures: falls risk (Short-Form Physiological Profile Assessment), seven-day pedometer count, choice stepping reaction time, co-ordinated stability

Time points when outcomes were assessed: at baseline and at month 12 cises were progressed regularly to ensure that the intervention remained challenging"

\section{Risk of bias}

Bias Authors' judgement Support for judgement

Random sequence genera- Low risk tion (selection bias)
"Random permuted blocks of 2 to 6 participants. The allocation sequence was computer generated before commencement of the study, and a set of consecutively numbered, sealed opaque envelopes containing the allocation was centrally generated for each stroke club"

"The experimental and control classes were held in different areas of the

Allocation concealment Low risk (selection bias) stroke club and at different times to minimize the risk of 'contamination' between the groups"

Blinding of outcome as- Low risk
sessment (detection bias) All outcomes

\begin{abstract}
"The participants and therapists delivering the intervention could not be blinded to intervention group allocation. Falls were recorded from self-report calendars. All other outcome measures were collected by an assessor who was blinded to group allocation. Blinding was ensured using several strategies. Participants were asked not to reveal details of their program to the assessors, and assessments were collected outside the times for exercise classes"
\end{abstract}

$\begin{array}{ll}\begin{array}{l}\text { Incomplete outcome data } \\ \text { (attrition bias) }\end{array} & \text { Dropouts accounted for } \\ \text { All outcomes } & \text { At month 12, } 11 \text { participants were lost to follow-up in the experimental group: } \\ & \text { incontinence }(n=1) \text {, moved }(n=2) \text {, carer illness }(n=2) \text { and illness }(n=6) \text {. At }\end{array}$


Dean 2006 (Continued)

month 12, seven participants were lost to follow-up in the control group: died

$(n=3)$, illness $(n=2)$ and refused classes/reassessment $(n=2)$

"of 18 withdrawals, only 1 was related to the intervention: 1 participant withdrew as the experimental exercise exacerbated an incontinence problem"

$\begin{array}{ll}\begin{array}{l}\text { Free of systematic differ- } \\ \text { ences in baseline charac- }\end{array} & \text { Low risk } \\ \text { at baseline, the groups were similar in terms of demographic characteristics } \\ \text { and other comorbidities" }\end{array}$
teristics of groups compared?

\begin{tabular}{lll}
\hline $\begin{array}{l}\text { Did authors adjust for } \\
\text { baseline differences in } \\
\text { their analyses? }\end{array}$ & Low risk & \\
\hline Other bias & Unclear risk & No information provided \\
\hline
\end{tabular}

Dean 2007

\section{Study characteristics}

\begin{tabular}{ll}
\hline Methods & Study design: RCT \\
& $\begin{array}{l}\text { Method of randomisation: "Randomisation was concealed from the recruiter and assessor by using } \\
\text { sealed opaque envelopes containing the allocation, which was generated earlier by a person indepen- } \\
\text { dent of the study using random number tables, blocked to ensure equal numbers of experimental and } \\
\text { control participants" }\end{array}$ \\
\hline
\end{tabular}

Participants

Number of participants: $n=12$

Inclusion criteria: diagnosis of first stroke resulting in hemiplegia within the previous three months, no orthopaedic problems that would interfere with the ability to perform seated reaching tasks, no visual problems that would interfere with reaching to pick up objects or reading, score of at least three on item three (sitting balance) of the Motor Assessment Scale for Stroke (Carr 1985), able to reach with intact arm a distance equivalent to $140 \%$ of arm's length, no major cognitive or perceptual problems identified using the short portable mental status questionnaire (Pfeiffer 1975), no left neglect identified using the Letter Cancellation Test (Wilson 1987), able to give informed consent and able to understand instructions

Interventions $\quad$ (1) Experimental group $(n=6)$

"During the training period participants in both groups received all regular physiotherapy intervention other than training to improve sitting. All participants continued to attend other multidisciplinary rehabilitation services"

"Participants in the experimental group were given the sitting training protocol designed by Dean 1997. Designed to improve sitting by reaching beyond arm's length using the unaffected hand whilst focusing on: (1) smooth coordinated motion of the trunk and arm to get the hand to the object; (2) appropriate loading of the affected foot; and (3) preventing the use of maladaptive strategies such as widening the base of support. While reaching beyond arm's length, reach distance, direction, thigh support, seat height, and task were varied systematically. Training was progressed over the 2-week period by increasing the reach distance and the number of repetitions"

The individual components delivered are listed in Table 3. Based on the individual components, this intervention is categorised as comprising functional task training

Length of intervention period: two weeks 
Dean 2007 (Continued)

Number of sessions and length of individual sessions: 10 sessions, with each session lasting approximately 30 minutes

Intervention provider: physiotherapist or supervised undergraduate physiotherapy students

(2) Control group group $(n=6)$

"Participants in the control group completed a sham sitting training protocol designed to improve attention (Dean 1997). Sham training was performed so that participants would consider themselves in volved in a training program, which would eliminate any effect due to placebo. This training involved participants completing a series of 11 cognitive-manipulative tasks. Participants were seated at a table, well supported in a chair with back and armrests, with their forearms resting on the table. The workspace was confined so that reach distance was less than $50 \%$ of arm's length which minimised perturbations to balance. Training was progressed over the 2-week period by increasing the number of repetitions and cognitive difficulty of the cognitive-manipulative tasks. Therefore, this training was sham sitting training because the perturbations to balance were minimal and were unlikely to lead to improvements in sitting"

The individual components delivered are listed in Table 3. Based on the individual components, this intervention is categorised as comprising attention control (cognitive training)

Length of intervention period: two weeks

Number of sessions and length of individual sessions: 10 sessions, with each session lasting approximately 30 minutes

Intervention provider: physiotherapist or supervised undergraduate physiotherapy student

This study was classified as intervention (functional task training) versus attention control (cognition) (Table 5)

Measures of postural control and balance: sitting ability (maximum reach distance), sitting quality
Measures of voluntary movements: 10-Metre Walk test (comfortable speed)
Other secondary outcome measures: carryover to mobility (standing up and walking)
Time points when outcomes were assessed: "prospective randomised design with pre-, post-, and fol-
low-up tests (six months later)"

\section{Notes}

\section{Risk of bias}

\begin{tabular}{lll}
\hline Bias & Authors' judgement & Support for judgement \\
\hline $\begin{array}{l}\text { Random sequence genera- } \\
\text { tion (selection bias) }\end{array}$ & Low risk & $\begin{array}{l}\text { Randomisation was concealed from the recruiter and assessor by using sealed } \\
\text { opaque envelopes containing the allocation, which was generated earlier by a } \\
\text { person independent of the study using random number tables, blocked to en- } \\
\text { sure equal numbers of experimental and control participants }\end{array}$ \\
\hline
\end{tabular}

\begin{tabular}{lll}
\hline $\begin{array}{l}\text { Allocation concealment } \\
\text { (selection bias) }\end{array}$ & Low risk & $\begin{array}{l}\text { "Concealment of allocation from the recruiter and blinded assessor was suc- } \\
\text { cessful" }\end{array}$ \\
\hline $\begin{array}{l}\text { Blinding of outcome as- } \\
\text { sessment (detection bias) }\end{array}$ & Low risk & $\begin{array}{l}\text { The third study author remained blinded to group allocation and collected the } \\
\text { outcomes measures post training and six months later. The collection of some } \\
\text { outcome measures required two persons, one of whom was not blinded. To re- } \\
\text { duce bias, the blinded assessor (third study author) gave all instructions and } \\
\text { measured outcomes that were not collected by the computer }\end{array}$ \\
\hline
\end{tabular}

Incomplete outcome data Low risk $\quad$ Dropouts accounted for
(attrition bias)


Dean 2007 (Continued)

All outcomes
"All 12 participants received intervention as allocated and completed post testing. Only 9 participants (5 Experimental and 4 Control) were available for six month follow up measures. Reasons for loss to follow-up were: 1 refusal (Experimental), 1 death (Control), and 1 no longer residing at address and unable to be contacted (Control)"
Free of systematic differences in baseline characteristics of groups compared?
Unclear risk

"With respect to sitting ability, both groups were similar at baseline with a maximum reach distance of approximately $1.1 \mathrm{~m} "$

"For one of the quality of sitting measures, reach movement time, the experimental group reached faster than the control group at baseline"

"For the other quality of sitting measure, average peak vertical force through the affected foot during the forward and across reaches, both groups were similar at baseline"

"For walking, the experimental group walked faster than the control group at baseline with three of the control group unable to walk"

Did authors adjust for $\quad$ Unclear risk $\quad$ No adjustment made
baseline differences in

their analyses?

Other bias Unclear risk No information provided

Deng 2011

\section{Study characteristics}

Study design: RCT
Methods of randomisation: based on the "unbalance index minimum principle," age, nature of lesion,
side of lesion, commencement of treatment time and cognitive deficits

Participants Number of participants: $\mathrm{n}=100$

Inclusion criteria: first ever stroke, confirmed by CT scan or MRI, satisfied 1995 evaluative standard revised by the Fourth [National] Academic Conference of Cerebral Vascular Disease, Glasgow Coma Score > eight, persistent motor deficits, participant or family consent, willing to sign on informed consent sheet

Exclusion criteria: severe active liver disease, insufficient function of the liver and/or kidney, cardiovascular issues, malignant tumour(s), cardiorespiratory issues, inability to comply with investigators, haemorrhage in the eyes and home inaccessible to visiting personnel

Interventions

(1) Intervention group $(n=50)$

"Both groups received conventional therapy and nursing care in the Department of Neurology"

"The intervention group were also given ADL system intervention. The team looking after the intervention group comprised therapists and rehabilitation nurses. The therapists used Brunnstrom stages of motor recovery to deliver the intervention training, the rehabilitation nurses provided guidance on patient self-care, based on the assessment of patient self-care needs and self-care ability, through 3 care systems, using the methods of full substitution, guidance, encouragement and provision of environment and education to improve the patients' ADL ability"

"ADL system intervention content:

(a) Acute phase (flaccid paralysis phase): 
The aim of training is to prevent disuse, prevent possible complications so as to create the conditions for initial training. Initial bedside activities for ADL training include: maintaining positions which prevent spasms; changing body positions; passive ranging exercises through normal joint ranges of motion; passive massages; active bed training: mainly focused on training of truncal muscles, including bridging exercises, leg exercises, hip exercises, self-assisted upper limb extensor exercises, shoulder exercises, rolling to both sides, sitting up from non-affected and affected sides etc.

(b) Early recovery phase (spastic phase):

The aim of training is to reduce muscle tension, resolve spasticity and exercise in isolated movements, detailed content includes: sitting balance training at levels 1, 2, 3; sit-stand training; sitting knee and ankle dorsiflexion exercises; standing balance training at levels 1, 2, 3; supported and assisted stepping exercises

(c) Middle and late recovery phase (equivalent to recovery phase):

When spasms are more or less under control, the aim of training is to generate fine, co-ordinate, quick random movements. Detailed content includes: continuation of gait re-education and postural correction, so as to allow further improvement of body function; upper limb and hand isolated movement training"

The individual components delivered are listed in Table 3. Based on the individual components, this intervention is categorised as comprising functional task training, musculoskeletal intervention (active) and musculoskeletal intervention (passive)

Length of intervention period: six weeks

Number of sessions and length of individual sessions: intervention frequency was 60 minutes/session, two/wk. Therapists provided training for the participants 45 minutes/session, one/d, five/wk. Rehabilitation nurses provided ADL supervision and guidance at least 60 minutes/d, five/wk. After discharge from the hospital, therapists continued to provide training during participants' follow-up visits, frequency unchanged; rehabilitation nurses provided home ADL system

Intervention provider: therapists and rehabilitation nurses

(2) Control group $(n=50)$

"... received conventional therapy and nursing care in the Department of Neurology"

The individual components delivered are listed in Table 3. Based on the individual components, this intervention is categorised as comprising no intervention

Length of intervention period: not stated

Number of sessions and length of individual sessions: not stated

Intervention provider: not stated

This study is classified as intervention (functional task training, musculoskeletal (active), musculoskeletal (passive)) versus no treatment (Table 4)

Measure of motor function: Fugl-Meyer Assessment (FMA)
Other secondary outcomes: Stroke Impact Scale
Time points when outcomes were assessed: "outcome assessments for both groups were completed
before and after 6 weeks of intervention"

Notes Original study translated from Chinese to English

\section{Risk of bias}


Deng 2011 (Continued)

Random sequence genera- Low risk "Based on the unbalance index minimum principle, age, nature of lesion, side tion (selection bias) of lesion, commencement of treatment time and cognitive deficits, 100 patients were randomly divided into intervention $(n=50)$ and control $(n=50)$ groups"

\begin{tabular}{lll}
\hline $\begin{array}{l}\text { Allocation concealment } \\
\text { (selection bias) }\end{array}$ & Unclear risk information provided
\end{tabular}

\begin{tabular}{lll}
\hline $\begin{array}{l}\text { Blinding of outcome as- } \\
\text { sessment (detection bias) }\end{array}$ & Unclear risk & No information provided \\
All outcomes & \\
\hline $\begin{array}{l}\text { Incomplete outcome data } \\
\text { (attrition bias) }\end{array}$ & Unclear risk & No dropouts described \\
All outcomes & \\
\hline
\end{tabular}

Free of systematic differ- Low risk "Age, gender, nature of lesion, commencement of treatment time and cogniences in baseline charac- $\quad$ tive deficits etc $(p>0.05)$ for baseline differences" teristics of groups compared?

\begin{tabular}{lll}
\hline $\begin{array}{l}\text { Did authors adjust for } \\
\text { baseline differences in } \\
\text { their analyses? }\end{array}$ & Low risk & \\
\hline Other bias & Unclear risk & No information provided
\end{tabular}

\section{Duncan 1998}

\section{Study characteristics}

\begin{tabular}{ll}
\hline Methods & $\begin{array}{l}\text { Study design: RCT } \\
\text { Method of randomisation: "Randomization was done in blocks of 10. Before initiation of this study, a } \\
\text { random list was generated by group assignments" }\end{array}$ \\
\hline Participants & $\begin{array}{l}\text { Number of participants: } \mathrm{n}=20 \\
\text { Inclusion criteria: } 30 \text { to } 90 \text { days post stroke, Fugl-Meyer score of } 40 \text { to } 90 \text {, Orpington prognostic score } \\
\text { two to } 52, \text { ambulatory with supervision or assistive device, or both, living at home (less than } 50 \text { miles } \\
\text { from Kansas), no medical condition that would limit participation, Mini Mental State score greater than } \\
18 \text { and able to follow three-step command }\end{array}$ \\
\hline
\end{tabular}

Interventions

(1) Mixed group $(n=10)$

Home-based programme aiming to improve "strength, balance and endurance and to encourage more use of the affected extremity"

Assistive and resistive exercises; proprioceptive neuromuscular facilitation (PNF); Theraband exercises; balance exercises; functional activities for the affected upper extremity; progressive walking programme; progressive bicycle ergometer exercise

"The study investigator and co-investigator observed at least 1 therapy session for each subject to ensure standard application of interventions"

Treatments followed a detailed written protocol for intervention

The individual components delivered are listed in Table 3. Based on the individual components, this intervention is categorised as comprising cardiopulmonary interventions, functional task training, musculoskeletal interventions (active) and neurophysiological interventions 
Duncan 1998 (Continued)

Length of intervention period: eight weeks and instructed to continue programme on own for further four weeks

Number of sessions and length of individual sessions: approximately 1.5 hours, three/wk

Intervention provider: physical therapist and occupational therapist

(2) Control group $(\mathrm{n}=10)$

Usual care: "the therapy programmes received by the control group varied in intensity, frequency and duration"

Three participants received physiotherapy; seven had physiotherapy and occupational therapy

Types of exercise interventions given were balance training (60\%), progressive resistive exercises (40\%), bimanual activities (50\%) and facilitative exercises (30\%)

The individual components delivered are listed in Table 3. Based on the individual components, this intervention is categorised as comprising usual care (functional task training, musculoskeletal (active) and neurophysiological intervention)

Length of intervention period: "Participants in this group were visited by a research assistant every 2 weeks to assess the patients' exercise and activity level. Duration of surveillance was 12 weeks"

Number of sessions and length of individual sessions: average number of visits for physiotherapy and occupational therapy patients was 39 , average duration was 44 minutes

Intervention provider: physical therapist and occupational therapist

This study was classified as intervention (functional task training, musculoskeletal (active) and neurophysiological) versus usual care (functional task training, musculoskeletal (active) and neurophysiological) (Table 5). The intervention group also received cardiopulmonary intervention

Outcomes

Measures of independence in ADL: Barthel Index; Lawton Instrumental ADL

Measures of functional independence: Fugl-Meyer Motor score

Measures of postural control and balance: Berg Balance Scale

Measures of voluntary movement: timed 10-Metre Walk; Six-Minute Walk test

Other measures: Orpington Prognostic Scale; Medical Outcomes Study-36 Health Status Measure; Jeb-

sen test of hand function

Time points when outcomes were assessed: "Baseline and postintervention assessments; follow-up testing for postintervention results was performed 12 weeks after the baseline function assessment"

Notes

\section{Risk of bias}

Bias Authors' judgement Support for judgement

Random sequence genera- Low risk tion (selection bias)
"After baseline assessments, the subjects were randomly assigned to the experimental or control group. Randomization was done in blocks of 10. Before initiation of this study, a random list was generated by group assignments. Only a laboratory technician who had no input into subject selection or recruitment was aware of group assignment. After baseline assessment, the technician assigned the subject to the experimental or the control group"

\begin{tabular}{ll}
\hline $\begin{array}{l}\text { Allocation concealment } \\
\text { (selection bias) }\end{array}$ & Low risk
\end{tabular}




\section{Duncan 1998 (Continued)}

Incomplete outcome data (attrition bias)

All outcomes
Low risk No dropouts

\begin{tabular}{lll}
$\begin{array}{l}\text { Free of systematic differ- } \\
\text { ences in baseline charac- }\end{array}$ & Low risk & Baseline demographics con \\
teristics of groups com- & & \\
pared? & & \\
\hline $\begin{array}{l}\text { Did authors adjust for } \\
\text { baseline differences in } \\
\text { their analyses? }\end{array}$ & Low risk & No information provided
\end{tabular}

Other bias High risk $\quad$ Participants in the control group received "usual care"

All control group participants received physiotherapy and seven of 10 received occupational therapy

The exercises given to control group participants appear to have similarities to those given to the intervention group

Some of the control group had greater contact with therapists than those in the intervention group

Some possibility of contamination between groups was noted, but action was taken to avoid this, with therapists seeing only the intervention group

Duncan 2003

\section{Study characteristics}

\begin{tabular}{ll}
\hline Methods & $\begin{array}{l}\text { Study design: RCT } \\
\text { Method of randomisation: blocked randomisation (block size six), random number generator and } \\
\text { sealed envelopes }\end{array}$ \\
\hline
\end{tabular}

\section{Participants}

Number of participants: $n=100$

Inclusion criteria: stroke within 30 to 150 days, able to walk $25 \mathrm{~m}$ independently, mild to moderate stroke deficits, MMSE greater than 16

Exclusion criteria: subarachnoid haemorrhage, lethargic, obtunded or comatose; uncontrolled blood pressure, hepatic or renal failure, NYHA III/IV heart failure, known limited life expectancy or prestroke disability in self care and lived in a nursing home before the stroke

Exercise programme at home aimed at improving strength, balance, endurance, upper limb use Included a variety of techniques from different theoretical 'approaches'

Techniques included ROM (range of movement exercises), PNF (proprioceptive neuromuscular facilitation), task-specific training

Structured protocols for the exercise tasks, criteria for progression and guidelines for reintroducing therapy after intercurrent illness

The individual components delivered are listed in Table 3. Based on the individual components, this intervention is categorised as comprising cardiopulmonary interventions, functional task training, musculoskeletal interventions (active) and neurophysiological interventions 
Number of sessions and length of individual sessions: 36 sessions of 90 minutes

Intervention provider: supervised by physiotherapist or occupational therapist

(2) Control group $(n=50)$

Usual care: Participants in the usual care group had services as prescribed by their physicians. Treating therapists for usual care participants completed a treatment log. "In the usual care group, $46 \%$ of the subjects did not receive any postacute rehabilitation services from physical or occupational therapy. Two thirds were provided recommendations for an unsupervised exercise program. Among the usual care group members who did receive therapy, participants received an average of 8.7 (SD 5.3) physical therapy visits and 10.4 (SD 7.1) occupational therapy visits. Physical and occupational therapy services were received separately as prescribed by their physicians"

The therapy that participants received was primarily directed at strength, balance, endurance, upper extremity, range of motion, mobility and ADL/IADL

The individual components delivered are listed in Table 3. Based on the individual components, this intervention is categorised as comprising usual care (functional task training, musculoskeletal intervention (active))

Length of intervention period: varied

Number of sessions and length of individual sessions: visited by researcher every two weeks

Intervention provider: physiotherapists and occupational therapists

This study was classified as intervention (functional task training, musculoskeletal interventions (active) and neurophysiological interventions) versus usual care (functional task training, musculoskeletal (active)) (Table 5). The intervention group also received cardiopulmonary intervention

Outcomes

Measures of functional independence: Fugl-Meyer (LL)

Measures of postural control and balance: Berg Balance Scale score

Measures of voluntary movement: gait velocity

Measures of muscle strength: ankle and knee strength

Time points when outcomes were assessed: "primary outcomes of the study were assessed at 3 months, immediately after the intervention"

Notes

\section{Risk of bias}

Bias Authors' judgement Support for judgement

Random sequence genera- Low risk tion (selection bias)

Blocked randomisation (block size six), random number generator and sealed envelopes

"After baseline assessments, the subjects were randomly assigned to the intervention or control group through the use of a random number generator with a block size of 6 and sealed envelopes"

\begin{tabular}{|c|c|c|}
\hline $\begin{array}{l}\text { Allocation concealment } \\
\text { (selection bias) }\end{array}$ & Low risk & No information provided \\
\hline $\begin{array}{l}\text { Blinding of outcome as- } \\
\text { sessment (detection bias) }\end{array}$ & Low risk & The assessor was blinded \\
\hline All outcomes & & $\begin{array}{l}\text { "Outcome assessment was performed by research staff blinded to treatment } \\
\text { assignment. Participants were instructed to avoid mentioning anything re- } \\
\text { garding their study experience to the assessors. Participants were not blinded }\end{array}$ \\
\hline
\end{tabular}




\begin{tabular}{ll}
\hline $\begin{array}{l}\text { Incomplete outcome data } \\
\text { (attrition bias) }\end{array}$ & Low risk \\
All outcomes & Dropouts accounted for \\
\hline
\end{tabular}

\begin{tabular}{|c|c|c|}
\hline $\begin{array}{l}\text { Free of systematic differ- } \\
\text { ences in baseline charac- } \\
\text { teristics of groups com- } \\
\text { nared? }\end{array}$ & Low risk & $\begin{array}{l}\text { No significant differences in baseline characteristics of intervention, control } \\
\text { and dropout groups except on the Wolf Motor Function test ("significantly dif- } \\
\text { ferent in those who did vs did not drop out") }\end{array}$ \\
\hline
\end{tabular}
pared?

\begin{tabular}{|c|c|c|}
\hline $\begin{array}{l}\text { Did authors adjust for } \\
\text { baseline differences in } \\
\text { their analyses? }\end{array}$ & Low risk & No information provided \\
\hline Other bias & High risk & $\begin{array}{l}\text { Participants in the intervention group received greater contact with therapists } \\
\text { than participants in the control group, providing a potential source of perfor- } \\
\text { mance bias. However, } 54 \% \text { of the control group did receive rehabilitation from } \\
\text { physiotherapists and occupational therapists during the study period. This } \\
\text { 'usual care' may have made the control and treatment groups similar in the re- } \\
\text { habilitation they received, potentially reducing the effect of the intervention }\end{array}$ \\
\hline
\end{tabular}

\section{Fan 2006}

\section{Study characteristics}

Study design: RCT
Methods
groups"

Participants Number of participants: $\mathrm{n}=82$ (of whom two in the control group died)

Inclusion criteria: using the 1995 evaluative standard revised by the Fourth [National] Academic Conference of Cerebral Vascular Disease, assessed whether stroke type was ischaemic or haemorrhagic and confirmed by CT scan or MRI, willing to provide informed consent, medically stable within one week, Glasgow Coma Scale score greater than eight, aged between 40 and 80 years and had motor deficits

Exclusion criteria: active liver disease, compromised liver or kidney function, cardiovascular disorder, malignant tumour(s), history of intellectual disorders, cardiorespiratory issue(s), paralysis of four limbs, ischaemic or haemorrhagic stroke outwith three weeks, inaccessible homes located in the outskirts, psychological illness(es) and 'deaf, mute' individuals

\section{Interventions $\quad$ (1) Treated group $(\mathrm{n}=42)$}

"Therapists delivering the rehabilitative treatment all underwent the same training. For acute stroke patients, up to one month after stroke, the first phase of treatment was undertaken, mainly carried out in the Department of Neurology ward, and while given conventional medical treatment, these patients were given early bedside rehabilitative treatment after becoming medically stable, focusing on physiotherapy, with rehabilitative treatment commencing within 1 week after patients were medically stable; for subacute stroke patients, the second (beyond 1 to 3 months after stroke) and third phase (beyond 3 to 6 months after stroke) of treatment [were] undertaken, and based on the patient's condition and level of functional recovery, patients were transferred to rehabilitation ward/centre for further rehabilitation, or discharged home, where a therapist would conduct home visits to guide the patient, and help the patient conduct necessary functional training, until the end of follow-up. Here, 'stage one rehabilitation' referred to the patient's early conventional medicine treatment at the hospital's emergency or Department of Neurology ward, as well as early stage rehabilitative treatment, 'stage two rehabilita- 
tion' referred to the patient's rehabilitative treatment at the rehabilitation ward/centre, 'stage three rehabilitation' referred to the continuation of rehabilitative treatment at the community or home setting.

The rehabilitative treatment method combined physiotherapy and occupational therapy into a holistic method: early stage of treatment was focused on physiotherapy, progressing to occupational therapy. Physiotherapy treatment included: (1) supine and sitting positions to combat spasticity; (2) passive ranging exercise of all affected limb joints, including mobilisation of the shoulder girdle, starting from small to large ranges of motion, to avoid causing pain to the patient; (3) rolling practice (from affected and non-affected sides); (4) bridging exercises; (5) exercises in ankle dorsiflexion and wrist extension; (6) outwith therapy time, sitting practice: headrest of bed lifted to 30 degrees, and, if participant could tolerate the angle for longer than 30 minutes, the angle was increased by another 10 degrees the following day, until participant could tolerate 90 degrees for longer than 30 minutes, which would then be followed by lying-to-sitting up training (from non-affected and affected sides); (7) sitting balance training (sitting on edge of bed); (8) sit-to-stand training; (9) standing balance training; (10) gait training, etc. During the flaccid phase, focus was on postural correction, passive activities and active/passive practice, rolling, lying-to-sitting on edge of bed. During the spasticity phase, focus was on relaxation practice for spastic muscles, antispasticity manual techniques and muscle training for non-spastic muscles, as well as practising exercises in isolated movements. Occupational therapy treatment was based largely on the participant's functional ability at each stage; the appropriate intervention would be given, namely, feeding, grooming, donning, bed-to-wheelchair and wheelchair-to-bed transfer and other ADLs, as well as practice of woodwork, sewing and other handicrafts and ring-insertion games, jigsaws and other leisure activities.

Stage one rehabilitation included antispasticity positioning, passive training of limbs, active training of non-affected limbs under guidance and deep breathing, as well as training of abdominal muscles, sitting up from lying, sitting balance and standing up training, etc, to train the participant's ability to get up from the bed (treatment was done), one/d, 45 minutes/session, five/wk, during the training period, the participant's family or nursing workers were taught the correct supplementary exercises and methods of care concurrently, to achieve partial training out with therapy times, while reducing the damage to affected limbs due to inappropriate nursing care; stage two rehabilitation mainly consisted of standing training, standing balance, single-leg standing, gait training and up-and-down stair training etc, to train the participant's ability to ambulate (treatment was done) $2 x /$ day, 30-45 minutes/session, $5 x /$ week; stage three rehabilitation mainly consisted of feeding, donning, grooming, personal hygiene management and other ADL ability training (treatment was done) 2x/day, 30-45 minutes/session, five to seven days/wk. Stage one early rehabilitation and stage two rehabilitation within rehabilitation ward/centre were delivered by therapists, while concurrently teaching participant's family or nursing workers how to assist the participant in training; during stage three community rehabilitation, therapists conducted fortnightly home visits, and while delivering occupational therapy and necessary physiotherapy, also taught the participant's family or nursing workers how to help the participant train, leaving them to assist the participant in completing the remaining daily necessary training."

The individual components delivered are listed in Table 3. Based on the individual components, this intervention is categorised as comprising functional task training, musculoskeletal intervention (active) and musculoskeletal intervention (passive)

Length of intervention period: not stated

Number of sessions and length of individual sessions: dependent on stage of rehabilitation

Stage one rehabilitation: one/d, 45 minutes/session, five/wk

Stage two rehabilitation: two/d, 30 to 45 minutes/session, five/wk

Stage three rehabilitation: two/d, 30 to 45 minutes/session, five to seven days/wk

Intervention provider: therapists (participant's family or nursing worker outwith therapy times)

(2) Control group $(n=40)$ (of whom two died)

"Patients in the control group were not given any standardised rehabilitation therapy, but were given the same usual medical treatment as the treated group. It was noted that some patients self-trained after verbal advice from their doctor, while some patients' families assisted the patient in movement based on their own knowledge, and the possibility that some patients underwent certain rehabilitation

Physical rehabilitation approaches for the recovery of function and mobility following stroke (Review) 
Fan 2006 (Continued)

treatment after seeking help from other rehabilitation organisations upon discharge could not be excluded"

The individual components delivered are listed in Table 3. Based on the individual components, this intervention is categorised as comprising no intervention

Length of intervention period: not stated

Number of sessions and length of individual sessions: not stated

Intervention provider: not stated

This study was classified as intervention (functional task training and musculoskeletal (active), musculoskeletal (passive)) versus no treatment (Table 4)

\section{Outcomes}

Other secondary outcomes: Functional Comprehensive Assessment

Time points when outcomes were assessed: "at recruitment, 1 month after stroke, 3 months after stroke, 6 months after stroke"

\begin{tabular}{ll}
\hline Notes & Original study translated from Chinese to English \\
& No data suitable for analysis \\
\hline
\end{tabular}

\section{Risk of bias}

\begin{tabular}{lll}
\hline Bias & Authors' judgement & Support for judgement \\
\hline $\begin{array}{l}\text { Random sequence genera- } \\
\text { tion (selection bias) }\end{array}$ & Low risk & Stratified by type of stroke-ischaemic or haemorrhagic \\
\hline $\begin{array}{l}\text { Allocation concealment } \\
\text { (selection bias) }\end{array}$ & Unclear risk & No information given \\
\hline $\begin{array}{l}\text { Blinding of outcome as- } \\
\text { sessment (detection bias) } \\
\text { All outcomes }\end{array}$ & Low risk & $\begin{array}{l}\text { All outcome assessments undertaken by the same assessor, assessor did not } \\
\text { deliver any therapy }\end{array}$ \\
\hline
\end{tabular}

\begin{tabular}{l}
\hline Incomplete outcome data Low risk Two participants from the control group died. All dropouts were accounted for \\
(attrition bias) \\
All outcomes
\end{tabular}

Free of systematic differ- Low risk Study commented no obvious difference in baseline characteristics
ences in baseline charac-
teristics of groups com-
pared?

\begin{tabular}{lll}
\hline $\begin{array}{l}\text { Did authors adjust for } \\
\text { baseline differences in } \\
\text { their analyses? }\end{array}$ & Low risk & \\
\hline Other bias & Unclear risk & No information provided \\
\hline
\end{tabular}

Fang 2003

\section{Study characteristics}

Methods Study design: RCT


Fang 2003 (Continued)

Method of randomisation: "Randomization was achieved through computer-generated random numbers in sealed envelopes"

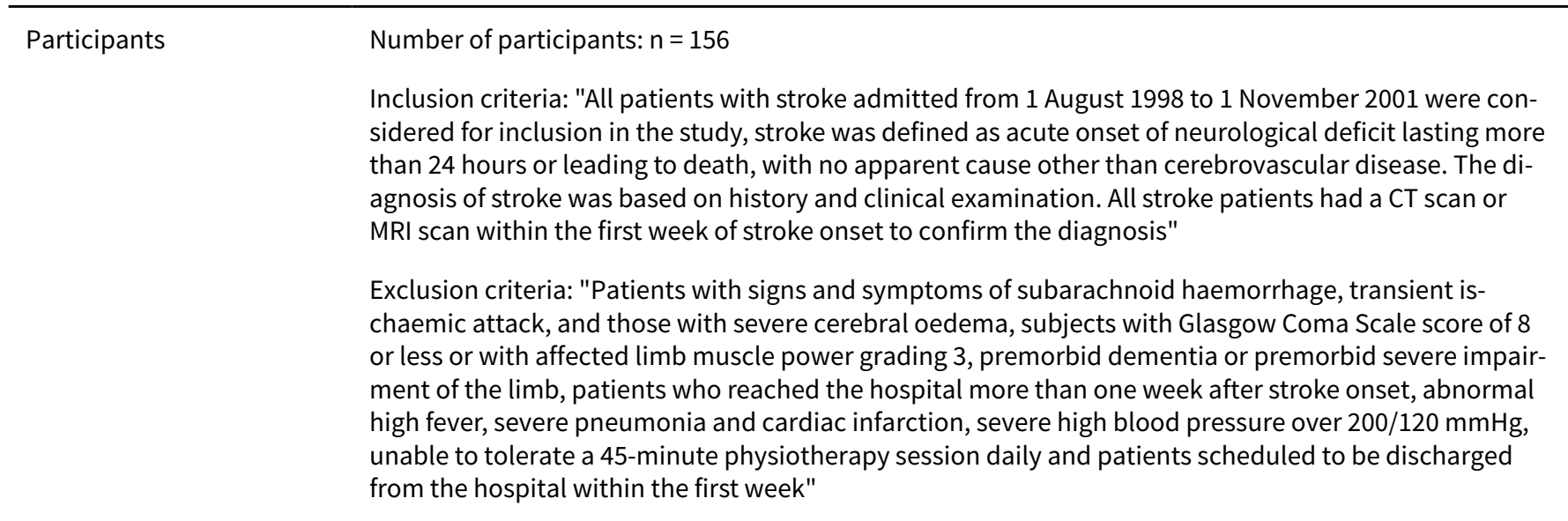

Interventions

(1) Additional early physiotherapy (AEP) intervention group $(n=78)$

"The early therapy included Bobath techniques and passive movements training of the affected limb, and was initiated within the first week after stroke onset. Passive movement training included a series [of] movements of the joints of completely paralytic limbs to prevent contracture and malformation"

The individual components delivered are listed in Table 3. Based on the individual components, this intervention is categorised as comprising musculoskeletal intervention (passive) and neurophysiological intervention

Length of intervention period: four weeks

Number of sessions and length of individual sessions: 45 minutes/d, five days/wk

Intervention provider: ".. two experienced rehabilitation therapists from the department of rehabilitation in the hospital"

(2) Routine therapy (RT) group $(n=78)$

"... routine therapy group received no professional or regular physiotherapy during the whole hospitalization period"

The individual components delivered are listed in Table 3. Based on the individual components, this intervention is categorised as comprising no intervention

Length of intervention period: no intervention

Number of sessions and length of individual sessions: no intervention

Intervention provider: no intervention

This study is classified as intervention (musculoskeletal (passive), neurophysiological) versus no treatment (Table 4)

Outcomes $\quad$ Measures of independence in ADL: modified Barthel Index

Measures of functional independence: Fugl-Meyer Assessment (upper limb and lower limb)

Other measures: Glasgow Coma Scale (GCS), Mini-Mental State Examination (MMSE), Clinical Neurological Deficit Scale (CNDS)

Time points when outcomes were assessed: "follow-up assessments of the above outcome measures were performed 30 days and six months respectively since stroke onset" 
Fang 2003 (Continued)

Notes
"Stroke related symptoms and complications in each group were treated with multidisciplinary approaches in the stroke centre by a special team. No special cognitive or acupuncture therapy was administered"

This study had high numbers of dropouts from the treatment group (28/78 from treatment group during treatment period) as compared with no dropouts from the control group. Large numbers were lost to follow-up from both groups for the six-month follow-up (with only 12/50 and 14/78 included at six months)

\section{Risk of bias}

\begin{tabular}{lll}
\hline Bias & Authors' judgement & Support for judgement \\
\hline $\begin{array}{l}\text { Random sequence genera- } \\
\text { tion (selection bias) }\end{array}$ & Low risk & $\begin{array}{l}\text { "Randomization was achieved through computer-generated random numbers } \\
\text { in sealed envelopes" }\end{array}$ \\
\hline $\begin{array}{l}\text { Allocation concealment } \\
\text { (selection bias) }\end{array}$ & Unclear risk & No information provided \\
\hline $\begin{array}{l}\text { Blinding of outcome as- } \\
\text { sessment (detection bias) } \\
\text { All outcomes }\end{array}$ & Low risk & "Therapists were blinded to patients' groupings" \\
& $\begin{array}{l}\text { "... evaluations were performed in the rehabilitation clinic and general outpa- } \\
\text { tients department by two trained neurologists who were blinded to the group- } \\
\text { ing of the subjects" }\end{array}$ \\
\hline
\end{tabular}

\section{Incomplete outcome data High risk} (attrition bias)

All outcomes
28 in the AEP group were not able to tolerate a 45-minute physiotherapy session daily with or without deteriorating illness and were lost to follow-up at six weeks, and a further 102 were lost at six months

High numbers of dropouts in the intervention group at 30 days and in both groups at six months. "Our study is weakened by the large loss of patients in the group receiving additional therapy"

\section{Free of systematic differ- Low risk} ences in baseline characteristics of groups compared?

"... were no differences in age, sex and educational status. The groups were comparable regarding the frequency of previous stroke, type of stroke, incontinence or not, aphasia and psychiatric disturbances"

"no differences between the AEP and RT groups in conscious level, cognitive state, motor function, stroke severity, independence of daily living at prerehabilitation"

Did authors adjust for Low risk No information provided
baseline differences in their analyses?

\begin{tabular}{lll}
\hline Other bias $\quad$ Unclear risk $\quad$ No information provided
\end{tabular}

Fang 2004 old

\section{Study characteristics}

$\begin{array}{ll}\text { Methods } & \text { Study design: RCT } \\ \text { Method of randomisation: not stated }\end{array}$

Participants

Number of participants: $\mathrm{n}=70$ 
Fang 2004 old (Continued)

Inclusion criteria: "Using the 1995 evaluative standard revised by the Fourth [National] Academic Conference of Cerebral Vascular Disease, participants were selected from hospital admissions between January 1996 and December 2001"

Interventions

(1) Rehabilitation group $(\mathrm{n}=25)$

"Both groups used conventional medication, without using acupuncture. In addition, the 'rehabilitation group' used the Bobath technique to deliver massage to paretic limbs, passive ranging exercises. In supine, professional therapists helped exercise the paretic limbs, 1/day, 45min/session, starting 0-7 days after stroke, for a treatment duration of 3 days"

The individual components delivered are listed in Table 3. Based on the individual components, this intervention is categorised as comprising musculoskeletal intervention (passive)

Length of intervention period: three days

Number of sessions and length of individual sessions: one/d, 45 minutes/session

Intervention provider: therapists

(2) Control group $(n=45)$

"Both groups used conventional medication, without using acupuncture. Control group did not undertake this intervention"

The individual components delivered are listed in Table 3. Based on the individual components, this intervention is categorised as comprising no intervention

Length of intervention period: not stated

Number of sessions and length of individual sessions: not stated

Intervention provider: not stated

This study was classified as intervention (musculoskeletal (passive)) versus no treatment (Table 4)

\begin{tabular}{ll}
\hline Outcomes & Measures of independence in ADL: modified Barthel Index \\
& Measure of motor function: Fugl-Meyer Assessment (FMA) (upper and lower limbs) \\
Other secondary outcome measures: Glasgow Coma Score, Neurological Functional Deficit Score, Mini \\
Mental State Examination \\
Time points when outcomes were assessed: "before intervention, 30 days after intervention, 6 months \\
after intervention" \\
Original study translated from Chinese to English \\
Although the description of the intervention included a reference to 'Bobath,' a consensus decision was \\
made by the review authors to not categorise this as a neurophysiological component, as the reference \\
to 'Bobath' appeared to pertain only to the delivery of massage, which was not in line with our review \\
definitions of neurophysiological interventions
\end{tabular}

\section{Risk of bias}

\begin{tabular}{lll}
\hline Bias & Authors' judgement & Support for judgement \\
\hline $\begin{array}{l}\text { Random sequence genera- } \\
\text { tion (selection bias) }\end{array}$ & Unclear risk & No information provided \\
\hline $\begin{array}{l}\text { Allocation concealment } \\
\text { (selection bias) }\end{array}$ & Unclear risk & No information provided \\
\hline
\end{tabular}


Fang 2004 old (Continued)

Blinding of outcome as- Low risk Outcome assessment conducted by two assessors who were blinded to treatsessment (detection bias)

All outcomes

\section{ment allocation}

Incomplete outcome data High risk $\quad$ No explanation given for mismatch of numbers of participants
(attrition bias)

(attrition bias)

\begin{tabular}{lll}
\hline Free of systematic differ- & Unclear risk & Authors have stated no baseline differences, but methods of analysis are not \\
ences in baseline charac- & sound
\end{tabular}
teristics of groups compared?

\begin{tabular}{lll}
\hline $\begin{array}{l}\text { Did authors adjust for } \\
\text { baseline differences in } \\
\text { their analyses? }\end{array}$ & Unclear risk & No information provided \\
\hline Other bias & Unclear risk & No information provided \\
\hline
\end{tabular}

Fang 2004 young

\section{Study characteristics}

\begin{tabular}{ll}
\hline Methods & Study design: RCT \\
& Method of randomisation: not stated \\
\hline Participants & Number of participants: $\mathrm{n}=58$ \\
& $\begin{array}{l}\text { Inclusion criteria: "Using the } 1995 \text { evaluative standard revised by the Fourth [National] Academic Con- } \\
\text { ference of Cerebral Vascular Disease, participants were selected from hospital admissions between } \\
\text { January } 1996 \text { and December 2001" }\end{array}$
\end{tabular}

Interventions (1) Rehabilitation group $(\mathrm{n}=50)$

"Both groups used conventional medication, without using acupuncture. In addition, the 'rehabilitation group' used the Bobath technique to deliver massage to paretic limbs, passive ranging exercises. In supine, professional therapists helped exercise the paretic limbs, 1/day, 45min/session, starting 0-7 days after stroke, for a treatment duration of 3 days"

The individual components delivered are listed in Table 3. Based on the individual components, this intervention is categorised as comprising musculoskeletal intervention (passive)

Length of intervention period: three days

Number of sessions and length of individual sessions: one/d, 45 minutes/session

Intervention provider: therapists

(2) Control group $(n=78)$

"Both groups used conventional medication, without using acupuncture. Control group did not undertake this intervention"

The individual components delivered are listed in Table 3. Based on the individual components, this intervention is categorised as comprising no intervention

Length of intervention period: not stated 
Fang 2004 young (Continued)

Number of sessions and length of individual sessions: not stated

Intervention provider: not stated

This study was classified as intervention (musculoskeletal (passive)) versus no treatment (Table 4)

\begin{tabular}{ll}
\hline Outcomes & Measures of independence in ADL: modified Barthel Index \\
& Measure of motor function: Fugl-Meyer Assessment (FMA) (upper and lower limbs) \\
Other secondary outcome measures: Glasgow Coma Score, Neurological Functional Deficit Score, Mini \\
Mental State Examination \\
Time points when outcomes were assessed: "before intervention, 30 days after intervention, 6 months \\
after intervention" \\
Original study translated from Chinese to English \\
Although the description of the intervention included a reference to 'Bobath,' a consensus decision was \\
made by the review authors to not categorise this as a neurophysiological component, as the reference \\
to 'Bobath' appeared to pertain only to the delivery of massage, which was not in line with our review \\
definitions of neurophysiological interventions
\end{tabular}

\section{Risk of bias}

\begin{tabular}{|c|c|c|}
\hline Bias & Authors' judgement & Support for judgement \\
\hline $\begin{array}{l}\text { Random sequence genera- } \\
\text { tion (selection bias) }\end{array}$ & Unclear risk & No information provided \\
\hline $\begin{array}{l}\text { Allocation concealment } \\
\text { (selection bias) }\end{array}$ & Unclear risk & No information provided \\
\hline $\begin{array}{l}\text { Blinding of outcome as- } \\
\text { sessment (detection bias) } \\
\text { All outcomes }\end{array}$ & Low risk & $\begin{array}{l}\text { Outcome assessment conducted by two assessors who were blinded to treat- } \\
\text { ment allocation }\end{array}$ \\
\hline $\begin{array}{l}\text { Incomplete outcome data } \\
\text { (attrition bias) } \\
\text { All outcomes }\end{array}$ & High risk & No explanation given for mismatch of numbers of participants \\
\hline $\begin{array}{l}\text { Free of systematic differ- } \\
\text { ences in baseline charac- } \\
\text { teristics of groups com- } \\
\text { pared? }\end{array}$ & Unclear risk & $\begin{array}{l}\text { Authors have stated no baseline differences, but methods of analysis were not } \\
\text { sound }\end{array}$ \\
\hline $\begin{array}{l}\text { Did authors adjust for } \\
\text { baseline differences in } \\
\text { their analyses? }\end{array}$ & Unclear risk & No information provided \\
\hline Other bias & Unclear risk & No information provided \\
\hline
\end{tabular}

\section{Study characteristics}

Methods Study design: RCT


Exclusion criteria: "History of stroke, onset of transient cerebral ischemia, reversible and ischemic nerve disorder, subarachnoid hemorrhage, serious complications and bilateral lesions"

"Rehabilitation group received rehabilitation therapy after primary assay, including Bobath, middle-frequency electrotherapy, and auxiliary acupuncture and massage. Bobath method was the focus of movement training, such as position treatment such as lateral lying down for minutes during relaxing paralysis; to support sitting position with affected limbs before spasmodic paralysis, active and passive movements of joints, flexion and extension of limbs, anteversion and rotation of torso, to place the affected limb on health limb; lying down-sitting-standing gait training"

The individual components delivered are listed in Table 3. Based on the individual components, this intervention is categorised as comprising functional task training, modality, musculoskeletal intervention (passive), musculoskeletal intervention (active) and neurophysiological intervention

Length of intervention period: not stated

Number of sessions and length of individual sessions: "once a day, 30-45 minutes for each time" Intervention provider: not stated

(2) Control group ( $\mathrm{n}=28-$ see notes below)

"All patients received the same routine therapy but the control group received no rehabilitation therapy"

The individual components delivered are listed in Table 3. Based on the individual components, this intervention is categorised as comprising no intervention

Length of intervention period: no intervention

Number of sessions and length of individual sessions: no intervention

Intervention provider: no intervention

This study is classified as intervention (functional task training, musculoskeletal (active), musculoskeletal (passive), neurophysiological) versus no treatment (Table 4) The intervention group also received modality

Outcomes Measures of participation: ADL; "ADL was assayed for all participants"

Time points when outcomes were assessed: "primary assay should be done within 7 days after onset, while the final assay [should] be carried out 3 months after onset"

Notes Abstract only-limited information available

Mismatch in number of participants reported (total of 40 , but when broken down into the two groups, 20 in the rehabilitation group and 28 in the control group). Need to contact study authors to clarify this point

Middle-frequency therapy is well described in the methods

No data suitable for analysis

\section{Risk of bias}


Ge 2003 (Continued)

\title{
Bias Authors' judgement Support for judgement
}

Random sequence genera- Unclear risk No information provided

tion (selection bias)

"40 patients with acute stroke admitted during 2002-01/06 were randomly divided into two groups"

\begin{tabular}{|c|c|c|}
\hline $\begin{array}{l}\text { Allocation concealment } \\
\text { (selection bias) }\end{array}$ & Unclear risk & No information provided \\
\hline
\end{tabular}

Blinding of outcome as- Unclear risk No information provided

sessment (detection bias)

All outcomes

Incomplete outcome data Unclear risk $\quad$ No dropouts, but a mismatch in participant numbers needs clarification
(attrition bias)

All outcomes

\begin{tabular}{lll}
\hline $\begin{array}{l}\text { Free of systematic differ- } \\
\text { ences in baseline charac- } \\
\text { teristics of groups com- } \\
\text { pared? }\end{array}$ & Low risk & $\begin{array}{l}\text { "No significant difference" in the baseline ADL score between groups, and age- } \\
\text { gender similar across groups }\end{array}$ \\
\hline $\begin{array}{l}\text { Did authors adjust for } \\
\text { baseline differences in } \\
\text { their analyses? }\end{array}$ & Low risk & No information provided \\
\hline $\begin{array}{l}\text { Other bias } \\
\text { Unclear risk }\end{array}$ & $\begin{array}{l}\text { Very little information available about length of intervention provided to the } \\
\text { treatment group }\end{array}$ \\
\hline
\end{tabular}

Gelber 1995

\section{Study characteristics}

\begin{tabular}{ll}
\hline Methods & $\begin{array}{l}\text { Study design: RCT } \\
\text { Method of randomisation: "patients were randomised to one of two treatment arms" }\end{array}$ \\
\hline Participants & $\begin{array}{l}\text { Number of participants: } \mathrm{n}=27 \\
\text { Inclusion criteria: pure motor hemiparetic ischaemic stroke, less than one month post stroke; no cogni- } \\
\text { tive, language, visual, sensory or bilateral deficits; no history of stroke and no premorbid use of walking } \\
\text { stick }\end{array}$ \\
\hline
\end{tabular}

Interventions

\begin{abstract}
(1) Neurodevelopmental Technique (NDT) group $(n=15)$
"Inhibition of abnormal muscle tone and initiation of normal (good quality) motor movements with progression through developmental sequences prior to advancing to functional activities. Therapy techniques included tone inhibition and weight bearing activities, and encouraged patients to use their affected side. Resistance exercises and use of abnormal reflexes and mass movements were avoided." All therapists had received training and evaluation in use of the approaches and were given 'strict guidelines' for treatment.
\end{abstract}

The individual components delivered are listed in Table 3. Based on the individual components, this intervention is categorised as comprising functional task training and neurophysiological intervention

Length of intervention period: "continued for the duration of the inpatient and outpatient rehabilitation programmes"

Number of sessions and length of individual sessions: not stated 
Intervention provider: "these treatment approaches were used by both the physiotherapists and occupational therapists who treated the patients, and were used throughout the patients' time as both inpatients and outpatients. All interventions were administered according to allocated treatment group. The nursing staff reinforced any practice of techniques that patients were to carry out outside their treatment sessions"

(2) Orthopaedic group (traditional functional retraining: TFR) $(n=12)$

"Practicing functional tasks as early as possible even in the presence of spasticity or abnormal postures"

"Passive range of movement; resistive exercises; assistive devices and bracing allow use of unaffected side to perform functional tasks. Therapists had all received training and evaluation in the use of the approaches, and were given 'strict guidelines' for treatment"

The individual components delivered are listed in Table 3. Based on the individual components, this intervention is categorised as comprising assistive devices, functional task training, musculoskeletal interventions (active) and musculoskeletal interventions (passive)

Length of intervention period: "continued for the duration of the inpatient and outpatient rehabilitation programmes"

Number of sessions and length of individual sessions: not stated

Intervention provider: as above

This study is classified as active intervention one (functional task training, neurophysiological) versus active intervention two (functional task training, musculoskeletal (active), musculoskeletal (passive)) (Table 6). Intervention group two also received assistive devices

Measures of Independence in ADL: Functional Independence Measure
Measures of voluntary movement: parameters of gait
Other measures: length of stay and inpatient hospital costs; Box and Block test; 9-Hole Peg test
Time points when outcomes were assessed: "All of the outcome measures were evaluated at admis-
sion, discharge, six months and at twelve months follow-up"

Notes

\section{Risk of bias}

Bias Authors' judgement Support for judgement

Random sequence genera- Unclear risk No information provided ("patients were randomised to one of two treatment tion (selection bias) arms")

\begin{tabular}{ll}
$\begin{array}{l}\text { Allocation concealment } \\
\text { (selection bias) }\end{array}$ & Unclear risk information provided \\
\hline
\end{tabular}

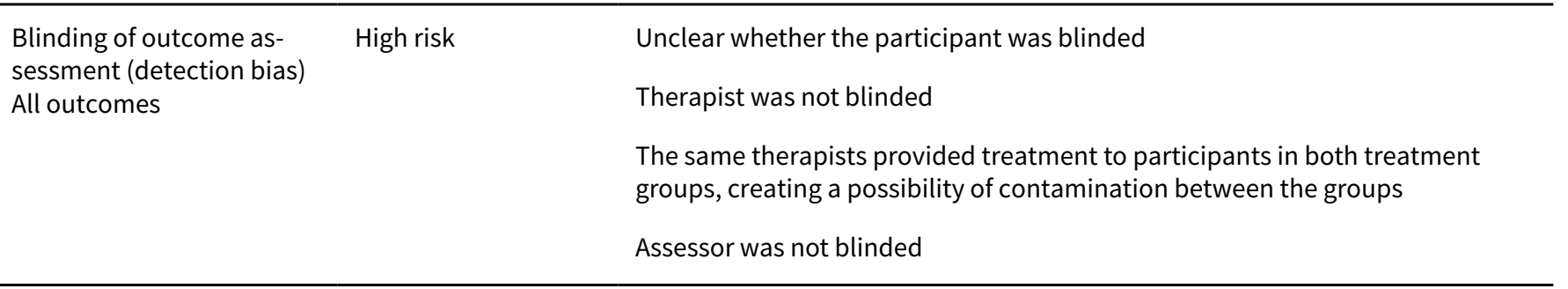

\begin{tabular}{|c|c|c|}
\hline $\begin{array}{l}\text { Incomplete outcome data } \\
\text { (attrition bias) }\end{array}$ & Unclear risk & Dropouts accounted for \\
\hline
\end{tabular}


16/27 at follow-up assessments (23/27 for Functional Independence Measure, carried out by telephone)

\begin{tabular}{|c|c|c|}
\hline $\begin{array}{l}\text { Free of systematic differ- } \\
\text { ences in baseline charac- } \\
\text { teristics of groups com- } \\
\text { pared? }\end{array}$ & Low risk & $\begin{array}{l}\text { "NDT and TFR treated patients did not differ with respect to age, gender, side } \\
\text { of stroke or days from stroke to entry in the study" }\end{array}$ \\
\hline $\begin{array}{l}\text { Did authors adjust for } \\
\text { baseline differences in } \\
\text { their analyses? }\end{array}$ & Low risk & No information provided \\
\hline Other bias & Unclear risk & $\begin{array}{l}\text { The nursing staff reinforced any practice of techniques that participants were } \\
\text { to carry out outside of their treatment sessions; this difference in nursing care } \\
\text { may introduce performance bias }\end{array}$ \\
\hline
\end{tabular}

Green 2002

\section{Study characteristics}

\begin{tabular}{ll} 
Methods & $\begin{array}{l}\text { Study design: RCT } \\
\text { Method of randomisation: blocked randomisation (numbered, sealed, opaque envelopes prepared } \\
\text { from random number tables. Assignment by independent person) }\end{array}$ \\
\hline Participants & $\begin{array}{l}\text { Number of participants: } \mathrm{n}=170 \\
\text { Inclusion criteria: aged over } 50 \text { years, stroke longer than one year previously and persisting mobility } \\
\text { problems } \\
\text { Exclusion criteria: non-stroke mobility problem, dementia, severe co-morbidity, bed bound and physio- } \\
\text { therapy in previous six months }\end{array}$ \\
\hline
\end{tabular}

Interventions (1) Mixed: community physiotherapy using a problem-solving approach $(n=85)$

"Physiotherapy treatment was done by an established community physiotherapy service (13 staff) as part of their usual work"

Community physiotherapists assessed using a 'problem solving approach' and administered interventions according to the problem identified

Physiotherapy interventions included: "gait re-education, exercise therapy, balance re-education, counselling and advice, neurological mobilisations, functional exercises, posture re-education, other interventions"

The individual components delivered are listed in Table 3. Based on the individual components, this intervention is categorised as comprising functional task training

Length of intervention period: maximum of 13 weeks

Number of sessions and length of individual sessions: minimum of three contacts per participant ("median number of treatments per patient was three (IQR 2 to 7 , range 0 to 22) and the mean duration of every treatment was $44 \mathrm{~min} ")$

Intervention provider: physiotherapists

(2) Control: no intervention $(n=85)$

No treatment

The individual components delivered are listed in Table 3. Based on the individual components, this intervention is categorised as comprising no intervention 
Number of sessions and length of individual sessions: no intervention

Intervention provider: no intervention

This study is classified as intervention (functional task training) versus no treatment (Table 4)

Measures of Independence in ADL: Barthel Index
Measures of functional independence: Rivermead Mobility Index, Frenchay Activities Index
Measures of voluntary movement: gait speed
Other measures: Hospital Anxiety and Depression Scale; Depression, General Health Questionnaire 28;
number of participants who had falls
Time points when outcomes were assessed: "After baseline assessment, follow-up assessments were 3-
monthly until 9 months"

Notes

\section{Risk of bias}

\begin{tabular}{lll}
\hline Bias & Authors' judgement & Support for judgement \\
\hline $\begin{array}{l}\text { Random sequence genera- } \\
\text { tion (selection bias) }\end{array}$ & Low risk & $\begin{array}{l}\text { Blocked randomisation (numbered, sealed, opaque envelopes prepared from } \\
\text { random number tables. Assignment by independent person) }\end{array}$ \\
\hline
\end{tabular}

\begin{tabular}{|c|c|c|}
\hline $\begin{array}{l}\text { Allocation concealment } \\
\text { (selection bias) }\end{array}$ & Low risk & Assignment was by independent person \\
\hline $\begin{array}{l}\text { Blinding of outcome as- } \\
\text { sessment (detection bias) } \\
\text { All outcomes }\end{array}$ & Low risk & $\begin{array}{l}\text { Unclear whether the participant was blinded } \\
\text { Therapist was not blinded } \\
\text { Assessor was blinded }\end{array}$ \\
\hline $\begin{array}{l}\text { Incomplete outcome data } \\
\text { (attrition bias) } \\
\text { All outcomes }\end{array}$ & Low risk & $\begin{array}{l}\text { Dropouts accounted for } \\
161 / 170 \text { completed intervention } \\
151 / 170 \text { at six-month assessment }\end{array}$ \\
\hline $\begin{array}{l}\text { Free of systematic differ- } \\
\text { ences in baseline charac- } \\
\text { teristics of groups com- } \\
\text { pared? }\end{array}$ & Low risk & "The characteristics of the two groups were reasonably similar at baseline" \\
\hline $\begin{array}{l}\text { Did authors adjust for } \\
\text { baseline differences in } \\
\text { their analyses? }\end{array}$ & Low risk & No information provided \\
\hline Other bias & Unclear risk & No information provided \\
\hline
\end{tabular}

\section{Holmgren 2006}

\section{Study characteristics}

Methods Study design: single-centre, single-blinded RCT


Holmgren 2006 (Continued)

Method of randomisation: "Randomization of subjects into the intervention (IG) or control group (CG) was conducted with a minimization software program, MiniM (29) to avoid baseline risk factor imbalances between the two groups. Two variables were taken into account: cognition, using the Mini Mental State Examination, MMSE and fall risk, using the Fall Risk Index"

Participants Number of participants: $\mathrm{n}=34$

Inclusion criteria: first ever or recurrent ischaemic or haemorrhagic stroke three to six months before enrolment and randomisation, age $\geq 55$ years, ability to walk 10 metres with or without a walking device, ability to understand and comply with instructions in Swedish and at risk of fall (at the time of enrolment)

Exclusion criteria: ability to walk outdoors independently, without personal assistance or walking device, severe aphasia or severe vision or hearing impairment, medical condition that a physician determined was inconsistent with study participation (e.g. cancer or severe congestive heart failure with expected short remaining life expectancy, recurrent stroke within three months before study start) and if the individual lived farther than $100 \mathrm{~km}$ away from the training facilities ("this was considered as too far away, since it would take too much time and energy away from the individual each day of the intervention")

Interventions

(1) Intervention group $(n=15)$

"The focus of the exercise was on physical activity and functional performance (based on the HIFE program), to improve the subjects' lower-limb strength, balance and gait ability. The program includes lower-limb strength (e.g. chair stand) and balance exercises (e.g. weight shifting outside support surface), standing (e.g. knee bend) and walking (e.g. obstacle crossing course)"

"The two daily training sessions were divided between exercise according to the HIFE program and implementing of the same in to everyday life activities, e.g. walking outdoors or sweep the yard. All exercises were performed at a high intensity, if possible, for each subject. 'High intensity' was defined as (i) strength exercises comprising at least two sets of exercises with $8-12$ repetitions (maximum), (ii) the balance exercises were close to the subjects' balance maximum, and (iii) the subject did not rest more than necessary, all according to the HIFE program"

"In addition, there was a 1-hour educational discussion session, per week. These discussions were about the increased risks of complications after stroke, such as falls. During the last week of intervention, an individualized home-based exercise program was designed by the physiotherapist. This home exercise program was part of the intervention program and consisted of a maximum of three different exercises that were based on the exercises performed during the 5-week intervention. It was easy to adjust the intensity of all the exercises so that they could be modified as the subject progressed. The instructions were to perform this home-based exercise program three times a week at least until the 3month follow-up"

The individual components delivered are listed in Table 3. Based on the individual components; this intervention is categorised as comprising functional task training and musculoskeletal intervention (active)

Length of intervention period: five weeks. In addition, participants were instructed to continue to perform the home-based exercise programme three times a week at least until the three-month follow-up

Number of sessions and length of individual sessions: "the exercise sessions, which lasted approximately 45 min each, were performed six times each week, a total of 30 exercise sessions over 5 weeks. Subjects also received a 45-min session per day of activities related to real-life situations. In addition, one day each week there was a 1-hour educational discussion session, a total of five educational sessions over 5 weeks"

Intervention provider: physiotherapist and occupational therapist

(2) Control group $(n=19)$

Education only 
"The group discussions were about communication difficulties, fatigue, depressive symptoms, mood swings, personality changes and dysphagia, all more or less hidden dysfunctions after stroke and how to cope with these difficulties. There was no special focus on the risks of falling in these discussions"

The individual components delivered are listed in Table 3. Based on the individual components, this intervention is categorised as comprising no intervention

Length of intervention period: five weeks

Number of sessions and length of individual sessions: "met once a week for 1 hour of educational session"

Intervention provider: occupational therapist

This study is classified as intervention (functional task training, musculoskeletal (active)) versus no treatment (Table 4)

Measures of independence in ADL: Barthel Index
Measures of postural control and balance: Berg Balance Scale
Measures of participation: Frenchay Activities Index last three months (FAI-3) tertially
Other secondary outcome measures: Falls Efficacy Scale-International (FES-I) and number of falls
Time points when outcomes were assessed: "Assessments were done at baseline, post-intervention, 3
and 6-month follow-up"

Notes SD computed from confidence intervals and $P$ value

Risk of bias

\begin{tabular}{lll}
\hline Bias & Authors' judgement & Support for judgement \\
\hline $\begin{array}{ll}\text { Random sequence genera- } \\
\text { tion (selection bias) }\end{array}$ & Low risk & "Randomization of subjects into the intervention (IG) or control group (CG) \\
& $\begin{array}{l}\text { was conducted with a minimization software program, MiniM (29) to avoid } \\
\text { baseline risk factor imbalances between the two groups. Two variables were } \\
\text { taken into account: cognition, using the Mini Mental State Examination, MMSE } \\
\text { and fall risk, using the Fall Risk Index" }\end{array}$ \\
&
\end{tabular}

\begin{tabular}{ll}
\hline $\begin{array}{l}\text { Allocation concealment } \quad \text { Low risk } \\
\text { (selection bias) }\end{array}$ & $\begin{array}{l}\text { "Randomization procedure was conducted by the two principal investigators; } \\
\text { these two were involved neither in the assessments nor in the intervention } \\
\text { group or control group. Both investigators were blinded to allocation at the } \\
\text { time of randomization, which was made possible by using code numbers for } \\
\text { each participant" }\end{array}$ \\
\end{tabular}

\begin{tabular}{|c|c|c|}
\hline $\begin{array}{l}\text { Blinding of outcome as- } \\
\text { sessment (detection bias) } \\
\text { All outcomes }\end{array}$ & Low risk & $\begin{array}{l}\text { "All assessments were done by blinded staff, who were instructed that if they } \\
\text { had any reason to believe that they had revealed a subject's group they should } \\
\text { make an adverse event report. The staff in the intervention did not take part in } \\
\text { any of the assessments" }\end{array}$ \\
\hline
\end{tabular}

\begin{tabular}{|c|c|c|}
\hline Incomplete outcome data & Low risk & Dropouts accounted for \\
\hline All outcomes & & $\begin{array}{l}\text { "All but one subject completed the entire program, although two subjects } \\
\text { dropped out during follow-up; the reason for drop-out was worsening overall } \\
\text { medical condition in all three cases" }\end{array}$ \\
\hline
\end{tabular}

\begin{tabular}{|c|c|c|}
\hline $\begin{array}{l}\text { Free of systematic differ- } \\
\text { ences in baseline charac- } \\
\text { teristics of groups com- }\end{array}$ & Low risk & $\begin{array}{l}\text { "There were no significant differences in the baseline characteristics of the } \\
\text { two groups except from the TOAST pathogenesis classification of ischemic } \\
\text { stroke" }\end{array}$ \\
\hline
\end{tabular}
pared? 
Holmgren 2006 (Continued)

Did authors adjust for baseline differences in their analyses?
Low risk No information provided

Other bias Unclear risk No information provided

Hou 2006

\section{Study characteristics}

\begin{tabular}{|c|c|}
\hline Methods & $\begin{array}{l}\text { Study design: RCT } \\
\text { Method of randomisation: not stated }\end{array}$ \\
\hline Participants & $\begin{array}{l}\text { Number of participants: } \mathrm{n}=80 \\
\text { Inclusion criteria: "Using the } 1995 \text { evaluative standard revised by the Fourth [National] Academic Con- } \\
\text { ference of Cerebral Vascular Disease, and confirmation of a first ever stroke from CT or MRI scan, } 80 \text { par- } \\
\text { ticipants were selected" (not explicitly stated as inclusion or exclusion criteria but the text also men- } \\
\text { tioned "that participants were medically stable within two week of stroke onset; had GCS score of }>8 \text {; } \\
\text { had deficits in motor function; aged between } 40-80 \text { years old") }\end{array}$ \\
\hline
\end{tabular}

Exclusion criteria: "Active liver/kidney disease, paralysis of four limbs, deaf and/or mute and issues with coordinating assessment or inaccessible homes"

Interventions

(1) Rehabilitation group $(n=40)$

"Both groups of patients received conventional clinical treatment and care. Rehabilitation group, besides conventional clinical treatment, also strictly followed 'fifteen' research topics 'cerebrovascular disease three level rehabilitation programme' in carrying out rehabilitation; control group did not perform any standard rehabilitation

Rehabilitation group patients, on entering the group, immediately commenced level one rehabilitation programme (within neurology ward), comprising anti-spasticity positioning on bed; breathing exercises; passive ranging exercises on limbs of affected side; use of neural stimulation technique (mainly Rood technique and Brunnstrom technique); active ranging exercises of limbs of non-affected side; lying to sitting training; sitting-balance training; ADL training on bed; neural network and functional electrical stimulation etc. Based on individual situation, selectively performed, $1-2 /$ day, $30-40 \mathrm{~min} / \mathrm{ses}-$ sion, 5 days/week"

"Over time, patient's condition gradually improved, and they were transferred from neurology ward to rehabilitation zone or rehabilitation centre to continue rehabilitation, i.e. level two rehabilitation, with content based on patient's condition to further intensify the measures taken in level one rehabilitation; sit to stand training; transference training; use of neural stimulation technique (mainly Bobath technique and PNF technique); standing-balance training; weight-bearing exercises on affected limbs; gait training and stair (up and down) training, while concurrently adding in relevant occupational therapy. Therapy was done at least 2/day, $40 \mathrm{~min} / \mathrm{session}, 5$ - 6 days/week"

"After a period of level two rehabilitation, most patients were discharged to their homes or community, and thus level three rehabilitation referred to patient's continued rehabilitation at home or in the community setting. Mainly involved therapist making regular home visits, to aid the patient in performing some necessary functional training, for example further enhancing exercise ability; guiding patient on how to adapt to the home living environment; how to independently complete ADLs, etc. Therapy was done usually once every $1-2$ weeks until six months post stroke"

'Three level rehabilitation' training always required participant's family members or nurse to be present to learn the key points of the movements, to allow participant to receive some training outwith therapy time. In particular, the participant, after discharge from hospital, still had to perform rehabilitative training with assistance from family members or nurse 
Hou 2006 (Continued)

The individual components delivered are listed in Table 3. Based on the individual components, this intervention is categorised as comprising functional task training, modality, musculoskeletal (active), musculoskeletal (passive) and neurophysiological intervention

Length of intervention period: six months

Number of sessions and length of individual sessions: dependent on phase of treatment (as above)

Intervention provider: "therapists; outwith therapy time, patient's family members and nurses assisted patients with rehabilitative training"

(2) Control group $(n=40)$

Received conventional clinical treatment and care

The individual components delivered are listed in Table 3. Based on the individual components, this intervention is categorised as comprising no intervention

Length of intervention period: no intervention

Number of sessions and length of individual sessions: no intervention

Intervention provider: no intervention

This study is classified as intervention (functional task training, musculoskeletal (active), musculoskeletal (passive), neurophysiological) versus no treatment (Table 4). The intervention group also received modality

Measures of Independence in ADL: Barthel Index
Other secondary outcome measures: Brunnstrom
Time points when outcomes were assessed: at group allocation, one month, three months and six
months after stroke

Notes Original study translated from Chinese to English

\section{Risk of bias}

\begin{tabular}{|c|c|c|}
\hline Bias & Authors' judgement & Support for judgement \\
\hline $\begin{array}{l}\text { Random sequence genera- } \\
\text { tion (selection bias) }\end{array}$ & Unclear risk & No information provided \\
\hline $\begin{array}{l}\text { Allocation concealment } \\
\text { (selection bias) }\end{array}$ & Unclear risk & No information provided \\
\hline $\begin{array}{l}\text { Blinding of outcome as- } \\
\text { sessment (detection bias) } \\
\text { All outcomes }\end{array}$ & Low risk & Outcome assessors did not deliver intervention \\
\hline $\begin{array}{l}\text { Incomplete outcome data } \\
\text { (attrition bias) } \\
\text { All outcomes }\end{array}$ & Low risk & No dropouts \\
\hline $\begin{array}{l}\text { Free of systematic differ- } \\
\text { ences in baseline charac- } \\
\text { teristics of groups com- } \\
\text { pared? }\end{array}$ & Low risk & $\begin{array}{l}\text { No significant difference }(P \text { value }>0.05) \text { for gender, age, days after stroke, type } \\
\text { of stroke and Brunnstrom score for upper limb, hand and lower limb at base- } \\
\text { line }\end{array}$ \\
\hline
\end{tabular}


Hou 2006 (Continued)

Did authors adjust for baseline differences in their analyses?
Low risk No information provided

Other bias Unclear risk No information provided

Howe 2005

\section{Study characteristics}

$\begin{array}{ll}\text { Methods } & \text { Study design: RCT } \\ & \text { Method of randomisation: "Group allocation was via randomized permutated blocks," by telephone }\end{array}$

Participants Number of participants: $\mathrm{n}=35$

Inclusion criteria: aged over 18 years, acute vascular stroke and previously independently mobile indoors and in personal ADL

Exclusion criteria: other neurological pathology, drugs or conditions affecting balance, impaired consciousness, dementia, unable to tolerate therapy, 'pusher' syndrome and severe perceptual problems

Interventions

(1) Additional therapy $(n=17)$

Exercises aimed at improving lateral weight transference in sitting and standing. Incorporated elements of motor learning, including repetition (practice) of self initiated goal-oriented activities with, where appropriate, manual guidance and verbal encouragement (feedback). Specific techniques are detailed with an appendix to the published paper. Participants in this group received the same usual care as participants in the usual care group

The individual components delivered are listed in Table 3, Based on the individual components, this intervention is categorised as comprising functional task training

Length of intervention period: four weeks

Number of sessions and length of individual sessions: "as their usual care, 217 sessions, total duration 7135 min." Participants received 12 additional therapy sessions-total of six additional hours over the intervention period ("between them received 181 additional treatment sessions, mean 10.6 sessions, each of 30 min duration, total $5430 \mathrm{~min} ")$

Intervention provider: delivered by trained physiotherapy assistants

(2) Usual care $(n=18)$

"Physiotherapists reported that usual care was loosely based on 'neurophysiological' principles, however, their choice of specific physical interventions during each session was determined on an individual basis based on the symptomatic presentation of the patient at the time"

The individual components delivered are listed in Table 3. Based on the individual components, this intervention is categorised as comprising usual care (neurophysiological)

Length of intervention period: four weeks

Number of sessions and length of individual sessions: "Patients in the usual care group $(n=18)$ received 255 sessions of therapy, total duration 8643 min"

Intervention provider: physiotherapists

This study is classified as intervention (functional task training) versus usual care (neurophysiological) (Table 5) stand 
Howe 2005 (Continued)

Time points when outcomes were assessed: "at baseline, four weeks (retest) and eight weeks (follow-up)"

Notes

No outcomes included in analysis; all outcomes were specific to goal of lateral weight transference

\section{Risk of bias}

\begin{tabular}{lll}
\hline Bias & Authors' judgement & Support for judgement \\
\hline $\begin{array}{ll}\text { Random sequence genera- } \\
\text { tion (selection bias) }\end{array}$ & Low risk & "were randomly allocated to a usual care only group, or to the treatment \\
& group" \\
& "Group allocation was via randomized permutated blocks. The project manag- \\
& er held details of assignment and revealed these to the recruiting physiother- \\
& apist via telephone only when the patient was due to be allocated to a group. \\
& The code was not broken until all patients had completed the study and all \\
analysis was complete"
\end{tabular}

\begin{tabular}{ll}
\hline $\begin{array}{l}\text { Allocation concealment } \\
\text { (selection bias) }\end{array}$ & "The project manager held details of assignment and revealed these to the re- \\
& cruiting physiotherapist via telephone only when the patient was due to be al- \\
located to a group"
\end{tabular}

\begin{tabular}{|c|c|c|}
\hline \multirow[t]{2}{*}{$\begin{array}{l}\text { Blinding of outcome as- } \\
\text { sessment (detection bias) } \\
\text { All outcomes }\end{array}$} & Low risk & $\begin{array}{l}\text { Participant was not blinded } \\
\text { Therapist was not blinded }\end{array}$ \\
\hline & & $\begin{array}{l}\text { Assessor was blinded (outcome measures "were undertaken by a blind inde- } \\
\text { pendent observer") }\end{array}$ \\
\hline
\end{tabular}

\begin{tabular}{|c|c|c|}
\hline $\begin{array}{l}\text { Incomplete outcome data } \\
\text { (attrition bias) } \\
\text { All outcomes }\end{array}$ & Low risk & $\begin{array}{l}\text { Dropouts accounted for } \\
33 / 35 \text { completed intervention } \\
31 / 35 \text { had eight-week follow-up }\end{array}$ \\
\hline $\begin{array}{l}\text { Free of systematic differ- } \\
\text { ences in baseline charac- } \\
\text { teristics of groups com- } \\
\text { pared? }\end{array}$ & Low risk & $\begin{array}{l}\text { "The groups did not differ (statistically significantly) in any of these character- } \\
\text { istics" }\end{array}$ \\
\hline $\begin{array}{l}\text { Did authors adjust for } \\
\text { baseline differences in } \\
\text { their analyses? }\end{array}$ & Low risk & No information provided \\
\hline Other bias & Unclear risk & $\begin{array}{l}\text { The standard physiotherapy and other usual care procedures could have } \\
\text { changed as a direct or indirect result of the additional study intervention } \\
\text { Participants could have passed on information about their additional treat- } \\
\text { ment both to the therapists providing standard care and to other participants } \\
\text { in the study }\end{array}$ \\
\hline
\end{tabular}

Hu 2007 haem

\section{Study characteristics}

\begin{tabular}{ll}
\hline Methods & Study design: RCT \\
& Method of randomisation: "Randomisation done after classifying into cerebral infarction group or \\
& haemorrhage group"
\end{tabular}

haemorrhage group" 
Hu 2007 haem (Continued)

$\begin{array}{ll}\text { Participants } & \text { Number of participants: } \mathrm{n}=352 \\ \text { Inclusion criteria: } \text { not stated }\end{array}$

Interventions

(1) Test group ( $n=178$ haemorrhagic)

"Patients from both groups received the same routine neurological intervention, but the treated group received additional standardised tertiary rehabilitation (STR), with details of training content outlined in references (Research Group 2007)"

Length of intervention period: six months

Number of sessions and length of individual sessions: not stated

Intervention provider: not stated

The individual components delivered are listed in Table 4. Based on the individual components, this intervention is categorised as comprising components not stated

(2) Control group $(n=174)$

Received routine neurological intervention

Length of intervention period: not stated

Number of sessions and length of individual sessions: not stated

Intervention provider: not stated

The individual components delivered are listed in Table 3. Based on the individual components, this intervention is categorised as comprising no intervention

This study is classified as intervention (functional task training, musculoskeletal (passive)) versus no treatment (Table 4)

Outcomes Measures of motor function: Fugl-Meyer Assessment

Time points when outcomes were assessed: at enrolment, after one month after stroke, after three months after stroke and six months after stroke

Notes

Data analysis conducted by dividing participants by type of stroke. Dropouts not accounted for. Number of participants with data for extraction varied

Original study translated from Chinese to English

\section{Risk of bias}

\begin{tabular}{lll}
\hline Bias & Authors' judgement & Support for judgement \\
\hline $\begin{array}{l}\text { Random sequence genera- } \\
\text { tion (selection bias) }\end{array}$ & Unclear risk & No information provided \\
\hline $\begin{array}{l}\text { Allocation concealment } \\
\text { (selection bias) }\end{array}$ & Unclear risk & No information provided \\
\hline $\begin{array}{l}\text { Blinding of outcome as- } \\
\text { sessment (detection bias) } \\
\text { All outcomes }\end{array}$ & Low risk & Outcome assessors did not deliver intervention and were blinded \\
\hline $\begin{array}{l}\text { Incomplete outcome data } \\
\text { (attrition bias) } \\
\text { All outcomes }\end{array}$ & High risk & Unaccounted for or unexplained dropouts/lost data \\
\hline \hline
\end{tabular}


Hu 2007 haem (Continued)

Free of systematic differ- Low risk No significant differences between groups for time of enrolment, gender, age, ences in baseline characteristics of groups compared?

$$
\text { side of stroke, etc }
$$

\begin{tabular}{lll}
\hline $\begin{array}{l}\text { Did authors adjust for } \\
\text { baseline differences in } \\
\text { their analyses? }\end{array}$ & Low risk & No information provided \\
\hline Other bias & Unclear risk & No information provided \\
\hline
\end{tabular}

Hu 2007 isch

\section{Study characteristics}

\begin{tabular}{ll}
\hline Methods & Study design: RCT \\
& $\begin{array}{l}\text { Method of randomisation: randomisation done after classifying into cerebral infarction group or haem- } \\
\text { orrhage group }\end{array}$
\end{tabular}

Participants $\quad$ Number of participants: $\mathrm{n}=965$

Inclusion criteria: not stated

Interventions (1) Test group ( $\mathrm{n}=485$ ischaemic only)

"Patients from both groups received the same routine neurological intervention, but the treated group received additional standardised tertiary rehabilitation (STR), with details of training content outlined in references (Research Group 2007)"

Length of intervention period: six months

Number of sessions and length of individual sessions: not stated

Intervention provider: not stated

The individual components delivered are listed in Table 4. Based on the individual components, this intervention is categorised as comprising components not stated

(2) Control group $(n=480)$

Received routine neurological intervention

Length of intervention period: not stated

Number of sessions and length of individual sessions: not stated

Intervention provider: not stated

The individual components delivered are listed in Table 3. Based on the individual components, this intervention is categorised as comprising no intervention

This study is classified as intervention (functional task training, musculoskeletal (passive)) versus no treatment (Table 4)

Outcomes Measures of motor function: Fugl-Meyer Assessment

Time points when outcomes were assessed: at enrolment, after one month after stroke, after three months after stroke and six months after stroke 
Hu 2007 isch (Continued)

Notes
Data analysis conducted by dividing participants by type of stroke. Dropouts not accounted for. Number of participants with data for extraction varied

Original study translated from Chinese to English

\section{Risk of bias}

\begin{tabular}{|c|c|c|}
\hline Bias & Authors' judgement & Support for judgement \\
\hline $\begin{array}{l}\text { Random sequence genera- } \\
\text { tion (selection bias) }\end{array}$ & Unclear risk & No information provided \\
\hline $\begin{array}{l}\text { Allocation concealment } \\
\text { (selection bias) }\end{array}$ & Unclear risk & No information provided \\
\hline $\begin{array}{l}\text { Blinding of outcome as- } \\
\text { sessment (detection bias) } \\
\text { All outcomes }\end{array}$ & Low risk & Outcome assessors did not deliver intervention and were blinded \\
\hline $\begin{array}{l}\text { Incomplete outcome data } \\
\text { (attrition bias) } \\
\text { All outcomes }\end{array}$ & High risk & Unaccounted for or unexplained dropouts/lost data \\
\hline $\begin{array}{l}\text { Free of systematic differ- } \\
\text { ences in baseline charac- } \\
\text { teristics of groups com- } \\
\text { pared? }\end{array}$ & Low risk & $\begin{array}{l}\text { No significant differences between groups for time of enrolment, gender, age, } \\
\text { side of stroke, etc }\end{array}$ \\
\hline $\begin{array}{l}\text { Did authors adjust for } \\
\text { baseline differences in } \\
\text { their analyses? }\end{array}$ & Low risk & No information provided \\
\hline Other bias & Unclear risk & No information provided \\
\hline
\end{tabular}

Huang 2003

\section{Study characteristics}

\begin{tabular}{|c|c|}
\hline Methods & $\begin{array}{l}\text { Study design: RCT } \\
\text { Method of randomisation: stratified randomisation }\end{array}$ \\
\hline Participants & $\begin{array}{l}\text { Number of participants: } \mathrm{n}=50 \\
\text { Inclusion criteria: "Diagnosis of stroke, confirmed by CT or MRI scan, medically stable within a week, } \\
\text { did not exceed } 2 \text { weeks on enrolment to study, aged } 40-80 \text { years, Glasgow Coma Score }>8 \text { and deficits } \\
\text { in limb function" }\end{array}$ \\
\hline & $\begin{array}{l}\text { Exclusion criteria: "Absence of malignant tumour(s), high blood pressure, no psychological conditions, } \\
\text { not deaf, not mute and not have paralysis of four limbs" }\end{array}$ \\
\hline
\end{tabular}

Interventions

(1) Rehabilitation group $(n=25)$

"Both groups received routine treatment (such as medicine). For the participants in the treatment group, exercise therapy combined Bobath, Rood, Motor Relearning Program and proprioceptive neuromuscular facilitation (PNF) techniques; in terms of electrotherapy, early use of electrical stimulation, acupuncture is carried out, with additional appropriate electrical stimulation for participants with 
shoulder-hand syndrome. Recovery of each motor function was targeted by using sequential and progressive therapy, using the following methods: (i) correct positioning: teaching patient's family and caregivers the correct limb positioning of limbs in supine position, lateral position with affected limbs at lower side and healthy limbs at upper side, and lateral position with affected limbs at upper side and healthy limbs at lower side, requesting changing of body position every two hours. (ii) Rolling practice: with both hands intertwined and both elbows extended in front of the body, participants practised rolling to the left and right, with knees in flexion. (iii) Self-assisted bed exercises: with both hands intertwined, participants extended both elbows in front of the body, overhead, to the left and right, touched the nose and did bridging exercises etc. (iv) Passive ranging bed exercises: upper limb: scapula, shoulder, elbow, wrist joints; truncal extension, shoulder retraction: lower limb: hip, knee, talo-crural, metatarsal joints. (v) Techniques to facilitate muscle contractions. (vi) Lung clearance: percussions to the posterior segments of the lungs. (vii) Upright sitting training: gradually increasing the inclining angle of the bed, participants sat upright for 30 minutes. Once this was accomplished, the inclining angle is increased by 10 degrees until the participant could sit on the edge of bed. (viii) Stimulation to the muscles of the face, tongue and lips: opening of mouth, bulging of cheeks, gritting of teeth, extending the tongue, placing the tongue on the upper palate, iced cotton swabs (or placing ice cubes in the buccal cavity) and stimulation of taste. (ix) Breathing control practice: participants were requested to take a deep breath, slowly exhale and then relax. (x) Sitting on the edge of bed training: participants practised pushing up from side lying to sitting on the edge of bed without the inclining angle of the bed increased to 90 degrees. (xi) Sitting balance: postural correction, balance training while sitting on the edge of bed, including perturbations to the front, back, left and right. (xii) Exercises in sitting: to further train sitting balance, participants reached forwards, sideways, touched the nose, pointed to objects with intertwined hands and extended arms; lower limb strengthening exercises, taught to participant's family and caregivers so they could supervise practice of the exercises several times a day. (xiii) Positioning from bed to wheelchair (or chair) training. (xiv) Sit to stand practice: Training of standing tolerance (beside the bed) commenced early to allow re-gaining of gravitational sense, re-gaining of control of muscles working against gravity, normalisation of blood pressure, correct standing balance as well as to overcome postural hypotension. Typically, participants with ischemic stroke were expected to be able to sit on the edge of bed within 3-4 days of rehabilitation, commence standing training within 2 weeks, with the level of assistance given depending on the medical status of the participants; participants with haemorrhagic stroke should aim to sit on the edge of bed within 2 weeks of rehabilitation and commencement of standing training within 4 weeks. (xv) Participants with contractures were treated accordingly. (xvi) Neuromuscular facilitation techniques. (xvii) Gait training. (xviii) Stair practice (up and down)"

The individual components delivered are listed in Table 3. Based on the individual components, this intervention is categorised as comprising functional task training, modality, musculoskeletal intervention (active), musculoskeletal intervention (passive) and neurophysiological intervention

Length of intervention period: 30 days

Number of sessions and length of individual sessions: each session lasting at least 45 minutes, one/d

Intervention provider: "one to one sessions with a therapist"

(2) Control group $(n=25)$

Participants in the control group received routine treatment (such as medicine) only

The individual components delivered are listed in Table 3. Based on the individual components, this intervention is categorised as comprising no treatment

Length of intervention period: no intervention

Number of sessions and length of individual sessions: no intervention

Intervention provider: no intervention

This study is classified as intervention (functional task training, musculoskeletal (active), musculoskeletal (passive), neurophysiological) versus no treatment (Table 4). The intervention group also received modality 
Measures of motor function: simplified Fugl-Meyer

Other secondary outcome measures: cognitive ability rating (translated)

Time points when outcomes were assessed: at enrolment and 30 days after enrolment

Notes Original study translated from Chinese to English

\section{Risk of bias}

\begin{tabular}{|c|c|c|}
\hline Bias & Authors' judgement & Support for judgement \\
\hline $\begin{array}{l}\text { Random sequence genera- } \\
\text { tion (selection bias) }\end{array}$ & Low risk & $\begin{array}{l}\text { Stratified randomisation done, randomisation done by participants' onset of } \\
\text { stroke }\end{array}$ \\
\hline $\begin{array}{l}\text { Allocation concealment } \\
\text { (selection bias) }\end{array}$ & Unclear risk & Insufficient details \\
\hline $\begin{array}{l}\text { Blinding of outcome as- } \\
\text { sessment (detection bias) } \\
\text { All outcomes }\end{array}$ & Low risk & $\begin{array}{l}\text { Outcome assessors did not deliver intervention and were blinded to group al- } \\
\text { location }\end{array}$ \\
\hline $\begin{array}{l}\text { Incomplete outcome data } \\
\text { (attrition bias) } \\
\text { All outcomes }\end{array}$ & Unclear risk & No dropouts reported \\
\hline $\begin{array}{l}\text { Free of systematic differ- } \\
\text { ences in baseline charac- } \\
\text { teristics of groups com- } \\
\text { pared? }\end{array}$ & Low risk & $\begin{array}{l}\text { No obvious difference between groups for time since stroke, gender, age, side } \\
\text { and type of stroke, etc }\end{array}$ \\
\hline $\begin{array}{l}\text { Did authors adjust for } \\
\text { baseline differences in } \\
\text { their analyses? }\end{array}$ & Low risk & No information provided \\
\hline Other bias & Unclear risk & No information provided \\
\hline
\end{tabular}

Hui-Chan 2009

\section{Study characteristics}

\begin{tabular}{|c|c|}
\hline Methods & $\begin{array}{l}\text { Study design: "single-blinded, randomised, placebo-controlled trial" } \\
\text { Method of randomisation: "subjects were allocated randomly, using a computer program" }\end{array}$ \\
\hline \multirow[t]{3}{*}{ Participants } & $\begin{array}{l}\text { Number of participants: } n=54 \text { (109 in whole study-but only } 54 \text { in groups relevant to this review: see } \\
\text { notes) }\end{array}$ \\
\hline & $\begin{array}{l}\text { Inclusion criteria: "sustained a single stroke more than } 1 \text { year previously, were able to walk } 10 \mathrm{~m} \text { unas- } \\
\text { sisted, with or without walking aids, and had a Composite Spasticity Score of } \geq 10 \text { in their ankle plan- } \\
\text { tarflexors" }\end{array}$ \\
\hline & $\begin{array}{l}\text { Exclusion criteria: "medical comorbidity, e.g. unstable cardiopulmonary disease (acute myocardial in- } \\
\text { farction, wearing a cardiac pacemaker, shortness of breath, tachycardia), uncontrolled diabetes melli- } \\
\text { tus, or pre-existing neurological disorders such as multiple sclerosis, receptive dysphasia, or cognitive } \\
\text { impairment (denoted by scoring < } 7 \text { out of } 10 \text { on the Abbreviated Mental Test)" }\end{array}$ \\
\hline
\end{tabular}

\begin{tabular}{ll} 
Interventions & (1) PLBO + TRT group $(n=25)$ \\
\hline
\end{tabular}


Hui-Chan 2009 (Continued)

"received 60 minutes of placebo-TENS from TENS devices with the electrical circuit disconnected inside, followed by 60 minutes of TRT which included six exercises: (i) loading exercise on the affected leg, (ii) stepping up exercise with the affected leg, (iii) stepping down exercise with the unaffected leg, (iv) heel lifts from a dorsiflexed position when standing, (v) standing up from a chair, walking a short distance, and returning to the chair, and (vi) walking with rhythmic auditory cues generated by a metronome"

The individual components delivered are listed in Table 3. Based on the individual components, this intervention is categorised as comprising functional task training

Length of intervention period: four weeks

Number of sessions and length of individual sessions: "home rehabilitation programme daily, 5 days a week"

Intervention provider: "The treatment compliance and safety of the programme [were] closely monitored by the physiotherapist in charge"

(2) Control group $(n=29)$

"received no treatment"

The individual components delivered are listed in Table 3. Based on the individual components, this intervention is categorised as no intervention

Length of intervention period: no intervention

Number of sessions and length of individual sessions: no intervention

Intervention provider: no intervention

This study is classified as intervention (functional task training) versus no treatment (Table 4)

Measures of postural control and balance: Timed Up and Go (TUG) test
Measures of voluntary movements: gait velocity, Six-Minute Walk test
Measures of tone and spasticity: Composite Spasticity Scale
Other secondary outcome measures: surface electromyography and torque measurements
Time points when outcomes were assessed: "assessor blinded to the treatment allocation assessed
the subjects at four time intervals: before and after 2 and 4 weeks of treatment, and 4 weeks after treat-
ment"

Notes This study also included a TENS group $(n=28)$ and a TENS + TRT group $(n=27)$ that we judged not to be relevant to this review, and no data relating to these groups have been extracted

\section{Risk of bias}

\begin{tabular}{lll}
\hline Bias & Authors' judgement & Support for judgement \\
\hline $\begin{array}{l}\text { Random sequence genera- } \\
\text { tion (selection bias) }\end{array}$ & Low risk & "subjects were allocated randomly, using a computer program" \\
\hline $\begin{array}{l}\text { Allocation concealment } \\
\text { (selection bias) }\end{array}$ & Unclear risk & No information provided \\
\hline $\begin{array}{l}\text { Blinding of outcome as- } \\
\text { sessment (detection bias) } \\
\text { All outcomes }\end{array}$ & Low risk & $\begin{array}{l}\text { "assessor blinded to the treatment allocation assessed the subjects at four } \\
\text { time intervals" }\end{array}$ \\
\hline
\end{tabular}


Hui-Chan 2009 (Continued)

Incomplete outcome data (attrition bias)

Unclear risk

All outcomes
"Eight (7.3\%) subjects dropped out from the study" (NB: This is eight of the whole study size of 109.) Reasons for, or groups of, dropouts were not reported

\begin{tabular}{|c|c|c|}
\hline $\begin{array}{l}\text { Free of systematic differ- } \\
\text { ences in baseline charac- } \\
\text { teristics of groups com- } \\
\text { pared? }\end{array}$ & Low risk & $\begin{array}{l}\text { "No significant differences were found between the groups in the five baseline } \\
\text { outcome measurements (age, gender, weight, height, and the type, side and } \\
\text { duration of their strokes)" }\end{array}$ \\
\hline
\end{tabular}

pared?

Did authors adjust for $\quad$ Low risk information provided
baseline differences in
their analyses?

\begin{tabular}{|c|c|c|}
\hline Other bias & Unclear risk & No information provided \\
\hline
\end{tabular}

Jiang 2006

\title{
Study characteristics
}

\begin{tabular}{|c|c|}
\hline Methods & $\begin{array}{l}\text { Study design: RCT } \\
\text { Method of randomisation: stratified randomisation }\end{array}$ \\
\hline Participants & $\begin{array}{l}\text { Number of participants: } \mathrm{n}=82 \text { (baseline data available); } \mathrm{n}=79 \text { (data extraction at six months) } \\
\text { Inclusion criteria: "According to the evaluative standard revised by the } 1995 \text { Fourth National Academ- } \\
\text { ic Conference of Cerebral Vascular Disease, ischaemic and haemorrhagic stroke were diagnosed, con- } \\
\text { firmed by CT or MRI scan, willingly signed informed consent forms, medically stable within a week, } \\
\text { Glasgow Coma Score > } 8 \text {, aged between } 40 \text { to } 80 \text { years and possessed deficits in limb function" } \\
\text { Exclusion criteria: "Active liver disease, impaired liver or kidney function, cardiovascular conditions, } \\
\text { malignant tumours, history of cognitive issues, impaired respiratory function, paralysis of four limbs; } \\
\text { ischaemic or haemorrhagic stroke exceeding } 3 \text { weeks, pre-existing cerebral vascular disease resulting } \\
\text { in functional deficits, unable to manage home visits, psychological conditions and deafness and/or } \\
\text { muteness" }\end{array}$ \\
\hline
\end{tabular}

Interventions $\quad$ (1) Treated group $(n=42)$

"Enrolled patients were classed by infarct cerebral accident (ICA) or hemorrhagic cerebral accident (HCA), and randomised into treated or control group"

\begin{abstract}
"From stroke onset to 1 month after stroke, during stage 1 rehabilitation, the patients in the treated group mainly had treatment in the Department of Neurology ward, and while being given usual medical treatment in the Department of Neurology, were given early bed side rehabilitation therapy once medically stable, and commenced rehabilitation therapy 1 week after the patient's symptoms stabilised; from the second month after stroke to the end of the third month after stroke (stage 2 rehabilitation), and from the fourth month after stroke to the end of the sixth month after stroke (stage 3 rehabilitation), patients were transferred to the rehabilitation ward/centre for continued treatment depending on the patient's condition and functional recovery status, or transferred to home, where therapists would guide and assist patients in undertaking the necessary functional training, until the end of follow-up. Here, 'stage one rehabilitation' refers to patient's early stage emergency visit to hospital or conventional medicine treatment as well as early stage rehabilitative treatment, 'stage two rehabilitation' refers to patient's rehabilitative treatment at the rehabilitation ward/centre, 'stage three rehabilitation' refers to the continuation of rehabilitative treatment within community or home setting"

"The rehabilitative treatment method combined physiotherapy (PT) and occupational therapy (OT) into a holistic method. Stage one rehabilitation included anti-spasticity positioning, passive training of limbs, active training of non-affected limbs under guidance, deep breathing as well as training of ab-
\end{abstract}


dominal muscles, sitting up from lying, sitting balance and standing up training etc, in order to train the patient's ability to get up from the bed, (treatment is done) 1 /day, $45 \mathrm{~min} / \mathrm{session}$, 5/week, during the training period, the patient's family or nursing workers were taught the correct supplementary exercises and methods of care concurrently, in order to achieve partial training out with therapy times, while also reducing the damage to affected limbs due to inappropriate nursing care; stage two rehabilitation mainly consisted of standing training, standing balance, single-leg standing, gait training and up-and-down stair training etc, in order to train the patient's ability to ambulate, (treatment is done) 2/day, $30-45 \mathrm{~min} / \mathrm{session}$, 5/week; stage three rehabilitation mainly consisted of: feeding, donning, grooming, personal hygiene management and other ADL ability training, (treatment is done) 2/day, 30 - $45 \mathrm{~min} / \mathrm{session}, 5$ - 7 days/week. Stage one early rehabilitation and stage two rehabilitation within rehabilitation ward/centre, was delivered by therapists, while concurrently teaching patient's family or nursing workers how to assist the patient in training; some patients during the second stage community rehabilitation had therapists conducting home visits to provide guidance on rehabilitation treatment 1 /week, teaching patient's family or nursing workers how to assist the patient in training while treating the patient, leaving them to assist the patient to complete the remaining bulk of the training in the week; during stage three community rehabilitation, therapists conducted fortnightly home visits, and while delivering occupational therapy and necessary physiotherapy, also taught the patient's family or nursing workers how to help the patient train, leaving them to assist the patient in completing the remaining daily necessary training"

The individual components delivered are listed in Table 3. Based on the individual components, this intervention is categorised as comprising functional task training, modality and musculoskeletal intervention (passive)

Length of intervention period: six months

Number of sessions and length of individual sessions: "stage one rehabilitation-1/day, $45 \mathrm{~min} / \mathrm{session}$, $5 \mathrm{x} /$ week; stage two rehabilitation-2/day, 30 - $45 \mathrm{~min} /$ session, $5 \mathrm{x} /$ week and stage three rehabilitation2/day, $30-45 \mathrm{~min} /$ session, $5-7$ days/week"

Intervention provider: "Stage one early rehabilitation and stage two rehabilitation within rehabilitation ward/centre, was delivered by therapists, while concurrently teaching patient's family or nursing workers how to assist the patient in training; some patients during the second stage community rehabilitation had therapists conducting home visits to provide guidance on rehabilitation treatment $1 x /$ week, teaching patient's family or nursing workers how to assist the patient in training while treating the patient, leaving them to assist the patient to complete the remaining bulk of the training in the week; during stage three community rehabilitation, therapists conducted fortnightly home visits, and while delivering occupational therapy and necessary physiotherapy, also taught the patient's family or nursing workers how to help the patient train, leaving them to assist the patient in completing the remaining daily necessary training"

(2) Control group $(n=40)$

"Patients in the control group were not given standardised rehabilitation therapy, but were given the same usual medical treatment as the treated group. It was noted that some patients self-trained after verbal advice from their doctor, while some patients' families assisted the patient in movement based on their own knowledge, and the possibility that some patients underwent certain rehabilitation treatment after seeking help from other rehabilitation organisations upon discharge cannot be excluded"

The individual components delivered are listed in Table 3. Based on the individual components, this intervention is categorised as comprising no treatment

Length of intervention period: not stated

Number of sessions and length of individual sessions: not stated

Intervention provider: not stated

This study is classified as intervention (functional task training, musculoskeletal (passive)) versus no treatment (Table 4). The intervention group also received modality 


Notes Original study translated from Chinese to English

No data suitable for analysis

\section{Risk of bias}

\begin{tabular}{|c|c|c|}
\hline Bias & Authors' judgement & Support for judgement \\
\hline $\begin{array}{l}\text { Random sequence genera- } \\
\text { tion (selection bias) }\end{array}$ & Low risk & Stratified randomisation \\
\hline $\begin{array}{l}\text { Allocation concealment } \\
\text { (selection bias) }\end{array}$ & Unclear risk & No information provided \\
\hline $\begin{array}{l}\text { Blinding of outcome as- } \\
\text { sessment (detection bias) } \\
\text { All outcomes }\end{array}$ & Low risk & $\begin{array}{l}\text { Outcome assessor did not deliver intervention and was blinded to group allo- } \\
\text { cation }\end{array}$ \\
\hline $\begin{array}{l}\text { Incomplete outcome data } \\
\text { (attrition bias) } \\
\text { All outcomes }\end{array}$ & Low risk & Dropouts accounted for (only three dropouts) \\
\hline $\begin{array}{l}\text { Free of systematic differ- } \\
\text { ences in baseline charac- } \\
\text { teristics of groups com- } \\
\text { pared? }\end{array}$ & Low risk & $\begin{array}{l}\text { No obvious difference between groups for time since stroke, gender, age, side } \\
\text { and type of stroke, etc }\end{array}$ \\
\hline $\begin{array}{l}\text { Did authors adjust for } \\
\text { baseline differences in } \\
\text { their analyses? }\end{array}$ & Low risk & No information provided \\
\hline Other bias & Unclear risk & $\begin{array}{l}\text { It was noted that some participants self-trained after receiving verbal advice } \\
\text { from their doctor, while some participants' families assisted the participant in } \\
\text { movement based on their own knowledge, and the possibility that some par- } \\
\text { ticipants underwent certain rehabilitation treatment after seeking help from } \\
\text { other rehabilitation organisations upon discharge cannot be excluded }\end{array}$ \\
\hline
\end{tabular}

Jing 2006

\section{Study characteristics}

\begin{tabular}{ll}
\hline Methods & Study design: RCT \\
& Method of randomisation: "Randomisation done using SPSS 12.0 software" \\
\hline Participants & Number of participants: $\mathrm{n}=160$ \\
& Inclusion criteria: "(1) Stroke diagnosis according to the evaluative standard revised by the 1995 Fourth \\
& National Academic Conference of Cerebral Vascular Disease; (2) confirmed by CT or MRI scan; (3) first \\
& ever stroke, within 3 months of stroke; (4) cognitively sound, able to cooperate, understood and agree- \\
able to intervention" & \\
& Exclusion criteria: "incomplete patient information, huge lapses in memory loss"
\end{tabular}

Interventions (1) Exercise and occupational therapy group ( $n=120)$


"For the exercise + occupational therapy participants, while following the principles of the intervention of the exercise therapy group, the following intervention was done: early rolling, donning, feeding, transfers etc and re-learning and practising ADL activities, focusing on activities of choice for therapy, focusing on the dexterity of affected limbs, through active and active-assisted means of training, and compensating with the non-affected limb training etc. Emphasised on activities with the largest ADL limitation. In the ward, patient's family and nurse supervised ADL activities, rendering as little assistance as possible. $45-60 \mathrm{~min} /$ day, one to one therapy session with an occupational therapist"

The individual components delivered are listed in Table 3. Based on the individual components, this intervention is categorised as comprising functional task training, musculoskeletal intervention (active), musculoskeletal intervention (passive) and neurophysiological intervention

Length of intervention period: not stated

Number of sessions and length of individual sessions: 45 to 60 minutes/d

Intervention provider: occupational therapist

(2) Exercise therapy group $(n=40)$

"For the exercise therapy participants, after becoming medically stable, while concurrently receiving conventional treatment, had the following intervention: positioning of the unaffected limbs, passive ranging exercises of the joints, bridging exercises, neuromuscular facilitation technique, sitting balance, standing balance and gait training etc"

The individual components delivered are listed in Table 3. Based on the individual components, this intervention is categorised as comprising functional task training, musculoskeletal intervention (passive) and neurophysiological intervention

Length of intervention period: on average seven weeks

Number of sessions and length of individual sessions: 40 to 50 minutes/d

Intervention provider: one-to-one therapy session with an exercise therapist

This study is classified as active intervention one (functional task training, musculoskeletal (active), musculoskeletal (passive), neurophysiological) versus active intervention two (functional task training, musculoskeletal (passive), neurophysiological) (Table 6)

\begin{tabular}{|c|c|}
\hline \multirow[t]{3}{*}{ Outcomes } & Measures of Independence in ADL: Barthel Index \\
\hline & Measures of motor function: Fugl-Meyer Assessment \\
\hline & $\begin{array}{l}\text { Time points when outcomes were assessed: within } 24 \text { hours of commencement of therapy, every two } \\
\text { weeks thereafter }\end{array}$ \\
\hline \multirow[t]{2}{*}{ Notes } & Original study translated from Chinese to English \\
\hline & $\begin{array}{l}\text { As the two active treatment groups were classified as including similar treatment components, data } \\
\text { from this study have not been included within the comparisons of one active intervention versus an- } \\
\text { other active intervention }\end{array}$ \\
\hline
\end{tabular}

\section{Risk of bias}

Authors' judgement Support for judgement

Random sequence genera- Unclear risk tion (selection bias)
"Random number generation using SPSS 12.0 software." No reason is provided for the unequal distribution between groups (120 and 40)

$\begin{array}{lll}\begin{array}{l}\text { Allocation concealment } \\ \text { (selection bias) }\end{array} & \text { Unclear risk } & \text { No information provided }\end{array}$

(selection bias) 
Jing 2006 (Continued)

Blinding of outcome as- $\quad$ Low risk $\quad$ Blind assessment
sessment (detection bias)

All outcomes

Incomplete outcome data Unclear risk No dropouts reported
(attrition bias)
All outcomes

All outcomes

\begin{tabular}{|c|c|c|}
\hline Free of systematic differ- & Low risk & $\begin{array}{l}\text { No obvious difference between groups for time since stroke, gender, age, side } \\
\text { and type of stroke }\end{array}$ \\
\hline
\end{tabular}
teristics of groups compared?

\begin{tabular}{lll}
\hline $\begin{array}{l}\text { Did authors adjust for } \\
\text { baseline differences in } \\
\text { their analyses? }\end{array}$ & Low risk & No information provided \\
\hline Other bias & Unclear risk & No information provided \\
\hline
\end{tabular}

Kim 2011

\section{Study characteristics}

\begin{tabular}{ll}
\hline Methods & Study design: RCT \\
& Method of randomisation: not stated \\
& "0 patients diagnosed with hemiplegia due to stroke were randomly assigned" \\
\hline Participants & Number of participants: $\mathrm{n}=40$ \\
& $\begin{array}{l}\text { Inclusion criteria: "The study subjects were selected from among patients diagnosed with stroke who } \\
\text { could walk by themselves without being helped by others or could walk at least } 10 \text { m using a walk- } \\
\text { ing aid, scored at least } 24 \text { points in the mini-mental state examination-K (MMSE-K), had spasticity of } \\
\text { Grade } 2 \text { or lower in the affected lower extremity as evaluated by the Modified Ashworth Scale (MAS), } \\
\text { had no orthopaedic problem that could affect the treatment, and could receive training for } 30 \text { minutes } \\
\text { or longer" }\end{array}$
\end{tabular}

Interventions
(1) PNF group $(n=20)$
"Trunk stability exercise using proprioceptive neuromuscular facilitation (PNF)"
"The experimental group received therapeutic intervention of PNF using SR and RS for 10 minutes dur- ing 30 minutes of general therapeutic exercise, implemented five times a week for six weeks"
"The PNF provided to the experimental group was implemented after the exercise programs were ex- plained and demonstrated by professionally trained therapists so that the subjects would sufficiently understand the exercise programs"

The individual components delivered are listed in Table 3. Based on the individual components, this intervention is categorised as comprising musculoskeletal (active), musculoskeletal (passive) and neurophysiological intervention

Length of intervention period: six weeks

Number of sessions and length of individual sessions: "intervention of PNF using SR (stabilising reversal) and RS (rhythmic stabilisation) for 10 minutes during 30 minutes of general therapeutic exercise, implemented five times a week" 
Kim 2011 (Continued)

Intervention provider: "professionally trained therapists"

(2) Control group $(n=20)$

"The control group received only general therapeutic exercise for 30 minutes, five times a week for six weeks. The general therapeutic exercise was composed of stretching exercises and exercises for the range of motion of joints"

The individual components delivered are listed in Table 3. Based on the individual components, this intervention is categorised as comprising musculoskeletal intervention (active) and musculoskeletal intervention (passive)

Length of intervention period: six weeks

Number of sessions and length of individual sessions: 30 minutes, five times a week

Intervention provider: not stated

This study is classified as active intervention one (musculoskeletal (active), musculoskeletal (passive), neurophysiological) versus active intervention two (musculoskeletal (active), musculoskeletal (passive)) (Table 6)

Outcomes

Measures of postural control and balance: Functional Reach Test (FRT)

Other secondary outcome measures: EMG measures of four muscles (soleus, tibialis anterior, hamstring, quadriceps)

Time points when outcomes were assessed: "pre- and post-experiment measurements were made of the FRT"

Notes

\section{Risk of bias}

Bias Authors' judgement Support for judgement

\begin{tabular}{lll}
\hline Random sequence genera- & Unclear risk & No information provided \\
tion (selection bias) &
\end{tabular}

\begin{tabular}{lll}
\hline $\begin{array}{l}\text { Allocation concealment } \\
\text { (selection bias) }\end{array}$ & Unclear risk & No information provided
\end{tabular}
(selection bias)

$\begin{array}{lll}\text { Blinding of outcome as- } & \text { Unclear risk } & \text { No information provided } \\ \text { sessment (detection bias) } & \end{array}$
sessment (detection bias)

All outcomes

\begin{tabular}{ll}
\hline Incomplete outcome data Unclear risk & No dropouts reported \\
(attrition bias) &
\end{tabular}

All outcomes

Free of systematic differ- Low risk Baseline demographics comparable
ences in baseline charac-
teristics of groups com-
pared?

\begin{tabular}{lll}
\hline $\begin{array}{l}\text { Did authors adjust for } \\
\text { baseline differences in } \\
\text { their analyses? }\end{array}$ & Low risk & \\
\hline Other bias & Unclear risk & No information provided \\
\hline
\end{tabular}




\section{Study characteristics}

$\begin{array}{ll}\text { Methods } & \text { Study design: RCT } \\ \text { Method of randomisation: not stated ("participants were randomly assigned to two groups") }\end{array}$

Participants Number of participants: $n=20$

Inclusion criteria: "ability to walk $10 \mathrm{~m}$ independently using an aid or orthotic with or without supervision or aid, and minimum score of 20 in the Korean Mini-Mental State Examination (K-MMSE)"

Exclusion criteria: "joint contraction, pain or fracture of the musculoskeletal system, and hemianopsia"

Interventions
(1) Experimental group $(n=10)$

"Subjects in both groups underwent conservative physical therapy"

"The experimental group also participated in task-oriented training for 1 hour per day, 3 days a week, for 4 weeks. The training consists of 10 walking-related tasks designed to strengthen the lower extremities, and enhance the walking balance, speed and distance in a progressive manner. The 10 tasks were (i) step-ups, (ii) balance beam, (iii) kicking a ball, (iv) stand up and walk, (v) obstacle course, (vi) treadmill, (vii) walk and carry, (viii) speed walk, (ix) walk backwards, and (x) stairs. Before commencing training, the subjects warmed up for 5 minutes to improve their range of motion and flexibility. Each item was practiced for 5 minutes, and 1 minute of rest time was allowed between each item"

The individual components delivered are listed in Table 3. Based on the individual components, this intervention is categorised as comprising functional task training, musculoskeletal intervention (active) and musculoskeletal intervention (passive)

Length of intervention period: four weeks

Number of sessions and length of individual sessions: conservative physical training for one hour per day, five days a week. in addition to task-oriented training for one hour per day, three days per week

Intervention provider: "supervised by a physical or occupational therapist"

(2) Control group $(n=10)$

"Conservative physical therapy consisted of joint mobilization, muscle strengthening, and balance training"

The individual components delivered are listed in Table 3. Based on the individual components, this intervention is categorised as comprising usual care (functional task training, musculoskeletal intervention (active) and musculoskeletal intervention (passive))

Length of intervention period: four weeks

Number of sessions and length of individual sessions: conservative physical training for one hour per day, five days a week

Intervention provider: not stated

This study is classified as intervention (functional task training, musculoskeletal (active), musculoskeletal (passive)) versus usual care (functional task training, musculoskeletal (active), musculoskeletal (passive)) (Table 5)

Outcomes Measures of postural control and balance: Berg Balance Scale, Timed-Up and Go test (TUG),Trunk Impairment Scale

Measures of voluntary movements: 10-Metre Walk test 
Kim 2012 (Continued)

Time points when outcomes were assessed: "measured before and after the 4 weeks of therapy"

Notes

\section{Risk of bias}

\begin{tabular}{|c|c|c|}
\hline Bias & Authors' judgement & Support for judgement \\
\hline $\begin{array}{l}\text { Random sequence genera- } \\
\text { tion (selection bias) }\end{array}$ & Unclear risk & "participants were randomly assigned to two groups" \\
\hline $\begin{array}{l}\text { Allocation concealment } \\
\text { (selection bias) }\end{array}$ & Unclear risk & No information provided \\
\hline $\begin{array}{l}\text { Blinding of outcome as- } \\
\text { sessment (detection bias) } \\
\text { All outcomes }\end{array}$ & Unclear risk & No information provided \\
\hline $\begin{array}{l}\text { Incomplete outcome data } \\
\text { (attrition bias) } \\
\text { All outcomes }\end{array}$ & Unclear risk & No dropouts reported \\
\hline $\begin{array}{l}\text { Free of systematic differ- } \\
\text { ences in baseline charac- } \\
\text { teristics of groups com- } \\
\text { pared? }\end{array}$ & Unclear risk & $\begin{array}{l}\text { Limited baseline demographics } \\
\text { Pretraining outcome measures similar across groups except on the TUG test }\end{array}$ \\
\hline $\begin{array}{l}\text { Did authors adjust for } \\
\text { baseline differences in } \\
\text { their analyses? }\end{array}$ & Unclear risk & No information provided \\
\hline Other bias & High risk & $\begin{array}{l}\text { Although both groups received active interventions, the dose was substantial- } \\
\text { ly less for the second group }\end{array}$ \\
\hline
\end{tabular}

Kwakkel 2002

\section{Study characteristics}

Methods Study design: RCT with three treatment groups

Method of randomisation: "within the first 14 days poststroke, patients were randomly assigned to one of the 3 treatment conditions. Randomisation (permuted blocks of 9), with random number tables for every participating hospital, was applied"

Pumber of participants: $\mathrm{n}=53$
Inclusion criteria: "Primary, first-ever stroke in the territory of the middle cerebral artery as revealed
by CT or MRI, aged between 30 to 80 years, impaired lower extremities (LE) and upper extremities (UE)
motor function as assessed with the Motricity Index (i.e. scores < 100 points for each paretic limb), un-
able to walk without assistance on admission, no complicating medical history on the basis of review
of medical records such as cardiac, pulmonary or neurological disorders. No severe deficits in commu-
nication, memory, or understanding and gave written or verbal informed consent and were sufficiently
motivated to participate"

Interventions $\quad$ (1) Lower extremities (LE) group $(n=17)$ 
"focused on the recovery of tasks such as turning over and maintaining sitting and standing balance. In addition, the LE intervention was designed to improve the symmetry in interlimb coordination during walking"

"The guidelines were based on evidence-based practice patterns derived from findings reported in 165 intervention studies in the field of stroke rehabilitation. We used what we believe is an eclectic approach based on research indicating that subjects' practice of motor skills needs to be both task and context specific"

The individual components delivered are listed in Table 3. Based on the individual components, this intervention is categorised as comprising functional task training

Length of intervention period: for a period of 20 weeks post stroke ("from week 20 onward, type of treatment and its duration [were] determined by the physical therapists and occupational therapists involved, on average 3 times half an hour a week")

Number of sessions and length of individual sessions: 30 minutes of LE training, five days a week. In addition, all three groups participated daily in a basic treatment programme of 15 minutes of LE exercises and 15 minutes of UE exercises, as well as a weekly 90-minute session of ADL training administered by an occupational therapist

Intervention provider: physical therapists and occupational therapists

(2) Upper extremities (UE) group $(n=18)$

"focused on the improvement of grasping, reaching, leaning, and dressing and hair combing"

The individual components delivered are listed in Table 3. Based on the individual components, this intervention is categorised as comprising upper limb training

Length of intervention period: for a period of 20 weeks post stroke ("from week 20 onward, type of treatment and its duration [were] determined by the physical therapists and occupational therapists involved, on average 3 times half an hour a week")

Number of sessions and length of individual sessions: 30 minutes of LE training, five days a week. In addition, all three groups participated daily in a basic treatment programme of 15 minutes of LE exercises and 15 minutes of UE exercises, as well as a weekly 90 -minute session of ADL training administered by an occupational therapist

Intervention provider: physical therapists and occupational therapists

(3) Control group $(n=18)$

"Immobilisation of the paretic LE and UE by means of an inflatable pressure splint, which was applied for 30 minutes in a lying position, 5 days a week"

The individual components delivered are listed in Table 3. Based on the individual components, this intervention is categorised as comprising no treatment

Length of intervention period: for a period of 20 weeks post stroke ("from week 20 onward, type of treatment and its duration [were] determined by the physical therapists and occupational therapists involved, on average 3 times half an hour a week")

Number of sessions and length of individual sessions: 30 minutes of LE training, five days a week. In addition, all three groups participated daily in a basic treatment programme of 15 minutes of LE exercises and 15 minutes of UE exercises, as well as a weekly 90 -minute session of ADL training administered by an occupational therapist

Intervention provider: physical therapists and occupational therapists

This study is classified as intervention (functional task training) versus attention control (upper limb) (Table 5) and intervention (functional task training) versus no treatment (Table 4) 
Kwakkel 2002 (Continued)

Other secondary outcome measures: mean continuous relative phase

Time points when outcomes were assessed: "patients were assessed during the first 10 weeks on a weekly basis and biweekly from week 10 to week 20 . With exception of kinematic measurements, final assessment took place at 26 weeks poststroke"

Notes

Intervention two comprised upper limb training. Although upper limb training might be classed as a subcomponent of functional task training, for the purposes of comparisons in this review we have categorised this intervention as an attention control. This is because the upper limb training was delivered alone (i.e. no other subcomponents of functional task training were delivered), and therefore no active treatment was aimed at lower limb or balance outcomes

No outcomes included in analysis because it was unclear at which time points the data were collected

\section{Risk of bias}

\begin{tabular}{|c|c|c|}
\hline Bias & Authors' judgement & Support for judgement \\
\hline $\begin{array}{l}\text { Random sequence genera- } \\
\text { tion (selection bias) }\end{array}$ & Low risk & $\begin{array}{l}\text { "Randomisation (permuted blocks of 9), with random number tables for every } \\
\text { participating hospital, was applied" }\end{array}$ \\
\hline $\begin{array}{l}\text { Allocation concealment } \\
\text { (selection bias) }\end{array}$ & Low risk & "Concealed allocation was done by the use of sealed envelopes" \\
\hline $\begin{array}{l}\text { Blinding of outcome as- } \\
\text { sessment (detection bias) } \\
\text { All outcomes }\end{array}$ & Low risk & $\begin{array}{l}\text { All measurements were carried out by an independent observer who had more } \\
\text { than } 15 \text { years of experience in the use of these measurement instruments }\end{array}$ \\
\hline $\begin{array}{l}\text { Incomplete outcome data } \\
\text { (attrition bias) } \\
\text { All outcomes }\end{array}$ & Low risk & No dropouts \\
\hline $\begin{array}{l}\text { Free of systematic differ- } \\
\text { ences in baseline charac- } \\
\text { teristics of groups com- } \\
\text { pared? }\end{array}$ & Low risk & $\begin{array}{l}\text { "No differences in subject characteristics were found amongst the } 3 \text { treatment } \\
\text { groups at either the time of onset or the first gait assessment" }\end{array}$ \\
\hline $\begin{array}{l}\text { Did authors adjust for } \\
\text { baseline differences in } \\
\text { their analyses? }\end{array}$ & Low risk & No systematic differences \\
\hline Other bias & Unclear risk & No information provided \\
\hline
\end{tabular}

Kwakkel 2008

\section{Study characteristics}

Methods

\author{
Study design: RCT
}

Method of randomisation: "Participants were stratified by rehabilitation centre, and randomisation took place using an 'online' minimisation procedure"
Number of participants: $\mathrm{n}=250$

Inclusion criteria: verified stroke according to the WHO definition, able to walk a minimum of $10 \mathrm{~m}$ without physical assistance (functional ambulation categories $\geq$ three), discharged home from a rehabilitation centre, needed to continue physiotherapy during outpatient care to improve walking compe- 
tency or physical condition, or both, able to give informed consent and be motivated to participate in a 12-week intensive programme of physiotherapy

Exclusion criteria: cognitive deficits as evaluated by the mini-mental state examination ( $<24$ points), unable to communicate (< four points on the Utrechts Communicatie Onderzoek, UCO) and lived farther than $30 \mathrm{~km}$ from the rehabilitation centre

Interventions

Outcomes
(1) Circuit training $(n=126)$

"Graded task oriented circuit training programme ('warming up (5 minutes), circuit training (60 minutes), evaluation and a short break (10 minutes), and group game (15 minutes)') twice a week over a 12 week period ( 24 sessions)"

"Training included eight different workstations, intended to improve meaningful tasks relating to walking competency"

The individual components delivered are listed in Table 3. Based on the individual components, this intervention is categorised as comprising functional task training and musculoskeletal intervention (active)

Length of intervention period: 12 weeks

Number of sessions and length of individual sessions: "90 minute session twice a week (24 sessions). The circuit training group received 4461 treatment sessions, average treatment time per session was 72 (SD 39) minutes"

Intervention provider: physiotherapist and sports therapist

(2) Usual physiotherapy $(n=124)$

"Patients allocated to the control group received usual outpatient physiotherapy, mainly one to one treatments tailored to the patient with a physiotherapist who had not been on the circuit training course at one of the participating rehabilitation centres. Sessions designed to improve control of standing balance, physical condition, and walking competency were provided according to Dutch physiotherapy guidelines"

The individual components delivered are listed in Table 3. Based on the individual components, this intervention is categorised as comprising usual care (cardiopulmonary intervention, functional task training and musculoskeletal intervention (active))

Length of intervention period: not stated

Number of sessions and length of individual sessions: "no additional restrictions with respect to content, time, or duration of the physiotherapy. The group received 4378 with an average of 34 (SD 10) minutes per session"

Intervention provider: physiotherapist

This study is classified as intervention (functional task training, musculoskeletal (active)) versus usual care (functional task training, musculoskeletal (active)) (Table 5). Both groups received cardiopulmonary intervention

Measures of motor function: Rivermead Mobility Index (RMI)

Measures of postural control and balance: timed balance test, Timed Up and Go, modified stairs test

Measures of voluntary movements: Six-Minute Walk test, five-metre comfortable walking speed test, functional ambulation categories

Measures of participation: Nottingham extended activities of daily living (NEADL)

Other secondary outcome measures: Stroke Impact Scale, Falls Efficacy Scale (FES), Hospital Anxiety and Depression Scale (HADS), Fatigue Severity Scale (FSS), Letter cancellation task, the Motricity index (MI-arm and MI-leg) 
Time points when outcomes were assessed: "measured all outcomes before randomisation at baseline and after 12 and 24 weeks"

Notes

Full details of this trial, called FIT-Stroke, have been reported elsewhere (see Van de Port, I, Wevers L, Roelse H, van Kats L, Lindeman E, Kwakkel G. Cost-effectiveness of a structured progressive task-oriented circuit class training programme to enhance walking competency after stroke: the protocol of the FIT-Stroke trial. BMC Neurol 2009;9:43, for more details on trial methodology and randomisation)

\section{Risk of bias}

\begin{tabular}{lll}
\hline Bias & Authors' judgement & Support for judgement \\
\hline $\begin{array}{l}\text { Random sequence genera- } \\
\text { tion (selection bias) }\end{array}$ & Unclear risk & $\begin{array}{l}\text { "Patients were randomly allocated to circuit training or usual physiotherapy, } \\
\text { after stratification by rehabilitation centre, with an online randomisation pro- } \\
\text { cedure" }\end{array}$ \\
\hline $\begin{array}{l}\text { Allocation concealment } \\
\text { (selection bias) }\end{array}$ & Unclear risk & Not stated \\
\hline
\end{tabular}

\begin{tabular}{|c|c|c|}
\hline $\begin{array}{l}\text { Blinding of outcome as- } \\
\text { sessment (detection bias) } \\
\text { All outcomes }\end{array}$ & Low risk & $\begin{array}{l}\text { "Three trained research assistants (LW, HK, LK), who were blinded to treat- } \\
\text { ment allocation, measured all outcomes before randomisation at baseline and } \\
\text { after } 12 \text { and } 24 \text { weeks in face to face meetings at the patient's own home or at } \\
\text { the rehabilitation centre" }\end{array}$ \\
\hline
\end{tabular}

$\begin{array}{ll}\begin{array}{l}\text { Incomplete outcome data Low risk } \\ \text { (attrition bias) }\end{array} & \text { Dropouts accounted for } \\ \text { All outcomes } & \text { "Of the } 250 \text { included patients, one patient in the circuit training group and } \\ & \text { seven in the usual care group were excluded from the analysis. Reasons were } \\ & \text { withdrawal from participation }(\mathrm{n}=3) \text {, death from cancer }(\mathrm{n}=2) \text {, and recurrent } \\ & \text { stroke }(\mathrm{n}=2), \text { while one patient missed the } 12 \text { week assessment visit because of } \\ & \text { change of address. No patients were lost to follow-up after } 12 \text { weeks" }\end{array}$

\begin{tabular}{lll}
\hline $\begin{array}{l}\text { Free of systematic differ- } \\
\text { ences in baseline charac- }\end{array}$ & Unclear risk & $\begin{array}{l}\text { Significant baseline differences in favour of the circuit training group for a few } \\
\text { secondary outcomes }\end{array}$
\end{tabular}
teristics of groups com pared?

\begin{tabular}{ll}
\hline $\begin{array}{l}\text { Did authors adjust for } \\
\text { baseline differences in } \\
\text { their analyses? }\end{array}$ & Low risk \\
\hline Other bias & $\begin{array}{l}\text { Significant baseline differences in favour of the circuit training group for a few } \\
\text { secondary outcomes. All analyses, however, were adjusted for these co-vari- } \\
\text { ates at baseline }\end{array}$ \\
& $\begin{array}{l}\text { "Patients with only mild to moderate stroke were selected, which limits the } \\
\text { generalisability of our trial. Able to recruit only a quarter of all patients who } \\
\text { were discharged from one of the participating rehabilitation centres. These } \\
\text { centres receive about 10\% of all patients with stroke discharged from hospi- } \\
\text { tals in the Netherlands. About a third of all patients discharged from these re- } \\
\text { habilitation centres showed no or insufficient problems with walking and an- } \\
\text { other third were too ill to be included in the present study" } \\
\text { "The combination of workstations in FIT-Stroke represents an arbitrary selec- } \\
\text { tion. Our workstations were selected for safety, clinical relevance in terms of } \\
\text { activities, simplicity of execution, and feasibility, without additional costs to } \\
\text { the physiotherapy department. Unclear whether a different combination of } \\
\text { workstations would have resulted in other outcomes. In our opinion, the work- } \\
\text { stations for task oriented circuit training should at least be task specific, inten- } \\
\text { sive, and graded in time" }\end{array}$ \\
\end{tabular}


Langhammer 2000

\section{Study characteristics}

\begin{tabular}{ll}
\hline Study design: RCT \\
Method of randomisation: double-blind randomisation (stratified according to sex and side of lesion) \\
and sealed coding
\end{tabular}

\begin{tabular}{ll}
\hline Participants & Number of participants: $\mathrm{n}=61$ \\
Inclusion criteria: first stroke, verified clinically and by CT scan, no subarachnoid bleeding, no tumours, \\
no severe medical conditions and not more than four points on each MAS section
\end{tabular}

Interventions

(1) Neurophysiological (Bobath) $(n=28)$

A "theoretical framework in a reflex-hierarchical theory"

Physiotherapists attended workshops and discussed the treatment approaches and were provided with a manual, based on the supporting texts, which described the key philosophy of the approaches

Techniques were not described

The individual components delivered are listed in Table 3. Based on the individual components, this intervention is categorised as comprising neurophysiological intervention

Length of intervention period: "as long as they were hospitalized"

Number of sessions and length of individual sessions: five days/wk for a minimum of 40 minutes, while an inpatient. Folowing discharge, attempts were made to ensure that physiotherapy continued based on the assigned approach, and physiotherapists involved in the treatment were able to discuss treatments with hospital physiotherapists and project leaders

Intervention provider: physiotherapists

(2) Motor learning $(n=33)$

"Based in system theory, and is basically task-oriented"

Physiotherapists attended workshops and discussed the treatment approaches and were provided with a manual, based on the supporting texts, that described the key philosophy of the approaches

Techniques were not described

The individual components delivered are listed in Table 3. Based on the individual components, this intervention is categorised as comprising functional task training

Length of intervention period: "as long as they were hospitalized"

Number of sessions and length of individual sessions: as above

Intervention provider: physiotherapists

This study is classified as active intervention one (neurophysiological) versus active intervention two (functional task training) (Table 6)

Outcomes
Measures of Independence in ADL: Barthel Index
Measures of functional independence: MAS; Sodring Motor Evaluation
Other secondary outcome measures: Nottingham Health Profile
Other secondary outcome measures: length of stay, use of assistive devices, discharge destination
Time points when outcomes were assessed: "patients were tested three times: (1) three days after ad- mission to the hospital, (2) two weeks thereafter, and (3) three months post stroke" 
Langhammer 2000 (Continued)

Risk of bias

\begin{tabular}{lll}
\hline Bias & Authors' judgement & Support for judgement \\
\hline $\begin{array}{l}\text { Random sequence genera- } \\
\text { tion (selection bias) }\end{array}$ & Low risk & $\begin{array}{l}\text { Double-blind randomisation (stratified according to sex and side of lesion) and } \\
\text { sealed coding }\end{array}$ \\
\hline $\begin{array}{l}\text { Allocation concealment } \\
\text { (selection bias) }\end{array}$ & Low risk & $\begin{array}{l}\text { "The study was double blind, and the code was sealed until the last test was } \\
\text { performed at three months follow-up" }\end{array}$
\end{tabular}

Blinding of outcome as- Low risk

sessment (detection bias)

All outcomes

\section{Unclear whether the participant was blinded}

Therapist was not blinded ("Information concerning the physiotherapy used was known only by the therapists who treated the patients and the secretary of the ward, who was in charge of the randomization")

Assessor was blinded ("The tests were conducted by the project leader who had no information about which group the patient belonged to")

\begin{tabular}{|c|c|c|}
\hline $\begin{array}{l}\text { Incomplete outcome data } \\
\text { (attrition bias) } \\
\text { All outcomes }\end{array}$ & Low risk & $\begin{array}{l}\text { Dropouts accounted for } \\
29 / 33 \text { in motor learning group and } 24 / 28 \text { in Bobath group completed interven- } \\
\text { tion }\end{array}$ \\
\hline $\begin{array}{l}\text { Free of systematic differ- } \\
\text { ences in baseline charac- } \\
\text { teristics of groups com- } \\
\text { pared? }\end{array}$ & Low risk & $\begin{array}{l}\text { "Mean age of the patients was } 78 \text { years (range } 49-95 \text { years, SD 9), with no sig- } \\
\text { nificant difference between the two groups. Marital status was also similar" } \\
\text { "There were no significant differences in MAS, SMES or Barthel ADL Index be- } \\
\text { tween the two groups in the acute stage" }\end{array}$ \\
\hline $\begin{array}{l}\text { Did authors adjust for } \\
\text { baseline differences in } \\
\text { their analyses? }\end{array}$ & Low risk & No information provided \\
\hline Other bias & Unclear risk & $\begin{array}{l}\text { The same therapists provided treatment to participants in both treatment } \\
\text { groups, creating the possibility of contamination between groups. Treatment } \\
\text { following hospital discharge may not have been administered according to the } \\
\text { randomisation process, potentially introducing performance bias to the post- } \\
\text { discharge results }\end{array}$ \\
\hline
\end{tabular}

\section{Study characteristics}

Methods
Study design: "longitudinal randomized controlled stratified trial"
Method of randomisation: "At discharge from the acute hospital, patients were randomized to one of
two different groups by a person not involved with the patients or the treatment in the ward. Random-
ization was performed with a die: patients with uneven numbers went to group 1, an intensive exercise
group, and those with even numbers to group 2, a regular exercise group. Stratification was accord-
ing to gender and hemisphere lesion: the first male patient with a right hemisphere lesion and with an
uneven number was allocated to the intensive exercise group, and the next male patient with a right
hemisphere lesion was allocated to the regular exercise group. The procedure with the die was then
used when the third male patient with a right hemisphere lesion entered the stroke unit and so on. A
corresponding procedure was followed for female patients"

Participants Number of participants: $n=75$


Langhammer 2007 (Continued)

Inclusion criteria: "Inclusion criteria were first-time-ever stroke with neurological signs, computer tomography-confirmed stroke and voluntary participation"

Exclusion criteria: "more than one stroke incident, subarachnoid bleeding, tumour, other serious illness, and brainstem or cerebellar stroke"

Interventions

(1) Intensive exercise $(n=35)$

"The subsequent training for the intensive exercise group included a functional exercise programme with emphasis on high intensity of endurance, strength and balance. The individualized training programmes were aimed at functional improvements but with variations, for example: getting up from a chair, walking indoors, Nordic walking outdoors, stationary bicycling, and stair walking, where the physiotherapist monitored the levels of intensity through Borg's Scale or through the pulse rate. A protocol with suggestions of types of exercises and levels of intensity was developed in discussion with all physiotherapists involved. This protocol was intended as a guideline. The goal of these exercises was to improve and maintain motor function, activities of daily living and grip strength. Patients in the intensive exercise group were also encouraged to maintain a high activity level apart from that in the training sessions"

The individual components delivered are listed in Table 3. Based on the individual components, this intervention is categorised as comprising cardiopulmonary intervention, functional task training and musculoskeletal intervention (active)

Length of intervention period: 12 months

Number of sessions and length of individual sessions: "Arrangements were made for patients allocated to the intensive exercise group to have physiotherapy during four periods, with a minimum of 20 hours every third month, in the first year after the stroke. The intervention sessions started immediately after discharge, two or three times a week if the patient was at home or attending a private physiotherapy practice, and daily if he or she was in a rehabilitation ward. This intervention was repeated after three months, six months and one year"

Intervention provider: physiotherapists

(2) Regular exercise $(n=40)$

"If the patients in the regular exercise group were considered to be in need of follow-up treatment or rehabilitation they were assigned to that, but not on a regular basis. No specific treatment was recommended to this group. On the other hand, the same encouragement to maintain a high activity level besides the training, if any, was given to the regular exercise group"

The individual components delivered are listed in Table 3. Based on the individual components, this intervention is categorised as comprising usual care

Length of intervention period: 12 months

Number of sessions and length of individual sessions: "The regular exercise group patients were given follow-up treatment according to their needs, as considered by the rehabilitation staff at the stroke unit/rehabilitation department and by the rehabilitation team in the community after discharge"

Intervention provider: physiotherapists

This study is classified as active intervention one (functional task training, musculoskeletal (active)) versus usual care (Table 5). The intervention group also received cardiopulmonary intervention

Outcomes

Measures of independence in ADL: Barthel index

Measures of motor function: motor assessment scale

Other secondary outcome measures: grip strength 
Langhammer 2007 (Continued)

Time points when outcomes were assessed: "patients were tested on admission, at discharge, and three months, six months and one year after stroke by an experienced investigator, blinded to group allocation"

Notes

During the acute phase of rehabilitation at the hospital, both groups received functional task-oriented training tailored to their specific needs. The amount of training was equal in the two groups, with two periods per day, the two periods comprising a total of one hour of physiotherapy in combination with other specialised therapies according to the participant's needs. At discharge, participants were randomly assigned to two separate groups-an intensive exercise group and a regular exercise group, as described above

\section{Risk of bias}

Bias Authors' judgement Support for judgement

Random sequence genera- Low risk tion (selection bias)
"Randomization was performed with a die: patients with uneven numbers went to group 1, an intensive exercise group, and those with even numbers to group 2, a regular exercise group. Stratification was according to gender and hemisphere lesion: the first male patient with a right hemisphere lesion and with an uneven number was allocated to the intensive exercise group, and the next male patient with a right hemisphere lesion was allocated to the regular exercise group. The procedure with the die was then used when the third male patient with a right hemisphere lesion entered the stroke unit and so on. A corresponding procedure was followed for female patients"

Allocation concealment Low risk
(selection bias)
(selection bias)
"At discharge from the acute hospital, patients were randomized to one of two different groups by a person not involved with the patients or the treatment in the ward"

\begin{tabular}{|c|c|c|}
\hline $\begin{array}{l}\text { Blinding of outcome as- } \\
\text { sessment (detection bias) } \\
\text { All outcomes }\end{array}$ & Low risk & $\begin{array}{l}\text { The protocol was sealed for } 1.5 \text { years from the start of the study until the last } \\
\text { included participant was tested at one year of follow-up. The study was an in- } \\
\text { tention-to-treat trial with the aim of being double-blind, that is, neither the in- } \\
\text { vestigator nor the participants knew to which group participants were allocat- } \\
\text { ed }\end{array}$ \\
\hline
\end{tabular}

\begin{tabular}{ll}
\hline $\begin{array}{l}\text { Incomplete outcome data } \\
\text { (attrition bias) }\end{array}$ & Low risk \\
$\begin{array}{l}\text { All outcomes } \\
\text { Of these } 75 \text { initially included in the study, four died and four withdrew during } \\
\text { the acute stage }\end{array}$
\end{tabular}

\begin{tabular}{lll}
\hline $\begin{array}{l}\text { Free of systematic differ- } \\
\text { ences in baseline charac- } \\
\text { teristics of groups com- } \\
\text { pared? }\end{array}$ & Low risk & $\begin{array}{l}\text { "no significant differences between the groups regarding age, hemisphere le- } \\
\text { sion, marital status at baseline, or admission to the stroke unit" }\end{array}$ \\
\hline $\begin{array}{l}\text { Did authors adjust for } \\
\text { baseline differences in } \\
\text { their analyses? }\end{array}$ & Unclear risk & No information provided \\
\hline Other bias & Unclear risk & No information provided \\
\hline
\end{tabular}

\section{Lennon 2006}

\section{Study characteristics}

Methods Study design: RCT


Lennon 2006 (Continued)

Method of randomisation: "after giving informed consent, patients were randomised using sealed envelopes"

\begin{tabular}{ll}
\hline Participants & Number of participants: $\mathrm{n}=61$ \\
Inclusion criteria: "All consecutive patients within 8 to 14 days poststroke were included if medically \\
stable with a first-ever stroke and they were able to stand or walk with assistance"
\end{tabular}

Interventions

(1) Bobath group $(n=30)$

"Conventional Bobath therapy"

The individual components delivered are listed in Table 3. Based on the individual components, this intervention is categorised as comprising neurophysiological intervention

Length of intervention period: four weeks

Number of sessions and length of individual sessions: five sessions/wk (total of 20 sessions) lasting 40 minutes

Intervention provider: not stated

(2) Gait-specific group $(\mathrm{n}=31)$

"introducing more walking practice into Bobath therapy (spending $50 \%$ of the daily physiotherapy session working on walking practice)"

The individual components delivered are listed in Table 3. Based on the individual components, this intervention is categorised as comprising functional task training and neurophysiological intervention

Length of intervention period: four weeks

Number of sessions and length of individual sessions: five sessions/wk (total of 20 sessions) lasting 40 minutes

Intervention provider: not stated

This study is classified as active intervention one (neurophysiological) versus active intervention two (functional task training, neurophysiological) (Table 6)

$\begin{array}{ll}\text { Outcomes } & \text { Measures of Independence in ADL: Barthel Index } \\ & \text { Measures of motor function: Motor Assessment Scale, modified Rivermead Mobility Index } \\ & \text { Measures of postural control and balance: Step test } \\ & \text { Measures of voluntary movements: 10-Metre Walk test } \\ & \text { Time points when outcomes were assessed: "All measures were performed by a blinded assessor at } \\ \text { baseline, post intervention, at } 3 \text { and } 6 \text { months post stroke" } & \text { Abstracts only } \\ \text { Notes } & \text { Data not suitable for analysis }\end{array}$

\section{Risk of bias}

Bias Authors' judgement Support for judgement

Random sequence genera- Low risk "patients were randomised using sealed envelopes"
tion (selection bias)


Lennon 2006 (Continued)

\begin{tabular}{l}
$\begin{array}{l}\text { Allocation concealment } \\
\text { (selection bias) }\end{array} \quad$ Unclear risk No information provided \\
\hline
\end{tabular}

Blinding of outcome as- Low risk Blinded assessor

sessment (detection bias)

All outcomes

Incomplete outcome data Low risk No dropouts
(attrition bias)
All outcomes

Free of systematic differ- Unclear risk No information provided
ences in baseline characteristics of groups compared?

Did authors adjust for Unclear risk No information provided
baseline differences in
their analyses?

\begin{tabular}{ll}
\hline Other bias $\quad$ Unclear risk $\quad$ No information provided \\
\hline
\end{tabular}

\title{
Study characteristics
}

\begin{tabular}{ll}
\hline Methods & Study design: RCT \\
& Method of randomisation: not stated \\
\hline Participants & Number of participants: $\mathrm{n}=61$ \\
& $\begin{array}{l}\text { Inclusion criteria: "Satisfied } 1986 \text { 2nd National Conference of Cerebral Vascular Disease assessment } \\
\text { guidelines and confirmed by CT or MRI scan of ischaemic or haemorrhagic stroke" }\end{array}$ \\
\hline
\end{tabular}

Interventions (1) Early rehabilitation group $(n=30)$

\begin{abstract}
"Basic treatment for the two groups: use of medication to prevent cerebral oedema during the acute phase, dosage and time dependent on the medical condition. In addition, the early rehabilitation group used Bobath technique for training. Commencement of training: ischaemia, between 24 hours and 8 days after stroke onset; haemorrhage, 48 hours and 10 days after stroke onset; medically stable. Main method of rehabilitation was as follows: (I) supine: (i) maintain anti-spasticity positions, with regular positional change. ii) Passive ranging of all joints. iii) Active rolling, moving. iv) Bridging exercise training. v) Truncal movement training. vi) Independently complete transfer from supine to sitting up. (II) Sitting: (i) Crawling or kneeling position training. (ii) Sitting balance training - 3 stages. (iii) Independently complete transfer from sitting to standing up. (III) Standing: (i) Standing balance training. (ii) Affected lower limb load training. (iii) Practice of knee flexion. (iv) Gait training: In standing, while loading onto the affected lower limb, step forward and backward in increasing amount of angle with the nonaffected lower limb. Without extending the hip, move the knee forward, dorsiflex ankle, and heel strike"

The individual components delivered are listed in Table 3. Based on the individual components, this intervention is categorised as comprising functional task training, musculoskeletal intervention (active), musculoskeletal intervention (passive) and neurophysiological intervention
\end{abstract}

Length of intervention period: one month

Number of sessions and length of individual sessions: "noon and afternoon/daily, $30 \mathrm{~min} / \mathrm{session"}$ 
Li 1999 (Continued)

Intervention provider: not stated

(2) Control group $(n=31)$

"Basic treatment for the two groups: use of medication to prevent cerebral oedema during the acute phase, dosage and time dependent on the medical condition. Control group did not have rehabilitation practice"

The individual components delivered are listed in Table 3. Based on the individual components, this intervention is categorised as comprising no intervention

Length of intervention period: no intervention

Number of sessions and length of individual sessions: no intervention

Intervention provider: no intervention

This study is classified as intervention (functional task training, musculoskeletal (active), musculoskeletal (passive), neurophysiological) versus no treatment (Table 4)

Measures of Independence in ADL: Barthel Index
Measures of motor function: Fugl-Meyer Assessment
Other secondary outcome measures: neurological deficit score
Time points when outcomes were assessed: before rehabilitation practice and after one month of inter-
vention

Notes Original study translated from Chinese to English

\section{Risk of bias}

\begin{tabular}{|c|c|c|}
\hline Bias & Authors' judgement & Support for judgement \\
\hline $\begin{array}{l}\text { Random sequence genera- } \\
\text { tion (selection bias) }\end{array}$ & Unclear risk & No information provided \\
\hline $\begin{array}{l}\text { Allocation concealment } \\
\text { (selection bias) }\end{array}$ & Unclear risk & No information provided \\
\hline $\begin{array}{l}\text { Blinding of outcome as- } \\
\text { sessment (detection bias) } \\
\text { All outcomes }\end{array}$ & Unclear risk & No information provided \\
\hline $\begin{array}{l}\text { Incomplete outcome data } \\
\text { (attrition bias) } \\
\text { All outcomes }\end{array}$ & Unclear risk & No dropouts described \\
\hline $\begin{array}{l}\text { Free of systematic differ- } \\
\text { ences in baseline charac- } \\
\text { teristics of groups com- } \\
\text { pared? }\end{array}$ & High risk & $\begin{array}{l}\text { Early rehabilitation group had a higher co-morbidity score than the con- } \\
\text { trol group at baseline. No difference for age and past history rating between } \\
\text { groups. No mention of other variables tested for baseline differences }\end{array}$ \\
\hline $\begin{array}{l}\text { Did authors adjust for } \\
\text { baseline differences in } \\
\text { their analyses? }\end{array}$ & High risk & No adjustment mentioned \\
\hline Other bias & Unclear risk & No information provided \\
\hline
\end{tabular}




\section{Study characteristics}

Methods Study design: RCT

Method of randomisation: not stated ("Total 174 patients were selected in this study, and these patients were randomly divided")

Participants

Number of participants: $n=174$

Inclusion criteria: not stated ("359 patients with paralysis after cerebral hemorrhage were admitted from March 2001 to May 2002")
(1) Rehabilitation group $(\mathrm{n}=87)$

"received regular nursing and rehabilitation nursing"

"In the early phase, passive activity for affected side and active activity for health side [were] suggested, such as combing the hair and hitting with health hands, raising the legs, clipping legs, contacting hands and feet"

"During acute phase, turning over every 1-2 hours was practiced under supine position. Lateral recumbent position was avoided to prevent compression of limbs. During lateral position, pillow was used to support the affected side and the health upper limbs and raise elbow. During the lateral position with the health side, elbow joint was stretched with palm toward health side, and the lower health limbs were extended backwardly. Exercise of limbs included the gentle pressing, massage, malaxtion from the distal end to proximal end twice a day, 20 min each time. Massage with safflower of regions surrounding should joint and from forearms to fingers was performed. Doctors should make patients believe their limbs were capable of moving for patients who were clear. The active movement dominated by the big nerves was suggested when patients showed signs of limbs activity, including raising hands, shoulder and leg and antielbow extension, hitting palms along the diagonal direction"

"During rehabilitation phase, patients were asked to sit by the bed with the health hands holding the bed, and legs dropping, and nurses standing by the affected side to prevent inclination toward the affected side. Once patients were capable of sitting stably by self, sitting exercise was initiated with body against bed, health hands holding bed, and nurses sitting by the side. Sitting exercises lasted from a few seconds to minutes, during which, patients were asked to swing affected limbs, 5 min each time and times and duration can be increased gradually. Walking exercise was initiated if patients were capable of sitting for 10-15 min without assistance. First patients were asked to do stepping on under the help of crutches. During exercise, center of gravity was gradually shifted to the affected side. Patients were asked to support the center of gravity under the nurses assistance, then began the walking with health limbs till patients could take care of themselves"

"Psychological rehabilitation, support, encourage, assiliation were given during the different psychological stage to make them exercise actively under good environment"

The individual components delivered are listed in Table 3. Based on the individual components, this intervention is categorised as comprising functional task training, musculoskeletal intervention (active) and musculoskeletal intervention (passive)

Length of intervention period: unclear: "The average hospitalisation was ( $20 \pm 9)$ days and $(31 \pm 11)$ days for rehabilitation group and control group respectively. Therapeutic effect of rehabilitation group was significantly superior to that of control group 15 days after treatment"

Number of sessions and length of individual sessions: dependent on patient condition (see above) Intervention provider: nursing staff

(2) Control group $(n=87)$

"received the general nursing" 
Li 2003 (Continued)

The individual components delivered are listed in Table 3. Based on the individual components, this intervention is categorised as comprising no intervention

Length of intervention period: no intervention

Number of sessions and length of individual sessions: no intervention

Intervention provider: no intervention

This study is classified as intervention (functional task training, musculoskeletal (active), musculoskeletal (passive)) versus no treatment (Table 4)

\begin{tabular}{ll} 
Outcomes & Other outcome measures: 'Score of Neural Defection,' average length of hospitalisation \\
& Time points when outcomes were assessed: before and after treatment ... "15 days after treatment" \\
\hline Notes & Abstract only \\
& No data suitable for analysis \\
& This paper was written in English, and the extracts above are direct quotes
\end{tabular}

\section{Risk of bias}

\begin{tabular}{|c|c|c|}
\hline Bias & Authors' judgement & Support for judgement \\
\hline $\begin{array}{l}\text { Random sequence genera- } \\
\text { tion (selection bias) }\end{array}$ & Unclear risk & No information provided \\
\hline $\begin{array}{l}\text { Allocation concealment } \\
\text { (selection bias) }\end{array}$ & Unclear risk & No information provided \\
\hline $\begin{array}{l}\text { Blinding of outcome as- } \\
\text { sessment (detection bias) } \\
\text { All outcomes }\end{array}$ & Unclear risk & No information provided \\
\hline $\begin{array}{l}\text { Incomplete outcome data } \\
\text { (attrition bias) } \\
\text { All outcomes }\end{array}$ & Unclear risk & No dropouts described \\
\hline $\begin{array}{l}\text { Free of systematic differ- } \\
\text { ences in baseline charac- } \\
\text { teristics of groups com- } \\
\text { pared? }\end{array}$ & Low risk & $\begin{array}{l}\text { "There was no significant difference in age, gender, course of disease, and } \\
\text { score of neural defection at admission" }\end{array}$ \\
\hline $\begin{array}{l}\text { Did authors adjust for } \\
\text { baseline differences in } \\
\text { their analyses? }\end{array}$ & Low risk & No information provided \\
\hline Other bias & Unclear risk & No information provided \\
\hline
\end{tabular}

\section{Study characteristics}

\begin{tabular}{ll}
\hline Methods & Study design: RCT \\
& Method of randomisation: "divided by draw method"
\end{tabular}


Li 2005 (Continued)

Participants

Number of participants: $\mathrm{n}=61$

Inclusion criteria: "Satisfied the 1995 Fourth National Conference on Cerebral Vascular Disease classification guidelines and 1982 International Meeting on Neurology assessment guidelines, first ever stroke, less than 70 years of age exclusive, one month within stroke onset inclusive and participant's informed consent"

Exclusion criteria: "Previous stroke, does not include transient ischaemic attack, transient ischaemic attack, with neurological deficits, conjunctival haemorrhage etc, severe complications and dementia"

Interventions
(1) Motor relearning group $(n=31)$

"Motor relearning group strictly followed the Motor Relearning Program for rehabilitation. Consisting [of] seven components, each component was subdivided into the following 4 subsections for practice: (i) observation, analysis, comparison, and description of normal activity patterns and using the observation and comparison of occupational activity limitation to analyse the missing basic components and abnormal performance. (ii) Practising the missing exercise components, including practice explanation, instruction, language, visual feedback and manual guidance. (iii) ADL practice, including explanation, instruction, practice, language, visual feedback and manual guidance, re-assessing, encouraging agility. (iv) Change in practice schedule, including scheduled and block practice, practice requiring self-supervision, creating a positive learning environment, involving the participation of family members and relevant personnel"

"Training method: (i) Upper limb function training: stimulate muscle activity and training control of reaching to objects -> maintaining muscle length, prevent contractures -> stimulate muscle control in the hand and training exercise control -> transferring from practice to ADL. (ii) Actinal surface function training: practise swallowing, facial exercise, improve breathing control -> transferring from practice to ADL. (iii) Lying to sitting on the edge of bed training -> transferring from practice to ADL. (iv) Sitting balance training: training postural adjustment during gravitational shifts -> increasing the complexity of training $>>$ transferring from practice to ADL. (v) Sit to stand and stand to sit training. (vi) Standing balance training: bilateral lower limb loading training $->$ hip alignment practice $->$ initiation of quadriceps contraction practice -> training postural adjustment during gravitational shifts -> increasing difficulty $>$ transferring from practice to ADL. (vii) Gait training: standing training-> stepping practice -> ambulatory training -> increasing difficulty $->$ transferring from practice to ADL"

"Rehabilitation sequentially and progressively used the above seven components for intervention, adapting to the individual participant's treatment progress"

The individual components delivered are listed in Table 3. Based on the individual components, this intervention is categorised as comprising functional task training and modality

Length of intervention period: not stated

Number of sessions and length of individual sessions: "45 min/session, 1/day, till the end of the study" Intervention provider: not stated

(2) Neurodevelopmental therapy group $(n=30)$

"Followed a combination of Bobath, proprioceptive neuromuscular facilitation (PNF) technique, Brunnstrom and Rood for rehabilitation"

"Neurodevelopmental therapy intervention: Using Bobath as the main component, intervention included 3 stages to treat: retardation stage, spasm stage and relative recovery stage: maintaining the correct supine position -> rolling practice -> sitting up from bed practice -> sitting balance training -> transfers training $->$ sit to stand training $>>$ standing balance training $>>$ gait training $\rightarrow$ ADL training. The above followed the sequence and principles of neurodevelopment"

The individual components delivered are listed in Table 3. Based on the individual components, this intervention is categorised as comprising functional task training, modality, musculoskeletal intervention (passive) and neurophysiological intervention

Length of intervention period: not stated 
Number of sessions and length of individual sessions: "45 min/session, 1/day, till the end of the study"

Intervention provider: not stated

This study is classified as active intervention one (functional task training) versus active intervention two (functional task training, musculoskeletal (passive), neurophysiological) (Table 6). Both intervention groups also received modality

Measures of Independence in ADL: Barthel Index
Other secondary outcome measures: stroke lesion score evaluation form
Time points when outcomes were assessed: day one of hospital admission and every month thereafter.
For participants who were discharged before one month, assessment was done on discharge

Notes Original study translated from Chinese to English

\section{Risk of bias}

\begin{tabular}{|c|c|c|}
\hline Bias & Authors' judgement & Support for judgement \\
\hline $\begin{array}{l}\text { Random sequence genera- } \\
\text { tion (selection bias) }\end{array}$ & Unclear risk & No information provided \\
\hline $\begin{array}{l}\text { Allocation concealment } \\
\text { (selection bias) }\end{array}$ & High risk & Design and implementation of study conducted by first study author \\
\hline $\begin{array}{l}\text { Blinding of outcome as- } \\
\text { sessment (detection bias) } \\
\text { All outcomes }\end{array}$ & Low risk & Outcome assessor blinded \\
\hline $\begin{array}{l}\text { Incomplete outcome data } \\
\text { (attrition bias) } \\
\text { All outcomes }\end{array}$ & Unclear risk & No dropouts described \\
\hline $\begin{array}{l}\text { Free of systematic differ- } \\
\text { ences in baseline charac- } \\
\text { teristics of groups com- } \\
\text { pared? }\end{array}$ & Low risk & No significant baseline differences \\
\hline $\begin{array}{l}\text { Did authors adjust for } \\
\text { baseline differences in } \\
\text { their analyses? }\end{array}$ & Low risk & No information provided \\
\hline Other bias & Unclear risk & No information provided \\
\hline
\end{tabular}

\section{Liao 2006}

\section{Study characteristics}

\begin{tabular}{ll}
\hline Methods & $\begin{array}{l}\text { Study design: RCT } \\
\text { Method of randomisation: participants randomly divided into the two groups by the time of hospital } \\
\text { admission }\end{array}$ \\
\hline Participants & Number of participants: $\mathrm{n}=96$
\end{tabular}


Liao 2006 (Continued)

Inclusion criteria: "(1) Satisfied the 1995 Fourth National Conference on Cerebral Vascular Disease classification guidelines, confirmed by CT or MRI scan for the first ever stroke, (2) deficits in motor function, without any serious cognitive impairment, (3) aged between 40 to 80, no severe heart, liver, kidney or other organ diseases, (4) Glasgow Coma Scale > 8, medically stable within 1 week"

Interventions

(1) Treatment group $(n=48)$

"Both groups of patients were treated with conventional medicine to reduce oedema in brain, nourish brain and nervous system, improve blood circulation in brain, etc, treatment group besides conventional rehabilitation also received trunk control function training therapy"

"Conventional rehabilitation method: (i) regular change in body positioning and maintaining limbs in correct positions; (ii) Passive ranging exercises on affected limbs; (iii) Facilitative training of affected limbs; (iv) Bedside sitting-balance training: affected upper limb maintained in anti-spasticity position to prop against the bed and progressing from sitting with support to sitting without support, and by repeatedly training the patient to use head and trunk to shift towards the centre, sitting-balance was induced; (v) Sit to stand balance training: Patient clasped hands Bobath style, extended upper limbs, leaned head and trunk forward, placed both feet on ground, and extended torso, hip and knee to stand up, and in process of standing up, affected lower limb should fully bear weight; (vi) gait training: As patient's standing-balance and weight-bearing ability of affected lower limb increased, patient could undertake ambulatory training between parallel bars or assisted gait training, after which progressing to unaided gait training; (vii) ADLs training"

"Trunk control function training: (I) Training method while in lying position: (i). Therapist placed both hands on both sides of patient's hypochondrium, and in line with breathing motion, pushed down and centrally on thorax; (ii). Therapist placed palm on patient's abdomen, and in line with breathing motion, pushed up and inwards; (iii). With arms folded, independently extend the left and right shoulder forward, to train the twisting of upper trunk, and then with bent knees, keeping knees together, rotated pelvis to the right and left; (iv). Both legs or single-leg bridging exercise. (II) Training method while in sitting position: (i). Keeping both knees and hip bent and kept together, arms crossed to embrace knees, and moving forwards and backward; (ii). While sitting on edge of bed, patient supported knee with both hands, therapist used both hands to control patient's trunk to perform forward and backward pelvis motion, followed by extension and flexion of trunk on affected side via active assistive ranging exercise; (iii). While sitting on stool, with both hands propped on stool, trunk was twisted towards non-affected side and twisted towards affected side, followed by training in shifting of body weight towards non-affected side of the trunk; (iv). While sitting on side of bed, keeping knees and hip bent, both lower limbs lifted off the ground, to train trunk-balance. (III) Training method while in standing position: (i). Patient placed both hands on treatment table while in standing position, therapist used one hand to lightly push patients' buttocks, while other hand controlled trunk, to train torso extension; (ii). Therapist placed one hand on patient's buttocks, one hand on the abdomen, to train forwards and backwards motion of the pelvis; (iii). With both hands holding exercise bar, twisting and extension exercises were performed"

The individual components delivered are listed in Table 3. Based on the individual components, this intervention is categorised as comprising functional task training and musculoskeletal intervention (passive) and neurophysiological intervention

Length of intervention period: $23.8 \pm 6.7$ days of treatment on average for this group

Number of sessions and length of individual sessions: once a day, each time 45 minutes, every week six times

Intervention provider: not stated

(2) Control group $(n=48)$

Control group received conventional rehabilitation as described above

The individual components delivered are listed in Table 3. Based on the individual components, this intervention is categorised as comprising functional task training and musculoskeletal intervention (passive)

Length of intervention period: $24.6 \pm 6.5$ days of treatment on average for this group 
Liao 2006 (Continued)

Number of sessions and length of individual sessions: once a day, each time 45 minutes, every week six times

Intervention provider: not stated

This study is classified as active intervention one (functional task training, musculoskeletal (passive), neurophysiological) versus active intervention two (functional task training, musculoskeletal (passive)) (Table 6)

\begin{tabular}{ll} 
Outcomes & Measures of motor function: Fugl-Meyer (balance ability and motor function of lower extremity) \\
Other secondary outcome measures: Sheikh (truncal control) \\
Time points when outcomes were assessed: before and after intervention \\
\hline Notes & Original study translated from Chinese to English \\
& $\begin{array}{l}\text { Note: Treatment group has been classified as neurophysiological based on the description of handling } \\
\text { techniques provided in the paper. No reference is made to Bobath or Davies. The categorisation will be } \\
\text { explored in a sensitivity analysis }\end{array}$
\end{tabular}

\section{Risk of bias}

\begin{tabular}{|c|c|c|}
\hline Bias & Authors' judgement & Support for judgement \\
\hline $\begin{array}{l}\text { Random sequence genera- } \\
\text { tion (selection bias) }\end{array}$ & Unclear risk & No information provided \\
\hline $\begin{array}{l}\text { Allocation concealment } \\
\text { (selection bias) }\end{array}$ & Unclear risk & No information provided \\
\hline $\begin{array}{l}\text { Blinding of outcome as- } \\
\text { sessment (detection bias) } \\
\text { All outcomes }\end{array}$ & Unclear risk & No information provided \\
\hline $\begin{array}{l}\text { Incomplete outcome data } \\
\text { (attrition bias) } \\
\text { All outcomes }\end{array}$ & Unclear risk & No dropouts described \\
\hline $\begin{array}{l}\text { Free of systematic differ- } \\
\text { ences in baseline charac- } \\
\text { teristics of groups com- } \\
\text { pared? }\end{array}$ & Low risk & No significant baseline differences \\
\hline $\begin{array}{l}\text { Did authors adjust for } \\
\text { baseline differences in } \\
\text { their analyses? }\end{array}$ & Low risk & No information provided \\
\hline Other bias & Unclear risk & No information provided \\
\hline
\end{tabular}

Lincoln 2003

\section{Study characteristics}

Study design: RCT
Method of randomisation: blocked randomisation. Computer-generated random sequence of numbers
in opaque sealed envelopes opened sequentially by researcher


Lincoln 2003 (Continued)

Participants
Number of participants: $\mathrm{n}=120$

Inclusion criteria: stroke less than two weeks previously

Exclusion criteria: excluded if unconscious on admission, unable to toilet self before stroke, unable to tolerate more than 30 minutes of physical tasks and living farther than $25 \mathrm{~km}$ from hospital or if no informed consent given

Interventions

(1) Neurophysiological (Bobath) $(n=60)$

"Treatment delivered by different groups of physiotherapists using prepared written guidelines, consisting of theoretical concepts for practice and main clinical objectives, based on their own knowledge and experience and their interpretation of the literature"

Techniques were not described. Bobath-based treatment was delivered by the unit's existing physiotherapists, who had used this approach routinely before the start of the study

Prepared written guidelines were available

The individual components delivered are listed in Table 3. Based on the individual components, this intervention is categorised as comprising neurophysiological intervention

Length of intervention period: "treatment continued for as long as was needed"....approach continued as outpatient if necessary.. Amount matched to "typical amount" given by existing ward physiotherapists

Number of sessions and length of individual sessions: median of 23 minutes per weekday

Intervention provider: more time was spent with physiotherapist and physiotherapy assistant together for this treatment group. Stated that occupational therapists also used this approach

(2) Motor learning $(n=60)$

"Treatment delivered by different groups of physiotherapists using prepared written guidelines, consisting of theoretical concepts for practice and main clinical objectives, based on their own knowledge and experience and their interpretation of the literature"

Techniques were not described. Motor-learning treatment was delivered by two physiotherapists, who previously had 'insufficient experience of treatment' but who were given training

Prepared written guidelines were available

The individual components delivered are listed in Table 3. Based on the individual components, this intervention is categorised as comprising functional task training

Length of intervention period: "treatment continued for as long as was needed"....approach continued as outpatient if necessary. Amount matched to "typical amount" given by existing ward physiotherapists

Number of sessions and length of individual sessions: median of 23 minutes per weekday

Intervention provider: more time was spent with physiotherapy assistant alone in this treatment group. Stated that occupational therapists also used this approach

This study is classified as active intervention one (neurophysiological) versus active intervention two (functional task training) (Table 6)

Outcomes

Measures of Independence in ADL: Barthel Index; EADL

Measures of functional independence: Motor Assessment Scale; Rivermead Motor Assessment

Measures of voluntary movement: 10-Metre Walk test

Measures of tone/spasticity: Modified Ashworth Scale

Other measures: Nine-Hole Peg test; Nottingham Sensory Assessment; length of stay 
Lincoln 2003 (Continued)

Time points when outcomes were assessed: "Measures were performed by a blinded assessor at base-

line, and then at 1,3 , and 6 months after baseline"

Notes

\section{Risk of bias}

\begin{tabular}{lll}
\hline Bias & Authors' judgement & Support for judgement \\
\hline $\begin{array}{l}\text { Random sequence genera- } \\
\text { tion (selection bias) }\end{array}$ & Low risk & $\begin{array}{l}\text { Computer-generated random sequence of numbers in opaque sealed en- } \\
\text { velopes opened sequentially by researcher }\end{array}$ \\
& $\begin{array}{l}\text { ("Allocation to treatment groups was by a computer generated random se- } \\
\text { quence provided by a therapist not involved with the trial, with notification } \\
\text { delivered in opaque, sealed envelopes. Blocked randomisation was used to } \\
\text { ensure approximately equal numbers of patients in each group at any time. } \\
\text { Patients were screened consecutively on admission to the ward and those that } \\
\text { met the inclusion criteria were referred for initial assessment. After the initial } \\
\text { assessment was completed, a research therapist opened the next envelope } \\
\text { and informed the therapists providing the treatments of the group allocation") }\end{array}$
\end{tabular}

\begin{tabular}{|c|c|c|}
\hline $\begin{array}{l}\text { Allocation concealment } \\
\text { (selection bias) }\end{array}$ & Low risk & $\begin{array}{l}\text { Allocation to treatment groups was by a computer-generated random se- } \\
\text { quence provided by a therapist not involved with the trial }\end{array}$ \\
\hline
\end{tabular}

Blinding of outcome as- Low risk
sessment (detection bias)

All outcomes
Participant not blinded ("Patients were asked not to mention their treatment or therapist to the assessor")

\section{Therapist not blinded}

Assessor was blinded (Outcome assessments were completed at one, three and six months after random allocation by an assessor who was blind to the group allocation) ("To ensure masking, assessments of inpatients occurred in a room separate from the ward and patients were brought to the assessor there whenever possible. For later examination of the success of masking, the assessor recorded a guess of the patient's group allocation at each assessment")

\begin{tabular}{ll}
\hline $\begin{array}{l}\text { Incomplete outcome data } \\
\text { (attrition bias) }\end{array}$ & Low risk \\
All outcomes & Dropouts were accounted for \\
& $\begin{array}{l}52 / 60 \text { in Bobath group and } 47 / 60 \text { in motor learning group remained at one } \\
\text { month; } 43 / 60 \text { and } 42 / 60 \text { completed assessments, respectively, at three } \\
\text { months; and } 45 / 60 \text { and } 42 / 60, \text { respectively, at six months }\end{array}$ \\
\hline
\end{tabular}

\begin{tabular}{|c|c|c|}
\hline $\begin{array}{l}\text { Free of systematic differ- } \\
\text { ences in baseline charac- } \\
\text { teristics of groups com- } \\
\text { pared? }\end{array}$ & Low risk & $\begin{array}{l}\text { "The groups were not significantly different in age, gender, side of stroke, type } \\
\text { of lesion, or cognitive impairments" }\end{array}$ \\
\hline $\begin{array}{l}\text { Did authors adjust for } \\
\text { baseline differences in } \\
\text { their analyses? }\end{array}$ & Low risk & No information provided \\
\hline Other bias & Unclear risk & $\begin{array}{l}\text { Some possibility of contamination between groups, as physiotherapists pro- } \\
\text { viding the motor learning intervention were previously using Bobath therapy } \\
\text { and therefore may have reverted to using some Bobath techniques } \\
\text { Also some possibility of contamination due to participants being inpatients on } \\
\text { the same unit: the study authors state: "some aspects of the treatments could } \\
\text { not be implemented because both treatments were occurring on the same re- } \\
\text { habilitation wards and there was a risk of treatment contamination" }\end{array}$ \\
\hline
\end{tabular}




\section{Study characteristics}

\begin{tabular}{ll}
\hline Methods & Study design: RCT \\
& Method of randomisation: not stated \\
\hline Participants & Number of participants: $\mathrm{n}=120$ \\
& $\begin{array}{l}\text { Inclusion criteria: "There were } 120 \text { hemiplegic patients with cerebral apoplexy from July } 2000 \text { to Feb- } \\
\text { ruary } 2001 \text { (not counting those with serious heart, lung, kidney, and stomach complication and distur- } \\
\text { bance of consciousness). We made a diagnosis according to WHO standards after using CT or MRI head } \\
\text { diagnosing without exception" }\end{array}$ \\
\hline
\end{tabular}

Interventions

(1) Rehabilitation group $(n=60)$

"All the 120 patients were treated according to endoneurological routine. On this basis we made recovery training for the rehabilitation group using modern technology. In accordance with patients condition, we took appropriate recovery measures (PT, OT), such as favourable limb position in bed, particular passive movement, healthy limb active movement and sick limb movement with the help of the healthy, sitting position balancing training, dressing and eating, speaking and ADL training"

"The rehabilitation group started to accept the treatment in 3-5 days after attack when the patients had been conscious, vital signs had been smooth, nervous signs had not advanced within 48 hours"

The individual components delivered are listed in Table 3. Based on the individual components, this intervention is categorised as comprising functional task training, musculoskeletal intervention (active) and musculoskeletal intervention (passive)

Length of intervention period: 15 days

Number of sessions and length of individual sessions: "four times a day, 30 minutes at every turn" Intervention provider: not stated

(2) Control group $(n=60)$

No intervention

The individual components delivered are listed in Table 3. Based on the individual components, this intervention is categorised as comprising no intervention

Length of intervention period: no intervention

Number of sessions and length of individual sessions: no intervention

Intervention provider: no intervention

This study was classified as intervention (functional task training, musculoskeletal (active), musculoskeletal (passive)) versus no treatment (Table 4) 
Liu 2003 (Continued)

Measures of motor function: Fugl-Meyer Assessment

State time points when outcomes were assessed: "we evaluated from the very beginning of treatment and on the fifteenth day of treatment"

Notes Abstract only

\section{Risk of bias}

Bias Authors' judgement Support for judgement

Random sequence genera- Unclear risk No information provided

tion (selection bias)

\begin{tabular}{lll}
\hline $\begin{array}{l}\text { Allocation concealment } \\
\text { (selection bias) }\end{array}$ & Unclear risk & No information provided
\end{tabular}

Blinding of outcome as- Unclear risk No information provided

sessment (detection bias)

All outcomes

\begin{tabular}{l}
\hline Incomplete outcome data Unclear risk $\quad$ No dropouts described \\
(attrition bias) \\
All outcomes
\end{tabular}

\begin{tabular}{|c|c|c|}
\hline $\begin{array}{l}\text { Free of systematic differ- } \\
\text { ences in baseline charac- } \\
\text { teristics of groups com- } \\
\text { pared? }\end{array}$ & Low risk & $\begin{array}{l}\text { Reported baseline demographics (age, gender and type of stroke) similar be- } \\
\text { tween the two groups. Baseline measures ('pretreatment') FMA and BI scores } \\
\text { for the two groups are comparable }\end{array}$ \\
\hline
\end{tabular}

\begin{tabular}{lll}
\hline $\begin{array}{l}\text { Did authors adjust for } \\
\text { baseline differences in } \\
\text { their analyses? }\end{array}$ & Low risk & \\
\hline Other bias & Unclear risk & No information provided \\
\hline
\end{tabular}

McClellan 2004

\section{Study characteristics}

\begin{tabular}{|c|c|}
\hline Methods & $\begin{array}{l}\text { Study design: RCT } \\
\text { Method of randomisation: numbered, sealed, opaque envelopes }\end{array}$ \\
\hline Participants & $\begin{array}{l}\text { Number of participants: } \mathrm{n}=26 \\
\text { Inclusion criteria: stroke less than } 18 \text { months previously, aged over } 45 \text { years, living in community, score } \\
\text { greater than zero and less than six on item five of MAS, and less than six on items seven and eight of } \\
\text { MAS }\end{array}$ \\
\hline Interventions & $\begin{array}{l}\text { (1) Motor learning }(n=15) \\
\text { "Intervention was standardised by prescribing the first five exercises that the subject could perform } \\
\text { successfully from a list of } 23 \text { predetermined exercises. The exercises were arranged loosely hierarchi- } \\
\text { cally, based on their challenge to balance...exercises were progressed systematically..." }\end{array}$ \\
\hline & $\begin{array}{l}\text { The individual components delivered are listed in Table } 3 \text {. Based on the individual components, this in- } \\
\text { tervention is categorised as comprising functional task training }\end{array}$ \\
\hline
\end{tabular}


Number of sessions and length of individual sessions: instructed to practise twice per day with videotape. Telephoned after one week. Returned for exercise review at end of weeks two and four. Record of practice kept for six weeks

Intervention provider: exercises prescribed by physiotherapist

(2) Placebo control (motor learning, upper limb) $(n=11)$

Similar to above, but aimed at improving function of the affected upper limb

The individual components delivered are listed in Table 3. Based on the individual components, this intervention is categorised as comprising upper limb training

Length of intervention period: six weeks

Number of sessions and length of individual sessions: instructed to practise twice per day with videotape. Telephoned after one week. Returned for exercise review at end of weeks two and four. Record of practice kept for six weeks

Intervention provider: exercises prescribed by physiotherapist

This study is classified as intervention (functional task training) versus attention control (upper limb) (Table 5)

Measures of functional independence: MAS (item five)
Measures of balance and postural control: Functional Reach Test
Time points when outcomes were assessed: "were measured prior to, immediately after, and two
months after intervention"

Notes

Risk of bias

\begin{tabular}{|c|c|c|}
\hline Bias & Authors' judgement & Support for judgement \\
\hline $\begin{array}{l}\text { Random sequence genera- } \\
\text { tion (selection bias) }\end{array}$ & Low risk & $\begin{array}{l}\text { "To ensure allocation was concealed, randomisation was by numbered, } \\
\text { sealed, opaque envelopes" }\end{array}$ \\
\hline $\begin{array}{l}\text { Allocation concealment } \\
\text { (selection bias) }\end{array}$ & Low risk & No information provided \\
\hline \multirow[t]{3}{*}{$\begin{array}{l}\text { Blinding of outcome as- } \\
\text { sessment (detection bias) } \\
\text { All outcomes }\end{array}$} & Low risk & $\begin{array}{l}\text { Participant was blinded to study aims ("To increase the likelihood that sub- } \\
\text { jects were blind to group allocation, neither the exact purpose of the research } \\
\text { nor the types of exercises that subjects would be receiving were specified and } \\
\text { both mobility and upper limb function [were] measured") }\end{array}$ \\
\hline & & Therapist not blinded \\
\hline & & $\begin{array}{l}\text { Assessor was blinded ("Outcome measures were collected at Weeks } 0,6 \text {, and } \\
14 \text { by a measurer blinded to group allocation") }\end{array}$ \\
\hline $\begin{array}{l}\text { Incomplete outcome data } \\
\text { (attrition bias) }\end{array}$ & Low risk & Dropouts accounted for \\
\hline All outcomes & & $\begin{array}{l}23 / 26 \text { completed intervention } \\
21 / 26 \text { assessed at six weeks }\end{array}$ \\
\hline
\end{tabular}

Free of systematic differ- Low risk Characteristics of the experimental group and the control group are similar
ences in baseline charac- 
McClellan 2004 (Continued) teristics of groups compared?

\begin{tabular}{|c|c|c|}
\hline $\begin{array}{l}\text { Did authors adjust for } \\
\text { baseline differences in } \\
\text { their analyses? }\end{array}$ & Low risk & No information provided \\
\hline Other bias & Unclear risk & $\begin{array}{l}\text { Compliance with the home exercise regime is a potential confounding variable } \\
\text { This was measured and, on average, participants recorded that they practised } \\
75 \% \text { of the times that they were instructed to do so }\end{array}$ \\
\hline
\end{tabular}

Mudge 2009

\section{Study characteristics}

Study design: single-blind RCT
Method of randomisation: "prospective, randomized, single-blind, attention controlled clinical trial of
circuit-based rehabilitation in adults at least 6 months after stroke"
'Participants were randomly assigned to the exercise or control group through the use of comput-
er-generated random numbers by an individual not associated with the study. Randomization was re-
vealed to each participant by the principal investigator after the second baseline assessment"

Pumber of participants: $\mathrm{n}=58$
Inclusion criteria: "1 or more strokes more than 6 months earlier, discharged from rehabilitation, and
were able to walk independently (with an aid if necessary). Some residual gait difficulty was required,
as defined by a score of less than 2 on at least 1 of the walking items of the physical functioning scale of
the 36-Item Short Form Health Survey"
Exclusion criteria: "Progressive neurologic disease, other significant health problems that adversely af-
fected walking ability, more than 2 falls in the previous 6 months and unstable cardiac conditions, un-
controlled hypertension, or congestive heart failure"

"There were 15 stations in the circuit, which were graded to each participant's ability and progressed as tolerated. Each station contained either a task-oriented gait or standing balance activity, or strengthening of a lower extremity muscle in a way designed to improve gait. Details of the content of each station and examples of progressions are provided in an Appendix. The total exercise time was 30 minutes, although sessions lasted between 50 to 60 minutes, including stretching. Participants spent 2 minutes at each station of the circuit, with time allowed to move between stations and receive instructions for the next station. Details about exercise intensity and/or repetitions performed at each station were recorded for each participant"

The individual components delivered are listed in Table 3. Based on the individual components, this intervention is categorised as comprising functional task training and musculoskeletal intervention (active)

Length of intervention period: four weeks

Number of sessions and length of individual sessions: in 12 group circuit exercise sessions three times a week

Intervention provider: "groups contained up to 9 participants and were led by 1 of the investigators (S.M.) assisted by 2 physiotherapy students"

(2) Control group $(n=27)$ 
"The control group was run by an occupational therapist and consisted of 4 social and 4 educational sessions. Detailed content of the sessions is available in Appendix. The duration of the control group sessions was designed to match the duration of the intervention sessions in order to control for possible effects of dosage"

"Matching for duration and not number of sessions was a pragmatic choice based on resources, allowing 1 intervention session a weekday to be scheduled over the 4-week intervention period"

The individual components delivered are listed in Table 3. Based on the individual components, this intervention is categorised as attention control (social)

Length of intervention period: four weeks

Number of sessions and length of individual sessions: eight 90-minute session weeks in groups of up to eight

Intervention provider: occupational therapist

This study is classified as intervention (functional task training, musculoskeletal (active)) versus attention control (Table 5)

Measures of motor function: Rivermead Mobility Index
Measures of voluntary movement: Six-Minute Walk test, Timed 10-Metre Walk test
Other secondary outcome measures: Physical Activity and Disability Scale (PADS), Activities-Specific
Balance and Confidence Scale, mean number of steps/d (measured by the StepWatch Activity Monitor)
Time points when outcomes were assessed: "Two baseline testing sessions 3 weeks apart were per-
formed to ensure that participant measures were stable. The testing sessions were repeated immedi-
ately after the group sessions (postintervention) and at 3 months (follow-up). All tests were performed
once, and all testing sessions were identical"

Notes

Data provided are means and ranges. For analysis, standard deviations have been estimated by calculating (upper range - lower range)/four

\section{Risk of bias}

\begin{tabular}{lll}
\hline Bias & Authors' judgement & Support for judgement \\
\hline $\begin{array}{l}\text { Random sequence genera- } \\
\text { tion (selection bias) }\end{array}$ & Low risk & $\begin{array}{l}\text { "randomly assigned to the exercise or control group through the use of com- } \\
\text { puter-generated random numbers by an individual not associated with the } \\
\text { study" }\end{array}$ \\
\hline $\begin{array}{l}\text { Allocation concealment } \\
\text { (selection bias) }\end{array}$ & Low risk & $\begin{array}{l}\text { "Participants were not blind because they were aware of their own group al- } \\
\text { location, which was revealed after the second testing session. Participants } \\
\text { were instructed not to discuss group allocation with the assessor. The testing } \\
\text { sessions were carried out in the same rehabilitation clinic as the intervention } \\
\text { groups but were scheduled at different times to maintain blinding of the asses- } \\
\text { sor" }\end{array}$
\end{tabular}

\begin{tabular}{ll}
\hline $\begin{array}{l}\text { Blinding of outcome as- } \\
\text { sessment (detection bias) }\end{array}$ & Low risk
\end{tabular}$\quad \begin{aligned} & \text { "Outcome assessment was performed by an independent physiotherapist } \\
& \text { blind to treatment assignment" }\end{aligned}$
All outcomes

\begin{tabular}{lll}
$\begin{array}{l}\text { Incomplete outcome data } \\
\text { (attrition bias) } \\
\text { All outcomes }\end{array}$ & Low risk \\
\hline $\begin{array}{l}\text { Free of systematic differ- } \\
\text { ences in baseline charac- }\end{array}$ & Low risk & $\begin{array}{l}\text { "There was no significant difference between the baseline characteristics of } \\
\text { the } 2 \text { groups" }\end{array}$
\end{tabular}


Mudge 2009 (Continued) teristics of groups compared?

Did authors adjust for $\quad$ Low risk No information provided
baseline differences in

Other bias Unclear risk No information provided

Mudie 2002

\section{Study characteristics}

\begin{tabular}{ll}
\hline Methods & $\begin{array}{l}\text { Study design: RCT } \\
\text { Method of randomisation: random number tables; random numbers assigned alternately to group; ran- } \\
\text { dom number drawn by independent person and participant assigned to appropriate group }\end{array}$ \\
\hline Participants & $\begin{array}{l}\text { Number of participants: } \mathrm{n}=40 \\
\text { Inclusion criteria: recent stroke, asymmetrical in sitting and capacity for relearning } \\
\text { Exclusion criteria: pain, existing co-morbidities and previous balance training }\end{array}$ \\
\hline
\end{tabular}

Interventions

(1) Feedback only $(n=10)$

"Visual rather than auditory signals from the balance performance monitor (BPM) were used during training"

The individual components delivered are listed in Table 3. Based on the individual components, this intervention is categorised as comprising functional task training and modality

Length of intervention period: six weeks

Number of sessions and length of individual sessions: "training sessions for each of the three approaches extended for 30 minutes"

Intervention provider: "conducted by staff occupational therapists"

(2) Motor learning (task related training) $(n=10)$

Sitting; reaching to encourage weight-shift

The individual components delivered are listed in Table 3. Based on the individual components, this intervention is categorised as comprising functional task training

Length of intervention period: six weeks

Number of sessions and length of individual sessions: 30 minutes per day. Unclear how many sessions/wk. Received trial intervention in addition to 'standard' treatment

Intervention provider: "task-specific reaching and BPM training were conducted by staff occupational therapists"

(3) Neurophysiological (Bobath) $(n=10)$

Treatment protocol based on Bobath practices (devised by Bobath trained staff physiotherapists). Protocol focused on increasing trunk and pelvic range of movement, normalising muscle tone, maintaining appropriate balance responses. Series of postures and postural manoeuvres involving weight shift, pelvic tilting, trunk movements; verbally and manually facilitated by therapists

The individual components delivered are listed in Table 3. Based on the individual components, this intervention is categorised as comprising neurophysiological intervention 
Number of sessions and length of individual sessions: 30 minutes per day. Unclear how many sessions/wk. Received trial intervention in addition to 'standard' treatment

Intervention provider: Bobath-trained staff physiotherapists

(4) Control (no treatment) $(n=10)$

"control group participated in standard physiotherapy and occupational therapy programmes as did the three treatment groups in addition to their specific training"

The individual components delivered are listed in Table 3. Based on the individual components, this intervention is categorised as comprising usual care (not stated)

Length of intervention period: six weeks

Number of sessions and length of individual sessions: received 'standard' treatment

Intervention provider: physiotherapists and occupational therapists

This study is classified as active intervention one (functional task training) versus active intervention two (neurophysiological) (Table 6) versus usual care (not stated) (Table 5)

Outcomes $\quad$ Measures of independence in ADL: Barthel Index

Measures of postural control and balance: symmetry in sitting, weight distribution in sitting

Time points when outcomes were assessed: "Measurements were performed using the BPM daily before treatment sessions, two weeks after cessation of treatment and 12 weeks post study"

\section{Notes}

Intervention for group (one) based on components from motor learning theory, but as this intervention is feedback only (and trials of feedback only have been excluded from this review), the data from this group will not be used

\section{Risk of bias}

Bias Authors' judgement Support for judgement

Random sequence genera- Low risk tion (selection bias)
Random number tables; random numbers assigned alternately to group; random number drawn by independent person and participant assigned to appropriate group ("Forty numbers from a random numbers table were sequentially drawn from a box by a clinician independent of the study. The numbers were written alternately in columns headed with the training regimes of the four groups until all 40 numbers were placed. The slips of paper containing the random numbers were replaced in an opaque canister that was kept in a locked filing cabinet in the senior investigator's office")

Allocation concealment Low risk
(selection bias)

"On admission of a patient to the study, an independent person drew a number from the container and the patient was allocated to the treatment group with the matching number"

\section{Blinding of outcome as- Low risk} sessment (detection bias)

All outcomes

\begin{abstract}
Unclear whether participant was blind
Therapist not blinded, although blinded to data ("The therapists administering the training had no access to weight distribution data of subjects before or during training. Monitoring by the senior investigator ensured that the research assistants measuring and recording the data remained blind to the patients' training regime and that therapists remained blind to the measurement
\end{abstract} data")

Assessor was blinded ("Testing and recording of results [were] conducted by grant-funded research assistants (occupational therapy students and assis- 
tants) who were independent of the rehabilitation wards from which the subjects came and unaware of the treatment subjects were to receive")

"Group allocation was more difficult to hide from the assistants (successful in approximately $75 \%$ of cases) but blinding of therapists to measurement data was successful in every case"

\begin{tabular}{|c|c|c|}
\hline $\begin{array}{l}\text { Incomplete outcome data } \\
\text { (attrition bias) }\end{array}$ & Low risk & Dropouts accounted for \\
\hline All outcomes & & $33 / 40$ completed intervention \\
\hline
\end{tabular}

Free of systematic differ- Low risk "no significant difference in ages between the four stroke groups"
ences in baseline characteristics of groups compared?

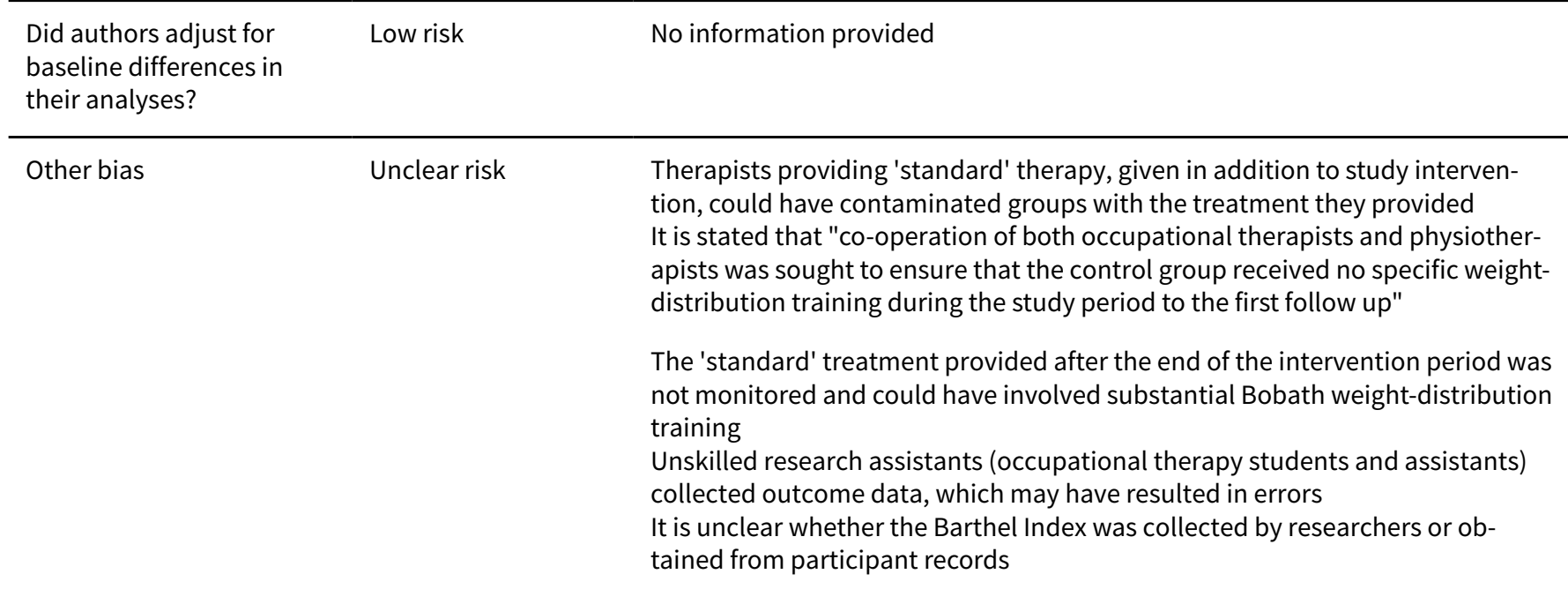

\section{Study characteristics}

\begin{tabular}{ll}
\hline Methods & Study design: RCT \\
& Method of randomisation: not stated \\
\hline Participants & Number of participants: $n=68$ \\
Inclusion criteria: not stated & (1) Comprehensive rehabilitation training group ( $\mathrm{n}=34)$ \\
& "Participants from both groups received conventional neurological treatment. The comprehensive re- \\
& habilitation training group additionally received Bobath and Brunnstrom focused exercise therapy, em- \\
phasising on standing balance and lower limb exercise control ability training. For participants weak in \\
Interventions \\
first and second metatarsal, so as to stimulate dorsiflexion, this method could be repeatedly used; dur- \\
ing ambulation, the affected limb was supported by an elastic bandage into 90 degrees of ankle dorsi- \\
flexion. Training of the muscles at the shin area used the FZ-1 model to deliver low frequency electrical \\
stimulation pulse, with intensity selected as appropriate, $1 /$ day, 20 min/session, $20-40 x$ of treatment on \\
average, with those requiring more than $20 x$ of treatment having a 10 day rest before commencement \\
of the next session"
\end{tabular}


Ni 1997 (Continued)

The individual components delivered are listed in Table 3. Based on the individual components, this intervention is categorised as comprising assistive devices, functional task training, modality and neurophysiological intervention

Length of intervention period: "2 months of treatment on average"

Number of sessions and length of individual sessions: two/d, 30 to 45 minutes/session

Intervention provider: not stated

(2) Control group $(n=34)$

Conventional neurological treatment

The individual components delivered are listed in Table 3. Based on the individual components, this intervention is categorised as comprising no intervention

Length of intervention period: no intervention

Number of sessions and length of individual sessions: no intervention

Intervention provider: no intervention

This study is classified as intervention (functional task training, neurophysiological) versus no treatment (Table 4). The intervention group also received assistive devices and modality

\begin{tabular}{ll} 
Outcomes & Measures of Independence in ADL: Functional Independence Measure (FIM) \\
& Measures of motor function: Fugl-Meyer Assessment \\
& Other secondary outcome measures: Brunnstrom \\
& Time points when outcomes were assessed: "1 week after stroke onset or before commencement of in- \\
tervention and 3 months after stroke" & \\
\hline Notes & Original study translated from Chinese to English
\end{tabular}

Risk of bias

\begin{tabular}{|c|c|c|}
\hline Bias & Authors' judgement & Support for judgement \\
\hline $\begin{array}{l}\text { Random sequence genera- } \\
\text { tion (selection bias) }\end{array}$ & Unclear risk & No information provided \\
\hline $\begin{array}{l}\text { Allocation concealment } \\
\text { (selection bias) }\end{array}$ & Unclear risk & No information provided \\
\hline $\begin{array}{l}\text { Blinding of outcome as- } \\
\text { sessment (detection bias) } \\
\text { All outcomes }\end{array}$ & Unclear risk & No information provided \\
\hline $\begin{array}{l}\text { Incomplete outcome data } \\
\text { (attrition bias) } \\
\text { All outcomes }\end{array}$ & Unclear risk & No dropouts described \\
\hline $\begin{array}{l}\text { Free of systematic differ- } \\
\text { ences in baseline charac- } \\
\text { teristics of groups com- } \\
\text { pared? }\end{array}$ & Unclear risk & Did not report significance \\
\hline
\end{tabular}


Ni 1997 (Continued)

Did authors adjust for baseline differences in their analyses?
Unclear risk No information provided

Other bias Unclear risk No information provided

Pan 2004

\section{Study characteristics}

\begin{tabular}{ll}
\hline Methods & Study design: RCT \\
& Method of randomisation: "by participants drawing lots" \\
\hline Participants & Number of participants: $\mathrm{n}=96$ \\
& Inclusion criteria: not stated \\
\hline
\end{tabular}

Interventions

(1) Rehabilitation group $(n=48)$

"Both groups received usual medical care and acupuncture. Participants in the treatment group commenced therapy once medically stable, where participants with ischemic stroke commenced therapy within 5 days of hospitalisation and participants with haemorrhagic stroke commencing therapy between 7 to 14 days of hospitalisation"

"Treatment intervention: (i) Regular change in bed position: for participants not able to turn in bed independently, turning was done every two hours in the supine position, lateral position with affected limbs at lower side and healthy limbs at upper side, and lateral position with affected limbs at upper side and healthy limbs at lower side. (ii) Optimal placement of limbs in functional positions. (iii) Joint movement: Daily movement of each joint 2-3 times/day, 5-10 times/session. Upon gaining consciousness, participants were encouraged to do active assisted exercises of shoulder flexion with intertwined hands and lower limb bridging exercises with attention to pelvic control, until participants were able to do the exercises actively. (iv) Lying to sitting and sitting balance training: participants first shifted to edge of bed, dangled both legs off the bed with the unaffected leg supported the top affected leg, and pushed into sitting position with the unaffected arm. For sitting balance training, participants practised reaching for/placing objects from one side to another, increasing the amount of reach as much as possible. Participants were also trained to self-correct their sitting balance by subjecting them to perturbations in all directions. (v) Sit to stand and standing balance training: participants adopted the Bobath method of intertwining both arms, forward extension of arms, head and trunk, shifted body weight to the arms in order to lift the pelvis, hips, knee and stand up. If needed, therapist assisted by pushing the affected knee forward and putting one hand on the unaffected pelvis to help lift it. Participants stood in parallel bars, supporting with the unaffected arm and with assistance from the doctor or family, and gradually increased the time in standing until 30 mins could be attained. Participants progressed to sit to stand practice from using a high chair to a low chair for added challenge. (vi) Gait re-education and stair training (up and down). (vii) ADL practice: inculcated ADL tasks practice into therapy sessions, 3-4x/day, $30 \mathrm{~min} /$ session. Family of participants were instructed to assist in practice"

The individual components delivered are listed in Table 3. Based on the individual components, this intervention is categorised as comprising assistive devices, functional task training, modality, musculoskeletal intervention (active) and musculoskeletal intervention (passive)

Length of intervention period: not stated

Number of sessions and length of individual sessions: dependent on participant's condition (see above)

Intervention provider: therapist and "family of participants were instructed to assist in practice"

(2) Control group $(n=48)$ 
Pan 2004 (Continued)

"Received usual medical care and acupuncture"

The individual components delivered are listed in Table 3. Based on the individual components, this intervention is categorised as comprising no intervention

Length of intervention period: no intervention

Number of sessions and length of individual sessions: no intervention

Intervention provider: no intervention

This study is classified as intervention (functional task training, musculoskeletal (active), musculoskeletal (passive)) versus no treatment (Table 4). The intervention group also received assistive devices and modality

Measures of independence in ADL: Barthel Index
Measures of motor function: Fugl-Meyer Assessment (upper and lower limbs)
Other secondary outcome measures: neurological deficit (CNS)
Time points when outcomes were assessed: "24 hrs before commencement of intervention and 3-4
weeks after intervention"

Notes Original study translated from Chinese to English

\section{Risk of bias}

\begin{tabular}{|c|c|c|}
\hline Bias & Authors' judgement & Support for judgement \\
\hline $\begin{array}{l}\text { Random sequence genera- } \\
\text { tion (selection bias) }\end{array}$ & Unclear risk & $\begin{array}{l}\text { States that randomisation by participants drawing lots, but no further details } \\
\text { provided }\end{array}$ \\
\hline $\begin{array}{l}\text { Allocation concealment } \\
\text { (selection bias) }\end{array}$ & Unclear risk & No information provided \\
\hline $\begin{array}{l}\text { Blinding of outcome as- } \\
\text { sessment (detection bias) } \\
\text { All outcomes }\end{array}$ & Unclear risk & No information provided \\
\hline $\begin{array}{l}\text { Incomplete outcome data } \\
\text { (attrition bias) } \\
\text { All outcomes }\end{array}$ & Unclear risk & No dropouts described \\
\hline $\begin{array}{l}\text { Free of systematic differ- } \\
\text { ences in baseline charac- } \\
\text { teristics of groups com- } \\
\text { pared? }\end{array}$ & Low risk & No significant baseline differences \\
\hline $\begin{array}{l}\text { Did authors adjust for } \\
\text { baseline differences in } \\
\text { their analyses? }\end{array}$ & Low risk & No information provided \\
\hline Other bias & Unclear risk & No information provided \\
\hline
\end{tabular}

\section{Study characteristics}


Pang 2003 (Continued)

Methods

Study design: RCT

Method of randomisation: not stated

"All the patients were randomly divided into rehabilitation group and control group"

Participants Number of participants: $n=86$

Inclusion criteria: "diagnosed according to diagnostic criteria established in the Fourth National Cerebral Vascular Disease Conference in 1995"

Interventions
(1) Rehabilitation group $(\mathrm{n}=50)$

"Both groups received routine treatment in department of internal neurology. Cerebral edema [was] controlled by $20 \%$ mannitol and support treatment. In rehabilitation group, superearly stage, flaccid paralysis and recovery stage rehabilitation care were performed based on routine internal medicine treatment"

"(i) Superearly stage care: Carefully observe and keep stable life signs and improve basical care within the first 3 days. Keep functional position, including flexion and abduct of shoulder joint, extension of the elbow, dorsal extension of wrist, extension or light flexion of fingers, flexion of knee and hip joint and keep ankle joint in medium position. Turn the body over in regular time and change position, healthy side lateral recumbent position and injured side lateral recumbent position. Still, the patients were required to take injured side lateral recumbent position as much as possible to simulate feeling of the injury side and is advantageble to motion of the healthy side of the body"

"(ii) Flaccid paralysis stage care: After 3 weeks' basic treatment, proximal to distal massaging can be taken based on stable life signs and maintaining functional position. After that, proper short time passive flexion and extension were practiced within the limit of the various articular motion. Motion sequence is started from the bigger joint to the smaller one and stick to from little to large range, proper and step by step principle. Violence was avoided to prevent soft tissue injured. Motion is according to the fatigue status of the patients, with 2 or 3 times a day and 30 min each time"

"(iii) Early rehabilitation care: It is important to perform ADL exercise 3 to 4 weeks after injury. The patients were required to practice initiative and passive motion, including position transversion, balance and functional exercise of upper extremities and hand, overload exercise of the lower extremities and flexion and extension of the hip, knee and ankle joint and language exercise, with 1 time per day and 30 to 60 min each time"

The individual components delivered are listed in Table 3. Based on the individual components, this intervention is categorised as comprising functional task training and musculoskeletal intervention (passive)

Length of intervention period: four weeks

Number of sessions and length of individual sessions: dependent on phase of recovery

Intervention provider: not stated

(2) Control group $(n=36)$

"Patients in the control group were taken routine care during the experiment"

The individual components delivered are listed in Table 3. Based on the individual components, this intervention is categorised as comprising no intervention

Length of intervention period: no intervention

Number of sessions and length of individual sessions: no intervention

Intervention provider: no intervention 
Pang 2003 (Continued)

This study is classified as intervention (functional task training, musculoskeletal (passive)) versus no treatment (Table 4)

Outcomes
Measures of independence in ADL: Barthel Index
Time points when outcomes were assessed: "BI comparison between two groups when were received"
and "BI comparison between two group[s] 4 weeks after reception"

and "BI comparison between two group[s] 4 weeks after reception"

Notes Abstract only

This paper was written in English, and the extracts above are direct quotes

\section{Risk of bias}

\begin{tabular}{|c|c|c|}
\hline Bias & Authors' judgement & Support for judgement \\
\hline $\begin{array}{l}\text { Random sequence genera- } \\
\text { tion (selection bias) }\end{array}$ & Unclear risk & $\begin{array}{l}\text { "All the patients were randomly divided into rehabilitation group and control } \\
\text { group" }\end{array}$ \\
\hline $\begin{array}{l}\text { Allocation concealment } \\
\text { (selection bias) }\end{array}$ & Unclear risk & No information provided \\
\hline $\begin{array}{l}\text { Blinding of outcome as- } \\
\text { sessment (detection bias) } \\
\text { All outcomes }\end{array}$ & Unclear risk & No information provided \\
\hline $\begin{array}{l}\text { Incomplete outcome data } \\
\text { (attrition bias) } \\
\text { All outcomes }\end{array}$ & Unclear risk & No dropouts described \\
\hline $\begin{array}{l}\text { Free of systematic differ- } \\
\text { ences in baseline charac- } \\
\text { teristics of groups com- } \\
\text { pared? }\end{array}$ & Low risk & Baseline demographics comparable between the two groups \\
\hline $\begin{array}{l}\text { Did authors adjust for } \\
\text { baseline differences in } \\
\text { their analyses? }\end{array}$ & Low risk & No information provided \\
\hline Other bias & Unclear risk & No information provided \\
\hline
\end{tabular}

Pang 2006

\section{Study characteristics}

\begin{tabular}{|c|c|}
\hline \multirow[t]{2}{*}{ Methods } & Study design: RCT \\
\hline & Method of randomisation: "according to the enrolled sequence with 3 cases in 1 group" \\
\hline \multirow[t]{3}{*}{ Participants } & Number of participants: $n=80$ \\
\hline & $\begin{array}{l}\text { Inclusion criteria: "Satisfied the } 1995 \text { Fourth National Conference on Cerebral Vascular Disease classifi- } \\
\text { cation guidelines, ischaemic stroke as confirmed by CT or MRI scan and informed consent from partici- } \\
\text { pants" }\end{array}$ \\
\hline & $\begin{array}{l}\text { Exclusion criteria: "Impaired consciousness, unable to comply with assessment and/or treatment, sen- } \\
\text { sory aphasia, European Stroke Scale score }>80 \text {, history of stroke, severe psychological and/or cognitive }\end{array}$ \\
\hline
\end{tabular}


Pang 2006 (Continued)

issues, severe impairment in cardiac liver and kidney function and nil consent from participant or family members"

Interventions

(1) Treatment group $(n=41)$

"The treatment group received cocktail treatment in addition to conventional therapy. Cocktail treatment comprised notoginseng saponin, rehabilitation training, electroacupuncture and hyperbaric oxygen treatment. Notoginseng saponin treatment: Injections given since allocation to treatment group, saline (or 50g/L glucose) $200 \mathrm{~mL}+0.4 \mathrm{~g}$, for 14 consecutive days. Hyperbaric oxygen treatment: using 202 $\mathrm{kPa}$ (gradual pressure increment time $20 \mathrm{~min}$, suction $202 \mathrm{kPa}$ oxygen $20 \mathrm{~min}$, thereafter gradual pressure decrement time $20 \mathrm{~min}$ ), treatment time: 1 /day, $60 \mathrm{~min} /$ session, 5/week, for a total of $10 \mathrm{x}$ "

"Bobath treatment method: using Bobath method of therapy for active, passive rehabilitation training, $1 /$ day, $30 \mathrm{~min} /$ session, $5 /$ week, for a total of $10 x^{\prime \prime \prime}$

"Electroacupuncture treatment: stimulated selected acupuncture points hegu, quchi, zusanli, sanyinjiao, stimulation frequency $0.5 \mathrm{~Hz}$, intermittent, 2-wave amplitude, amplitude $10 \mathrm{~V}$, pulse width $400 \mu \mathrm{s}$, intensity $20 \mathrm{~mA}$. Treatment time arranged as: $1 /$ day, $30 \mathrm{~min} /$ session, $5 /$ week, for a total of $10 \mathrm{x} "$

The individual components delivered are listed in Table 3. Based on the individual components, this intervention is categorised as comprising modality and neurophysiological intervention

Length of intervention period: See above

Number of sessions and length of individual sessions: See above

Intervention provider: not stated

(2) Control group $(n=37)$

"Received conventional therapy (expectant therapy, anti-platelet aggregation, decompression by dehydration, neurotrophy, prevention and cure of complications etc)"

The individual components delivered are listed in Table 3. Based on the individual components, this intervention is categorised as comprising no intervention

Length of intervention period: no intervention

Number of sessions and length of individual sessions: no intervention

Intervention provider: no intervention

This study is classified as intervention (neurophysiological) versus no treatment (Table 4). The intervention group also received modality

Outcomes

Measures of independence in ADL: Barthel Index

Other secondary outcome measures: European Stroke Scale (ESS)

Time points when outcomes were assessed: "before and after intervention"

Notes Original study translated from Chinese to English

Note: Treatment group received both notoginseng saponin treatment and hyperbaric oxygen treatment as well as the physiotherapy components

\section{Risk of bias}

\begin{tabular}{lll}
\hline Bias & Authors' judgement & Support for judgement \\
\hline $\begin{array}{l}\text { Random sequence genera- } \\
\text { tion (selection bias) }\end{array}$ & Unclear risk & $\begin{array}{l}\text { "according to the enrolled sequence with } 3 \text { cases in } 1 \text { group". No further de- } \\
\text { tails provided }\end{array}$ \\
\hline
\end{tabular}


Pang 2006 (Continued)

Allocation concealment High risk Implementation of study design was done by first study author
(selection bias)

\begin{tabular}{|c|c|c|}
\hline $\begin{array}{l}\text { Blinding of outcome as- } \\
\text { sessment (detection bias) }\end{array}$ & High risk & Outcome assessment was done by fourth and fifth study authors \\
\hline
\end{tabular}

sessment (detection bias)

All outcomes

\begin{tabular}{ll}
\hline $\begin{array}{l}\text { Incomplete outcome data } \\
\text { (attrition bias) }\end{array}$ & Low risk \\
All outcomes & Two participants withdrew from control group \\
\hline
\end{tabular}

\begin{tabular}{lll}
\hline $\begin{array}{l}\text { Free of systematic differ- } \\
\text { ences in baseline charac- } \\
\text { teristics of groups com- } \\
\text { pared? }\end{array}$ & Unclear risk & \\
\hline $\begin{array}{l}\text { Did authors adjust for } \\
\text { baseline differences in } \\
\text { their analyses? }\end{array}$ & Unclear risk & No information provided \\
\hline Other bias & High risk & $\begin{array}{l}\text { Treatment group received both notoginseng saponin treatment and hyperbar- } \\
\text { ic oxygen treatment as well as the physiotherapy components }\end{array}$ \\
\hline
\end{tabular}

\section{Pollock 1998}

\section{Study characteristics}

\begin{tabular}{ll} 
Methods & $\begin{array}{l}\text { Study design: } \mathrm{RCT} \\
\text { Method of randomisation: blocked randomisation, with two control (neurophysiology): one interven- } \\
\text { tion (mixed); sealed opaque envelopes numbered and opened sequentially }\end{array}$ \\
\hline Participants & $\begin{array}{l}\text { Number of participants: } \mathrm{n}=28 \\
\text { Inclusion criteria: diagnosis of stroke less than six weeks previously, attending regular physiotherapy } \\
\text { sessions, able to achieve one minute of independent sitting balance, unable to achieve } 10 \text { independent } \\
\text { steps, no known disabilities, pathology or neurological deficit that affected mobility before the current } \\
\text { hospital admission and able to understand the nature of the study and give informed consent }\end{array}$ \\
\hline
\end{tabular}

Interventions

(1) Additional motor learning $(\mathrm{n}=9)$

Independent practice of context-specific tasks

Supervised practice of seated reaching tasks

The individual components delivered are listed in Table 3. Based on the individual components, this intervention is categorised as comprising functional task training

Length of intervention period: four weeks

Number of sessions and length of individual sessions: addition of one hour per day, five days per week, in addition to usual care

Intervention provider: physiotherapists

(2) Usual care (Bobath) $(\mathrm{n}=19)$

Based on assessment by treating physiotherapist: routine care

The individual components delivered are listed in Table 3. Based on the individual components, this intervention is categorised as usual care (neurophysiological intervention) 
Pollock 1998 (Continued)

Length of intervention period: four weeks

Number of sessions and length of individual sessions: usual care, normally once per day, five days per week

Intervention provider: physiotherapists

This study is classified as intervention (functional task training) versus usual care (neurophysiological) (Table 5)

Measures of independence in ADL: Barthel Index
Measures of postural control and balance: symmetry during sitting, standing, rising to stand, sitting
down; weight transference during reaching
Time points when outcomes were assessed: at end of intervention (six weeks)

Notes Participants who were discharged from hospital before the end of the study period (six weeks) were not
followed up, resulting in considerable numbers of dropouts from the study

\section{Risk of bias}

\begin{tabular}{lll}
\hline Bias & Authors' judgement & Support for judgement \\
\hline $\begin{array}{l}\text { Random sequence genera- } \\
\text { tion (selection bias) }\end{array}$ & Low risk & $\begin{array}{l}\text { Blocked randomisation, with two control (neurophysiology): one intervention } \\
\text { (mixed); sealed opaque envelopes numbered and opened sequentially }\end{array}$ \\
\hline $\begin{array}{l}\text { Allocation concealment } \\
\text { (selection bias) }\end{array}$ & Low risk & No information provided \\
\hline $\begin{array}{l}\text { Blinding of outcome as- } \\
\text { sessment (detection bias) } \\
\text { All outcomes }\end{array}$ & High risk & Participant not blinded \\
& & Therapist not blinded \\
\end{tabular}

Incomplete outcome data High risk
(attrition bias)

All outcomes

\section{Dropouts accounted for}

$11 / 19$ in the control group and five of nine in the intervention group completed final assessment

"This pilot study had low numbers of participants, and a relatively high number of withdrawals"

\begin{tabular}{|c|c|c|}
\hline $\begin{array}{l}\text { Free of systematic differ- } \\
\text { ences in baseline charac- }\end{array}$ & Unclear risk & $\begin{array}{l}\text { Difference in gender distribution between the groups, but other characteristics } \\
\text { similar }\end{array}$ \\
\hline
\end{tabular}
teristics of groups compared?

Did authors adjust for $\quad$ Unclear risk No information provided
baseline differences in
their analyses?

\begin{tabular}{ll}
\hline Other bias & High risk \\
& $\begin{array}{l}\text { The control group received no additional treatment, and the treatment group } \\
\text { received the intervention away from the ward and from control group par- } \\
\text { ticipants; therefore it is unlikely that there was any contamination between } \\
\text { groups }\end{array}$ \\
Attendance at the practice sessions was voluntary and varied considerably be- \\
tween participants
\end{tabular}




\section{Study characteristics}

\begin{tabular}{ll}
\hline Methods & Study design: $\mathrm{RCT}$ \\
& Method of randomisation: "by participants drawing lots" \\
\hline Participants & Number of participants: $\mathrm{n}=42$ \\
& Inclusion criteria: not stated \\
\hline
\end{tabular}

"Participants in the treatment group received therapy from a mix of approaches: (i) Exercises focused on the affected limbs with appropriate progression, $20 \mathrm{~min} / \mathrm{session}, 1 /$ day. (ii) Neuromuscular facilitation techniques, including Rood, PNF, Brunnstrom, Bobath and functional stimulative techniques, 20 min/session, 1x/day. (iii) Electrical stimulation: using Auto Move AM800, current set at between 0-60mA into $2.5 \mathrm{k} \Omega$; set at $100-400 \mu \mathrm{s}$; frequency $10-100 \mathrm{~Hz}$; continuous stimulation time at between $2-20 \mathrm{~s}$; resting time between stimulation 2-50s. Intensity is set to as tolerated by participants, with each treatment lasting $20 \mathrm{~min} /$ session, $1 /$ day. (iv) ADL practice: 1 /day. (v) Rehabilitation included limb positioning, regular change in body position in bed etc. (vi) Acupuncture, 1/day"

The individual components delivered are listed in Table 3. Based on the individual components, this intervention is categorised as comprising functional task training, modality, musculoskeletal intervention (passive) and neurophysiological intervention

Length of intervention period: not stated

Number of sessions and length of individual sessions: session length dependent on technique applied (see above)

Intervention provider: "Exercises were assisted by a nurse or family under supervision by a therapist"

(2) Control group $(n=19)$

"The control group received routine rehabilitative instruction only"

The individual components delivered are listed in Table 3. Based on the individual components, this intervention is categorised as comprising no intervention

Length of intervention period: no intervention

Number of sessions and length of individual sessions: no intervention

Intervention provider: no intervention

This study is classified as intervention (functional task training, musculoskeletal (passive), neurophysiological) versus no treatment (Table 4). The intervention group also received modality

Time points when outcomes were assessed: before and one, three and six months after intervention

Notes

Original study translated from Chinese to English 
Qian 2004 (Continued)

No data suitable for analysis

\section{Risk of bias}

Bias Authors' judgement Support for judgement

Random sequence genera- Unclear risk tion (selection bias)

Randomisation done by participants drawing lots. No further details provided

Allocation concealment High risk Implementation of study design was done by study authors

(selection bias)

Blinding of outcome as- Low risk Outcome assessment was blinded

sessment (detection bias)

All outcomes

Incomplete outcome data Unclear risk $\quad$ No dropouts reported
(attrition bias)

All outcomes

$\begin{array}{ll}\text { Free of systematic differ- } & \text { Low risk } \quad \text { No significant baseline differences } \\ \text { ences in baseline charac- } & \end{array}$

teristics of groups com-

pared?

$\begin{array}{lll}\text { Did authors adjust for } & \text { Low risk } & \text { No information provided } \\ \text { baseline differences in } & \end{array}$

their analyses?

Other bias Unclear risk No information provided

Qian 2005

\section{Study characteristics}

\begin{tabular}{|c|c|}
\hline \multirow[t]{2}{*}{ Methods } & Study design: RCT \\
\hline & Method of randomisation: "by participants drawing lots" \\
\hline \multirow[t]{3}{*}{ Participants } & Number of participants: $n=42$ \\
\hline & $\begin{array}{l}\text { Inclusion criteria: "Stroke diagnosis confirmed by clinical assessment and CT scan, participant's in- } \\
\text { formed consent" }\end{array}$ \\
\hline & Exclusion criteria: "Severe premorbid illness, cognitive impairment and severe complications" \\
\hline \multirow[t]{2}{*}{ Interventions } & (1) Therapy group $(n=20)$ \\
\hline & $\begin{array}{l}\text { "Regular rehabilitation therapy: (i) therapeutic exercise for hemiplegia, with therapeutic exercises cor- } \\
\text { responding to different stages, } 20 \text { min each time, once a day. (ii) neuro-muscular stimulative technique, } \\
\text { including Rood technique, PNF technique, Bobath technique etc, } 20 \text { min each time, once a day. (iii) gait } \\
\text { training, } 20 \text { min each time, once a day. (iv) ADL training, } 20 \text { min each time, once a day. (v) functional } \\
\text { electrical stimulation, using Beijing-produced J18A1 model computerised pulse therapy device, with } \\
\text { specifications: mid-frequency pulse frequency } 4 \mathrm{kHz} \text {, low-frequency pulse frequency } 1 / 5-150 \mathrm{~Hz} \text {; tune } \\
\text { shape of wave to square wave, exponential wave, triangle wave; peak output current: } 50 \mathrm{~mA}<\mathrm{Current} \\
<100 \mathrm{~mA} \text { (resistance } 500 \Omega \text { ); tuning method: continuous, pause, rest, change settings; strength control: } \\
\text { continuous control; working voltage: main electricity } 220 \mathrm{~V} \text {, power } \leq 15 \mathrm{~W} \text {. Two working electrodes were }\end{array}$ \\
\hline
\end{tabular}


Qian 2005 (Continued)

placed on two ends of muscle to be stimulated, at the tendons, strength set at patient's limit of tolerance, with treatment time being 20 min each time, once a day. (vi) acupuncture, once a day"

"In addition flexor reflexes were used to provide therapy for the lower limbs, as described: the therapist used a hand or hard object, without damaging the skin, to stimulate the affected side's sole on the plantar side, such that the patient felt a tolerable discomfort, and on lower extremities of patient's affected side, hip flexion, knee flexion and ankle dorsiflexion would be induced; force would increase from light to heavy, with focus on active participation by patient; each time lasting 10s, 30s between each practice; repeated practice for 20 min, once a day"

The individual components delivered are listed in Table 3. Based on the individual components, this intervention is categorised as comprising functional task training, modality, neurophysiological intervention

Length of intervention period: not stated

Number of sessions and length of individual sessions: session length dependent on technique applied (see above)

Intervention provider: therapist

(2) Control group $(n=20)$

"Received regular rehabilitation therapy (as above)"

The individual components delivered are listed in Table 3. Based on the individual components, this intervention is categorised as comprising usual care (functional task training, modality and neurophysiological intervention)

Length of intervention period: not stated

Number of sessions and length of individual sessions: session length dependent on technique applied (see above)

Intervention provider: therapist

This study is classified as intervention (functional task training, neurophysiological) versus usual care (functional task training, neurophysiological) (Table 5). Both intervention groups also received modality

Outcomes Measures of motor function: Fugl-Meyer Assessment (lower limb)

Time points when outcomes were assessed: before and one month after intervention

Notes $\quad$ Original study translated from Chinese to English

\section{Risk of bias}

Bias Authors' judgement Support for judgement

$\begin{aligned} & \text { Random sequence genera- } \\ & \text { tion (selection bias) }\end{aligned}$
Unclear risk Randomisation done by participants drawing lots. No further details provided

\begin{tabular}{lll}
\hline $\begin{array}{l}\text { Allocation concealment } \\
\text { (selection bias) }\end{array}$ & High risk & Implementation of study design was done by first study author \\
\hline $\begin{array}{l}\text { Blinding of outcome as- } \\
\text { sessment (detection bias) } \\
\text { All outcomes }\end{array}$ & Low risk & Outcome assessment was blinded \\
\hline
\end{tabular}

Incomplete outcome data Unclear risk $\quad$ No dropouts described
(attrition bias)


Qian 2005 (Continued)

All outcomes

Free of systematic differ- Low risk No significant baseline differences
ences in baseline charac-
teristics of groups com-
pared?

\begin{tabular}{lll}
\hline $\begin{array}{l}\text { Did authors adjust for } \\
\text { baseline differences in } \\
\text { their analyses? }\end{array}$ & Low risk & \\
\hline Other bias & Unclear risk & No information provided \\
\hline
\end{tabular}

Richards 1993

\section{Study characteristics}

\begin{tabular}{ll} 
Methods & $\begin{array}{l}\text { Study design: RCT } \\
\text { Method of randomisation: sealed envelopes, opened remotely by telephone request } \\
\text { Blocked randomisation, stratified according to prognostic category, with randomly varying block size }\end{array}$ \\
\hline Participants & $\begin{array}{l}\text { Number of participants: } \mathrm{n}=27 \\
\text { Inclusion criteria: middle cerebral artery infarct, confirmed by CT scan, living less than } 50 \mathrm{~km} \text { from Que- } \\
\text { bec, between } 40 \text { and } 80 \text { years old, zero to seven days since onset of stroke, no other neurological prob- } \\
\text { lems, no major medical problems that would interfere with rehabilitation, not independent in ambula- } \\
\text { tion and not unconscious at onset }\end{array}$ \\
\hline
\end{tabular}

Interventions (1) Early-mixed $(\mathrm{n}=10)$

Intensive and focused

"Goal was to promote gait relearning through locomotor activities that were adapted to the individual level of motor recovery"

Techniques included tilt table; limb load monitor, resistive exercises, with isokinetic exercises; treadmill training

The individual components delivered are listed in Table 3. Based on the individual components, this intervention is categorised as comprising functional task training, modality, musculoskeletal intervention (active) and musculoskeletal intervention (passive)

Length of intervention period: whilst inpatient

Number of sessions and length of individual sessions: 'intensive'

Intervention provider: physiotherapists

(2) Early-neurophysiological (Bobath) $(n=8)$

Techniques not described but communication with the study author confirms this intervention as 'neurodevelopmental or Bobath'

The individual components delivered are listed in Table 3. Based on the individual components, this intervention is categorised as comprising neurophysiological intervention

Length of intervention period: whilst inpatient

Number of sessions and length of individual sessions: 'intensive'

Intervention provider: physiotherapists 
(3) Conventional-neurophysiological (Bobath) $(\mathrm{n}=9)$

The individual components delivered are listed in Table 3. Based on the individual components, this intervention is categorised as comprising usual care (neurophysiological intervention)

Length of intervention period: whilst inpatient

Number of sessions and length of individual sessions: as usual care (less than treatment groups)

Intervention provider: physiotherapists

This study is classified as active Intervention one (functional task training, musculoskeletal (active), musculoskeletal (passive)) versus active intervention two (neurophysiological) (Table 6) versus usual care (neurophysiological) (Table 5). Active intervention group one also received modality

Measures of independence in ADL: Barthel Index
Measures of functional independence: Fugl-Meyer Motor Assessment
Measures of postural control and balance: Berg Balance Scale
Measures of voluntary movement: temporal gait parameters
Other secondary outcome measures: Canadian Stroke Scale
Time points when outcomes were assessed: "Gait movements and muscle activations were evaluated
in the Motor Evaluation Laboratory six weeks, three months, and six months after stroke"

Notes

Analysis based on comparison of neurophysiological (early) with mixed (early), as these two groups are comparable in terms of timing and intensity

\section{Risk of bias}

\begin{tabular}{lll}
\hline Bias & Authors' judgement & Support for judgement \\
\hline $\begin{array}{l}\text { Random sequence genera- } \\
\text { tion (selection bias) }\end{array}$ & Low risk & Sealed envelopes, opened remotely by telephone request \\
\hline $\begin{array}{l}\text { Allocation concealment } \\
\text { (selection bias) }\end{array}$ & Low risk & No information provided \\
\hline $\begin{array}{l}\text { Blinding of outcome as- } \\
\text { sessment (detection bias) } \\
\text { All outcomes }\end{array}$ & Low risk & Unclear whether participant was blinded \\
& & Therapist not blinded \\
\hline
\end{tabular}

\begin{tabular}{|c|c|c|}
\hline $\begin{array}{l}\text { Incomplete outcome data } \\
\text { (attrition bias) } \\
\text { All outcomes }\end{array}$ & Unclear risk & $\begin{array}{l}23 / 27 \text { completed intervention } \\
\text { Dropouts not accounted for } \\
\text { Participants with missing data were dropped from analysis }\end{array}$ \\
\hline
\end{tabular}

\begin{tabular}{|c|c|c|}
\hline $\begin{array}{l}\text { Free of systematic differ- } \\
\text { ences in baseline charac- } \\
\text { teristics of groups com- } \\
\text { pared? }\end{array}$ & Unclear risk & No information provided \\
\hline $\begin{array}{l}\text { Did authors adjust for } \\
\text { baseline differences in } \\
\text { their analyses? }\end{array}$ & Unclear risk & No information provided \\
\hline Other bias & Unclear risk & $\begin{array}{l}\text { The same two therapists provided treatment to both treatment groups, creat- } \\
\text { ing the possibility of contamination between groups }\end{array}$ \\
\hline
\end{tabular}


Salbach 2004

\section{Study characteristics}

\begin{tabular}{ll}
\hline Methods & Study design: RCT \\
& Method of randomisation: stratified blocked randomisation, stratified according to three levels of walk- \\
& ing deficit \\
& Computer-generated numbers in sealed opaque envelopes, managed by person not involved in the \\
study
\end{tabular}

\section{Participants}

Number of participants: $\mathrm{n}=91$

Inclusion criteria: clinical diagnosis of stroke, less than one year post stroke, residual walking deficit but able to walk $10 \mathrm{~m}$ (with or without aid or supervision), discharged from physical rehabilitation and living in community

Interventions

(1) Motor learning (mobility) $(n=44)$

Task-orientated training of walking

"Standardised programme, supervised by a physical or occupational therapist, of 10 walking-related tasks designed to strengthen the lower extremities and enhance walking balance, speed and distance in a progressive manner"

The individual components delivered are listed in Table 3. Based on the individual components, this intervention is categorised as comprising cardiopulmonary intervention, functional task training and musculoskeletal intervention (active)

Length of intervention period: six weeks

Number of sessions and length of individual sessions: 18 sessions given three times per week. Recommended that participants carry over walking component of the programme to home

Intervention provider: physical or occupational therapist

(2) Placebo control (motor learning, upper limb) $(n=47)$

Functional upper extremity tasks, done in sitting

The individual components delivered are listed in Table 3. Based on the individual components, this intervention is categorised as comprising upper limb training

Length of intervention period: six weeks

Number of sessions and length of individual sessions: 18 sessions given three times per week. Recommended that participants carry over walking component of the programme to home

Intervention provider: physical or occupational therapist

This study is classified as intervention (functional task training, musculoskeletal (active)) versus attention control (upper limb) (Table 5). The intervention group also received cardiopulmonary intervention

Outcomes Measures of postural control and balance: Berg Balance Scale

Measures of voluntary movement: gait speed

Time points when outcomes were assessed: "evaluations were conducted by trained evaluators at baseline, and on completion of the intervention (mean four days)"

Notes

Intervention two comprised upper limb training. Although upper limb training might be classed as a subcomponent of functional task training, for the purposes of comparisons in this review, we have categorised this intervention as an attention control. This is because the upper limb training was delivered alone (i.e. no other subcomponents of functional task training were delivered), and therefore no active treatment was aimed at lower limb or balance outcomes 
Salbach 2004 (Continued)

Risk of bias

\begin{tabular}{|c|c|c|}
\hline Bias & Authors' judgement & Support for judgement \\
\hline $\begin{array}{l}\text { Random sequence genera- } \\
\text { tion (selection bias) }\end{array}$ & Low risk & $\begin{array}{l}\text { Computer-generated numbers in sealed opaque envelopes, managed by per- } \\
\text { son not involved in the study }\end{array}$ \\
\hline $\begin{array}{l}\text { Allocation concealment } \\
\text { (selection bias) }\end{array}$ & Low risk & No information provided \\
\hline $\begin{array}{l}\text { Blinding of outcome as- } \\
\text { sessment (detection bias) } \\
\text { All outcomes }\end{array}$ & High risk & $\begin{array}{l}\text { Participant not blinded } \\
\text { Therapist not blinded } \\
\text { Assessor was blinded } \\
\text { However, unblinding of the outcome evaluators occurred for } 18 \text { of } 42 \text { mobility } \\
\text { and } 16 \text { of } 43 \text { upper extremity evaluations }\end{array}$ \\
\hline $\begin{array}{l}\text { Incomplete outcome data } \\
\text { (attrition bias) } \\
\text { All outcomes }\end{array}$ & Unclear risk & $\begin{array}{l}\text { Dropouts accounted for } \\
\text { 84/91 completed intervention }\end{array}$ \\
\hline $\begin{array}{l}\text { Free of systematic differ- } \\
\text { ences in baseline charac- } \\
\text { teristics of groups com- } \\
\text { pared? }\end{array}$ & Unclear risk & No information provided \\
\hline $\begin{array}{l}\text { Did authors adjust for } \\
\text { baseline differences in } \\
\text { their analyses? }\end{array}$ & Unclear risk & No information provided \\
\hline
\end{tabular}

Shin 2011

\section{Study characteristics}

\begin{tabular}{ll}
\hline Methods & Study design: RCT \\
& Method of randomisation: "subjects were randomly allocated to one of into two groups" \\
\hline Participants & Number of participants: $\mathrm{n}=21$ \\
& Inclusion criteria: "Subjects who were between 6 months and 5 years since diagnosis of stroke, subjects \\
& with hemiplegia of the lower extremities" \\
& Exclusion criteria: "subjects who could not ride a bicycle or perform functional exercise due to arthri- \\
tis, low-back pain, or degenerative joint disease; subjects who were receiving medical treatment due \\
to other symptoms; and subjects who could not follow the instructions due to low perceptive abilities, \\
cognitive disorder, or communication disorder"
\end{tabular}

Interventions

(1) Combined exercise training group $(n=11)$

"Exercise combined with aerobic and functional strengthening exercises for balance"

"The first exercise was 30 min of functional strength training, consisting of six sub-categories: bridge exercise, lifting toes, and ankles, sitting and standing, stretching out the arms while standing, step ex- 
ercise, and stairs exercise. Bridge exercise is lifting pelvis using the legs, from bending hips and knees with supine. Lifting toes and ankles is dorsiflexion and plantar flexion of the hemiplegic leg in the sitting position. Sitting and standing is standing from sitting and sitting again until the hip touches chair. Stretching out the arms while standing is stretching out the arms upward, downward, right-side, leftside and diagonally. Step exercise is shifting of weight bearing to a leg on a step. The hemiplegic leg and non-hemiplegic leg are placed in turn on the step and the location of step alternates from the front to one side of the subject. Stairs exercise is walking up stairs with the hemiplegic leg supporting the body weight and walking down stairs with the non-hemiplegic leg support body weight. Before exercise, 5 minutes warming-up exercise of breathing exercise and stretching were conducted. Each exercise was repeated at medium intensity without fatigue ten to fifteen times. The second exercise for the combined exercise training group was aerobic exercise. Treadmill walking and riding a bicycle were conducted for fifteen minutes each. Treadmill walking started at $0.5 \mathrm{~m} / \mathrm{s}$ and the initial 5 minutes was on adaption period. In the next 10 minutes walking velocity increased or walking was done with less support from the hand-rail. A stationary bicycle was used for the bicycle riding exercise. At the beginning a patient started with a velocity which he/she could feel comfortable with. As time went by the velocity was increased. The intensity of the two aerobic exercises was determined by checking the heart rate"

The individual components delivered are listed in Table 3. Based on the individual components, this intervention is categorised as comprising cardiopulmonary intervention, functional task training and musculoskeletal intervention (active)

Length of intervention period: four weeks

Number of sessions and length of individual sessions: 60 minutes per day, five times a week

Intervention provider: physical therapist ("aerobic exercise was conducted with the assistance of a caregiver or family member under the supervision of a physical therapist")

(2) Conventional exercise group $(n=10)$

"Special instructions were not given to the physical therapist in charge, and the conventional training was done as usual. The therapist focused on re-educating normal movement during functional activities that were meaningful to the patients. Training was composed of balance exercise, posture control exercise, and gait exercise. Keeping normal movement of the pelvis for balance and posture control was emphasized. The therapist judged that working on increasing anterior and posterior pelvic tilt would improve weight transfer and hip extension during gait, leading to improvements in selective distal control of the knee and the foot. Trunk control and alignment can affect muscle tone, range of motion, and control of the limb. For the upper limbs, treatment was conducted focusing on movement of the scapular. For balance exercise, weight transfer exercise and reaching exercise were alternately performed on the affected side and the unaffected side in the sitting or standing position. Bridging exercise was performed to strengthen the trunk muscles. Selective movement of each joint of the shoulder, elbow, knee and ankle joint was performed to facilitate upper and lower limb movement. For gait exercise, training in weight transfer during gait was conducted by planting the unaffected side foot at the front and back of the body. Gait training was divided between the stance phase and the swing phase, and exercises for each phase were performed. In addition, stair climbing practice and gait training for crossing obstacles were conducted. Patients did not do the same exercise every training day but suitable exercises were selected according to the goals of each patient and the therapist. The intensity of each exercise was decided by the therapist considering each patient's capacity for exercise"

The individual components delivered are listed in Table 3. Based on the individual components, this intervention is categorised as comprising functional task training, musculoskeletal intervention (active) and neurophysiological intervention

Length of intervention period: four weeks

Number of sessions and length of individual sessions: 60 minutes per day, five times a week Intervention provider: physical therapist 
Shin 2011 (Continued)

This study is classified as active intervention one (functional task training, musculoskeletal (active)) versus active intervention two (functional task training, musculoskeletal (active), neurophysiological) (Table 6). Active intervention group one also received cardiopulmonary intervention

\begin{tabular}{ll}
\hline Outcomes & $\begin{array}{l}\text { Measures of postural control and balance: Berg Balance Scale (dynamic balance), force platform (static } \\
\text { balance) }\end{array}$ \\
& $\begin{array}{l}\text { Time points when outcomes were assessed: "start of the intervention..and after completion of the 4- } \\
\text { week intervention" }\end{array}$ \\
\hline Notes & $\begin{array}{l}\text { Note: The conventional exercise group was categorised as comprising 'neurophysiological' compo- } \\
\text { nents, as a description of facilitation of 'normal movement' was provided. However, this was refer- } \\
\text { enced to Bobath/Davies }\end{array}$
\end{tabular}

\section{Risk of bias}

\begin{tabular}{|c|c|c|}
\hline Bias & Authors' judgement & Support for judgement \\
\hline $\begin{array}{l}\text { Random sequence genera- } \\
\text { tion (selection bias) }\end{array}$ & Unclear risk & "subjects were randomly allocated to one of two groups" \\
\hline $\begin{array}{l}\text { Allocation concealment } \\
\text { (selection bias) }\end{array}$ & Unclear risk & No information provided \\
\hline $\begin{array}{l}\text { Blinding of outcome as- } \\
\text { sessment (detection bias) } \\
\text { All outcomes }\end{array}$ & Unclear risk & No information provided \\
\hline $\begin{array}{l}\text { Incomplete outcome data } \\
\text { (attrition bias) } \\
\text { All outcomes }\end{array}$ & Unclear risk & No dropouts described \\
\hline $\begin{array}{l}\text { Free of systematic differ- } \\
\text { ences in baseline charac- } \\
\text { teristics of groups com- } \\
\text { pared? }\end{array}$ & Low risk & $\begin{array}{l}\text { "the pre-intervention dynamic balance of the two groups was not significantly } \\
\text { different" }\end{array}$ \\
\hline $\begin{array}{l}\text { Did authors adjust for } \\
\text { baseline differences in } \\
\text { their analyses? }\end{array}$ & Low risk & No information provided \\
\hline Other bias & Unclear risk & No information provided \\
\hline
\end{tabular}

\section{Stephenson 2004}

\section{Study characteristics}

\begin{tabular}{ll}
\hline Methods & Study design: RCT with three groups \\
& Method of randomisation: not stated ("Subjects were randomized into one of three groups") \\
\hline Participants & $\begin{array}{l}\text { Number of participants: } \mathrm{n}=18 \\
\text { Inclusion criteria: not stated ("Eighteen subjects with chronic }(>6 \text { mo) stroke participated in this } \\
\text { study") }\end{array}$ \\
\hline Interventions & (1) Body weight support treadmill training $(\mathrm{n}=6)$
\end{tabular}


"whole task approach"

"gait training on a treadmill while an overhead harness supported a percentage of the subject's body weight $(<30 \%) "$

The individual components delivered are listed in Table 3. Based on the individual components, this intervention is categorised as comprising functional task training

Length of intervention period: four weeks

Number of sessions and length of individual sessions: 20 minutes, three times a week

Intervention provider: not stated

(2) Proprioceptive neuromuscular facilitation (PNF) training $(n=6)$

"part task approach"

"received PNF resisted mat activities and PNF gait training"

The individual components delivered are listed in Table 3. Based on the individual components, this intervention is categorised as comprising functional task training and neurophysiological intervention

Length of intervention period: four weeks

Number of sessions and length of individual sessions: 20 minutes, three times a week

Intervention provider: not stated

(3) Control group $(n=6)$

"no physical therapy interventions between pre- and post-tests"

The individual components delivered are listed in Table 3. Based on the individual components, this intervention is categorised as comprising no intervention

Length of intervention period: no intervention

Number of sessions and length of individual sessions: no intervention

Intervention provider: no intervention

This study is classified as intervention (functional task training, neurophysiological) versus no treatment (Table 4)

Notes Abstract only

Body weight support treadmill training is not relevant to this review (studies of treadmill training have been excluded), and this intervention therefore is not included within this review

\section{Risk of bias}

\section{Bias}

Random sequence generation (selection bias)

\section{Authors' judgement Support for judgement}

No information provided

"Subjects were randomized into one of three groups" 
Stephenson 2004 (Continued)

\begin{tabular}{l}
$\begin{array}{l}\text { Allocation concealment } \\
\text { (selection bias) }\end{array}$ \\
\hline
\end{tabular}

\begin{tabular}{lll}
\hline Blinding of outcome as- & Unclear risk & No information provided \\
sessment (detection bias) &
\end{tabular}

All outcomes

\begin{tabular}{l}
\hline Incomplete outcome data $\quad$ Unclear risk $\quad$ No dropouts described \\
(attrition bias) \\
All outcomes
\end{tabular}

Free of systematic differ- Unclear risk No information provided
ences in baseline characteristics of groups compared?

Did authors adjust for Unclear risk No information provided
baseline differences in
their analyses?

\begin{tabular}{|c|c|c|}
\hline Other bias & Unclear risk & No information provided \\
\hline
\end{tabular}

Tang 2009

\section{Study characteristics}

\begin{tabular}{ll}
\hline Methods & Study design: RCT \\
& Method of randomisation: not stated \\
\hline Participants & Number of participants: $\mathrm{n}=70$ \\
Inclusion criteria: "Satisfied the Fourth National Conference on Cerebral Vascular Disease classification \\
guidelines and first ever stroke as confirmed by CT or MRI scan" \\
Exclusion criteria: "Obvious barriers to understanding and severe pathological changes to organs"
\end{tabular}

Interventions (1) Observation group $(\mathrm{n}=35)$

"Both groups received standard pharmacotherapy and motor function training using mainly Bobath method: including positioning of non-affected limbs and training on bed; transfer training; stepping training and gait re-education; occupational training and ADL training. In addition, the observation group also received sensory function training

Superficial sensation training: (i) Using tip of large-headed pin with constant force to lightly poke the skin on affected side, and compare with non-affected side; (ii) Using cotton swab to lightly touch the affected side's skin and mucosa; (iii) Using towel soaked in hot water $\left(40^{\circ} \mathrm{C}-50^{\circ} \mathrm{C}\right)$ and cold water $\left(5^{\circ} \mathrm{C}-\right.$ $10^{\circ} \mathrm{C}$ ) to wipe and train sensation of temperature; (iv) During the early period especially during flaccid paralysis period, light tapping, hitting, light touching, rapid brushing, etc, performed on affected limbs. Training could be done initially with eyes closed, but if there was obvious difficulty, could be trained with eyes opened instead, with training done with eyes closed after there is improvement, such that there is repeated training, following eyes closed -> eyes opened -> eyes closed sequence"

"Deep sensation training: (i) Maintenance of non-affected limb position during early period, with appropriate increase in time spent lying on affected side, giving extra protection of affected limbs when changing position; (ii) Using fingers, therapist lightly held patient's affected side's finger or toe to perform passive ranging exercises, or placed affected limbs in a particular position, to allow patient to feel the position of his limbs, while staying motionless, training repeatedly until the patient himself 
could complete certain movements with affected limb, being especially useful for low muscle tone and poor control of lower limbs; (iii) While sitting or standing, paying attention to weight borne by affected limbs, and while performing active or passive ranging exercises, could also use an elastic strap to wrap and place pressure on joint being moved"

"Touch sensation training: once patient had recovered sensation on fingers, this training could immediately commence. (i) Patient closed eyes and used touch to identify common objects such as key, pen, toothbrush, button, etc, and if unable to identify, could also allow touching with eyes opened or using non-affected hand to touch; (ii) Plastic pieces, paper, cloth and fur, etc, were mixed together, for patient to identify using touch with eyes closed, and if there is error in identification, could similarly use non-affected hand or open eyes; (iii) Patient showed a picture, and then asked to find a similar object in a black box"

"Balance training: using balance assessment training system, based on patient's condition, either sitting or standing position was used, initially using corresponding pressure sensor, patient focused on the display unit during training, adjusted their own position according to change in display lights on display unit, to undergo training on maintenance of centre of gravity, shifting of centre of gravity, single-leg weight-bearing, etc. After becoming stable with eyes opened, this training could also be performed with eyes closed"

The individual components delivered are listed in Table 3. Based on the individual components, this intervention is categorised as comprising functional task training, musculoskeletal intervention (passive) and neurophysiological intervention

Length of intervention period: eight weeks

Number of sessions and length of individual sessions: one time a day, each time 45 minutes

Intervention provider: therapist

(2) Control group $(n=35)$

"Control group used standard pharmacotherapy and motor function training using mainly Bobath method: including positioning of non-affected limbs and training on bed; changing of position training; ambulatory training and gait re-education; occupational training and ADL training"

The individual components delivered are listed in Table 3. Based on the individual components, this intervention is categorised as comprising functional task training, musculoskeletal intervention (passive) and neurophysiological intervention

Length of intervention period: not stated

Number of sessions and length of individual sessions: not stated

Intervention provider: not stated

This study is classified as intervention (functional task training, musculoskeletal (passive), neurophysiological) versus usual care (functional task training, musculoskeletal (passive), neurophysiological) (Table 5)

Outcomes Measures of motor function: Fugl-Meyer Assessment (sensory, motor)

Time points when outcomes were assessed: before and after eight weeks of intervention

Notes Original study translated from Chinese to English

\section{Risk of bias}

\begin{tabular}{lll}
\hline Bias & Authors' judgement & Support for judgement \\
\hline $\begin{array}{l}\text { Random sequence genera- } \\
\text { tion (selection bias) }\end{array}$ & Unclear risk & No information provided \\
\hline
\end{tabular}


Tang 2009 (Continued)

$\begin{aligned} & \text { Allocation concealment } \\ & \text { (selection bias) }\end{aligned}$
Unclear risk

\begin{tabular}{|c|c|c|}
\hline $\begin{array}{l}\text { Blinding of outcome as- } \\
\text { sessment (detection bias) } \\
\text { All outcomes }\end{array}$ & Unclear risk & No information provided \\
\hline
\end{tabular}

\begin{tabular}{l}
\hline $\begin{array}{l}\text { Incomplete outcome data } \\
\text { (attrition bias) }\end{array}$ \\
All outcomes
\end{tabular}

\begin{tabular}{lll}
$\begin{array}{l}\text { Free of systematic differ- } \\
\text { ences in baseline charac- } \\
\text { teristics of groups com- }\end{array}$ & Low risk \\
pared? & \\
\hline $\begin{array}{l}\text { Did authors adjust for } \\
\text { baseline differences in } \\
\text { their analyses? }\end{array}$ & Low risk & No information provided \\
\hline Other bias & Unclear risk & No information provided \\
\hline
\end{tabular}

\section{Study characteristics}

Methods
Study design: RCT
Method of randomisation: "2 research centers in Germany and the United States were selected by a
random number table. Patients were randomly assigned to either the experimental (RAS; $\mathrm{n}=43 ;$ male
$=22$, female $=21$ ) or control (neurodevelopmental technique/Bobath; $\mathrm{n}=35 ;$ male $=19, \mathrm{female}=16)$
training group (see Table 1$)$. Treatment allocation was accomplished by computerized random number
generators in both centers"

Participants
Number of participants: $n=78$
Inclusion criteria: not stated ("from an eligible catchment pool of 155 patients")
"Patients entered the study within 4 weeks of onset, as soon as they could complete 5 stride cycles with handheld assistance by the therapist, that is, with no more than support of the forearm, wrist, and el- bow at approximately 90 degrees of elbow flexion on the nonparetic side. Handheld assistance was available to all patients throughout training when needed"

Interventions

(1) Rhythmic auditory stimulation $(n=43)$

"RAS training followed established protocols using a metronome and specifically prepared music tapes in digital MIDI format to ensure temporal precision and tempo stability as well as full capacity for frequency modulation of the stimulus based on patient needs. After an initial cadence assessment, cuing frequencies were matched to the gait cadence for the first quarter of the session. During the second quarter, cue frequencies were increased in $5 \%$ increments as kinematically indicated without compromising postural and dynamic stability. During the third quarter, adaptive gait patterns, for example, ramp or step walking, were practiced. The last quarter was spent fading the cues intermittently to train for independent carryover"

The individual components delivered are listed in Table 3. Based on the individual components, this intervention is categorised as comprising functional task training

Length of intervention period: three weeks 
Thaut 2007 (Continued)

Number of sessions and length of individual sessions: "gait training daily for 30 minutes, 5 times per week"

Intervention provider: "Four gait therapists for each group conducted the training to ensure consistency in training protocols and procedures. Each center had its own independently trained pool of therapists"

(2) Neurodevelopmental therapy (NDT)/Bobath-based training $(n=35)$

"control group trained the same amount of time and distance, following NDT and Bobath principles as well as using similar instructions about gait parameters to practice, but without rhythmic auditory cuing"

The individual components delivered are listed in Table 3. Based on the individual components, this intervention is categorised as comprising functional task training and neurophysiological intervention

Length of intervention period: three weeks

Number of sessions and length of individual sessions: "gait training daily for 30 minutes, 5 times per week"

Intervention provider: "Four gait therapists for each group conducted the training to ensure consistency in training protocols and procedures. Each center had its own independently trained pool of therapists"

This study is classified as active intervention one (functional task training) versus active intervention two (functional task training, neurophysiological) (Table 6)

Measures of independence in ADL: Barthel Index
Measures of motor function: Fugl-Meyer Assessment
Measures of voluntary movement: gait velocity, stride length, cadence and symmetry
Time points when outcomes were assessed: "All patients were tested 1 day before the training sessions
started and 1 day after the last training session"
"All available participant data after removing dropout participants were analyzed in an intention-to-
treat analysis"

\section{Risk of bias}

Bias Authors' judgement Support for judgement

Random sequence genera- Low risk "selected by a random number table" tion (selection bias)

$\begin{array}{ll}\begin{array}{l}\text { Allocation concealment } \\ \text { (selection bias) }\end{array} & \begin{array}{l}\text { "Treatment allocation was accomplished by computerized random number } \\ \text { generators in both centers. Random numbers for the allocation-to-treatment } \\ \text { sequence were concealed from the recruiter and the therapists carrying out } \\ \text { the training. Patients were informed of the } 2 \text { possible treatment allocations } \\ \text { but blinded to the aims of an experimental versus control condition" }\end{array} \\ & \text { "Therapists were not blinded to the treatment conditions of the study. Howev- } \\ \text { er, because both conditions are considered full treatment conditions, no per- } \\ \text { formance bias was expected" }\end{array}$


Thaut 2007 (Continued)

$\begin{array}{ll}\begin{array}{l}\text { Incomplete outcome data } \\ \text { (attrition bias) }\end{array} & \text { Droph risk } \\ \text { All outcomes } & \text { "Dropout rate in one center was } 23 \% \text { of initially included patients. There was } \\ & \text { a } 10 \% \text { dropout rate in the other center. Dropout reasons were due to hospital } \\ \text { transfer, early discharge, medical complication, or unspecified personal rea- } & \text { sons" }\end{array}$

\begin{tabular}{|c|c|c|}
\hline $\begin{array}{l}\text { Free of systematic differ- } \\
\text { ences in baseline charac- } \\
\text { teristics of groups com- } \\
\text { pared? }\end{array}$ & Low risk & $\begin{array}{l}\text { "Lesion site was closely matched in both groups. Mild to moderate sensory } \\
\text { dysfunction was present in all middle cerebral artery distribution strokes. Both } \\
\text { groups had lower limb spasticity, most pronounced in knee flexors/extensors, } \\
\text { plantar flexion, and hip flexors/extensors, as typical for a stage } 4 \text { or early stage } \\
3 \text { on the Brunnstrom hemiplegia recovery scale" }\end{array}$ \\
\hline
\end{tabular}

\begin{tabular}{lll}
\hline Did authors adjust for $\quad$ Low risk information provided & No
\end{tabular}

baseline differences in

their analyses?

\begin{tabular}{lll}
\hline Other bias & Unclear risk & No information provided \\
\hline
\end{tabular}

\section{Torres-Arreola 2009}

\section{Study characteristics}

\begin{tabular}{ll}
\hline Methods & Study design: RCT \\
& $\begin{array}{l}\text { Method of randomisation: "Each patient was randomly allocated to one strategy after they had given } \\
\text { written informed consent and completed questionnaires and they were randomised by the coordinator } \\
\text { of the study using consecutive opaque envelopes, which were chosen by the patients or their relatives" }\end{array}$ \\
\hline Participants & Number of participants: $\mathrm{n}=110$ \\
& $\begin{array}{l}\text { Inclusion criteria: "age } 40 \text { years or older; diagnosis of first event and ischaemic stroke, mild and moder- } \\
\text { ate neurological damage. Age criterion was decided on frequency and type of stroke, as haemorrhagic } \\
\text { type is more frequent in younger patients" }\end{array}$ \\
\hline
\end{tabular}

Interventions (1) Strategy one (S1) $(\mathrm{n}=59)$

"The team of two trained nurses (see notes) applied one of the two rehabilitation strategies to each patient, beginning 72 hours after initial hospitalisation, when the patient's clinical condition had been stabilised"

"The first strategy (S1) included two parts: education and physiotherapy. The education portion consisted of providing information on general care such as feeding, hygiene and mobilisation through a Manual of Physical Rehabilitation for the patient with Stroke and also verbally and through a practical rehabilitation training session provided by the nurse to the caregiver. This was an individual plan of daily rehabilitation according to the stage of Brunnstrom's classification that the caregiver had to perform. The Manual was drafted by the research group, especially for this study. The physiotherapy part of the strategy was designed and applied based on the basal condition of the patients and their stage of physical recovery. The latter was defined using the Brunnstrom scale (I-III), which reflects voluntary activity, movement, coordination, postural stability, muscular tonicity and sphincter control (further details supplied in Table 1). The physiotherapy provided by nurse was divided into three phases. The intensive phase consisted of daily physical therapy initiated at the hospital and continued for two weeks postdischarge through in-home visits. The intermediate phase consisted of bi-weekly inhome visits during the following two weeks (weeks three and four postdischarge). Finally, the support phase consisted of weekly in-home visits during the following three months, up to four months postdischarge" 
The individual components delivered are listed in Table 3. Based on the individual components, this intervention is categorised as comprising assistive devices, functional task training, musculoskeletal intervention (active) and musculoskeletal intervention (passive)

Length of intervention period: four months

Number of sessions and length of individual sessions: "average number of visits for the S1 group was 15 and the average duration of each visit had to be about 90 minutes"

Intervention provider: nurse (see notes) and caregiver

(2) Strategy two (S2) ( $=51)$

"The second strategy (S2) consisted of education alone. The information and individual plan of rehabilitation provided by the nurse to the patient and caregiver were the same as in the S1 group. This intervention also began at the hospital and was continued in-home with weekly visits"

The individual components delivered are listed in Table 3. Based on the individual components, this intervention is categorised as comprising no intervention

Length of intervention period: four months

Number of sessions and length of individual sessions: "The average number of visits in this group was eight and the average duration of each visit was approximately 45 minutes"

Intervention provider: nurse and caregiver

This study is classified as intervention (functional task training, musculoskeletal (active), musculoskeletal (passive)) versus no treatment (Table 4). The intervention group also received assistive devices

Outcomes Measures of independence in ADL: Barthel Index

Measures of participation: Frenchay Activities Index

Other secondary outcome measures: MMSE, Canadian Neurological Scale

Time points when outcomes were assessed: "Before randomisation, the patient and/or relatives were interviewed in the hospital to evaluate the patient's basic ADL (Barthel index) and social activities (Frenchay index), which were used as the main outcome variables"

"Follow-up measurements of the basic and social ADL and the cognitive state of the stroke patient were obtained at one, three and six months postdischarge"

Further details on intervention/intervention provider: "Two general nurses received two months of theoretical ( 80 hours) and practical training ( 80 hours) in using the intervention strategy, as taught by a physical medicine and rehabilitation doctor, a physiotherapist and a specialised nurse. The theoretical part included information about stroke, general aspects of physical rehabilitation, stages of recovery and physiotherapy according to Brunnstrom's stage. The practical part was performed with healthy volunteers and later with stroke patients in the hospital. In addition, as a product of this phase of the study, the Stroke Rehabilitation Manual for Nurses was elaborated; this had to be used in the rehabilitation strategy. The physical medicine and rehabilitation doctor and physiotherapist established the minimal criteria to consider that the nurse was sufficiently trained to perform physiotherapy"

\section{Risk of bias}

\section{Bias Authors' judgement Support for judgement}

Random sequence genera- Low risk "randomised by the coordinator of the study using consecutive opaque ention (selection bias) velopes, which were chosen by the patients or their relatives" 
Torres-Arreola 2009 (Continued)

\begin{tabular}{l}
$\begin{array}{l}\text { Allocation concealment Low risk } \\
\text { (selection bias) }\end{array}$ \\
\hline
\end{tabular}

\begin{tabular}{|c|c|c|}
\hline $\begin{array}{l}\text { Blinding of outcome as- } \\
\text { sessment (detection bias) } \\
\text { All outcomes }\end{array}$ & Low risk & $\begin{array}{l}\text { "All outcome variables were gathered by a team of nurses who were different } \\
\text { from the intervention team and were blinded to the randomised group alloca- } \\
\text { tion" }\end{array}$ \\
\hline
\end{tabular}

\begin{tabular}{|c|c|c|}
\hline $\begin{array}{l}\text { Incomplete outcome data } \\
\text { (attrition bias) }\end{array}$ & High risk & $\begin{array}{l}\text { Dropouts accounted for but no explanation given for participants lost to fol- } \\
\text { low-up }\end{array}$ \\
\hline All outcomes & & $\begin{array}{l}\text { "Sixty-seven patients ( } 61 \% \text { ) completed the study, five died because of stroke } \\
\text { complications and } 38 \text { were lost during the follow-up because of other reasons" }\end{array}$ \\
\hline
\end{tabular}

$\begin{array}{lll}\begin{array}{l}\text { Free of systematic differ- } \\ \text { ences in baseline charac- }\end{array} & \text { High risk } & \text { "When we compared patients enrolled in S1 vs. S2, we noted increases in the } \\ \text { teristics of groups com- } & \begin{array}{l}\text { rates of aphasia, dysphasia and/or unconsciousness, and Brunnstrom's stage I } \\ \text { in the patients of group S1" }\end{array}\end{array}$
pared?

"No differences were found in the patients' characteristics of those who completed the follow-up and those who did not"

Did authors adjust for $\quad$ Unclear risk No information provided
baseline differences in
their analyses?

\begin{tabular}{lll}
\hline Other bias & Unclear risk & No information provided \\
\hline
\end{tabular}

Verheyden 2006

\section{Study characteristics}

Study design: RCT
Methods
ization into an experimental or control group. Randomization was done by a person who was not in-
volved in the assessment or treatment of the patients"

Number of participants: $\mathrm{n}=33$
Inclusion criteria: "recruited .. if they attended the inpatient stroke rehabilitation program and had a
hemiparesis that was stroke related. Stroke diagnosis was confirmed by the consultant appointed at
the rehabilitation center on the basis of CT or MRI imaging. Patients who suffered from an earlier stroke
were only allowed in the study if they were fully recovered"
Exclusion criteria: "80 years of age or older, were not able to understand the instructions, had other dis-
orders that could affect motor performance, or obtained a maximum trunk performance score at the
start of the study. Trunk performance was evaluated by means of the Trunk Impairment Scale (TIS)"

Interventions

(1) Experimental group $(n=17)$

"Patients in the experimental and control groups received the conventional multidisciplinary stroke rehabilitation program provided by the rehabilitation center"

"In addition to the conventional treatment, patients from the experimental group received 30 minutes of extra training, 4 times a week, for 5 weeks. In total, 10 hours of additional training were given. The additional exercises consisted of selective movements of the upper and lower part of the trunk in supine and sitting. Supine exercises, with the legs bent and the feet resting on the treatment table, included selective anterior-posterior movements of the pelvis, extension of the hips (bridging), and rotation of the trunk initiated from the upper and lower part of the trunk. Exercises in a sitting position 
included: flexion and extension of the trunk (the patient flexes and extends the trunk without moving the trunk forwards or backwards); flexion and extension of the lumbar part of the spine (this involves selective anteflexion and retroflexion of the lower part of the trunk); flexion and extension of the hips with the trunk extended (with an extended trunk, the movement is initiated in the hips and the patient brings the extended trunk forwards and backwards); lateral flexion of the trunk initiated from the shoulder and pelvic girdle (from the shoulder girdle means that the patient touches the exercise table with one elbow and returns to the starting position, from the pelvic girdle means that the patient lifts one side of the pelvis and returns to the starting position); rotation from the upper and lower part of the trunk (from the upper part of the trunk means that the patient moves each shoulder forwards and backwards, from the lower part of the trunk means that the patient, while sitting in the upright position, moves each knee forwards and backwards); and finally shuffling forwards and backwards on an exercise table (the participant shifts the weight from one side to the other and moves forwards and backwards on the exercise table). Exercises were gradually introduced and the number of repetitions was determined by the therapist on the basis of the patients' performance"

The individual components delivered are listed in Table 3. Based on the individual components, this intervention is categorised as comprising functional task training, musculoskeletal intervention (active) and neurophysiological intervention

Length of intervention period: five weeks

Number of sessions and length of individual sessions: "mean number of physiotherapy sessions = 23; $\mathrm{SD}=4$; mean number of occupational therapy sessions $=22 ; \mathrm{SD}=4$ " "30 minutes of extra training, 4 times a week. In addition to this conventional intervention - total of 10 hours of additional training were given"

Intervention provider: "therapists"

(2) Control group $(n=16)$

"The conventional treatment program is patient-specific and consists mainly of physiotherapy, occupational therapy, and nursing care. Neuropsychological and speech therapy are provided if needed. Therapists combine elements from different neurological treatment concepts but the main emphasis is on the neurodevelopmental treatment concept and on motor relearning strategies"

The individual components delivered are listed in Table 3. Based on the individual components, this intervention is categorised as comprising usual care (functional task training and neurophysiological intervention)

Length of intervention period: five weeks

Number of sessions and length of individual sessions: "mean number of physiotherapy sessions = 24; $\mathrm{SD}=6$; mean number of occupational therapy sessions $=24 ; \mathrm{SD}=6 "$

Intervention provider: "therapists"

This study is classified as intervention (functional task training, musculoskeletal (active), neurophysiological) versus usual care (functional task training, neurophysiological) (Table 5)

Time points when outcomes were assessed: "pre-treatment and post-treatment"

Notes No data suitable for analysis

\section{Risk of bias}

\section{Bias}

Authors' judgement Support for judgement

Random sequence genera- Unclear risk tion (selection bias)
Method of randomisation not described: "divided by simple randomization into an experimental or control group" 
Verheyden 2006 (Continued)

\begin{tabular}{l}
$\begin{array}{l}\text { Allocation concealment } \\
\text { (selection bias) }\end{array} \quad \begin{array}{l}\text { "Randomization was done by a person who was not involved in the assess- } \\
\text { ment or treatment of the patients" }\end{array}$ \\
\hline
\end{tabular}

Blinding of outcome as- Low risk "assessor-blinded randomized controlled trial"

sessment (detection bias)

All outcomes

$\begin{array}{ll}\begin{array}{l}\text { Incomplete outcome data } \\ \text { (attrition bias) }\end{array} & \text { Low risk } \\ \text { All outcomes } & \text { "no dropouts during the course of the study, but } 2 \text { patients in the experimental } \\ & \text { group had } 3 \text { and } 4 \text { fewer hours of additional therapy sessions because of ear- } \\ & \text { ly discharge from the rehabilitation center }(20 \text { and } 21 \text { days after inclusion in } \\ \text { the study). In the control group, } 3 \text { patients were discharged after } 21,23, \text { and } 25 \\ \text { days, respectively. All participants were evaluated before discharge from the } \\ \text { rehabilitation center and included in the analysis" }\end{array}$

\begin{tabular}{ll}
$\begin{array}{l}\text { Free of systematic differ- } \\
\text { ences in baseline charac- }\end{array}$ & Low risk \\
$\begin{array}{l}\text { teristics of groups com- } \\
\text { pared? }\end{array}$ & $\begin{array}{l}\text { "No significant differences were found pretreatment between the } 2 \text { groups for } \\
\text { the collected demographic variables, stroke-related parameters, clinical mea- } \\
\text { sures, number of therapy sessions received, and primary outcome measure } \\
\text { used" }\end{array}$ \\
\hline
\end{tabular}

Did authors adjust for Low risk No information provided
baseline differences in
their analyses?

\begin{tabular}{|c|c|c|}
\hline Other bias & Unclear risk & No information provided \\
\hline
\end{tabular}

Verma 2011

\section{Study characteristics}

Study design: assessor-blinded RCT
Method of randomisation: "the patients were randomly assigned to either the experimental group ( $\mathrm{n}$
$=15$ ) or the control group $(\mathrm{n}=15)$ using computer-generated random numbers After the blocks were
numbered, a random-number generator program was used to select numbers that established the se-
quence in which blocks were allocated to either one or the other group. The intervention assignments
were enclosed in sealed envelopes, which were opaque and sequentially numbered. A resident physi-
cian at the study site conducted the random-number program. However, the resident physician was
blinded to the research protocol and was not involved in the trial"

Participants Number of participants: $\mathrm{n}=30$

Inclusion criteria: first episode of unilateral stroke with hemiparesis during the last month, functional ambulation classification level II and above, ability to understand instructions (Hindi Mental State Examination [HMSE] > 24), ambulatory before stroke, ability to cope with the intensive training programme, ability for mental imaging (Movement Imagery Questionnaire-revised second version [MIQRS] $\geq 25$ ) and National Institutes of Health Stroke Scale (NIHSS) score $<14$

Exclusion criteria: history of any other neurological pathology such as Parkinson disease and epilepsy, conditions affecting balance, neglect, dementia, impaired vision, impaired conscious level, concomitant medical illness, musculoskeletal conditions affecting lower limbs, cardiovascular instability (resting systolic blood pressure $>200 \mathrm{~mm} \mathrm{Hg}$ and resting diastolic blood pressure $>100 \mathrm{~mm} \mathrm{Hg}$ ) and/or serious cardiac conditions (hospitalization for heart disease within three months, active angina, serious cardiac arrhythmias, hypertrophic cardiomyopathy, severe aortic stenosis)

Interventions $\quad$ (1) Experimental group $(\mathrm{n}=15)$


"The program included different workstations and was intended to improve the meaningful tasks related to walking competency, such as balance control, stair walking, turning, transfers, and speed walking. Further, each session consisted of a continuous practice of standing and walking-related tasks on specified workstations with a minimal break"

"Motor imagery comprised imagining walking abilities and tasks related to a real-life situation"

The individual components delivered are listed in Table 3. Based on the individual components, this intervention is categorised as comprising functional task training

Length of intervention period: two weeks

Number of sessions and length of individual sessions: "15 minutes of motor imagery followed by 25 minutes of task-oriented circuit class training for a total of 40 minutes, 7 days per week for 2 weeks (14 sessions)"

Intervention provider: "Task-oriented circuit class training was provided to groups comprising up to 4 patients at any one time with a physiotherapist or occupational therapist for supervision"

(2) Control group $(n=15)$

"Conventional post stroke lower extremity rehabilitation program based on the Bobath's neurodevelopmental technique"

The individual components delivered are listed in Table 3. Based on the individual components, this intervention is categorised as comprising neurophysiological intervention

Length of intervention period: two weeks

Number of sessions and length of individual sessions: "The control group program was matched for duration, number, and frequency of the sessions with the experimental group program"

Intervention provider: not stated

This study is classified as active intervention one (functional task training) versus active intervention two (neurophysiological) (Table 6)

Outcomes Measures of independence in ADL: Barthel Index

Measures of voluntary movements: Functional Ambulation Classification, Rivermead Visual Gait Assessment, 10-Metre Walk test, Six-Minute Walk test, step length, stride length, step width and cadence

Time points when outcomes were assessed: "All the participants were assessed using the outcome measures at baseline (preintervention), week 2 (postintervention) except for Barthel Index (BI), and at week 6 (follow-up)"

Notes

\section{Risk of bias}

\section{Bias}

Authors' judgement Support for judgement

Random sequence genera- Low risk tion (selection bias)

"Computer-generated random numbers. After the blocks were numbered, a random-number generator program was used to select numbers that established the sequence in which blocks were allocated to either one or the other group. The intervention assignments were enclosed in sealed envelopes, which were opaque and sequentially numbered"

"A resident physician at the study site conducted the random-number program. However, the resident physician was blinded to the research protocol and was not involved in the trial" 
Verma 2011 (Continued)

Blinding of outcome as- Low risk_ "The experimental and control interventions were given by 2 independent sessment (detection bias) therapists. The subjects were blinded for intervention of interest"

All outcomes

\begin{tabular}{|c|c|c|}
\hline $\begin{array}{l}\text { Incomplete outcome data } \\
\text { (attrition bias) } \\
\text { All outcomes }\end{array}$ & Low risk & $\begin{array}{l}\text { Dropouts accounted for } \\
\text { (One participant was lost to follow-up, from the experimental group because } \\
\text { of a second stroke) }\end{array}$ \\
\hline
\end{tabular}

\begin{tabular}{lll}
\hline $\begin{array}{l}\text { Free of systematic differ- } \\
\text { ences in baseline charac- } \\
\text { teristics of groups com- } \\
\text { pared? }\end{array}$ & Low risk & $\begin{array}{l}\text { "The groups did not significantly differ in any of the demographic and baseline } \\
\text { clinical characteristics" }\end{array}$ \\
\hline $\begin{array}{l}\text { Did authors adjust for } \\
\text { baseline differences in } \\
\text { their analyses? }\end{array}$ & Low risk & No information provided \\
\hline Other bias & Unclear risk & No information provided \\
\hline
\end{tabular}

Wade 1992

\section{Study characteristics}

\begin{tabular}{ll}
\hline Methods & $\begin{array}{l}\text { Study design: RCT } \\
\text { Method of randomisation: permuted blocks of } 10 \text {, using random number tables }\end{array}$ \\
\hline Participants & $\begin{array}{l}\text { Number of participants: } \mathrm{n}=94 \\
\text { Inclusion criteria: first stroke more than one year previously and mobility problems (fall within last } \\
\text { three months, used a walking aid, limited mobility or slow gait speed) }\end{array}$ \\
\hline
\end{tabular}

Interventions

(1) Mixed (problem solving, community physiotherapy) ( $n=49)$

"'Problem solving' approach: patients were assessed with particular reference to their mobility, and problem areas were identified. Realistic, achievable goals were discussed with the patient and carers and then the physiotherapist intervened if required"

Re-education of abnormal components of gait

Practice walking inside and outside

For standing balance: exercises to stimulate reactions, obstacle courses, practice on uneven surfaces

Reeducation of sitting to standing

Equipment: removal, provision, maintenance, adjustment

Activities of daily living: advice, referral to community occupational therapist

Demonstrate participant's ability to participant/carer

Graduated exercise programme

The individual components delivered are listed in Table 3. Based on the individual components, this intervention is categorised as comprising assistive devices, cardiopulmonary intervention and functional task training

Length of intervention period: not stated 
Wade 1992 (Continued)

Number of sessions and length of individual sessions: range one to 11 visits, mean number of visits = four (standard deviation 2.5). Time (including travel and administration) ranged from one hour $10 \mathrm{~min}-$ utes to three hours 10 minutes

Intervention provider: physiotherapist and occupational therapist

(2) No treatment $(n=45)$

The individual components delivered are listed in Table 3. Based on the individual components, this intervention is categorised as comprising no intervention

Length of intervention period: no intervention

Number of sessions and length of individual sessions: no intervention

Intervention provider: no intervention

This study is classified as intervention (functional task training) versus no treatment (Table 4). The intervention group also received assistive devices and cardiopulmonary intervention

Outcomes Measures of independence in ADL: Barthel Index, Frenchay Activities Index, Nottingham EADL Scale Measures of functional independence: Rivermead Mobility Assessment

Measures of voluntary movement: gait speed

Time points when outcomes were assessed: "Once accepted into the trial, each patient was assessed by an independent (non-treating) physiotherapist immediately, one to six weeks later, and then about three, six, and nine months after the second assessment"

Notes

Cross-over design: Participants in the control group received treatment after three-month assessment This study was initially excluded from this review, as the review authors assessed, based on the abstract, that this study explored timing of intervention

Comments from peer reviewers for the 2007 version led to the inclusion of this trial

\section{Risk of bias}

\begin{tabular}{lll}
\hline Bias & Authors' judgement & Support for judgement \\
\hline $\begin{array}{l}\text { Random sequence genera- } \\
\text { tion (selection bias) }\end{array}$ & Low risk & Permuted blocks of 10, using random number tables \\
\hline $\begin{array}{l}\text { Allocation concealment } \\
\text { (selection bias) }\end{array}$ & Low risk & No information provided \\
\hline $\begin{array}{l}\text { Blinding of outcome as- } \\
\text { sessment (detection bias) } \\
\text { All outcomes }\end{array}$ & Low risk & Participant not blinded-not possible \\
& & Therapist not blinded-not possible \\
\hline
\end{tabular}

$\begin{array}{ll}\begin{array}{l}\text { Incomplete outcome data } \\ \text { (attrition bias) }\end{array} & \text { Unclear risk } \\ \text { All outcomes } & \text { Dropouts accounted for } \\ & 89 / 94 \text { completed intervention and had three-month follow-up } \\ & \begin{array}{l}\text { All participants included in the analysis unless they died or had not reached } \\ \text { last follow-up point } \\ \text { For some outcomes (e.g. gait speed), numbers are less because not all could } \\ \text { perform the test }\end{array}\end{array}$

Free of systematic differ- Unclear risk No information provided
ences in baseline characteristics of groups compared? 
Wade 1992 (Continued)

Did authors adjust for baseline differences in their analyses?
Unclear risk No information provided

$\begin{array}{ll}\text { Other bias } & \text { Initial recruitment was from final follow-up from Oxford Community Stroke } \\ & \text { Project, but not enough participants were recruited. Additional participants } \\ & \text { were recruited by contacting a rehabilitation centre, asking general prac- } \\ \text { titioners, making a radio appeal and contacting community workers and } & \text { through self referral. Participants recruited from the Oxford Community Stroke } \\ & \text { Project were less disabled than those recruited in other ways. However, the } \\ \text { two groups were similar at randomisation }\end{array}$

Wang 2004a

\section{Study characteristics}

Methods Study design: RCT

Method of randomisation: according to the assessment time, in a 2:1 (rehabilitation:control) ratio

Participants Number of participants: $\mathrm{n}=105$ (at recruitment); $\mathrm{n}=98$ (data available after treatment)

Inclusion criteria: "Aged between 42-78 years old, commenced therapy (approximately) within 3 days of stroke onset, satisfied the 1995 Fourth National Conference on Cerebral Vascular Disease classification guidelines and stroke as confirmed by CT or MRI scan"

Exclusion criteria: "Stroke onset exceeding 1 week and severe impaired cognitive and/or speech issues"

Interventions

(1) Rehabilitation group ( $\mathrm{n}=70$ before dropout; $\mathrm{n}=66$ (data available: see Table 5 )

"Both groups received conventional treatment during the critical period, with treatment group commencing early-stage physical rehabilitation once medically stable. Based on the condition of each patient, a treatment plan was developed prior to treatment. Treatment method utilised exercise therapy, including techniques and equipment, locations included bedside and treatment room, treatment was conducted by therapist. Mainly utilised motor relearning methods, including: (i) Positioning of healthy limbs. (ii) Passive ranging exercises on joints of limbs on affected side. (iii) Functional training of upper limbs, including stimulation of muscle motion as well as training of control of extension, separation of fingers, prevention of contracture, etc. (iv) Training of rolling on bed, as well as training of lying to bedside sitting, training of bedside sitting balance, sit-to-stand training. (v) Upright training (bed). (vi) Bedside standing and sitting training as well as bedside standing balance training. (vii) Gait training. (viii) Weight-bearing and weight-assisted training, etc. Throughout entire treatment period, emphasis on correct training posture and relaxation training of spastic muscles, anti-spasticity techniques and muscle training of non-spastic muscles. Family members were taught the correct complementary training and care methods, while concurrently focusing on psychological treatment, during the course of the training, frequently use language that will boost the patient's confidence in recovery"

The individual components delivered are listed in Table 3. Based on the individual components, this intervention is categorised as comprising functional task training and musculoskeletal intervention (passive)

Length of intervention period: 30 days

Number of sessions and length of individual sessions: one to two/d, 45 minutes/session, with exercise time and intensity reduced as appropriate if participant lacked the capability

Intervention provider: therapist

(2) Control group ( $\mathrm{n}=35$ before dropout, $\mathrm{n}=32$ data available: see Table 5) 
Wang 2004a (Continued)

"Received conventional treatment during the critical period but did not receive any rehabilitation treatment"

The individual components delivered are listed in Table 3. Based on the individual components, this intervention is categorised as comprising no intervention

Length of intervention period: no intervention

Number of sessions and length of individual sessions: no intervention

Intervention provider: no intervention

This study is classified as intervention (functional task training, musculoskeletal (passive)) versus no treatment (Table 4)

\section{Outcomes}

Measures of motor function: Fugl-Meyer Assessment (upper limb, lower limb)

Other secondary outcome measures: somatosensory evoked potential (SEP)

Time points when outcomes were assessed: "Each patient, within 2 days of being medically stable, would undergo 1st assessment, and 30 days later would undergo the 2 nd assessment"

Notes Original study translated from Chinese to English

\section{Risk of bias}

\begin{tabular}{|c|c|c|}
\hline Bias & Authors' judgement & Support for judgement \\
\hline $\begin{array}{l}\text { Random sequence genera- } \\
\text { tion (selection bias) }\end{array}$ & Unclear risk & $\begin{array}{l}\text { "According to the assessment time, in a 2:1 (rehabilitation:control) ratio"-no } \\
\text { further details provided }\end{array}$ \\
\hline $\begin{array}{l}\text { Allocation concealment } \\
\text { (selection bias) }\end{array}$ & Unclear risk & No information provided \\
\hline $\begin{array}{l}\text { Blinding of outcome as- } \\
\text { sessment (detection bias) } \\
\text { All outcomes }\end{array}$ & Low risk & Blinded outcome assessment \\
\hline $\begin{array}{l}\text { Incomplete outcome data } \\
\text { (attrition bias) } \\
\text { All outcomes }\end{array}$ & Low risk & $\begin{array}{l}\text { Dropouts accounted for } \\
\text { Dropouts due to financial reasons or inability to adhere to study design ( } n=4 \\
\text { dropouts in the rehabilitation group and } n=3 \text { in the control group) }\end{array}$ \\
\hline $\begin{array}{l}\text { Free of systematic differ- } \\
\text { ences in baseline charac- } \\
\text { teristics of groups com- } \\
\text { pared? }\end{array}$ & Low risk & No significant baseline differences \\
\hline $\begin{array}{l}\text { Did authors adjust for } \\
\text { baseline differences in } \\
\text { their analyses? }\end{array}$ & Low risk & No information provided \\
\hline Other bias & Unclear risk & No information provided \\
\hline
\end{tabular}

\section{Study characteristics}


Wang 2004b (Continued)

Methods
Study design: RCT

Method of randomisation: not stated
Participants
Number of participants: $n=50$

Inclusion criteria: "Satisfied the 1995 Fourth National Conference on Cerebral Vascular Disease classification guidelines, confirmed by CT or MRI scan, first ever stroke, within 4 months since stroke onset, spastic hemiplegic limbs and between 1-3 for Ashworth assessment"

Exclusion criteria: "Impairment in consciousness, psychology, cognition, agnosia, apraxia, Parkinson's disease, epilepsy, electrolyte imbalance, cardiac pacemaker, severe malnutrition, severe cardiorespiratory disease and participants with poor adherence"
Interventions
(1) Treatment group $(n=25)$

"Both groups were given routine drug treatment and basic rehabilitative training, looking over brain circulation, anti-coagulation, nutrition and rest, electrolyte balance, prevention of various secondary complications; maintaining optimal limb positioning, passive and active joint range of motion, tissue massage etc. Patients in the treatment group had additional neural facilitation combined with the use of the muscular spasm machine, following the characteristics of the stages of spasticity. Prior to neural facilitation, patients had to undergo relaxation. Neural facilitation training included prone positioning, slow traction to relax tensed muscles; striking spastic muscles including both agonists and antagonists to restore the appropriate muscular balance; traction to muscles and gentle striking to the muscle belly, guiding the affected limbs to exercise, so as to stimulate a balancing reaction, overcoming over activated muscles and compensatory movements; using co-contraction principles, allowing resistive forces exerted during flexion and extension of the non-affected upper limb to illicit flexion and extension of the affected upper limb, and assisting or encouraging the patient to actively flex and extend the limb; making use of asymmetric tonic neck reflex mechanisms, reducing the tone in both upper and lower limbs and stimulating limb movement by rotating the patient's neck, holding on to the lower limbs while moving them with momentum, or through reverse action by moving the non-affected shoulder and elbow joints rhythmically so as to reduce muscle tension; making use of body weight to optimise ankle joint integrity; stretching the thumb and externally rotating the forearm to reduce tension in the wrist joint and finger flexors; using cold and hot sensations etc to stimulate and activate contraction of relevant muscles, suppress spasticity etc, compressing the joint to reduce tension, reducing spasticity etc. Low-frequency pulse current treatment: using a Beijing manufactured $\mathrm{KX}-3 \mathrm{~A}$ model for spasticity treatment"

The individual components delivered are listed in Table 3. Based on the individual components, this intervention is categorised as comprising modality, musculoskeletal intervention (passive) and musculoskeletal (active) and neurophysiological intervention

Length of intervention period: four weeks

Number of sessions and length of individual sessions: 30 to 45 minutes/session, one session/d, five/wk

Intervention provider: doctor, nurse

(2) Control group $(n=25)$

"Both groups were given routine drug treatment and basic rehabilitative training, looking over brain circulation, anti-coagulation, nutrition and rest, electrolyte balance, prevention of various secondary complications; maintaining optimal limb positioning, passive and active joint range of motion, tissue massage etc"

The individual components delivered are listed in Table 3. Based on the individual components, this intervention is categorised as usual care (musculoskeletal intervention (passive))

Length of intervention period: not stated

Number of sessions and length of individual sessions: not stated

Intervention provider: not stated 
Wang 2004b (Continued)

This study was classified as intervention (musculoskeletal (active), musculoskeletal (passive), neurophysiological) versus usual care (musculoskeletal (passive)) (Table 5). The intervention group also received modality

Outcomes

Measures of motor function: Fugl-Meyer Assessment

Measures of tone or spasticity: Ashworth Scale

Time points when outcomes were assessed: at enrolment and after four weeks of intervention

\section{Risk of bias}

\begin{tabular}{|c|c|c|}
\hline Bias & Authors' judgement & Support for judgement \\
\hline $\begin{array}{l}\text { Random sequence genera- } \\
\text { tion (selection bias) }\end{array}$ & Unclear risk & No information provided \\
\hline $\begin{array}{l}\text { Allocation concealment } \\
\text { (selection bias) }\end{array}$ & High risk & Study implementation by authors \\
\hline $\begin{array}{l}\text { Blinding of outcome as- } \\
\text { sessment (detection bias) } \\
\text { All outcomes }\end{array}$ & High risk & Outcome assessment by authors \\
\hline $\begin{array}{l}\text { Incomplete outcome data } \\
\text { (attrition bias) } \\
\text { All outcomes }\end{array}$ & Low risk & No dropouts \\
\hline $\begin{array}{l}\text { Free of systematic differ- } \\
\text { ences in baseline charac- } \\
\text { teristics of groups com- } \\
\text { pared? }\end{array}$ & Low risk & No significant baseline differences \\
\hline $\begin{array}{l}\text { Did authors adjust for } \\
\text { baseline differences in } \\
\text { their analyses? }\end{array}$ & Low risk & No information provided \\
\hline Other bias & Unclear risk & No information provided \\
\hline
\end{tabular}

Wang 2005

\section{Study characteristics}

\begin{tabular}{ll}
\hline Methods & $\begin{array}{l}\text { Study design: RCT } \\
\text { Method of randomisation: stratified into "patients with spasticity" (Brunnström stage two or three) and } \\
\text { "patients with relative recovery" (Brunnström stage four or five) } \\
\text { Sealed envelopes, independent person }\end{array}$ \\
\hline Participants & $\begin{array}{l}\text { Number of participants: } \mathrm{n}=44 \\
\text { Inclusion criteria: "Hemiparesis secondary to CVA, LE Brunnström motor recovery } 2 \text { to } 5 \text { and able to } \\
\text { communicate and co-operate" }\end{array}$ \\
\hline Interventions & (1) Neurophysiological (Bobath) \\
"Based on Bobath philosophy"
\end{tabular}


Treatment was "individualised, constantly modified according to subject response"

Techniques included facilitating normal movement patterns and retraining normal alignment through appropriate sensory and proprioceptive input, direct manual facilitation, key point control, verbal and visual feedback

The individual components delivered are listed in Table 3. Based on the individual components, this intervention is categorised as comprising neurophysiological intervention

Length of intervention period: four weeks

Number of sessions and length of individual sessions: 40 minutes, five sessions per week for 20 sessions Intervention provider: Bobath-trained therapists

(2) Orthopaedic

Passive, assistive, active and progressive resistive exercise

Multiple repetitions of practice of functional activities: rolling, sitting up, transfer and gait

Gait training using parallel bars

The individual components delivered are listed in Table 3. Based on the individual components, this intervention is categorised as comprising functional task training, musculoskeletal intervention (active) and musculoskeletal intervention (passive)

Length of intervention period: four weeks

Number of sessions and length of individual sessions: 40 minutes, five sessions per week for 20 sessions Intervention provider: physical therapists

This study is classified as active intervention one (neurophysiological) versus active intervention two (functional task training, musculoskeletal (active), musculoskeletal (passive)) (Table 6)

Measures of functional independence: MAS, Stroke Assessment Impairment Set
Measures of postural control and balance: Berg Balance Scale
Measures of spasticity/tone: Stroke Assessment Impairment Set (SAIS, tone)
Time points when outcomes were assessed: "The patients were assessed twice: once before and once
after treatment"

Notes

\section{Risk of bias}

\begin{tabular}{lll}
\hline Bias & Authors' judgement & Support for judgement \\
\hline $\begin{array}{l}\text { Random sequence genera- } \\
\text { tion (selection bias) }\end{array}$ & Low risk & $\begin{array}{l}\text { "Sealed envelopes, independent person. The random assignment was } \\
\text { achieved by an independent person who chose one of the sealed envelopes } 30 \\
\text { min before the start of the intervention" }\end{array}$ \\
\hline $\begin{array}{l}\text { Allocation concealment } \\
\text { (selection bias) }\end{array}$ & Low risk & Independent person \\
\hline $\begin{array}{l}\text { Blinding of outcome as- } \\
\text { sessment (detection bias) } \\
\begin{array}{l}\text { All outcomes } \\
\text { m }\end{array}\end{array}$ & Low risk & Participant not blinded \\
\end{tabular}



ated by a separate physical therapist who was not involved in the treatment programme and did not know about the patient's group"

Incomplete outcome data Low risk No dropouts
(attrition bias)
All outcomes

\begin{tabular}{|c|c|c|}
\hline $\begin{array}{l}\text { Free of systematic differ- } \\
\text { ences in baseline charac- } \\
\text { teristics of groups com- } \\
\text { pared? }\end{array}$ & Unclear risk & $\begin{array}{l}\text { Baseline characteristics are reported but are not compared statistically. Un- } \\
\text { clear whether the groups were similar at baseline for all characteristics }\end{array}$ \\
\hline $\begin{array}{l}\text { Did authors adjust for } \\
\text { baseline differences in } \\
\text { their analyses? }\end{array}$ & Unclear risk & No information provided \\
\hline \multirow[t]{2}{*}{ Other bias } & Unclear risk & $\begin{array}{l}\text { Participants were all inpatients and presumably were able to see the treat- } \\
\text { ment given to participants in the other treatment group, which is a potential } \\
\text { source of contamination }\end{array}$ \\
\hline & & $\begin{array}{l}\text { Different therapists did apply the two interventions (four therapists in total), } \\
\text { so different aspects related to their personal delivery of the intervention could } \\
\text { be a potential confounder. However, it is not clear whether two therapists } \\
\text { each applied one intervention or whether all four therapists applied both in- } \\
\text { terventions }\end{array}$ \\
\hline
\end{tabular}

Wang 2006

\section{Study characteristics}

\begin{tabular}{ll}
\hline Methods & Study design: RCT \\
& Method of randomisation: not stated \\
\hline Participants & Number of participants: $\mathrm{n}=80$ \\
& $\begin{array}{l}\text { Inclusion criteria: "Satisfied the } 1995 \text { Fourth National Conference on Cerebral Vascular Disease classifi- } \\
\text { cation guidelines, confirmed by CT or MRI scan as first ever stroke, medically stable within } 3 \text { weeks after } \\
\text { stroke onset, Glasgow Coma Scale }>8 \text {, aged } 40-80 \text { years old and deficits in limb function" }\end{array}$ \\
& $\begin{array}{l}\text { Exclusion criteria: "Active liver, kidney disease(s), paraplegia, deaf, mute, unable to accommodate as- } \\
\text { sessment and inaccessible homes" }\end{array}$ \\
\hline
\end{tabular}

Interventions $\quad$ (1) Rehabilitation group $(n=40)$

"Both groups of patients received conventional clinical treatment and care. Treatment group besides receiving conventional clinical treatment, also strictly followed 'fifteen' research topic 'cerebrovascular disease 3-phase rehabilitation intervention' to undergo rehabilitative training. On entering the group, treatment group patients immediately commenced phase 1 rehabilitative treatment plan (in neurology ward), including anti-spasticity positioning on bed; breathing exercises; passive ranging exercises on each joint on affected side; nerve and muscle stimulation technique (mainly Rood technique and Brunnstrom technique); active ranging exercises of healthy limbs; lying to sitting training; sitting balance training; ADL training on bed, etc"

"With progression of time, patients' physical ability gradually improved, and were transferred from neurology to rehabilitation zone to undergo rehabilitative treatment, i.e.. phase 2 rehabilitative treatment, with continued strengthening of phase 1 rehabilitation measures as per the patient's condition; sit to stand training; transference training; deployment of nerve and muscle stimulation techniques 
(mainly Bobath technique and PNF technique); standing balance training; affected limbs weight-bearing training; gait training and stair-climbing training, while concurrently inserting appropriate occupational therapy"

"After a period of phase 2 rehabilitative treatment, most patients were discharged to their homes or community to continue rehabilitative treatment, i.e. phase 3 rehabilitative treatment. Mainly involved therapist making regular house visits to provide guidance, help patient to undergo required functional training, until the course of disease has reached 6 months mark"

The individual components delivered are listed in Table 3. Based on the individual components, this intervention is categorised as comprising functional task training and musculoskeletal intervention (passive), neurophysiological intervention

Length of intervention period: till six months after stroke

Number of sessions and length of individual sessions: not stated

Intervention provider: therapists

(2) Control group $(n=40)$

"Received clinical treatment and unguided self-training. The control group did not do any standard rehabilitative training"

The individual components delivered are listed in Table 3. Based on the individual components, this intervention is categorised as comprising no intervention

Length of intervention period: no intervention

Number of sessions and length of individual sessions: no intervention

Intervention provider: no intervention

This study is classified as intervention (functional task training, musculoskeletal (passive), neurophysiological) versus no treatment (Table 4)

\section{Outcomes}

Measures of tone or spasticity: Modified Ashworth Scale

Other secondary outcome measures: Brunnstrom

Time points when outcomes were assessed: on enrolment, one month after stroke, three months after stroke and six months after stroke

\section{Notes}

Original study translated from Chinese to English

No data suitable for analysis

\section{Risk of bias}

\section{Bias}

\section{Authors' judgement Support for judgement}

Random sequence genera- Unclear risk No information provided

tion (selection bias)

\begin{tabular}{ll}
\hline $\begin{array}{l}\text { Allocation concealment } \\
\text { (selection bias) }\end{array}$ & Unclear risk information provided
\end{tabular}

\begin{tabular}{lll}
\hline $\begin{array}{l}\text { Blinding of outcome as- } \\
\text { sessment (detection bias) }\end{array}$ & Unclear risk & $\begin{array}{l}\text { Outcome assessors were not involved in delivering the intervention, but it is } \\
\text { not clear whether they were blinded to allocation of groups }\end{array}$
\end{tabular}

All outcomes not clear whether they were blinded to allocation of groups

Incomplete outcome data Unclear risk No dropouts described
(attrition bias)


Wang 2006 (Continued)

All outcomes

Free of systematic differ- Low risk No significant baseline differences
ences in baseline charac-
teristics of groups com-
pared?

\begin{tabular}{lll}
\hline $\begin{array}{l}\text { Did authors adjust for } \\
\text { baseline differences in } \\
\text { their analyses? }\end{array}$ & Low risk & \\
\hline Other bias & Unclear risk & No information provided \\
\hline
\end{tabular}

\section{Study characteristics}

\begin{tabular}{ll}
\hline Methods & Study design: RCT \\
& Method of randomisation: not stated ("80 cases were randomly [divided] into 2 groups, control and \\
& training group'")
\end{tabular}

Participants Number of participants: $\mathrm{n}=80$

Inclusion criteria: "CVA suffers within 3 months after onset with no obvious cognitive problems (able to follow the instructions) and no previous mobility limitations"

"each group received physical therapy...selective trunk activities were emphasized in exercise group as well"

"The intervention was basically Bobath orientated, the experimental group focused on exercises of trunk activities (based on PM Davis "Right in the middle" Springer-Verlag New York 1990)"

"Both groups received rehabilitation retraining. The 'training' group emphasized on truncal training. In supine: a chest support exercises: participant leaning forward, therapist placed hands on both sides of chest, compressing inwards and downwards by following the breathing rhythm. B. facilitating abdominal breathing exercises. C. lower truncal rotation. D. supine to prone position. E. shifting between sitting and supine position. In sitting: a. sitting on the therapy bed, participant hugged knees with both hands and rocked forwards and backwards. B. sitting on bed with knee extended, both hands touching the knees (therapists maintained ankle joints in dorsiflexion). C. sitting on bed with knee extended, therapists gently hold on to participants' hands, adding in truncal rotation while lying down. D. sitting on a stool, both hands hugging the shoulders, hip move accordingly. E. sitting on a stool, add in truncal rotation. F. sitting on a stool, Place non-affected ankle to affected knee, both hands clasped to touch non-affected knee, shift bodyweight left and right"

"In standing: a. Standing in front of a table the same height as the hips, both arms crossed, both shoulder blades leaning on table, slowing raise shoulders till straight. B. both hands clasped to raise ball from different heights. C. facing the wall, both hands clasped to draw arcs. D. Standing facing the therapist, push shoulders against therapist's hands. Using therapy ball to train truncal flexion and extension, rotation and side flexion"

The individual components delivered are listed in Table 3. Based on the individual components, this intervention is categorised as comprising neurophysiological

Length of intervention period: 12 weeks (mean 68 days) 
Wei 1998 (Continued)

Number of sessions and length of individual sessions: one/d, 45 minutes to one hour/session, five times per week

Intervention provider: not stated

(2) Control group $(n=40)$

"each group received physical therapy"

The individual components delivered are listed in Table 3. Based on the individual components, this intervention is categorised as comprising usual care (components not described)

Length of intervention period: 12 weeks (mean 68 days)

Number of sessions and length of individual sessions: five times per week

Intervention provider: not stated

This study is classified as intervention (neurophysiological) versus usual care (not described) (Table 5)

\begin{tabular}{ll}
\hline Outcomes & $\begin{array}{l}\text { Measures of motor function: Fugl-Meyer Assessment } \\
\text { State time points when outcomes were assessed: "assessment scores were recorded before and after } \\
\text { the experiment period" }\end{array}$ \\
\hline Notes & Abstract only \\
& Further details supplied by the study author (personal communication) \\
\hline
\end{tabular}

\section{Risk of bias}

\begin{tabular}{|c|c|c|}
\hline Bias & Authors' judgement & Support for judgement \\
\hline $\begin{array}{l}\text { Random sequence genera- } \\
\text { tion (selection bias) }\end{array}$ & Unclear risk & $\begin{array}{l}\text { No information provided } \\
\text { " } 80 \text { cases were randomly [divided] into } 2 \text { groups, control and training group" }\end{array}$ \\
\hline $\begin{array}{l}\text { Allocation concealment } \\
\text { (selection bias) }\end{array}$ & Unclear risk & No information provided \\
\hline $\begin{array}{l}\text { Blinding of outcome as- } \\
\text { sessment (detection bias) } \\
\text { All outcomes }\end{array}$ & Unclear risk & No information provided \\
\hline $\begin{array}{l}\text { Incomplete outcome data } \\
\text { (attrition bias) } \\
\text { All outcomes }\end{array}$ & Low risk & No dropouts described \\
\hline $\begin{array}{l}\text { Free of systematic differ- } \\
\text { ences in baseline charac- } \\
\text { teristics of groups com- } \\
\text { pared? }\end{array}$ & Low risk & Baseline demographics similar between groups \\
\hline $\begin{array}{l}\text { Did authors adjust for } \\
\text { baseline differences in } \\
\text { their analyses? }\end{array}$ & Low risk & No information provided \\
\hline Other bias & Unclear risk & No information provided \\
\hline
\end{tabular}


Wellmon 1997

\section{Study characteristics}

\begin{tabular}{ll}
\hline Methods & $\begin{array}{l}\text { Study design: RCT } \\
\text { Method of randomisation: not stated }\end{array}$ \\
\hline Participants & $\begin{array}{l}\text { Number of participants: } \mathrm{n}=21 \\
\text { Inclusion criteria: disorder of unilateral movement in lower limb, CVA less than } 150 \text { days previously, } \\
\text { able to stand unsupported for longer than } 30 \text { seconds, able to walk farther than } 7 \mathrm{~m} \text {, able to under- } \\
\text { stand visual/verbal commands, medically stable enough for } 20 \text { minutes of treatment, more than zero } \\
\text { degrees passive ankle dorsiflexion and no hip, knee, ankle, foot pain }\end{array}$
\end{tabular}

Interventions

(1) Motor learning

Repetitive practice of context-specific task

Repetitive practice of stepping task

The individual components delivered are listed in Table 3. Based on the individual components, this intervention is categorised as comprising functional task training

Length of intervention period: two days

Number of sessions and length of individual sessions: addition of 20 minutes, twice a day for two days. Participants had received routine physiotherapy, based on motor learning principles, although no routine physiotherapy was given on the two days of the intervention

Intervention provider: not stated

(2) Control (no treatment)

The individual components delivered are listed in Table 3. Based on the individual components, this intervention is categorised as comprising no intervention

Length of intervention period: no intervention

Number of sessions and length of individual sessions: Participants had received routine physiotherapy, based on motor learning principles, although no routine physiotherapy was given on the two days of the intervention

Intervention provider: no intervention

This study is classified as intervention (functional task training) versus no treatment (Table 4)

Outcomes Measures of postural control and balance: standing symmetry; step length symmetry; single stance
symmetry

Notes No outcomes included in analysis

\section{Risk of bias}

\begin{tabular}{lll}
\hline Bias & Authors' judgement & Support for judgement \\
\hline $\begin{array}{l}\text { Random sequence genera- } \\
\text { tion (selection bias) }\end{array}$ & Unclear risk & $\begin{array}{l}\text { "were randomly assigned to either treatment or control group." No further de- } \\
\text { tails }\end{array}$ \\
\hline $\begin{array}{l}\text { Allocation concealment } \\
\text { (selection bias) }\end{array}$ & Unclear risk & No information provided \\
\hline $\begin{array}{l}\text { Blinding of outcome as- } \\
\text { sessment (detection bias) } \\
\text { All outcomes }\end{array}$ & High risk & Participant not blinded \\
\hline
\end{tabular}


Wellmon 1997 (Continued)

Assessor not blinded

\begin{tabular}{lll}
\hline $\begin{array}{l}\text { Incomplete outcome data } \\
\text { (attrition bias) } \\
\text { All outcomes }\end{array}$ & Low risk & \\
\hline $\begin{array}{l}\text { Free of systematic differ- } \\
\text { ences in baseline charac- } \\
\text { teristics of groups com- } \\
\text { pared? }\end{array}$ & Unclear risk & No information \\
\hline $\begin{array}{l}\text { Did authors adjust for } \\
\text { baseline differences in } \\
\text { their analyses? }\end{array}$ & Unclear risk & No information \\
\hline $\begin{array}{l}\text { Other bias } \\
\end{array}$ & High risk & $\begin{array}{l}\text { The amount of treatment was very limited (four sessions over two days); this } \\
\text { may have been insufficient to effect a change }\end{array}$ \\
\hline
\end{tabular}

Wu 2006

\section{Study characteristics}

\begin{tabular}{ll}
\hline Methods & Study design: RCT \\
& Method of randomisation: randomisation based on enrolment time \\
\hline Participants & Number of participants: $\mathrm{n}=100$ \\
& $\begin{array}{l}\text { Inclusion criteria: ischaemic or haemorrhagic stroke participants identified from the } 1995 \text { Fourth Na- } \\
\text { tional Conference on Cerebral Vascular Disease classification guidelines, confirmed by CT or MRI scan } \\
\text { as first ever stroke, provided informed consent, medically stable within } 24 \text { hours after stroke onset, } \\
\text { Glasgow Coma Scale score }>\text { eight, aged } 40 \text { to } 80 \text { years old with deficits in limb function }\end{array}$
\end{tabular}

Interventions

(1) Rehabilitation group $(\mathrm{n}=50)$

"Received clinical treatment and regular rehabilitation training. Standardised rehabilitation training commenced after the patients were cognitively and medically stable for 48 hours with no aggravation of existing stroke symptoms (within 21 days of stroke, as stage 1 rehabilitation); rehabilitation treatment from early recovery to transfer to Rehabilitation ward (within 3 months of stroke, as stage 2 rehabilitation); community rehabilitation (e.g. home ward) from middle to late recovery (within 4-6 months after stroke, as stage 3 rehabilitation), with the following method:

Prevention and treatment of spasticity: (i) appropriate positioning. (ii) Bobath method of holding hands: both hands intertwined, use the non-affected fingers to lock the affected fingers, with the affected thumb at the top, stretch forward, flex shoulders, lift arms to touch the forehead, hold for a few seconds before returning to original position, practising multiple times daily. (iii) active stretch: extend affected fingers, place $20 \mathrm{~cm}$ from body on a support, use body weight to stretch spastic muscles, for 3-5minutes per stretch, practising multiple times daily. (iv) Traction: apply traction techniques to affected spastic joints, on feeling resistance, continue with technique for at least 2-3 minutes on the same spot, relax, and repeat. (v) standing bed training: stand on a sloped plank in front of a ladder wall, correct foot inversion and foot, target lower limb spasticity, 5-8 minutes daily. (vi) critical control of Bobath technique, PNF's technique of upper limb stretch and lower limb flexion; both tonic labyrinthine reflex and the asymmetrical tonic neck reflex were also used in the rehabilitation process"

"On top of preventing and treating spasticity, appropriate therapy was given depending on the stroke phase, including: rolling practice on bed, bridging practice, lying to sitting and sitting balance training, sit-to-stand and standing balance training, gait and up-and-down stairs training, ADL practice, manual therapy, speech and language therapy, psychological counselling" 
Wu 2006 (Continued)

The individual components delivered are listed in Table 3. Based on the individual components, this intervention is categorised as comprising functional task training, musculoskeletal intervention (active), musculoskeletal intervention (passive) and neurophysiological intervention

Length of intervention period: six months

Number of sessions and length of individual sessions: dependent on stage of recovery (see above)

Intervention provider: not stated

(2) Control group $(n=50)$

"Received clinical treatment and unguided self-training"

The individual components delivered are listed in Table 3. Based on the individual components, this intervention is categorised as comprising no intervention

Length of intervention period: six months

Number of sessions and length of individual sessions: not stated

Intervention provider: not stated

This study is classified as intervention (functional task training, musculoskeletal (active), muscu-

loskeletal (passive), neurophysiological) versus no treatment (Table 4)

Measures of motor function: Fugl-Meyer Assessment

Measures of tone or spasticity: Modified Ashworth Scale

Time points when outcomes were assessed: "pre-treatment' and after 6 months of intervention"

\begin{tabular}{|c|c|c|}
\hline Notes & \multicolumn{2}{|c|}{ Original study translated from Chinese to English } \\
\hline \multicolumn{3}{|l|}{ Risk of bias } \\
\hline Bias & Authors' judgement & Support for judgement \\
\hline $\begin{array}{l}\text { Random sequence genera- } \\
\text { tion (selection bias) }\end{array}$ & Unclear risk & No information provided \\
\hline $\begin{array}{l}\text { Allocation concealment } \\
\text { (selection bias) }\end{array}$ & Unclear risk & No information provided \\
\hline $\begin{array}{l}\text { Blinding of outcome as- } \\
\text { sessment (detection bias) } \\
\text { All outcomes }\end{array}$ & Unclear risk & No information provided \\
\hline $\begin{array}{l}\text { Incomplete outcome data } \\
\text { (attrition bias) } \\
\text { All outcomes }\end{array}$ & Low risk & $\begin{array}{l}\text { Dropouts accounted for } \\
\text { Dropouts }(n=4) \text { attributed to three deaths and one failure to attend assess- } \\
\text { ment }\end{array}$ \\
\hline $\begin{array}{l}\text { Free of systematic differ- } \\
\text { ences in baseline charac- } \\
\text { teristics of groups com- } \\
\text { pared? }\end{array}$ & Low risk & No significant baseline differences \\
\hline
\end{tabular}


Wu 2006 (Continued)

Did authors adjust for baseline differences in their analyses?
Low risk No information provided

Other bias Unclear risk No information provided

Xiao 2003

\section{Study characteristics}

$\begin{array}{ll}\text { Methods } & \text { Study design: RCT } \\ & \text { Method of randomisation: first divided participants into ischaemic or haemorrhagic type of stroke, fol- } \\ \text { lowed by block randomisation }\end{array}$

Participants

Number of participants: $\mathrm{n}=134$

Inclusion criteria: "Cartoid artery system, ischaemic stroke participants, no past history of stroke or no obvious deficits if had stroke history, premorbid independence in ADL, hemiplegic limb 4th grade on muscle strength, informed consent obtained and followed the 1995 Fourth National Conference on Cerebral Vascular Disease classification guidelines"

Exclusion guidelines: "Subarachnoid haemorrhage or transient ischaemic attack, vertebrobasilar arterial thrombosis, obvious awareness issues during stroke onset, hemiplegic limb $>4$ th grade on muscle strength and severe organ diseases during stroke onset"

Interventions

(1) Intensive rehabilitation group $(n=67)$

"After classifying patients as ICA or HCA, patients were randomly allocated to either the intensive rehabilitation or conventional group. Both groups received conventional treatment and rehabilitation, with rehabilitative training up to 2 weeks in duration. The intensive rehabilitation group made use of: combined Bobath and PNF therapy. This was done 3/week, 30min/session; using UTU-500 ultrasound at the same time, 1/day, $30 \mathrm{~min} / \mathrm{session"}$

The individual components delivered are listed in Table 3. Based on the individual components, this intervention is categorised as comprising modality and neurophysiological intervention

Length of intervention period: up to two weeks in duration

Number of sessions and length of individual sessions: three/wk, 30 minutes/session; using UTU-500 ultrasound at the same time, one/d, 30 minutes/session

Intervention provider: not stated

(2) Conventional group $(n=67)$

"The conventional group made use of usual therapeutic and Bobath techniques"

The individual components delivered are listed in Table 3. Based on the individual components, this intervention is categorised as comprising usual care (neurophysiological intervention)

Length of intervention period: "up to 2 weeks in duration"

Number of sessions and length of individual sessions: three/wk, 30 minutes/session

Intervention provider: not stated

This study is classified as intervention (neurophysiological) versus usual care (neurophysiological) (Table 5). The intervention group also received modality 
Xiao 2003 (Continued)

Outcomes Other secondary outcome measures: neurological deficit score, total activity ability score, ADL score

Time points when outcomes were assessed: before and after intervention

Notes Original study translated from Chinese to English

No data suitable for analysis

\section{Risk of bias}

\begin{tabular}{|c|c|c|}
\hline Bias & Authors' judgement & Support for judgement \\
\hline $\begin{array}{l}\text { Random sequence genera- } \\
\text { tion (selection bias) }\end{array}$ & Unclear risk & $\begin{array}{l}\text { First divided participants into ischaemic or haemorrhagic type of stroke, fol- } \\
\text { lowed by block randomisation. No further details provided }\end{array}$ \\
\hline $\begin{array}{l}\text { Allocation concealment } \\
\text { (selection bias) }\end{array}$ & Unclear risk & No information provided \\
\hline $\begin{array}{l}\text { Blinding of outcome as- } \\
\text { sessment (detection bias) } \\
\text { All outcomes }\end{array}$ & Unclear risk & No information provided \\
\hline $\begin{array}{l}\text { Incomplete outcome data } \\
\text { (attrition bias) } \\
\text { All outcomes }\end{array}$ & Unclear risk & No dropouts described \\
\hline $\begin{array}{l}\text { Free of systematic differ- } \\
\text { ences in baseline charac- } \\
\text { teristics of groups com- } \\
\text { pared? }\end{array}$ & Low risk & No significant baseline differences \\
\hline $\begin{array}{l}\text { Did authors adjust for } \\
\text { baseline differences in } \\
\text { their analyses? }\end{array}$ & Low risk & No information provided \\
\hline Other bias & Unclear risk & No information provided \\
\hline
\end{tabular}

Xie 2003

\section{Study characteristics}

\begin{tabular}{ll}
\hline Methods & Study design: RCT \\
& $\begin{array}{l}\text { Method of randomisation: not stated ('"4 patients were randomly divided into two groups, with } 32 \text { in } \\
\text { each one") }\end{array}$ \\
\hline Participants & $\begin{array}{l}\text { Number of participants: } \mathrm{n}=64 \\
\text { Inclusion criteria: "Every patient was diagnosed through head CT examination and reached the stan- } \\
\text { dard established by 4th National Cerebrovascular Disease Conference" }\end{array}$ \\
\hline Interventions & (1) Rehabilitation group ( $\mathrm{n}=32$ ) \\
& "Following methods were adopted in acute phase: (i) Keeping limbs in function position; (ii) Passive \\
& joint motion within its maximal bound. Beginning from healthy side to hemiplegia one, from bigger \\
joint to smaller one. Paying more attention to elbow, finger and ankle, because they are vulnerable to \\
stiff. Each joint was moved five to six times in articular direction, practising two to three times each day.
\end{tabular}


Xie 2003 (Continued)

(iii) Relax of hemiplegia side could be achieved through gentle and regular massage. Pectoral muscles were massaged to lessen contracture and avoid shoulder dysfunction." "The massage was practised five to six times each day, and each massage lasted 15 - 20 min"

"The recover phase began one to three weeks after stroke attack. Integrated treatment could be adopted to deal with this phase. It included the rehabilitation of stiff joints and spastic muscles, the training of upper and lower limbs. Several treatments could be used in joint rehabilitation, including passive motion, intermittent or constant traction, muscle massage, drug and biological feedback treatment. Both positive and passive motions were adopted in upper limb training, stepping up and down stairs. The training was practised twice a day, with 30 min each time. ADL training could be divided into lying and sitting ADL training, so as to avoid deformity and correct abnormal motion mode"

The individual components delivered are listed in Table 3. Based on the individual components, this intervention is categorised as comprising functional task training and musculoskeletal intervention (passive)

Length of intervention period: not stated

Number of sessions and length of individual sessions: dependent on stage of recovery (see above)

Intervention provider: not stated

(2) Control group $(n=32)$

No intervention

The individual components delivered are listed in Table 3. Based on the individual components, this intervention is categorised as comprising no intervention

Length of intervention period: no intervention

Number of sessions and length of individual sessions: no intervention

Intervention provider: no intervention

This study is classified as intervention (functional task training, musculoskeletal (passive)) versus no treatment (Table 4)

Outcomes Measures of independence in ADL: Barthel Index

Time points when outcomes were assessed: "first assessment began within two days after patients vital sign steady, the other two assessments were adopted in 30 days and 60 days"

Notes Short Chinese study published in English (brief report only)

\section{Risk of bias}

\begin{tabular}{lll}
\hline Bias & Authors' judgement & Support for judgement \\
\hline $\begin{array}{l}\text { Random sequence genera- } \\
\text { tion (selection bias) }\end{array}$ & Unclear risk & No information provided \\
\hline $\begin{array}{l}\text { Allocation concealment } \\
\text { (selection bias) }\end{array}$ & Unclear risk & No information provided \\
\hline $\begin{array}{l}\text { Blinding of outcome as- } \\
\text { sessment (detection bias) } \\
\text { All outcomes }\end{array}$ & Unclear risk & No information provided \\
\hline $\begin{array}{l}\text { Incomplete outcome data } \\
\text { (attrition bias) } \\
\text { All outcomes }\end{array}$ & Unclear risk & No dropouts described \\
\hline
\end{tabular}


Xie 2003 (Continued)

Free of systematic differ- Low risk "No significant difference in age or score of neural function assessment could ences in baseline characbe found between the two groups" teristics of groups compared?

\begin{tabular}{lll}
\hline $\begin{array}{l}\text { Did authors adjust for } \\
\text { baseline differences in } \\
\text { their analyses? }\end{array}$ & Low risk & \\
\hline Other bias & Unclear risk & No information provided \\
\hline
\end{tabular}

\section{Study characteristics}

\begin{tabular}{ll}
\hline Methods & Study design: RCT \\
& Method of randomisation: "After dividing patients into ischaemic or haemorrhagic type of stroke, \\
randomisation principles were followed to divide patients into control and rehabilitation treatment \\
groups"
\end{tabular}

Participants Number of participants: $\mathrm{n}=70$

Inclusion criteria: "stroke participants identified from the 1995 Fourth National Conference on Cerebral Vascular Disease classification guidelines, confirmed by CT or MRI scan as first ever stroke, Glasgow coma scale $>8$, aged $40-80$ years old, deficits in limb function, not more than 3 weeks after stroke and provided informed consent"

Exclusion criteria: "active liver disease, impaired function of the liver, kidney, congestive heart failure, dementia, history of deafness, muteness, impaired respiratory system, paralysis of four limbs"

Interventions

(1) Rehabilitation treatment group $(n=35)$

"In addition to conventional drug treatment, therapists administered 6 months (average of $12.2 \pm 9.2$ days) of one-to-one rehabilitation training to patients in the rehabilitation treatment group. Approximately one month after stroke, these patients underwent Level 1 rehabilitation in the ward, 1/day, $45 \mathrm{~min} / \mathrm{session}$, with the therapists teaching the family members and nursing aides the correct method of assisting with exercises and nursing care, requesting them to facilitate practice of exercises outwith therapy time, 1-2/day"

"Training content included: 'putting good posture, turning the body over training, self-assistance exercises on the bed (plugging the two hands, bridge-like movement, shifting on the bed, controlling the coax), the passive motion of upper limb, trunk and lower limb, sit-decubitus training, standing training, concordant training of every joint, gait training and activities of daily living training etc. Additional intervention information in reported in the text includes: sitting and lying training; facial, tongue and lip muscle training; breathing control training; balance control training"

"During 2nd and 3rd month, participants transferred to Level 2 rehabilitation hospital to continue with rehabilitation training (Level 2 rehabilitation) depending on participant's medical condition and functional recovery status, with rehabilitation delivered by Level 2 rehabilitation hospital's therapists, 2-3/week, $45 \mathrm{~min} / \mathrm{session}$, with nursing aides and family members assisting participants with exercises everyday outwith therapy time; or participants were transferred home with therapists conducting home visits $2 /$ week, assisting participants with necessary functional training, till home visits ended (Level 3 rehabilitation)"

The individual components delivered are listed in Table 3. Based on the individual components, this intervention is categorised as comprising functional task training, musculoskeletal intervention (active) and musculoskeletal intervention (passive) 
Xie 2005 (Continued)

Length of intervention period: six months

Number of sessions and length of individual sessions: dependent on stage of recovery (see above)

Intervention provider: therapists

(2) Control group $(n=35)$

"The control group underwent similar conventional drug treatment as the rehabilitation treatment group, without any rehabilitation by therapists"

The individual components delivered are listed in Table 3. Based on the individual components, this intervention is categorised as comprising no intervention

Length of intervention period: no intervention

Number of sessions and length of individual sessions: no intervention

Intervention provider: no intervention

This study was classified as intervention (functional task training, musculoskeletal (active), musculoskeletal (passive)) versus no treatment (Table 4)

\begin{tabular}{ll}
\hline Outcomes & $\begin{array}{l}\text { Other secondary outcome measures: Chinese edition of the World Health Organization quality of life } \\
\text { scale }\end{array}$ \\
& $\begin{array}{l}\text { Time points when outcomes were assessed: "at the moment of selecting and } 1,3 \text { and } 6 \text { months of } \\
\text { progress" }\end{array}$ \\
\hline Notes & No outcomes included in analysis
\end{tabular}

\section{Risk of bias}

\begin{tabular}{lll}
\hline Bias & Authors' judgement & Support for judgement \\
\hline $\begin{array}{l}\text { Random sequence genera- } \\
\text { tion (selection bias) }\end{array}$ & Unclear risk & No information provided \\
\hline $\begin{array}{l}\text { Allocation concealment } \\
\text { (selection bias) }\end{array}$ & Unclear risk & No information provided \\
\hline $\begin{array}{l}\text { Blinding of outcome as- } \\
\text { sessment (detection bias) } \\
\text { All outcomes }\end{array}$ & Low risk & Single blinding of assessor \\
\hline $\begin{array}{l}\text { Incomplete outcome data } \\
\text { (attrition bias) }\end{array}$ & Unclear risk & Dropouts due to deaths \\
All outcomes & & \\
\hline
\end{tabular}

Free of systematic differ- Low risk No significant baseline differences
ences in baseline charac-
teristics of groups com-
pared?

\begin{tabular}{lll}
\hline $\begin{array}{l}\text { Did authors adjust for } \\
\text { baseline differences in } \\
\text { their analyses? }\end{array}$ & Low risk & No information provided \\
\hline Other bias & Unclear risk & No information provided \\
\hline
\end{tabular}


Xu 1999

\section{Study characteristics}

\begin{tabular}{ll}
\hline Methods & Study design: RCT \\
& Method of randomisation: not stated \\
\hline Participants & Number of participants: $\mathrm{n}=62$ \\
& Inclusion criteria: not stated \\
\hline
\end{tabular}

Interventions

(1) Rehabilitation treatment group $(n=32)$

"Patients in the 'rehabilitation treatment' group were given clinical treatment and regular recovery training. They used various exercise treatment techniques which had Bobath and Brunnstrom as their focus"

The individual components delivered are listed in Table 3. Based on the individual components, this intervention is categorised as comprising neurophysiological intervention

Length of intervention period: one month

Number of sessions and length of individual sessions: one-to-one treatment approach, two times/d, one hour/session

Intervention provider: not stated but "with participation from family"

(2) Control group $(n=30)$

"Received 'traditional clinical treatment'"

The individual components delivered are listed in Table 3. Based on the individual components, this intervention is categorised as comprising no intervention

Length of intervention period: no intervention

Number of sessions and length of individual sessions: no intervention

Intervention provider: no intervention

This study is classified as intervention (neurophysiological) versus no treatment (Table 4)

Outcomes

Measures of independence in ADL: Barthel Index

Other secondary outcome measures: "Degree of neural defect"

Time points when outcomes were assessed: before and after intervention

Notes Original study translated from Chinese to English

\section{Risk of bias}

\begin{tabular}{lll}
\hline Bias & Authors' judgement & Support for judgement \\
\hline $\begin{array}{l}\text { Random sequence genera- } \\
\text { tion (selection bias) }\end{array}$ & Unclear risk & No information provided \\
\hline $\begin{array}{l}\text { Allocation concealment } \\
\text { (selection bias) }\end{array}$ & Unclear risk & No information provided \\
\hline $\begin{array}{l}\text { Blinding of outcome as- } \\
\text { sessment (detection bias) }\end{array}$ & Unclear risk & No information provided \\
\hline
\end{tabular}


Xu 1999 (Continued)

All outcomes

\begin{tabular}{lll}
\hline $\begin{array}{l}\text { Incomplete outcome data } \\
\text { (attrition bias) } \\
\text { All outcomes }\end{array}$ & Unclear risk & \\
\hline $\begin{array}{l}\text { Free of systematic differ- } \\
\text { ences in baseline charac- } \\
\begin{array}{l}\text { teristics of groups com- } \\
\text { pared? }\end{array}\end{array}$ & Unclear risk & No information provided \\
\hline $\begin{array}{l}\text { Did authors adjust for } \\
\text { baseline differences in } \\
\text { their analyses? }\end{array}$ & Unclear risk & No information provided \\
\hline $\begin{array}{l}\text { Other bias } \\
\end{array}$ & Unclear risk & No information provided \\
\hline
\end{tabular}

\section{Xu 2003a}

\section{Study characteristics}

\begin{tabular}{ll}
\hline Methods & Study design: $\mathrm{RCT}$ \\
& Method of randomisation: not stated \\
& "186 patients were randomly divided into rehabilitation group $(\mathrm{n}=94)$ and control group $(\mathrm{n}=92) "$ \\
\hline Participants & Number of participants: $\mathrm{n}=186$ \\
& Inclusion criteria: "2000-11/2002-05, all cases conformed to 1995 Fourth National Cerebrovascular Dis- \\
& ease Diagnosis Standards, proved by CT" \\
& Exclusion criteria: "those of mild type or combined with mental disturbance, disorder of conscious- \\
& ness, mixed and sensory aphasia were excluded"
\end{tabular}

Interventions (1) Rehabilitation group $(\mathrm{n}=94)$

"rehabilitation treatment of acute phase advanced by Yu duisheng of rehabilitation research center (1997), Beijing"

"Early rehabilitation procedure included: (i) Design of position of healthy limbs (massage position) including supine position, lateral position with affected limbs at lower side and healthy limbs at upper side and lateral position with affected limbs at upper side and healthy limbs at lower side. (ii) Training of motion range of joints; (iii) Bridging training; (iv) Balance training including sitting position and erect position; (v) Walk training"

The individual components delivered are listed in Table 3. Based on the individual components, this intervention is categorised as comprising functional task training, musculoskeletal intervention (active) and musculoskeletal intervention (passive)

Length of intervention period: 21 days

Number of sessions and length of individual sessions: "once a day"

Intervention provider: not stated

(2) Control group ( $n=92)$

"Two groups received routine treatment in department of neurology" 
Xu 2003a (Continued)

\section{No treatment}

The individual components delivered are listed in Table 3. Based on the individual components, this intervention is categorised as comprising no intervention

Length of intervention period: no intervention

Number of sessions and length of individual sessions: no intervention

Intervention provider: no intervention

This study is classified as intervention (functional task training, musculoskeletal (active), musculoskeletal (passive)) versus no treatment (Table 4)

Outcomes
Measures of independence in ADL: Barthel Index
Measures of motor function: Fugl-Meyer 'Analysis'
Measures of voluntary movement: walking recovery rate
Time points when outcomes were assessed: "After first evaluation, rehabilitation group received reha-
bilitation treatment of acute phase advanced by Yu duisheng of rehabilitation research center (1997),
Beijing, once a day for 21 days and evaluated for the second time after treatment"

Notes Short Chinese study published in English-brief report only

\section{Risk of bias}

\section{Bias}

Random sequence genera- Unclear risk tion (selection bias)

\begin{tabular}{lll}
\hline $\begin{array}{l}\text { Allocation concealment } \\
\text { (selection bias) }\end{array}$ & Unclear risk & No information provided
\end{tabular}

Blinding of outcome as- Unclear risk No information provided
sessment (detection bias)

All outcomes

Incomplete outcome data Unclear risk $\quad$ No dropouts described
(attrition bias)

\section{Authors' judgement Support for judgement}

No information provided

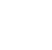


Xu 2003b (Continued)

Methods Study design: RCT

Method of randomisation: not stated ("the patients were divided randomly into ..")

Number of participants: $\mathrm{n}=180$

Inclusion criteria: "There were 180 in-patients recruited by Department of Neurology of our hospital, which were diagnosed as stroke and belonged to initial attack. All the diagnosis of patients accorded with the standards made by Fourth National Cerebrovascular Meeting"

Exclusion criteria: "The patients diagnosed as light type (score of TMA > 85) and combined with the disturbance of consciousness and sensory aphasia were excluded"

Interventions

(1) Rehabilitation group ( $\mathrm{n}=92)$

"The rehabilitation included facilitation of nerve and muscle, controlling of posture and functional training et al, one hour every day and continuously for four weeks"

The individual components delivered are listed in Table 3. Based on the individual components, this intervention is categorised as comprising functional task training, musculoskeletal intervention (passive) and neurophysiological intervention

Length of intervention period: four weeks

"The earliest rehabilitation training began after two days of disease and the latest began after ten days of disease (with the average of 6 days)"

Number of sessions and length of individual sessions: "one hour every day"

Intervention provider: not stated

(2) Control group $(n=88)$

No intervention

The individual components delivered are listed in Table 3. Based on the individual components, this intervention is categorised as comprising no intervention

Length of intervention period: no intervention

Number of sessions and length of individual sessions: no intervention

Intervention provider: no intervention

This study is classified as intervention (functional task training, musculoskeletal (passive), neurophysiological) versus no treatment (Table 4)

Outcomes

Measures of independence in ADL: Barthel Index

Measures of motor function: Fugl-Meyer Assessment

Time points when outcomes were assessed: "The assessment was given on the 28th day respectively before and after treatment"

Notes Short Chinese study published in English: brief report only

This paper was written in English, and the extracts above are direct quotes. No definition of TMA is provided.

\section{Risk of bias}


Xu 2003b (Continued)
Random sequence genera- Unclear risk
No information provided
tion (selection bias)
"the patients were divided randomly into rehabilitation group ( $n=92)$ and control group $(\mathrm{n}=88) "$

\begin{tabular}{lll}
\hline $\begin{array}{l}\text { Allocation concealment } \\
\text { (selection bias) }\end{array}$ & Unclear risk information provided
\end{tabular}

\begin{tabular}{lll}
\hline $\begin{array}{l}\text { Blinding of outcome as- } \\
\text { sessment (detection bias) } \\
\text { All outcomes }\end{array}$ & Unclear risk & \\
\hline $\begin{array}{l}\text { Incomplete outcome data } \\
\text { (attrition bias) }\end{array}$ & Unclear risk & No dropouts described \\
All outcomes & \\
\hline
\end{tabular}

\begin{tabular}{lll}
\hline $\begin{array}{l}\text { Free of systematic differ- } \\
\text { ences in baseline charac- } \\
\text { teristics of groups com- } \\
\text { pared? }\end{array}$ & Low risk & \\
\hline $\begin{array}{l}\text { Did authors adjust for } \\
\text { baseline differences in } \\
\text { their analyses? }\end{array}$ & Low risk & No information provided \\
\hline Other bias & Unclear risk & No information provided \\
\hline
\end{tabular}

\section{Study characteristics}

\begin{tabular}{ll}
\hline Methods & Study design: RCT \\
& Method of randomisation: not stated \\
\hline Participants & Number of participants: $\mathrm{n}=57$ \\
& $\begin{array}{l}\text { Inclusion criteria: "Ischaemic or haemorrhagic stroke, first ever stroke, aged less than } 80 \text { years old, con- } \\
\text { firmed by CT or MRI scan, Glasgow Coma Scale }>8 \text { and no physical deficits from other illnesses before } \\
\text { stroke onset" } \\
\text { Exclusion criteria: "Participants who underwent thrombolytic therapy or surgery for haemorrhage, sub- } \\
\text { arachnoid haemorrhage, transient ischaemic attack, reversible ischaemic neurological deficit (RIND), } \\
\text { worsening condition, new tissue ischaemia or haemorrhage, Mini mental state examination (MMSE) (il- } \\
\text { literacy } \leq 17, \text { primary learning } \leq 20, \text { secondary learning and other sections } \leq 24) \text { and severe loss of lan- } \\
\text { guage ability affecting communication" }\end{array}$ \\
\hline
\end{tabular}

Interventions (1) Rehabilitation group $(\mathrm{n}=30)$

"Both groups had similar medical treatment. The 'rehabilitation' group underwent comprehensive treatment techniques focused on Bobath, including the following content: (i) passive ranging of all affected limbs with shoulder (extension, circumduction, various range of motion involving the scapular and torso regions), hand (wrist extension and extension exercises of all finger joints), hip and foot joints, from proximal to distal joints, from small to larger range of motion, seeking to achieve the largest range of motion within pain-free threshold; (ii) rolling, sitting up training; (iii) bridging exercises; (iv) sitting and standing balance training; (v) getting up from bed training; (vi) gait training; (vii) ADL training" 
The individual components delivered are listed in Table 3. Based on the individual components, this intervention is categorised as comprising functional task training, musculoskeletal intervention (passive) and neurophysiological intervention

Length of intervention period: one month after stroke

Number of sessions and length of individual sessions: one/d, 40 to 50 minutes/session, five/wk

Intervention provider: not stated but "on commencement of intervention, participants were taught appropriate positioning and family members were taught basic exercises so they could supervise participants"

(2) Control group $(n=27)$

"Did not receive any rehabilitation training or guidance"

The individual components delivered are listed in Table 3. Based on the individual components, this intervention is categorised as comprising no intervention

Length of intervention period: no intervention

Number of sessions and length of individual sessions: no intervention

Intervention provider: no intervention

This study is classified as intervention (functional task training, musculoskeletal (passive), neurophysiological) versus no treatment (Table 4)

Measures of independence in ADL: Barthel Index
Measures of motor function: Fugl-Meyer Assessment
Other secondary outcome measures: "degree of deficit of neural function (DDNF)"
Time points when outcomes were assessed: within seven to 23 days after stroke and one month after
stroke

Notes Original study translated from Chinese to English

\section{Risk of bias}

\begin{tabular}{|c|c|c|}
\hline Bias & Authors' judgement & Support for judgement \\
\hline $\begin{array}{l}\text { Random sequence genera- } \\
\text { tion (selection bias) }\end{array}$ & Unclear risk & No information provided \\
\hline $\begin{array}{l}\text { Allocation concealment } \\
\text { (selection bias) }\end{array}$ & Unclear risk & No information provided \\
\hline $\begin{array}{l}\text { Blinding of outcome as- } \\
\text { sessment (detection bias) } \\
\text { All outcomes }\end{array}$ & Unclear risk & No information provided \\
\hline $\begin{array}{l}\text { Incomplete outcome data } \\
\text { (attrition bias) } \\
\text { All outcomes }\end{array}$ & Unclear risk & No dropouts described \\
\hline $\begin{array}{l}\text { Free of systematic differ- } \\
\text { ences in baseline charac- } \\
\text { teristics of groups com- } \\
\text { pared? }\end{array}$ & Low risk & No significant baseline differences \\
\hline
\end{tabular}


Xu 2004 (Continued)
Did authors adjust for
Low risk
No information provided baseline differences in their analyses?

Other bias Unclear risk No information provided

\title{
Xue 2006
}

\section{Study characteristics}

$\begin{array}{ll}\text { Methods } & \text { Study design: RCT } \\ & \text { Method of randomisation: not stated ("enrolled patients were randomly divided into training group } \\ (\mathrm{n}=78) \text { and control group }(\mathrm{n}=72) \text { at admission") }\end{array}$

\section{Participants}

\author{
Number of participants: $n=150$
}

Inclusion criteria: "patients were accorded with the diagnostic standards about stroke set by the Fifth National Academic Meeting for Cerebrovascular Disease (1996), confirmed by CT and MRI, and they were all informed with the interventions and the items of evaluation"

Exclusion criteria: "Patients with infarction of vertebral basilar artery, transient ischemic attack and subarachnoid hemorrhage were excluded"

"those in the training group received rehabilitation training by motor relearning program and Bobath technique"
"The rehabilitation training began after the vital signs became stable within 24 hours to 3 days after at- tack for the patients with cerebral infarction and 48 hours to five days after attack for those with cere- bral hemorrhage respectively"

\begin{abstract}
"The patients in the training group passively or actively learned, imitated and reinforced following the normal motor pattern of limbs. (i) Lying position: The patients should keep the anti-spasm posture in the supine position, contralateral and ipsilateral lying positions, and the postures should be changed regularly; The patients should exercise each joint passively; turn the body over and move actively; They should also perform the bringing training, and the weight loading training for the affected upper limb. (ii) Sitting position: The patients should finish the conversion from a lying position to a sitting one independently; They also took the training in crawling position or kneeling position, as well as training for sitting balance of grade 3. (iii) Standing position: The patients should finish the conversion from a sitting position to a standing one independently; They also took the training for standing balance, weight loading training for the affected lower limb. The dorsiflexion of ankle was extended repeatedly. The walking training should be performed when the affected lower limb could support 2/3 of body mass. (iv) Walking training: In a standing position supported by the affected lower limb, the unaffected one stepped in small range forward and backward; Each process of balancing and controlling standing position was trained by supporting the body mass by the affected lower limb; In swinging position, the independent movement of the ipsilateral knee joint was trained in alternation of flexion and extension, in order to reach the results of without raising coxa, but relaxing knee, stepping with flexing knee and the dorsiflexion of ankle when the heel touch the ground"
\end{abstract}

The individual components delivered are listed in Table 3. Based on the individual components, this intervention is categorised as comprising functional task training, musculoskeletal intervention (active), musculoskeletal intervention (passive) and neurophysiological intervention

Length of intervention period: one month

Number of sessions and length of individual sessions: "three times a day in the morning, at noon and in the evening respectively, 30 minutes for each time" 
Xue 2006 (Continued)

Intervention provider: not stated

(2) Control group $(n=72)$

"All the patients were given routine treatments, including managing blood pressure, maintaining the balance of hydrolyte and electrolure, reducing intracranial pressure by dehydration, and venous injection of citicoline"

The individual components delivered are listed in Table 3. Based on the individual components, this intervention is categorised as comprising no intervention

Length of intervention period: no intervention

Number of sessions and length of individual sessions: no intervention

Intervention provider: no intervention

This study is classified as intervention (functional task training, musculoskeletal (active), musculoskeletal (passive), neurophysiological) versus no treatment (Table 4)

Outcomes Measures of independence in ADL: Modified Barthel Index

Measures of motor function: Fugl-Meyer Assessment

Other secondary outcome measures: neurological deficit score

Time points when outcomes were assessed: "patients were evaluated by the professional group (the fourth and fifth authors) before treatment and 1 month after treatment respectively"

Notes This paper was written in English, and the extracts above are direct quotes

\section{Risk of bias}

Bias Authors' judgement Support for judgement

Random sequence genera- Unclear risk No information provided
tion (selection bias)

\begin{tabular}{lll}
\hline $\begin{array}{l}\text { Allocation concealment } \\
\text { (selection bias) }\end{array}$ & Unclear risk & No information provided \\
\hline $\begin{array}{l}\text { Blinding of outcome as- } \\
\text { sessment (detection bias) } \\
\text { All outcomes }\end{array}$ & High risk & $\begin{array}{l}\text { "patients were evaluated by the professional group (the fourth and fifth au- } \\
\text { thors) before treatment and } 1 \text { month after treatment respectively" }\end{array}$ \\
\hline $\begin{array}{l}\text { Incomplete outcome data } \\
\text { (attrition bias) } \\
\text { All outcomes }\end{array}$ & Low risk & No dropouts \\
& $\begin{array}{l}\text { "All the } 150 \text { patients with post-stroke hemiplegia were involved in the analysis, } \\
\text { no one missed" }\end{array}$ \\
\hline
\end{tabular}

\begin{tabular}{|c|c|c|}
\hline $\begin{array}{l}\text { Free of systematic differ- } \\
\text { ences in baseline charac- } \\
\text { teristics of groups com- } \\
\text { pared? }\end{array}$ & Low risk & $\begin{array}{l}\text { "the baseline data were comparable between the two groups, and there were } \\
\text { no significant differences }(P>0.05) "\end{array}$ \\
\hline $\begin{array}{l}\text { Did authors adjust for } \\
\text { baseline differences in } \\
\text { their analyses? }\end{array}$ & Low risk & No information provided \\
\hline Other bias & Unclear risk & No information provided \\
\hline
\end{tabular}




\section{Study characteristics}

\begin{tabular}{ll}
\hline Methods & Study design: RCT \\
& Method of randomisation: not stated \\
\hline Participants & Number of participants: $\mathrm{n}=78$ \\
& Inclusion criteria: not stated \\
\hline
\end{tabular}

Interventions

(1) Rehabilitation group $(n=40)$

"Both groups of patients received conventional neurological drug treatment, treatment group after initial assessment commenced rehabilitative treatment procedure. The rehabilitative training procedure was as follows: based on the extent of the patient's hemiplegia and affected limbs' functional status, procedure was divided into 3 phases: early phase, rehabilitative treatment on bed, and rehabilitative treatment after leaving the bed"

"(i) Early phase: commenced $48 \mathrm{~h}$ after patient was medically stable and neurological symptoms stopped progressing. Healthy limbs: therapists should be familiar with positioning of healthy limbs, and let family members understand the reason for doing so and to master these techniques. Upper limb: a pillow slightly higher than torso was placed below extended upper limb and maintained upper limb in supine position; Pelvis and lower limb: those with tendency for lower limb to buckle, while in lying position should have a pillow supporting the affected side of the limb to prevent excessive flexion. Pillows were used to prevent excessive abduction, external rotation. Participants with excessive ankle curvature or inversion used a splint board for positioning. Passive joint range of motion: from distal to proximal, 10x/joint, 2/day. Movement should be of ease, of short duration, used Brunnstrom technique's rapid traction, stimulation through overpressuring of joints, 2/day, 15min/session"

"(ii) rehabilitative treatment on bed: This phase commenced when participants possessed good sense of awareness and communication ability: using Bobath method of holding hands, with elbow extension, shoulder elevation to 90 degrees, using both upper limbs to lead during rolling, before turning the hips. After gaining independence in rolling, participants can commence bridging exercises, ankle dorsiflexion exercises. Sitting up from bed training: increase angle of bed to 30 degrees, once participants could tolerate longer than 30 mins in the angle, the angle of bed is increased by 10 degrees, until participants can maintain at 90 degrees for 30 mins. Lying to sitting and sitting balance training. The above were practised 2/day, $30 \mathrm{~min} / \mathrm{session}$, increasing to 3-4/day if participants had no discomfort"

"(iii) Rehabilitative treatment after leaving the bed: In the rehabilitation centre, standing upright training -> forward, backward, left and right weight shifting training $>>$ training by using the affected lower limb to support (single leg standing) -> limb loading of the affected lower limb (rising training, sitting down training, sit to stand training) -> ankle dorsiflexion $->$ anterior, posterior pelvic tilt training $->$ gait training with pelvic stabilisation (parallel bar $->$ flat ground $->$ slope -> stairs), 2/day, $1 \mathrm{hr} / \mathrm{session}$. Average length of treatment: 38 days"

The individual components delivered are listed in Table 3. Based on the individual components, this intervention is categorised as comprising assistive devices, functional task training and musculoskeletal intervention (passive)

Length of intervention period: 38 days

Number of sessions and length of individual sessions: session length dependent on technique applied (see above)

Intervention provider: not stated

(2) Control group $(n=38)$

"Received conventional neurological drug treatment but did not receive any standard rehabilitative treatment" 
Yan 2002 (Continued)

The individual components delivered are listed in Table 3. Based on the individual components, this intervention is categorised as comprising no intervention

Length of intervention period: no intervention

Number of sessions and length of individual sessions: no intervention

Intervention provider: no intervention

This study is classified as intervention (functional task training, musculoskeletal (passive)) versus no treatment (Table 4). The intervention group also received assistive devices

Measures of Independence in ADL: Barthel Index
Other secondary outcome measures: Brunnstrom, three-level balance rating
Time points when outcomes were assessed: within 24 hours before start of intervention and on the last
day of intervention

Notes $\quad$ Original study translated from Chinese to English

\section{Risk of bias}

\begin{tabular}{|c|c|c|}
\hline Bias & Authors' judgement & Support for judgement \\
\hline $\begin{array}{l}\text { Random sequence genera- } \\
\text { tion (selection bias) }\end{array}$ & Unclear risk & No information provided \\
\hline $\begin{array}{l}\text { Allocation concealment } \\
\text { (selection bias) }\end{array}$ & Unclear risk & No information provided \\
\hline $\begin{array}{l}\text { Blinding of outcome as- } \\
\text { sessment (detection bias) } \\
\text { All outcomes }\end{array}$ & Unclear risk & No information provided \\
\hline $\begin{array}{l}\text { Incomplete outcome data } \\
\text { (attrition bias) } \\
\text { All outcomes }\end{array}$ & Unclear risk & No dropouts described \\
\hline $\begin{array}{l}\text { Free of systematic differ- } \\
\text { ences in baseline charac- } \\
\text { teristics of groups com- } \\
\text { pared? }\end{array}$ & Low risk & No significant baseline differences \\
\hline $\begin{array}{l}\text { Did authors adjust for } \\
\text { baseline differences in } \\
\text { their analyses? }\end{array}$ & Low risk & No information provided \\
\hline Other bias & Unclear risk & No information provided \\
\hline
\end{tabular}

Yelnik 2008

\section{Study characteristics}

\begin{tabular}{ll}
\hline Methods & Study design: "Prospective, multicenter, randomised parallel-group trial with a single-blind evalua- \\
tion"
\end{tabular}


Yelnik 2008 (Continued)

Method of randomisation: "After patients gave informed consent, the trial statistician generated the randomisation sequence using random number tables. Randomization was stratified by center"

Participants Number of participants: $n=68$

Inclusion criteria: "Hemiplegia after a single hemispheric stroke due to an infarct or hemorrhage shown by computerized tomography or magnetic resonance imaging within 3 to 15 months prior to entry, at onset of stroke, subjects had to be unable to walk for at least 2 weeks, but not exceeding 3 months. Walking was defined as the ability to walk at least 50 meters with an orthosis or cane if needed (but without human assistance). Aged less than 80 years old, ambulatory, and living at home"

Exclusion criteria: "a previous history of walking disorder, cognitive disorders that prevented comprehension of the rehabilitation program, and history of a vestibular disorder"

Interventions
(1) NDT-based treatment $(n=35)$

"Based on global sensory motor rehabilitation derived from the neurodevelopmental concepts described by Bobath, more attention paid to the quality of the gesture and gait control, the spasticity, and abnormal movement inhibition than to the quantity of exercise and an increase of the difficulty from one session to another depending on the ability of the patients"

"Sessions 1 to 4: Exercises conducted on the Bobath platform, weight shifting, waist dissociation, pelvis control, crawling, turning over, four footing, and standing on the knees. Sessions 5 to 8: Exercises on the edge of the platform in sitting position, transfers from lying to sitting, sitting to standing, sitting on the platform to a chair, upper limb used for bearing. Analytic exercises for upper limb were associated for a maximum of one third of the session. Sessions 9 to 20: Walking in the corridor and on the steps, control of the weight bearing and shifting, quality of the heel strike, knee control, and waist dissociation"

"During the 20 sessions, visual deprivation, head movements, or training with unstable bases of support were forbidden for the progression of exercise difficulties"

The individual components delivered are listed in Table 3. Based on the individual components, this intervention is categorised as comprising functional task training and neurophysiological intervention

Length of intervention period: "Physical therapy had to begin within 7 days, and conducted 5 days a week for the following 4 weeks"

Number of sessions and length of individual sessions: total of 20 successive sessions conducted five days a week

"Each session lasted 60 to 70 minutes, depending on the rest required, and included 5 minutes for spasticity inhibition, 40 to 45 minutes of exercises specific to the session, 10 to 20 minutes of rest distributed throughout the session, and related to need"

Intervention provider: not stated

(2) Multisensorial $(n=33)$

"Physical rehabilitation based on the manipulation of the sensory information required to maintain balance, attention being paid to the amount of exercise, that is, duration and intensity, rather than the quality of the movement. Most of the exercises were conducted in visual deprivation, thus challenging the selection and synthesis by the brain of vestibular and somatosensory information"

"Each type of exercise was related to the patient's progress, with progression under visual control for repetition of the exercises, then as much under visual deprivation as possible, and using unstable planes and foam ground-sheet, tilting the head back, rightward and leftward. The duration of the exercises under visual deprivation was not exactly fixed and took approximately half of the session"

"The exercises had to be repeated for patients to learn them and moreover improve their performance in terms of duration or intensity by slowly increasing the difficulty. There were 4 types of modalities, conducted as follows: sessions 1 to 4, modality 1 ; sessions 5 to 8 , modality 2; sessions 9 to 20, by alternating modality 3 once and modality 4 twice. Modality 1: On the foam Bobath platform, four footing, 
Yelnik 2008 (Continued)

standing on the knees, anteroposterior and lateral weight shifting, moving objects with the upper limb, external destabilization. Modality 2: Sitting on the edge of the platform and sitting on a ball, weight shifting, upper limb movements, moving objects with the upper limb, external destabilizations. Modality 3: Static standing with feet together, tandem position, one foot standing, control of weight shifting, moving objects with upper limbs, external destabilizations. Modality 4: Walking with movements of the upper limbs, while speaking, with external destabilization, walking laterally and backward, 10 minutes of treadmill training without upper limb support, opening eyes at various speeds, closing eyes at constant speed. In each modality, the variations that can be used were head movements, foam support, unstable platform, rolling skate, irregular floor, and constant visual deprivation"

The individual components delivered are listed in Table 3. Based on the individual components, this intervention is categorised as comprising functional task training

Length of intervention period: "Physical therapy had to begin within 7 days, and conducted 5 days a week for the following 4 weeks"

Number of sessions and length of individual sessions: total of 20 successive sessions conducted five days a week

"Each session lasted 60 to 70 minutes, depending on the rest required and included 5 minutes for spasticity inhibition, 30 to 35 minutes to specific modalities, 10 minutes of walking and stepping, and 10 to 20 minutes of rest distributed throughout the session"

Intervention provider: not stated

This study is classified as active intervention one (functional task training, neurophysiological) versus active intervention two (functional task training) (Table 6)

Measures of postural control and balance: Berg Balance Scale, posturographic limits of stability

Measures of voluntary movements: speed of walking, percentage of double-stance phase, time to climb 10 steps and return, daily time of walking (minutes)

Measures of quality of life and social isolation: Nottingham Health Profile

Other secondary outcome measures: security sensation during walking, number of falls since stroke

Time points when outcomes were assessed: "The first visit for evaluation (day 0 [D0]) was conducted by one of the blinded evaluators. Posttreatment evaluation (day 30 [D30]) was carried out within 7 days of the end of the physical rehabilitation program. The second posttreatment evaluation (day 90 [D90]) was carried out 3 months after the first evaluation"

Data not suitable for analysis

\section{Risk of bias}

\begin{tabular}{lll}
\hline Bias & Authors' judgement & Support for judgement \\
\hline $\begin{array}{l}\text { Random sequence genera- } \\
\text { tion (selection bias) }\end{array}$ & Low risk & $\begin{array}{l}\text { "trial statistician generated the randomization sequence using random num- } \\
\text { ber tables. Randomization was stratified by center" }\end{array}$ \\
\hline $\begin{array}{l}\text { Allocation concealment } \\
\text { (selection bias) }\end{array}$ & Low risk & By trial statistician \\
\hline $\begin{array}{l}\text { Blinding of outcome as- } \\
\text { sessment (detection bias) } \\
\text { All outcomes }\end{array}$ & Low risk & $\begin{array}{l}\text { "the first visit for evaluation (day 0 [D0]) was conducted by one of the blinded } \\
\text { evaluators" }\end{array}$ \\
\hline
\end{tabular}


Yelnik 2008 (Continued)

$\begin{array}{ll}\begin{array}{l}\text { Incomplete outcome data Low risk } \\ \text { (attrition bias) }\end{array} & \text { Dropouts accounted for } \\ \text { All outcomes } & \text { "In the NDT-based group, } 1 \text { patient had to stop after } 5 \text { sessions of physical } \\ & \text { therapy for carotid surgery, which had not been planned. He could not be as- } \\ & \text { sessed. In the multisensorial group, } 1 \text { patient was lost to follow-up between } \\ \text { D30 and D90 and another because of an adverse event unrelated to the treat- } \\ \text { ment" }\end{array}$

Free of systematic differ- Low risk "no differences were found at entry between groups"
ences in baseline characteristics of groups compared?

\begin{tabular}{|c|c|c|}
\hline $\begin{array}{l}\text { Did authors adjust for } \\
\text { baseline differences in } \\
\text { their analyses? }\end{array}$ & Low risk & No information provided \\
\hline Other bias & Unclear risk & $\begin{array}{l}\text { Deviations from protocol reported } \\
\text { "Two patients had fewer than } 16 \text { physical therapy sessions ( } 12 \text { and } 15) \text {, but } \\
\text { they could be assessed at D } 30 \text { and D90. For } 6 \text { patients, the time lag for the first } \\
\text { assessment was longer than } 45 \text { days }(46,47,2 \cdot 48 \text {, and } 2.49 \text { days)" }\end{array}$ \\
\hline
\end{tabular}

Yin 2003a

\section{Study characteristics}

Study design: RCT with three groups
Method of randomisation: not stated
"among them, randomly selected 30 persons as the rehabilitation group, and another 30 randomly se-
lected persons were grouped into rehabilitation with intermediate frequency"

Participants Number of participants: $n=90$

Inclusion criteria: "All the patients selected were coincident with the diagnosis criteria of the second national cerebral vascular disease conference and diagnosed by CT or MRI. The patients had a stable life signs and were 2 weeks after stroke"

Exclusion criteria: "abnormal in liver, kidney, heart and lung function"

Interventions

(1) Rehabilitation group $(\mathrm{n}=30)$

"the routine drugs in three groups were same"

"Patients in the rehabilitation group were administered with rehabilitation exercise and general education of rehabilitation"

"We employed Bobath method on hemiplegia extremities. Pay attention to the position of the suffered extremities. When the patients lying on the healthy side, suffered upper limb were padded with a pillow and the upper limb were straight, with center of the palm down. The wrists were lifted with a pad. When the patients lied with the suffered side, suffered should extended forward, elbow extended and center of the palm down. For cases with the increased strength in flexor muscle, finger differentiation board or pad [was] put in the center of the hand and keep the finger straight forward as much as possible. The lower [limbs] were lightly flexioned, with back of the foot and lower leg [kept] perpendicular as much as possible. When the patients lying with back, suffered upper limb were in lightly extension conditions, with elbow lightly flexed, support shoulder and hand with pad to keep center of the palm up and keep upper limb posterior rotation position to prevent scapula down and dislocation of shoulder 
Yin 2003a (Continued)

joint. Lower limb, pelvis and hip were supported with pad to keep perpendicular conditions between back of the foot and lower leg...Turn exercise, sitting exercise and standing exercise were according to regulations of Bobath adult hemiplegia exercise"

The individual components delivered are listed in Table 3. Based on the individual components, this intervention is categorised as comprising assistive devices, functional task training, musculoskeletal intervention (passive) and neurophysiological intervention

Length of intervention period: not stated

Number of sessions and length of individual sessions: "rehabilitation therapy lasted for 40 min with once a day"

Intervention provider: not stated

(2) Rehabilitation with therapy with intermediate frequency $(n=30)$

The individual components delivered are listed in Table 3. Based on the individual components, this intervention is categorised as comprising assistive devices, functional task training, musculoskeletal intervention (passive) and neurophysiological intervention

Length of intervention period: not stated

Number of sessions and length of individual sessions: not stated

Intervention provider: not stated

(3) Control group $(n=30)$

"another 30 persons in internal neurology department were selected as the control group with pharmacy treatment alone"

"No rehabilitation exercise or intermediate frequency therapy were administered in control group"

The individual components delivered are listed in Table 3. Based on the individual components, this intervention is categorised as comprising no intervention

Length of intervention period: no intervention

Number of sessions and length of individual sessions: no intervention

Intervention provider: no intervention

This study is classified as intervention (functional task training, musculoskeletal (passive), neurophysiological) versus no treatment (Table 4). The intervention group also received assistive devices

Outcomes Measures of motor function: Fugl-Meyer Assessment

Time points when outcomes were assessed: "the patients in three groups were evaluated at the beginning, 2 weeks, 4 weeks and 8 weeks after therapy and the patients in control group were evaluated in their family"

Notes Short Chinese study published in English

Not clear how the dose varied between groups (1) and (2): both groups used for analysis, with control group 'shared' between

Mean and SD computed from categorical data

\section{Risk of bias}


Yin 2003a (Continued)

Random sequence genera- High risk tion (selection bias)

\begin{abstract}
Not stated
"among them, randomly selected 30 persons as the rehabilitation group, and another 30 randomly selected persons were grouped into rehabilitation with intermediate frequency"

Unclear how the control group was selected: "another 30 persons in internal neurology department were selected as the control group with pharmacy treatment alone"
\end{abstract}

\begin{tabular}{lll}
\hline $\begin{array}{l}\text { Allocation concealment } \\
\text { (selection bias) }\end{array}$ & Unclear risk & No information provided
\end{tabular}

\begin{tabular}{lll}
\hline $\begin{array}{l}\text { Blinding of outcome as- } \\
\text { sessment (detection bias) }\end{array}$ & Unclear risk & No information provided
\end{tabular}

All outcomes

\begin{tabular}{|c|c|c|}
\hline $\begin{array}{l}\text { Incomplete outcome data } \\
\text { (attrition bias) } \\
\text { All outcomes }\end{array}$ & Unclear risk & No dropouts described \\
\hline $\begin{array}{l}\text { Free of systematic differ- } \\
\text { ences in baseline charac- } \\
\text { teristics of groups com- } \\
\text { pared? }\end{array}$ & Low risk & $\begin{array}{l}\text { Limited information available regarding the baseline characteristics. Gender } \\
\text { and age are similar in the rehabilitation groups but there is a potential gen- } \\
\text { der bias in the control group. However, baseline Fugl-Meyer scores are similar } \\
\text { across all groups }\end{array}$ \\
\hline
\end{tabular}

\begin{tabular}{ll}
\hline $\begin{array}{l}\text { Did authors adjust for } \\
\text { baseline differences in }\end{array}$ & Low risk
\end{tabular}
their analyses?

Other bias Unclear risk No information provided

Zhang 1998

\section{Study characteristics}

\begin{tabular}{ll}
\hline Methods & Study design: RCT \\
& Method of randomisation: not stated ("participants were randomly allocated to 2 groups") \\
\hline Participants & Number of participants: $\mathrm{n}=56$ \\
& $\begin{array}{l}\text { Inclusion criteria: "patients were diagnosed using clinical appearance and cerebral CT as having cere- } \\
\text { bro-vascular disease" }\end{array}$
\end{tabular}

Interventions
(1) Early rehabilitation group $(\mathrm{n}=29)$
"The intervention group..underwent early rehabilitation therapy in conjunction with normal medical therapy'"
"Stage 1 (lying exercises): The patient is without any independent movement, and is in a state of flaccid hemiplegia. The patient is placed in a functional position and lightly massaged on the hands and limbs. Limbs are passively extended and retracted in small and large movements. This is in addition to rolling the patient on the bed and single and double leg bridge movements. The goal is to gradually lead the patient to balancing the trunk in a sitting position"
"Stage 2 (sitting exercises): When the patient gains some muscle strength and muscle groups can react and work in groups, continue the exercises described above until the goals in stage one are achieved. In


the meantime, add balancing exercises as well as resistance training in the sitting position. At the same time, stimulate sensory input by using cold and hot compresses and light taps to the antagonistic muscles. Gradually lead the patient into training for balancing while standing up"

"Stage 3 (standing exercises): When the patient is at the stage where his limbs spasm and can freely initiate movement in groups, continue the exercises in stage 2 until goals are met as well as correcting and controlling irregular positions and increasing normal movement and co-ordination. In order to prepare for walking, exercise the extensor carpi muscles, supination of forearm, standing with support, standing with weights on the lower limbs and walking with support"

"Stage 4 (walking exercises): when the patient's spasming eases, continue to complete the goals set out in stage 3 as well as walking indoors and on stairs, correcting walking posture, co-ordination and completing ADL activities"

"Patients about to be discharged from hospital were also trained to perform ADL. For individuals with slow recovery of function, we encouraged them to overcome dependence on others and actively participate in social activities. For some patients, it was necessary to advise occupational training"

"Psychological therapy: Patients with depression, pessimism, loss of confidence and unwillingness to cooperate with therapy were promptly given psychological care, allowing the patient to achieve optimal psychological status, as a proactive approach from the patient to rehabilitation is essential"

The individual components delivered are listed in Table 3. Based on the individual components, this intervention is categorised as comprising functional task training, musculoskeletal intervention (active), musculoskeletal intervention (passive) and neurophysiological intervention

Length of intervention period: not stated

Number of sessions and length of individual sessions: "Therapy was conducted in a one-to-one approach in one hour sessions once daily"

Intervention provider: not stated but does mention that "therapy was conducted... with the help of the patient's family"

(2) Control group $(n=27)$

"underwent conventional neurological medical therapy"

The individual components delivered are listed in Table 3. Based on the individual components; this intervention is categorised as comprising no intervention

Length of intervention period: no intervention

Number of sessions and length of individual sessions: no intervention

Intervention provider: no intervention

This study is classified as intervention (functional task training, musculoskeletal (active), musculoskeletal (passive), neurophysiological) versus no treatment (Table 4)

Outcomes

Measures of independence in ADL: Barthel Index

Measures of motor function: Fugl-Meyer Assessment

Other secondary outcome measures: "recovery of mobility was assessed according to the Brunnstrom 6 classification"

Time points when outcomes were assessed: "assessments of all patients' condition[s] were conducted 3 months after onset of symptoms"

Notes Study translated by Cochrane Stroke Group

\section{Risk of bias}


Zhang 1998 (Continued)

\begin{tabular}{|c|c|c|}
\hline Bias & Authors' judgement & Support for judgement \\
\hline \multirow{2}{*}{$\begin{array}{l}\text { Random sequence genera- } \\
\text { tion (selection bias) }\end{array}$} & \multirow[t]{2}{*}{ Unclear risk } & No information provided \\
\hline & & "participants were randomly allocated to 2 groups" \\
\hline $\begin{array}{l}\text { Allocation concealment } \\
\text { (selection bias) }\end{array}$ & Unclear risk & No information provided \\
\hline $\begin{array}{l}\text { Blinding of outcome as- } \\
\text { sessment (detection bias) } \\
\text { All outcomes }\end{array}$ & Unclear risk & No information provided \\
\hline $\begin{array}{l}\text { Incomplete outcome data } \\
\text { (attrition bias) } \\
\text { All outcomes }\end{array}$ & Unclear risk & No dropouts described \\
\hline $\begin{array}{l}\text { Free of systematic differ- } \\
\text { ences in baseline charac- } \\
\text { teristics of groups com- } \\
\text { pared? }\end{array}$ & Unclear risk & $\begin{array}{l}\text { Limited information available regarding baseline demographics, although } \mathrm{BI} \\
\text { and FMA scores at baseline are similar between the two groups }\end{array}$ \\
\hline $\begin{array}{l}\text { Did authors adjust for } \\
\text { baseline differences in } \\
\text { their analyses? }\end{array}$ & Unclear risk & No information provided \\
\hline Other bias & Unclear risk & No information provided \\
\hline
\end{tabular}

Zhang 2004

\section{Study characteristics}

Methods

\author{
Study design: RCT
}

Method of randomisation: not stated
Number of participants: $\mathrm{n}=1078$

Inclusion criteria: "Ischaemic or haemorrhagic stroke, confirmed by CT or MRI scan, satisfied the Fourth National Conference on Cerebral Vascular Disease classification guidelines, first ever stroke, aged below 85 years old, Glasgow Coma Scale > 8 and deficits in limb function"

Exclusion criteria: "Subarachnoid haemorrhage and transient ischaemic attack, aggravation of medical condition or progressing ischaemia or haemorrhage and impaired function of the heart, lungs, liver, kidney and other vital organs etc"

"Building a tertiary rehabilitation network: Establish a tertiary rehabilitation network (3-tier medical, rehabilitation network) from several hospitals' Department of Neurology-rehabilitation centres (or merge hospitals' Department of Rehabilitation)-community rehabilitation organisations (or home therapy) from several cities in the whole country

Patients from the rehabilitation group first stayed at the hospital's Department of Neurology, receiving 28 days of critical phase routine medical care and early rehabilitation. Based on ADL ability, patients who were ADL independent, who discharged to home, transferred to community rehabilitation organisations to undertake their rehabilitative training, to further improve their exercise ability, integration ability and ADL ability. Patients who were unable to reach ADL independence or still had moderate to 
severe functional disability after the early rehabilitation period, were transferred to rehabilitation centres or the tertiary rehabilitation network hospitals' Department of Rehabilitation to undertake specific strengthening training, to minimise the patient's functional disability. This phase usually took approximately 2 months. After which, patients transferred to community rehabilitation organisations to undertake further rehabilitative training, to consolidate effects from previous rehabilitation, and to continue rehabilitation for other persisting functional disabilities. Patients in the control group discharged home after 28 days of routine treatment and early rehabilitation, were given pre-arranged guidance from professionals, and undertook rehabilitative training on their own or with assistance from their family. Community rehabilitation in certain regions was not developed, were unable to provide community rehabilitation, thus patients had to return to the tertiary rehabilitation network Department of Rehabilitation to continue training. Each tertiary rehabilitation network utilised uniform inclusion criteria to select patients, with randomisation to the rehabilitation or control group. Personnel involved in the tertiary rehabilitation network included doctors, therapists, assessors who undertook training sessions to ensure uniformity at all centres. All tertiary rehabilitation network centres used a uniform rehabilitation training method and outcome assessment"

"Tertiary rehabilitation network treatment: Treatment during the critical phase was guided by Zhonghua Medical Association's recommendations. Rehabilitative training focused on physiotherapy (PT) and occupational therapy (OT), with speech and language therapy and psychological therapy given at centres with the capabilities. Physiotherapists delivered therapy uniformly, using Bobath techniques and methods from the motor relearning program mainly, with added use of PNF techniques, Brunnstrom etc. Therapy and training followed the patient's stroke recovery characteristics, adjusting to the patient's actual functional status, and delivered systematically and progressively"

"Training content: During the period when patient was confined in bed, passive ranging, optimal limb positioning, rolling, sitting up, bridging exercises in bed, sitting on bed, sitting balance training etc was given. When patient could achieve sitting for $30 \mathrm{~min}$ without postural hypotension or other symptoms, the patient could use a wheelchair to undertake training in the gym. Training content mainly included: active-passive exercises, standing and standing balance training, lower limb weight training and shifting of centre of mass training, stepping practice and gait correction, ankle dorsiflexion, forearm pronation and supination, finger grasping etc. Family members were taught rehabilitative training methods, and at the same time, patient and family underwent health counselling, given a health education booklet, shown a video on neurological recovery etc, with the aim of furthering the patient's recovery"

The individual components delivered are listed in Table 3. Based on the individual components, this intervention is categorised as comprising functional task training, musculoskeletal intervention (active), musculoskeletal intervention (passive) and neurophysiological intervention

Length of intervention period: six months

Number of sessions and length of individual sessions: dependent on stage of recovery (see above)

Intervention provider: doctors and therapists

(2) Control group $(n=463)$

"The control group received conventional neurological medical therapy only. Patients in the control group discharged home after 28 days of routine treatment and early rehabilitation, were given prearranged guidance from professionals, and undertook rehabilitative training on their own or with assistance from their family"

The individual components delivered are listed in Table 3. Based on the individual components, this intervention is categorised as comprising no intervention

Length of intervention period: six months

Number of sessions and length of individual sessions: no intervention

Intervention provider: no intervention

This study is classified as intervention (functional task training, musculoskeletal (active), musculoskeletal (passive), neurophysiological) versus no treatment (Table 4) 
Zhang 2004 (Continued)

Outcomes

Measures of independence in ADL: Barthel Index

Measures of motor function: Fugl-Meyer Assessment

Measures of tone or spasticity: Ashworth Scale

Measures of quality of life and social isolation: SF-36

Other secondary outcome measures: Loewenstein Occupational Therapy Cognitive Assessment (LOTCA), NIHSS, WAB (speech assessment), Hamilton (depression assessment)

Time points when outcomes were assessed: within one week after stroke and at the end of each month for six months

Notes Original study translated from Chinese to English

\section{Risk of bias}

\begin{tabular}{|c|c|c|}
\hline Bias & Authors' judgement & Support for judgement \\
\hline $\begin{array}{l}\text { Random sequence genera- } \\
\text { tion (selection bias) }\end{array}$ & Unclear risk & No information provided \\
\hline $\begin{array}{l}\text { Allocation concealment } \\
\text { (selection bias) }\end{array}$ & Unclear risk & No information provided \\
\hline $\begin{array}{l}\text { Blinding of outcome as- } \\
\text { sessment (detection bias) } \\
\text { All outcomes }\end{array}$ & Unclear risk & No information provided \\
\hline $\begin{array}{l}\text { Incomplete outcome data } \\
\text { (attrition bias) } \\
\text { All outcomes }\end{array}$ & Unclear risk & $\begin{array}{l}\text { Dropouts: } 19 \text { deaths and } 157 \text { dropouts (SARS epidemic prevented follow-up in } \\
\text { "more than } 50 \text { percent of the } 157 \text { cases" and no information reported in the re- } \\
\text { maining cases) }\end{array}$ \\
\hline $\begin{array}{l}\text { Free of systematic differ- } \\
\text { ences in baseline charac- } \\
\text { teristics of groups com- } \\
\text { pared? }\end{array}$ & Unclear risk & No statistical analysis provided \\
\hline $\begin{array}{l}\text { Did authors adjust for } \\
\text { baseline differences in } \\
\text { their analyses? }\end{array}$ & Unclear risk & No information provided \\
\hline Other bias & Unclear risk & No information provided \\
\hline
\end{tabular}

Zhao 2002

\section{Study characteristics}

\begin{tabular}{ll}
\hline Methods & Study design: RCT \\
& Method of randomisation: not stated \\
\hline Participants & Number of participants: $\mathrm{n}=180$ \\
& Inclusion criteria: not stated \\
\hline
\end{tabular}


Zhao 2002 (Continued)

Interventions
(1) Rehabilitation nursing group $(n=100)$

"The rehabilitation nursing group received conventional treatment and rehabilitative treatment. During acute cerebral oedema, rehabilitation commenced with bedside therapy, postural positioning and passive ranging exercises. 7-14 days, after reduction in cerebral oedema, active training became the focus, including six stages: (i) active or passive ranging bed exercises; (ii) sit up from bed and sitting on the edge of bed balance training; (iii) sit-to-stand training; (iv) standing balance training; (v) flat ground gait training; (vi) up-and-down stair and ADL training (e.g. donning, grooming, feeding, showering etc). After discharging to home, rehabilitation focused on maintaining joint range of motion and ADL, seeking the assistance of family members. After discharge, both groups had follow up visits to the hospital fortnightly, and monthly after a period of 3 months"

The individual components delivered are listed in Table 3. Based on the individual components, this intervention is categorised as comprising functional task training, musculoskeletal intervention (active) and musculoskeletal intervention (passive)

Length of intervention period: total therapy duration $31.6 \pm 11.2$ days

Number of sessions and length of individual sessions: five/wk, with the first phase having $30 \mathrm{~min}$ utes/session, and 45 minutes/session thereafter

Intervention provider: 1:1 nurse-to-participant ratio

(2) Control group $(n=80)$

"Received conventional treatment, including control of hypertension, lowering intracranial pressure, anti-coagulant treatment, neuro-regenerative treatment and self-treatment, with treatment duration being 29.1 days \pm 8.4 days"

The individual components delivered are listed in Table 3. Based on the individual components, this intervention is categorised as comprising no intervention

Length of intervention period: treatment duration $29.1 \pm 8.4$ days

Number of sessions and length of individual sessions: no intervention

Intervention provider: no intervention

This study is classified as intervention (functional task training, musculoskeletal (active), musculoskeletal (passive)) versus no treatment (Table 4)

Measures of motor function: Fugl-Meyer Assessment

Other secondary outcome measures: degree of neurological deficit (translated)

Time points when outcomes were assessed: before intervention and at one week, three weeks, five weeks, three months, six months and 12 months after stroke

\section{Risk of bias}

Bias Authors' judgement Support for judgement

Random sequence genera- Unclear risk No information provided
tion (selection bias)

$\begin{array}{lll}\begin{array}{l}\text { Allocation concealment } \\ \text { (selection bias) }\end{array} & \text { Unclear risk } & \text { No information provided }\end{array}$


Zhao 2002 (Continued)

Blinding of outcome as-
sessment (detection bias) $\quad$ Low risk $\quad$ Blinded assessment

All outcomes

Incomplete outcome data Unclear risk No dropouts described
(attrition bias)
All outcomes

\begin{tabular}{lll}
\hline $\begin{array}{l}\text { Free of systematic differ- } \\
\text { ences in baseline charac- } \\
\text { teristics of groups com- }\end{array}$ & Low risk & \\
pared? & & \\
\hline $\begin{array}{l}\text { Did authors adjust for } \\
\text { baseline differences in } \\
\text { their analyses? }\end{array}$ & Low risk & No information provided \\
\hline Other bias & Unclear risk & No information provided \\
\hline
\end{tabular}

Zhao 2003

\section{Study characteristics}

\begin{tabular}{ll}
\hline Methods & Study design: RCT \\
& Method of randomisation: not stated ("All the patients were divided into two groups randomly") \\
\hline Participants & Number of participants: $\mathrm{n}=300$ \\
& $\begin{array}{l}\text { Inclusion criteria: "all the cases accorded with the diagnosis criteria instituted on the Fourth Cere- } \\
\text { brovascular Disease Academic Conference in } 1995 \text { and were confirmed by clinic, CT or cerebral MR. All } \\
\text { the cases suffered with different levels of paralysis, without conscious disturbance" }\end{array}$
\end{tabular}

Interventions (1) Rehabilitation group $(\mathrm{n}=150)$

"Both groups received drug therapy after hospitalization. Rehabilitation group began to receive rehabilitation as soon as state of illness was stable"

"Patients in Atonia stage received posture transfer exercise with active and passive exercises. In spasm stage, patients received exercise of inhibiting spasms, limb weight loading exercise, trunk control exercise, exercise for bilateral limbs coordination, limb normal motor pattern exercise and exercises for movement coordination. Patients in recovery stage received exercise for speed, mental movement training, walking training, up and down stair exercise and ADL exercise"

The individual components delivered are listed in Table 3. Based on the individual components, this intervention is categorised as comprising functional task training, musculoskeletal intervention (active) and musculoskeletal intervention (passive)

Length of intervention period: "(PT) :.. 10 days as a treatment course, persisting 2 courses. (OT):.. 10 days as a treatment course, persisting 2 courses"

Number of sessions and length of individual sessions: "(PT): 1 time per day, 40 mins per time, (OT): 1 time per day, 30-40 minutes per day"

Intervention provider: not stated

(2) Control group $(n=150)$

No intervention 
The individual components delivered are listed in Table 3. Based on the individual components, this intervention is categorised as comprising no intervention

Length of intervention period: no intervention

Number of sessions and length of individual sessions: no intervention

Intervention provider: no intervention

This study is classified as intervention (functional task training, musculoskeletal (active), musculoskeletal (passive)) versus no treatment (Table 4)

\begin{tabular}{ll}
\hline Outcomes & Measures of Independence in ADL: Barthel Index \\
& $\begin{array}{l}\text { Time points when outcomes were assessed: "patients were evaluated by the same doctor before and } \\
\text { after treatment" }\end{array}$ \\
\hline Notes & Short Chinese study published in English-brief report only \\
& Mean and standard deviations computed from categorical data
\end{tabular}

\section{Risk of bias}

\begin{tabular}{|c|c|c|}
\hline Bias & Authors' judgement & Support for judgement \\
\hline $\begin{array}{l}\text { Random sequence genera- } \\
\text { tion (selection bias) }\end{array}$ & Unclear risk & No information provided \\
\hline $\begin{array}{l}\text { Allocation concealment } \\
\text { (selection bias) }\end{array}$ & Unclear risk & No information provided \\
\hline $\begin{array}{l}\text { Blinding of outcome as- } \\
\text { sessment (detection bias) } \\
\text { All outcomes }\end{array}$ & Unclear risk & No information provided \\
\hline $\begin{array}{l}\text { Incomplete outcome data } \\
\text { (attrition bias) } \\
\text { All outcomes }\end{array}$ & Unclear risk & No dropouts described \\
\hline $\begin{array}{l}\text { Free of systematic differ- } \\
\text { ences in baseline charac- } \\
\text { teristics of groups com- } \\
\text { pared? }\end{array}$ & Low risk & "There is no difference in age, gender and side of hemiplegia between groups" \\
\hline $\begin{array}{l}\text { Did authors adjust for } \\
\text { baseline differences in } \\
\text { their analyses? }\end{array}$ & Low risk & No information provided \\
\hline Other bias & Unclear risk & No information provided \\
\hline
\end{tabular}

Zhu 2001

\section{Study characteristics}

\begin{tabular}{ll}
\hline Methods & Study design: RCT \\
Method of randomisation: not stated
\end{tabular}


Zhu 2001 (Continued)

Participants Number of participants: $\mathrm{n}=125$

Inclusion criteria: "Satisfied the 1995 Fourth National Conference on Cerebral Vascular Disease classification guidelines, confirmed by CT or MRI scan as first ever stroke; Functional deficit of limbs; Aged between 45-70 years old, no major heart, liver, kidney and other organ diseases; Glasgow Coma Scale $\geq 8$ within 4-7 days for ischaemic stroke, within 10-14 days for haemorrhagic stroke, vital signs stable"

Interventions
(1) Rehabilitation group $(n=72)$

"Both groups received conventional medical treatment during the critical acute phase. Participants in the treatment group commenced rehabilitation once medically stable. Rehabilitation comprised exercise therapy, occupational therapy and electro therapy etc. Exercise therapy consisted of techniques mainly from the Motor Relearning Program and Bobath, customised to the impairment of the individual participants and progressed appropriately. Exercise therapy included: (i) Passive ranging exercises of all joints of the affected limbs (including scapula), starting from small to large movements, without any pain caused to the participants; (ii) Rolling practice to both sides; (iii) bridging exercises; (iv) Exercises involving the wrist and ankle joints; (v) outwith therapy, participants trained in upright sitting, beginning at an inclination angle of 30 degrees. Once participants could tolerate the angle for $30 \mathrm{~min}$, the inclination was increased by 10 degrees until participants could sit upright at 90 degrees for $30 \mathrm{mins}$. Thereafter, participants commenced sitting on the edge of bed exercises and lying to sitting training; (vi) sitting on the edge of bed balance training; (vii) sit to stand practice; (viii) standing balance training; (ix) gait training etc. Throughout therapy, participants' family were taught the exercises, so that they could assist with practice outwith therapy. Electrotherapy was conducted for the affected limbs. Psychological therapy was given throughout to strengthen participant's motivation to recover"

"Although the control group was not given any therapy, some participants exercised based on the doctor's advice, and some participants' family assisted with exercises from their own knowledge. Upon discharge, participants from the treatment group continued with outpatient therapy or home therapy or assistance with daily exercises by their family under telephone supervision at least 3/week"

The individual components delivered are listed in Table 3. Based on the individual components, this intervention is categorised as comprising functional task training, modality, musculoskeletal intervention (passive) and neurophysiological intervention

Length of intervention period: not stated (outcome assessment done three months after stroke)

Number of sessions and length of individual sessions: rehabilitation programme: exercise therapy and occupational therapy one/d, 45 minutes/session, five/wk, electrotherapy one/d, 20 minutes/session, five/wk

Intervention provider: not stated

(2) Control group $(n=53)$

"Received conventional medical treatment during the critical acute phase"

The individual components delivered are listed in Table 3. Based on the individual components, this intervention is categorised as comprising no intervention

Length of intervention period: no intervention

Number of sessions and length of individual sessions: no intervention

Intervention provider: no intervention

This study is classified as intervention (functional task training, musculoskeletal (passive), neurophysiological) versus no treatment (Table 4). The intervention group also received modality

Outcomes

Time points when outcomes were assessed: "Within 2 days of being medically stable or at the commencement of training as first assessment, the second assessment being 3 months after stroke" 
Zhu 2001 (Continued)

Notes Original study translated from Chinese to English

\section{Risk of bias}

\begin{tabular}{|c|c|c|}
\hline Bias & Authors' judgement & Support for judgement \\
\hline $\begin{array}{l}\text { Random sequence genera- } \\
\text { tion (selection bias) }\end{array}$ & Unclear risk & No information provided \\
\hline $\begin{array}{l}\text { Allocation concealment } \\
\text { (selection bias) }\end{array}$ & Unclear risk & No information provided \\
\hline $\begin{array}{l}\text { Blinding of outcome as- } \\
\text { sessment (detection bias) } \\
\text { All outcomes }\end{array}$ & Unclear risk & No information provided \\
\hline $\begin{array}{l}\text { Incomplete outcome data } \\
\text { (attrition bias) } \\
\text { All outcomes }\end{array}$ & Unclear risk & No dropouts described \\
\hline $\begin{array}{l}\text { Free of systematic differ- } \\
\text { ences in baseline charac- } \\
\text { teristics of groups com- } \\
\text { pared? }\end{array}$ & Low risk & No statistically significant differences in baseline values \\
\hline $\begin{array}{l}\text { Did authors adjust for } \\
\text { baseline differences in } \\
\text { their analyses? }\end{array}$ & Low risk & No information provided \\
\hline Other bias & Unclear risk & No information provided \\
\hline
\end{tabular}

\section{Zhu 2004b}

\section{Study characteristics}

\begin{tabular}{ll} 
Methods & Study design: RCT \\
& $\begin{array}{l}\text { Method of randomisation: "All } 52 \text { patients firstly are brought into two blocks: primary cerebral infarc- } \\
\text { tion and primary cerebral haemorrhage then are divided into treated group and controlled group ran- } \\
\text { domly" }\end{array}$ \\
\hline Number of participants: $\mathrm{n}=52$ \\
Inclusion criteria: "Satisfied the 1995 Fourth National Conference on Cerebral Vascular Disease classifi- \\
cation guidelines, ischaemic or haemorrhagic stroke, confirmed by CT or MRI scan as first ever stroke, \\
aged between 40-80 years old, within 1 week of being medically stable, Glasgow Coma Score > 8, func- \\
tional deficit of limbs and provided informed consent" \\
Exclusion criteria: "Active liver disease, impaired liver, kidney function, congestive heart failure, ma- \\
lignant tumours, history of dementia, impaired respiratory system, paralysis of 4 limbs, more than 3 \\
weeks post stroke, rural residence preventing re-assessments, history of psychological disorders and \\
deafness and/or muteness"
\end{tabular}

Interventions

(1) Treated group $(n=26)$ 
"The intervention combined physiotherapy (PT) and occupational therapy (OT): The earlier stage of recovery (from stroke onset to one month after stroke) focused on physiotherapy, and the later stage on occupational therapy

PT included: (i) anti-spasticity positioning in supine and sitting; (ii) passive ranging of all joints of affected limbs from small to larger range, within pain-free thresholds, including scapular activity; (iii) rolling from both sides; (iv) bridging practice; (v) wrist extension and ankle dorsiflexion training; (vi) Outwith therapy, participants trained sitting tolerance, starting from a bed height of 30 degrees, and increasing the height by 10 degrees each day if participants were able to cope for 30 mins until 30 mins at 90 degrees is achieved; (vii) Sitting balance training on the bedside; (viii) sit to stand training; (ix) Standing balance training. (x) Gait training etc"

"In the flaccid phase, participants focused on correct positioning, passive exercises and active-passive training, rolling, lying to sitting; in the spastic phase, participants focused on relaxation practice (biofeedback technique), anti-spasticity techniques and training of non-spastic muscles, and isolation training. OT: Depending on participant's ability, feeding, washing, donning, transfer from bed to wheelchair and vice versa and other ADL training, deep breathing and abdominal training, supine to sitting up, sitting balance and sit to stand training etc, 1/day, 45min/session, 5/week"

"During the intervention, family members were taught how to facilitate training and care so that participants could get practice even out with therapy, and to prevent injuries due to inappropriate handling of the affected limbs"

"Second stage of rehabilitation (from the second to end of third month after stroke) mainly involved standing training, standing balance training, single leg standing, gait training and stairs training etc, in order to resolve the participant's ambulatory ability, 2/day, 30-45min/session, 5 days/week; third stage of rehabilitation (from the fourth to the end of the sixth month after stroke) focused on feeding, donning, washing, hygiene issues and other ADL training, 2/day, 30-40min/session, 5-7days/week"

The individual components delivered are listed in Table 3. Based on the individual components, this intervention is categorised as comprising functional task training, musculoskeletal intervention (active), musculoskeletal intervention (passive)

Length of intervention period: six months

Number of sessions and length of individual sessions: dependent on stage of recovery (see above)

Intervention provider: therapists

(2) Controlled group $(n=26)$

"The control group was not given any therapy. However, some participants exercised based on the doctor's advice, and some participants' family assisted with exercises from their own knowledge. Participants might also have sought help from other rehabilitative services upon discharge"

The individual components delivered are listed in Table 3. Based on the individual components, this intervention is categorised as comprising no intervention

Length of intervention period: no intervention

Number of sessions and length of individual sessions: no intervention

Intervention provider: no intervention

This study is classified as intervention (functional task training, musculoskeletal (active), musculoskeletal (passive)) versus no treatment (Table 4)

Time points when outcomes were assessed: at enrolment and end of one, three and six months after stroke 
Zhu 2004b (Continued)

No outcomes included in analysis

\section{Risk of bias}

Bias Authors' judgement Support for judgement

Random sequence genera- Low risk tion (selection bias)

"All 52 patients firstly are brought into two blocks: primary cerebral infarction and primary cerebral haemorrhage then are divided into treated group and controlled group randomly"

\begin{tabular}{lll}
\hline $\begin{array}{l}\text { Allocation concealment } \\
\text { (selection bias) }\end{array}$ & Unclear risk & No information provided \\
\hline
\end{tabular}

\begin{tabular}{|c|c|c|}
\hline $\begin{array}{l}\text { Blinding of outcome as- } \\
\text { sessment (detection bias) } \\
\text { All outcomes }\end{array}$ & Low risk & Blinded assessment \\
\hline $\begin{array}{l}\text { Incomplete outcome data } \\
\text { (attrition bias) } \\
\text { All outcomes }\end{array}$ & Unclear risk & No dropouts described \\
\hline $\begin{array}{l}\text { Free of systematic differ- } \\
\text { ences in baseline charac- } \\
\text { teristics of groups com- } \\
\text { pared? }\end{array}$ & Low risk & No statistically significant differences in baseline values \\
\hline $\begin{array}{l}\text { Did authors adjust for } \\
\text { baseline differences in } \\
\text { their analyses? }\end{array}$ & Low risk & No information provided \\
\hline Other bias & Unclear risk & No information provided \\
\hline
\end{tabular}

\section{Zhu 2006}

\section{Study characteristics}

\begin{tabular}{ll}
\hline Methods & Study design: RCT \\
& Method of randomisation: "Randomised according to the time of hospital admission" \\
\hline Participants & Number of participants: $\mathrm{n}=70$ \\
& $\begin{array}{l}\text { Inclusion criteria: "Ischaemic or haemorrhagic stroke, confirmed by CT or MRI scan as first ever stroke, } \\
\text { aged between } 55-80 \text { years old, functional deficit of limbs, no severe cognitive issues, no severe diseases } \\
\text { of the heart liver, kidney and other organs, within } 1 \text { week of being medically stable and Glasgow Coma } \\
\text { Scale }>8 \text { " }\end{array}$ \\
\hline
\end{tabular}

"Both groups of patients received the same drug therapy, including treatment for cerebral edema, brain care and improving blood circulation. 3 - 7 days after becoming medically stable, the test group underwent rehabilitative therapy using Bobath technique, Rood technique, as well as ADL training, etc, with training conducted by rehabilitation nurses, rehabilitative therapy once a day, $1 \mathrm{hr}$ each time, 5 times a week"

"The contents were as follows: 
Physiotherapy: (i) On-bed positioning of healthy limbs, with regular turning over; (ii) Passive ranging exercises of joints of limbs on affected side, including passive ranging exercise of scapular, motion progressing from proximal joints to distal joints, range of motion progressed from small to large, within pain-free thresholds, while concurrently, patients were encouraged to use healthy limbs to aid motion of affected limbs, for example exercise involving crossing both sides and lifting, lower limbs bridgestyle exercise; (iii) Utilising Rood technique to brush, tap, pat etc arbitrary exercises to stimulate affected limbs; (iv) Sitting exercise involving lifting headrest, headrest gradually lifted, maintaining each position 30min, repeating training with 10 degree increments until able to sit upright at bedside; (v) Bedside sitting balance training: correct sitting posture, starting from static balance to dynamic balance training, torso back-and-forth, side-to-side and rotation training, and finally training of maintaining balance while being pushed externally; (vi) Sit-to-stand balance training, patients holding hands Bobath-style, extending upper limbs, head and torso leaning forward, moving center-of-gravity forward, torso, hip and knee extending until standing, during standing process, body weight distributed equally on both sides, and then undergoing training of moving body weight back-and-forth, side-to-side; (vii) Gait training, after patient's standing balance and affected limbs weight bearing ability improved, starting from gait training between parallel bars to gait training using walking stick and eventually progressing to training of stair climbing and descending"

"Occupational therapy: (i) For patients with difficulty swallowing, training was done to stimulate face, tongue and lips, opening and closing of lips, opening and closing of lower jaw, tongue pushing upper palate, extension of tongue, etc, or using ice-cold cotton bud to stimulate swallowing reflex; (ii) Activity involving the palm and all joints of the fingers as well as agility, coordination and dexterity of the fingers training; (iii) ADL training, including brushing, feeding, washing, donning, passing bowels etc, encouraging the completion of tasks using the affected limbs, or breaking the tasks into components and getting participants to train specific components"

The individual components delivered are listed in Table 3. Based on the individual components, this intervention is categorised as comprising functional task training, musculoskeletal intervention (passive) and neurophysiological intervention

Length of intervention period: not stated

Number of sessions and length of individual sessions: once a day, one hour each time, five times a week Intervention provider: rehabilitation nurses

(2) Controlled group $(n=35)$

Both groups of patients received the same drug therapy, including treatment for cerebral oedema, brain care and improving blood circulation

The individual components delivered are listed in Table 3. Based on the individual components, this intervention is categorised as comprising no intervention

Length of intervention period: no intervention

Number of sessions and length of individual sessions: no intervention

Intervention provider: no intervention

This study is classified as intervention (functional task training, musculoskeletal (passive), neurophysiological) versus no treatment (Table 4)

Outcomes

Measures of independence in ADL: Modified Barthel Index

Measures of motor function: Fugl-Meyer Assessment (simplified)

Other secondary outcome measures: Brunnstrom Grading Scale

Time points when outcomes were assessed: before and after intervention

Notes Original study translated from Chinese to English

Physical rehabilitation approaches for the recovery of function and mobility following stroke (Review) Copyright ( 2023 The Cochrane Collaboration. Published by John Wiley \& Sons, Ltd. 
Zhu 2006 (Continued)

Risk of bias

\begin{tabular}{|c|c|c|}
\hline Bias & Authors' judgement & Support for judgement \\
\hline $\begin{array}{l}\text { Random sequence genera- } \\
\text { tion (selection bias) }\end{array}$ & High risk & "Randomised according to the time of hospital admission" \\
\hline $\begin{array}{l}\text { Allocation concealment } \\
\text { (selection bias) }\end{array}$ & High risk & "Randomised according to the time of hospital admission" \\
\hline $\begin{array}{l}\text { Blinding of outcome as- } \\
\text { sessment (detection bias) } \\
\text { All outcomes }\end{array}$ & Unclear risk & No information provided \\
\hline $\begin{array}{l}\text { Incomplete outcome data } \\
\text { (attrition bias) } \\
\text { All outcomes }\end{array}$ & Unclear risk & No dropouts described \\
\hline $\begin{array}{l}\text { Free of systematic differ- } \\
\text { ences in baseline charac- } \\
\text { teristics of groups com- } \\
\text { pared? }\end{array}$ & Low risk & No statistically significant differences in baseline values \\
\hline $\begin{array}{l}\text { Did authors adjust for } \\
\text { baseline differences in } \\
\text { their analyses? }\end{array}$ & Low risk & No information provided \\
\hline Other bias & Unclear risk & No information provided \\
\hline
\end{tabular}

Zhu 2007 haem

\section{Study characteristics}

\begin{tabular}{ll}
\hline Methods & $\begin{array}{l}\text { Study design: RCT } \\
\text { Method of randomisation: "Stratified block randomisation" }\end{array}$ \\
\hline Participants & Number of participants: $\mathrm{n}=78$ \\
& $\begin{array}{l}\text { Inclusion criteria: "Provided informed consent, medically stable for }>48 \text { hours, Glasgow Coma Scale }>8 \\
\text { and functional deficit of limbs" }\end{array}$ \\
& $\begin{array}{l}\text { Exclusion criteria: "Active liver disease, impaired liver, kidney function, congestive heart failure, im- } \\
\text { paired respiratory system, malignant tumours, history of dementia, history of psychological disorders, } \\
\text { paralysis of } 4 \text { limbs, more than } 4 \text { weeks post stroke, history of previous stroke which resulted in func- } \\
\text { tional deficit of limbs, unable to allow re-assessments to take place and deafness and/or muteness" }\end{array}$ \\
\hline
\end{tabular}

Interventions

(1) Cerebral haemorrhage rehabilitation group $(n=12)$

"All patients received routine clinical treatment and care. Treatment group was transferred to rehabilitation centre after becoming medically stable, and under guidance from the therapists, utilised physiotherapy and occupational therapy to undergo integrated rehabilitative treatment; patients on being discharged to their homes, were visited by therapists who would teach the required training, until the follow-up ended"

"Key training contents included: (i) From 1 week after medical stabilisation till one month after onset of stroke, focus was on bed and bed-side exercises, including anti-spasticity positioning, passive exercis- 
es on affected limbs and neuro-muscular stimulative technique, active exercises on non-affected limbs, truncal muscle control training, shifting on bed, rolling (affected side, non-affected side), sitting up, sitting-balance training, sitting position-standing position transfer as well as eating, grooming, dressing etc ADL training, once a day, 45 min each time, 5 days a week. (ii) From start of 2 nd month till end of 3rd month after onset of stroke, focus was on standing training, including standing-balance training, single-leg weight bearing, gait and stair climbing/descending, and other trainings, while providing guidance on toileting, bed-chair transferring, indoors or outdoors walking, use of stairs, washing and other practical ADLs; twice a day, 45 min each time, 5 days a week. Because muscle tone could increase gradually during this period, it was necessary to increase the intensity of trainings to reduce muscle tone and inhibit abnormal exercise patterns. For some patients who returned to community setting, therapists would conduct weekly home-visits to guide the patient on rehabilitative treatment. (iii) Once training had commenced, therapists concurrently taught the patients' family members or caregiver on the correct assistive training methods and care methods, such that they could provide some training outwith therapy time, while also reducing the secondary damage due to inappropriate care. (iv) $\mathrm{Pa}$ tients learned to monitor their own body for discomfort, and report on time to therapist and caregiver"

The individual components delivered are listed in Table 3. Based on the individual components, this intervention is categorised as comprising functional task training and musculoskeletal intervention (passive)

Length of intervention period: not stated

Number of sessions and length of individual sessions: dependent on stage of recovery (see above)

Intervention provider: therapists

(2) Cerebral haemorrhage control group $(n=10)$

"Control group was not given standard rehabilitative treatment, but were allowed to perform activities independently under doctor's advice or with assistance from nurses"

The individual components delivered are listed in Table 3. Based on the individual components, this intervention is categorised as comprising no intervention

Length of intervention period: no intervention

Number of sessions and length of individual sessions: no intervention

Intervention provider: no intervention

This study is classified as intervention (functional task training, musculoskeletal (passive)) versus no treatment (Table 4)

Measures of motor function: Fugl-Meyer Assessment

Time points when outcomes were assessed: at allocation to groups and end of one and three months after stroke

Notes

Original study translated from Chinese to English

Data provided are means and ranges. For analysis, standard deviations have been estimated by calculating (upper range - lower range)/four

\section{Risk of bias}

\begin{tabular}{lll}
\hline Bias & Authors' judgement & Support for judgement \\
\hline $\begin{array}{l}\text { Random sequence genera- } \\
\text { tion (selection bias) }\end{array}$ & Low risk & $\begin{array}{l}\text { "Stratified block randomisation" (divided by type of stroke before allocation to } \\
\text { intervention or control groups) }\end{array}$ \\
\hline
\end{tabular}


Zhu 2007 haem (Continued)

$\begin{aligned} & \text { Allocation concealment } \\ & \text { (selection bias) }\end{aligned}$
Unclear risk

\begin{tabular}{lll}
\hline $\begin{array}{l}\text { Blinding of outcome as- } \\
\text { sessment (detection bias) }\end{array}$ & Low risk & Blinded assessment \\
All outcomes & \\
\hline $\begin{array}{l}\text { Incomplete outcome data } \\
\text { (attrition bias) }\end{array}$ & Unclear risk & No dropouts described \\
All outcomes & &
\end{tabular}

\begin{tabular}{lll}
\hline $\begin{array}{l}\text { Free of systematic differ- } \\
\text { ences in baseline charac- } \\
\text { teristics of groups com- }\end{array}$ & Now risk \\
pared? & \\
\hline $\begin{array}{l}\text { Did authors adjust for } \\
\text { baseline differences in } \\
\text { their analyses? }\end{array}$ & Low risk & No information provided \\
\hline Other bias & Unclear risk & No information provided \\
\hline
\end{tabular}

Zhu 2007 isch

\title{
Study characteristics
}

\begin{tabular}{ll}
\hline Methods & Study design: RCT \\
& Method of randomisation: "Stratified block randomisation" \\
\hline Participants & Number of participants: $\mathrm{n}=78$ \\
& $\begin{array}{l}\text { Inclusion criteria: "Provided informed consent, medically stable for }>48 \text { hours, Glasgow Coma Scale }>8 \\
\text { and functional deficit of limbs" }\end{array}$ \\
& $\begin{array}{l}\text { Exclusion criteria: "Active liver disease, impaired liver, kidney function, congestive heart failure, im- } \\
\text { paired respiratory system, malignant tumours, history of dementia, history of psychological disorders, } \\
\text { paralysis of } 4 \text { limbs, more than } 4 \text { weeks post stroke, history of previous stroke which resulted in func- } \\
\text { tional deficit of limbs, unable to allow re-assessments to take place and deafness and/or muteness" }\end{array}$
\end{tabular}

"All patients received routine clinical treatment and care. Treatment group was transferred to rehabilitation centre after becoming medically stable, and under guidance from the therapists, utilised physiotherapy and occupational therapy to undergo integrated rehabilitative treatment; patients on being discharged to their homes, were visited by therapists who would teach the required training, until the follow-up ended"

\begin{abstract}
"Key training contents included: (i) From 1 week after medical stabilisation till one month after onset of stroke, focus was on bed and bed-side exercises, including anti-spasticity positioning, passive exercises on affected limbs and neuro-muscular stimulative technique, active exercises on non-affected limbs, truncal muscle control training, shifting on bed, rolling (affected side, non-affected side), sitting up, sitting-balance training, sitting position-standing position transfer as well as eating, grooming, dressing etc ADL training, once a day, 45 min each time, 5 days a week. (ii) From start of 2 nd month till end of 3rd month after onset of stroke, focus was on standing training, including standing-balance training, single-leg weight bearing, gait and stair climbing/descending, and other trainings, while providing guidance on toileting, bed-chair transferring, indoors or outdoors walking, use of stairs, washing and other practical ADLs; twice a day, 45 min each time, 5 days a week. Because muscle tone could increase grad-
\end{abstract}


ually during this period, it was necessary to increase the intensity of trainings to reduce muscle tone and inhibit abnormal exercise patterns. For some patients who returned to community setting, therapists would conduct weekly home-visits to guide the patient on rehabilitative treatment. (iii) Once training had commenced, therapists concurrently taught the patients' family members or caregiver on the correct assistive training methods and care methods, such that they could provide some training outwith therapy time, while also reducing the secondary damage due to inappropriate care. (iv) $\mathrm{Pa}$ tients learned to monitor their own body for discomfort, and report on time to therapist and caregiver"

The individual components delivered are listed in Table 3. Based on the individual components, this intervention is categorised as comprising functional task training and musculoskeletal intervention (passive)

Length of intervention period: not stated

Number of sessions and length of individual sessions: dependent on stage of recovery (see above) Intervention provider: therapists

(2) Cerebral infarction control group $(n=28)$

"Control group was not given standard rehabilitative treatment, but were allowed to perform activities independently under doctor's advice or with assistance from nurses"

The individual components delivered are listed in Table 3. Based on the individual components, this intervention is categorised as comprising no intervention

Length of intervention period: no intervention

Number of sessions and length of individual sessions: no intervention

Intervention provider: no intervention

This study is classified as intervention (functional task training, musculoskeletal (passive)) versus no treatment (Table 4)

\begin{tabular}{|c|c|}
\hline \multirow[t]{3}{*}{ Outcomes } & Measures of Independence in ADL: Barthel Index \\
\hline & Measures of motor function: Fugl-Meyer Assessment \\
\hline & $\begin{array}{l}\text { Time points when outcomes were assessed: at allocation to groups and end of one and three months } \\
\text { after stroke }\end{array}$ \\
\hline \multirow[t]{2}{*}{ Notes } & Original study translated from Chinese to English \\
\hline & $\begin{array}{l}\text { Data provided are means and ranges. For analysis, standard deviations have been estimated by calcu- } \\
\text { lating (upper range - lower range)/four }\end{array}$ \\
\hline
\end{tabular}

\section{Risk of bias}

\begin{tabular}{lll}
\hline Bias & Authors' judgement & Support for judgement \\
\hline $\begin{array}{l}\text { Random sequence genera- } \\
\text { tion (selection bias) }\end{array}$ & Low risk & $\begin{array}{l}\text { "Stratified block randomisation (divided by type of stroke before allocation to } \\
\text { intervention or control groups)" }\end{array}$ \\
\hline $\begin{array}{l}\text { Allocation concealment } \\
\text { (selection bias) }\end{array}$ & Unclear risk & No information provided \\
\hline $\begin{array}{l}\text { Blinding of outcome as- } \\
\text { sessment (detection bias) } \\
\text { All outcomes }\end{array}$ & Low risk & Blinded assessment \\
\hline
\end{tabular}

Incomplete outcome data Unclear risk

No dropouts described

(attrition bias)

Physical rehabilitation approaches for the recovery of function and mobility following stroke (Review) 
Zhu 2007 isch (Continued)

All outcomes

Free of systematic differences in baseline characteristics of groups compared?

$\begin{array}{lll}\text { Did authors adjust for } & \text { Low risk } & \text { No information provided } \\ \text { baseline differences in } & \end{array}$
their analyses?

Other bias Unclear risk No information provided

\title{
Study characteristics
}

\section{Methods}

\author{
Study design: "RCT with 3 treatment groups"
}

Method of randomisation: "Research team randomly assigned participants to one of three groups: (1) acupuncture alone, (2) physiotherapy alone, or (3) combined acupuncture and physiotherapy. The team's data management center generated the randomization numbers with SAS9.1.3 (Statistical Analysis System provided by SAS Institute Inc, Cary, North Carolina). Each of the seven sites had a designated research assistant who was responsible for obtaining a random number for each participant from a web-based, password-protected Internet site and who actually assigned the participant to one of the three treatment groups"

\section{Participants}

\section{Number of participants: $\mathrm{n}=274$}

Inclusion criteria: recent (longer than two weeks and less than three months) history of ischaemic stroke that clinical signs and imaging confirmed, admission to a specialist stroke unit as an inpatient, participants of either sex, age from 45 to 70 years, ability to give informed consent, participants also had to "score better than 10 on the Neurologic Defect Scale (NDS), which ranges from 0 to 45, with 45 as most severe"

Exclusion criteria: haemorrhagic cerebrovascular disease, vascular disease and dysfunction, history of dementia or other mental illness, cancer, diseases transmissible by blood, severe disease of the heart, liver, kidney, hematopoietic system or endocrine system, severe visual or hearing impairment, history of previous acupuncture, fear of needling

"The research team based the physiotherapy, a conventional rehabilitative method for stroke, on the Bobath approach, which intends to restore normal movement and improve muscle strength"

The individual components delivered are listed in Table 3. Based on the individual components, this intervention is categorised as comprising neurophysiological intervention

Length of intervention period: four weeks

Number of sessions and length of individual sessions: "six 60-minute sessions per week of standard physiotherapy and six 45-minute sessions per week of occupational therapy"

Intervention provider: "qualified therapists. A physiotherapist tailored the treatment protocol to each participant's needs, based on recovery stage"

(2) Acupuncture $(\mathrm{n}=91)$

"All participants received conventional care as needed, including psychological counselling, standard nursing care, and daily medical evaluation. Attending physicians, blinded to the participant's treat- 
ment assignment, prescribed medications when appropriate, including drugs for controlling blood glucose concentration, blood lipid levels, and blood pressure. The study allowed antiplatelet agents and anticoagulants at the discretion of the attending physician"

"Well-trained, qualified, experienced acupuncturists performed the acupuncture treatments, with participants in the supine position. The acupuncturists used sterile, disposable needles: 30 -gauge $(0.3 \mathrm{~mm}$ in diameter), $40-\mathrm{mm}$-long needles for limb points and 32-gauge ( $0.25 \mathrm{~mm}$ in diameter), $25-\mathrm{mm}$-long needles on the head. When the participant felt de qi-the sensation characterized by heaviness, distension, soreness, or numbness-the acupuncturist kept the needles in situ for 30 minutes without manual or electrical stimulation. The acupuncturists followed the recommendations of a standard acupuncture textbook for the depth and angle of insertion into each acupoint. The acupuncturist needled three primary scalp points on the stroke side: the first, 2 in above the ear apex and the others, 1 in anterior and 1 in posterior to the first. The acupuncturist selected secondary acupoints based on traditional Chinese medicine (TCM) theory. Patients with flaccid paralysis received Quchi (LI11), Waiguan (TE5), and Hegu (LI4) for the upper limb and Futu (ST32), Zusanli (ST36), and Taichong (LR3) for the lower limb. Patients with spastic paralysis received Jiquan (HT1), Chize (LU5), and Neiguan (PC6) for the upper limb and Yinlingquan (SP9) and Sanyinjiao (SP6) for the lower limb"

The individual components delivered are listed in Table 3. Based on the individual components, this intervention is categorised as comprising modality

Length of intervention period: four weeks

Number of sessions and length of individual sessions: each session lasted at least 30 minutes. "Participants received treatments once a day except on Sundays"

Intervention provider: "Well-trained, qualified, experienced acupuncturists"

(3) Combination therapy $(n=97)$

"Received both acupuncture and physiotherapy" (as previously described). "The acupuncture session took place randomly before or after the physiotherapy session and during the same half-day"

The individual components delivered are listed in Table 3. Based on the individual components, this intervention is categorised as comprising modality and neurophysiological intervention

Length of intervention period: four weeks

Number of sessions and length of individual sessions: "participants received treatments once a day except on Sundays"

Intervention provider: "qualified therapists"

This study is classified as active Intervention one (neurophysiological) versus active intervention two (acupuncture) (Table 6)

Measures of independence in ADL scales: Modified Barthel Index

Measures of motor function: Fugl-Meyer Assessment

Other secondary outcome measures: Neurologic Defect Scale (NDS)

Time points when outcomes were assessed: "research team evaluated all patients at baseline, after 2 weeks, and after 4 weeks"

Notes

For analysis, we have just used Groups (1) and (2). As Group 2 did not receive any active physical rehabilitation in addition to acupuncture, we will explore the categorisation of this group with sensitivity analysis

\section{Risk of bias}


Zhuang 2012 (Continued)

Random sequence genera- Low risk tion (selection bias)

"The team's data management center generated the randomization numbers with SAS9.1.3 (Statistical Analysis System provided by SAS Institute Inc, Cary, North Carolina). Each of the seven sites had a designated research assistant who was responsible for obtaining a random number for each participant from a web-based, password-protected Internet site and who actually assigned the participant to one of the three treatment groups"

$\begin{array}{ll}\begin{array}{l}\text { Allocation concealment } \\ \text { (selection bias) }\end{array} & \text { Low risk } \\ \text { that they were receiving with their therapists" }\end{array}$

\begin{tabular}{|c|c|c|}
\hline $\begin{array}{l}\text { Blinding of outcome as- } \\
\text { sessment (detection bias) } \\
\text { All outcomes }\end{array}$ & Low risk & $\begin{array}{l}\text { "Physicians who performed the outcome assessments were blinded to treat- } \\
\text { ment assignments. The principal investigator was blinded to treatment assign- } \\
\text { ment and was not involved in treating the patients" }\end{array}$ \\
\hline
\end{tabular}

\begin{tabular}{|c|c|c|}
\hline Incomplete outcome data & Low risk & Dropouts accounted for \\
\hline All outcomes & & $\begin{array}{l}\text { "Of those who dropped out, nine left the hospital, eight discontinued treat- } \\
\text { ment, two dropped out due to poor health, one (from the acupuncture group) } \\
\text { suffered a second stoke and one (from the physiotherapy group) died due to a } \\
\text { respiratory tract infection" }\end{array}$ \\
\hline
\end{tabular}

\begin{tabular}{|c|c|c|}
\hline $\begin{array}{l}\text { Free of systematic differ- } \\
\text { ences in baseline charac- } \\
\text { teristics of groups com- } \\
\text { pared? }\end{array}$ & Low risk & $\begin{array}{l}\text { "At baseline, no significant differences existed between the three groups in } \\
\text { terms of gender, age, or length or severity of disease }(P>0.05) "\end{array}$ \\
\hline $\begin{array}{l}\text { Did authors adjust for } \\
\text { baseline differences in } \\
\text { their analyses? }\end{array}$ & Low risk & No information provided \\
\hline Other bias & Unclear risk & $\begin{array}{l}\text { "Due to the lack of a sham-acupuncture, placebo-control group, the research } \\
\text { team cannot rule out the possible placebo effect of acupuncture" }\end{array}$ \\
\hline
\end{tabular}

ADL: activities of daily living.

AEP: additional early physiotherapy.

BI: Barthel Index.

BPM: balance performance monitor.

CG: control group.

CNDS: Clinical Neurological Deficit Scale.

CNS: central nervous system.

CPT: computed physiotherapy.

CT: computed tomography.

CVA: cerebrovascular accident.

DDNF: degree of deficit of neural function.

EADL: extended activities of daily living.

EG: experimental group.

EMG: electromyograph.

ESS: European Stroke Scale.

FAl-3: Frenchay Activities Index.

FCA: Functional Comprehensive Assessment.

FES-I: Falls Efficacy Scale-International/

FIM: Functional Independence Measure.

FMA: Fugl-Meyer Assessment.

FRT: Functional Reach test.

FSS: Fatigue Severity Scale.

FST: functional strength training.GCS: Glasgow Coma Scale.

HADS: Hospital Anxiety and Depression Scale.

HCA: haemorrhagic cerebral accident.

HIFE: High Intensity Functional Exercises.

Physical rehabilitation approaches for the recovery of function and mobility following stroke (Review)

Copyright (c) 2023 The Cochrane Collaboration. Published by John Wiley \& Sons, Ltd. 
HMSI: Hindi Mental State Examination.

IADL: instrumental activities of daily living.

ICA: infarct cerebral accident.

IG: intervention group.

IQR: interquartile range.

JTHFT: Jebsen Taylor Hand Function Test.

LE: lower extremity.

LL: lower limb.

LOTCA: Loewenstein Occupational Therapy Cognitive Assessment.

m: metre.

MAS: Modified Ashworth Scale.

MAS: Motor Assessment Scale.

MI: Motricity Index.

MIDI: Musical Instrument Digital Interface.

MIQ-RS: Movement Imagery Questionnaire-revised second version.

MMSE: Mini Mental State Examination.

MRI: magnetic resonance imaging.

$\mathrm{n}$ : number of participants.

NDS: Neurologic Defect Scale.

NDT: neurodevelopmental treatment.

NEADL: Nottingham extended activities of daily living.

NIHSS: National Institutes of Health Stroke Scale.

NYHA: New York Heart Association.

OT: occupational therapy.

PADS: Physical Activity and Disability Scale.

PLBO: placebo.

PNF: proprioceptive neuromuscular facilitation.

PT: physiotherapy.

$\mathrm{RCT}$ : randomised controlled trial.

RIND: reversible ischaemic neurological deficit.

RMI: Rivermead Mobility Index.

ROM: range of movement.

RS: rhythmic stabilisation.

RT: routine therapy.

SAIS: Stroke Assessment Impairment Set.

SARS: severe acute respiratory syndrome.

SEP: somatosensory evoked potential.

SMES: Sodring Motor Evaluation of Stroke patients

SPSS: Statistical Package for the Social Sciences.

SR: stabilising reversal.

TENS: transelectrical nerve stimulation.

TFR: traditional functional retraining.

TIA: transient ischaemic attack.

TIS: Trunk Impairment Scale.

TRT: treatment.

TUG: Timed Up and Go test.

UE: upper extremity.

WAB: Wester Aphasia Battery.

WHO: World Health Organization.

Characteristics of excluded studies [ordered by study ID]

\section{Study Reason for exclusion}

Acalha 2010

Design: unclear. ("Thirteen chronic stroke patients were divided into experimental group-EG ( $\mathrm{n}=$ 7) and control group-CG $(n=6)$.") Abstract only. Clarification of randomisation and intervention sought but not obtained

de Paula Oliveira 2007

Design: RCT. Abstract only. Clarification of intervention sought but not obtained. Study excluded because of insufficient information available regarding intervention 


\begin{tabular}{ll}
\hline Study & Reason for exclusion \\
\hline Dean 2000a & Repeated case study $(\mathrm{n}=3)$; confirmed by correspondence with the author \\
\hline Dickstein 1986 & $\begin{array}{l}\text { Cohort design, not RCT: quasi-randomisation of participants (based on administrative procedures) } \\
\text { to one of } 13 \text { physiotherapists; however, each physiotherapist provided treatment interventions in } \\
\text { a predetermined (not randomised) order (first five participants received treatment } \text { A, next five par- } \\
\text { ticipants treatment } \mathrm{B} \text {, next five participants treatment } \mathrm{C} \text { ); this study was therefore assessed to be a } \\
\text { cohort design rather than a randomised trial }\end{array}$
\end{tabular}

\begin{tabular}{ll}
\hline Eng 2003 & Repeated measures design; not RCT \\
\hline English 2003 & $\begin{array}{l}\text { Alternating allocation, not RCT: "Patients admitted into a stroke unit during particular time periods } \\
\text { were allocated to either arm of the trial, e.g. weeks } 1 \text { to } 6 \text { to treatment group, weeks } 7 \text { to } 12 \text { to usual } \\
\text { care and so on" }\end{array}$
\end{tabular}

Gong $2003 \quad \begin{aligned} & \text { Design: unclear (further information required). Clarification of randomisation sought but not ob- } \\ & \text { tained }\end{aligned}$

Gregson $2003 \quad$ Design: single-centre, single-blind RCT. Clinical trial protocol only available. Results presented at a conference in July 2005. No publication intended as of 22 November 2011. Clarification of intervention sought but not obtained. Study excluded because of insufficient information regarding intervention

\begin{tabular}{|c|c|}
\hline Hesse 1998 & Single-participant design \\
\hline Inaba 1973 & $\begin{array}{l}\text { Compared three orthopaedic approaches; excluded from this version of the review; quasi-randomi- } \\
\text { sation }\end{array}$ \\
\hline Karaduman 2001 & Study not randomised (confirmed by correspondence with study author) \\
\hline Khanna 2003 & This study was never carried out (confirmed by correspondence with study author) \\
\hline Kim 2001 & Specific strength training intervention (i.e. component, not approach) \\
\hline Krutulyte 2003 & $\begin{array}{l}\text { Design: unclear (further information required). Clarification of randomisation sought but not ob- } \\
\text { tained }\end{array}$ \\
\hline Li 2004 & Trial of a specific balance training intervention (i.e. component, not approach) \\
\hline Lin 2004 & Trial of timing of intervention (i.e. component, not approach) \\
\hline Liu 2008 & Quasi-randomised study \\
\hline Meng 2005 & $\begin{array}{l}\text { Design: unclear (further information required). Clarification of randomisation sought but not ob- } \\
\text { tained }\end{array}$ \\
\hline Ng 2005 & $\begin{array}{l}\text { Design: unclear (further information required). Clarification of randomisation sought but not ob- } \\
\text { tained }\end{array}$ \\
\hline Nissan-Lavi 2009 & $\begin{array}{l}\text { British Library unable to locate or supply the document. We were also unable to contact the study } \\
\text { author to obtain a copy of the paper }\end{array}$ \\
\hline Ozdemir 2001 & Quasi-randomised study \\
\hline Pomeroy 2001a & This study was never carried out (confirmed by correspondence with study author) \\
\hline Pyoria 2007 & Controlled clinical trial, not a randomised controlled trial \\
\hline
\end{tabular}




\begin{tabular}{|c|c|}
\hline Study & Reason for exclusion \\
\hline Qiu 2004 & Trial of a specific balance training intervention (i.e. component, not approach) \\
\hline Richards 2004 & Compares two different intensities of a mixed approach \\
\hline Salter 1991 & Collected data retrospectively from participant charts; had not used preplanned data collection \\
\hline Stern 1970 & Quasi-randomised study \\
\hline Stuart 2008 & Non-randomised controlled study \\
\hline Thielman 2004 & Treatment intervention and outcomes concentrated on upper limb \\
\hline Wagenaar 1990 & $\begin{array}{l}\text { Compared two neurophysiological approaches; excluded from this version of the review; qua- } \\
\text { si-randomisation }\end{array}$ \\
\hline Wang 2005b & $\begin{array}{l}\text { Study not randomised ("Total of } 100 \text { patients with CVA were selected and divided into test and con- } \\
\text { trol group, } 50 \text { cases each") }\end{array}$ \\
\hline Wolny 2003 & $\begin{array}{l}\text { Study not randomised ("Two 20-subject groups-the experimental one and the control one, partici- } \\
\text { pated") }\end{array}$ \\
\hline Wood 1994 & Study never carried out (confirmed by communication with study author) \\
\hline \multirow[t]{2}{*}{ Xu 2008} & Design: RCT \\
\hline & $\begin{array}{l}\text { Abstract only available. Further details sought regarding the intervention but not obtained. Study } \\
\text { excluded because of insufficient information available regarding intervention }\end{array}$ \\
\hline Yin 2003b & Study not randomised \\
\hline \multirow[t]{2}{*}{ Yu 2008} & Design: RCT \\
\hline & $\begin{array}{l}\text { Abstract only available. Further details sought regarding the intervention but not obtained. Study } \\
\text { excluded because of insufficient information available regarding intervention }\end{array}$ \\
\hline Zhong 2006 & $\begin{array}{l}\text { Design: unclear (further information required). Clarification of randomisation sought but not ob- } \\
\text { tained }\end{array}$ \\
\hline Zhou 2003 & $\begin{array}{l}\text { Study not randomised ("Patients were divided into treatment group }(n=50) \text { and control group }(n= \\
\text { 50).") }\end{array}$ \\
\hline Zhu 2004a & Quasi-randomised study \\
\hline
\end{tabular}

$\mathrm{n}$ : number of participants.

$\mathrm{RCT}$ : randomised controlled trial.

Characteristics of studies awaiting classification [ordered by study ID]

Giraux 2008

\begin{tabular}{ll}
\hline Methods & Design: multi-centre RCT \\
\hline Participants & Estimated enrolment: $\mathrm{n}=240$ \\
& $\begin{array}{l}\text { Inclusion criteria: "(1) Patients must be aged between } 18 \text { to } 75 \text { years, with a first ischemic or hem- } \\
\text { orrhagic stroke at least six months ago and no longer than two years ago, without a subsequent }\end{array}$
\end{tabular}


Giraux 2008 (Continued)

stroke. It must have caused initially complete hemiplegia of the right or left hemibody, but they must be able [to] walk alone with or without technical assistance over a distance of at least $10 \mathrm{~m}$. They must be able to change direction too and (2) They must have a functional ambulation classification score between 4 and 6 during the inclusion"

Exclusion criteria: "(1) We will exclude patients with a neurological history other than a stroke, a psychiatric illness, or an associated debilitating disease, (2) They must not have an associated cerebella syndrome or a clinical brainstem attack and (3) We will refuse patients who are pregnant, who have not signed the written consent and who aren't entitled to a social security scheme"

Interventions

"(1) Active comparator: patients who continue physical therapy sessions during two months. Intervention: behavioural-two physical therapy sessions per week for two months"

"(2) No intervention: patients who stop physical therapy sessions during two months. Intervention: behavioural-patients who stop two physical therapy sessions per week for two months"

Outcomes

Primary outcome measures: average number of steps/d recorded over three days in an outpatient setting. To be measured at day three and then six months later

Secondary outcome measures: Six-Minute Walking test, Wade's test, Rivermead Mobility Index score and Barthel Index. These will be measured at day zero and at day 55

Notes Clarification of intervention sought but not obtained

Li 2000

Methods Design: "prospective RCT"

Participants

"The interview group was targeted from 200 stroke patients who participated and were discharged from the prospective randomised controlled trial between 1995 to 1997. All cases were confirmed to be first onset by clinical and CT or MRI diagnosis. This did not include less severe or very severe cases"

Interventions

"The control group $(n=100)$ only received conventional neurological treatment, while the rehabilitation group underwent an early rehabilitation program commencing on an average of 9 days after diagnosis with bedside exercises, in addition to the treatment"

Outcomes

"The interview was conducted in the form of calls and letters, with the time of interview being 18 months after treatment. The interview content included the patient's survival, functional mobility, environment patient was mobile in, ADL ability, quality of life $(\mathrm{QOL})$ and any secondary injury. The latter included joint pain, joint range of motion, limb or hand swelling, muscle atrophy, pressure ulcers, lung and urinary tract infection, with 1 point given for presence of secondary injury and no points for the absence of injury"

Notes

Original study translated from Chinese to English. This study is a follow-up from an earlier study undertaken in 1998. British Library unable to supply this earlier publication, and, despite extensive searching, the reviewers have not been able to find any other known UK locations for it. Clarification of intervention from the earlier RCT was sought from the study authors but was not obtained

Matsumoto 2010

Methods Design: single-blind, parallel RCT

Participants

Target sample size: $\mathrm{n}=50$ 
Inclusion criteria: (1) between 20 and 80 years old, (2) post-stroke patients whose onset was between one and six months

Exclusion criteria: (1) severe higher brain dysfunction, (2) severe dementia and (3) loss of consciousness

\begin{tabular}{ll}
\hline Interventions & Trunk facilitation technique and without trunk facilitation technique. No further details available \\
\hline Outcomes & Primary outcomes: muscle strength, Functional Assessment for Control Trunk, Berg Balance Scale, \\
& Functional Reach Test, 10-Metre Gait Measurement, Functional Independence Measure
\end{tabular}

Notes Clarification of intervention sought but not obtained

\section{Richardson 2011}

\begin{tabular}{|c|c|}
\hline Methods & Design: single-blinded randomised controlled trial \\
\hline \multirow[t]{3}{*}{ Participants } & $\begin{array}{l}\text { Delivered to persons with stroke }+/-18 \text { years, community dwelling and able to walk } 100 \text { metres in } \\
\text { dependently. Still recruiting participants }\end{array}$ \\
\hline & $\begin{array}{l}\text { Inclusion criteria: (1) adults }>18 \text { years, ( } 2 \text { ) living in the community, (3) able to ambulate }>10 \text { metr } \\
\text { with or without an assistive device, (4) able to tolerate } 60 \text { minutes of activity with rest intervals, } \\
\text { have clearance from a physician to participate in the programme, (6) can independently follow in } \\
\text { structions and ( } 7 \text { ) are not involved in active rehabilitation }\end{array}$ \\
\hline & $\begin{array}{l}\text { Exclusion criteria: (1) musculoskeletal contraindications to exercise, (2) unstable cardiovascular } \\
\text { conditions, ( } 3 \text { ) unstable medical conditions and (4) significant cognitive impairment }\end{array}$ \\
\hline
\end{tabular}

Interventions

"12 week intervention comprised of group and individual exercise programs and an 8 week, Living with Stroke Education program (1 hr/week). The intervention was delivered by kinesiologists (YMCA) with consultation from physiotherapists (Hamilton Health Sciences)"

Outcomes Primary outcomes: Six-Minute Walk Test (6MWT), Hand Grip Strength and Rapid Assessment of Physical Activity

Secondary outcomes: Patient Activation Measure

Participants were assessed at baseline, 12 weeks and 24 weeks by a blind assessor

Notes Clarification of intervention sought but not obtained

Further details about the trial are available at www.clinicaltrials.gov (NCT01194102)

Sanchez-Sanchez 2011

\begin{tabular}{ll}
\hline Methods & Design: randomised double-blind controlled trial \\
\hline Participants & "25 patients (mean age $77.20 \pm 7 . \mathrm{SS}$ ) were included in the study and 11 completed the protocol \\
(control group $\mathrm{n}=4$, target group $\mathrm{n}=7$ )" & \\
& Inclusion criteria: "Patients over 60 years old who had suffered a single stroke episode with residual \\
hemiparesis, ability to walk before stroke and to be clinically stable enough to begin physiothera- & \\
py" & \\
Exclusion criteria: "Blindness, prosthetics or significant osteoarthritis of the lower limbs, serious \\
cardiac disease and severe cognitive impairment"
\end{tabular}


Sanchez-Sanchez 2011 (Continued)

Interventions

"Control and target groups were treated with conventional physiotherapy for stroke, but we added specific techniques to the target group depending on patient's functional level"

Outcomes

"Outcome measures were balance on Berg Balance Scale: walking ability on gait speed and HS Functional Ambulation Classification (FACHS), and functional ability on Barthel Index. Assessment was done at baseline, on the fourth and the twelfth week"

Notes Abstract only. Clarification of intervention sought but not obtained

Wang 2005a

Methods Design: unclear ("were divided randomly into rehabilitation group and control group"). Both groups received normal treatment and general nursing, based on which rehabilitation group received rehabilitation training

\begin{tabular}{ll}
\hline Participants & "62 post-stroke hemiplegic patients" \\
\hline Interventions & $\begin{array}{l}\text { "Both groups received normal treatment and general nursing, based on which rehabilitation group } \\
\text { received rehabilitation training. The therapy of movement was divided into four stages, which in- } \\
\text { cluded posture in lying training, eating training, standing training and walking training. We de- } \\
\text { signed several examination methods in activity of daily life such as beating ball, picking up beans, } \\
\text { stirring abacus, taking off clothes, using dishware, etc" }\end{array}$ \\
\hline Outcomes & $\begin{array}{l}\text { "Six-period opinion method and Modified Bathel Index were used to evaluate motor function of } \\
\text { limbs and activity of daily life in the first week of hospitalization and before discharge" }\end{array}$ \\
\hline Notes & $\begin{array}{l}\text { British Library unable to supply this publication, and, despite extensive searching, the review au- } \\
\text { thors have not been able to find any other known UK locations for it. Clarification of intervention } \\
\text { was sought from the study authors but was not obtained }\end{array}$ \\
\hline
\end{tabular}

Wang 2012

\begin{tabular}{ll}
\hline Methods & $\begin{array}{l}\text { Design: unclear ("A multi-center intervention study was conducted in five sub-centers of three } \\
\text { cities in China. We randomly evaluated the awareness of rehabilitation and situation of receiving } \\
\text { rehabilitation services in the stroke survivors") }\end{array}$
\end{tabular}

Participants "Three hundred and forty-two patients successfully completed three-month rehabilitation training in the community health neighbourhood service center"

Interventions $\quad$ "The intervention measures comprised a rehabilitation information package and a new rehabilita-
tion exercise program that is a simplified form of Conductive Education"

Outcomes

"We used the Fugl-Meyer Assessment (FM) to evaluate motor function, and Barthel index (BI) to evaluate activities of daily living (ADL)"

Notes

Abstract only. Clarification of randomisation and intervention sought but not obtained

Methods Design: randomised double-blinded controlled trial


Yau 2010 (Continued)

Participants $\quad$ "Thirty-nine (21 male, 18 female) stroke patients with mild mobility limitation, were recruited within one-week of onset and randomized to augmented therapy group (ATG) or control group (CG)"

Interventions

"Subjects from both groups received additional exercise sessions for three days. Exercise program for ATG was based on task-oriented strength training of the lower limb while those for the CG contained dexterity exercises within arm-reach"

Outcomes

Outcome measures included modified functional ambulation classification, modified Rivermead Mobility Index, functional reach, five times sit to stand, step test, Berg Balance Scale (BBS), Timed Up and Go test and gait speed (comfortable and maximum) were performed at recruitment and after intervention

Participant compliance and any adverse events were also recorded

\begin{tabular}{ll}
\hline Methods & Design: unclear ("divided randomly into 2 groups: the treatment group and the control group") \\
\hline Participants & "80 cases with acute brain vascular disease" \\
\hline Interventions & $\begin{array}{l}\text { "The treatment group was treated with three grades regular rehabilitation treatment whereas the } \\
\text { control received no rehabilitation treatment unless treated with acupuncture or massage by pa- } \\
\text { tients themselves. Both groups received routine treatment of internal medicine" }\end{array}$ \\
\hline Outcomes & $\begin{array}{l}\text { "Both groups were evaluated with simplified Fugl-[Meyer] (FM) scale at the beginning and the end } \\
\text { of the treatment" }\end{array}$ \\
\hline Notes & Abstract only. Clarification of intervention sought but not obtained \\
\hline
\end{tabular}

Characteristics of ongoing studies [ordered by study ID]

\section{Cross 2009}

Study name

Methods

Methods

Participants

Participants

Interventions

\section{Outcomes}

The FeSTivaLS trial protocol: a randomised evaluation of the efficacy of functional strength training on enhancing walking and upper limb function later post stroke

\section{Randomised, observer-blind trial with embedded qualitative investigation of participants' views of} functional strength training

\section{"Participants ( $n=58$ ), six months to five years after stroke with difficulty using their paretic upper} and lower limbs for everyday functional activity"

\begin{tabular}{l} 
(outcome) and six weeks thereafter (follow-up). Primary outco \\
Functional Ambulation Categories (FAC) and the Action Resear \\
\hline Starting date \\
\hline \hline Physical rehabilitation approaches for the recovery of function and mobility following stroke (Review) \\
Copyright $\odot 2023$ The Cochrane Collaboration. Published by John Wiley \& Sons, Ltd.
\end{tabular}

"All will be randomized to either: (1) functional strength training-upper limb or (2) functional strength training-lower limb delivered in their own homes for fours days each week for six weeks. FST involves repetitive progressive resisted exercise during goal directed functional activities. The therapist's main input is to provide verbal prompting and feedback" 
Cross 2009 (Continued)

Contact information
Dr Kathryn Mares, School of Allied Health Professions, University of East Anglia, Queen's Building, Norwich Research Park, Norwich, NR4 7TJ, UK

E-mail:k.mares@uea.ac.uk

Notes

The trial is registered on the Current Controlled Trials database (ISRCTN71632550). The full protocol has also been published (see Mares et al (2013) (Cross 2009))

\section{Kumaran 2010}

Study name

Effectiveness and feasibility of a task and context-based exercise programme in stroke patients: a randomised controlled trial

\begin{tabular}{|c|c|}
\hline Methods & Randomised, parallel-group, active-controlled trial \\
\hline \multirow[t]{3}{*}{ Participants } & "Total sample size = 202; sample size from India = 202" \\
\hline & $\begin{array}{l}\text { Inclusion criteria: "(1) first stroke discharged from hospital; (2) ischemic stroke (3) aged between } 30 \\
\text { years and } 65 \text { years; (4) both sexes; (5) a minimum of three months post stroke duration; }(6) \text { the abil- } \\
\text { ity to ambulate at least } 5 \text { meters with supervision or guarding; ( } 7 \text { ) the ability to understand instruc- } \\
\text { tions and follow simple commands" }\end{array}$ \\
\hline & $\begin{array}{l}\text { Exclusion criteria: "(1) patient with a present history of severe, uncontrolled, or unstable cardiac } \\
\text { disease; (2) other systemic disorders for which exercise is contraindicated; ( } 3 \text { ) terminally ill; (4) } \\
\text { hearing and visually challenged; (5) any other coexisting conditions that would interfere with out- } \\
\text { come assessments or participation in treatment regimens" }\end{array}$ \\
\hline Interventions & $\begin{array}{l}\text { "Randomized controlled trial evaluating the effectiveness and feasibility of a task and context } \\
\text { based exercise program in stroke patients. patients in control will receive conventional physiother- } \\
\text { apy exercises whereas patients in experimental group will receive task and context based exercise } \\
\text { program. both the group will receive exercise program thrice weekly, for a period of } 12 \text { weeks. pri- } \\
\text { mary outcome measure is stroke impact scale score" }\end{array}$ \\
\hline
\end{tabular}

Outcomes

Primary outcome measures: Stroke Impact Scale score

Secondary outcome measures: Motricity Index score, gait velocity by 5-Metre Timed Walk test, Berg Balance Scale score, walking distance by Six-Minute Walk test, impact on participation and autonomy questionnaire score, falls efficacy scale-international score

Time points: pre eight weeks, post eight weeks, post 12 weeks, post 16 weeks

\begin{tabular}{ll}
\hline Starting date & Registered on 27 April 2010; date of first enrolment 16 August 2011 \\
\hline Contact information & Senthil Kumaran, Associate Professor, Department of Physiotherapy, MCOAHS, Manipal University, \\
& Udupi, Karnataka 576104, India \\
& E-mail:senthil.kumaran@manipal.edu
\end{tabular}

\section{DATA AND ANALYSES}


Comparison 1. Intervention versus no treatment: immediate outcomes

\begin{tabular}{|c|c|c|c|c|}
\hline Outcome or subgroup title & No. of studies & $\begin{array}{l}\text { No. of partici- } \\
\text { pants }\end{array}$ & Statistical method & Effect size \\
\hline 1.1 Independence in ADL scales & 28 & 3423 & $\begin{array}{l}\text { Std. Mean Difference (IV, Random, } \\
95 \% \mathrm{CI} \text { ) }\end{array}$ & $0.78[0.58,0.97]$ \\
\hline 1.1.1 Functional task training & 2 & 250 & $\begin{array}{l}\text { Std. Mean Difference (IV, Random, } \\
95 \% \mathrm{CI} \text { ) }\end{array}$ & $-0.06[-0.30,0.19]$ \\
\hline $\begin{array}{l}\text { 1.1.2 Functional task training + } \\
\text { musculoskeletal }\end{array}$ & 9 & 967 & $\begin{array}{l}\text { Std. Mean Difference (IV, Random, } \\
95 \% \mathrm{CI} \text { ) }\end{array}$ & $0.97[0.67,1.27]$ \\
\hline 1.1.3 Neurophysiological & 2 & 140 & $\begin{array}{l}\text { Std. Mean Difference (IV, Random, } \\
95 \% \mathrm{CI})\end{array}$ & $0.79[0.45,1.14]$ \\
\hline $\begin{array}{l}\text { 1.1.4 Neurophysiological + mus- } \\
\text { culoskeletal }\end{array}$ & 1 & 128 & $\begin{array}{l}\text { Std. Mean Difference (IV, Random, } \\
95 \% \mathrm{CI} \text { ) }\end{array}$ & $0.02[-0.34,0.37]$ \\
\hline $\begin{array}{l}\text { 1.1.5 Functional training + neuro- } \\
\text { physiological }\end{array}$ & 0 & 0 & $\begin{array}{l}\text { Std. Mean Difference (IV, Random, } \\
95 \% \mathrm{CI} \text { ) }\end{array}$ & Not estimable \\
\hline $\begin{array}{l}\text { 1.1.6 Functional training + neuro- } \\
\text { physiological + musculoskeletal }\end{array}$ & 12 & 1838 & $\begin{array}{l}\text { Std. Mean Difference (IV, Random, } \\
95 \% \mathrm{CI} \text { ) }\end{array}$ & $0.96[0.66,1.27]$ \\
\hline 1.1.7 Musculoskeletal & 2 & 100 & $\begin{array}{l}\text { Std. Mean Difference (IV, Random, } \\
95 \% \mathrm{CI} \text { ) }\end{array}$ & $0.06[-0.34,0.45]$ \\
\hline 1.2 Motor function scales & 27 & 4558 & $\begin{array}{l}\text { Std. Mean Difference (IV, Random, } \\
95 \% \mathrm{CI} \text { ) }\end{array}$ & $0.81[0.58,1.04]$ \\
\hline 1.2.1 Functional task training & 2 & 250 & $\begin{array}{l}\text { Std. Mean Difference (IV, Random, } \\
95 \% \mathrm{CI} \text { ) }\end{array}$ & $0.14[-0.31,0.58]$ \\
\hline $\begin{array}{l}\text { 1.2.2 Functional task training + } \\
\text { musculoskeletal }\end{array}$ & 10 & 2175 & $\begin{array}{l}\text { Std. Mean Difference (IV, Random, } \\
95 \% \mathrm{CI} \text { ) }\end{array}$ & $1.13[0.61,1.66]$ \\
\hline 1.2.3 Neurophysiological & 0 & 0 & $\begin{array}{l}\text { Std. Mean Difference (IV, Random, } \\
95 \% \mathrm{CI} \text { ) }\end{array}$ & Not estimable \\
\hline $\begin{array}{l}\text { 1.2.4 Neurophysiological + mus- } \\
\text { culoskeletal }\end{array}$ & 1 & 128 & $\begin{array}{l}\text { Std. Mean Difference (IV, Random, } \\
95 \% \mathrm{CI} \text { ) }\end{array}$ & $0.17[-0.19,0.52]$ \\
\hline $\begin{array}{l}\text { 1.2.5 Functional training + neuro- } \\
\text { physiological }\end{array}$ & 1 & 68 & $\begin{array}{l}\text { Std. Mean Difference (IV, Random, } \\
95 \% \mathrm{CI} \text { ) }\end{array}$ & $1.49[0.95,2.03]$ \\
\hline $\begin{array}{l}\text { 1.2.6 Functional training + neuro- } \\
\text { physiological + musculoskeletal }\end{array}$ & 11 & 1837 & $\begin{array}{l}\text { Std. Mean Difference (IV, Random, } \\
95 \% \mathrm{CI} \text { ) }\end{array}$ & $0.76[0.54,0.97]$ \\
\hline 1.2.7 Musculoskeletal & 2 & 100 & $\begin{array}{l}\text { Std. Mean Difference (IV, Random, } \\
95 \% \mathrm{CI} \text { ) }\end{array}$ & $0.22[-0.21,0.64]$ \\
\hline 1.3 Balance (Berg Balance Scale) & 1 & 34 & $\begin{array}{l}\text { Std. Mean Difference (IV, Random, } \\
95 \% \mathrm{CI} \text { ) }\end{array}$ & $-0.04[-0.71,0.64]$ \\
\hline $\begin{array}{l}\text { 1.3.1 Functional task training + } \\
\text { musculoskeletal }\end{array}$ & 1 & 34 & $\begin{array}{l}\text { Std. Mean Difference (IV, Random, } \\
95 \% \mathrm{CI} \text { ) }\end{array}$ & $-0.04[-0.71,0.64]$ \\
\hline
\end{tabular}




\begin{tabular}{|c|c|c|c|c|}
\hline Outcome or subgroup title & No. of studies & $\begin{array}{l}\text { No. of partici- } \\
\text { pants }\end{array}$ & Statistical method & Effect size \\
\hline 1.3.2 Functional task training & 0 & 0 & $\begin{array}{l}\text { Std. Mean Difference (IV, Random, } \\
95 \% \mathrm{CI} \text { ) }\end{array}$ & Not estimable \\
\hline 1.3.3 Neurophysiological & 0 & 0 & $\begin{array}{l}\text { Std. Mean Difference (IV, Random, } \\
95 \% \mathrm{Cl} \text { ) }\end{array}$ & Not estimable \\
\hline $\begin{array}{l}\text { 1.3.4 Neurophysiological + mus- } \\
\text { culoskeletal }\end{array}$ & 0 & 0 & $\begin{array}{l}\text { Std. Mean Difference (IV, Random, } \\
95 \% \mathrm{Cl} \text { ) }\end{array}$ & Not estimable \\
\hline $\begin{array}{l}\text { 1.3.5 Functional training + neuro- } \\
\text { physiological }\end{array}$ & 0 & 0 & $\begin{array}{l}\text { Std. Mean Difference (IV, Random, } \\
95 \% \mathrm{CI} \text { ) }\end{array}$ & Not estimable \\
\hline $\begin{array}{l}\text { 1.3.6 Functional training + neuro- } \\
\text { physiological + musculoskeletal }\end{array}$ & 0 & 0 & $\begin{array}{l}\text { Std. Mean Difference (IV, Random, } \\
95 \% \mathrm{CI} \text { ) }\end{array}$ & Not estimable \\
\hline 1.3.7 Musculoskeletal & 0 & 0 & $\begin{array}{l}\text { Std. Mean Difference (IV, Random, } \\
95 \% \mathrm{Cl} \text { ) }\end{array}$ & Not estimable \\
\hline 1.4 Gait velocity & 3 & 292 & $\begin{array}{l}\text { Std. Mean Difference (IV, Random, } \\
95 \% \mathrm{CI} \text { ) }\end{array}$ & $0.05[-0.18,0.28]$ \\
\hline 1.4.1 Functional task training & 3 & 292 & $\begin{array}{l}\text { Std. Mean Difference (IV, Random, } \\
95 \% \mathrm{CI} \text { ) }\end{array}$ & $0.05[-0.18,0.28]$ \\
\hline $\begin{array}{l}\text { 1.4.2 Functional task training + } \\
\text { musculoskeletal }\end{array}$ & 0 & 0 & $\begin{array}{l}\text { Std. Mean Difference (IV, Random, } \\
95 \% \mathrm{Cl} \text { ) }\end{array}$ & Not estimable \\
\hline 1.4.3 Neurophysiological & 0 & 0 & $\begin{array}{l}\text { Std. Mean Difference (IV, Random, } \\
95 \% \mathrm{CI} \text { ) }\end{array}$ & Not estimable \\
\hline $\begin{array}{l}\text { 1.4.4 Neurophysiological + mus- } \\
\text { culoskeletal }\end{array}$ & 0 & 0 & $\begin{array}{l}\text { Std. Mean Difference (IV, Random, } \\
95 \% \mathrm{CI} \text { ) }\end{array}$ & Not estimable \\
\hline $\begin{array}{l}\text { 1.4.5 Functional training + neuro- } \\
\text { physiological }\end{array}$ & 0 & 0 & $\begin{array}{l}\text { Std. Mean Difference (IV, Random, } \\
95 \% \mathrm{Cl} \text { ) }\end{array}$ & Not estimable \\
\hline $\begin{array}{l}\text { 1.4.6 Functional training + neuro- } \\
\text { physiological + musculoskeletal }\end{array}$ & 0 & 0 & $\begin{array}{l}\text { Std. Mean Difference (IV, Random, } \\
95 \% \mathrm{Cl} \text { ) }\end{array}$ & Not estimable \\
\hline 1.4.7 Musculoskeletal & 0 & 0 & $\begin{array}{l}\text { Std. Mean Difference (IV, Random, } \\
95 \% \mathrm{Cl} \text { ) }\end{array}$ & Not estimable \\
\hline 1.5 Length of stay & 3 & 318 & $\begin{array}{l}\text { Mean Difference (IV, Random, 95\% } \\
\mathrm{CI})\end{array}$ & $\begin{array}{l}-2.85[-10.47 \\
4.76]\end{array}$ \\
\hline 1.5.1 Functional task training & 0 & 0 & $\begin{array}{l}\text { Mean Difference (IV, Random, 95\% } \\
\mathrm{Cl} \text { ) }\end{array}$ & Not estimable \\
\hline $\begin{array}{l}\text { 1.5.2 Functional task training + } \\
\text { musculoskeletal }\end{array}$ & 3 & 318 & $\begin{array}{l}\text { Mean Difference (IV, Random, 95\% } \\
\mathrm{Cl} \text { ) }\end{array}$ & $\begin{array}{l}-2.85[-10.47 \\
4.76]\end{array}$ \\
\hline 1.5.3 Neurophysiological & 0 & 0 & $\begin{array}{l}\text { Mean Difference (IV, Random, 95\% } \\
\mathrm{Cl} \text { ) }\end{array}$ & Not estimable \\
\hline
\end{tabular}




\begin{tabular}{|c|c|c|c|c|}
\hline Outcome or subgroup title & No. of studies & $\begin{array}{l}\text { No. of partici- } \\
\text { pants }\end{array}$ & Statistical method & Effect size \\
\hline $\begin{array}{l}\text { 1.5.4 Neurophysiological + mus- } \\
\text { culoskeletal }\end{array}$ & 0 & 0 & $\begin{array}{l}\text { Mean Difference (IV, Random, 95\% } \\
\mathrm{CI})\end{array}$ & Not estimable \\
\hline $\begin{array}{l}\text { 1.5.5 Functional training + neuro- } \\
\text { physiological }\end{array}$ & 0 & 0 & $\begin{array}{l}\text { Mean Difference (IV, Random, 95\% } \\
\mathrm{CI})\end{array}$ & Not estimable \\
\hline $\begin{array}{l}\text { 1.5.6 Functional training + neuro- } \\
\text { physiological + musculoskeletal }\end{array}$ & 0 & 0 & $\begin{array}{l}\text { Mean Difference (IV, Random, 95\% } \\
\mathrm{CI})\end{array}$ & Not estimable \\
\hline 1.5.7 Musculoskeletal & 0 & 0 & $\begin{array}{l}\text { Mean Difference (IV, Random, 95\% } \\
\mathrm{CI} \text { ) }\end{array}$ & Not estimable \\
\hline
\end{tabular}


Analysis 1.1. Comparison 1: Intervention versus no treatment: immediate outcomes, Outcome 1: Independence in ADL scales

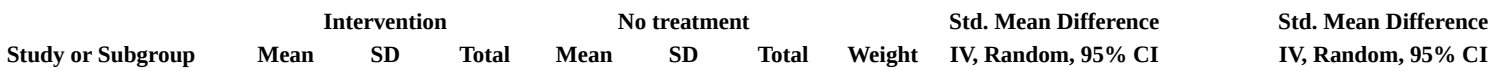

\begin{tabular}{|c|c|c|c|c|c|c|c|}
\hline \multicolumn{8}{|c|}{ 1.1.1 Functional task training } \\
\hline Green 2002 (1) & 18 & 2.1 & 81 & 18 & 2.1 & 80 & $4.1 \%$ \\
\hline Wade 1992 & 16.2 & 3.1 & 48 & 16.7 & 3.2 & 41 & $3.8 \%$ \\
\hline Subtotal (95\% CI) & & & 129 & & & 121 & $7.8 \%$ \\
\hline \multicolumn{8}{|c|}{ Heterogeneity: $\mathrm{Tau}^{2}=0.00 ; \mathrm{Chi}^{2}=0.35, \mathrm{df}=1(\mathrm{P}=0.55) ; \mathrm{I}^{2}=0 \%$} \\
\hline \multicolumn{8}{|c|}{ Test for overall effect: $\mathrm{Z}=0.44(\mathrm{P}=0.66)$} \\
\hline \multicolumn{8}{|c|}{ 1.1.2 Functional task training + musculoskeletal } \\
\hline Chen 2006 & 56.9 & 9.89 & 25 & 49.8 & 9.87 & 20 & $3.1 \%$ \\
\hline Liu 2003 & 48.5 & 13.8 & 60 & 32.2 & 14.6 & 60 & $3.9 \%$ \\
\hline Pan 2004 & 73.63 & 20.33 & 48 & 48.23 & 16.22 & 48 & $3.7 \%$ \\
\hline Xie 2003 & 13 & 5 & 32 & 8 & 5 & 32 & $3.4 \%$ \\
\hline Xu 2003a & 57 & 15 & 94 & 41 & 15 & 92 & $4.1 \%$ \\
\hline Yan 2002 & 65.8 & 17.2 & 40 & 45.8 & 18.3 & 38 & $3.6 \%$ \\
\hline Zhao 2003 (2) & 3.16 & 0.86 & 150 & 2.87 & 1 & 150 & $4.3 \%$ \\
\hline Zhu 2007 haem (3) & 42.5 & 10 & 12 & 27.5 & 12.5 & 10 & $2.2 \%$ \\
\hline Zhu 2007 isch (3) & 50 & 21.25 & 28 & 30 & 18.75 & 28 & $3.3 \%$ \\
\hline
\end{tabular}

Subtotal (95\% CI) 489

$0.00[-0.31,0.31]$

$-0.16[-0.58,0.26]$

$\mathbf{- 0 . 0 6}[-0.30,0.19]$

作 ${ }^{2}=0.14 ; \mathrm{Chi}^{2}=33.40, \mathrm{df}=8(\mathrm{P}<0.0001) ; \mathrm{I}^{2}=76 \%$

Test for overall effect: $\mathrm{Z}=6.41(\mathrm{P}<0.00001)$

\subsubsection{Neurophysiological}

Xu 1999

70.72

18.07

$82.59 \quad 16.83$

41

56.58

16.19

$37 \quad 3.6 \%$

Subtotal (95\% CI)

73

15.34

$30 \quad 3.4 \%$

$67 \quad 7.0 \%$

$0.81[0.35,1.28]$

$0.77[0.25,1.29]$

$0.79[0.45,1.14]$

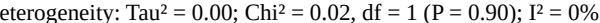

Test for overall effect: $\mathrm{Z}=4.51(\mathrm{P}<0.00001)$

1.1.4 Neurophysiological + musculoskeletal

$\begin{array}{lll}\text { Fang } 2003 & 47.67 \quad 28.75\end{array}$

Subtotal (95\% CI)

50
50

47.16

28.73

$78 \quad 3.9 \%$

$0.02[-0.34,0.37]$

$0.02[-0.34,0.37]$

Heterogeneity: Not applicable

Test for overall effect: $\mathrm{Z}=0.10(\mathrm{P}=0.92)$

1.1.5 Functional training + neurophysiological

Subtotal (95\% CI)

$\mathbf{0}$

Heterogeneity: Not applicable

Test for overall effect: Not applicable

1.1.6 Functional training + neurophysiological + musculoskeletal

$\begin{array}{lllll}\text { Chen } 2004 & 4.49 & 5.83 & 39 & 2.95\end{array}$

$\begin{array}{lllll}\text { Chu } 2003 & 47.67 & 11.12 & 30 & 30.18\end{array}$

Hou 2006

Huang 2003

$4.88 \quad 0.81$

0.81

$40 \quad 3.9$

4.69

10.22

$39 \quad 3.7 \%$

$0.29[-0.16,0.73]$

$1.61[1.02,2.21]$

$1.07[0.60,1.54]$

$1.65[1.00,2.30]$

$1.64[1.06,2.23]$

$0.56[0.15,0.97]$

$1.10[0.79,1.41]$

$0.36[-0.17,0.88]$

$1.66[1.29,2.03]$

$1.15[0.58,1.72]$

$0.51[0.37,0.64]$

$0.27[-0.20,0.74]$

$0.96[0.66,1.27]$

Wu 2006

Xu 2004

Zhang 1998

Zhang 2004

Subtotal (95\% CI)

$\begin{array}{rrrrr}84 & 33 & 439 & 69 & 26 \\ 54.12 & 30.36 & 35 & 45.87 & 29.83\end{array}$ $\mathbf{9 1 5}$

$35 \quad 3.6 \%$

$923 \quad 43.1 \%$

IV, Random, 95\% CI

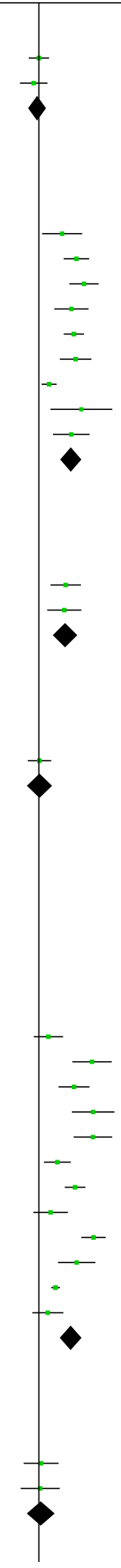

1.1.7 Musculoskeletal

Fang 2004 old

$\begin{array}{rrrr}45 & 29.08 & 24 & 42.97\end{array}$

29.81

$32 \quad 3.4 \%$

$0.07[-0.46,0.60]$

Fang 2004 young

$\begin{array}{lll}50.71 & 28.78 & 21 \\ & & 45\end{array}$

$49.57 \quad 27.38$

$23 \quad 3.2 \%$

$0.04[-0.55,0.63]$

Heterogeneity: $\mathrm{Tau}^{2}=0.00 ; \mathrm{Chi}^{2}=0.00, \mathrm{df}=1(\mathrm{P}=0.94) ; \mathrm{I}^{2}=0 \%$

0.06 [-0.34, 0.45]

Tect for nvarall offert. $7=\cap 78(\mathrm{P}=\cap 78)$ 


\section{Analysis 1.1. (Continued)}

Heterogeneity: Tau $^{2}=0.00 ; \mathrm{Chi}^{2}=0.00, \mathrm{df}=1(\mathrm{P}=0.94) ; \mathrm{I}^{2}=0 \%$

Test for overall effect: $\mathrm{Z}=0.28(\mathrm{P}=0.78)$

Total (95\% CI)

1701

Heterogeneity: Tau $^{2}=0.21 ; \mathrm{Chi}^{2}=176.00, \mathrm{df}=27(\mathrm{P}<0.00001) ; \mathrm{I}^{2}=85 \%$

Test for overall effect: $\mathrm{Z}=7.90(\mathrm{P}<0.00001)$

Test for subgroup differences: $\mathrm{Chi}^{2}=52.00, \mathrm{df}=5(\mathrm{P}<0.00001), \mathrm{I}^{2}=90.4 \%$

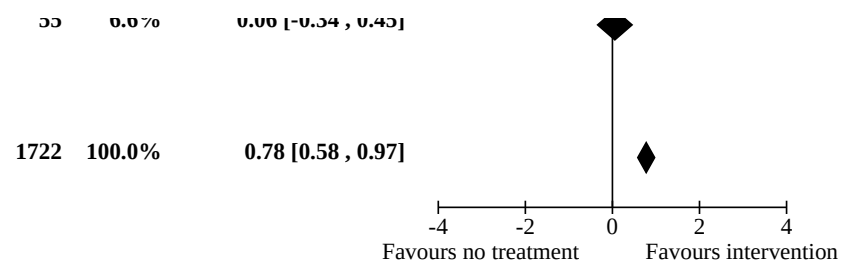

Footnotes

(1) Standard deviations estimated from range ((max - min range)/4)

(2) Mean and SD computed from categorical data.

(3) Estimated $\mathrm{SD}=(\max -\min$ range $) / 4$. 
Analysis 1.2. Comparison 1: Intervention versus no treatment: immediate outcomes, Outcome 2: Motor function scales

\begin{tabular}{|c|c|c|c|c|c|c|c|c|c|c|}
\hline \multirow[b]{2}{*}{ Study or Subgroup } & \multicolumn{3}{|c|}{ Intervention } & \multicolumn{3}{|c|}{ No treatment } & \multirow[b]{2}{*}{ Weight } & \multirow{2}{*}{$\begin{array}{l}\text { Std. Mean Difference } \\
\text { IV, Random, 95\% CI }\end{array}$} & \multirow{2}{*}{$\begin{array}{l}\text { Std. Mean Difference } \\
\text { IV, Random, } 95 \% \text { CI }\end{array}$} & \\
\hline & Mean & SD & Total & Mean & SD & Total & & & & \\
\hline \multicolumn{11}{|c|}{ 1.2.1 Functional task training } \\
\hline Green 2002 (1) & 11 & 2.9 & 81 & 10 & 2.9 & 80 & $3.9 \%$ & $0.34[0.03,0.65]$ & {$[-$} & \\
\hline Wade 1992 & 12.2 & 4.3 & 48 & 12.7 & 4.2 & 41 & $3.7 \%$ & $-0.12[-0.53,0.30]$ & 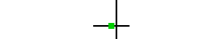 & \\
\hline Subtotal (95\% CI) & & & 129 & & & 121 & $7.6 \%$ & $0.14[-0.31,0.58]$ & & \\
\hline \multicolumn{11}{|c|}{ Heterogeneity: Tau $^{2}=0.07 ;$ Chi $^{2}=3.00, \mathrm{df}=1(\mathrm{P}=0.08) ; \mathrm{I}^{2}=67 \%$} \\
\hline \multicolumn{10}{|c|}{ Test for overall effect: $\mathrm{Z}=0.59(\mathrm{P}=0.55)$} & \\
\hline \multicolumn{11}{|c|}{ 1.2.2 Functional task training + musculoskeletal } \\
\hline Deng 2011 & 55.98 & 12.52 & 50 & 40.64 & 11.64 & 50 & $3.6 \%$ & $1.26[0.83,1.69]$ & - & \\
\hline Hu 2007 haem & 44 & 27 & 178 & 32 & 24 & 174 & $4.1 \%$ & $0.47[0.26,0.68]$ & - & \\
\hline Hu 2007 isch & 47 & 27 & 485 & 37 & 26 & 480 & $4.1 \%$ & $0.38[0.25,0.50]$ & 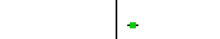 & \\
\hline Liu 2003 & 6.2 & 1.3 & 60 & 3.2 & 2.1 & 60 & $3.7 \%$ & $1.71[1.29,2.13]$ & - & \\
\hline Pan 2004 & 25.62 & 7.33 & 48 & 16.66 & 8.76 & 48 & $3.6 \%$ & $1.10[0.67,1.53]$ & $\rightarrow$ & \\
\hline Wang 2004a & 68.15 & 20.12 & 66 & 58.69 & 19.13 & 32 & $3.7 \%$ & $0.47[0.05,0.90]$ & & \\
\hline Xu 2003a & 23 & 11 & 94 & 18 & 12 & 92 & $3.9 \%$ & $0.43[0.14,0.72]$ & - & \\
\hline Zhao 2002 & 52.4 & 4.21 & 100 & 38.1 & 1.89 & 80 & $3.4 \%$ & $4.21[3.68,4.74]$ & & $\rightarrow$ \\
\hline Zhu 2007 haem (1) & 7.5 & 4 & 12 & 6.5 & 4.25 & 10 & $2.7 \%$ & $0.23[-0.61,1.08]$ & & \\
\hline Zhu 2007 isch (1) & 14.5 & 5 & 28 & 8.5 & 5.5 & 28 & $3.3 \%$ & $1.13[0.56,1.69]$ & & \\
\hline Subtotal (95\% CI) & & & 1121 & & & 1054 & $36.1 \%$ & $1.13[0.61,1.66]$ & & \\
\hline \multicolumn{11}{|c|}{ Heterogeneity: Tau $^{2}=0.66 ; \mathrm{Chi}^{2}=237.13, \mathrm{df}=9(\mathrm{P}<0.00001) ; \mathrm{I}^{2}=96 \%$} \\
\hline \multicolumn{11}{|c|}{ Test for overall effect: $\mathrm{Z}=4.22(\mathrm{P}<0.0001)$} \\
\hline \multicolumn{11}{|c|}{ 1.2.3 Neurophysiological } \\
\hline Subtotal (95\% CI) & & & $\mathbf{0}$ & & & $\mathbf{0}$ & & Not estimable & & \\
\hline \multicolumn{11}{|c|}{ Heterogeneity: Not applicable } \\
\hline \multicolumn{10}{|c|}{ Test for overall effect: Not applicable } & \\
\hline \multicolumn{10}{|c|}{ 1.2.4 Neurophysiological + musculoskeletal } & \\
\hline Fang 2003 & 19.73 & 10.03 & 50 & 18.05 & 9.92 & 78 & $3.8 \%$ & $0.17[-0.19,0.52]$ & - & \\
\hline Subtotal (95\% CI) & & & 50 & & & 78 & $3.8 \%$ & $0.17[-0.19,0.52]$ & & \\
\hline Heterogeneity: Not a & cable & & & & & & & & & \\
\hline Test for overall effec & $=0.92(\mathrm{P}=$ & $.36)$ & & & & & & & & \\
\hline 1.2.5 Functional tra & g + neurop & ysiologic & & & & & & & & \\
\hline Ni 1997 & 26.12 & 6.26 & 34 & 17.12 & 5.7 & 34 & $3.4 \%$ & $1.49[0.95,2.03]$ & $-\square$ & \\
\hline Subtotal (95\% CI) & & & 34 & & & 34 & $3.4 \%$ & $1.49[0.95,2.03]$ & & \\
\hline Heterogeneity: Not a & cable & & & & & & & & & \\
\hline Test for overall effec & $=5.39(\mathrm{P}<$ & .00001) & & & & & & & & \\
\hline 1.2.6 Functional tra & g + neurop & ysiologic & al + musci & uloskeletal & & & & & & \\
\hline Chu 2003 & 21.47 & 5.36 & 30 & 13.29 & 3.85 & 28 & $3.2 \%$ & $1.72[1.11,2.33]$ & & \\
\hline Huang 2003 & 72.12 & 22.34 & 25 & 49.12 & 17.69 & 25 & $3.2 \%$ & $1.12[0.52,1.72]$ & - & \\
\hline Li 1999 & 70.4 & 28.4 & 30 & 41.3 & 28.6 & 31 & $3.4 \%$ & $1.01[0.47,1.54]$ & -- & \\
\hline Wu 2006 & 71.48 & 23.28 & 48 & 59.6 & 26.89 & 48 & $3.7 \%$ & $0.47[0.06,0.87]$ & - & \\
\hline Xu 2003b & 21 & 16 & 92 & 18 & 12 & 88 & $3.9 \%$ & $0.21[-0.08,0.50]$ & 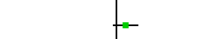 & \\
\hline Xue 2006 & 72 & 28 & 78 & 43 & 28 & 72 & $3.8 \%$ & $1.03[0.69,1.37]$ & - & \\
\hline Yin 2003a (2) & 4.19 & 4.84 & 30 & 2.43 & 5.1 & 14 & $3.1 \%$ & $0.35[-0.29,0.99]$ & - & \\
\hline Yin 2003a (3) & 3.68 & 4.94 & 30 & 2.43 & 5.1 & 15 & $3.2 \%$ & $0.25[-0.38,0.87]$ & - & \\
\hline Zhang 1998 & 64.94 & 20.67 & 29 & 43.49 & 14.57 & 27 & $3.3 \%$ & $1.18[0.60,1.75]$ & $\longrightarrow$ & \\
\hline Zhang 2004 & 92 & 33 & 439 & 67 & 31 & 463 & $4.1 \%$ & $0.78[0.65,0.92]$ & $=$ & \\
\hline Zhu 2001 & 12.82 & 5.31 & 72 & 8.2 & 5 & 53 & $3.8 \%$ & $0.89[0.51,1.26]$ & - & \\
\hline Zhu 2006 & 22.84 & 10.53 & 35 & 19.36 & 10.87 & 35 & $3.6 \%$ & $0.32[-0.15,0.79]$ & - & \\
\hline Subtotal $(95 \%$ CI) & & & 938 & & & 899 & $42.4 \%$ & $0.76[0.54,0.97]$ & $\theta$ & \\
\hline Heterogeneity: Tau² & 9; $\mathrm{Chi}^{2}=3$ & $57, \mathrm{df}=1$ & $11(\mathrm{P}<0.0$ & $001) ; I^{2}=7$ & & & & & & \\
\hline Test for overall effec & $=6.79(\mathrm{P}<$ & .00001) & & & & & & & & \\
\hline 1.2.7 Musculoskelet & & & & & & & & & & \\
\hline Fang 2004 old & 21.13 & 10.17 & 24 & 16.97 & 9.74 & 32 & $3.4 \%$ & $0.41[-0.12,0.95]$ & $L$ & \\
\hline Fang 2004 young & 18.14 & 9.87 & 21 & 18.35 & 10.29 & 23 & $3.3 \%$ & $-0.02[-0.61,0.57]$ & - & \\
\hline Subtotal (95\% CI) & & & 45 & & & 55 & $6.7 \%$ & $0.22[-0.21,0.64]$ & & \\
\hline
\end{tabular}




\section{Analysis 1.2. (Continued)}

40
Heterogeneity: Tau $^{2}=0.01 ; \mathrm{Chi}^{2}=1.14, \mathrm{df}=1(\mathrm{P}=0.29) ; \mathrm{I}^{2}=12 \%$

Test for overall effect: $\mathrm{Z}=1.00(\mathrm{P}=0.32)$
Total (95\% CI)
2317
Heterogeneity: $\mathrm{Tau}^{2}=0.32 ; \mathrm{Chi}^{2}=318.66, \mathrm{df}=27(\mathrm{P}<0.00001) ; \mathrm{I}^{2}=92 \%$
Test for overall effect: $\mathrm{Z}=7.02(\mathrm{P}<0.00001)$
Test for subgroup differences: $\mathrm{Chi}^{2}=29.20, \mathrm{df}=5(\mathrm{P}<0.0001), \mathrm{I}^{2}=82.9 \%$
Footnotes
(1) Standard deviations estimated from range ((max - min range)/4)
(2) Intervention group 2 vs no treatment. Mean and SD computed from categorical data.
(3) Intervention group 1 vs no treatment. Mean and SD computed from categorical data.

$2241 \quad 100.0 \%$

$0.81[0.58,1.04]$

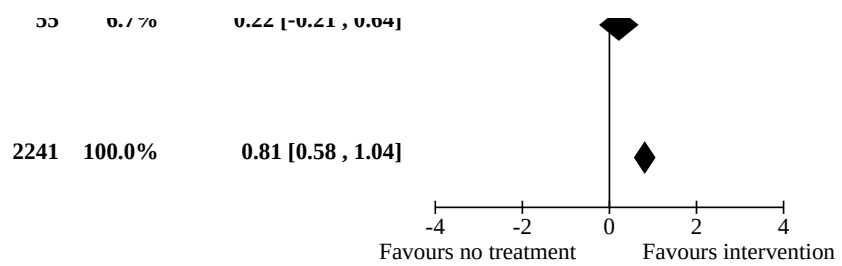

Analysis 1.3. Comparison 1: Intervention versus no treatment:
immediate outcomes, Outcome 3: Balance (Berg Balance Scale)

\begin{tabular}{|c|c|c|c|c|c|c|c|c|c|}
\hline \multirow{2}{*}{\multicolumn{4}{|c|}{$\begin{array}{c}\text { Intervention } \\
\end{array}$}} & \multicolumn{3}{|c|}{ No treatment } & \multirow{2}{*}{\multicolumn{2}{|c|}{$\begin{array}{l}\text { Std. Mean Difference } \\
\text { IV, Random, } 95 \% \text { CI }\end{array}$}} & \multirow{2}{*}{$\begin{array}{l}\text { Std. Mean Difference } \\
\text { IV, Random, } 95 \% \text { CI }\end{array}$} \\
\hline Study or Subgroup & & SD & Total & Mean & SD & Total & & & \\
\hline
\end{tabular}

1.3.1 Functional task training + musculoskeletal

$\begin{array}{llllllllll}\text { Holmgren } 2006(1) & 45.2 & 8.8483 & 15 & 45.5 & 7.4691 & 19 & 100.0 \% & -0.04[-0.71,0.64]\end{array}$

$\begin{array}{lllll}\text { Subtotal }(95 \% \mathrm{CI}) & 15 & 19 & \mathbf{1 0 0 . 0} \% & \mathbf{- 0 . 0 4}[-0.71,0.64]\end{array}$

Heterogeneity: Not applicable

Test for overall effect: $\mathrm{Z}=0.10(\mathrm{P}=0.92)$

1.3.2 Functional task training

Subtotal (95\% CI)

Not estimable

Heterogeneity: Not applicable

Test for overall effect: Not applicable

1.3.3 Neurophysiological

Subtotal (95\% CI)

0

$\mathbf{0}$

Not estimable

Heterogeneity: Not applicable

Test for overall effect: Not applicable

1.3.4 Neurophysiological + musculoskeletal

Subtotal (95\% CI)

Heterogeneity: Not applicable

Test for overall effect: Not applicable

1.3.5 Functional training + neurophysiological

Subtotal (95\% CI)

Not estimable

Heterogeneity: Not applicable

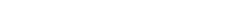

Test for overall effect: Not applicable

1.3.6 Functional training + neurophysiological + musculoskeletal

Subtotal (95\% CI)

Not estimable

Heterogeneity: Not applicable

Test for overall effect: Not applicable

1.3.7 Musculoskeletal

Subtotal (95\% CI)

Heterogeneity: Not applicable

Test for overall effect: Not applicable

Total (95\% CI)

Heterogeneity: Not applicable

Test for overall effect: $\mathrm{Z}=0.10(\mathrm{P}=0.92)$

Test for subgroup differences: Not applicable

Footnotes

(1) SD calcuated from CI and p-value.
IV, Random, 95\% CI 
Analysis 1.4. Comparison 1: Intervention versus no treatment: immediate outcomes, Outcome 4: Gait velocity

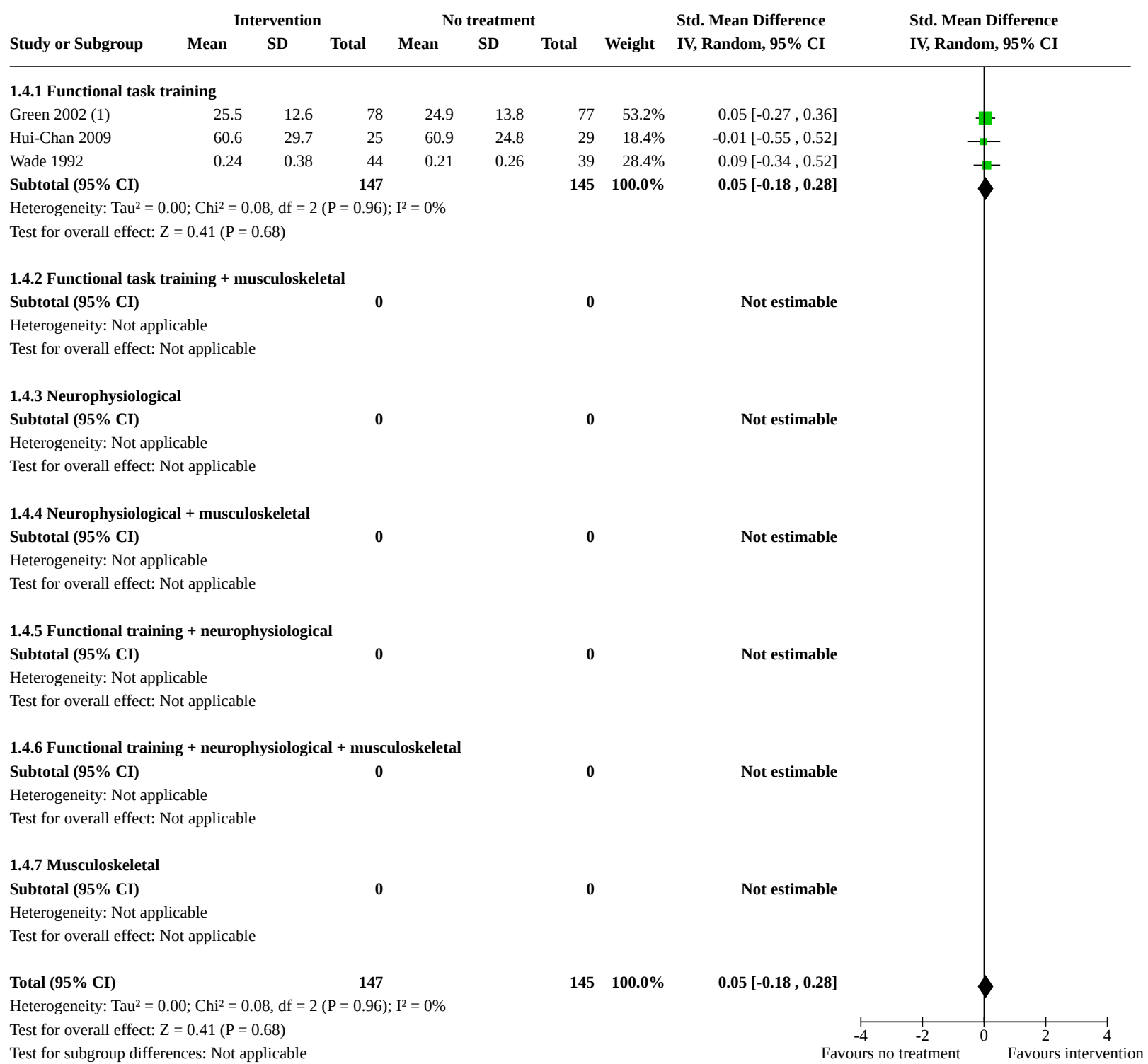

Footnotes

(1) Standard deviations estimated from range ((max - min range)/4) 
Analysis 1.5. Comparison 1: Intervention versus no treatment: immediate outcomes, Outcome 5: Length of stay

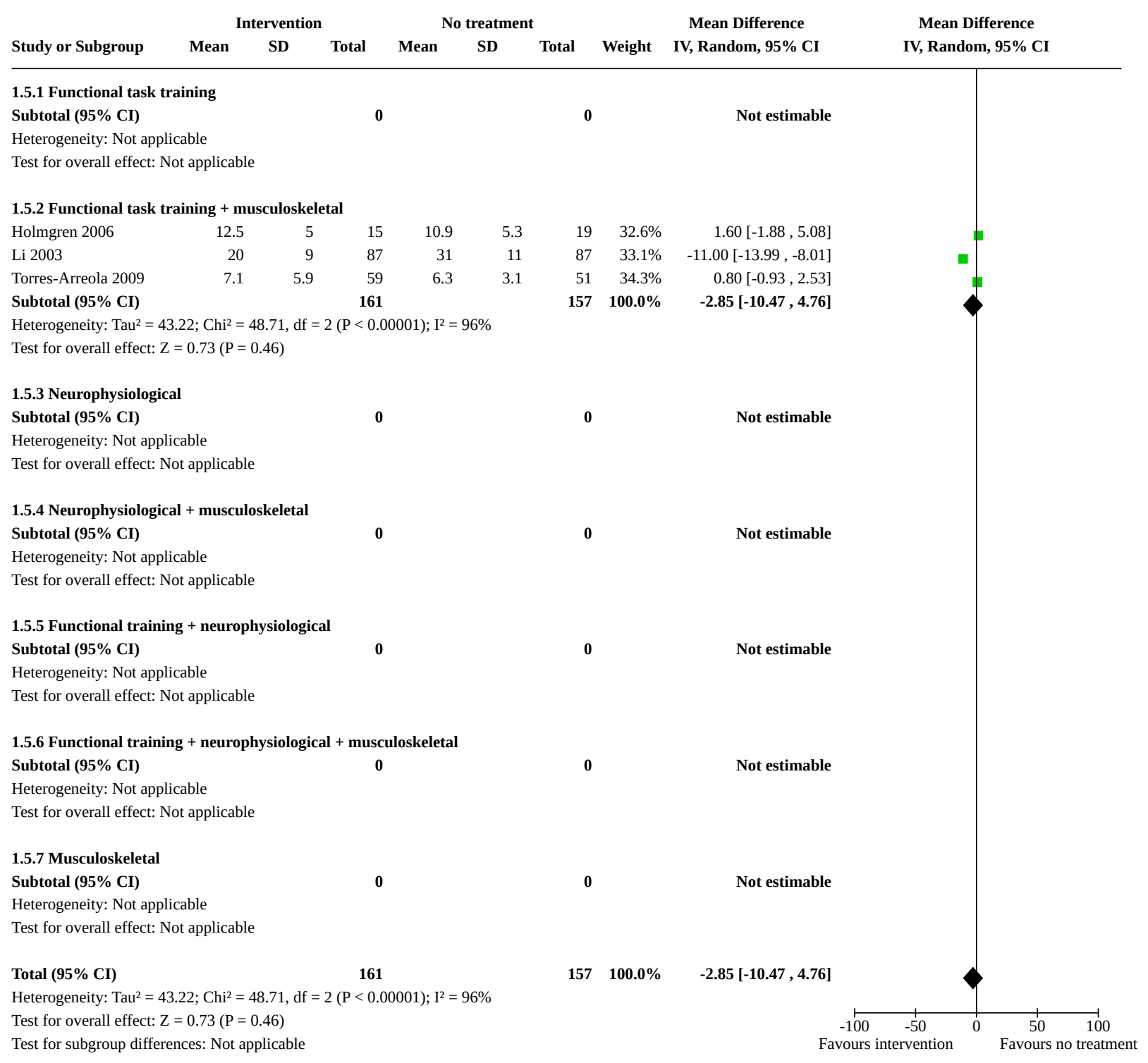

Comparison 2. Intervention versus usual care or attention control: immediate outcomes

\begin{tabular}{|c|c|c|c|c|}
\hline Outcome or subgroup title & No. of studies & $\begin{array}{l}\text { No. of partici- } \\
\text { pants }\end{array}$ & Statistical method & Effect size \\
\hline 2.1 Independence in ADL scales & 6 & 260 & $\begin{array}{l}\text { Std. Mean Difference (IV, Random, } \\
95 \% \mathrm{Cl})\end{array}$ & $0.04[-0.27,0.35]$ \\
\hline 2.1.1 Functional task training & 2 & 31 & $\begin{array}{l}\text { Std. Mean Difference (IV, Random, } \\
95 \% \mathrm{Cl} \text { ) }\end{array}$ & $-0.39[-1.16,0.38]$ \\
\hline $\begin{array}{l}\text { 2.1.2 Functional task training + } \\
\text { musculoskeletal }\end{array}$ & 3 & 184 & $\begin{array}{l}\text { Std. Mean Difference (IV, Random, } \\
95 \% \mathrm{Cl} \text { ) }\end{array}$ & $0.15[-0.41,0.71]$ \\
\hline 2.1.3 Neurophysiological & 2 & 25 & $\begin{array}{l}\text { Std. Mean Difference (IV, Random, } \\
95 \% \mathrm{CI} \text { ) }\end{array}$ & $-0.22[-1.04,0.61]$ \\
\hline
\end{tabular}




\begin{tabular}{|c|c|c|c|c|}
\hline Outcome or subgroup title & No. of studies & $\begin{array}{l}\text { No. of partici- } \\
\text { pants }\end{array}$ & Statistical method & Effect size \\
\hline $\begin{array}{l}\text { 2.1.4 Neurophysiological + mus- } \\
\text { culoskeletal }\end{array}$ & 0 & 0 & $\begin{array}{l}\text { Std. Mean Difference (IV, Random, } \\
95 \% \mathrm{CI})\end{array}$ & Not estimable \\
\hline $\begin{array}{l}2.1 .5 \text { Functional training + neu- } \\
\text { rophysiological }\end{array}$ & 0 & 0 & $\begin{array}{l}\text { Std. Mean Difference (IV, Random, } \\
95 \% \mathrm{CI} \text { ) }\end{array}$ & Not estimable \\
\hline $\begin{array}{l}\text { 2.1.6 Functional training + neu- } \\
\text { rophysiological + musculoskele- } \\
\text { tal }\end{array}$ & 1 & 20 & $\begin{array}{l}\text { Std. Mean Difference (IV, Random, } \\
95 \% \mathrm{CI} \text { ) }\end{array}$ & $0.08[-0.80,0.96]$ \\
\hline 2.1.7 Musculoskeletal & 0 & 0 & $\begin{array}{l}\text { Std. Mean Difference (IV, Random, } \\
95 \% \mathrm{CI})\end{array}$ & Not estimable \\
\hline 2.2 Motor function scales & 13 & 967 & $\begin{array}{l}\text { Std. Mean Difference (IV, Random, } \\
95 \% \mathrm{CI})\end{array}$ & $0.42[0.24,0.61]$ \\
\hline 2.2.1 Functional task training & 1 & 21 & $\begin{array}{l}\text { Std. Mean Difference (IV, Random, } \\
95 \% \mathrm{CI})\end{array}$ & $-0.34[-1.21,0.53]$ \\
\hline $\begin{array}{l}\text { 2.2.2 Functional task training + } \\
\text { musculoskeletal }\end{array}$ & 5 & 483 & $\begin{array}{l}\text { Std. Mean Difference (IV, Random, } \\
95 \% \mathrm{CI} \text { ) }\end{array}$ & $0.24[-0.01,0.50]$ \\
\hline 2.2.3 Neurophysiological & 2 & 90 & $\begin{array}{l}\text { Std. Mean Difference (IV, Random, } \\
95 \% \mathrm{CI})\end{array}$ & $0.86[0.42,1.29]$ \\
\hline $\begin{array}{l}\text { 2.2.4 Neurophysiological + mus- } \\
\text { culoskeletal }\end{array}$ & 1 & 50 & $\begin{array}{l}\text { Std. Mean Difference (IV, Random, } \\
95 \% \mathrm{CI})\end{array}$ & $0.65[0.08,1.22]$ \\
\hline $\begin{array}{l}2.2 .5 \text { Functional training + neu- } \\
\text { rophysiological }\end{array}$ & 1 & 42 & $\begin{array}{l}\text { Std. Mean Difference (IV, Random, } \\
95 \% \mathrm{CI})\end{array}$ & $1.09[0.43,1.74]$ \\
\hline $\begin{array}{l}\text { 2.2.6 Functional training + neu- } \\
\text { rophysiological + musculoskele- } \\
\text { tal }\end{array}$ & 4 & 281 & $\begin{array}{l}\text { Std. Mean Difference (IV, Random, } \\
95 \% \mathrm{CI})\end{array}$ & $0.46[0.21,0.70]$ \\
\hline 2.2.7 Musculoskeletal & 0 & 0 & $\begin{array}{l}\text { Std. Mean Difference (IV, Random, } \\
95 \% \mathrm{CI})\end{array}$ & Not estimable \\
\hline $\begin{array}{l}2.3 \text { Balance (Berg Balance } \\
\text { Scale) }\end{array}$ & 5 & 246 & $\begin{array}{l}\text { Std. Mean Difference (IV, Random, } \\
95 \% \mathrm{CI})\end{array}$ & $0.31[0.05,0.56]$ \\
\hline 2.3.1 Functional task training & 0 & 0 & $\begin{array}{l}\text { Std. Mean Difference (IV, Random, } \\
95 \% \mathrm{CI})\end{array}$ & Not estimable \\
\hline $\begin{array}{l}\text { 2.3.2 Functional task training + } \\
\text { musculoskeletal }\end{array}$ & 3 & 124 & $\begin{array}{l}\text { Std. Mean Difference (IV, Random, } \\
95 \% \mathrm{CI})\end{array}$ & $0.31[-0.04,0.67]$ \\
\hline 2.3.3 Neurophysiological & 1 & 10 & $\begin{array}{l}\text { Std. Mean Difference (IV, Random, } \\
95 \% \mathrm{CI})\end{array}$ & $0.60[-0.71,1.91]$ \\
\hline $\begin{array}{l}\text { 2.3.4 Neurophysiological + mus- } \\
\text { culoskeletal }\end{array}$ & 0 & 0 & $\begin{array}{l}\text { Std. Mean Difference (IV, Random, } \\
95 \% \mathrm{CI})\end{array}$ & Not estimable \\
\hline $\begin{array}{l}\text { 2.3.5 Functional training + neu- } \\
\text { rophysiological }\end{array}$ & 0 & 0 & $\begin{array}{l}\text { Std. Mean Difference (IV, Random, } \\
95 \% \mathrm{CI})\end{array}$ & Not estimable \\
\hline
\end{tabular}




\begin{tabular}{|c|c|c|c|c|}
\hline Outcome or subgroup title & No. of studies & $\begin{array}{l}\text { No. of partici- } \\
\text { pants }\end{array}$ & Statistical method & Effect size \\
\hline $\begin{array}{l}\text { 2.3.6 Functional training + neu- } \\
\text { rophysiological + musculoskele- } \\
\text { tal }\end{array}$ & 2 & 112 & $\begin{array}{l}\text { Std. Mean Difference (IV, Random, } \\
95 \% \mathrm{CI})\end{array}$ & $0.28[-0.10,0.65]$ \\
\hline 2.3.7 Musculoskeletal & 0 & 0 & $\begin{array}{l}\text { Std. Mean Difference (IV, Random, } \\
95 \% \mathrm{CI} \text { ) }\end{array}$ & Not estimable \\
\hline 2.4 Gait velocity & 14 & 1126 & $\begin{array}{l}\text { Std. Mean Difference (IV, Random, } \\
95 \% \mathrm{CI})\end{array}$ & $0.46[0.32,0.60]$ \\
\hline 2.4.1 Functional task training & 2 & 30 & $\begin{array}{l}\text { Std. Mean Difference (IV, Random, } \\
95 \% \mathrm{CI} \text { ) }\end{array}$ & $0.68[-0.51,1.86]$ \\
\hline $\begin{array}{l}\text { 2.4.2 Functional task training + } \\
\text { musculoskeletal }\end{array}$ & 9 & 865 & $\begin{array}{l}\text { Std. Mean Difference (IV, Random, } \\
95 \% \mathrm{CI})\end{array}$ & $0.45[0.25,0.65]$ \\
\hline 2.4.3 Neurophysiological & 1 & 10 & $\begin{array}{l}\text { Std. Mean Difference (IV, Random, } \\
95 \% \mathrm{CI} \text { ) }\end{array}$ & $-0.06[-1.32,1.21]$ \\
\hline $\begin{array}{l}\text { 2.4.4 Neurophysiological + mus- } \\
\text { culoskeletal }\end{array}$ & 0 & 0 & $\begin{array}{l}\text { Std. Mean Difference (IV, Random, } \\
95 \% \mathrm{CI})\end{array}$ & Not estimable \\
\hline $\begin{array}{l}\text { 2.4.5 Functional training + neu- } \\
\text { rophysiological }\end{array}$ & 0 & 0 & $\begin{array}{l}\text { Std. Mean Difference (IV, Random, } \\
95 \% \mathrm{CI})\end{array}$ & Not estimable \\
\hline $\begin{array}{l}\text { 2.4.6 Functional training + neu- } \\
\text { rophysiological + musculoskele- } \\
\text { tal }\end{array}$ & 3 & 221 & $\begin{array}{l}\text { Std. Mean Difference (IV, Random, } \\
95 \% \mathrm{CI})\end{array}$ & $0.45[0.17,0.72]$ \\
\hline 2.4.7 Musculoskeletal & 0 & 0 & $\begin{array}{l}\text { Std. Mean Difference (IV, Random, } \\
95 \% \mathrm{CI})\end{array}$ & Not estimable \\
\hline 2.5 Length of stay & 2 & 105 & $\begin{array}{l}\text { Mean Difference (IV, Random, 95\% } \\
\mathrm{CI} \text { ) }\end{array}$ & $\begin{array}{l}-10.36[-48.09 \\
27.36]\end{array}$ \\
\hline 2.5.1 Functional task training & 0 & 0 & $\begin{array}{l}\text { Mean Difference (IV, Random, 95\% } \\
\mathrm{CI})\end{array}$ & Not estimable \\
\hline $\begin{array}{l}\text { 2.5.2 Functional task training + } \\
\text { musculoskeletal }\end{array}$ & 2 & 105 & $\begin{array}{l}\text { Mean Difference (IV, Random, 95\% } \\
\mathrm{CI})\end{array}$ & $\begin{array}{l}-10.36[-48.09 \\
27.36]\end{array}$ \\
\hline 2.5.3 Neurophysiological & 0 & 0 & $\begin{array}{l}\text { Mean Difference (IV, Random, 95\% } \\
\mathrm{Cl} \text { ) }\end{array}$ & Not estimable \\
\hline $\begin{array}{l}\text { 2.5.4 Neurophysiological + mus- } \\
\text { culoskeletal }\end{array}$ & 0 & 0 & $\begin{array}{l}\text { Mean Difference (IV, Random, 95\% } \\
\mathrm{Cl} \text { ) }\end{array}$ & Not estimable \\
\hline $\begin{array}{l}\text { 2.5.5 Functional training + neu- } \\
\text { rophysiological }\end{array}$ & 0 & 0 & $\begin{array}{l}\text { Mean Difference (IV, Random, 95\% } \\
\mathrm{Cl} \text { ) }\end{array}$ & Not estimable \\
\hline $\begin{array}{l}\text { 2.5.6 Functional training + neu- } \\
\text { rophysiological + musculoskele- } \\
\text { tal }\end{array}$ & 0 & 0 & $\begin{array}{l}\text { Mean Difference (IV, Random, 95\% } \\
\mathrm{CI} \text { ) }\end{array}$ & Not estimable \\
\hline
\end{tabular}




\begin{tabular}{lllll}
\hline Outcome or subgroup title & No. of studies & $\begin{array}{l}\text { No. of partici- } \\
\text { pants }\end{array}$ & Statistical method & Effect size \\
\hline 2.5.7 Musculoskeletal & 0 & 0 & $\begin{array}{l}\text { Mean Difference (IV, Random, 95\% } \\
\text { Cl) }\end{array}$ & Not estimable \\
\hline
\end{tabular}




\section{Analysis 2.1. Comparison 2: Intervention versus usual care or attention control: immediate outcomes, Outcome 1: Independence in ADL scales}

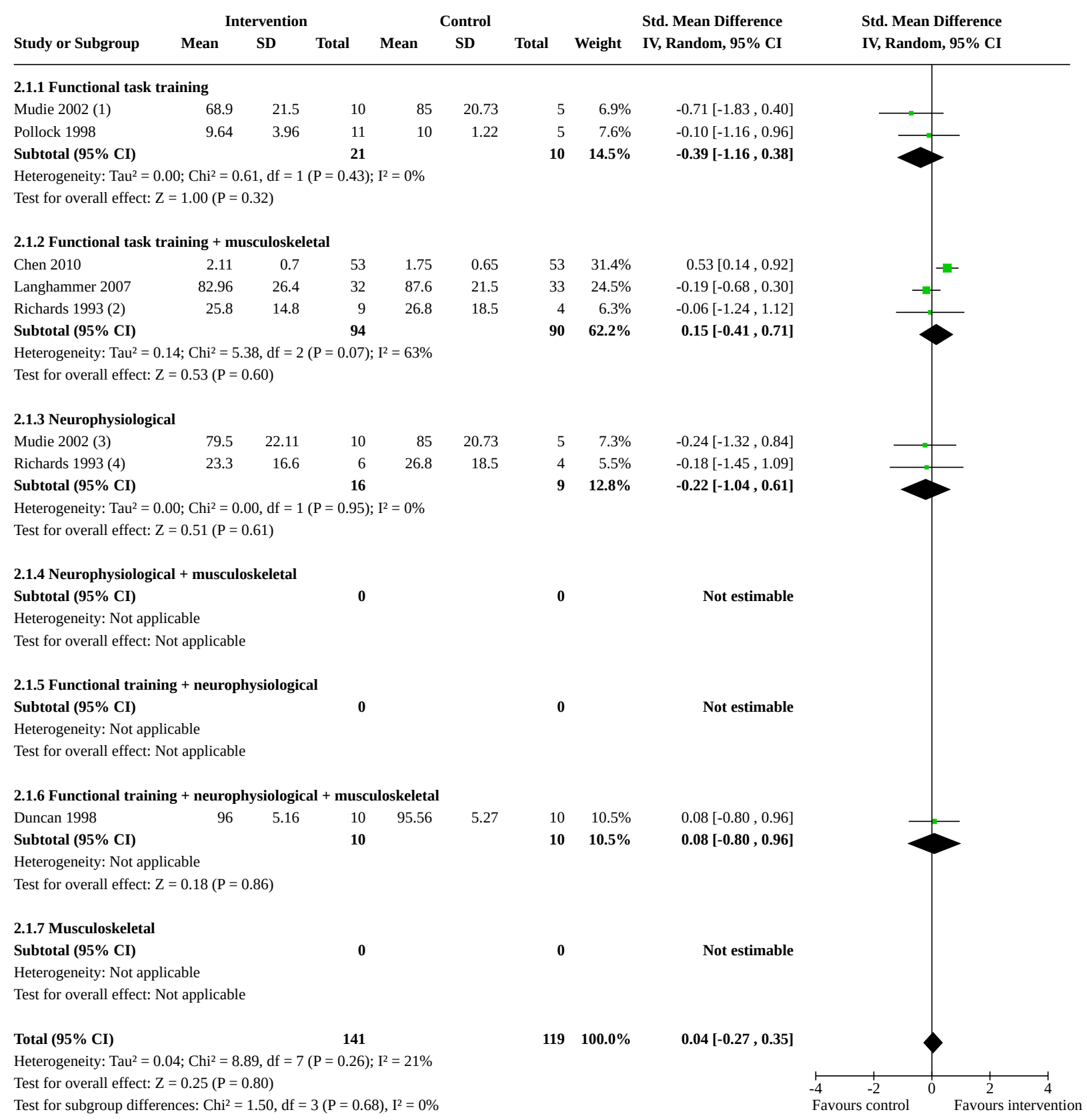

\section{Footnotes}

(1) Intervention group 1 vs usual care. Control group shared.

(2) Intervention group 2 vs usual care.

(3) Intervention group 2 vs usual care. Control group shared.

(4) Intervention group 1 vs usual care. 


\section{Analysis 2.2. Comparison 2: Intervention versus usual care or attention control: immediate outcomes, Outcome 2: Motor function scales}

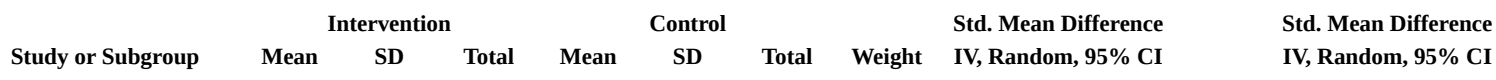

$\begin{array}{lllllllll}\text { 2.2.1 Functional task training } & & & & & & & & \\ \text { McClellan } 2004 & 4.3 & 1.2 & 12 & 4.7 & 1 & 9 & 3.6 \% & -0.34[-1.21,0.53] \\ \text { Subtotal (95\% CI) } & & & \mathbf{1 2} & & & \mathbf{9} & \mathbf{3 . 6 \%} & \mathbf{- 0 . 3 4}[-\mathbf{1 . 2 1}, \mathbf{0 . 5 3}]\end{array}$

Heterogeneity: Not applicable

Test for overall effect: $\mathrm{Z}=0.77(\mathrm{P}=0.44)$

2.2.2 Functional task training + musculoskeletal

$\begin{array}{lrrrr}\text { Chen 2010 } & 2.57 & 0.6 & 53 & 2.23 \\ \text { Kwakkel 2008 } & 13.47 & 1.44 & 125 & 12.82 \\ \text { Langhammer 2007 } & 36.4 & 13.9 & 32 & 38.9 \\ \text { Mudge 2009 (1) } & 14 & 1.5 & 31 & 14 \\ \text { Richards 1993 (2) } & 23.7 & 6.7 & 9 & 20 \\ \text { Subtotal (95\% CI) } & & & \mathbf{2 5 0} & \end{array}$

Heterogeneity: $\mathrm{Tau}^{2}=0.03 ; \mathrm{Chi}^{2}=6.39, \mathrm{df}=4(\mathrm{P}=0.17) ; \mathrm{I}^{2}=37 \%$

Test for overall effect: $\mathrm{Z}=1.86(\mathrm{P}=0.06)$

\subsubsection{Neurophysiological}

Wei 1998

22.7

$\begin{array}{rrrr}22.7 & 9.2 & 6 & 20 \\ 19.13 & 4.86 & 40 & 14.5\end{array}$

Subtotal (95\% CI)

46

Heterogeneity: $\mathrm{Tau}^{2}=0.00 ; \mathrm{Chi}^{2}=1.00, \mathrm{df}=1(\mathrm{P}=0.32) ; \mathrm{I}^{2}=0 \%$

Test for overall effect: $\mathrm{Z}=3.87(\mathrm{P}=0.0001)$

2.2.4 Neurophysiological + musculoskeletal

Wang 2004b $\quad 65.15 \quad 19.1$

Subtotal (95\% CI)

$\begin{array}{lll}65.15 & 19.1 & 25\end{array}$

$25 \quad 52.93$

17.8

$25 \quad 6.6 \%$

$0.65[0.08,1.22]$

Heterogeneity: Not applicable

Test for overall effect: $\mathrm{Z}=2.24(\mathrm{P}=0.03)$

2.2.5 Functional training + neurophysiological

Subtotal (95\% CI)

$24.14 \quad 8.35$

23
23

15.86

$\begin{array}{rrr}0.78 & 53 & 10.0 \% \\ 1.9 & 117 & 13.4 \% \\ 12.7 & 32 & 7.9 \% \\ 1.25 & 27 & 7.5 \% \\ 10.7 & 4 & 2.1 \% \\ & \mathbf{2 3 3} & \mathbf{4 0 . 9} \%\end{array}$

$0.49[0.10,0.87]$

$0.39[0.13,0.64]$

$-0.19[-0.68,0.31]$

$0.00[-0.52,0.52]$

$0.43[-0.76,1.63]$

$0.24[-0.01,0.50]$

Heterogeneity: Not applicable

Test for overall effect: $\mathrm{Z}=3.25(\mathrm{P}=0.001)$

2.2.6 Functional training + neurophysiological + musculoskeletal

$\begin{array}{llll}\text { Cooke } 2006(4) & 37.7 & 8.6 & 36\end{array}$

$\begin{array}{lllll}\text { Cooke } 2006(2) & 36.6 & 10.4 & 31 & 34.6\end{array}$

$\begin{array}{lllll}\text { Duncan } 1998 & 26.1 & 2.51 & 10 & 22.6\end{array}$

$\begin{array}{lllll}\text { Duncan } 2003 & 26.84 & 3.7 & 44 & 25.46\end{array}$

$\begin{array}{lllll}\text { Tang } 2009 & 69.51 & 10.93 & 35 & 61.53\end{array}$

Subtotal (95\% CI)

156

Heterogeneity: $\mathrm{Tau}^{2}=0.00 ; \mathrm{Chi}^{2}=2.88, \mathrm{df}=4(\mathrm{P}=0.58) ; \mathrm{I}^{2}=0 \%$

Test for overall effect: $\mathrm{Z}=3.67(\mathrm{P}=0.0002)$

\subsubsection{Musculoskeletal}

Subtotal (95\% CI)

$\mathbf{0}$

Heterogeneity: Not applicable

Test for overall effect: Not applicable

Total (95\% CI)

512

Heterogeneity: $\mathrm{Tau}^{2}=0.05 ; \mathrm{Chi}^{2}=23.98, \mathrm{df}=14(\mathrm{P}=0.05) ; \mathrm{I}^{2}=42 \%$

Test for overall effect: $\mathrm{Z}=4.48(\mathrm{P}<0.00001)$

Test for subgroup differences: $\mathrm{Chi}^{2}=13.21, \mathrm{df}=5(\mathrm{P}=0.02), \mathrm{I}^{2}=62.2 \%$

$10.8 \quad 16 \quad 6.3 \%$

$10.8 \quad 16 \quad 6.1 \%$

$\begin{array}{lll}4.7 & 10 & 3.2 \%\end{array}$

$3.5 \quad 48 \quad 9.4 \%$

$11.62 \quad 35 \quad 8.0 \%$

$12533.1 \%$
$0.25[-1.02,1.52]$

$0.94[0.48,1.40]$

$0.86[0.42,1.29]$

\section{Footnotes}

(1) Standard deviation estimated from range ((max-min range)/4)

(2) Intervention group 1 vs usual care. Control participants shared.

(3) Intervention group 2 vs usual care. Control participants shared.

(4) Intervention 2 group vs usual care. Control participants shared.

$0.33[-0.26,0.92]$ $0.19[-0.42,0.79]$ $0.89[-0.04,1.82]$ $0.38[-0.03,0.79]$ $0.70[0.22,1.18]$ $0.46[0.21,0.70]$

Not estimable

$\mathbf{0}$

$455 \quad 100.0 \%$

$0.42[0.24,0.61]$

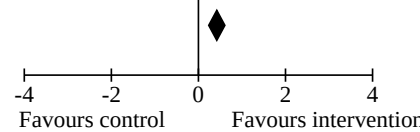




\section{Analysis 2.3. Comparison 2: Intervention versus usual care or attention control: immediate outcomes, Outcome 3: Balance (Berg Balance Scale)}

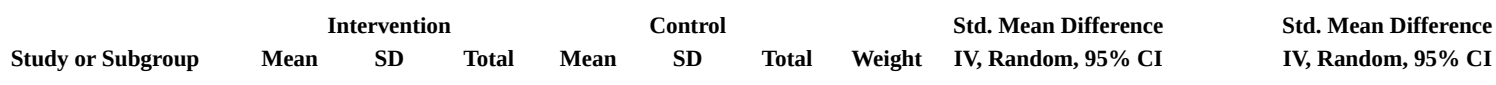

2.3.1 Functional task training

Subtotal (95\% CI)

Heterogeneity: Not applicable

Test for overall effect: Not applicable

2.3.2 Functional task training + musculoskeletal

Kim 2012

Richards 1993 (1)

Salbach 2004

$\begin{array}{rr}50.1 & 4.12 \\ 33.2 & 18.2 \\ 44 & 11\end{array}$

Subtotal (95\% CI)

Heterogeneity: $\mathrm{Tau}^{2}=0.00 ; \mathrm{Chi}^{2}=0.74, \mathrm{df}=2(\mathrm{P}=0.69) ; \mathrm{I}^{2}=0 \%$

Test for overall effect: $\mathrm{Z}=1.71(\mathrm{P}=0.09)$

2.3.3 Neurophysiological

Richards 1993 (2)

40

Subtotal (95\% CI)

$40 \quad 16.1$

6

28.4

19.7

$4 \quad 3.7 \%$

$4 \quad 3.7 \%$

$0.60[-0.71,1.91]$

$0.60[-0.71,1.91]$

(

Test for overall effect: $\mathrm{Z}=0.89(\mathrm{P}=0.37)$

2.3.4 Neurophysiological + musculoskeletal

Subtotal (95\% CI)

0

Heterogeneity: Not applicable

Test for overall effect: Not applicable

2.3.5 Functional training + neurophysiological

Subtotal (95\% CI)

Heterogeneity: Not applicable

Test for overall effect: Not applicable

2.3.6 Functional training + neurophysiological + musculoskeletal

$\begin{array}{lllll}\text { Duncan } 1998 & 46.9 & 3.63 & 10 & 45.8\end{array}$

$\begin{array}{lllll}\text { Duncan } 2003 & 47.16 & 7.2 & 44 & 44.8\end{array}$

Subtotal (95\% CI)

54

$44.8-5.39$

Heterogeneity: $\mathrm{Tau}^{2}=0.00 ; \mathrm{Chi}^{2}=0.01, \mathrm{df}=1(\mathrm{P}=0.91) ; \mathrm{I}^{2}=0 \%$

Test for overall effect: $\mathrm{Z}=1.45(\mathrm{P}=0.15)$

\subsubsection{Musculoskeletal}

Subtotal (95\% CI)

$\mathbf{0}$

Heterogeneity: Not applicable

Test for overall effect: Not applicable

Total $(95 \%$ CI)

123

Heterogeneity: $\mathrm{Tau}^{2}=0.00 ; \mathrm{Chi}^{2}=0.97, \mathrm{df}=5(\mathrm{P}=0.96) ; \mathrm{I}^{2}=0 \%$

Test for overall effect: $\mathrm{Z}=2.37(\mathrm{P}=0.02)$

Test for subgroup differences: $\mathrm{Chi}^{2}=0.22, \mathrm{df}=2(\mathrm{P}=0.90), \mathrm{I}^{2}=0 \%$

Not estimable

Not estimable

$0.23[-0.65,1.11]$

$0.29[-0.13,0.70]$

$0.28[-0.10,0.65]$

$48 \quad 37.9 \%$

Not estimable

$\mathbf{0}$

$123 \quad 100.0 \%$

$0.31[0.05,0.56]$

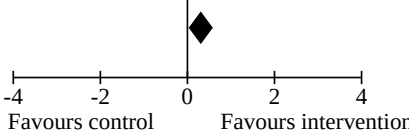

Footnotes

(1) Intervention group 1 vs usual care. Control participants shared.

(2) Intervention group 2 vs usual care. Control participants shared. 
Analysis 2.4. Comparison 2: Intervention versus usual care or attention control: immediate outcomes, Outcome 4: Gait velocity

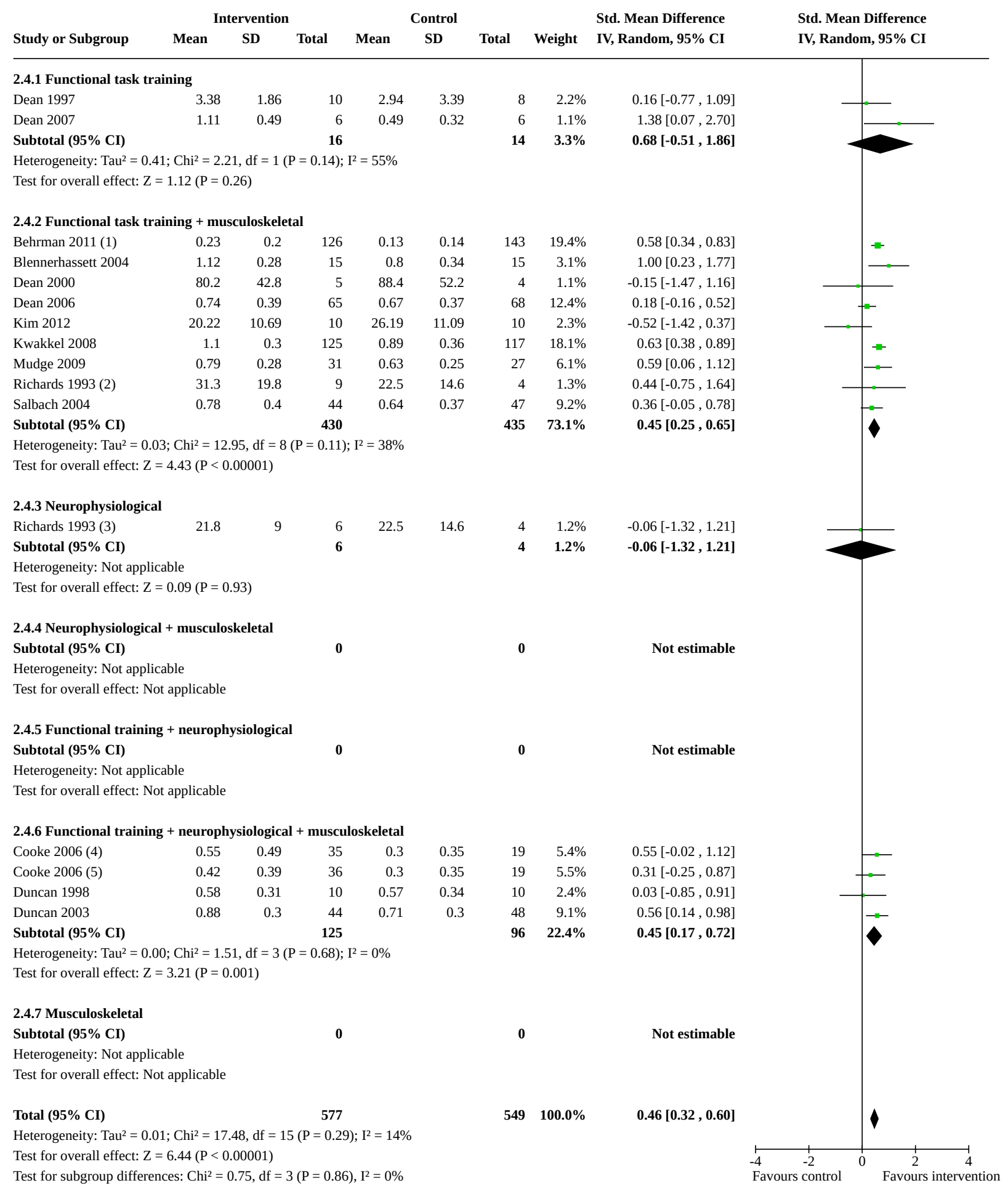

Footnotes

(1) Data entered are "walking speed increases"

(2) Intervention group 1 vs usual care. Control participants shared.

(3) Intervention group 2 vs usual care. Control participants shared.

(4) Intervention 1 vs usual care. Control participants shared.

(5) Intervention 2 vs usual care. Control participants shared. 


\section{Analysis 2.4. (Continued)}

(4) Intervention 1 vs usual care. Control participants shared.

(5) Intervention 2 vs usual care. Control participants shared.

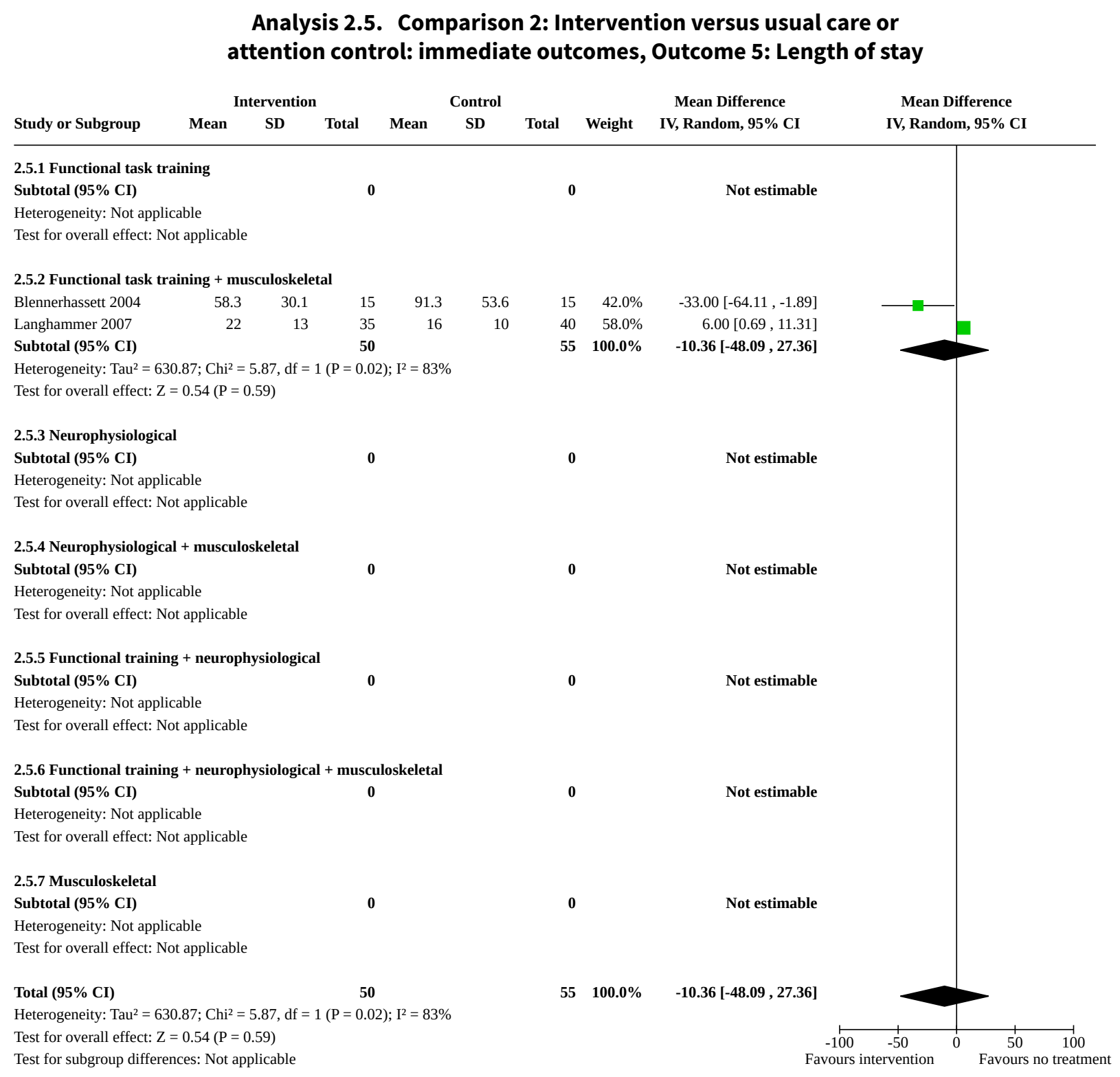

Comparison 3. One active intervention versus another active intervention: immediate outcomes

\begin{tabular}{lllll}
\hline Outcome or subgroup title & No. of studies & $\begin{array}{l}\text { No. of partici- } \\
\text { pants }\end{array}$ & Statistical method & Effect size \\
\hline 3.1 Independence in ADL scales & 7 & & $\begin{array}{l}\text { Std. Mean Difference (IV, } \\
\text { Random, 95\% Cl) }\end{array}$ & Subtotals only \\
\hline
\end{tabular}




\begin{tabular}{|c|c|c|c|c|}
\hline Outcome or subgroup title & No. of studies & $\begin{array}{l}\text { No. of partici- } \\
\text { pants }\end{array}$ & Statistical method & Effect size \\
\hline $\begin{array}{l}\text { 3.1.1 Includes functional training versus } \\
\text { does not include functional training }\end{array}$ & 4 & 186 & $\begin{array}{l}\text { Std. Mean Difference (IV, } \\
\text { Random, } 95 \% \mathrm{CI} \text { ) }\end{array}$ & $-0.03[-0.37,0.32]$ \\
\hline $\begin{array}{l}3.1 .2 \text { Includes neurophysiological versus } \\
\text { does not include neurophysiological }\end{array}$ & 7 & 451 & $\begin{array}{l}\text { Std. Mean Difference (IV, } \\
\text { Random, } 95 \% \mathrm{CI} \text { ) }\end{array}$ & $-0.02[-0.26,0.22]$ \\
\hline $\begin{array}{l}\text { 3.1.3 Includes musculoskeletal versus } \\
\text { does not include musculoskeletal }\end{array}$ & 3 & 103 & $\begin{array}{l}\text { Std. Mean Difference (IV, } \\
\text { Random, } 95 \% \mathrm{CI} \text { ) }\end{array}$ & $-0.12[-0.58,0.34]$ \\
\hline 3.2 Motor function scales & 8 & & $\begin{array}{l}\text { Std. Mean Difference (IV, } \\
\text { Random, } 95 \% \mathrm{CI} \text { ) }\end{array}$ & Subtotals only \\
\hline $\begin{array}{l}\text { 3.2.1 Includes functional training versus } \\
\text { does not include functional training }\end{array}$ & 4 & 188 & $\begin{array}{l}\text { Std. Mean Difference (IV, } \\
\text { Random, } 95 \% \mathrm{CI} \text { ) }\end{array}$ & $-0.16[-0.59,0.28]$ \\
\hline $\begin{array}{l}\text { 3.2.2 Includes neurophysiological versus } \\
\text { does not include neurophysiological }\end{array}$ & 8 & 506 & $\begin{array}{l}\text { Std. Mean Difference (IV, } \\
\text { Random, } 95 \% \mathrm{CI} \text { ) }\end{array}$ & $0.17[-0.05,0.39]$ \\
\hline $\begin{array}{l}\text { 3.2.3 Includes musculoskeletal versus } \\
\text { does not include musculoskeletal }\end{array}$ & 4 & 81 & $\begin{array}{l}\text { Std. Mean Difference (IV, } \\
\text { Random, } 95 \% \mathrm{CI} \text { ) }\end{array}$ & $-0.08[-0.53,0.36]$ \\
\hline 3.3 Balance (Berg Balance Scale) & 4 & & $\begin{array}{l}\text { Std. Mean Difference (IV, } \\
\text { Random, } 95 \% \mathrm{CI} \text { ) }\end{array}$ & Subtotals only \\
\hline $\begin{array}{l}\text { 3.3.1 Includes functional training versus } \\
\text { does not include functional training }\end{array}$ & 2 & 36 & $\begin{array}{l}\text { Std. Mean Difference (IV, } \\
\text { Random, } 95 \% \mathrm{CI} \text { ) }\end{array}$ & $-0.16[-0.82,0.51]$ \\
\hline $\begin{array}{l}\text { 3.3.2 Includes neurophysiological versus } \\
\text { does not include neurophysiological }\end{array}$ & 4 & 83 & $\begin{array}{l}\text { Std. Mean Difference (IV, } \\
\text { Random, } 95 \% \mathrm{CI} \text { ) }\end{array}$ & $-0.01[-0.44,0.43]$ \\
\hline $\begin{array}{l}\text { 3.3.3 Includes musculoskeletal versus } \\
\text { does not include musculoskeletal }\end{array}$ & 2 & 36 & $\begin{array}{l}\text { Std. Mean Difference (IV, } \\
\text { Random, } 95 \% \mathrm{CI} \text { ) }\end{array}$ & $0.14[-0.52,0.80]$ \\
\hline 3.4 Gait velocity & 7 & & $\begin{array}{l}\text { Std. Mean Difference (IV, } \\
\text { Random, } 95 \% \mathrm{CI} \text { ) }\end{array}$ & Subtotals only \\
\hline $\begin{array}{l}\text { 3.4.1 Includes functional training versus } \\
\text { does not include functional training }\end{array}$ & 3 & 144 & $\begin{array}{l}\text { Std. Mean Difference (IV, } \\
\text { Random, } 95 \% \mathrm{CI} \text { ) }\end{array}$ & $0.43[-0.37,1.22]$ \\
\hline $\begin{array}{l}\text { 3.4.2 Includes neurophysiological versus } \\
\text { does not include neurophysiological }\end{array}$ & 7 & 278 & $\begin{array}{l}\text { Std. Mean Difference (IV, } \\
\text { Random, } 95 \% \mathrm{CI} \text { ) }\end{array}$ & $-0.12[-0.95,0.70]$ \\
\hline $\begin{array}{l}\text { 3.4.3 Includes musculoskeletal versus } \\
\text { does not include musculoskeletal }\end{array}$ & 3 & 45 & $\begin{array}{l}\text { Std. Mean Difference (IV, } \\
\text { Random, } 95 \% \mathrm{Cl} \text { ) }\end{array}$ & $-0.47[-1.67,0.74]$ \\
\hline 3.5 Length of stay & 3 & & $\begin{array}{l}\text { Mean Difference (IV, Ran- } \\
\text { dom, } 95 \% \mathrm{CI} \text { ) }\end{array}$ & Subtotals only \\
\hline $\begin{array}{l}\text { 3.5.1 Includes functional training versus } \\
\text { does not include functional training }\end{array}$ & 1 & 53 & $\begin{array}{l}\text { Mean Difference (IV, Ran- } \\
\text { dom, } 95 \% \mathrm{CI} \text { ) }\end{array}$ & $\begin{array}{l}-13.00[-20.80 \\
-5.20]\end{array}$ \\
\hline $\begin{array}{l}3.5 .2 \text { Includes neurophysiological versus } \\
\text { does not include neurophysiological }\end{array}$ & 3 & 141 & $\begin{array}{l}\text { Mean Difference (IV, Ran- } \\
\text { dom, } 95 \% \mathrm{Cl} \text { ) }\end{array}$ & $\begin{array}{l}11.36[1.52 \\
21.19]\end{array}$ \\
\hline
\end{tabular}




\begin{tabular}{lllll}
\hline Outcome or subgroup title & No. of studies & $\begin{array}{l}\text { No. of partici- } \\
\text { pants }\end{array}$ & Statistical method & Effect size \\
\hline $\begin{array}{l}\text { 3.5.3 Includes musculoskeletal versus } \\
\text { does not include musculoskeletal }\end{array}$ & 2 & 88 & $\begin{array}{l}\text { Mean Difference (IV, Ran- } \\
\text { dom, 95\% CI) }\end{array}$ & $\begin{array}{l}8.71[-12.92, \\
30.34]\end{array}$ \\
\hline
\end{tabular}

\section{Analysis 3.1. Comparison 3: One active intervention versus another active intervention: immediate outcomes, Outcome 1: Independence in ADL scales}

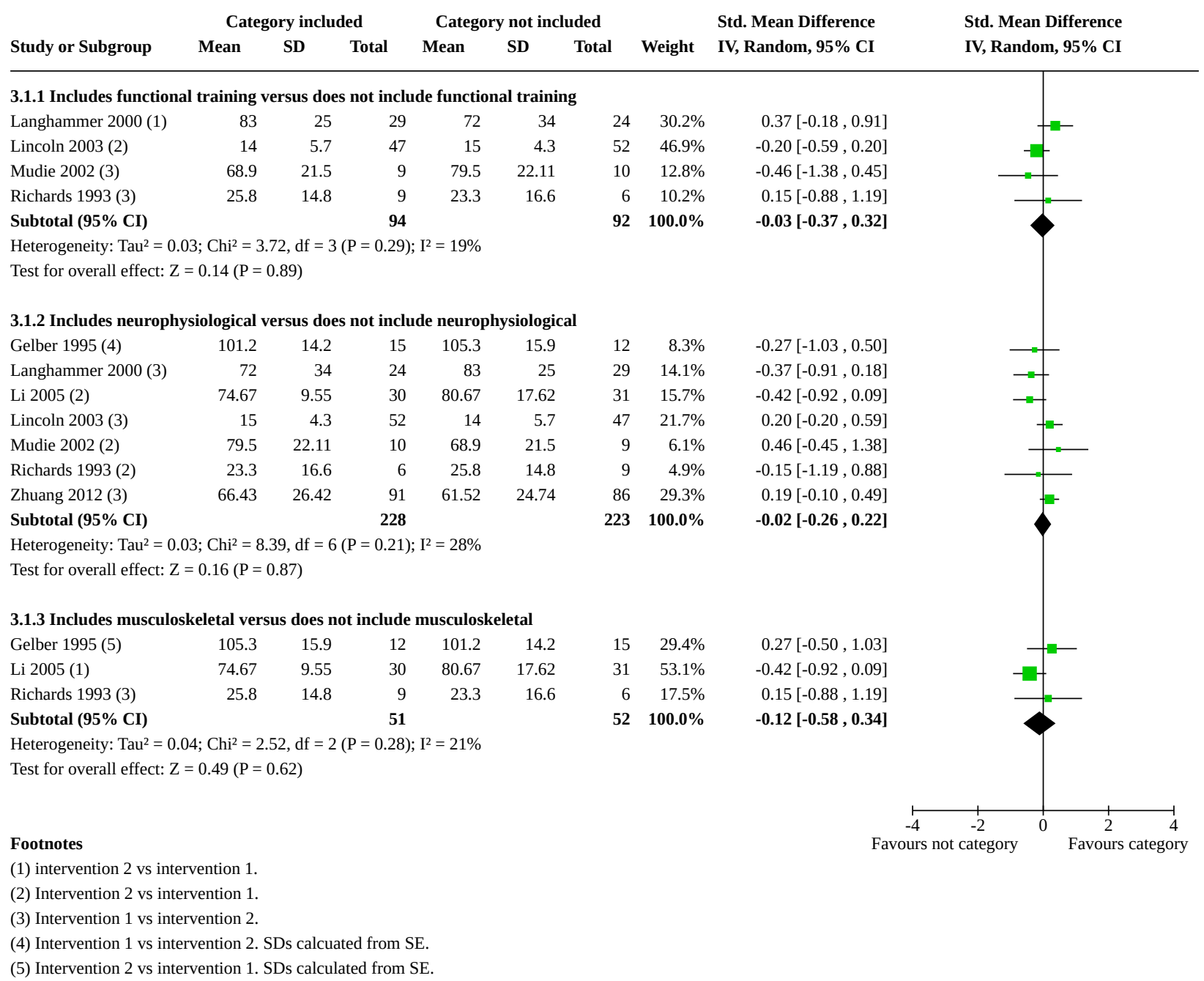




\section{Analysis 3.2. Comparison 3: One active intervention versus another active} intervention: immediate outcomes, Outcome 2: Motor function scales

\begin{tabular}{|c|c|c|c|c|c|c|c|c|}
\hline \multirow[b]{2}{*}{ Study or Subgroup } & \multicolumn{3}{|c|}{ Category included } & \multicolumn{3}{|c|}{ Category not included } & \multirow[b]{2}{*}{ Weight } & \multirow{2}{*}{$\begin{array}{l}\text { Std. Mean Difference } \\
\text { IV, Random, } 95 \% \text { CI }\end{array}$} \\
\hline & Mean & SD & Total & Mean & SD & Total & & \\
\hline \multicolumn{9}{|c|}{ 3.2.1 Includes functional training versus does not include functional training } \\
\hline Langhammer 2000 (1) & 37 & 12 & 29 & 33 & 15 & 24 & $30.4 \%$ & $0.29[-0.25,0$ \\
\hline Lincoln 2003 (1) & 5 & 5 & 47 & 7 & 5 & 52 & $38.9 \%$ & $-0.40[-0.80,0$. \\
\hline Richards 1993 (2) & 23.7 & 6.7 & 9 & 22.7 & 9.2 & 6 & $13.6 \%$ & $0.12[-0.91,1$ \\
\hline Wang 2005 (1) & 15.33 & 4.59 & 11 & 18.82 & 5.84 & 10 & $17.1 \%$ & $-0.64[-1.52,0$. \\
\hline Subtotal $(95 \%$ CI) & & & 96 & & & 92 & $100.0 \%$ & $-0.16[-0.59,0$. \\
\hline
\end{tabular}

Heterogeneity: $\mathrm{Tau}^{2}=0.09 ; \mathrm{Chi}^{2}=5.42, \mathrm{df}=3(\mathrm{P}=0.14) ; \mathrm{I}^{2}=45 \%$

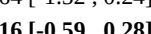

Test for overall effect: $\mathrm{Z}=0.72(\mathrm{P}=0.47)$

3.2.2 Includes neurophysiological versus does not include neurophysiological

$\begin{array}{lrrrrrrrr}\text { Bale 2008 (3) } & 3.1 & 0.53 & 10 & 3.125 & 0.53 & 8 & 5.0 \% & -0.04[-0.97,0.88] \\ \text { Gelber 1995 (4) } & 101.2 & 14.2 & 15 & 105.3 & 15.9 & 12 & 7.2 \% & -0.27[-1.03,0.50] \\ \text { Langhammer 2000 (2) } & 33 & 15 & 24 & 37 & 12 & 29 & 12.5 \% & -0.29[-0.84,0.25] \\ \text { Liao 2006 (2) } & 22.63 & 8.42 & 48 & 18.46 & 8.94 & 48 & 18.9 \% & 0.48[0.07,0.88] \\ \text { Lincoln 2003(2) } & 7 & 5 & 52 & 5 & 5 & 47 & 19.4 \% & 0.40[-0.00,0.80] \\ \text { Richards 1993 (1) } & 22.7 & 9.2 & 6 & 23.7 & 6.7 & 9 & 4.2 \% & -0.12[-1.16,0.91] \\ \text { Wang 2005(2) } & 18.82 & 5.84 & 10 & 15.33 & 4.59 & 11 & 5.5 \% & 0.64[-0.24,1.52] \\ \text { Zhuang 2012(2) } & 65.93 & 22.48 & 91 & 63.5 & 24.45 & 86 & 27.3 \% & 0.10[-0.19,0.40] \\ \text { Subtotal (95\% CI) } & & & \mathbf{2 5 6} & & & \mathbf{2 5 0} & \mathbf{1 0 0 . 0 \%} & \mathbf{0 . 1 7}[-\mathbf{0 . 0 5} \mathbf{0 . 3 9}]\end{array}$

Heterogeneity: $\mathrm{Tau}^{2}=0.02 ; \mathrm{Chi}^{2}=9.27, \mathrm{df}=7(\mathrm{P}=0.23) ; \mathrm{I}^{2}=24 \%$

$0.17[-0.05,0.39]$

Test for overall effect: $\mathrm{Z}=1.50(\mathrm{P}=0.13)$

3.2.3 Includes musculoskeletal versus does not include musculoskeletal

$\begin{array}{lrrrrrrrr}\text { Bale 2008 (3) } & 3.125 & 0.53 & 8 & 3.1 & 0.53 & 10 & 22.7 \% & 0.04[-0.88,0.97] \\ \text { Gelber 1995(5) } & 105.3 & 15.9 & 12 & 101.2 & 14.2 & 15 & 33.7 \% & 0.27[-0.50,1.03] \\ \text { Richards 1993 (2) } & 22.7 & 9.2 & 6 & 23.7 & 6.7 & 9 & 18.4 \% & -0.12[-1.16,0.91] \\ \text { Wang 2005(1) } & 15.33 & 4.59 & 11 & 18.82 & 5.84 & 10 & 25.2 \% & -0.64[-1.52,0.24] \\ \text { Subtotal (95\% CI) } & & & \mathbf{3 7} & & & \mathbf{4 4} & \mathbf{1 0 0 . 0 \%} & \mathbf{- 0 . 0 8 [ - 0 . 5 3 , 0 . 3 6 ]}\end{array}$

Heterogeneity: $\mathrm{Tau}^{2}=0.00 ; \mathrm{Chi}^{2}=2.42, \mathrm{df}=3(\mathrm{P}=0.49) ; \mathrm{I}^{2}=0 \%$

Test for overall effect: $\mathrm{Z}=0.37(\mathrm{P}=0.71)$

Footnotes

(1) Intervention 2 vs intervention 1.

(2) Intervention 1 vs intervention 2.

(3) Mean and SD calculated from categorical data.

(4) Intervention 1 vs intervention 2. SDs calculated from SE.

(5) Intervention 2 vs intervention 1. SDs calculated from SE.

Std. Mean Difference

IV, Random, 95\% CI 


\section{Analysis 3.3. Comparison 3: One active intervention versus another active} intervention: immediate outcomes, Outcome 3: Balance (Berg Balance Scale)

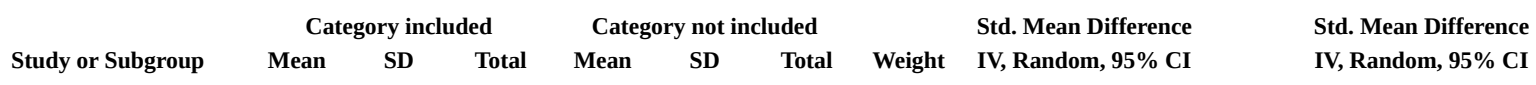

3.3.1 Includes functional training versus does not include functional training

Wang 2005 (2)

$20.42 \quad 4.64$

9
11

20.55

12.2

$6 \quad 40.2 \%$

Subtotal $(95 \%$ CI)

20

Heterogeneity: $\mathrm{Tau}^{2}=0.00 ; \mathrm{Chi}^{2}=0.26, \mathrm{df}=1(\mathrm{P}=0.61) ; \mathrm{I}^{2}=0 \%$

Test for overall effect: $\mathrm{Z}=0.46(\mathrm{P}=0.64)$

3.3.2 Includes neurophysiological versus does not include neurophysiological

Brock 2005 (3)

$47.3 \quad 4.6$

1247.4

5
18.2
7.5
4.64

$14 \quad 31.7 \%$

Richards 1993 (2)

40

16.1

6

33.2

$9 \quad 17.3 \%$

Wang 2005 (4)

$20.55 \quad 12.2$

Subtotal (95\% CI)

38

20.42

4.64

Heterogeneity: $\mathrm{Tau}^{2}=0.00 ; \mathrm{Chi}^{2}=0.84, \mathrm{df}=3(\mathrm{P}=0.84) ; \mathrm{I}^{2}=0 \%$

Test for overall effect: $\mathrm{Z}=0.03(\mathrm{P}=0.98)$

3.3.3 Includes musculoskeletal versus does not include musculoskeletal

\begin{tabular}{|c|c|c|c|c|c|c|c|c|}
\hline Richards 1993 (1) & 40 & 16.1 & 6 & 33.2 & 18.2 & 9 & $40.2 \%$ & $0.37[-0.68,1.41]$ \\
\hline Wang 2005 (2) & 20.42 & 4.64 & 11 & 20.55 & 12.2 & 10 & $59.8 \%$ & $-0.01[-0.87,0.8$ \\
\hline Subtotal $(95 \%$ CI $)$ & & & 17 & & & 19 & $100.0 \%$ & $0.14[-0.52,0.8$ \\
\hline
\end{tabular}

Subtotal (95\% CI)

$19 \quad 100.0 \%$

$11 \quad 25.4 \%$

$11 \quad 25.7 \%$

$45 \quad 100.0 \%$

$-0.02[-0.79,0.75]$

$0.37[-0.68,1.41]$

$-0.26[-1.13,0.60]$

$0.01[-0.84,0.87]$

$-0.01[-0.44,0.43]$
$-0.37[-1.41,0.68]$

$01[-0.87,0.84]$

$-0.16[-0.82,0.51]$

Heterogeneity: $\mathrm{Tau}^{2}=0.00 ; \mathrm{Chi}^{2}=0.31, \mathrm{df}=1(\mathrm{P}=0.58) ; \mathrm{I}^{2}=0 \%$

Test for overall effect: $\mathrm{Z}=0.41(\mathrm{P}=0.68)$

\section{Footnotes}

(1) Intervention 1 vs intervention 2.

(2) Intervention 2 vs intervention 1

(3) intervention 1 vs intervention 2.

(4) Intervention 1 vs intervention 2
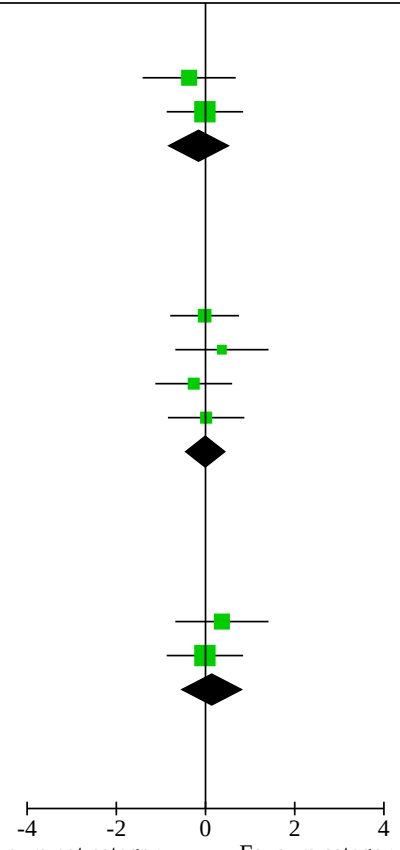

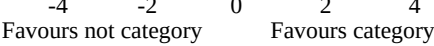




\section{Analysis 3.4. Comparison 3: One active intervention versus another} active intervention: immediate outcomes, Outcome 4: Gait velocity

\begin{tabular}{|c|c|c|c|c|c|c|c|c|c|}
\hline \multirow{2}{*}{ Study or Subgroup } & \multicolumn{3}{|c|}{ Category included } & \multicolumn{3}{|c|}{ Category not included } & & \multirow{2}{*}{$\begin{array}{l}\text { Std. Mean Difference } \\
\text { IV, Random, 95\% CI }\end{array}$} & \multirow{2}{*}{$\begin{array}{l}\text { Std. Mean Difference } \\
\text { IV, Random, 95\% CI }\end{array}$} \\
\hline & Mean & SD & Total & Mean & SD & Total & Weight & & \\
\hline
\end{tabular}

3.4.1 Includes functional training versus does not include functional training

Lincoln 2003 (1)

Richards 1993 (2)

$\begin{array}{ll}0.64 & 0.39 \\ 31.3 & 19.8 \\ 0.58 & 0.14\end{array}$

$\begin{array}{rr}47 & 0.69 \\ 9 & 21.8 \\ 15 & 0.43\end{array}$

0.45

IV, Random, 95\% CI

IV, Random, 95\%

Verma $2011(2)$

$\begin{array}{rr}6 & 25.6 \%\end{array}$

$0.14 \quad 15 \quad 32.5 \%$

Subtotal (95\% CI)

$73 \quad 100.0 \%$

$0.54[-0.52,1.60]$

$1.04[0.27,1.81]$

Heterogeneity: $\mathrm{Tau}^{2}=0.35 ; \mathrm{Chi}^{2}=7.45, \mathrm{df}=2(\mathrm{P}=0.02) ; \mathrm{I}^{2}=73 \%$

Test for overall effect: $\mathrm{Z}=1.06(\mathrm{P}=0.29)$

3.4.2 Includes neurophysiological versus does not include neurophysiological

Bale 2008 (1)

Brock 2005 (2)

$\begin{array}{ll}0.46 & 0.3\end{array}$

$\begin{array}{lll}10 & 0.36 & 0.2\end{array}$

$56.8 \quad 28.3$

0.36
36.2

0.2
27.9

$8 \quad 13.9 \%$

Gelber 1995 (3)

$0.52 \quad 0.2$

0.21

0.09

Lincoln 2003 (2)

$0.69 \quad 0.45$

0.64

0.39

Richards 1993 (1)

21.8

31.3

19.8

Thaut 2007 (1)

$20.3 \quad 6.5$

Verma 2011 (1)

$0.43 \quad 0.14$

Subtotal (95\% CI)

Heterogeneity: $\mathrm{Tau}^{2}=1.04 ; \mathrm{Chi}^{2}=53.44, \mathrm{df}=6(\mathrm{P}<0.00001) ; \mathrm{I}^{2}=89 \%$

Test for overall effect: $\mathrm{Z}=0.29(\mathrm{P}=0.77)$

3.4.3 Includes musculoskeletal versus does not include musculoskeletal

$\begin{array}{lrrrrrrr}\text { Bale 2008 (2) } & 0.36 & 0.2 & 8 & 0.46 & 0.3 & 10 & 36.9 \% \\ \text { Gelber 1995 (4) } & 0.21 & 0.09 & 6 & 0.52 & 0.2 & 6 & 28.2 \% \\ \text { Richards 1993 (2) } & 31.3 & 19.8 & 9 & 21.8 & 9 & 6 & 34.8 \% \\ \text { Subtotal (95\% CI) } & & & \mathbf{2 3} & & & \mathbf{2 2} & \mathbf{1 0 0 . 0 \%}\end{array}$

$-0.36[-1.30,0.57]$

$-1.85[-3.29,-0.40]$

$0.54[-0.52,1.60]$

$-0.47[-1.67,0.74]$

$0.71[-0.09,1.51]$

$1.85[0.40,3.29]$

$0.12[-0.28,0.51]$

$-0.54[-1.60,0.52]$

$-1.75[-2.28,-1.22]$

$-1.04[-1.81,-0.27]$

$-0.12[-0.95,0.70]$

Heterogeneity: $\mathrm{Tau}^{2}=0.79 ; \mathrm{Chi}^{2}=6.83, \mathrm{df}=2(\mathrm{P}=0.03) ; \mathrm{I}^{2}=71 \%$

Test for overall effect: $\mathrm{Z}=0.76(\mathrm{P}=0.45)$

Footnotes

(1) Intervention 2 vs intervention 1

Favours not category $\quad 0 \quad$ Favours category

(2) Intervention 1 vs intervention 2.

(3) Intervention 1 vs intervention 2. SDs calculated from SE.

(4) Intervention 2 vs intervention 1. SDs calculated from SE. 


\section{Analysis 3.5. Comparison 3: One active intervention versus another active intervention: immediate outcomes, Outcome 5: Length of stay}

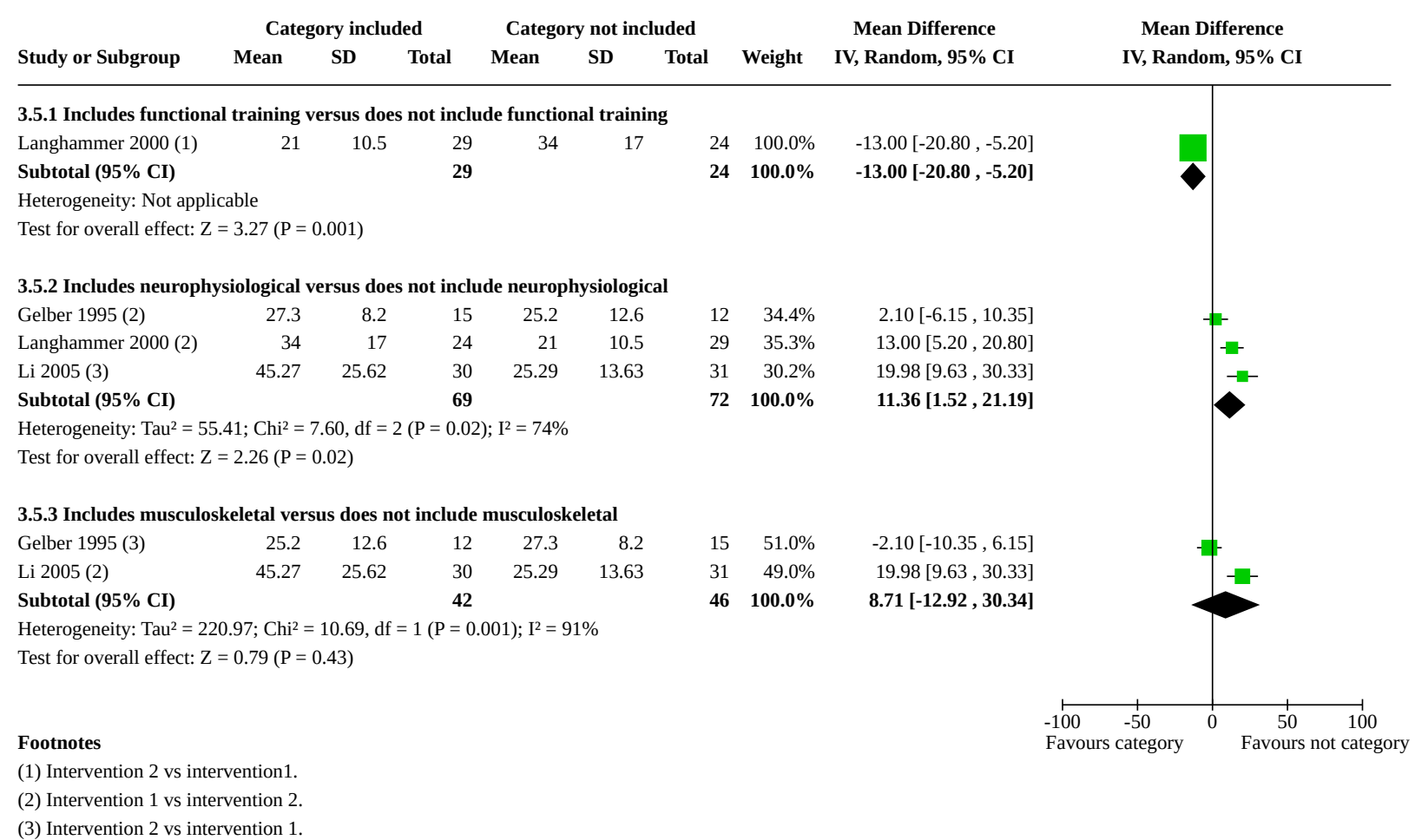

Comparison 4. Intervention versus no treatment: persisting outcomes

\begin{tabular}{|c|c|c|c|c|}
\hline Outcome or subgroup title & No. of studies & $\begin{array}{l}\text { No. of partici- } \\
\text { pants }\end{array}$ & Statistical method & Effect size \\
\hline 4.1 Independence in ADL scales & 10 & 540 & $\begin{array}{l}\text { Std. Mean Difference (IV, Random, } \\
95 \% \mathrm{CI} \text { ) }\end{array}$ & $0.58[0.11,1.04]$ \\
\hline 4.1.1 Functional task training & 2 & 232 & $\begin{array}{l}\text { Std. Mean Difference (IV, Random, } \\
95 \% \mathrm{Cl})\end{array}$ & $-0.07[-0.33,0.19]$ \\
\hline $\begin{array}{l}\text { 4.1.2 Functional task training + } \\
\text { musculoskeletal }\end{array}$ & 4 & 178 & $\begin{array}{l}\text { Std. Mean Difference (IV, Random, } \\
95 \% \mathrm{Cl} \text { ) }\end{array}$ & $1.13[0.44,1.82]$ \\
\hline 4.1.3 Neurophysiological & 0 & 0 & $\begin{array}{l}\text { Std. Mean Difference (IV, Random, } \\
95 \% \mathrm{Cl} \text { ) }\end{array}$ & Not estimable \\
\hline $\begin{array}{l}\text { 4.1.4 Neurophysiological + mus- } \\
\text { culoskeletal }\end{array}$ & 1 & 26 & $\begin{array}{l}\text { Std. Mean Difference (IV, Random, } \\
95 \% \mathrm{Cl} \text { ) }\end{array}$ & $0.14[-0.63,0.91]$ \\
\hline $\begin{array}{l}\text { 4.1.5 Functional training + neuro- } \\
\text { physiological }\end{array}$ & 0 & 0 & $\begin{array}{l}\text { Std. Mean Difference (IV, Random, } \\
95 \% \mathrm{CI} \text { ) }\end{array}$ & Not estimable \\
\hline $\begin{array}{l}\text { 4.1.6 Functional training + neuro- } \\
\text { physiological + musculoskeletal }\end{array}$ & 1 & 78 & $\begin{array}{l}\text { Std. Mean Difference (IV, Random, } \\
95 \% \mathrm{CI} \text { ) }\end{array}$ & $1.02[0.54,1.49]$ \\
\hline 4.1.7 Musculoskeletal & 2 & 26 & $\begin{array}{l}\text { Std. Mean Difference (IV, Random, } \\
95 \% \mathrm{Cl})\end{array}$ & $0.09[-0.70,0.89]$ \\
\hline
\end{tabular}




\begin{tabular}{|c|c|c|c|c|}
\hline Outcome or subgroup title & No. of studies & $\begin{array}{l}\text { No. of partici- } \\
\text { pants }\end{array}$ & Statistical method & Effect size \\
\hline 4.2 Motor function scales & 10 & 1829 & $\begin{array}{l}\text { Std. Mean Difference (IV, Random, } \\
95 \% \mathrm{CI} \text { ) }\end{array}$ & $1.06[0.37,1.75]$ \\
\hline 4.2.1 Functional task training & 2 & 234 & $\begin{array}{l}\text { Std. Mean Difference (IV, Random, } \\
95 \% \mathrm{Cl} \text { ) }\end{array}$ & $-0.07[-0.33,0.18]$ \\
\hline $\begin{array}{l}\text { 4.2.2 Functional task training + } \\
\text { musculoskeletal }\end{array}$ & 5 & 1543 & $\begin{array}{l}\text { Std. Mean Difference (IV, Random, } \\
95 \% \mathrm{Cl})\end{array}$ & $2.07[0.99,3.15]$ \\
\hline 4.2.3 Neurophysiological & 0 & 0 & $\begin{array}{l}\text { Std. Mean Difference (IV, Random, } \\
95 \% \mathrm{Cl} \text { ) }\end{array}$ & Not estimable \\
\hline $\begin{array}{l}\text { 4.2.4 Neurophysiological + mus- } \\
\text { culoskeletal }\end{array}$ & 1 & 26 & $\begin{array}{l}\text { Std. Mean Difference (IV, Random, } \\
95 \% \mathrm{Cl} \text { ) }\end{array}$ & $0.10[-0.67,0.87]$ \\
\hline $\begin{array}{l}\text { 4.2.5 Functional training + neuro- } \\
\text { physiological }\end{array}$ & 0 & 0 & $\begin{array}{l}\text { Std. Mean Difference (IV, Random, } \\
95 \% \mathrm{CI} \text { ) }\end{array}$ & Not estimable \\
\hline $\begin{array}{l}\text { 4.2.6 Functional training + neuro- } \\
\text { physiological + musculoskeletal }\end{array}$ & 0 & 0 & $\begin{array}{l}\text { Std. Mean Difference (IV, Random, } \\
95 \% \mathrm{Cl} \text { ) }\end{array}$ & Not estimable \\
\hline 4.2.7 Musculoskeletal & 2 & 26 & $\begin{array}{l}\text { Std. Mean Difference (IV, Random, } \\
95 \% \mathrm{CI} \text { ) }\end{array}$ & $0.07[-0.91,1.06]$ \\
\hline 4.3 Balance (Berg Balance Scale) & 1 & 34 & $\begin{array}{l}\text { Std. Mean Difference (IV, Random, } \\
95 \% \mathrm{CI} \text { ) }\end{array}$ & $-0.03[-0.70,0.65]$ \\
\hline 4.3.1 Functional task training & 0 & 0 & $\begin{array}{l}\text { Std. Mean Difference (IV, Random, } \\
95 \% \mathrm{Cl} \text { ) }\end{array}$ & Not estimable \\
\hline $\begin{array}{l}\text { 4.3.2 Functional task training }+ \\
\text { musculoskeletal }\end{array}$ & 1 & 34 & $\begin{array}{l}\text { Std. Mean Difference (IV, Random, } \\
95 \% \mathrm{CI} \text { ) }\end{array}$ & $-0.03[-0.70,0.65]$ \\
\hline 4.3.3 Neurophysiological & 0 & 0 & $\begin{array}{l}\text { Std. Mean Difference (IV, Random, } \\
95 \% \mathrm{Cl} \text { ) }\end{array}$ & Not estimable \\
\hline $\begin{array}{l}\text { 4.3.4 Neurophysiological + mus- } \\
\text { culoskeletal }\end{array}$ & 0 & 0 & $\begin{array}{l}\text { Std. Mean Difference (IV, Random, } \\
95 \% \mathrm{Cl} \text { ) }\end{array}$ & Not estimable \\
\hline $\begin{array}{l}\text { 4.3.5 Functional training + neuro- } \\
\text { physiological }\end{array}$ & 0 & 0 & $\begin{array}{l}\text { Std. Mean Difference (IV, Random, } \\
95 \% \mathrm{CI} \text { ) }\end{array}$ & Not estimable \\
\hline $\begin{array}{l}\text { 4.3.6 Functional training + neuro- } \\
\text { physiological + musculoskeletal }\end{array}$ & 0 & 0 & $\begin{array}{l}\text { Std. Mean Difference (IV, Random, } \\
95 \% \mathrm{CI} \text { ) }\end{array}$ & Not estimable \\
\hline 4.3.7 Musculoskeletal & 0 & 0 & $\begin{array}{l}\text { Std. Mean Difference (IV, Random, } \\
95 \% \mathrm{Cl} \text { ) }\end{array}$ & Not estimable \\
\hline 4.4 Gait velocity & 3 & 271 & $\begin{array}{l}\text { Std. Mean Difference (IV, Random, } \\
95 \% \mathrm{Cl} \text { ) }\end{array}$ & $-0.06[-0.29,0.18]$ \\
\hline 4.4.1 Functional task training & 3 & 271 & $\begin{array}{l}\text { Std. Mean Difference (IV, Random, } \\
95 \% \mathrm{Cl})\end{array}$ & $-0.06[-0.29,0.18]$ \\
\hline
\end{tabular}




\begin{tabular}{|c|c|c|c|c|}
\hline Outcome or subgroup title & No. of studies & $\begin{array}{l}\text { No. of partici- } \\
\text { pants }\end{array}$ & Statistical method & Effect size \\
\hline $\begin{array}{l}\text { 4.4.2 Functional task training + } \\
\text { musculoskeletal }\end{array}$ & 0 & 0 & $\begin{array}{l}\text { Std. Mean Difference (IV, Random, } \\
95 \% \mathrm{Cl} \text { ) }\end{array}$ & Not estimable \\
\hline 4.4.3 Neurophysiological & 0 & 0 & $\begin{array}{l}\text { Std. Mean Difference (IV, Random, } \\
95 \% \mathrm{CI} \text { ) }\end{array}$ & Not estimable \\
\hline $\begin{array}{l}\text { 4.4.4 Neurophysiological + mus- } \\
\text { culoskeletal }\end{array}$ & 0 & 0 & $\begin{array}{l}\text { Std. Mean Difference (IV, Random, } \\
95 \% \mathrm{CI} \text { ) }\end{array}$ & Not estimable \\
\hline $\begin{array}{l}\text { 4.4.5 Functional training + neuro- } \\
\text { physiological }\end{array}$ & 0 & 0 & $\begin{array}{l}\text { Std. Mean Difference (IV, Random, } \\
95 \% \mathrm{Cl} \text { ) }\end{array}$ & Not estimable \\
\hline $\begin{array}{l}\text { 4.4.6 Functional training + neuro- } \\
\text { physiological + musculoskeletal }\end{array}$ & 0 & 0 & $\begin{array}{l}\text { Std. Mean Difference (IV, Random, } \\
95 \% \mathrm{Cl})\end{array}$ & Not estimable \\
\hline 4.4.7 Musculoskeletal & 0 & 0 & $\begin{array}{l}\text { Std. Mean Difference (IV, Random, } \\
95 \% \mathrm{Cl})\end{array}$ & Not estimable \\
\hline
\end{tabular}




\section{Analysis 4.1. Comparison 4: Intervention versus no treatment: persisting outcomes, Outcome 1: Independence in ADL scales}

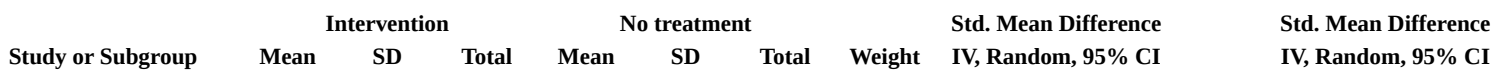

4.1.1 Functional task training

Green 2002 (1)

Wade 1992

$18 \quad 0.75$

Subtotal (95\% CI)

$16.2 \quad 3.4$

Test for overall effect: $\mathrm{Z}=0.53(\mathrm{P}=0.60)$

4.1.2 Functional task training + musculoskeletal

Holmgren 2006 (2)

Xie 2003

Zhu 2007 haem (1)

$\begin{array}{rrr}19.2 & 0.99 & 15 \\ 17 & 7 & 32 \\ 77.5 & 17.5 & 12\end{array}$

$15 \quad 18.5$

Zhu 2007 isch (1)

$82.5 \quad 15$

Subtotal (95\% CI)

87

$\begin{array}{ll}72 & 18\end{array}$

$47 \quad 16.8$

119

16.8

2.8

$74 \quad 12.2 \%$

$39 \quad 11.7 \%$

$113 \quad 23.8 \%$

$0.00[-0.32,0.32]$

$-0.19[-0.61,0.24]$

$\mathbf{- 0 . 0 7}[-0.33,0.19]$

Test for overall effect: $\mathrm{Z}=3.19(\mathrm{P}=0.001)$

4.1.3 Neurophysiological

Subtotal (95\% CI)

$\mathbf{0}$

Not estimable

Test for overall effect: Not applicable

4.1.4 Neurophysiological + musculoskeletal

Fang $2003 \quad 83.93 \quad 19.6$

Subtotal (95\% CI)

12

$80 \quad 32.96$

$149.5 \%$

$0.14[-0.63,0.91]$

Heterogeneity: Not applicable

$14 \quad 9.5 \%$

$0.14[-0.63,0.91]$

Test for overall effect: $\mathrm{Z}=0.35$ ( $\mathrm{P}=0.73$ )

4.1.5 Functional training + neurophysiological

Subtotal (95\% CI)

0

Not estimable

Test for overall effect: Not applicable

4.1.6 Functional training + neurophysiological + musculoskeletal

$\begin{array}{lllllll}10.64 & 5.02 & 39 & 5.13 & 5.68 & 39 & 11.4 \%\end{array}$

Subtotal (95\% CI)

39

$39 \quad 11.4 \%$

$1.02[0.54,1.49]$

$1.02[0.54,1.49]$

Test for overall effect: $\mathrm{Z}=4.22(\mathrm{P}<0.0001)$

\subsubsection{Musculoskeletal}

Fang 2004 old

Fang 2004 young

$\begin{array}{rr}85 & 15.2 \\ 83.13 & 23.4\end{array}$

$6 \quad 76.88$

36.74

$8 \quad 7.7 \%$

$0.26[-0.81,1.32]$

$-0.12[-1.32,1.08]$

Subtotal (95\% CI)

14

$12 \quad 14.7 \%$

$0.09[-0.70,0.89]$

Test for overall effect: $\mathrm{Z}=0.23(\mathrm{P}=0.82)$

Total (95\% CI)

271

Heterogeneity: $\mathrm{Tau}^{2}=0.44 ; \mathrm{Chi}^{2}=54.18, \mathrm{df}=9(\mathrm{P}<0.00001) ; \mathrm{I}^{2}=83 \%$

Test for overall effect: $\mathrm{Z}=2.42(\mathrm{P}=0.02)$

Test for subgroup differences: $\mathrm{Chi}^{2}=22.41, \mathrm{df}=4(\mathrm{P}=0.0002), \mathrm{I}^{2}=82.1 \%$

$269 \quad 100.0 \%$

$0.58[0.11,1.04]$

IV, Random, 95\% CI

\section{Footnotes}

(1) SDs estimated from range ((max-min range)/4).

(2) SDs calculated from CI and p-value.

(3) Data is BI category (walking). 


\section{Analysis 4.2. Comparison 4: Intervention versus no treatment: persisting outcomes, Outcome 2: Motor function scales}

\begin{tabular}{|c|c|c|c|c|c|c|c|c|}
\hline & \multicolumn{3}{|c|}{ Intervention } & \multicolumn{2}{|c|}{ No treatment } & & Std. Mean Difference & Std. Mean Difference \\
\hline & Mean & SD & Total & Mean & SD & Weight & IV, Random, 95\% CI & IV, Random, 95\% CI \\
\hline
\end{tabular}

4.2.1 Functional task training

Green 2002 (1)

Wade 1992

Subtotal (95\% CI)

$10 \quad 1.25$

$12.1 \quad 4.6$

$\begin{array}{ll}74 & 10 \\ 47 & 13\end{array}$

121

$10 \quad 1.25$

1.25
4

Heterogeneity: $\mathrm{Tau}^{2}=0.00 ; \mathrm{Chi}^{2}=0.57, \mathrm{df}=1(\mathrm{P}=0.45) ; \mathrm{I}^{2}=0 \%$

Test for overall effect: $\mathrm{Z}=0.57(\mathrm{P}=0.57)$

$\begin{array}{lrrr}\text { 4.2.2 Functional task training + musculoskeletal } \\ \text { Hu 2007 haem } & 80 & 21 & 17 \\ \text { Hu 2007 isch } & 75 & 23 & 47 \\ \text { Zhao 2002 } & 74.8 & 5.42 & 100 \\ \text { Zhu } 2007 \text { haem (1) } & 17.5 & 3.75 & 12 \\ \text { Zhu } 2007 \text { isch (1) } & 19 & 3.5 & 28\end{array}$

Subtotal (95\% CI)

Heterogeneity: $\mathrm{Tau}^{2}=1.42 ; \mathrm{Chi}^{2}=214.62, \mathrm{df}=4(\mathrm{P}<0.00001) ; \mathrm{I}^{2}=98 \%$

Test for overall effect: $\mathrm{Z}=3.77(\mathrm{P}=0.0002)$

\subsubsection{Neurophysiological}

Subtotal (95\% CI)

Heterogeneity: Not applicable

Test for overall effect: Not applicable

4.2.4 Neurophysiological + musculoskeletal

$\begin{array}{lll}\text { Fang } 2003 & 26.86 & 7.06\end{array}$

Subtotal (95\% CI)

$12 \quad 26 \quad 9.51$

12

55
55

44.9

26
26

$168 \quad 11.0 \%$

$469 \quad 11.1 \%$

$80 \quad 10.0 \%$

$10 \quad 9.4 \%$

$28 \quad 10.3 \%$

$755 \quad 51.7 \%$

IV, Random, 95\% CI

$1.06[0.83,1.28]$

$0.81[0.68,0.95]$

$6.19[5.48,6.90]$

$1.02[0.12,1.93]$

$1.42[0.83,2.01]$

$2.07[0.99,3.15]$

$14 \quad 9.8 \%$

$0.10[-0.67,0.87]$

$0.10[-0.67,0.87]$

Heterogeneity: Not applicable

Test for overall effect: $\mathrm{Z}=0.25(\mathrm{P}=0.80)$

4.2.5 Functional training + neurophysiological

Subtotal (95\% CI)

$\mathbf{0}$

Heterogeneity: Not applicable

Test for overall effect: Not applicable

4.2.6 Functional training + neurophysiological + musculoskeletal

Subtotal (95\% CI)

Heterogeneity: Not applicable

Test for overall effect: Not applicable

\subsubsection{Musculoskeletal}

Fang 2004 old

Fang 2004 young

$29.83 \quad 5.56$

$6 \quad 24.75$

10.59

Subtotal (95\% CI)

$24.63 \quad 7.56$

8

28.5

$\begin{array}{ll}8 & 8.7 \% \\ 4 & 8.3 \%\end{array}$

$0.54[-0.55,1.62]$

$-0.47[-1.69,0.75]$

Heterogeneity: $\mathrm{Tau}^{2}=0.16 ; \mathrm{Chi}^{2}=1.47, \mathrm{df}=1(\mathrm{P}=0.23) ; \mathrm{I}^{2}=32 \%$

$12 \quad 17.0 \%$

$0.07[-0.91,1.06]$

Test for overall effect: $\mathrm{Z}=0.15(\mathrm{P}=0.88)$

Total (95\% CI)

935

Heterogeneity: $\mathrm{Tau}^{2}=1.12 ; \mathrm{Chi}^{2}=283.12, \mathrm{df}=9(\mathrm{P}<0.00001) ; \mathrm{I}^{2}=97 \%$

Test for overall effect: $\mathrm{Z}=3.00(\mathrm{P}=0.003)$

Test for subgroup differences: $\mathrm{Chi}^{2}=14.43, \mathrm{df}=3(\mathrm{P}=0.002), \mathrm{I}^{2}=79.2 \%$

$894 \quad 100.0 \%$

$1.06[0.37,1.75]$

Footnotes

(1) SDs estimated from range ((max-min range)/4). 


\section{Analysis 4.3. Comparison 4: Intervention versus no treatment:} persisting outcomes, Outcome 3: Balance (Berg Balance Scale)

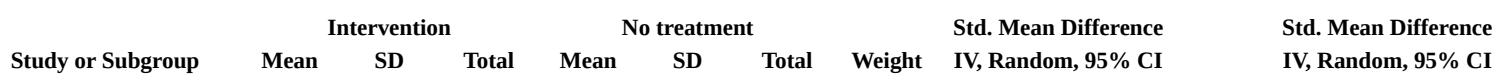

4.3.1 Functional task training

Subtotal (95\% CI)

Heterogeneity: Not applicable

Test for overall effect: Not applicable

4.3.2 Functional task training + musculoskeletal

Holmgren 2006 (1) $\quad 44.1 \quad 10.83$

Subtotal (95\% CI)

$10.83 \quad 15$
-15

Not estimable

Heterogeneity: Not applicable

Test for overall effect: $\mathrm{Z}=0.08(\mathrm{P}=0.94)$

4.3.3 Neurophysiological

Subtotal (95\% CI)

$44.4 \quad 11.41$

$19 \quad 100.0 \%$

$-0.03[-0.70,0.65]$

15

$19 \quad 100.0 \%$

$-0.03[-0.70,0.65]$

Heterogeneity: Not applicable

Test for overall effect: Not applicable

4.3.4 Neurophysiological + musculoskeletal

Subtotal (95\% CI)

Heterogeneity: Not applicable

Test for overall effect: Not applicable

4.3.5 Functional training + neurophysiological

Subtotal (95\% CI)

0

Heterogeneity: Not applicable

Test for overall effect: Not applicable

4.3.6 Functional training + neurophysiological + musculoskeletal

Subtotal (95\% CI)

Heterogeneity: Not applicable

Test for overall effect: Not applicable

\subsubsection{Musculoskeletal}

Subtotal (95\% CI)

Heterogeneity: Not applicable

Test for overall effect: Not applicable

Total (95\% CI)

15

Heterogeneity: Not applicable

Test for overall effect: $Z=0.08(P=0.94)$

Test for subgroup differences: Not applicable

Footnotes

(1) SD calculated from CI and p-value.
Not estimable
Not estimable
0

$19 \quad 100.0 \%$ 
Analysis 4.4. Comparison 4: Intervention versus no treatment: persisting outcomes, Outcome 4: Gait velocity

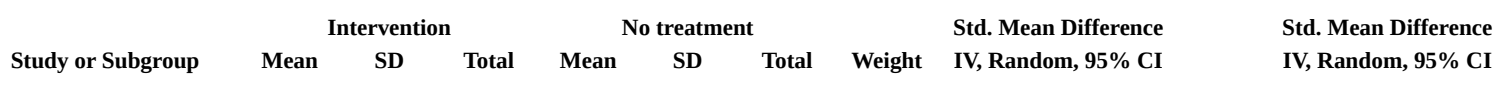

\begin{tabular}{lrlrrrrrr}
\hline 4.4.1 Functional task training & & & & & & & \\
Green 2002 (1) & 25.4 & 14.5 & 64 & 25.8 & 13.6 & 67 & $48.5 \%$ & $-0.03[-0.37,0.31]$ \\
Hui-Chan 2009 & 61.3 & 28.6 & 25 & 61.2 & 24.2 & 29 & $19.9 \%$ & $0.00[-0.53,0.54]$ \\
Wade 1992 & 0.205 & 0.29 & 47 & 0.246 & 0.318 & 39 & $31.5 \%$ & $-0.13[-0.56,0.29]$ \\
Subtotal (95\% CI) & & & $\mathbf{1 3 6}$ & & & $\mathbf{1 3 5}$ & $\mathbf{1 0 0 . 0 \%}$ & $\mathbf{- 0 . 0 6 [ - 0 . 2 9 , 0 . 1 8 ]}$
\end{tabular}

Heterogeneity: $\mathrm{Tau}^{2}=0.00 ; \mathrm{Chi}^{2}=0.20, \mathrm{df}=2(\mathrm{P}=0.90) ; \mathrm{I}^{2}=0 \%$

Test for overall effect: $\mathrm{Z}=0.45(\mathrm{P}=0.65)$

4.4.2 Functional task training + musculoskeletal

Subtotal (95\% CI)

Not estimable

Heterogeneity: Not applicable

Test for overall effect: Not applicable

4.4.3 Neurophysiological

Subtotal (95\% CI)

$\mathbf{0}$

0

Not estimable

Test for overall effect: Not applicable

4.4.4 Neurophysiological + musculoskeletal

Subtotal (95\% CI)

$\mathbf{0}$

Heterogeneity: Not applicable

Test for overall effect: Not applicable

4.4.5 Functional training + neurophysiological

Subtotal (95\% CI)

Heterogeneity: Not applicable

Test for overall effect: Not applicable

4.4.6 Functional training + neurophysiological + musculoskeletal

Subtotal (95\% CI)

Heterogeneity: Not applicable

$0 \quad$ Not estimable

Test for overall effect: Not applicable

\subsubsection{Musculoskeletal}

Subtotal (95\% CI)

Heterogeneity: Not applicable

(2.

Test for overall effect: Not applicable

Total (95\% CI)

136

Heterogeneity: $\mathrm{Tau}^{2}=0.00 ; \mathrm{Chi}^{2}=0.20, \mathrm{df}=2(\mathrm{P}=0.90) ; \mathrm{I}^{2}=0 \%$

Test for overall effect: $\mathrm{Z}=0.45(\mathrm{P}=0.65)$

Test for subgroup differences: Not applicable

0

Not estimable

Footnotes

(1) SDs estimated from range ((max-min range)/4).

Not estimable

Not estimable

$135 \quad 100.0 \% \quad-0.06[-0.29,0.18]$

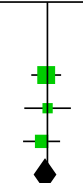

\section{Comparison 5. Intervention versus usual care or attention control: persisting outcomes}

\begin{tabular}{lllll}
\hline Outcome or subgroup title & No. of studies & $\begin{array}{l}\text { No. of partici- } \\
\text { pants }\end{array}$ & Statistical method & Effect size \\
\hline 5.1 Independence in ADL scales & 0 & 0 & $\begin{array}{l}\text { Std. Mean Difference (IV, Random, } \\
95 \% \mathrm{Cl})\end{array}$ & Not estimable \\
\hline $\begin{array}{l}\text { 5.1.1 Functional task training } \\
\text { T.1.2 Functional task training + }\end{array}$ & 0 & 0 & $\begin{array}{l}\text { Std. Mean Difference (IV, Random, } \\
95 \% \mathrm{Cl})\end{array}$ & Not estimable \\
\hline \begin{tabular}{l} 
musculoskeletal \\
\hline
\end{tabular} & 0 & 0 & $\begin{array}{l}\text { Std. Mean Difference (IV, Random, } \\
95 \% \mathrm{Cl})\end{array}$ & Not estimable \\
\hline
\end{tabular}

Physical rehabilitation approaches for the recovery of function and mobility following stroke (Review) 


\begin{tabular}{|c|c|c|c|c|}
\hline Outcome or subgroup title & No. of studies & $\begin{array}{l}\text { No. of partici- } \\
\text { pants }\end{array}$ & Statistical method & Effect size \\
\hline 5.1.3 Neurophysiological & 0 & 0 & $\begin{array}{l}\text { Std. Mean Difference (IV, Random, } \\
95 \% \mathrm{CI} \text { ) }\end{array}$ & Not estimable \\
\hline $\begin{array}{l}\text { 5.1.4 Neurophysiological + muscu- } \\
\text { loskeletal }\end{array}$ & 0 & 0 & $\begin{array}{l}\text { Std. Mean Difference (IV, Random, } \\
95 \% \mathrm{CI} \text { ) }\end{array}$ & Not estimable \\
\hline $\begin{array}{l}\text { 5.1.5 Functional training + neuro- } \\
\text { physiological }\end{array}$ & 0 & 0 & $\begin{array}{l}\text { Std. Mean Difference (IV, Random, } \\
95 \% \mathrm{CI} \text { ) }\end{array}$ & Not estimable \\
\hline $\begin{array}{l}\text { 5.1.6 Functional training + neuro- } \\
\text { physiological + musculoskeletal }\end{array}$ & 0 & 0 & $\begin{array}{l}\text { Std. Mean Difference (IV, Random, } \\
95 \% \mathrm{CI} \text { ) }\end{array}$ & Not estimable \\
\hline 5.2 Motor function scales & 3 & 160 & $\begin{array}{l}\text { Std. Mean Difference (IV, Random, } \\
95 \% \mathrm{CI} \text { ) }\end{array}$ & $-0.10[-0.42,0.23]$ \\
\hline 5.2.1 Functional task training & 1 & 23 & $\begin{array}{l}\text { Std. Mean Difference (IV, Random, } \\
95 \% \mathrm{CI})\end{array}$ & $-0.16[-0.99,0.66]$ \\
\hline $\begin{array}{l}\text { 5.2.2 Functional task training + } \\
\text { musculoskeletal }\end{array}$ & 1 & 58 & $\begin{array}{l}\text { Std. Mean Difference (IV, Random, } \\
95 \% \mathrm{CI})\end{array}$ & $0.00[-0.52,0.52]$ \\
\hline 5.2.3 Neurophysiological & 0 & 0 & $\begin{array}{l}\text { Std. Mean Difference (IV, Random, } \\
95 \% \mathrm{CI})\end{array}$ & Not estimable \\
\hline $\begin{array}{l}\text { 5.2.4 Neurophysiological + muscu- } \\
\text { loskeletal }\end{array}$ & 0 & 0 & $\begin{array}{l}\text { Std. Mean Difference (IV, Random, } \\
95 \% \mathrm{CI} \text { ) }\end{array}$ & Not estimable \\
\hline $\begin{array}{l}\text { 5.2.5 Functional training + neuro- } \\
\text { physiological }\end{array}$ & 0 & 0 & $\begin{array}{l}\text { Std. Mean Difference (IV, Random, } \\
95 \% \mathrm{CI} \text { ) }\end{array}$ & Not estimable \\
\hline $\begin{array}{l}\text { 5.2.6 Functional training + neuro- } \\
\text { physiological + musculoskeletal }\end{array}$ & 1 & 79 & $\begin{array}{l}\text { Std. Mean Difference (IV, Random, } \\
95 \% \mathrm{CI} \text { ) }\end{array}$ & $-0.16[-0.65,0.32]$ \\
\hline 5.3 Balance (Berg Balance Scale) & 0 & 0 & $\begin{array}{l}\text { Std. Mean Difference (IV, Random, } \\
95 \% \mathrm{CI})\end{array}$ & Not estimable \\
\hline 5.3.1 Functional task training & 0 & 0 & $\begin{array}{l}\text { Std. Mean Difference (IV, Random, } \\
95 \% \mathrm{CI})\end{array}$ & Not estimable \\
\hline $\begin{array}{l}\text { 5.3.2 Functional task training + } \\
\text { musculoskeletal }\end{array}$ & 0 & 0 & $\begin{array}{l}\text { Std. Mean Difference (IV, Random, } \\
95 \% \mathrm{CI})\end{array}$ & Not estimable \\
\hline 5.3.3 Neurophysiological & 0 & 0 & $\begin{array}{l}\text { Std. Mean Difference (IV, Random, } \\
95 \% \mathrm{CI})\end{array}$ & Not estimable \\
\hline $\begin{array}{l}\text { 5.3.4 Neurophysiological + muscu- } \\
\text { loskeletal }\end{array}$ & 0 & 0 & $\begin{array}{l}\text { Std. Mean Difference (IV, Random, } \\
95 \% \mathrm{CI} \text { ) }\end{array}$ & Not estimable \\
\hline $\begin{array}{l}\text { 5.3.5 Functional training + neuro- } \\
\text { physiological }\end{array}$ & 0 & 0 & $\begin{array}{l}\text { Std. Mean Difference (IV, Random, } \\
95 \% \mathrm{CI})\end{array}$ & Not estimable \\
\hline $\begin{array}{l}\text { 5.3.6 Functional training + neuro- } \\
\text { physiological + musculoskeletal }\end{array}$ & 0 & 0 & $\begin{array}{l}\text { Std. Mean Difference (IV, Random, } \\
95 \% \mathrm{CI})\end{array}$ & Not estimable \\
\hline
\end{tabular}




\begin{tabular}{|c|c|c|c|c|}
\hline Outcome or subgroup title & No. of studies & $\begin{array}{l}\text { No. of partici- } \\
\text { pants }\end{array}$ & Statistical method & Effect size \\
\hline 5.4 Gait velocity & 5 & 214 & $\begin{array}{l}\text { Std. Mean Difference (IV, Random, } \\
95 \% \mathrm{CI} \text { ) }\end{array}$ & $0.38[0.10,0.66]$ \\
\hline 5.4.1 Functional task training & 1 & 9 & $\begin{array}{l}\text { Std. Mean Difference (IV, Random, } \\
95 \% \mathrm{Cl} \text { ) }\end{array}$ & $1.15[-0.34,2.65]$ \\
\hline $\begin{array}{l}\text { 5.4.2 Functional task training + } \\
\text { musculoskeletal }\end{array}$ & 3 & 96 & $\begin{array}{l}\text { Std. Mean Difference (IV, Random, } \\
95 \% \mathrm{CI} \text { ) }\end{array}$ & $0.52[0.11,0.93]$ \\
\hline 5.4.3 Neurophysiological & 0 & 0 & $\begin{array}{l}\text { Std. Mean Difference (IV, Random, } \\
95 \% \mathrm{CI} \text { ) }\end{array}$ & Not estimable \\
\hline $\begin{array}{l}\text { 5.4.4 Neurophysiological + muscu- } \\
\text { loskeletal }\end{array}$ & 0 & 0 & $\begin{array}{l}\text { Std. Mean Difference (IV, Random, } \\
95 \% \mathrm{Cl} \text { ) }\end{array}$ & Not estimable \\
\hline $\begin{array}{l}\text { 5.4.5 Functional training + neuro- } \\
\text { physiological }\end{array}$ & 0 & 0 & $\begin{array}{l}\text { Std. Mean Difference (IV, Random, } \\
95 \% \mathrm{Cl} \text { ) }\end{array}$ & Not estimable \\
\hline $\begin{array}{l}\text { 5.4.6 Functional training + neuro- } \\
\text { physiological + musculoskeletal }\end{array}$ & 1 & 109 & $\begin{array}{l}\text { Std. Mean Difference (IV, Random, } \\
95 \% \mathrm{CI} \text { ) }\end{array}$ & $0.19[-0.21,0.58]$ \\
\hline
\end{tabular}




\section{Analysis 5.1. Comparison 5: Intervention versus usual care or attention control: persisting outcomes, Outcome 1: Independence in ADL scales}

\begin{tabular}{|c|c|c|c|c|c|c|c|}
\hline & & rven & & & & Std. Mean Difference & Std. Mean Difference \\
\hline tudy or Subgroup & Mean & SD & Mean & Total & Weight & IV, Random, 95\% CI & IV, Random, 95\% CI \\
\hline
\end{tabular}

5.1.1 Functional task training Subtotal (95\% CI)

Not estimable

Heterogeneity: Not applicable

Test for overall effect: Not applicable

5.1.2 Functional task training + musculoskeletal Subtotal (95\% CI)

$\mathbf{0}$

Not estimable

Heterogeneity: Not applicable

Test for overall effect: Not applicable

5.1.3 Neurophysiological

Subtotal (95\% CI)

0

0

Not estimable

Heterogeneity: Not applicable

Test for overall effect: Not applicable

5.1.4 Neurophysiological + musculoskeletal

Subtotal (95\% CI)

0

Heterogeneity: Not applicable

Test for overall effect: Not applicable

5.1.5 Functional training + neurophysiological Subtotal (95\% CI)

Not estimable

(eterogeneity: Not applicable

Test for overall effect: Not applicable

5.1.6 Functional training + neurophysiological + musculoskeletal Subtotal (95\% CI)

Heterogeneity: Not applicable

Test for overall effect: Not applicable

Total (95\% CI)

Heterogeneity: Not applicable

Test for overall effect: Not applicable

Test for subgroup differences: Not applicable
IV, Random, 95\% CI 


\section{Analysis 5.2. Comparison 5: Intervention versus usual care or attention control: persisting outcomes, Outcome 2: Motor function scales}

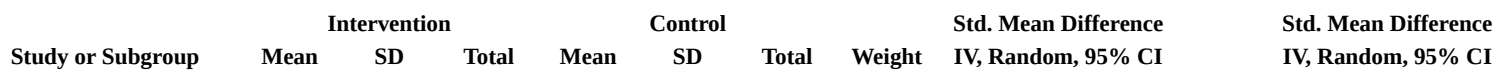

5.2.1 Functional task training

McClellan 2004

Subtotal (95\% CI)

Heterogeneity: Not applicable

Test for overall effect: $\mathrm{Z}=0.38(\mathrm{P}=0.70)$

5.2.2 Functional task training + musculoskeletal

$\begin{array}{llll}\text { Mudge } 2009(1) & 14 & 2.5 & 31 \\ \text { Subtotal (95\% CI) } & & & 31 \\ \text { Heterogeneity: Not applicable } & & \end{array}$

Heterogeneity: Not applicable

Test for overall effect: $\mathrm{Z}=0.00(\mathrm{P}=1.00)$

5.2.3 Neurophysiological

Subtotal (95\% CI)

$\mathbf{0}$

Heterogeneity: Not applicable

Test for overall effect: Not applicable

5.2.4 Neurophysiological + musculoskeletal

Subtotal (95\% CI)

Heterogeneity: Not applicable

Test for overall effect: Not applicable

5.2.5 Functional training + neurophysiological

Subtotal (95\% CI)

Heterogeneity: Not applicable

Test for overall effect: Not applicable

5.2.6 Functional training + neurophysiological + musculoskeletal

$\begin{array}{lllllllll}\text { Cooke 2006 (2) } & 39.9 & 7.2 & 28 & 39.7 & 5.7 & 11 & 21.8 \% & 0.03[-0.67,0.73] \\ \text { Cooke 2006 (3) } & 36.6 & 9.8 & 28 & 39.7 & 5.7 & 12 & 22.9 \% & -0.34[-1.03,0.34] \\ \text { Subtotal (95\% CI) } & & & \mathbf{5 6} & & & \mathbf{2 3} & \mathbf{4 4 . 6 \%} & \mathbf{- 0 . 1 6}[\mathbf{- 0 . 6 5 , 0 . 3 2}]\end{array}$

Subtotal (95\% CI)

$0.45) ; I^{2}=0 \%$

Heterogeneity: $\mathrm{Tau}^{2}=0.00 ; \mathrm{Chi}^{2}=0.56, \mathrm{~d}$
Test for overall effect: $\mathrm{Z}=0.65(\mathrm{P}=0.51)$

Total (95\% CI)

100

Heterogeneity: $\mathrm{Tau}^{2}=0.00 ; \mathrm{Chi}^{2}=0.79, \mathrm{df}=3(\mathrm{P}=0.85) ; \mathrm{I}^{2}=0 \%$

Test for overall effect: $\mathrm{Z}=0.59(\mathrm{P}=0.56)$

Test for subgroup differences: $\mathrm{Chi}^{2}=0.23, \mathrm{df}=2(\mathrm{P}=0.89), \mathrm{I}^{2}=0 \%$

$60 \quad 100.0 \%$

$-0.10[-0.42,0.23]$

$0.00[-0.52,0.52]$

$0.00[-0.52,0.52]$

Not estimable

Not estimable

Not estimable

Footnotes

(1) SDs estimated from range ((max-min range)/4)

(2) Intervention 2 vs usual care. Shared control data.

(3) Intervention 1 vs usual care. Shared control data.
IV, Random, 95\% CI 


\section{Analysis 5.3. Comparison 5: Intervention versus usual care or attention control: persisting outcomes, Outcome 3: Balance (Berg Balance Scale)}

\begin{tabular}{|c|c|c|c|c|c|c|c|}
\hline & & rven & & & & Std. Mean Difference & Std. Mean Difference \\
\hline tudy or Subgroup & Mean & SD & Mean & Total & Weight & IV, Random, 95\% CI & IV, Random, 95\% CI \\
\hline
\end{tabular}

5.3.1 Functional task training

Subtotal (95\% CI)

Not estimable

Heterogeneity: Not applicable

Test for overall effect: Not applicable

5.3.2 Functional task training + musculoskeletal

Subtotal (95\% CI)

$\mathbf{0}$

Not estimable

:

Test for overall effect: Not applicable

5.3.3 Neurophysiological

Subtotal (95\% CI)

0

Not estimable

Heterogeneity: Not applicable

Test for overall effect: Not applicable

5.3.4 Neurophysiological + musculoskeletal

Subtotal (95\% CI)

0

Heterogeneity: Not applicable

Test for overall effect: Not applicable

5.3.5 Functional training + neurophysiological

Subtotal (95\% CI)

Not estimable

(eterogeneity: Not applicable

Test for overall effect: Not applicable

5.3.6 Functional training + neurophysiological + musculoskeletal

Subtotal (95\% CI)

0

Heterogeneity: Not applicable

Test for overall effect: Not applicable

Total (95\% CI)

Heterogeneity: Not applicable

Test for overall effect: Not applicable

Test for subgroup differences: Not applicable
IV, Random, 95\% CI 


\section{Analysis 5.4. Comparison 5: Intervention versus usual care or} attention control: persisting outcomes, Outcome 4: Gait velocity

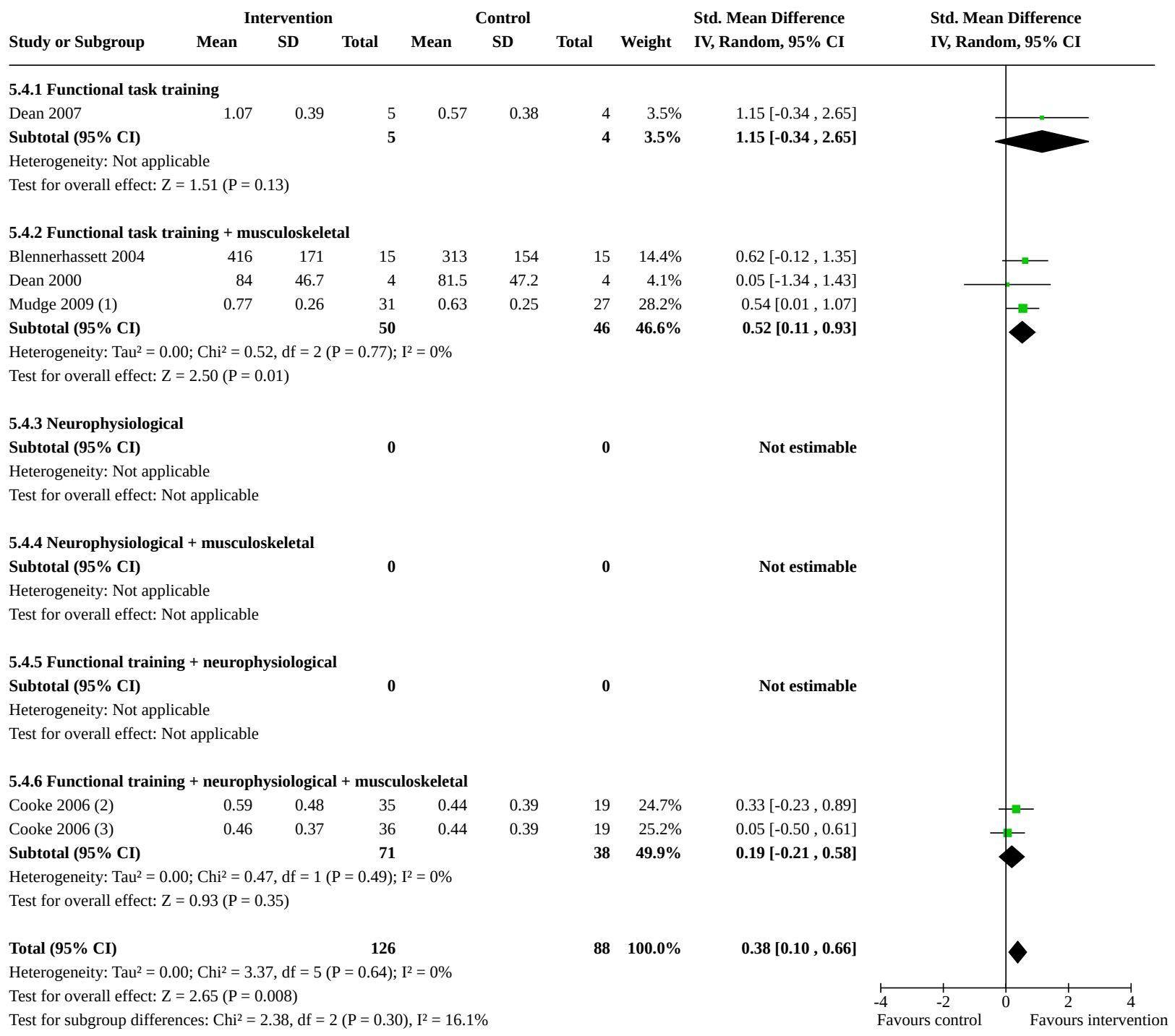

Footnotes

(1) SDs estimated from range ((max-min range)/4)

(2) Intervention 1 vs usual care. Control group shared.

(3) Intervention 2 vs usual care. Control group shared.

\section{Comparison 6. One active intervention versus another active intervention: persisting outcomes}

\begin{tabular}{lllll}
\hline Outcome or subgroup title & No. of studies & $\begin{array}{l}\text { No. of partici- } \\
\text { pants }\end{array}$ & Statistical method & Effect size \\
\hline 6.1 Independence in ADL scales & 2 & & $\begin{array}{l}\text { Std. Mean Difference (IV, } \\
\text { Random, 95\% CI) }\end{array}$ & Subtotals only \\
\hline $\begin{array}{l}\text { 6.1.1 Includes functional training versus } \\
\text { does not include functional training }\end{array}$ & 1 & 30 & $\begin{array}{l}\text { Std. Mean Difference (IV, } \\
\text { Random, 95\% CI) }\end{array}$ & $1.33[0.52,2.13]$ \\
\hline
\end{tabular}




\begin{tabular}{|c|c|c|c|c|}
\hline Outcome or subgroup title & No. of studies & $\begin{array}{l}\text { No. of partici- } \\
\text { pants }\end{array}$ & Statistical method & Effect size \\
\hline $\begin{array}{l}\text { 6.1.2 Includes neurophysiological versus } \\
\text { does not include neurophysiological }\end{array}$ & 2 & 57 & $\begin{array}{l}\text { Std. Mean Difference (IV, } \\
\text { Random, } 95 \% \mathrm{CI} \text { ) }\end{array}$ & $\begin{array}{l}-0.95[-1.67 \\
-0.22]\end{array}$ \\
\hline $\begin{array}{l}\text { 6.1.3 Includes musculoskeletal versus } \\
\text { does not include musculoskeletal }\end{array}$ & 1 & 27 & $\begin{array}{l}\text { Std. Mean Difference (IV, } \\
\text { Random, } 95 \% \mathrm{CI} \text { ) }\end{array}$ & $0.58[-0.19,1.36]$ \\
\hline 6.2 Motor function scales & 0 & 0 & $\begin{array}{l}\text { Std. Mean Difference (IV, } \\
\text { Random, } 95 \% \mathrm{CI} \text { ) }\end{array}$ & Not estimable \\
\hline $\begin{array}{l}\text { 6.2.1 Includes functional training versus } \\
\text { does not include functional training }\end{array}$ & 0 & 0 & $\begin{array}{l}\text { Std. Mean Difference (IV, } \\
\text { Random, } 95 \% \mathrm{CI} \text { ) }\end{array}$ & Not estimable \\
\hline $\begin{array}{l}\text { 6.2.2 Includes neurophysiological versus } \\
\text { does not include neurophysiological }\end{array}$ & 0 & 0 & $\begin{array}{l}\text { Std. Mean Difference (IV, } \\
\text { Random, } 95 \% \mathrm{CI} \text { ) }\end{array}$ & Not estimable \\
\hline $\begin{array}{l}6.2 .3 \text { Includes musculoskeletal versus } \\
\text { does not include musculoskeletal }\end{array}$ & 0 & 0 & $\begin{array}{l}\text { Std. Mean Difference (IV, } \\
\text { Random, } 95 \% \mathrm{CI} \text { ) }\end{array}$ & Not estimable \\
\hline 6.3 Balance (Berg Balance Scale) & 0 & 0 & $\begin{array}{l}\text { Std. Mean Difference (IV, } \\
\text { Random, } 95 \% \mathrm{CI} \text { ) }\end{array}$ & Not estimable \\
\hline $\begin{array}{l}\text { 6.3.1 Includes functional training versus } \\
\text { does not include functional training }\end{array}$ & 0 & 0 & $\begin{array}{l}\text { Std. Mean Difference (IV, } \\
\text { Random, } 95 \% \mathrm{CI} \text { ) }\end{array}$ & Not estimable \\
\hline $\begin{array}{l}\text { 6.3.2 Includes neurophysiological versus } \\
\text { does not include neurophysiological }\end{array}$ & 0 & 0 & $\begin{array}{l}\text { Std. Mean Difference (IV, } \\
\text { Random, } 95 \% \mathrm{CI} \text { ) }\end{array}$ & Not estimable \\
\hline $\begin{array}{l}\text { 6.3.3 Includes musculoskeletal versus } \\
\text { does not include musculoskeletal }\end{array}$ & 0 & 0 & $\begin{array}{l}\text { Std. Mean Difference (IV, } \\
\text { Random, } 95 \% \mathrm{CI} \text { ) }\end{array}$ & Not estimable \\
\hline 6.4 Gait velocity & 2 & & $\begin{array}{l}\text { Std. Mean Difference (IV, } \\
\text { Random, } 95 \% \mathrm{CI} \text { ) }\end{array}$ & Subtotals only \\
\hline $\begin{array}{l}\text { 6.4.1 Includes functional training versus } \\
\text { does not include functional training }\end{array}$ & 1 & 30 & $\begin{array}{l}\text { Std. Mean Difference (IV, } \\
\text { Random, } 95 \% \mathrm{CI} \text { ) }\end{array}$ & $1.14[0.36,1.92]$ \\
\hline $\begin{array}{l}\text { 6.4.2 Includes neurophysiological versus } \\
\text { does not include neurophysiological }\end{array}$ & 2 & 44 & $\begin{array}{l}\text { Std. Mean Difference (IV, } \\
\text { Random, } 95 \% \mathrm{CI} \text { ) }\end{array}$ & $\begin{array}{l}-0.82[-1.60 \\
-0.05]\end{array}$ \\
\hline $\begin{array}{l}\text { 6.4.3 Includes musculoskeletal versus } \\
\text { does not include musculoskeletal }\end{array}$ & 1 & 14 & $\begin{array}{l}\text { Std. Mean Difference (IV, } \\
\text { Random, } 95 \% \mathrm{CI} \text { ) }\end{array}$ & $0.33[-0.74,1.40]$ \\
\hline
\end{tabular}


Analysis 6.1. Comparison 6: One active intervention versus another active intervention: persisting outcomes, Outcome 1: Independence in ADL scales

\begin{tabular}{|c|c|c|c|c|c|c|c|c|c|}
\hline \multirow{2}{*}{ Study or Subgroup } & \multicolumn{3}{|c|}{ Category included } & \multicolumn{3}{|c|}{ Category not included } & & \multirow{2}{*}{$\begin{array}{l}\text { Std. Mean Difference } \\
\text { IV, Random, 95\% CI }\end{array}$} & \multirow{2}{*}{$\begin{array}{l}\text { Std. Mean Difference } \\
\text { IV, Random, 95\% CI }\end{array}$} \\
\hline & Mean & SD & Total & Mean & SD & Total & Weight & & \\
\hline
\end{tabular}

6.1.1 Includes functional training versus does not include functional training

Subtotal (95\% CI)

$90.67 \quad 5.93$

15

$74.67 \quad 15.52$

$15 \quad 100.0 \%$

$1.33[0.52,2.13]$

Heterogeneity: Not applicable

Test for overall effect: $\mathrm{Z}=3.24(\mathrm{P}=0.001)$

6.1.2 Includes neurophysiological versus does not include neurophysiological

$\begin{array}{lrrrrrrrr}\text { Gelber 1995 (1) } & 106.9 & 20.91 & 15 & 117.5 & 12.12 & 12 & 50.9 \% & -0.58[-1.36,0.19] \\ \text { Verma 2011 } & 74.67 & 15.52 & 15 & 90.67 & 5.93 & 15 & 49.1 \% & -1.33[-2.13,-0.52] \\ \text { Subtotal (95\% CI) } & & & \mathbf{3 0} & & & \mathbf{2 7} & \mathbf{1 0 0 . 0 \%} & \mathbf{- 0 . 9 5 [ - 1 . 6 7 , - 0 . 2 2}]\end{array}$

Subtotal (95\% CI)

$27 \quad 100.0 \%$

$-0.95[-1.67,-0.22]$

Heterogeneity: $\mathrm{Tau}^{2}=0.11 ; \mathrm{Chi}^{2}=1.69, \mathrm{df}=1(\mathrm{P}=0.19) ; \mathrm{I}^{2}=41 \%$

Test for overall effect: $\mathrm{Z}=2.56(\mathrm{P}=0.01)$

6.1.3 Includes musculoskeletal versus does not include musculoskeletal

\begin{tabular}{|c|c|c|c|c|c|c|c|c|}
\hline Gelber 1995 (2) & 117.5 & 12.12 & 12 & 106.9 & 20.91 & 15 & $100.0 \%$ & $0.58[-0.19,1.36]$ \\
\hline Subtotal (95\% CI) & & & 12 & & & 15 & $100.0 \%$ & $0.58[-0.19,1.36]$ \\
\hline
\end{tabular}

Heterogeneity: Not applicable

$0.58[-0.19,1.36]$

Test for overall effect: $\mathrm{Z}=1.47(\mathrm{P}=0.14)$

Test for subgroup differences: $\mathrm{Chi}^{2}=0.00, \mathrm{df}=2(\mathrm{P}<0.00001), \mathrm{I}^{2}=0 \%$

Footnotes

(1) Intervention 1 vs intervention 2. SDs estimated from range ((max-min range)/4).

(2) Intervention 2 vs intervention 1 . SDs estimated from range ((max-min range)/4).

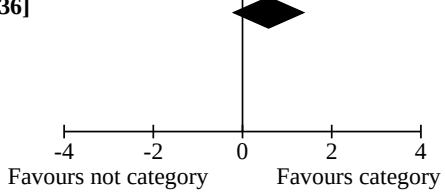

Analysis 6.2. Comparison 6: One active intervention versus another active intervention: persisting outcomes, Outcome 2: Motor function scales

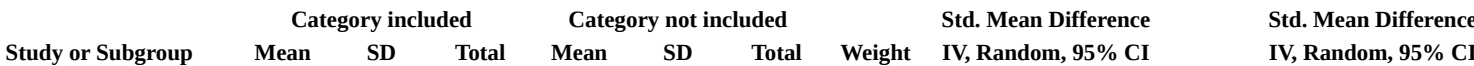

6.2.1 Includes functional training versus does not include functional training

Subtotal (95\% CI)

(0)

Not estimable

Heterogeneity: Not applicable

Test for overall effect: Not applicable

6.2.2 Includes neurophysiological versus does not include neurophysiological Subtotal (95\% CI)

$\mathbf{0}$

0

Not estimable

Heterogeneity: Not applicable

Test for overall effect: Not applicable

6.2.3 Includes musculoskeletal versus does not include musculoskeletal

Subtotal (95\% CI)

0

0

Not estimable

Heterogeneity: Not applicable

Test for overall effect: Not applicable

Total (95\% CI)

0

Not estimable

Heterogeneity: Not applicable

Test for overall effect: Not applicable

Test for subgroup differences: Not applicable 


\section{Analysis 6.3. Comparison 6: One active intervention versus another active} intervention: persisting outcomes, Outcome 3: Balance (Berg Balance Scale)

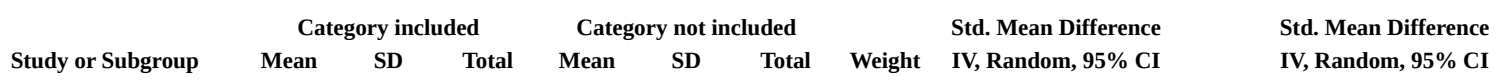

6.3.1 Includes functional training versus does not include functional training Subtotal (95\% CI)

Not estimable

Heterogeneity: Not applicable

Test for overall effect: Not applicable

6.3.2 Includes neurophysiological versus does not include neurophysiological Subtotal $(95 \% \mathrm{CI})$

0

Not estimable

Heterogeneity: Not applicable

Test for overall effect: Not applicable

6.3.3 Includes musculoskeletal versus does not include musculoskeletal Subtotal (95\% CI)

Heterogeneity: Not applicable

Test for overall effect: Not applicable

0

Not estimable

Heterogeneity: Not applicable

Test for overall effect: Not applicable

Test for subgroup differences: Not applicable

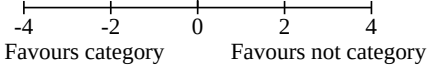

\section{Analysis 6.4. Comparison 6: One active intervention versus another active intervention: persisting outcomes, Outcome 4: Gait velocity}

\begin{tabular}{|c|c|c|c|c|c|c|c|c|c|}
\hline & \multicolumn{3}{|c|}{ Category included } & \multicolumn{3}{|c|}{ Category not included } & \multicolumn{2}{|r|}{ Std. Mean Difference } & Std. Mean Difference \\
\hline Study or Subgroup & Mean & SD & Total & Mean & SD & Total & Weight & IV, Random, 95\% CI & IV, Random, 95\% CI \\
\hline
\end{tabular}

6.4.1 Includes functional training versus does not include functional training

$\begin{array}{lllllllll}\text { Verma 2011 (1) } & 0.62 & 0.14 & 15 & 0.45 & 0.15 & 15 & 100.0 \% & 1.14[0.36,1.92] \\ \text { Subtotal (95\% CI) } & & & \mathbf{1 5} & & & \mathbf{1 5} & \mathbf{1 0 0 . 0 \%} & \mathbf{1 . 1 4}[\mathbf{0 . 3 6 , \mathbf { 1 . 9 2 }}]\end{array}$

Heterogeneity: Not applicable

Test for overall effect: $\mathrm{Z}=2.87(\mathrm{P}=0.004)$

6.4.2 Includes neurophysiological versus does not include neurophysiological

$\begin{array}{lrrrrrrrr}\text { Gelber 1995 (2) } & 0.3 & 0.34 & 8 & 0.42 & 0.34 & 6 & 39.4 \% & -0.33[-1.40,0.74] \\ \text { Verma 2011(3) } & 0.45 & 0.15 & 15 & 0.62 & 0.14 & 15 & 60.6 \% & -1.14[-1.92,-0.36] \\ \text { Subtotal (95\% CI) } & & & \mathbf{2 3} & & & \mathbf{2 1} & \mathbf{1 0 0 . 0 \%} & \mathbf{- 0 . 8 2}[\mathbf{- 1 . 6 0}, \mathbf{- 0 . 0 5}]\end{array}$

Heterogeneity: $\mathrm{Tau}^{2}=0.10 ; \mathrm{Chi}^{2}=1.44, \mathrm{df}=1(\mathrm{P}=0.23) ; \mathrm{I}^{2}=31 \%$

Test for overall effect: $\mathrm{Z}=2.08(\mathrm{P}=0.04)$

6.4.3 Includes musculoskeletal versus does not include musculoskeletal

$\begin{array}{lllllllll}\text { Gelber } 1995 \text { (4) } & 0.42 & 0.34 & 6 & 0.3 & 0.34 & 8 & 100.0 \% & 0.33[-0.74,1.40]\end{array}$

Subtotal $(95 \% \mathrm{CI})$

$8 \mathbf{1 0 0 . 0 \%}$

$0.33[-0.74,1.40]$

Heterogeneity: Not applicable

Test for overall effect: $\mathrm{Z}=0.61(\mathrm{P}=0.54)$

Test for subgroup differences: $\mathrm{Chi}^{2}=0.00, \mathrm{df}=2(\mathrm{P}<0.00001), \mathrm{I}^{2}=0 \%$

Footnotes

(1) Intervention 1 vs intervention 2.

(2) Intervention 1 vs intervention 2 . SDs estimated from range ((max-min range)/4).

(3) Intervention 2 vs intervention 1.

(4) Intervention 2 vs intervention 1. SDs estimated from range ((max-min range)/4). 
Comparison 7. Subgroups. Intervention versus no treatment: immediate outcome: independence in ADL

\begin{tabular}{|c|c|c|c|c|}
\hline $\begin{array}{l}\text { Outcome or subgroup ti- } \\
\text { tle }\end{array}$ & No. of studies & $\begin{array}{l}\text { No. of partici- } \\
\text { pants }\end{array}$ & Statistical method & Effect size \\
\hline 7.1 Time after stroke & 28 & 3423 & $\begin{array}{l}\text { Std. Mean Difference (IV, Random, 95\% } \\
\mathrm{Cl} \text { ) }\end{array}$ & $0.78[0.58,0.97]$ \\
\hline 7.1.1 < 30 days post stroke & 13 & 1195 & $\begin{array}{l}\text { Std. Mean Difference (IV, Random, 95\% } \\
\mathrm{CI})\end{array}$ & $0.86[0.61,1.11]$ \\
\hline $\begin{array}{l}7.1 .2<3 \text { months post } \\
\text { stroke }\end{array}$ & 1 & 70 & $\begin{array}{l}\text { Std. Mean Difference (IV, Random, 95\% } \\
\mathrm{Cl} \text { ) }\end{array}$ & $0.27[-0.20,0.74]$ \\
\hline $7.1 .3<1$ year post stroke & 0 & 0 & $\begin{array}{l}\text { Std. Mean Difference (IV, Random, 95\% } \\
\mathrm{CI} \text { ) }\end{array}$ & Not estimable \\
\hline 7.1.4 > 1 year post stroke & 3 & 295 & $\begin{array}{l}\text { Std. Mean Difference (IV, Random, 95\% } \\
\mathrm{Cl} \text { ) }\end{array}$ & $0.12[-0.29,0.53]$ \\
\hline 7.1.5 Time not stated & 11 & 1863 & $\begin{array}{l}\text { Std. Mean Difference (IV, Random, 95\% } \\
\mathrm{Cl} \text { ) }\end{array}$ & $0.89[0.56,1.22]$ \\
\hline $\begin{array}{l}7.2 \text { Study geographical lo- } \\
\text { cation }\end{array}$ & 28 & 3423 & $\begin{array}{l}\text { Std. Mean Difference (IV, Random, 95\% } \\
\mathrm{CI} \text { ) }\end{array}$ & $0.78[0.58,0.97]$ \\
\hline 7.2.1 Europe & 2 & 250 & $\begin{array}{l}\text { Std. Mean Difference (IV, Random, 95\% } \\
\mathrm{CI})\end{array}$ & $-0.06[-0.30,0.19]$ \\
\hline 7.2.2 Australia & 0 & 0 & $\begin{array}{l}\text { Std. Mean Difference (IV, Random, 95\% } \\
\mathrm{CI})\end{array}$ & Not estimable \\
\hline 7.2.3 Asia: China & 26 & 3173 & $\begin{array}{l}\text { Std. Mean Difference (IV, Random, 95\% } \\
\mathrm{Cl} \text { ) }\end{array}$ & $0.85[0.66,1.04]$ \\
\hline 7.2.4 Asia: other & 0 & 0 & $\begin{array}{l}\text { Std. Mean Difference (IV, Random, 95\% } \\
\mathrm{Cl} \text { ) }\end{array}$ & Not estimable \\
\hline 7.2.5 North America & 0 & 0 & $\begin{array}{l}\text { Std. Mean Difference (IV, Random, 95\% } \\
\mathrm{Cl} \text { ) }\end{array}$ & Not estimable \\
\hline 7.3 Dose of intervention & 28 & 3423 & $\begin{array}{l}\text { Std. Mean Difference (IV, Random, 95\% } \\
\mathrm{Cl} \text { ) }\end{array}$ & $0.78[0.58,0.97]$ \\
\hline $\begin{array}{l}7.3 .1>\text { once/d, with total } \\
\text { of } 60 \text { to } 120 \text { minutes }\end{array}$ & 8 & 711 & $\begin{array}{l}\text { Std. Mean Difference (IV, Random, 95\% } \\
\mathrm{Cl} \text { ) }\end{array}$ & $1.23[1.01,1.45]$ \\
\hline $\begin{array}{l}7.3 .2 \text { Once } / \mathrm{d}, 5 \text { to } 7 \times / w k \text {, } \\
\text { for } 30 \text { to } 60 \text { minutes }\end{array}$ & 12 & 1027 & $\begin{array}{l}\text { Std. Mean Difference (IV, Random, 95\% } \\
\mathrm{CI})\end{array}$ & $0.77[0.46,1.08]$ \\
\hline $7.3 .32 \times / w k$ & 2 & 173 & $\begin{array}{l}\text { Std. Mean Difference (IV, Random, 95\% } \\
\mathrm{CI})\end{array}$ & $0.32[-0.35,0.98]$ \\
\hline $\begin{array}{l}7.3 .41 \text { to } 11 \text { visits (to as- } \\
\text { sess/give exercises for self } \\
\text { practice) }\end{array}$ & 2 & 250 & $\begin{array}{l}\text { Std. Mean Difference (IV, Random, 95\% } \\
\mathrm{Cl} \text { ) }\end{array}$ & $-0.06[-0.30,0.19]$ \\
\hline 7.3.5 Dose not stated & 4 & 1262 & $\begin{array}{l}\text { Std. Mean Difference (IV, Random, 95\% } \\
\mathrm{Cl} \text { ) }\end{array}$ & $0.62[0.31,0.92]$ \\
\hline
\end{tabular}




\begin{tabular}{|c|c|c|c|c|}
\hline $\begin{array}{l}\text { Outcome or subgroup ti- } \\
\text { tle }\end{array}$ & No. of studies & $\begin{array}{l}\text { No. of partici- } \\
\text { pants }\end{array}$ & Statistical method & Effect size \\
\hline $\begin{array}{l}\text { 7.4 Provider of interven- } \\
\text { tion }\end{array}$ & 28 & 3423 & $\begin{array}{l}\text { Std. Mean Difference (IV, Random, 95\% } \\
\mathrm{Cl} \text { ) }\end{array}$ & $0.78[0.58,0.97]$ \\
\hline 7.4.1 Physiotherapist & 2 & 250 & $\begin{array}{l}\text { Std. Mean Difference (IV, Random, 95\% } \\
\mathrm{Cl} \text { ) }\end{array}$ & $-0.06[-0.30,0.19]$ \\
\hline 7.4.2 Therapist & 5 & 1158 & $\begin{array}{l}\text { Std. Mean Difference (IV, Random, 95\% } \\
\mathrm{CI} \text { ) }\end{array}$ & $0.79[0.32,1.26]$ \\
\hline 7.4.3 Therapist + family & 6 & 429 & $\begin{array}{l}\text { Std. Mean Difference (IV, Random, 95\% } \\
\mathrm{CI})\end{array}$ & $0.83[0.47,1.20]$ \\
\hline 7.4.4 Nurse & 2 & 128 & $\begin{array}{l}\text { Std. Mean Difference (IV, Random, 95\% } \\
\mathrm{Cl} \text { ) }\end{array}$ & $0.93[-0.39,2.24]$ \\
\hline 7.4.5 Not stated & 13 & 1458 & $\begin{array}{l}\text { Std. Mean Difference (IV, Random, 95\% } \\
\mathrm{CI} \text { ) }\end{array}$ & $0.87[0.59,1.15]$ \\
\hline $\begin{array}{l}\text { 7.5 Treatment compo- } \\
\text { nents included }\end{array}$ & 28 & & $\begin{array}{l}\text { Std. Mean Difference (IV, Random, 95\% } \\
\mathrm{Cl} \text { ) }\end{array}$ & Subtotals only \\
\hline $\begin{array}{l}\text { 7.5.1 Contains functional } \\
\text { training }\end{array}$ & 23 & 3055 & $\begin{array}{l}\text { Std. Mean Difference (IV, Random, 95\% } \\
\mathrm{Cl} \text { ) }\end{array}$ & $0.87[0.66,1.08]$ \\
\hline $\begin{array}{l}\text { 7.5.2 Contains neurophysi- } \\
\text { ological }\end{array}$ & 15 & 2106 & $\begin{array}{l}\text { Std. Mean Difference (IV, Random, 95\% } \\
\mathrm{CI} \text { ) }\end{array}$ & $0.87[0.61,1.14]$ \\
\hline $\begin{array}{l}\text { 7.5.3 Contains muscu- } \\
\text { loskeletal }\end{array}$ & 24 & 3033 & $\begin{array}{l}\text { Std. Mean Difference (IV, Random, 95\% } \\
\mathrm{Cl} \text { ) }\end{array}$ & $0.85[0.65,1.05]$ \\
\hline
\end{tabular}


Analysis 7.1. Comparison 7: Subgroups. Intervention versus no treatment: immediate outcome: independence in ADL, Outcome 1: Time after stroke

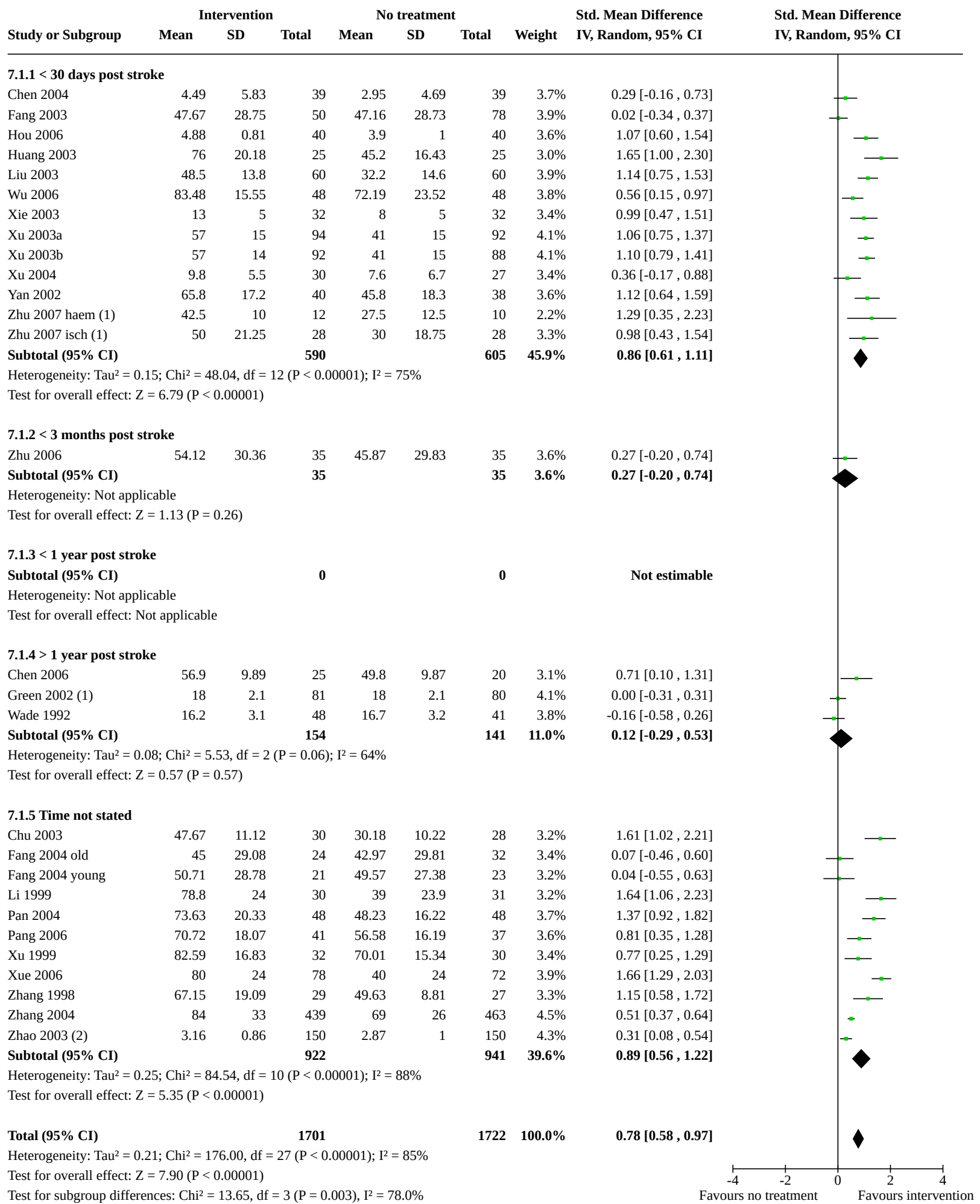

Footnotes

(1) SDs estimated from range ((max-min range)/4).

(2) Mean and SD computed from categorical data 


\section{Analysis 7.1. (Continued)}

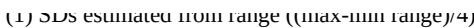

(2) Mean and SD computed from categorical data 
Analysis 7.2. Comparison 7: Subgroups. Intervention versus no treatment: immediate outcome: independence in ADL, Outcome 2: Study geographical location

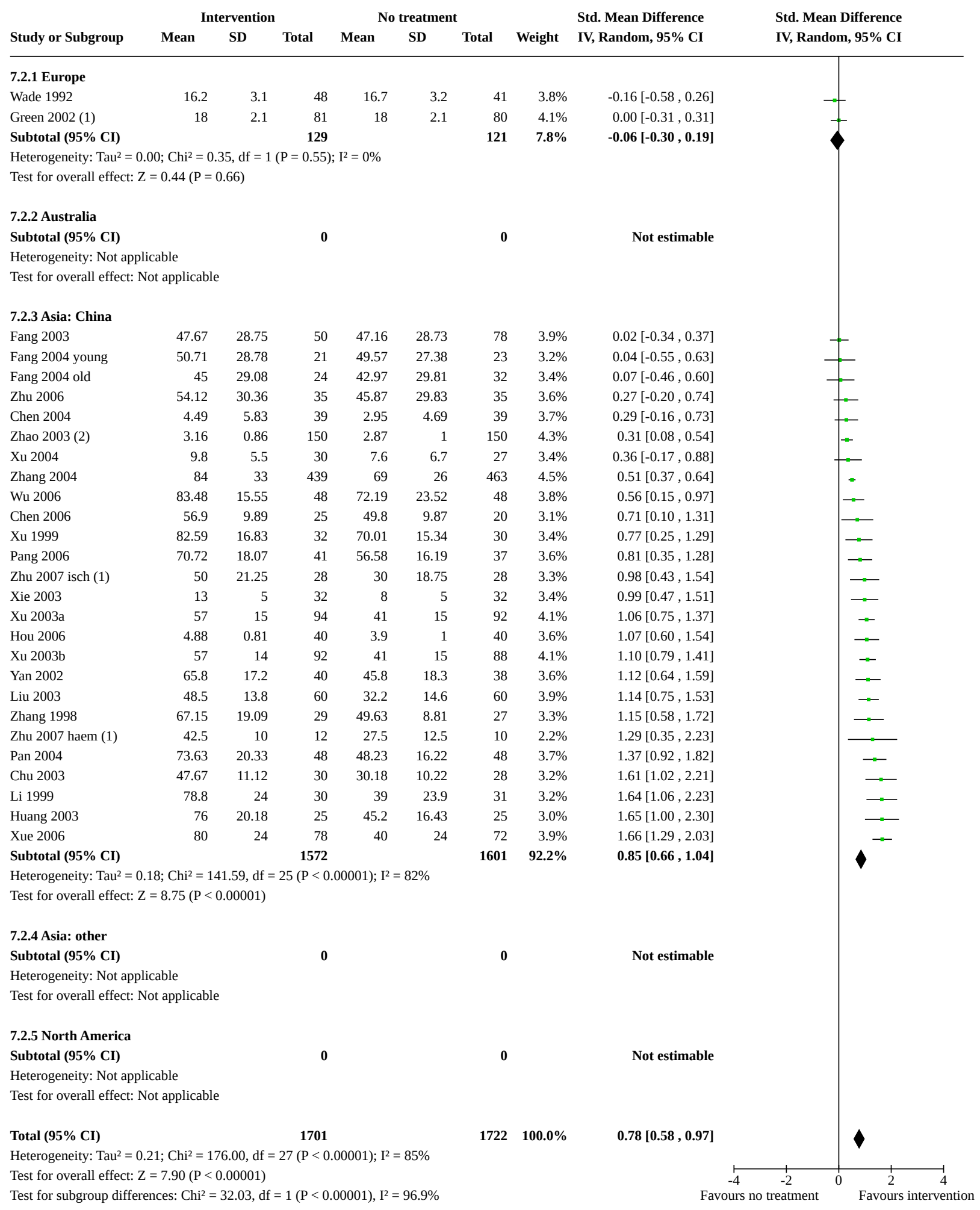

Footnotes

(1) SDs estimated from range ((max-min range)/4)

(2) Mean and SD computed from categorical data. 


\section{Analysis 7.2. (Continued)}

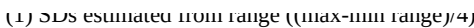

(2) Mean and SD computed from categorical data. 
Analysis 7.3. Comparison 7: Subgroups. Intervention versus no treatment: immediate outcome: independence in ADL, Outcome 3: Dose of intervention

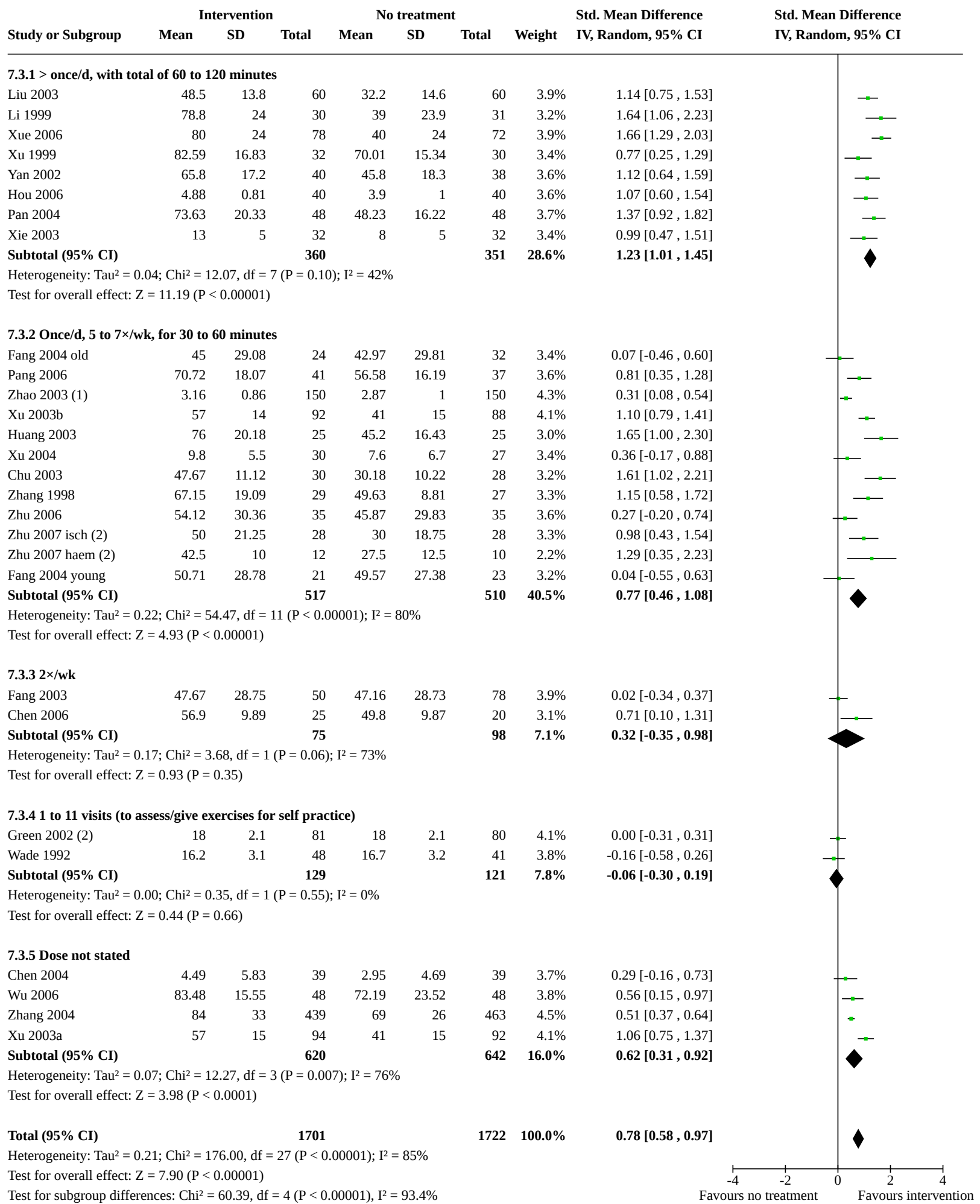

Footnotes

(1) Mean and SD calculated from categorical data.

(2) SDs estimated from range ((max-min range)/4) 


\section{Analysis 7.3. (Continued)}

(1) vileall allu

(2) SDs estimated from range ((max-min range)/4) 
Analysis 7.4. Comparison 7: Subgroups. Intervention versus no treatment: immediate outcome: independence in ADL, Outcome 4: Provider of intervention

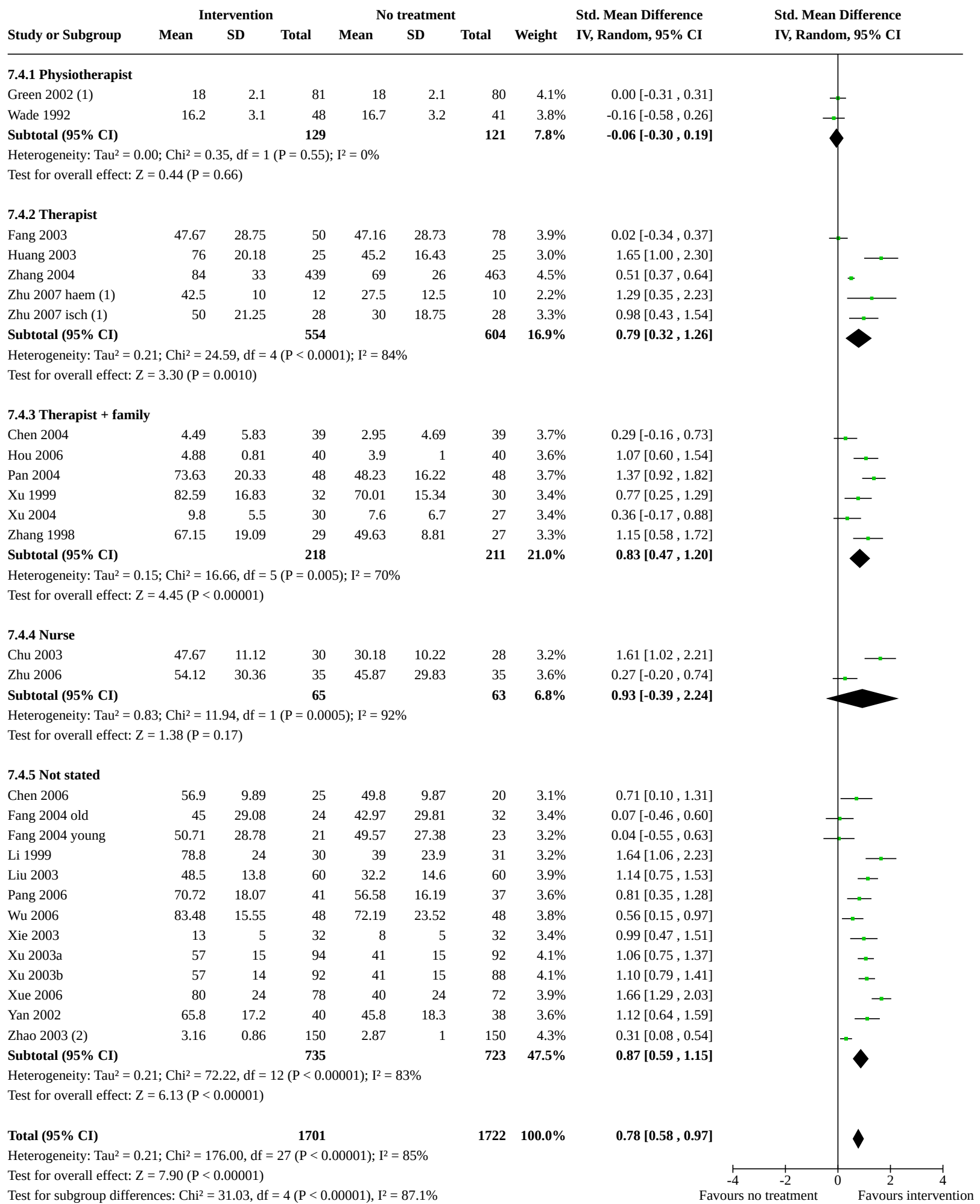

Footnotes

(1) SDs estimated from range ((max-min range)/4)

(2) Mean and SD computed from categorical data. 


\section{Analysis 7.4. (Continued)}

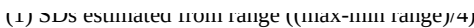

(2) Mean and SD computed from categorical data. 
Analysis 7.5. Comparison 7: Subgroups. Intervention versus no treatment: immediate outcome: independence in ADL, Outcome 5: Treatment components included

\begin{tabular}{|c|c|c|c|c|c|c|c|c|c|}
\hline \multirow[b]{2}{*}{ Study or Subgroup } & \multicolumn{3}{|c|}{ Intervention } & \multicolumn{3}{|c|}{ No treatment } & \multirow[b]{2}{*}{ Weight } & \multirow{2}{*}{$\begin{array}{l}\text { Std. Mean Difference } \\
\text { IV, Random, } 95 \% \text { CI }\end{array}$} & \multirow{2}{*}{$\begin{array}{l}\text { Std. Mean Difference } \\
\text { IV, Random, } 95 \% \text { CI }\end{array}$} \\
\hline & Mean & SD & Total & Mean & SD & Total & & & \\
\hline \multicolumn{10}{|c|}{ 7.5.1 Contains functional training } \\
\hline Chen 2004 & 4.49 & 5.83 & 39 & 2.95 & 4.69 & 39 & $4.4 \%$ & $0.29[-0.16,0.73]$ & \\
\hline Chen 2006 & 56.9 & 9.89 & 25 & 49.8 & 9.87 & 20 & $3.8 \%$ & $0.71[0.10,1.31]$ & $\ldots$ \\
\hline Chu 2003 & 47.67 & 11.12 & 30 & 30.18 & 10.22 & 28 & $3.8 \%$ & $1.61[1.02,2.21]$ & —- \\
\hline Green 2002 & 18 & 2.1 & 81 & 18 & 2.1 & 80 & $4.9 \%$ & $0.00[-0.31,0.31]$ & 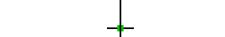 \\
\hline Hou 2006 & 4.88 & 0.81 & 40 & 3.9 & 1 & 40 & $4.4 \%$ & $1.07[0.60,1.54]$ & 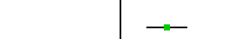 \\
\hline Huang 2003 & 76 & 20.18 & 25 & 45.2 & 16.43 & 25 & $3.7 \%$ & $1.65[1.00,2.30]$ & $\longrightarrow$ \\
\hline Li 1999 & 78.8 & 24 & 30 & 39 & 23.9 & 31 & $3.9 \%$ & $1.64[1.06,2.23]$ & 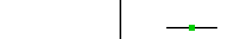 \\
\hline Liu 2003 & 48.5 & 13.8 & 60 & 32.2 & 14.6 & 60 & $4.7 \%$ & $1.14[0.75,1.53]$ & 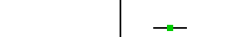 \\
\hline Pan 2004 & 73.63 & 20.33 & 48 & 48.23 & 16.22 & 48 & $4.4 \%$ & $1.37[0.92,1.82]$ & - \\
\hline Wade 1992 & 16.2 & 3.1 & 48 & 16.7 & 3.2 & 41 & $4.6 \%$ & $-0.16[-0.58,0.26]$ & 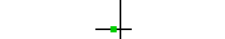 \\
\hline Wu 2006 & 83.48 & 15.55 & 48 & 72.19 & 23.52 & 48 & $4.6 \%$ & $0.56[0.15,0.97]$ & - \\
\hline Xie 2003 & 13 & 5 & 32 & 8 & 5 & 32 & $4.2 \%$ & $0.99[0.47,1.51]$ & -- \\
\hline Xu 2003a & 57 & 15 & 94 & 41 & 15 & 92 & $4.9 \%$ & $1.06[0.75,1.37]$ & -- \\
\hline Xu 2003b & 57 & 14 & 92 & 41 & 15 & 88 & $4.9 \%$ & $1.10[0.79,1.41]$ & - \\
\hline Xu 2004 & 9.8 & 5.5 & 30 & 7.6 & 6.7 & 27 & $4.1 \%$ & $0.36[-0.17,0.88]$ & \\
\hline Xue 2006 & 80 & 24 & 78 & 40 & 24 & 72 & $4.7 \%$ & $1.66[1.29,2.03]$ & -- \\
\hline Yan 2002 & 65.8 & 17.2 & 40 & 45.8 & 18.3 & 38 & $4.3 \%$ & $1.12[0.64,1.59]$ & 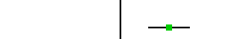 \\
\hline Zhang 1998 & 67.15 & 19.09 & 29 & 49.63 & 8.81 & 27 & $4.0 \%$ & $1.15[0.58,1.72]$ & — \\
\hline Zhang 2004 & 84 & 33 & 439 & 69 & 26 & 463 & $5.4 \%$ & $0.51[0.37,0.64]$ & $=$ \\
\hline Zhao 2003 (1) & 3.16 & 0.86 & 150 & 2.87 & 1 & 150 & $5.2 \%$ & $0.31[0.08,0.54]$ & - \\
\hline Zhu 2006 & 54.12 & 30.36 & 35 & 45.87 & 29.83 & 35 & $4.3 \%$ & $0.27[-0.20,0.74]$ & - \\
\hline Zhu 2007 haem (2) & 42.5 & 10 & 12 & 27.5 & 12.5 & 10 & $2.7 \%$ & $1.29[0.35,2.23]$ & \\
\hline Zhu 2007 isch (2) & 50 & 21.25 & 28 & 30 & 18.75 & 28 & $4.0 \%$ & $0.98[0.43,1.54]$ & \\
\hline Subtotal (95\% CI) & & & 1533 & & & 1522 & $100.0 \%$ & $0.87[0.66,1.08]$ & \\
\hline \multicolumn{10}{|c|}{ Heterogeneity: $\mathrm{Tau}^{2}=0.22 ; \mathrm{Chi}^{2}=152.92, \mathrm{df}=22(\mathrm{P}<0.00001) ; \mathrm{I}^{2}=86 \%$} \\
\hline \multicolumn{10}{|c|}{ Test for overall effect: $\mathrm{Z}=7.98(\mathrm{P}<0.00001)$} \\
\hline \multicolumn{10}{|c|}{ 7.5.2 Contains neurophysiological } \\
\hline Chen 2004 & 4.49 & 5.83 & 39 & 2.95 & 4.69 & 39 & $6.8 \%$ & $0.29[-0.16,0.73]$ & - \\
\hline Chu 2003 & 47.67 & 11.12 & 30 & 30.18 & 10.22 & 28 & $5.9 \%$ & $1.61[1.02,2.21]$ & 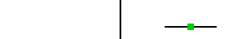 \\
\hline Fang 2003 & 47.67 & 28.75 & 50 & 47.16 & 28.73 & 78 & $7.3 \%$ & $0.02[-0.34,0.37]$ & - \\
\hline Hou 2006 & 4.88 & 0.81 & 40 & 3.9 & 1 & 40 & $6.6 \%$ & $1.07[0.60,1.54]$ & -- \\
\hline Huang 2003 & 76 & 20.18 & 25 & 45.2 & 16.43 & 25 & $5.6 \%$ & $1.65[1.00,2.30]$ & —- \\
\hline Li 1999 & 78.8 & 24 & 30 & 39 & 23.9 & 31 & $5.9 \%$ & $1.64[1.06,2.23]$ & — \\
\hline Pang 2006 & 70.72 & 18.07 & 41 & 56.58 & 16.19 & 37 & $6.7 \%$ & $0.81[0.35,1.28]$ & -- \\
\hline Wu 2006 & 83.48 & 15.55 & 48 & 72.19 & 23.52 & 48 & $7.0 \%$ & $0.56[0.15,0.97]$ & -- \\
\hline Xu 1999 & 82.59 & 16.83 & 32 & 70.01 & 15.34 & 30 & $6.4 \%$ & $0.77[0.25,1.29]$ & 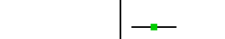 \\
\hline Xu 2003b & 57 & 14 & 92 & 41 & 15 & 88 & $7.5 \%$ & $1.10[0.79,1.41]$ & - \\
\hline Xu 2004 & 9.8 & 5.5 & 30 & 7.6 & 6.7 & 27 & $6.3 \%$ & $0.36[-0.17,0.88]$ & - \\
\hline Xue 2006 & 80 & 24 & 78 & 40 & 24 & 72 & $7.2 \%$ & $1.66[1.29,2.03]$ & - \\
\hline Zhang 1998 & 67.15 & 19.09 & 29 & 49.63 & 8.81 & 27 & $6.0 \%$ & $1.15[0.58,1.72]$ & - \\
\hline Zhang 2004 & 84 & 33 & 439 & 69 & 26 & 463 & $8.2 \%$ & $0.51[0.37,0.64]$ & $=$ \\
\hline Zhu 2006 & 54.12 & 30.36 & 35 & 45.87 & 29.83 & 35 & $6.6 \%$ & $0.27[-0.20,0.74]$ & - \\
\hline Subtotal (95\% CI) & & & 1038 & & & 1068 & $100.0 \%$ & $0.87[0.61,1.14]$ & \\
\hline \multicolumn{9}{|c|}{ Heterogeneity: $\mathrm{Tau}^{2}=0.22 ; \mathrm{Chi}^{2}=94.68, \mathrm{df}=14(\mathrm{P}<0.00001) ; \mathrm{I}^{2}=85 \%$} & \\
\hline \multicolumn{10}{|c|}{ Test for overall effect: $\mathrm{Z}=6.45(\mathrm{P}<0.00001)$} \\
\hline \multicolumn{10}{|c|}{ 7.5.3 Contains musculoskeletal } \\
\hline Chen 2004 & 4.49 & 5.83 & 39 & 2.95 & 4.69 & 39 & $4.3 \%$ & $0.29[-0.16,0.73]$ & $=$ \\
\hline Chen 2006 & 56.9 & 9.89 & 25 & 49.8 & 9.87 & 20 & $3.7 \%$ & $0.71[0.10,1.31]$ & —- \\
\hline Chu 2003 & 47.67 & 11.12 & 30 & 30.18 & 10.22 & 28 & $3.7 \%$ & $1.61[1.02,2.21]$ & —- \\
\hline Fang 2003 & 47.67 & 28.75 & 50 & 47.16 & 28.73 & 78 & $4.7 \%$ & $0.02[-0.34,0.37]$ & - \\
\hline Fang 2004 old & 45 & 29.08 & 24 & 42.97 & 29.81 & 32 & $4.0 \%$ & $0.07[-0.46,0.60]$ & - \\
\hline Fang 2004 young & 50.71 & 28.78 & 21 & 49.57 & 27.38 & 23 & $3.7 \%$ & $0.04[-0.55,0.63]$ & - \\
\hline Hou 2006 & 4.88 & 0.81 & 40 & 3.9 & 1 & 40 & $4.2 \%$ & $1.07[0.60,1.54]$ & $\rightarrow$ \\
\hline Huang 2003 & 76 & 20.18 & 25 & 45.2 & 16.43 & 25 & $3.5 \%$ & $1.65[1.00,2.30]$ & - \\
\hline Li 1999 & 78.8 & 24 & 30 & 39 & 23.9 & 31 & $3.7 \%$ & $1.64[1.06,2.23]$ & 一 \\
\hline Liu 2003 & 48.5 & 13.8 & 60 & 32.2 & 14.6 & 60 & $4.5 \%$ & $1.14[0.75,1.53]$ & - \\
\hline Pan 2004 & 73.63 & 20.33 & 48 & 48.23 & 16.22 & 48 & $4.3 \%$ & $1.37[0.92,1.82]$ & 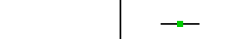 \\
\hline Wu 2006 & 83.48 & 15.55 & 48 & 72.19 & 23.52 & 48 & $4.5 \%$ & $0.56[0.15,0.97]$ & 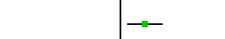 \\
\hline $\mathrm{X}$ is 0 กก? & 12 & 5 & 3) & 8 & 5 & 3) & $\Delta n \%$ & ก ११ [ก $\Delta 7 \quad 1511$ & \\
\hline
\end{tabular}




\section{Analysis 7.5. (Continued)}

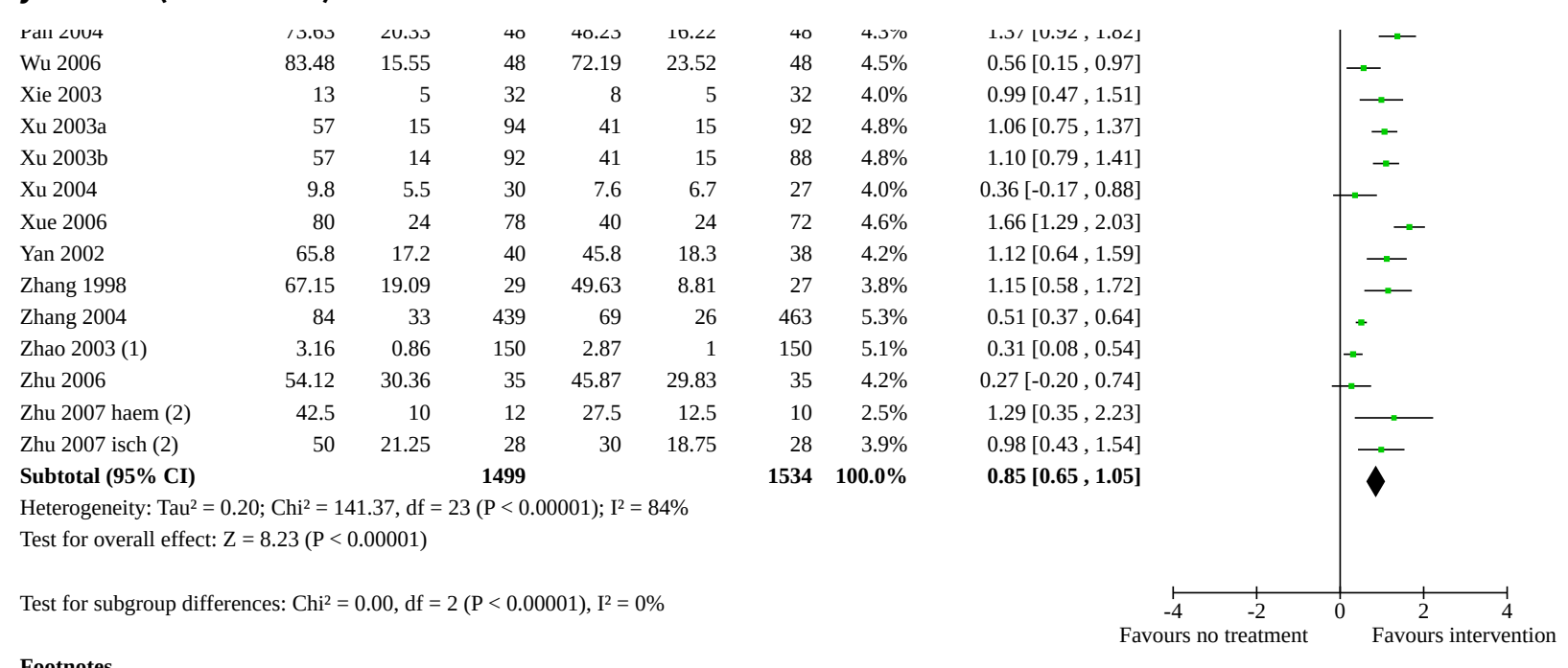

(1) Mean and SD calculated from categorical data.

(2) SDs estimated from range ((max-min range)/4)

Comparison 8. Subgroups. Intervention versus attention control or usual care: immediate outcome: independence in ADL

\begin{tabular}{|c|c|c|c|c|}
\hline Outcome or subgroup title & No. of studies & $\begin{array}{l}\text { No. of partici- } \\
\text { pants }\end{array}$ & Statistical method & Effect size \\
\hline 8.1 Time after stroke & 6 & 260 & $\begin{array}{l}\text { Std. Mean Difference (IV, Random, } \\
95 \% \mathrm{CI})\end{array}$ & $0.04[-0.27,0.35]$ \\
\hline $8.1 .1<30$ days post stroke & 2 & 129 & $\begin{array}{l}\text { Std. Mean Difference (IV, Random, } \\
95 \% \mathrm{CI})\end{array}$ & $0.42[0.07,0.77]$ \\
\hline 8.1.2 $<3$ months post stroke & 3 & 66 & $\begin{array}{l}\text { Std. Mean Difference (IV, Random, } \\
95 \% \mathrm{CI})\end{array}$ & $-0.20[-0.71,0.31]$ \\
\hline $8.1 .3<1$ year post stroke & 0 & 0 & $\begin{array}{l}\text { Std. Mean Difference (IV, Random, } \\
95 \% \mathrm{CI})\end{array}$ & Not estimable \\
\hline 8.1.4 > 1 year post stroke & 0 & 0 & $\begin{array}{l}\text { Std. Mean Difference (IV, Random, } \\
95 \% \mathrm{CI})\end{array}$ & Not estimable \\
\hline 8.1.5 Time not stated & 1 & 65 & $\begin{array}{l}\text { Std. Mean Difference (IV, Random, } \\
95 \% \mathrm{CI})\end{array}$ & $-0.19[-0.68,0.30]$ \\
\hline $\begin{array}{l}8.2 \text { Study geographical lo- } \\
\text { cation }\end{array}$ & 6 & 260 & $\begin{array}{l}\text { Std. Mean Difference (IV, Fixed, 95\% } \\
\mathrm{CI} \text { ) }\end{array}$ & $0.11[-0.14,0.36]$ \\
\hline 8.2.1 Europe & 2 & 81 & $\begin{array}{l}\text { Std. Mean Difference (IV, Fixed, 95\% } \\
\mathrm{CI} \text { ) }\end{array}$ & $-0.17[-0.62,0.27]$ \\
\hline 8.2.2 Australia & 1 & 30 & $\begin{array}{l}\text { Std. Mean Difference (IV, Fixed, 95\% } \\
\text { CI) }\end{array}$ & $-0.47[-1.24,0.31]$ \\
\hline
\end{tabular}




\begin{tabular}{|c|c|c|c|c|}
\hline Outcome or subgroup title & No. of studies & $\begin{array}{l}\text { No. of partici- } \\
\text { pants }\end{array}$ & Statistical method & Effect size \\
\hline 8.2.3 Asia: China & 1 & 106 & $\begin{array}{l}\text { Std. Mean Difference (IV, Fixed, 95\% } \\
\mathrm{Cl} \text { ) }\end{array}$ & $0.53[0.14,0.92]$ \\
\hline 8.2.4 Asia: other & 0 & 0 & $\begin{array}{l}\text { Std. Mean Difference (IV, Fixed, 95\% } \\
\mathrm{Cl} \text { ) }\end{array}$ & Not estimable \\
\hline 8.2.5 North America & 2 & 43 & $\begin{array}{l}\text { Std. Mean Difference (IV, Fixed, 95\% } \\
\mathrm{Cl} \text { ) }\end{array}$ & $-0.02[-0.63,0.60]$ \\
\hline 8.3 Dose of intervention & 6 & 260 & $\begin{array}{l}\text { Std. Mean Difference (IV, Random, } \\
95 \% \mathrm{CI})\end{array}$ & $0.04[-0.27,0.35]$ \\
\hline $\begin{array}{l}8.3 .1>\text { once/d, with total of } \\
60 \text { to } 120 \text { minutes }\end{array}$ & 0 & 0 & $\begin{array}{l}\text { Std. Mean Difference (IV, Random, } \\
95 \% \mathrm{Cl})\end{array}$ & Not estimable \\
\hline $\begin{array}{l}8.3 .2 \text { Once/d, } 5 \text { to } 7 \times / w k \text {, for } \\
30 \text { to } 60 \text { minutes }\end{array}$ & 2 & 46 & $\begin{array}{l}\text { Std. Mean Difference (IV, Random, } \\
95 \% \mathrm{Cl} \text { ) }\end{array}$ & $-0.34[-0.96,0.29]$ \\
\hline 8.3 .32 to $3 \times / w k$ & 2 & 85 & $\begin{array}{l}\text { Std. Mean Difference (IV, Random, } \\
95 \% \mathrm{Cl})\end{array}$ & $-0.13[-0.55,0.30]$ \\
\hline $\begin{array}{l}8.3 .41 \text { to } 11 \text { visits (to as- } \\
\text { sess/give exercises for self } \\
\text { practice) }\end{array}$ & 0 & 0 & $\begin{array}{l}\text { Std. Mean Difference (IV, Random, } \\
95 \% \mathrm{Cl} \text { ) }\end{array}$ & Not estimable \\
\hline 8.3.5 Dose not stated & 2 & 129 & $\begin{array}{l}\text { Std. Mean Difference (IV, Random, } \\
95 \% \mathrm{Cl} \text { ) }\end{array}$ & $0.42[0.07,0.77]$ \\
\hline 8.4 Provider of intervention & 6 & 260 & $\begin{array}{l}\text { Std. Mean Difference (IV, Random, } \\
95 \% \mathrm{Cl} \text { ) }\end{array}$ & $0.04[-0.27,0.35]$ \\
\hline 8.4.1 Physiotherapist & 4 & 124 & $\begin{array}{l}\text { Std. Mean Difference (IV, Random, } \\
95 \% \mathrm{Cl} \text { ) }\end{array}$ & $-0.12[-0.48,0.24]$ \\
\hline $\begin{array}{l}\text { 8.4.2 Bobath-trained phys- } \\
\text { iotherapist }\end{array}$ & 1 & 30 & $\begin{array}{l}\text { Std. Mean Difference (IV, Random, } \\
95 \% \mathrm{Cl} \text { ) }\end{array}$ & $-0.47[-1.24,0.31]$ \\
\hline 8.4.3 Not stated & 1 & 106 & $\begin{array}{l}\text { Std. Mean Difference (IV, Random, } \\
95 \% \mathrm{CI} \text { ) }\end{array}$ & $0.53[0.14,0.92]$ \\
\hline $\begin{array}{l}8.5 \text { Treatment components } \\
\text { included }\end{array}$ & 6 & & $\begin{array}{l}\text { Std. Mean Difference (IV, Fixed, 95\% } \\
\mathrm{CI} \text { ) }\end{array}$ & Subtotals only \\
\hline $\begin{array}{l}\text { 8.5.1 Contains functional } \\
\text { training }\end{array}$ & 6 & 244 & $\begin{array}{l}\text { Std. Mean Difference (IV, Fixed, 95\% } \\
\mathrm{CI} \text { ) }\end{array}$ & $0.12[-0.14,0.37]$ \\
\hline $\begin{array}{l}\text { 8.5.2 Contains neurophysio- } \\
\text { logical }\end{array}$ & 3 & 54 & $\begin{array}{l}\text { Std. Mean Difference (IV, Fixed, 95\% } \\
\text { Cl) }\end{array}$ & $-0.11[-0.64,0.43]$ \\
\hline $\begin{array}{l}\text { 8.5.3 Contains muscu- } \\
\text { loskeletal }\end{array}$ & 4 & 208 & $\begin{array}{l}\text { Std. Mean Difference (IV, Fixed, 95\% } \\
\text { CI) }\end{array}$ & $0.21[-0.07,0.48]$ \\
\hline
\end{tabular}


Analysis 8.1. Comparison 8: Subgroups. Intervention versus attention control or usual care: immediate outcome: independence in ADL, Outcome 1: Time after stroke

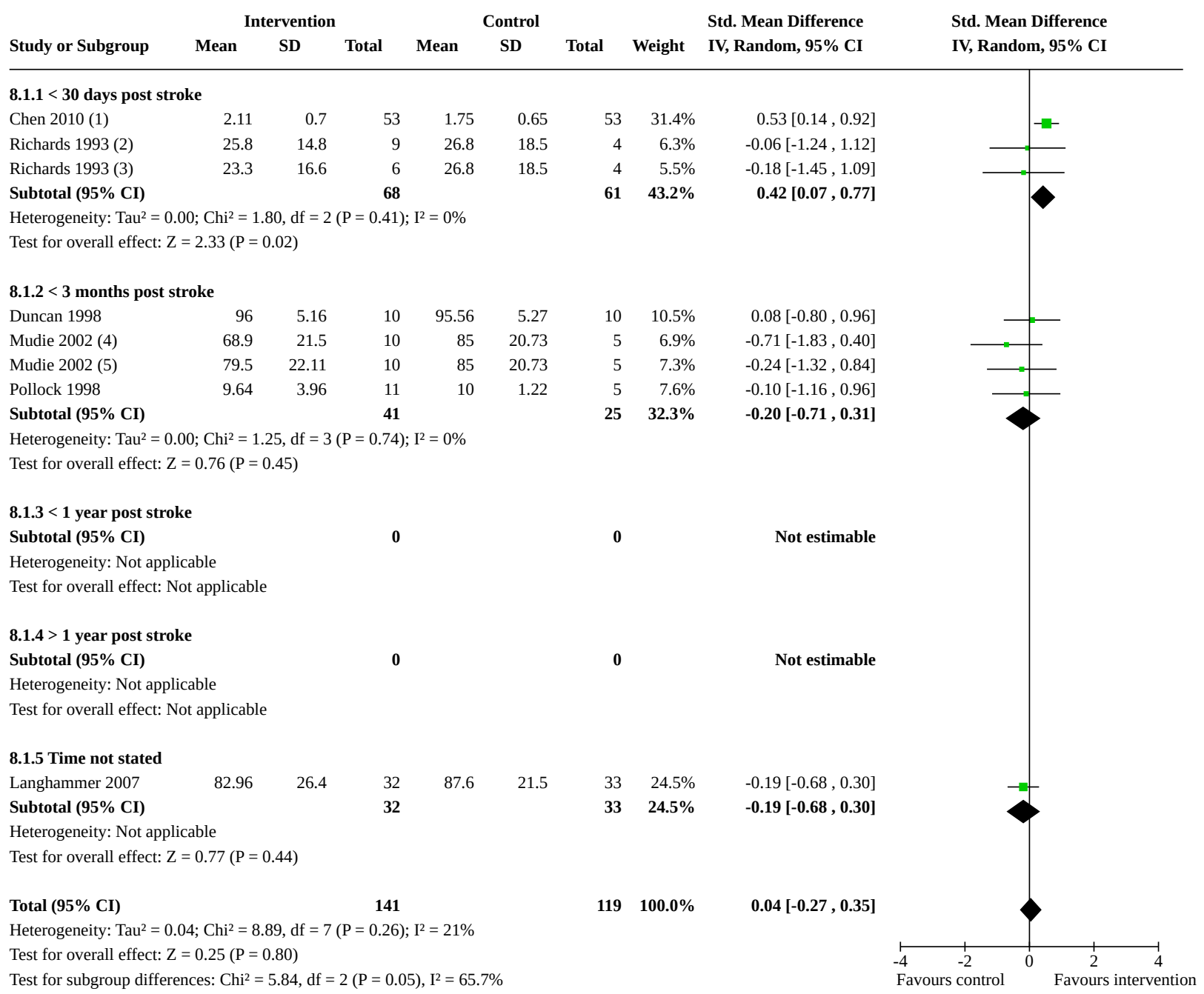

\section{Footnotes}

(1) Means and SD calculated from categorical data.

(2) Intervention group 1 vs usual care. Control group shared.

(3) Intervention group 2 vs usual care. Control group shared.

(4) Intervention 1 vs usual care. Control group shared.

(5) Intervention 2 vs usual care. Control group shared. 
Analysis 8.2. Comparison 8: Subgroups. Intervention versus attention control or usual care: immediate outcome: independence in ADL, Outcome 2: Study geographical location

\begin{tabular}{|c|c|c|c|c|c|c|c|c|c|}
\hline \multirow[b]{2}{*}{ Study or Subgroup } & \multicolumn{3}{|c|}{ Intervention } & \multicolumn{3}{|c|}{ Control } & \multirow{2}{*}{\multicolumn{2}{|c|}{$\begin{array}{l}\text { Std. Mean Difference } \\
\text { IV, Fixed, 95\% CI }\end{array}$}} & \multirow{2}{*}{$\begin{array}{l}\text { Std. Mean Difference } \\
\text { IV, Fixed, 95\% CI }\end{array}$} \\
\hline & Mean & SD & Total & Mean & SD & Total & & & \\
\hline \multicolumn{10}{|l|}{ 8.2.1 Europe } \\
\hline Langhammer 2007 & 82.96 & 26.4 & 32 & 87.6 & 21.5 & 33 & $26.2 \%$ & $-0.19[-0.68,0.30]$ & - \\
\hline Pollock 1998 & 9.64 & 3.96 & 11 & 10 & 1.22 & 5 & $5.6 \%$ & $-0.10[-1.16,0.96]$ & 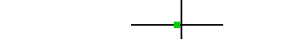 \\
\hline Subtotal (95\% CI) & & & 43 & & & 38 & $31.8 \%$ & $-0.17[-0.62,0.27]$ & \\
\hline \multicolumn{10}{|c|}{ Heterogeneity: $\mathrm{Chi}^{2}=0.02, \mathrm{df}=1(\mathrm{P}=0.88) ; \mathrm{I}^{2}=0 \%$} \\
\hline \multicolumn{10}{|c|}{ Test for overall effect: $\mathrm{Z}=0.77(\mathrm{P}=0.44)$} \\
\hline \multicolumn{10}{|l|}{ 8.2.2 Australia } \\
\hline Mudie 2002 (1) & 68.9 & 21.5 & 10 & 85 & 20.73 & 5 & $5.0 \%$ & $-0.71[-1.83,0.40]$ & - \\
\hline Mudie 2002 (2) & 79.5 & 22.11 & 10 & 85 & 20.73 & 5 & $5.4 \%$ & $-0.24[-1.32,0.84]$ & \\
\hline Subtotal (95\% CI) & & & 20 & & & 10 & $10.4 \%$ & $-0.47[-1.24,0.31]$ & \\
\hline \multicolumn{10}{|c|}{ Heterogeneity: $\mathrm{Chi}^{2}=0.36, \mathrm{df}=1(\mathrm{P}=0.55) ; \mathrm{I}^{2}=0 \%$} \\
\hline \multicolumn{10}{|c|}{ Test for overall effect: $\mathrm{Z}=1.18(\mathrm{P}=0.24)$} \\
\hline \multicolumn{10}{|l|}{ 8.2.3 Asia: China } \\
\hline Chen 2010 (3) & 2.11 & 0.7 & 53 & 1.75 & 0.65 & 53 & $41.4 \%$ & $0.53[0.14,0.92]$ & - \\
\hline Subtotal (95\% CI) & & & 53 & & & 53 & $41.4 \%$ & $0.53[0.14,0.92]$ & \\
\hline \multicolumn{10}{|c|}{ Heterogeneity: Not applicable } \\
\hline \multicolumn{10}{|c|}{ Test for overall effect: $\mathrm{Z}=2.68(\mathrm{P}=0.007)$} \\
\hline \multicolumn{10}{|l|}{ 8.2.4 Asia: other } \\
\hline Subtotal (95\% CI) & & & $\mathbf{0}$ & & & $\mathbf{0}$ & & Not estimable & \\
\hline \multicolumn{10}{|c|}{ Heterogeneity: Not applicable } \\
\hline \multicolumn{10}{|c|}{ Test for overall effect: Not applicable } \\
\hline \multicolumn{10}{|l|}{ 8.2.5 North America } \\
\hline Duncan 1998 & 96 & 5.16 & 10 & 95.56 & 5.27 & 10 & $8.1 \%$ & $0.08[-0.80,0.96]$ & \\
\hline Richards 1993 (1) & 25.8 & 14.8 & 9 & 26.8 & 18.5 & 4 & $4.5 \%$ & $-0.06[-1.24,1.12]$ & \\
\hline Richards 1993 (2) & 23.3 & 16.6 & 6 & 26.8 & 18.5 & 4 & $3.9 \%$ & $-0.18[-1.45,1.09]$ & \\
\hline Subtotal (95\% CI) & & & 25 & & & 18 & $16.4 \%$ & $-0.02[-0.63,0.60]$ & \\
\hline \multicolumn{10}{|c|}{ Heterogeneity: $\mathrm{Chi}^{2}=0.12, \mathrm{df}=2(\mathrm{P}=0.94) ; \mathrm{I}^{2}=0 \%$} \\
\hline \multicolumn{10}{|c|}{ Test for overall effect: $\mathrm{Z}=0.06(\mathrm{P}=0.95)$} \\
\hline Total (95\% CI) & & & 141 & & & 119 & $100.0 \%$ & $0.11[-0.14,0.36]$ & \\
\hline \multicolumn{10}{|c|}{ Heterogeneity: $\mathrm{Chi}^{2}=8.89, \mathrm{df}=7(\mathrm{P}=0.26) ; \mathrm{I}^{2}=21 \%$} \\
\hline Test for overall effect & $=0.88(\mathrm{P}=$ & $.38)$ & & & & & & & -2 \\
\hline Test for subgroup dif & ces: $\mathrm{Chi}^{2}=$ & 3.39, $\mathrm{df}=$ & $3(\mathrm{P}=0.0$ & 4), $\mathrm{I}^{2}=64$. & & & & & urs control \\
\hline
\end{tabular}

Footnotes

(1) Intervention 1 vs usual care. Control group shared.

(2) Intervention 2 vs usual care. Control group shared.

(3) Mean and SD calculated from categorical data. 
Analysis 8.3. Comparison 8: Subgroups. Intervention versus attention control or usual care: immediate outcome: independence in ADL, Outcome 3: Dose of intervention

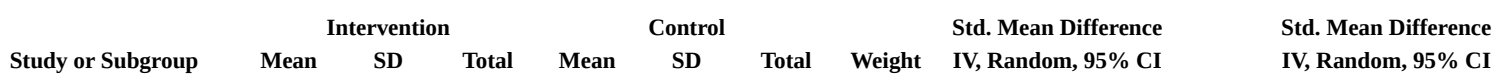

8.3.1 > once/d, with total of 60 to 120 minutes Subtotal (95\% CI)

Heterogeneity: Not applicable

Test for overall effect: Not applicable

8.3.2 Once/d, 5 to $7 \times /$ wk, for 30 to 60 minutes

Mudie 2002 (1) $\quad 68.9 \quad 21.5$

Mudie 2002 (2)

$79.5 \quad 22.11$

Pollock 1998

$9.64 \quad 3.96$

Subtotal (95\% CI)

(

10
10
11

$\begin{array}{ll}85 & 20.73 \\ 85 & 20.73\end{array}$

Heterogeneity: $\mathrm{Tau}^{2}=0.00 ; \mathrm{Chi}^{2}=0.66, \mathrm{df}=2(\mathrm{P}=0.72) ; \mathrm{I}^{2}=0 \%$

Test for overall effect: $\mathrm{Z}=1.06(\mathrm{P}=0.29)$

\subsubsection{2 to $3 \times / w k$}

Duncan 1998

Langhammer 2007

$\begin{array}{rr}96 & 5.16 \\ 82.96 & 26.4\end{array}$

5.16

$10 \quad 95.56$

Subtotal (95\% CI)

(8)

42

Heterogeneity: $\mathrm{Tau}^{2}=0.00 ; \mathrm{Chi}^{2}=0.28, \mathrm{df}=1(\mathrm{P}=0.60) ; \mathrm{I}^{2}=0 \%$

Test for overall effect: $\mathrm{Z}=0.58(\mathrm{P}=0.56)$

8.3.4 1 to 11 visits (to assess/give exercises for self practice)

Subtotal (95\% CI)

Heterogeneity: Not applicable

Test for overall effect: Not applicable

\subsubsection{Dose not stated}

Richards $1993(4)$

Richards 1993 (5)

2.11

25.8

23.3

0.7

14.8

53

$53 \quad 1.75$

26.8

0.65

18.5

Subtotal (95\% CI)

68

Heterogeneity: $\mathrm{Tau}^{2}=0.00 ; \mathrm{Chi}^{2}=1.80, \mathrm{df}=2(\mathrm{P}=0.41) ; \mathrm{I}^{2}=0 \%$

Test for overall effect: $\mathrm{Z}=2.33(\mathrm{P}=0.02)$

Total (95\% CI)

141

Heterogeneity: $\mathrm{Tau}^{2}=0.04 ; \mathrm{Chi}^{2}=8.89, \mathrm{df}=7(\mathrm{P}=0.26) ; \mathrm{I}^{2}=21 \%$

Test for overall effect: $\mathrm{Z}=0.25(\mathrm{P}=0.80)$

Test for subgroup differences: $\mathrm{Chi}^{2}=6.14, \mathrm{df}=2(\mathrm{P}=0.05), \mathrm{I}^{2}=67.4 \%$

$0.08[-0.80,0.96]$ $-0.19[-0.68,0.30]$ $-0.13[-0.55,0.30]$

$-0.24[-1.32,0.84]$

$-0.10[-1.16,0.96]$

$-0.34[-0.96,0.29]$

ndom, 95\% CI

\section{Footnotes}

(1) Intervention 1 vs usual care. Control group shared.

(2) Intervention 2 vs usual care. Control group shared.

(3) Mean and SD calculated from categorical data.

(4) Intervention group 1 vs usual care. Control group shared.

(5) Intervention group 2 vs usual care. Control group shared. 
Analysis 8.4. Comparison 8: Subgroups. Intervention versus attention control or usual care: immediate outcome: independence in ADL, Outcome 4: Provider of intervention

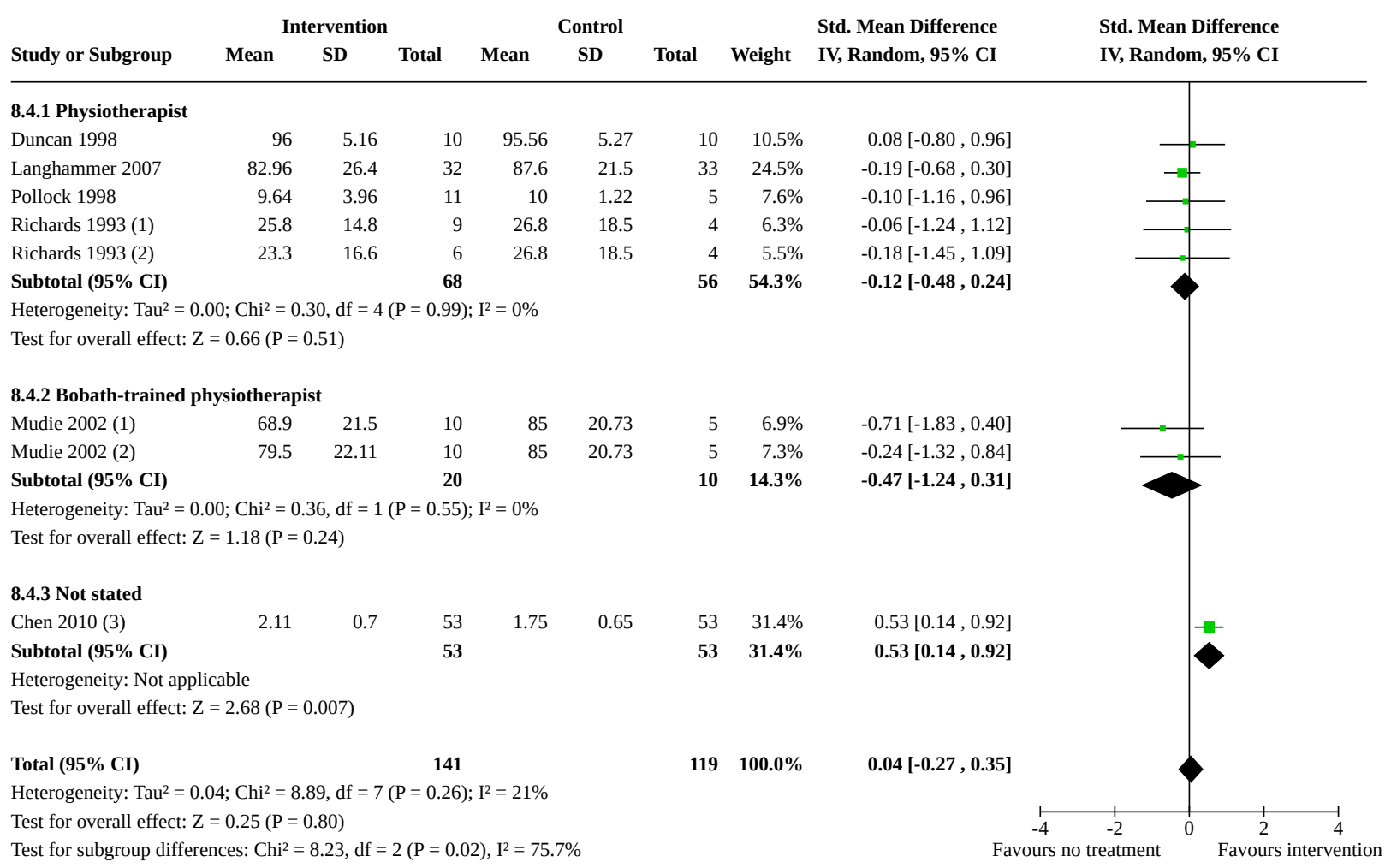

\section{Footnotes}

(1) Intervention 1 vs usual care. Control group shared.

(2) Intervention 2 vs usual care. Control group shared.

(3) Mean and SD calculated from categorical data. 
Analysis 8.5. Comparison 8: Subgroups. Intervention versus attention control or usual care: immediate outcome: independence in ADL, Outcome 5: Treatment components included

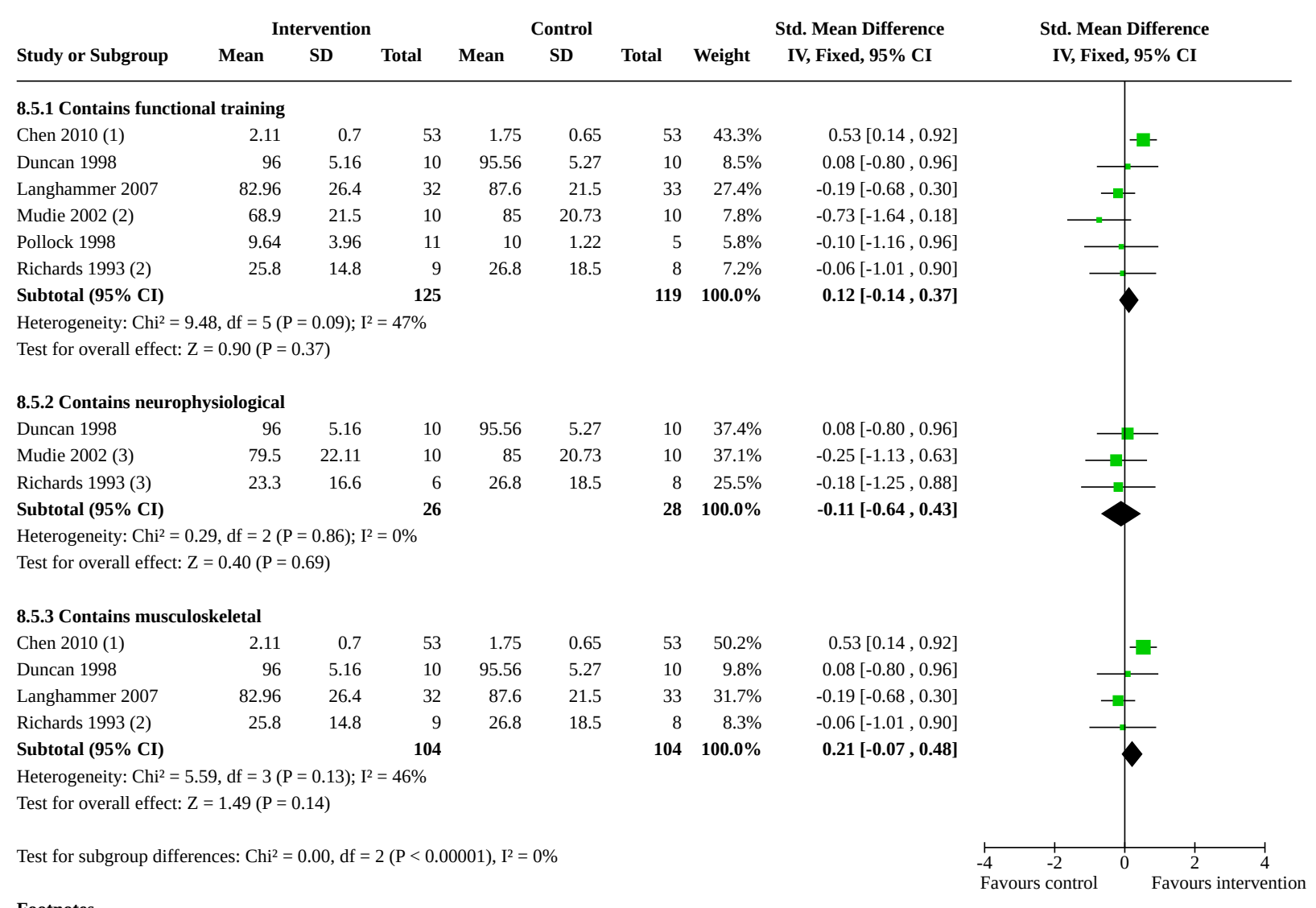

Footnotes

(1) Mean and SD calculated from categorical data.

(2) Intervention 1 vs usual care.

(3) Intervention 2 vs usual care.

Comparison 9. Subgroups. Intervention versus no treatment: immediate outcome: motor function

\begin{tabular}{|c|c|c|c|c|}
\hline $\begin{array}{l}\text { Outcome or subgroup ti- } \\
\text { tle }\end{array}$ & No. of studies & $\begin{array}{l}\text { No. of partici- } \\
\text { pants }\end{array}$ & Statistical method & Effect size \\
\hline 9.1 Time after stroke & 27 & 4558 & $\begin{array}{l}\text { Std. Mean Difference (IV, Random, 95\% } \\
\mathrm{CI} \text { ) }\end{array}$ & $0.81[0.58,1.04]$ \\
\hline $9.1 .1<30$ days post stroke & 15 & 2635 & $\begin{array}{l}\text { Std. Mean Difference (IV, Random, 95\% } \\
\mathrm{Cl} \text { ) }\end{array}$ & $0.68[0.47,0.89]$ \\
\hline $\begin{array}{l}9.1 .2<3 \text { months post } \\
\text { stroke }\end{array}$ & 1 & 70 & $\begin{array}{l}\text { Std. Mean Difference (IV, Random, 95\% } \\
\mathrm{Cl} \text { ) }\end{array}$ & $0.32[-0.15,0.79]$ \\
\hline $9.1 .3<1$ year post stroke & 0 & 0 & $\begin{array}{l}\text { Std. Mean Difference (IV, Random, 95\% } \\
\mathrm{Cl} \text { ) }\end{array}$ & Not estimable \\
\hline 9.1.4 > 1 year post stroke & 2 & 250 & $\begin{array}{l}\text { Std. Mean Difference (IV, Random, 95\% } \\
\mathrm{Cl} \text { ) }\end{array}$ & $0.14[-0.31,0.58]$ \\
\hline
\end{tabular}




\begin{tabular}{|c|c|c|c|c|}
\hline $\begin{array}{l}\text { Outcome or subgroup ti- } \\
\text { tle }\end{array}$ & No. of studies & $\begin{array}{l}\text { No. of partici- } \\
\text { pants }\end{array}$ & Statistical method & Effect size \\
\hline 9.1.5 Time not stated & 9 & 1603 & $\begin{array}{l}\text { Std. Mean Difference (IV, Random, 95\% } \\
\mathrm{Cl} \text { ) }\end{array}$ & $1.26[0.65,1.88]$ \\
\hline $\begin{array}{l}9.2 \text { Study geographical lo- } \\
\text { cation }\end{array}$ & 27 & 4558 & $\begin{array}{l}\text { Std. Mean Difference (IV, Random, 95\% } \\
\mathrm{CI} \text { ) }\end{array}$ & $0.81[0.58,1.04]$ \\
\hline 9.2.1 Europe & 2 & 250 & $\begin{array}{l}\text { Std. Mean Difference (IV, Random, 95\% } \\
\mathrm{CI} \text { ) }\end{array}$ & $0.14[-0.31,0.58]$ \\
\hline 9.2.2 Australia & 0 & 0 & $\begin{array}{l}\text { Std. Mean Difference (IV, Random, 95\% } \\
\mathrm{CI} \text { ) }\end{array}$ & Not estimable \\
\hline 9.2.3 Asia: China & 25 & 4308 & $\begin{array}{l}\text { Std. Mean Difference (IV, Random, 95\% } \\
\mathrm{CI} \text { ) }\end{array}$ & $0.87[0.63,1.10]$ \\
\hline 9.2.4 Asia: other & 0 & 0 & $\begin{array}{l}\text { Std. Mean Difference (IV, Random, 95\% } \\
\mathrm{CI} \text { ) }\end{array}$ & Not estimable \\
\hline 9.2.5 North America & 0 & 0 & $\begin{array}{l}\text { Std. Mean Difference (IV, Random, 95\% } \\
\mathrm{CI} \text { ) }\end{array}$ & Not estimable \\
\hline 9.3 Dose of intervention & 27 & 4558 & $\begin{array}{l}\text { Std. Mean Difference (IV, Random, 95\% } \\
\mathrm{Cl} \text { ) }\end{array}$ & $0.81[0.58,1.04]$ \\
\hline $\begin{array}{l}9.3 .1>\text { once/d, with total } \\
\text { of } 60 \text { to } 120 \text { minutes }\end{array}$ & 4 & 434 & $\begin{array}{l}\text { Std. Mean Difference (IV, Random, 95\% } \\
\mathrm{CI} \text { ) }\end{array}$ & $1.31[0.98,1.64]$ \\
\hline $\begin{array}{l}\text { 9.3.2 Once } / \mathrm{d}, 5 \text { to } 7 \times / w k \text {, } \\
\text { for } 30 \text { to } 60 \text { minutes }\end{array}$ & 13 & 1084 & $\begin{array}{l}\text { Std. Mean Difference (IV, Random, 95\% } \\
\mathrm{CI} \text { ) }\end{array}$ & $0.89[0.35,1.44]$ \\
\hline $9.3 .32 \times / w k$ & 3 & 289 & $\begin{array}{l}\text { Std. Mean Difference (IV, Random, 95\% } \\
\mathrm{CI} \text { ) }\end{array}$ & $0.80[0.08,1.52]$ \\
\hline $\begin{array}{l}\text { 9.3.4 } 1 \text { to } 11 \text { visits (to as- } \\
\text { sess/give exercises for self } \\
\text { practice) }\end{array}$ & 2 & 250 & $\begin{array}{l}\text { Std. Mean Difference (IV, Random, 95\% } \\
\mathrm{CI} \text { ) }\end{array}$ & $0.14[-0.31,0.58]$ \\
\hline 9.3.5 Dose not stated & 5 & 2501 & $\begin{array}{l}\text { Std. Mean Difference (IV, Random, 95\% } \\
\mathrm{CI} \text { ) }\end{array}$ & $0.52[0.32,0.71]$ \\
\hline $\begin{array}{l}\text { 9.4 Provider of interven- } \\
\text { tion }\end{array}$ & 27 & 4558 & $\begin{array}{l}\text { Std. Mean Difference (IV, Random, 95\% } \\
\mathrm{CI} \text { ) }\end{array}$ & $0.81[0.58,1.04]$ \\
\hline 9.4.1 Physiotherapist & 2 & 250 & $\begin{array}{l}\text { Std. Mean Difference (IV, Random, 95\% } \\
\mathrm{CI} \text { ) }\end{array}$ & $0.14[-0.31,0.58]$ \\
\hline 9.4.2 Therapist & 7 & 1356 & $\begin{array}{l}\text { Std. Mean Difference (IV, Random, 95\% } \\
\mathrm{CI} \text { ) }\end{array}$ & $0.74[0.45,1.03]$ \\
\hline 9.4.3 Therapist + family & 2 & 152 & $\begin{array}{l}\text { Std. Mean Difference (IV, Random, 95\% } \\
\mathrm{CI} \text { ) }\end{array}$ & $1.13[0.78,1.47]$ \\
\hline 9.4.4 Nurse & 3 & 308 & $\begin{array}{l}\text { Std. Mean Difference (IV, Random, 95\% } \\
\mathrm{Cl} \text { ) }\end{array}$ & $2.08[-0.27,4.43]$ \\
\hline
\end{tabular}




\begin{tabular}{lllll}
\hline $\begin{array}{l}\text { Outcome or subgroup ti- } \\
\text { tle }\end{array}$ & No. of studies & $\begin{array}{l}\text { No. of partici- } \\
\text { pants }\end{array}$ & Statistical method & Effect size \\
\hline $\begin{array}{llll}\text { 9.4.5 Not stated } \\
\text { (3) }\end{array}$ & 2492 & $\begin{array}{l}\text { Std. Mean Difference (IV, Random, 95\% } \\
\text { Cl) }\end{array}$ & $0.65[0.43,0.87]$ \\
\hline $\begin{array}{l}\text { 9.5 Treatment compo- } \\
\text { nents included }\end{array}$ & 27 & & $\begin{array}{l}\text { Std. Mean Difference (IV, Random, 95\% } \\
\text { Cl) }\end{array}$ & Subtotals only \\
\hline $\begin{array}{l}\text { 9.5.1 Contains functional } \\
\text { training }\end{array}$ & 24 & 4330 & $\begin{array}{l}\text { Std. Mean Difference (IV, Random, 95\% } \\
\text { CI) }\end{array}$ & $0.88[0.64,1.13]$ \\
\hline $\begin{array}{l}\text { 9.5.2 Contains neurophysi- } \\
\text { ological }\end{array}$ & 13 & 2033 & $\begin{array}{l}\text { Std. Mean Difference (IV, Random, 95\% } \\
\text { CI) }\end{array}$ & $0.76[0.54,0.98]$ \\
\hline $\begin{array}{l}\text { 9.5.3 Contains muscu- } \\
\text { loskeletal }\end{array}$ & 24 & 4240 & $\begin{array}{l}\text { Std. Mean Difference (IV, Random, 95\% } \\
\text { Cl) }\end{array}$ & $0.84[0.60,1.08]$ \\
\hline
\end{tabular}


Analysis 9.1. Comparison 9: Subgroups. Intervention versus no treatment: immediate outcome: motor function, Outcome 1: Time after stroke

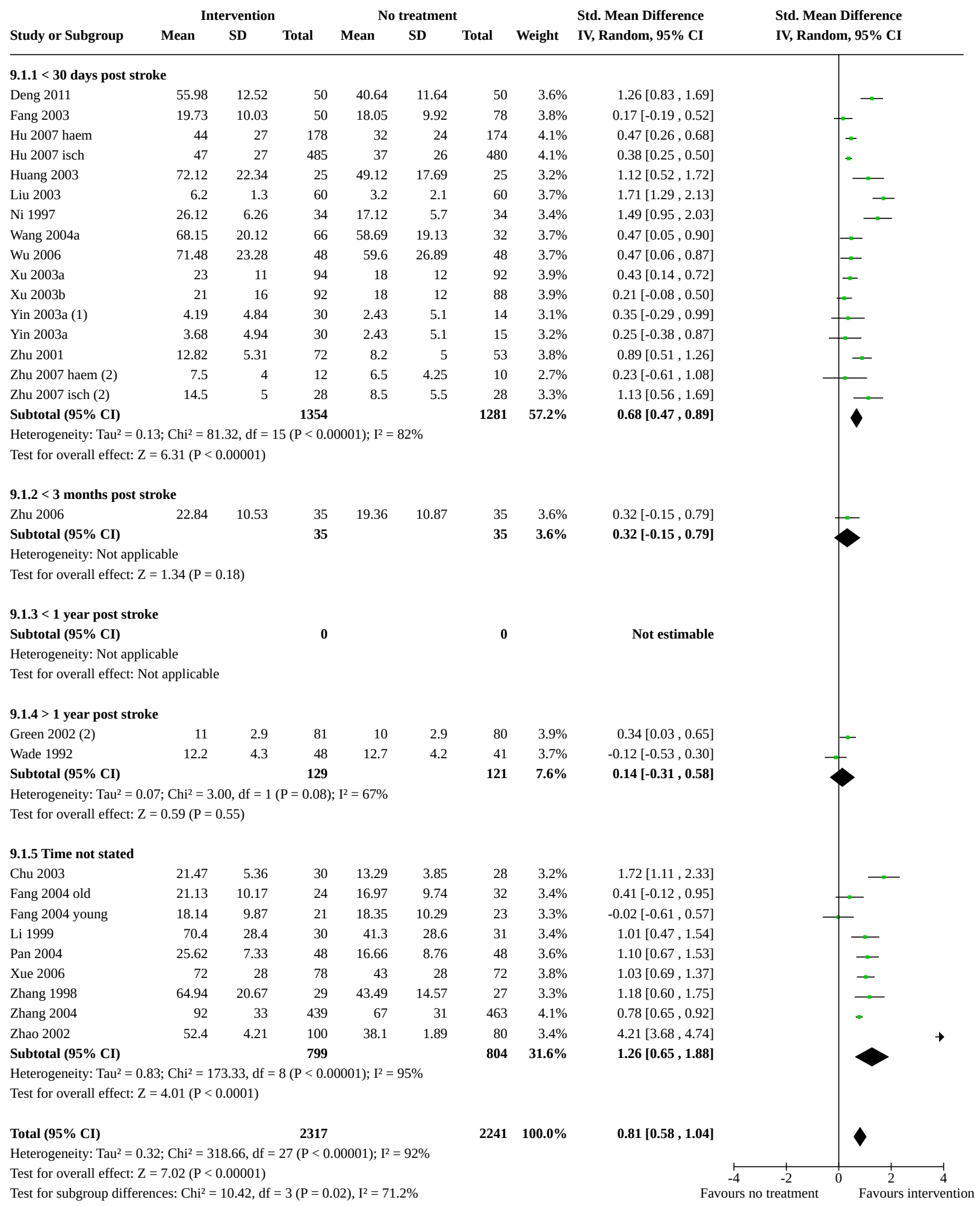

Footnotes

(1) Mean and SD calculated from categorical data.

(2) SDs estimated from range ((max-min range)/4) 


\section{Analysis 9.1. (Continued)}

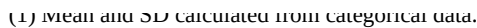

(2) SDs estimated from range ((max-min range)/4) 
Analysis 9.2. Comparison 9: Subgroups. Intervention versus no treatment: immediate outcome: motor function, Outcome 2: Study geographical location

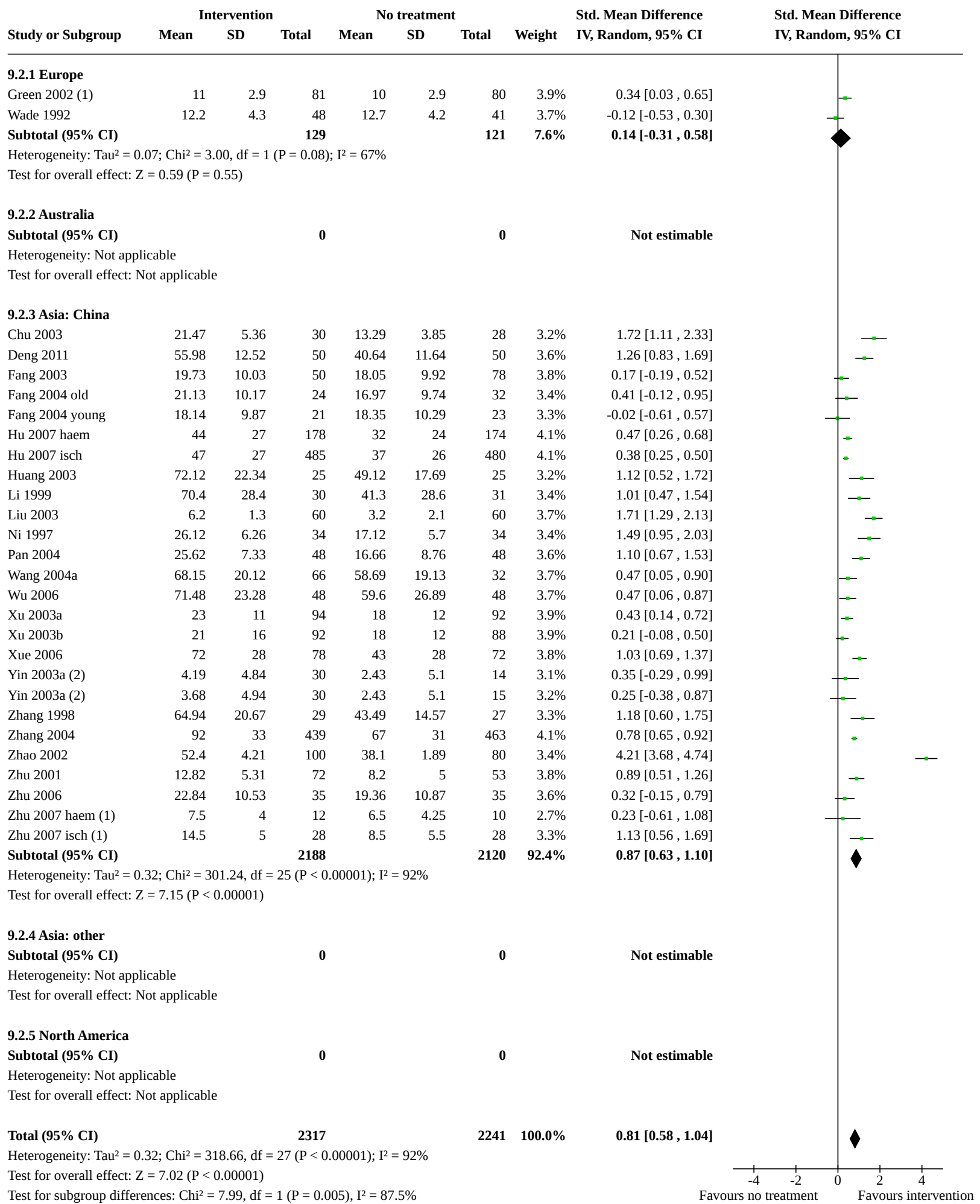

Footnotes

(1) SDs estimated from range ((max-min range)/4)

(2) Mean and SD calculated from categorical data. 


\section{Analysis 9.2. (Continued)}

(1) oעs esunlateu Huin Iallye ((III)

(2) Mean and SD calculated from categorical data. 
Analysis 9.3. Comparison 9: Subgroups. Intervention versus no treatment: immediate outcome: motor function, Outcome 3: Dose of intervention

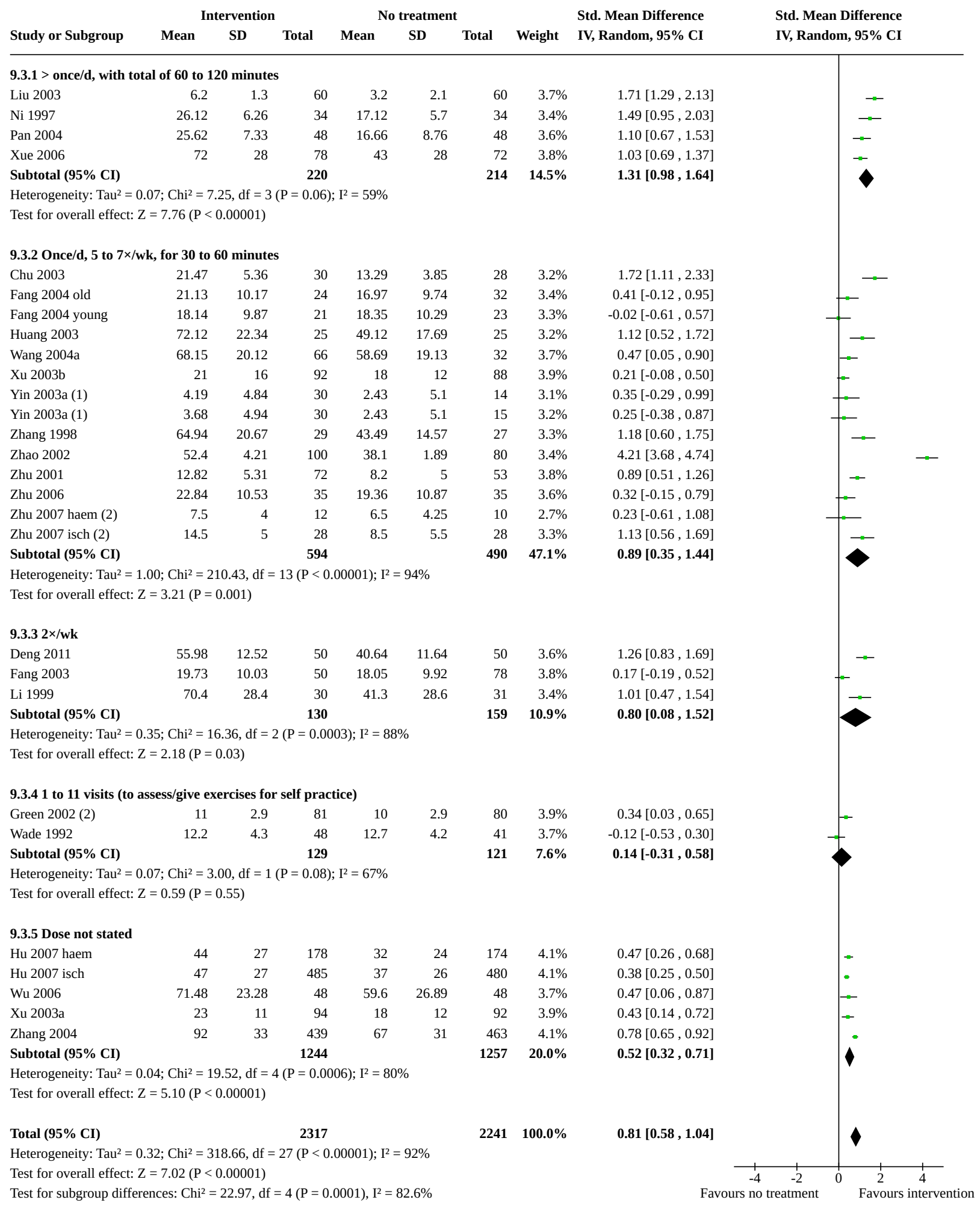

Footnotes

(1) Mean and SD calculated from categorical data.

(2) SDs estimated from range ((max-min range)/4) 


\section{Analysis 9.3. (Continued)}

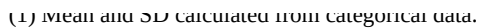

(2) SDs estimated from range ((max-min range)/4) 
Analysis 9.4. Comparison 9: Subgroups. Intervention versus no treatment: immediate outcome: motor function, Outcome 4: Provider of intervention

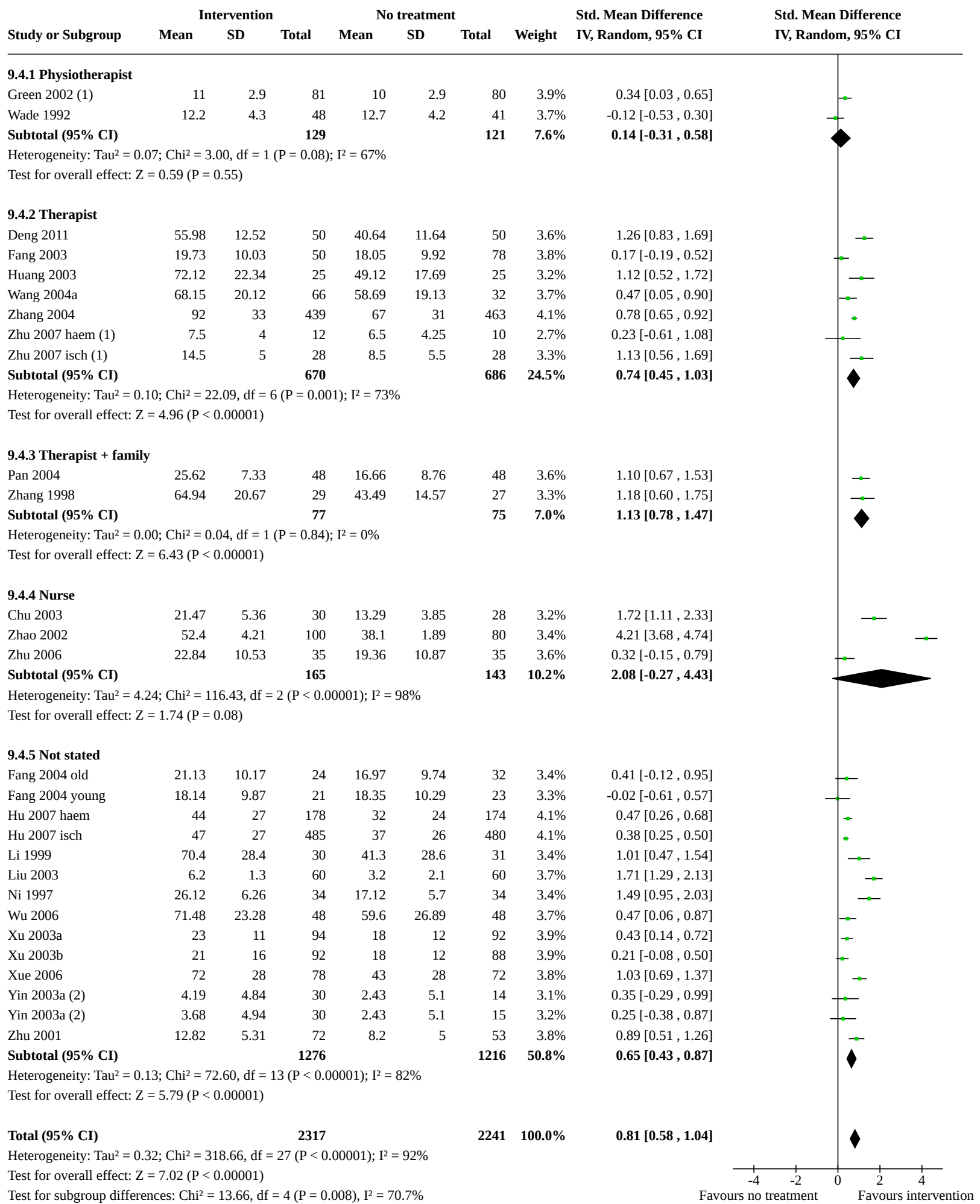

Footnotes

(1) SDs estimated from range ((max-min range)/4)

(2) Mean and SD computed from categorical data. 


\section{Analysis 9.4. (Continued)}

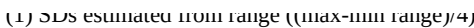

(2) Mean and SD computed from categorical data. 
Analysis 9.5. Comparison 9: Subgroups. Intervention versus no treatment: immediate outcome: motor function, Outcome 5: Treatment components included

\begin{tabular}{|c|c|c|c|c|c|c|c|c|c|c|}
\hline \multirow[b]{2}{*}{ Study or Subgroup } & \multicolumn{3}{|c|}{ Intervention } & \multicolumn{3}{|c|}{ No treatment } & \multirow[b]{2}{*}{ Weight } & \multirow{2}{*}{$\begin{array}{l}\text { Std. Mean Difference } \\
\text { IV, Random, } 95 \% \text { CI }\end{array}$} & \multirow{2}{*}{\multicolumn{2}{|c|}{$\begin{array}{l}\text { Std. Mean Difference } \\
\text { IV, Random, } 95 \% \text { CI }\end{array}$}} \\
\hline & Mean & SD & Total & Mean & SD & Total & & & & \\
\hline \multicolumn{11}{|c|}{ 9.5.1 Contains functional training } \\
\hline Chu 2003 & 21.47 & 5.36 & 30 & 13.29 & 3.85 & 28 & $3.6 \%$ & $1.72[1.11,2.33]$ & -- & \\
\hline Deng 2011 & 55.98 & 12.52 & 50 & 40.64 & 11.64 & 50 & $4.1 \%$ & $1.26[0.83,1.69]$ & - & \\
\hline Green 2002 (1) & 11 & 2.9 & 81 & 10 & 2.9 & 80 & $4.3 \%$ & $0.34[0.03,0.65]$ & - & \\
\hline Hu 2007 haem & 44 & 27 & 178 & 32 & 24 & 174 & $4.5 \%$ & $0.47[0.26,0.68]$ & - & \\
\hline Hu 2007 isch & 47 & 27 & 485 & 37 & 26 & 480 & $4.6 \%$ & $0.38[0.25,0.50]$ & $=$ & \\
\hline Huang 2003 & 72.12 & 22.34 & 25 & 49.12 & 17.69 & 25 & $3.6 \%$ & $1.12[0.52,1.72]$ & $\rightarrow$ & \\
\hline Li 1999 & 70.4 & 28.4 & 30 & 41.3 & 28.6 & 31 & $3.8 \%$ & $1.01[0.47,1.54]$ & $\rightarrow-$ & \\
\hline Liu 2003 & 6.2 & 1.3 & 60 & 3.2 & 2.1 & 60 & $4.1 \%$ & $1.71[1.29,2.13]$ & - & \\
\hline Ni 1997 & 26.12 & 6.26 & 34 & 17.12 & 5.7 & 34 & $3.8 \%$ & $1.49[0.95,2.03]$ & 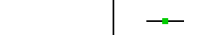 & \\
\hline Pan 2004 & 25.62 & 7.33 & 48 & 16.66 & 8.76 & 48 & $4.1 \%$ & $1.10[0.67,1.53]$ & - & \\
\hline Wade 1992 & 12.2 & 4.3 & 48 & 12.7 & 4.2 & 41 & $4.1 \%$ & $-0.12[-0.53,0.30]$ & $\rightarrow$ & \\
\hline Wang 2004a & 68.15 & 20.12 & 66 & 58.69 & 19.13 & 32 & $4.1 \%$ & $0.47[0.05,0.90]$ & - & \\
\hline Wu 2006 & 71.48 & 23.28 & 48 & 59.6 & 26.89 & 48 & $4.1 \%$ & $0.47[0.06,0.87]$ & $\rightarrow-$ & \\
\hline Xu 2003a & 23 & 11 & 94 & 18 & 12 & 92 & $4.4 \%$ & $0.43[0.14,0.72]$ & -- & \\
\hline Xu 2003b & 21 & 16 & 92 & 18 & 12 & 88 & $4.4 \%$ & $0.21[-0.08,0.50]$ & - & \\
\hline Xue 2006 & 72 & 28 & 78 & 43 & 28 & 72 & $4.3 \%$ & $1.03[0.69,1.37]$ & 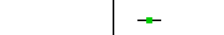 & \\
\hline Yin 2003a (2) & 4.19 & 4.84 & 30 & 2.43 & 5.1 & 14 & $3.5 \%$ & $0.35[-0.29,0.99]$ & $f=$ & \\
\hline Yin 2003a (2) & 3.68 & 4.94 & 30 & 2.43 & 5.1 & 15 & $3.6 \%$ & $0.25[-0.38,0.87]$ & - & \\
\hline Zhang 1998 & 64.94 & 20.67 & 29 & 43.49 & 14.57 & 27 & $3.7 \%$ & $1.18[0.60,1.75]$ & $\rightarrow$ & \\
\hline Zhang 2004 & 92 & 33 & 439 & 67 & 31 & 463 & $4.6 \%$ & $0.78[0.65,0.92]$ & $=$ & \\
\hline Zhao 2002 & 52.4 & 4.21 & 100 & 38.1 & 1.89 & 80 & $3.8 \%$ & $4.21[3.68,4.74]$ & & 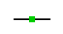 \\
\hline Zhu 2001 & 12.82 & 5.31 & 72 & 8.2 & 5 & 53 & $4.2 \%$ & $0.89[0.51,1.26]$ & - & \\
\hline Zhu 2006 & 22.84 & 10.53 & 35 & 19.36 & 10.87 & 35 & $4.0 \%$ & $0.32[-0.15,0.79]$ & - & \\
\hline Zhu 2007 haem (1) & 7.5 & 4 & 12 & 6.5 & 4.25 & 10 & $3.0 \%$ & $0.23[-0.61,1.08]$ & - & \\
\hline Zhu 2007 isch (1) & 14.5 & 5 & 28 & 8.5 & 5.5 & 28 & $3.7 \%$ & $1.13[0.56,1.69]$ & $\rightarrow$ & \\
\hline Subtotal (95\% CI) & & & 2222 & & & 2108 & $100.0 \%$ & $0.88[0.64,1.13]$ & 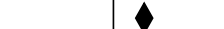 & \\
\hline \multicolumn{11}{|c|}{ Heterogeneity: $\mathrm{Tau}^{2}=0.33 ; \mathrm{Chi}^{2}=305.39, \mathrm{df}=24(\mathrm{P}<0.00001) ; \mathrm{I}^{2}=92 \%$} \\
\hline \multicolumn{11}{|c|}{ Test for overall effect: $\mathrm{Z}=7.14(\mathrm{P}<0.00001)$} \\
\hline \multicolumn{11}{|c|}{ 9.5.2 Contains neurophysiological } \\
\hline Chu 2003 & 21.47 & 5.36 & 30 & 13.29 & 3.85 & 28 & $5.8 \%$ & $1.72[1.11,2.33]$ & -- & \\
\hline Fang 2003 & 19.73 & 10.03 & 50 & 18.05 & 9.92 & 78 & $8.2 \%$ & $0.17[-0.19,0.52]$ & - & \\
\hline Huang 2003 & 72.12 & 22.34 & 25 & 49.12 & 17.69 & 25 & $5.9 \%$ & $1.12[0.52,1.72]$ & -- & \\
\hline Li 1999 & 70.4 & 28.4 & 30 & 41.3 & 28.6 & 31 & $6.5 \%$ & $1.01[0.47,1.54]$ & -- & \\
\hline Ni 1997 & 26.12 & 6.26 & 34 & 17.12 & 5.7 & 34 & $6.4 \%$ & $1.49[0.95,2.03]$ & 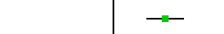 & \\
\hline Wu 2006 & 71.48 & 23.28 & 48 & 59.6 & 26.89 & 48 & $7.7 \%$ & $0.47[0.06,0.87]$ & - & \\
\hline Xu 2003b & 21 & 16 & 92 & 18 & 12 & 88 & $8.8 \%$ & $0.21[-0.08,0.50]$ & $=$ & \\
\hline Xue 2006 & 72 & 28 & 78 & 43 & 28 & 72 & $8.3 \%$ & $1.03[0.69,1.37]$ & - & \\
\hline Yin 2003a (3) & 4.19 & 4.84 & 30 & 2.43 & 5.1 & 14 & $5.6 \%$ & $0.35[-0.29,0.99]$ & - & \\
\hline Yin 2003a (3) & 3.68 & 4.94 & 30 & 2.43 & 5.1 & 15 & $5.7 \%$ & $0.25[-0.38,0.87]$ & $=$ & \\
\hline Zhang 1998 & 64.94 & 20.67 & 29 & 43.49 & 14.57 & 27 & $6.1 \%$ & $1.18[0.60,1.75]$ & -- & \\
\hline Zhang 2004 & 92 & 33 & 439 & 67 & 31 & 463 & $10.0 \%$ & $0.78[0.65,0.92]$ & $=$ & \\
\hline Zhu 2001 & 12.82 & 5.31 & 72 & 8.2 & 5 & 53 & $8.0 \%$ & $0.89[0.51,1.26]$ & - & \\
\hline Zhu 2006 & 22.84 & 10.53 & 35 & 19.36 & 10.87 & 35 & $7.1 \%$ & $0.32[-0.15,0.79]$ & - & \\
\hline Subtotal (95\% CI) & & & 1022 & & & 1011 & $100.0 \%$ & $0.76[0.54,0.98]$ & $\boldsymbol{\gamma}$ & \\
\hline \multicolumn{11}{|c|}{ Heterogeneity: $\mathrm{Tau}^{2}=0.12 ; \mathrm{Chi}^{2}=56.69, \mathrm{df}=13(\mathrm{P}<0.00001) ; \mathrm{I}^{2}=77 \%$} \\
\hline \multicolumn{11}{|c|}{ Test for overall effect: $\mathrm{Z}=6.72(\mathrm{P}<0.00001)$} \\
\hline \multicolumn{11}{|c|}{ 9.5.3 Contains musculoskeletal } \\
\hline Chu 2003 & 21.47 & 5.36 & 30 & 13.29 & 3.85 & 28 & $3.6 \%$ & $1.72[1.11,2.33]$ & 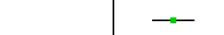 & \\
\hline Deng 2011 & 55.98 & 12.52 & 50 & 40.64 & 11.64 & 50 & $4.1 \%$ & $1.26[0.83,1.69]$ & - & \\
\hline Fang 2003 & 19.73 & 10.03 & 50 & 18.05 & 9.92 & 78 & $4.3 \%$ & $0.17[-0.19,0.52]$ & - & \\
\hline Fang 2004 old & 21.13 & 10.17 & 24 & 16.97 & 9.74 & 32 & $3.8 \%$ & $0.41[-0.12,0.95]$ & 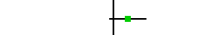 & \\
\hline Fang 2004 young & 18.14 & 9.87 & 21 & 18.35 & 10.29 & 23 & $3.7 \%$ & $-0.02[-0.61,0.57]$ & - & \\
\hline Hu 2007 haem & 44 & 27 & 178 & 32 & 24 & 174 & $4.5 \%$ & $0.47[0.26,0.68]$ & - & \\
\hline Hu 2007 isch & 47 & 27 & 485 & 37 & 26 & 480 & $4.6 \%$ & $0.38[0.25,0.50]$ & $=$ & \\
\hline Huang 2003 & 72.12 & 22.34 & 25 & 49.12 & 17.69 & 25 & $3.6 \%$ & $1.12[0.52,1.72]$ & - & \\
\hline Li 1999 & 70.4 & 28.4 & 30 & 41.3 & 28.6 & 31 & $3.8 \%$ & $1.01[0.47,1.54]$ & - & \\
\hline Liu 2003 & 6.2 & 1.3 & 60 & 3.2 & 2.1 & 60 & $4.1 \%$ & $1.71[1.29,2.13]$ & $\because$ & \\
\hline Pan 2004 & 25.62 & 7.33 & 48 & 16.66 & 8.76 & 48 & $4.1 \%$ & $1.10[0.67,1.53]$ & -- & \\
\hline Wano & 6815 & $m$ n 17 & 66 & 5869 & 1912 & 3) & $\Delta 1 \%$ & 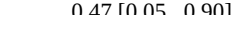 & & \\
\hline
\end{tabular}


Analysis 9.5. (Continued)

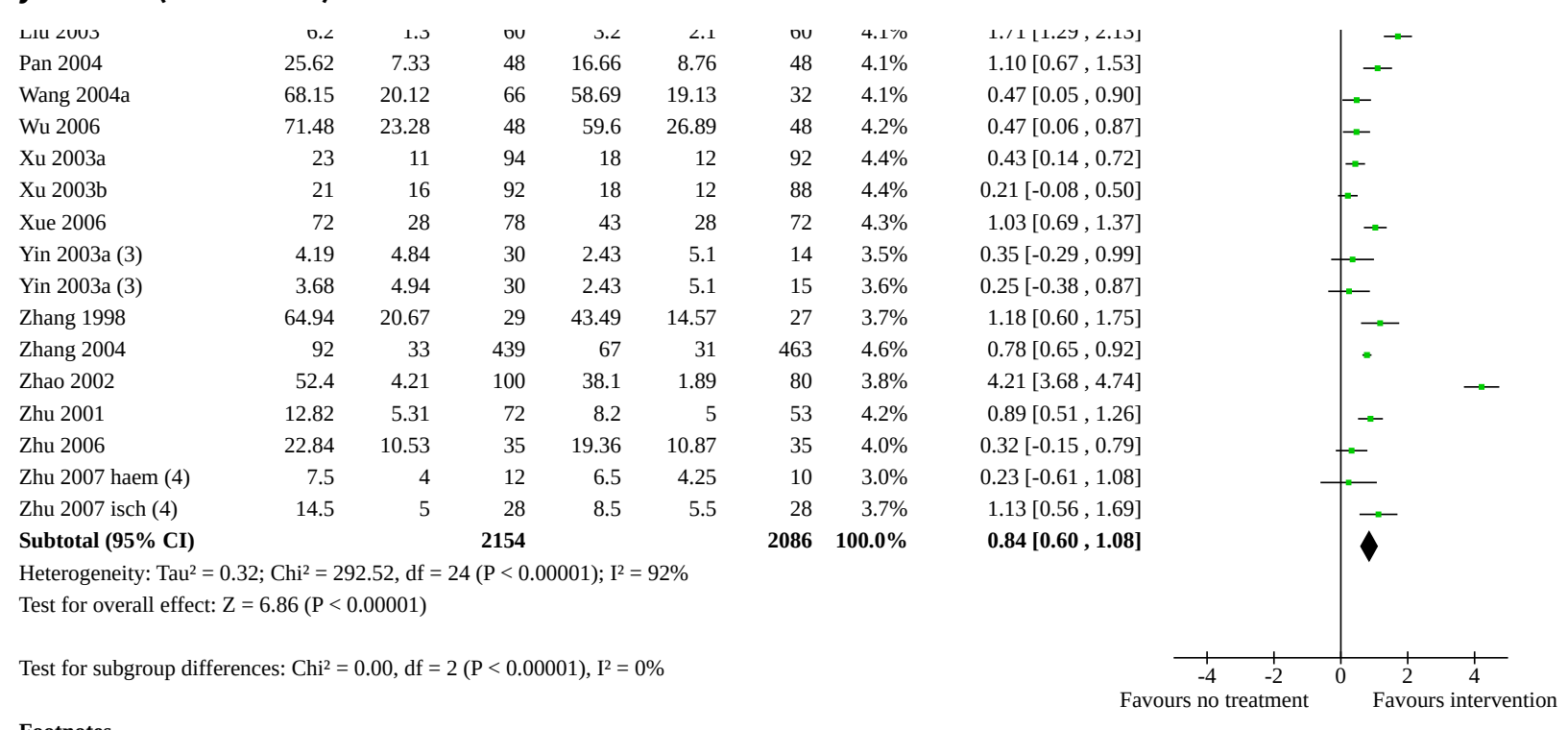

Footnotes

(1) SDs estimated from range ((max-min range)/4).

(2) Mean and SD calculated from categorical data.

(3) Mean and SD calculated from categorical data. Control group shared.

(4) Estimated SD = (max-min range) $/ 4$.

Comparison 10. Subgroups. Intervention versus attention control or usual care: immediate outcome: motor function

\begin{tabular}{|c|c|c|c|c|}
\hline $\begin{array}{l}\text { Outcome or subgroup ti- } \\
\text { tle }\end{array}$ & No. of studies & $\begin{array}{l}\text { No. of partici- } \\
\text { pants }\end{array}$ & Statistical method & Effect size \\
\hline 10.1 Time after stroke & 13 & 967 & $\begin{array}{l}\text { Std. Mean Difference (IV, Random, 95\% } \\
\mathrm{CI} \text { ) }\end{array}$ & $0.42[0.24,0.61]$ \\
\hline $\begin{array}{l}10.1 .1<30 \text { days post } \\
\text { stroke }\end{array}$ & 3 & 171 & $\begin{array}{l}\text { Std. Mean Difference (IV, Random, 95\% } \\
\text { CI) }\end{array}$ & $0.60[0.29,0.91]$ \\
\hline $\begin{array}{l}10.1 .2<3 \text { months post } \\
\text { stroke }\end{array}$ & 4 & 291 & $\begin{array}{l}\text { Std. Mean Difference (IV, Random, 95\% } \\
\mathrm{CI} \text { ) }\end{array}$ & $0.52[0.23,0.82]$ \\
\hline $10.1 .3<1$ year post stroke & 3 & 313 & $\begin{array}{l}\text { Std. Mean Difference (IV, Random, 95\% } \\
\mathrm{CI} \text { ) }\end{array}$ & $0.35[-0.04,0.74]$ \\
\hline 10.1.4 > 1 year post stroke & 1 & 58 & $\begin{array}{l}\text { Std. Mean Difference (IV, Random, 95\% } \\
\mathrm{CI} \text { ) }\end{array}$ & $0.00[-0.52,0.52]$ \\
\hline 10.1.5 Time not stated & 2 & 134 & $\begin{array}{l}\text { Std. Mean Difference (IV, Random, 95\% } \\
\mathrm{CI} \text { ) }\end{array}$ & $0.26[-0.61,1.13]$ \\
\hline $\begin{array}{l}\text { 10.2 Study geographical } \\
\text { location }\end{array}$ & 13 & 967 & Std. Mean Difference (IV, Fixed, 95\% Cl) & $0.42[0.29,0.55]$ \\
\hline 10.2.1 Europe & 3 & 405 & Std. Mean Difference (IV, Fixed, 95\% Cl) & $0.26[0.06,0.46]$ \\
\hline 10.2.2 Australia & 2 & 79 & Std. Mean Difference (IV, Fixed, 95\% Cl) & $-0.09[-0.53,0.36]$ \\
\hline
\end{tabular}




\begin{tabular}{|c|c|c|c|c|}
\hline $\begin{array}{l}\text { Outcome or subgroup ti- } \\
\text { tle }\end{array}$ & No. of studies & $\begin{array}{l}\text { No. of partici- } \\
\text { pants }\end{array}$ & Statistical method & Effect size \\
\hline 10.2.3 Asia: China & 5 & 348 & Std. Mean Difference (IV, Fixed, 95\% CI) & $0.72[0.50,0.94]$ \\
\hline 10.2.4 Asia: other & 0 & 0 & Std. Mean Difference (IV, Fixed, 95\% CI) & Not estimable \\
\hline 10.2.5 North America & 3 & 135 & Std. Mean Difference (IV, Fixed, 95\% CI) & $0.45[0.10,0.79]$ \\
\hline 10.3 Dose of intervention & 13 & 967 & $\begin{array}{l}\text { Std. Mean Difference (IV, Random, 95\% } \\
\mathrm{CI} \text { ) }\end{array}$ & $0.42[0.24,0.61]$ \\
\hline $\begin{array}{l}10.3 .1>\text { once } / \mathrm{d} \text {, with total } \\
\text { of } 60 \text { to } 120 \text { minutes }\end{array}$ & 0 & 0 & $\begin{array}{l}\text { Std. Mean Difference (IV, Random, 95\% } \\
\mathrm{Cl} \text { ) }\end{array}$ & Not estimable \\
\hline $\begin{array}{l}\text { 10.3.2 Once } / \mathrm{d}, 5 \text { to } 7 \times / \mathrm{wk} \text {, } \\
\text { for } 30 \text { to } 60 \text { minutes }\end{array}$ & 4 & 242 & $\begin{array}{l}\text { Std. Mean Difference (IV, Random, 95\% } \\
\mathrm{Cl} \text { ) }\end{array}$ & $0.83[0.57,1.09]$ \\
\hline 10.3 .33 to $4 \times / w k$ & 4 & 269 & $\begin{array}{l}\text { Std. Mean Difference (IV, Random, 95\% } \\
\mathrm{Cl} \text { ) }\end{array}$ & $0.29[0.04,0.53]$ \\
\hline 10.3.4 2×/wk & 3 & 327 & $\begin{array}{l}\text { Std. Mean Difference (IV, Random, 95\% } \\
\mathrm{Cl} \text { ) }\end{array}$ & $0.05[-0.44,0.53]$ \\
\hline 10.3.5 Dose not stated & 2 & 129 & $\begin{array}{l}\text { Std. Mean Difference (IV, Random, 95\% } \\
\mathrm{CI} \text { ) }\end{array}$ & $0.46[0.11,0.82]$ \\
\hline $\begin{array}{l}\text { 10.4 Provider of interven- } \\
\text { tion }\end{array}$ & 13 & 967 & $\begin{array}{l}\text { Std. Mean Difference (IV, Random, 95\% } \\
\mathrm{CI} \text { ) }\end{array}$ & $0.42[0.24,0.61]$ \\
\hline 10.4.1 Physiotherapist & 8 & 619 & $\begin{array}{l}\text { Std. Mean Difference (IV, Random, 95\% } \\
\mathrm{CI} \text { ) }\end{array}$ & $0.25[0.09,0.42]$ \\
\hline 10.4.2 Therapist & 2 & 112 & $\begin{array}{l}\text { Std. Mean Difference (IV, Random, 95\% } \\
\mathrm{CI} \text { ) }\end{array}$ & $0.84[0.45,1.23]$ \\
\hline 10.4.3 Nurse & 1 & 50 & $\begin{array}{l}\text { Std. Mean Difference (IV, Random, 95\% } \\
\mathrm{CI} \text { ) }\end{array}$ & $0.65[0.08,1.22]$ \\
\hline 10.4.4 Not stated & 2 & 186 & $\begin{array}{l}\text { Std. Mean Difference (IV, Random, 95\% } \\
\mathrm{CI} \text { ) }\end{array}$ & $0.69[0.25,1.14]$ \\
\hline $\begin{array}{l}\text { 10.5 Treatment compo- } \\
\text { nents included }\end{array}$ & 13 & & $\begin{array}{l}\text { Std. Mean Difference (IV, Random, 95\% } \\
\mathrm{Cl} \text { ) }\end{array}$ & Subtotals only \\
\hline $\begin{array}{l}10.5 .1 \text { Contains functional } \\
\text { training }\end{array}$ & 11 & 827 & $\begin{array}{l}\text { Std. Mean Difference (IV, Random, 95\% } \\
\mathrm{CI} \text { ) }\end{array}$ & $0.36[0.16,0.55]$ \\
\hline $\begin{array}{l}\text { 10.5.2 Contains neuro- } \\
\text { physiological }\end{array}$ & 8 & 467 & $\begin{array}{l}\text { Std. Mean Difference (IV, Random, 95\% } \\
\mathrm{CI} \text { ) }\end{array}$ & $0.61[0.40,0.81]$ \\
\hline $\begin{array}{l}\text { 10.5.3 Contains muscu- } \\
\text { loskeletal }\end{array}$ & 10 & 818 & $\begin{array}{l}\text { Std. Mean Difference (IV, Random, 95\% } \\
\mathrm{Cl} \text { ) }\end{array}$ & $0.36[0.20,0.52]$ \\
\hline
\end{tabular}


Analysis 10.1. Comparison 10: Subgroups. Intervention versus attention control or usual care: immediate outcome: motor function, Outcome 1: Time after stroke

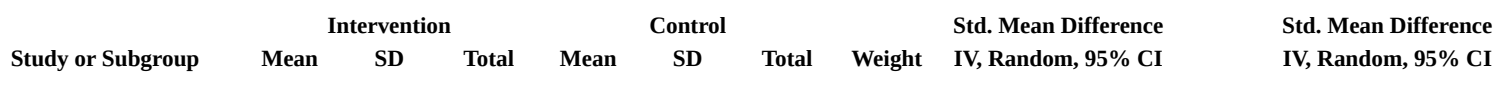

10.1.1 < 30 days post stroke

Chen 2010 (1)

Qian 2005

Richards 1993 (2)

Richards 1993 (3)

2.57

24.14

23.7

22.7

0.6

8.35

6.7

9.2

Subtotal (95\% CI)

Heterogeneity: Tau ${ }^{2}=0.00 ; \mathrm{Chi}^{2}=2.83, \mathrm{df}=3(\mathrm{P}=0.42) ; \mathrm{I}^{2}=0 \%$

Test for overall effect: $\mathrm{Z}=3.80(\mathrm{P}=0.0001)$

10.1.2 $<3$ months post stroke

Cooke 2006 (3)

Cooke 2006 (2)

\section{7}

Duncan 1998

\section{6}

$$
26.1
$$

8.6

10.4

36

34.6

Duncan 2003

26.84

2.51

Wei 1998

19.13

3.7

10

$10 \quad 22.6$

Subtotal (95\% CI)

$4.86 \quad 40$

161

25.46

14.5

Heterogeneity: $\mathrm{Tau}^{2}=0.04 ; \mathrm{Chi}^{2}=5.76, \mathrm{df}=4(\mathrm{P}=0.22) ; \mathrm{I}^{2}=31 \%$

Test for overall effect: $\mathrm{Z}=3.44(\mathrm{P}=0.0006)$

10.1.3 $<1$ year post stroke

McClellan 2004

13.47

$\begin{array}{rr}13.47 & 1.44 \\ 4.3 & 1.2\end{array}$

$1.44 \quad 125$

Wang 2004b

$65.15 \quad 19$.

Subtotal (95\% CI)

162

$25 \quad 12.82$

$12 \quad 4.7$

52.93

Heterogeneity: $\mathrm{Tau}^{2}=0.05 ; \mathrm{Chi}^{2}=3.51, \mathrm{df}=2(\mathrm{P}=0.17) ; \mathrm{I}^{2}=43 \%$

Test for overall effect: $\mathrm{Z}=1.74(\mathrm{P}=0.08)$

10.1.4 > 1 year post stroke

Mudge 2009 (4)

$14 \quad 1.5-31$

$0.78 \quad 53 \quad 10.0 \%$

$6.24 \quad 19 \quad 5.5 \%$

$10.7 \quad 4 \quad 2.1 \%$

10.7

$4 \quad 1.9 \%$

$8019.5 \%$

$0.49[0.10,0.87]$

$1.09[0.43,1.74]$

$0.43[-0.76,1.63]$

$0.25[-1.02,1.52]$

$0.60[0.29,0.91]$

Test for overall effect: $\mathrm{Z}=0.00(\mathrm{P}=1.00)$

10.1.5 Time not stated

Langhammer 2007

Tang 2009 (5)

36.4

13.9
0.93

$32 \quad 38.9$

61.53

12.7

11.62

$32 \quad 7.9 \%$

$35 \quad 8.0 \%$

$67 \quad 15.9 \%$

$-0.19[-0.68,0.31]$

$0.70[0.22,1.18]$

$0.26[-0.61,1.13]$

Heterogeneity: Tau $^{2}=0.33 ; \mathrm{Chi}^{2}=6.34, \mathrm{df}=1(\mathrm{P}=0.01) ; \mathrm{I}^{2}=84 \%$

Test for overall effect: $\mathrm{Z}=0.58(\mathrm{P}=0.56)$

Total (95\% CI)

512

Heterogeneity: $\mathrm{Tau}^{2}=0.05 ; \mathrm{Chi}^{2}=23.98, \mathrm{df}=14(\mathrm{P}=0.05) ; \mathrm{I}^{2}=42 \%$

Test for overall effect: $\mathrm{Z}=4.48(\mathrm{P}<0.00001)$

Test for subgroup differences: $\mathrm{Chi}^{2}=4.54, \mathrm{df}=4(\mathrm{P}=0.34), \mathrm{I}^{2}=11.9 \%$

$455 \quad 100.0 \%$

$0.42[0.24,0.61]$

$0.19[-0.42,0.79]$

$0.89[-0.04,1.82]$

$0.38[-0.03,0.79]$

$0.94[0.48,1.40]$

$0.52[0.23,0.82]$

$0.39[0.13,0.64]$

$-0.34[-1.21,0.53]$

$0.65[0.08,1.22]$

$0.35[-0.04,0.74]$

$0.00[-0.52,0.52]$

$0.00[-0.52,0.52]$

IV, Random, 95\% CI

\section{Footnotes}

(1) Mean and SD calculated from categorical data.

(2) Intervention 1 vs usual care. Control group shared.

(3) Intervention 2 vs usual care. Control group shared.

(4) SDs estimated from range ((max-min range)/4)

(5) FMA motor score used (not sensory score). 
Analysis 10.2. Comparison 10: Subgroups. Intervention versus attention control or usual care: immediate outcome: motor function, Outcome 2: Study geographical location

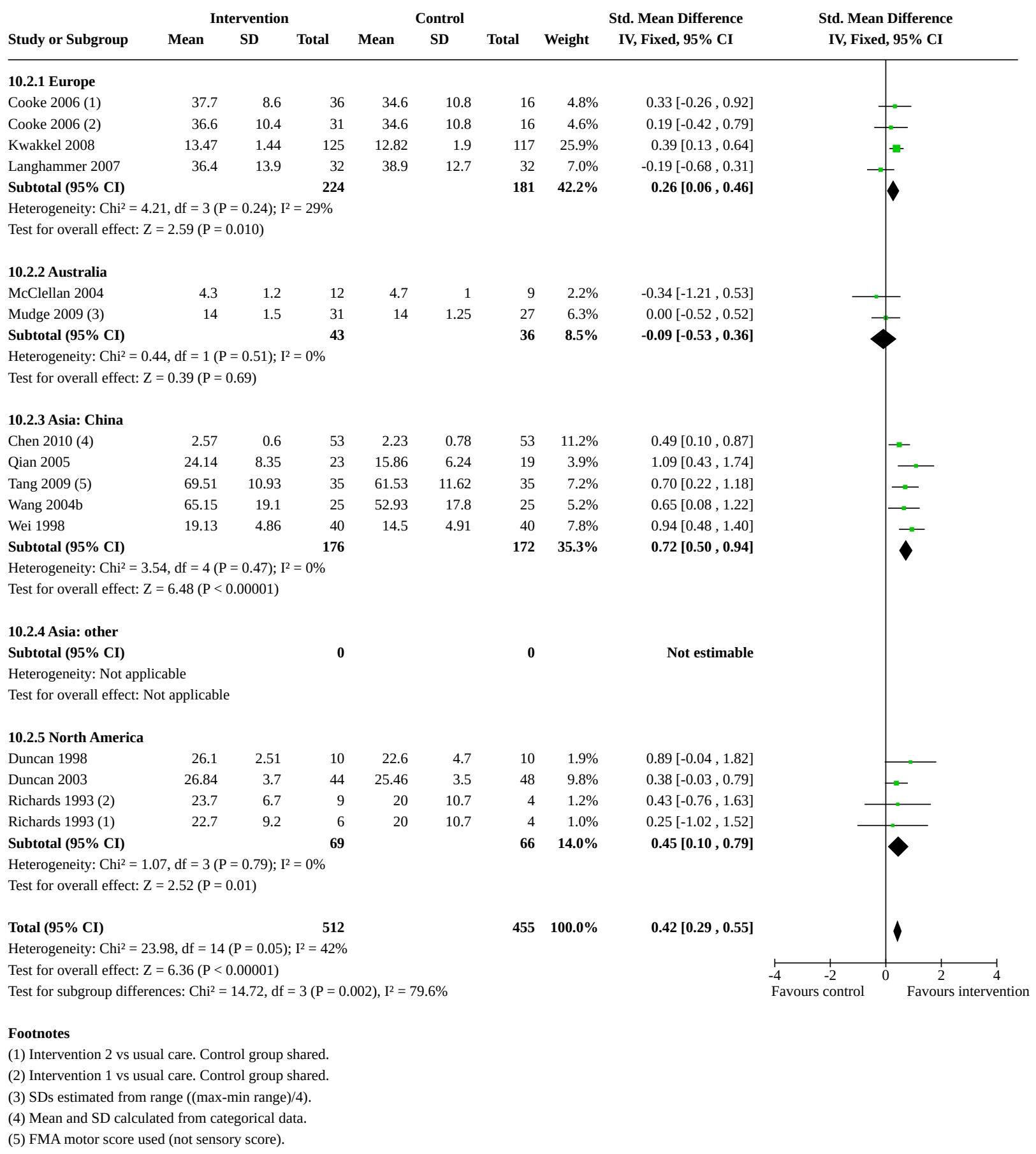




\section{Analysis 10.3. Comparison 10: Subgroups. Intervention versus attention control or usual care: immediate outcome: motor function, Outcome 3: Dose of intervention}

\begin{tabular}{|c|c|c|c|c|c|c|c|c|c|}
\hline & & erven & & & iontr & & & Std. Mean Difference & Std. Mean Difference \\
\hline Study or Subgroup & Mean & SD & Total & Mean & SD & Total & Weight & IV, Random, 95\% CI & IV, Random, 95\% CI \\
\hline
\end{tabular}

10.3.1 > once/d, with total of 60 to 120 minutes

Subtotal (95\% CI)

Heterogeneity: Not applicable

Test for overall effect: Not applicable

10.3.2 Once/d, 5 to $7 \times / w k$, for 30 to 60 minutes

Wang 2004b

Tang 2009 (1)

Wei 1998

Qian 2005

$65.15 \quad 19.1$

$69.51 \quad 10.93$

$\begin{array}{rr}69.51 & 10.93 \\ 19.13 & 4.86\end{array}$

25
35
40

$25 \quad 52.93$

17.8

11.62

$24.14 \quad 8.35$

Subtotal (95\% CI)

123

123

14.5

15.86

6.24

Heterogeneity: $\mathrm{Tau}^{2}=0.00 ; \mathrm{Chi}^{2}=1.46, \mathrm{df}=3(\mathrm{P}=0.69) ; \mathrm{I}^{2}=0 \%$

Test for overall effect: $\mathrm{Z}=6.16(\mathrm{P}<0.00001)$

10.3.3 3 to $4 \times /$ wk

Mudge 2009 (2)

Cooke 2006 (3)

Cooke 2006 (4)

Duncan 1998

Duncan 2003

Subtotal (95\% CI)

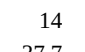

37.7

1.5

$8.6 \quad 36$

$31 \quad 14$

26.1

10.4

$31 \quad 34.6$

152

Heterogeneity: $\mathrm{Tau}^{2}=0.00 ; \mathrm{Chi}^{2}=3.13, \mathrm{df}=4(\mathrm{P}=0.54) ; \mathrm{I}^{2}=0 \%$

Test for overall effect: $\mathrm{Z}=2.28(\mathrm{P}=0.02)$

\subsection{4 $2 \times /$ wk}

McClellan 2004

Kwakkel 2008

Langhammer 2007

$\begin{array}{rrr}4.3 & 1.2 & 12 \\ 13.47 & 1.44 & 125\end{array}$

$12 \quad 4.7$

$125 \quad 12.82$

Subtotal (95\% CI)

169

38.9

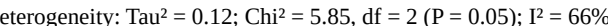

Test for overall effect: $\mathrm{Z}=0.19(\mathrm{P}=0.85)$

10.3.5 Dose not stated

2.57

Richards 1993 (4)

Richards 1993 (3)

2.57

22.7

Subtotal (95\% CI)

Heterogeneity: $\mathrm{Tau}^{2}=0.00 ; \mathrm{Chi}^{2}=0.12, \mathrm{df}=2(\mathrm{P}=0.94) ; \mathrm{I}^{2}=0 \%$

Test for overall effect: $\mathrm{Z}=2.56(\mathrm{P}=0.01)$

Total (95\% CI)

512

Heterogeneity: Tau$^{2}=0.05 ; \mathrm{Chi}^{2}=23.98, \mathrm{df}=14(\mathrm{P}=0.05) ; \mathrm{I}^{2}=42 \%$

Test for overall effect: $\mathrm{Z}=4.48(\mathrm{P}<0.00001)$

Test for subgroup differences: $\mathrm{Chi}^{2}=12.13, \mathrm{df}=3(\mathrm{P}=0.007), \mathrm{I}^{2}=75.3 \%$

$\begin{array}{rrr}1 & 9 & 3.6 \% \\ 1.9 & 117 & 13.4 \% \\ 12.7 & 32 & 7.9 \% \\ & \mathbf{1 5 8} & \mathbf{2 4 . 8 \%}\end{array}$

$-0.34[-1.21,0.53]$

$0.39[0.13,0.64]$

$-0.19[-0.68,0.31]$

$0.05[-0.44,0.53]$

\section{Footnotes}

(1) FMA motor score used (not sensory score).

(2) SDs estimated from range ((max-min range)/4)

(3) Intervention 2 vs usual care. Control group shared.

(4) Intervention 1 vs usual care. Control group shared.

(5) Mean and SD calculated from categorical data. 
Analysis 10.4. Comparison 10: Subgroups. Intervention versus attention control or usual care: immediate outcome: motor function, Outcome 4: Provider of intervention

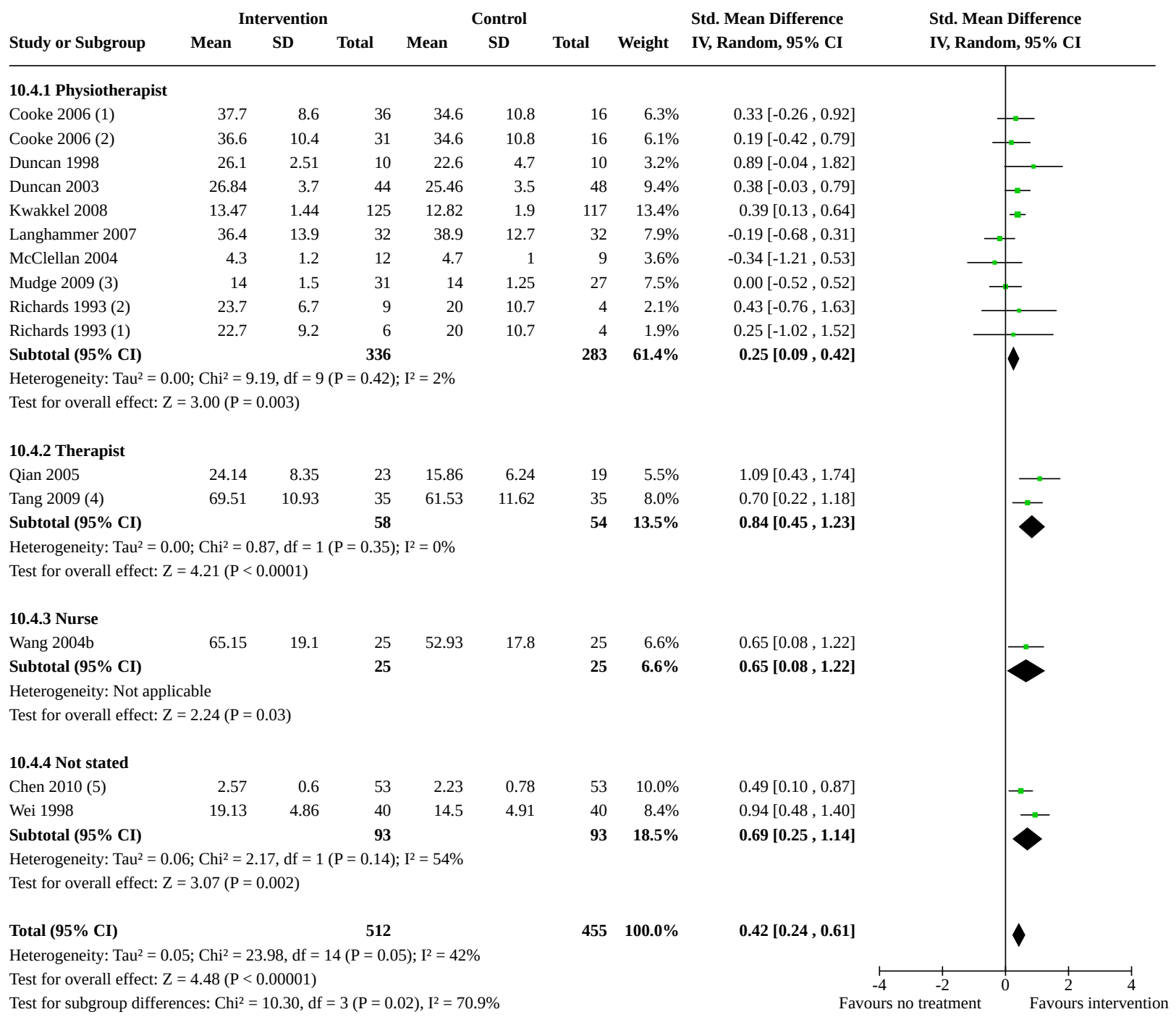

Footnotes

(1) Intervention 2 vs usual care. Control group shared.

(2) Intervention 1 vs usual care. Control group shared.

(3) SDs estimated from range ((max-min range)/4)

(4) FMA motor score used (not sensory score).

(5) Mean and SD calculated from categorical data. 
Analysis 10.5. Comparison 10: Subgroups. Intervention versus attention control or usual care: immediate outcome: motor function, Outcome 5: Treatment components included

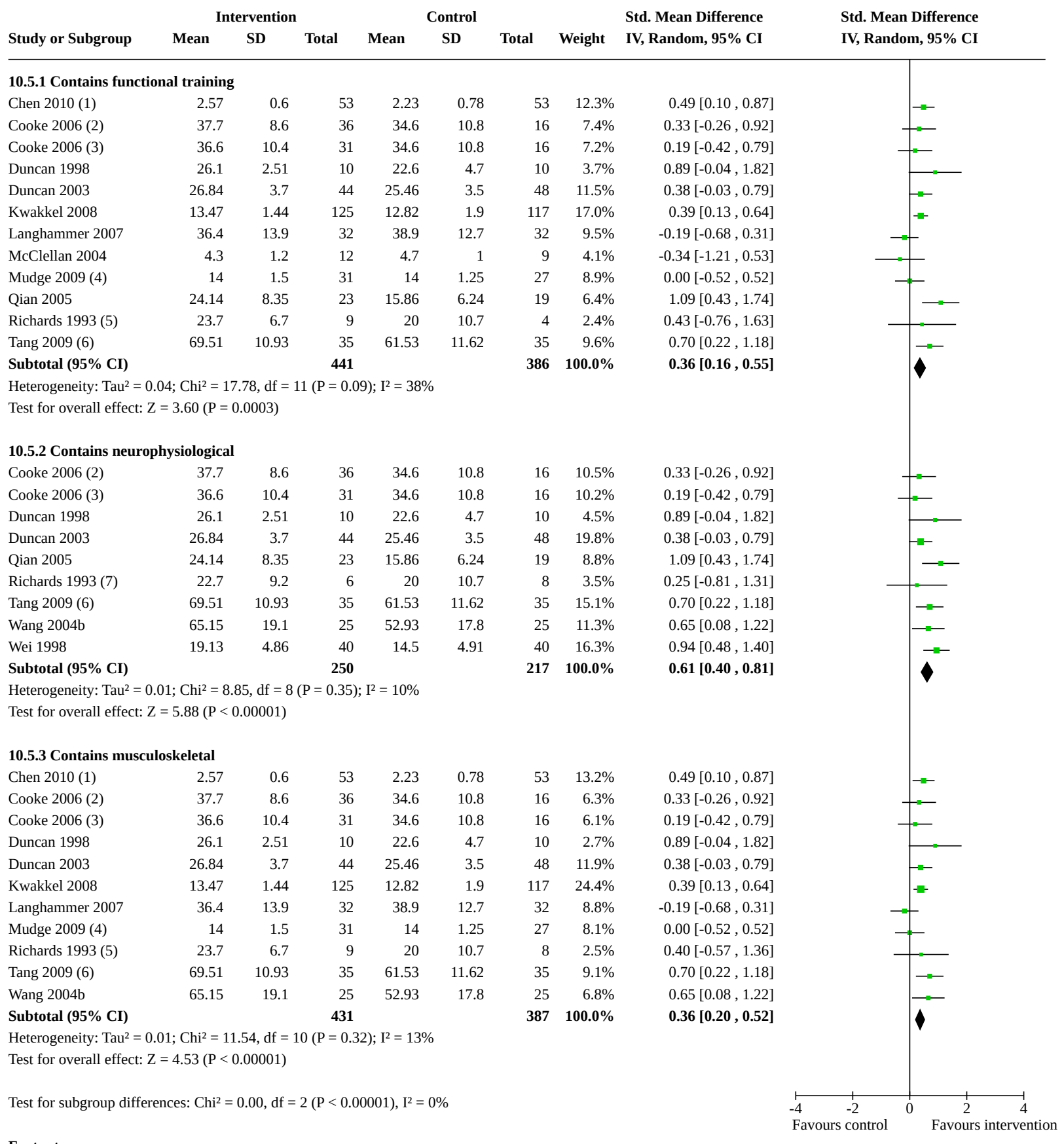

Footnotes

(1) Mean and SD calculated from categorical data.

(2) Intervention 2 vs usual care. Control group shared.

(3) Intervention 1 vs usual care. Control group shared.

(4) SDs estimated from range ((max-min range)/4).

(5) Intervention 1 vs usual care.

(6) FMA motor score used (not sensory score).

(7) Intervention 2 vs usual care. 
Comparison 11. Subgroups. One active intervention versus another active intervention: immediate outcomes: independence in ADL

\begin{tabular}{|c|c|c|c|c|}
\hline Outcome or subgroup title & No. of studies & $\begin{array}{l}\text { No. of partici- } \\
\text { pants }\end{array}$ & Statistical method & Effect size \\
\hline $\begin{array}{l}11.1 \text { Functional task training } \\
\text { components }\end{array}$ & 4 & & $\begin{array}{l}\text { Std. Mean Difference (IV, Random, } \\
95 \% \mathrm{CI})\end{array}$ & Subtotals only \\
\hline $\begin{array}{l}\text { 11.1.1 Described as motor re- } \\
\text { learning programme }\end{array}$ & 2 & 152 & $\begin{array}{l}\text { Std. Mean Difference (IV, Random, } \\
95 \% \mathrm{CI})\end{array}$ & $0.05[-0.50,0.60]$ \\
\hline $\begin{array}{l}\text { 11.1.2 One functional treatment } \\
\text { component }\end{array}$ & 1 & 15 & $\begin{array}{l}\text { Std. Mean Difference (IV, Random, } \\
95 \% \mathrm{CI})\end{array}$ & $0.15[-0.88,1.19]$ \\
\hline $\begin{array}{l}11.1 .3>1 \text { functional treatment } \\
\text { component }\end{array}$ & 1 & 19 & $\begin{array}{l}\text { Std. Mean Difference (IV, Random, } \\
95 \% \mathrm{CI})\end{array}$ & $-0.46[-1.38,0.45]$ \\
\hline $\begin{array}{l}11.2 \text { Neurophysiological com- } \\
\text { ponents }\end{array}$ & 6 & & $\begin{array}{l}\text { Std. Mean Difference (IV, Random, } \\
95 \% \mathrm{CI})\end{array}$ & Subtotals only \\
\hline 11.2.1 Described as Bobath & 5 & 325 & $\begin{array}{l}\text { Std. Mean Difference (IV, Random, } \\
95 \% \mathrm{CI})\end{array}$ & $-0.07[-0.41,0.26]$ \\
\hline $\begin{array}{l}11.2 .2 \text { Describes interventions } \\
\text { that may be Bobath }\end{array}$ & 2 & 46 & $\begin{array}{l}\text { Std. Mean Difference (IV, Random, } \\
95 \% \mathrm{CI})\end{array}$ & $0.05[-0.66,0.76]$ \\
\hline $\begin{array}{l}\text { 11.2.3 Proprioceptive neuro- } \\
\text { muscular facilitation (PNF) }\end{array}$ & 1 & 61 & $\begin{array}{l}\text { Std. Mean Difference (IV, Random, } \\
95 \% \mathrm{CI})\end{array}$ & $-0.42[-0.92,0.09]$ \\
\hline 11.2.4 Sensorimotor facilitation & 0 & 0 & $\begin{array}{l}\text { Std. Mean Difference (IV, Random, } \\
95 \% \mathrm{CI})\end{array}$ & Not estimable \\
\hline $\begin{array}{l}11.3 \text { Musculoskeletal compo- } \\
\text { nents }\end{array}$ & 3 & & $\begin{array}{l}\text { Std. Mean Difference (IV, Random, } \\
95 \% \mathrm{CI})\end{array}$ & Subtotals only \\
\hline $\begin{array}{l}\text { 11.3.1 Active movement + mus- } \\
\text { cle strengthening }\end{array}$ & 0 & 0 & $\begin{array}{l}\text { Std. Mean Difference (IV, Random, } \\
95 \% \mathrm{CI})\end{array}$ & Not estimable \\
\hline $\begin{array}{l}\text { 11.3.2 Active and active-assisted } \\
\text { movement }\end{array}$ & 0 & 0 & $\begin{array}{l}\text { Std. Mean Difference (IV, Random, } \\
95 \% \mathrm{CI})\end{array}$ & Not estimable \\
\hline 11.3.3 Muscle strengthening & 2 & 42 & $\begin{array}{l}\text { Std. Mean Difference (IV, Random, } \\
95 \% \mathrm{CI})\end{array}$ & $0.23[-0.39,0.84]$ \\
\hline 11.3.4 Passive only & 1 & 61 & $\begin{array}{l}\text { Std. Mean Difference (IV, Random, } \\
95 \% \mathrm{CI})\end{array}$ & $-0.42[-0.92,0.09]$ \\
\hline
\end{tabular}


Analysis 11.1. Comparison 11: Subgroups. One active intervention versus another active intervention: immediate outcomes: independence in ADL, Outcome 1: Functional task training components

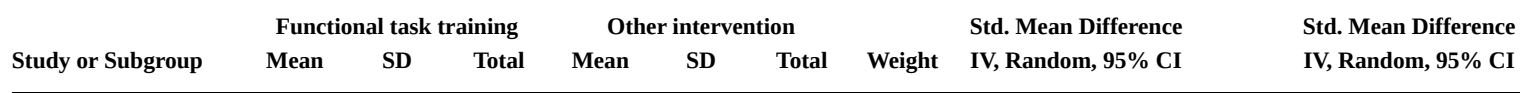

11.1.1 Described as motor relearning programme

$\begin{array}{lrrrrrrrr}\text { Langhammer 2000 (1) } & 83 & 25 & 29 & 72 & 34 & 24 & 44.3 \% & 0.37[-0.18,0.91] \\ \text { Lincoln 2003 (1) } & 14 & 5.7 & 47 & 15 & 4.3 & 52 & 55.7 \% & -0.20[-0.59,0.20] \\ \text { Subtotal (95\% CI) } & & & \mathbf{7 6} & & & \mathbf{7 6} & \mathbf{1 0 0 . 0 \%} & \mathbf{0 . 0 5}[-\mathbf{0 . 5 0 , 0 . 6 0 ]}\end{array}$

Heterogeneity: $\mathrm{Tau}^{2}=0.10 ; \mathrm{Chi}^{2}=2.71, \mathrm{df}=1(\mathrm{P}=0.10) ; \mathrm{I}^{2}=63 \%$

Test for overall effect: $\mathrm{Z}=0.19(\mathrm{P}=0.85)$

11.1.2 One functional treatment component

Richards 1993 (2) $\quad 25.8 \quad 14.8$

Subtotal (95\% CI)

$23.3 \quad 16.6$

$6 \quad 100.0 \%$

$0.15[-0.88,1.19]$

Heterogeneity: Not applicable

$6 \quad \mathbf{1 0 0 . 0} \%$

$0.15[-0.88,1.19]$

Test for overall effect: $\mathrm{Z}=0.29(\mathrm{P}=0.77)$

11.1.3 $>1$ functional treatment component

$\begin{array}{lllllllll}\text { Mudie } 2002(2) & 68.9 & 21.5 & 9 & 79.5 & 22.11 & 10 & 100.0 \% & -0.46[-1.38,0.45] \\ \text { Subtotal (95\% CI) } & & & \mathbf{9} & & & \mathbf{1 0} & \mathbf{1 0 0 . 0} \% & \mathbf{- 0 . 4 6}[-\mathbf{1 . 3 8 , 0 . 4 5}]\end{array}$

Heterogeneity: Not applicable

$10 \quad 100.0 \%$

$-0.46[-1.38,0.45]$

Test for overall effect: $\mathrm{Z}=0.99(\mathrm{P}=0.32)$

Test for subgroup differences: $\mathrm{Chi}^{2}=0.00, \mathrm{df}=2(\mathrm{P}<0.00001), \mathrm{I}^{2}=0 \%$

Footnotes

(1) Intervention 2 vs intervention 1.

(2) Intervention 1 vs intervention 2. 
Analysis 11.2. Comparison 11: Subgroups. One active intervention versus another active intervention: immediate outcomes: independence in ADL, Outcome 2: Neurophysiological components

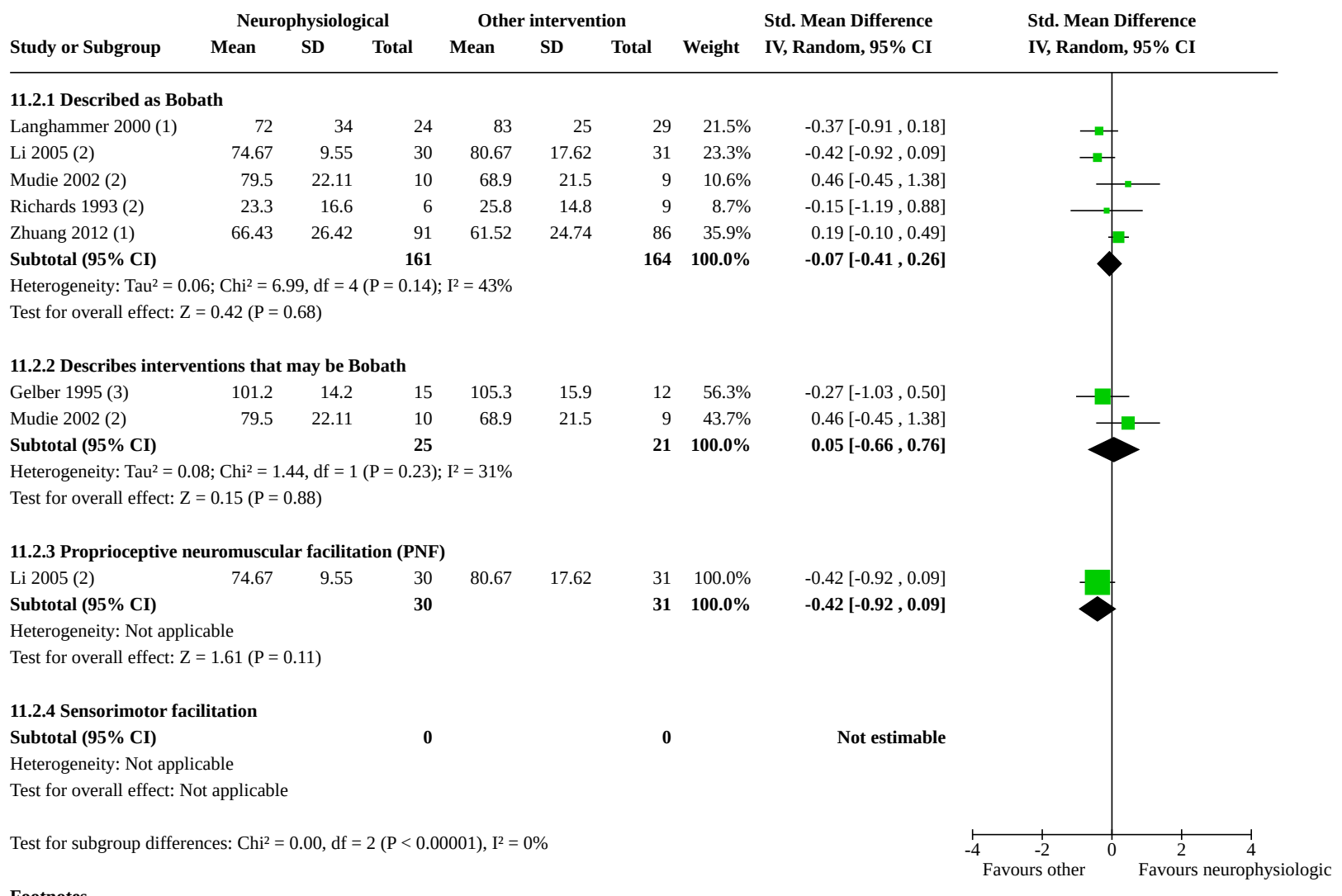

Dootnotes

(1) Intervention 1 vs intervention 2.

(2) Intervention 2 vs intervention 1.

(3) SDs calculated from SE. 
Analysis 11.3. Comparison 11: Subgroups. One active intervention versus another active intervention: immediate outcomes: independence in ADL, Outcome 3: Musculoskeletal components

\begin{tabular}{|c|c|c|c|c|c|c|c|c|}
\hline \multirow[b]{2}{*}{ Study or Subgroup } & \multicolumn{3}{|c|}{ Musculoskeletal } & \multicolumn{3}{|c|}{ Other intervention } & \multirow[b]{2}{*}{ Weight } & \multirow{2}{*}{$\begin{array}{l}\text { Std. Mean Difference } \\
\text { IV, Random, } 95 \% \text { CI }\end{array}$} \\
\hline & Mean & SD & Total & Mean & SD & Total & & \\
\hline \multicolumn{9}{|c|}{ 11.3.1 Active movement + muscle strengthening } \\
\hline & & & $\mathbf{0}$ & & & $\mathbf{0}$ & & Not estimable \\
\hline \multicolumn{9}{|c|}{ Heterogeneity: Not applicable } \\
\hline \multicolumn{9}{|c|}{ Test for overall effect: Not applicable } \\
\hline \multicolumn{9}{|c|}{ 11.3.2 Active and active-assisted movement } \\
\hline Subtotal (95\% CI) & & & $\mathbf{0}$ & & & $\mathbf{0}$ & & Not estimable \\
\hline \multicolumn{9}{|c|}{ Heterogeneity: Not applicable } \\
\hline \multicolumn{9}{|c|}{ Test for overall effect: Not applicable } \\
\hline \multicolumn{9}{|c|}{ 11.3.3 Muscle strengthening } \\
\hline Gelber 1995 (1) & 105.3 & 15.9 & 12 & 101.2 & 14.2 & 15 & $64.8 \%$ & $0.27[-0.50,1.03]$ \\
\hline Richards 1993 (2) & 25.8 & 14.8 & 9 & 23.3 & 16.6 & 6 & $35.2 \%$ & $0.15[-0.88,1.19]$ \\
\hline Subtotal (95\% CI) & & & 21 & & & 21 & $100.0 \%$ & $0.23[-0.39,0.84]$ \\
\hline \multicolumn{9}{|c|}{ Heterogeneity: $\mathrm{Tau}^{2}=0.00 ; \mathrm{Chi}^{2}=0.03, \mathrm{df}=1(\mathrm{P}=0.86) ; \mathrm{I}^{2}=0 \%$} \\
\hline \multicolumn{9}{|c|}{ Test for overall effect: $\mathrm{Z}=0.72(\mathrm{P}=0.47)$} \\
\hline \multicolumn{9}{|l|}{ 11.3.4 Passive only } \\
\hline Li 2005 (3) & 74.67 & 9.55 & 30 & 80.67 & 17.62 & 31 & $100.0 \%$ & $-0.42[-0.92,0.09]$ \\
\hline Subtotal $(95 \%$ CI) & & & 30 & & & 31 & $100.0 \%$ & $-0.42[-0.92,0.09]$ \\
\hline \multicolumn{9}{|c|}{ Heterogeneity: Not applicable } \\
\hline \multicolumn{9}{|c|}{ Test for overall effect: $\mathrm{Z}=1.61(\mathrm{P}=0.11)$} \\
\hline \multicolumn{9}{|c|}{ Test for subgroup differences: $\mathrm{Chi}^{2}=0.00, \mathrm{df}=1(\mathrm{P}<0.00001), \mathrm{I}^{2}=0 \%$} \\
\hline \multicolumn{9}{|l|}{ Footnotes } \\
\hline \multicolumn{9}{|c|}{ (1) SDs calculated from SE. } \\
\hline (2) Intervention 1 vs il & vention 2. & & & & & & & \\
\hline 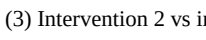 & IOn 1 & & & & & & & \\
\hline
\end{tabular}

Std. Mean Difference IV, Random, 95\% CI

Comparison 12. Subgroups. One active intervention versus another active intervention: immediate outcomes: motor function

\begin{tabular}{|c|c|c|c|c|}
\hline Outcome or subgroup title & No. of studies & $\begin{array}{l}\text { No. of partici- } \\
\text { pants }\end{array}$ & Statistical method & Effect size \\
\hline $\begin{array}{l}\text { 12.1 Functional task training } \\
\text { components }\end{array}$ & 4 & & $\begin{array}{l}\text { Std. Mean Difference (IV, Random, } \\
95 \% \mathrm{Cl} \text { ) }\end{array}$ & Subtotals only \\
\hline $\begin{array}{l}\text { 12.1.1 Described as motor re- } \\
\text { learning programme }\end{array}$ & 2 & 152 & $\begin{array}{l}\text { Std. Mean Difference (IV, Random, } \\
95 \% \mathrm{Cl} \text { ) }\end{array}$ & $-0.08[-0.75,0.60]$ \\
\hline $\begin{array}{l}\text { 12.1.2 One functional treatment } \\
\text { component }\end{array}$ & 1 & 15 & $\begin{array}{l}\text { Std. Mean Difference (IV, Random, } \\
95 \% \mathrm{Cl} \text { ) }\end{array}$ & $0.12[-0.91,1.16]$ \\
\hline $\begin{array}{l}12.1 .3>1 \text { functional treatment } \\
\text { component }\end{array}$ & 1 & 21 & $\begin{array}{l}\text { Std. Mean Difference (IV, Random, } \\
95 \% \mathrm{CI} \text { ) }\end{array}$ & $-0.64[-1.52,0.24]$ \\
\hline $\begin{array}{l}12.2 \text { Neurophysiological com- } \\
\text { ponents }\end{array}$ & 8 & & $\begin{array}{l}\text { Std. Mean Difference (IV, Random, } \\
95 \% \mathrm{Cl} \text { ) }\end{array}$ & Subtotals only \\
\hline 12.2.1 Described as Bobath & 6 & 383 & $\begin{array}{l}\text { Std. Mean Difference (IV, Random, } \\
95 \% \mathrm{Cl} \text { ) }\end{array}$ & $0.13[-0.10,0.36]$ \\
\hline $\begin{array}{l}\text { 12.2.2 Describes interventions } \\
\text { that may be Bobath }\end{array}$ & 4 & 162 & $\begin{array}{l}\text { Std. Mean Difference (IV, Random, } \\
95 \% \mathrm{Cl} \text { ) }\end{array}$ & $0.27[-0.12,0.66]$ \\
\hline
\end{tabular}




\begin{tabular}{|c|c|c|c|c|}
\hline Outcome or subgroup title & No. of studies & $\begin{array}{l}\text { No. of partici- } \\
\text { pants }\end{array}$ & Statistical method & Effect size \\
\hline $\begin{array}{l}\text { 12.2.3 Proprioceptive neuro- } \\
\text { muscular facilitation (PNF) }\end{array}$ & 0 & 0 & $\begin{array}{l}\text { Std. Mean Difference (IV, Random, } \\
95 \% \mathrm{CI} \text { ) }\end{array}$ & Not estimable \\
\hline 12.2.4 Sensorimotor facilitation & 1 & 18 & $\begin{array}{l}\text { Std. Mean Difference (IV, Random, } \\
95 \% \mathrm{Cl})\end{array}$ & $-0.04[-0.97,0.88]$ \\
\hline $\begin{array}{l}12.3 \text { Musculoskeletal compo- } \\
\text { nents }\end{array}$ & 4 & & $\begin{array}{l}\text { Std. Mean Difference (IV, Random, } \\
95 \% \mathrm{Cl} \text { ) }\end{array}$ & Subtotals only \\
\hline $\begin{array}{l}\text { 12.3.1 Active movement + mus- } \\
\text { cle strengthening }\end{array}$ & 1 & 21 & $\begin{array}{l}\text { Std. Mean Difference (IV, Random, } \\
95 \% \mathrm{Cl} \text { ) }\end{array}$ & $-0.64[-1.52,0.24]$ \\
\hline $\begin{array}{l}\text { 12.3.2 Active and active-assisted } \\
\text { movement }\end{array}$ & 0 & 0 & $\begin{array}{l}\text { Std. Mean Difference (IV, Random, } \\
95 \% \mathrm{Cl} \text { ) }\end{array}$ & Not estimable \\
\hline 12.3.3 Muscle strengthening & 3 & 60 & $\begin{array}{l}\text { Std. Mean Difference (IV, Random, } \\
95 \% \mathrm{CI} \text { ) }\end{array}$ & $0.10[-0.41,0.62]$ \\
\hline 12.3.4 Passive only & 0 & 0 & $\begin{array}{l}\text { Std. Mean Difference (IV, Random, } \\
95 \% \mathrm{Cl} \text { ) }\end{array}$ & Not estimable \\
\hline
\end{tabular}

Analysis 12.1. Comparison 12: Subgroups. One active intervention versus another active intervention: immediate outcomes: motor function, Outcome 1: Functional task training components

\begin{tabular}{|c|c|c|c|c|c|c|c|c|c|}
\hline \multirow{2}{*}{ Study or Subgroup } & \multicolumn{3}{|c|}{ Functional task training } & \multicolumn{3}{|c|}{ Other intervention } & & \multirow{2}{*}{$\begin{array}{l}\text { Std. Mean Difference } \\
\text { IV. Random, } 95 \% \text { CI }\end{array}$} & \multirow{2}{*}{$\begin{array}{l}\text { Std. Mean Difference } \\
\text { IV, Random, } 95 \% \text { CI }\end{array}$} \\
\hline & Mean & SD & Total & Mean & SD & Total & Weight & & \\
\hline
\end{tabular}

12.1.1 Described as motor relearning programme

\begin{tabular}{|c|c|c|c|c|c|c|c|c|}
\hline . & rnit & 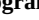 & & & & & & \\
\hline Langhammer 2000 (1) & 37 & 12 & 29 & 33 & 15 & 24 & $46.3 \%$ & $0.29[-0.25,0.84]$ \\
\hline Lincoln 2003 (2) & 5 & 5 & 47 & 7 & 5 & 52 & $53.7 \%$ & $-0.40[-0.80,0.00]$ \\
\hline Subtotal (95\% CI) & & & 76 & & & 76 & $100.0 \%$ & $-0.08[-0.75,0.60]$ \\
\hline
\end{tabular}

Heterogeneity: $\mathrm{Tau}^{2}=0.18 ; \mathrm{Chi}^{2}=4.02, \mathrm{df}=1(\mathrm{P}=0.04) ; \mathrm{I}^{2}=75 \%$

$-0.08[-0.75,0.60]$

Test for overall effect: $\mathrm{Z}=0.23(\mathrm{P}=0.82)$

12.1.2 One functional treatment component

\begin{tabular}{|c|c|c|c|c|c|c|c|c|}
\hline Richards 1993 (3) & 23.7 & 6.7 & 9 & 22.7 & 9.2 & 6 & $100.0 \%$ & $0.12[-0.91,1.16]$ \\
\hline Subtotal (95\% CI) & & & 9 & & & 6 & $100.0 \%$ & $0.12[-0.91,1.16]$ \\
\hline
\end{tabular}

Heterogeneity: Not applicable

Test for overall effect: $\mathrm{Z}=0.23(\mathrm{P}=0.82)$

12.1.3 > 1 functional treatment component

\begin{tabular}{|c|c|c|c|c|c|c|c|c|}
\hline Wang 2005 (2) & 15.33 & 4.59 & 11 & 18.82 & 5.84 & 10 & $100.0 \%$ & $-0.64[-1.52,0.24]$ \\
\hline Subtotal (95\% CI) & & & 11 & & & 10 & $100.0 \%$ & $-0.64[-1.52,0.24]$ \\
\hline
\end{tabular}

Heterogeneity: Not applicable

Test for overall effect: $\mathrm{Z}=1.42(\mathrm{P}=0.15)$

Test for subgroup differences: $\mathrm{Chi}^{2}=0.00, \mathrm{df}=2(\mathrm{P}<0.00001), \mathrm{I}^{2}=0 \%$

Footnotes

(1) intervention 2 vs intervention 1

(2) Intervention 2 vs intervention 1.

(3) Intervention 1 vs intervention 2. 
Analysis 12.2. Comparison 12: Subgroups. One active intervention versus another active intervention: immediate outcomes: motor function, Outcome 2: Neurophysiological components

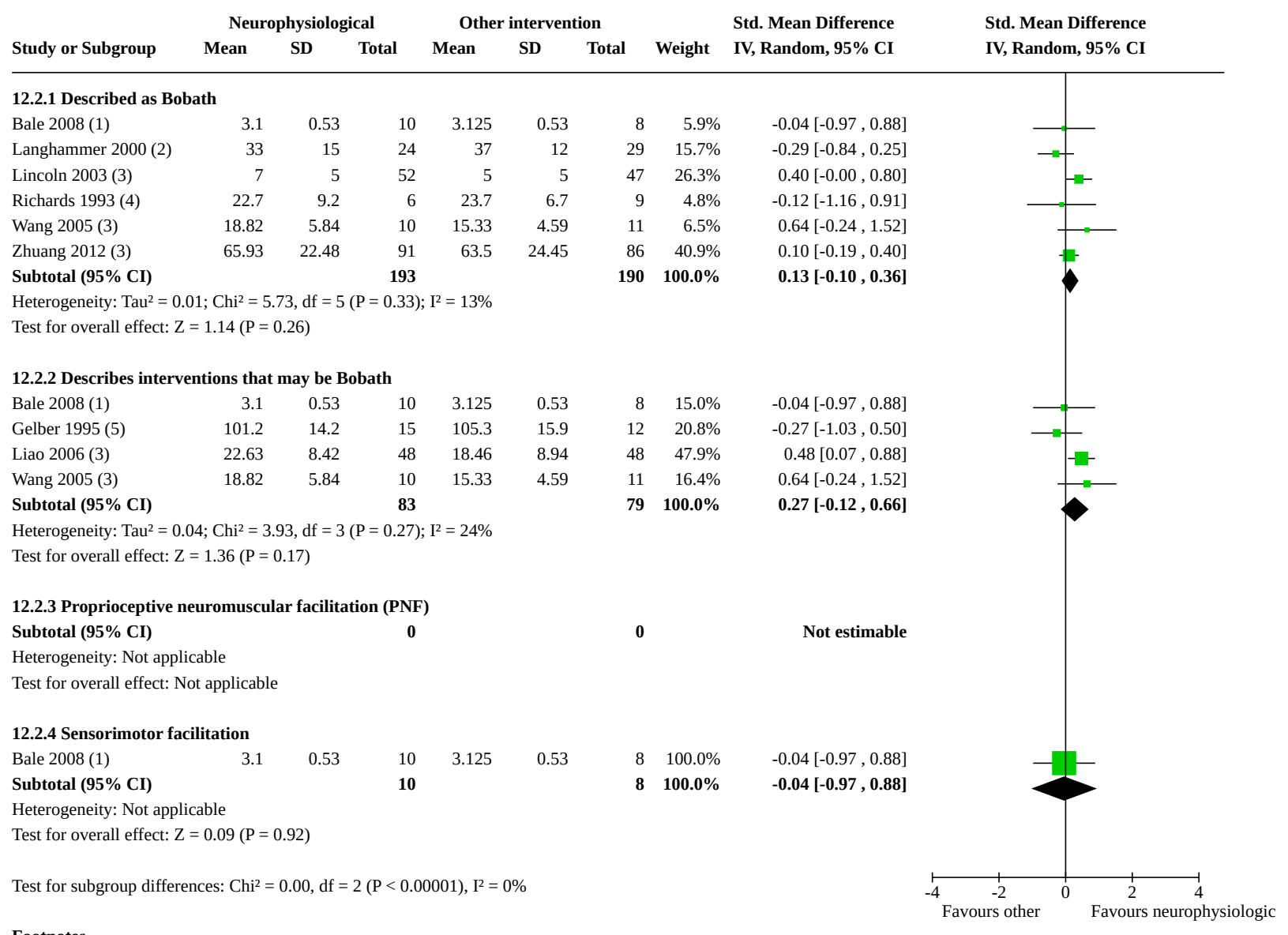

Footnotes

(1) Mean and SD calculated from categorical data.

(2) intervention 1 vs intervention 2

(3) Intervention 1 vs intervention 2.

(4) Intervention 2 vs intervention 1.

(5) Intervention 1 vs intervention 2. SD calculated from SE. 
Analysis 12.3. Comparison 12: Subgroups. One active intervention versus another active intervention: immediate outcomes: motor function, Outcome 3: Musculoskeletal components

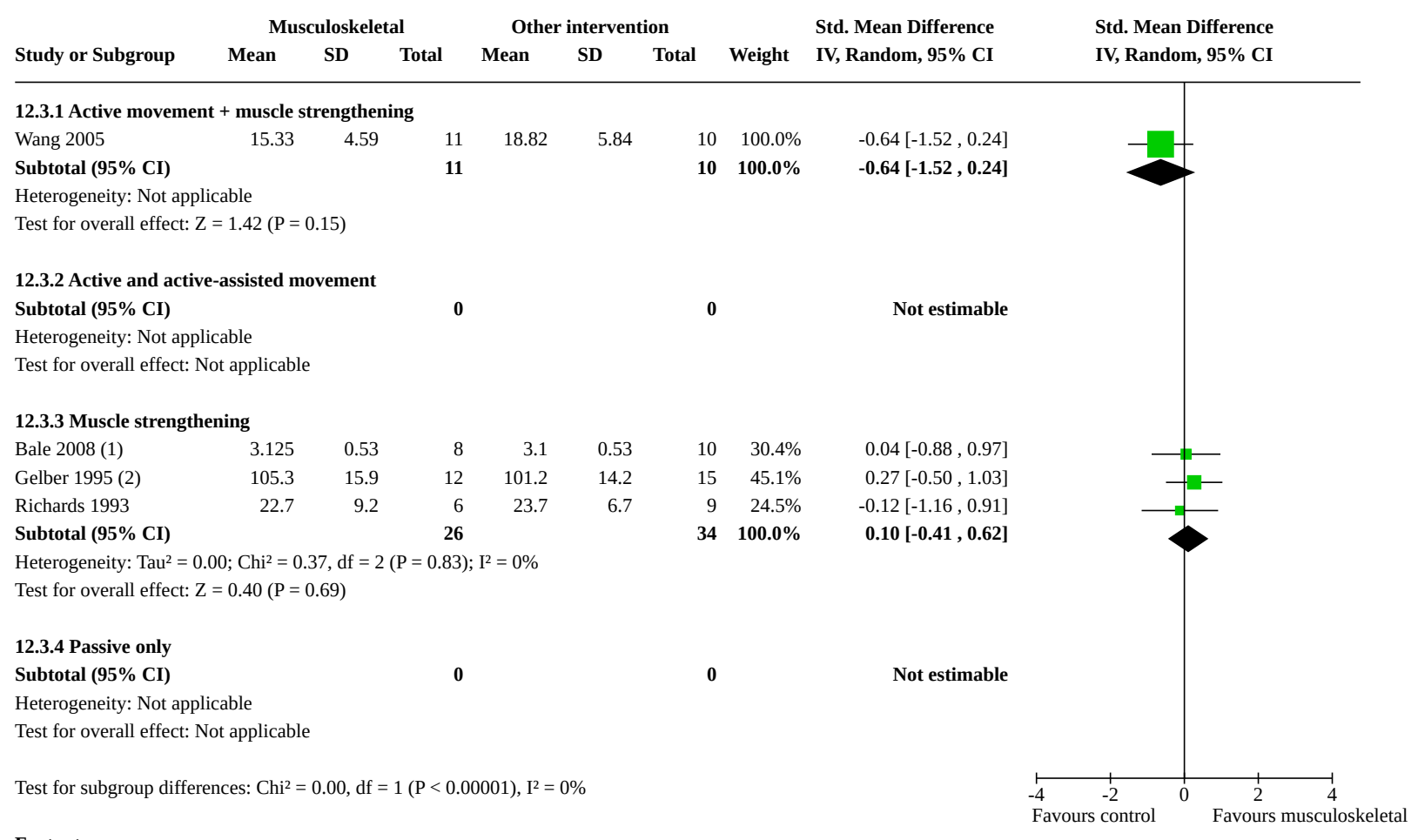

(1) Mean and SD calculated from categorical data.

(2) Intervention 2 vs intervention 1. SD calculated from SE.

ADDITIONAL TABLES

Table 1. Criteria for classification of neurophysiological and motor learning approaches

\begin{tabular}{|c|c|c|c|c|}
\hline $\begin{array}{l}\text { Name of ap- } \\
\text { proach }\end{array}$ & Philosophy/theory & Treatment principles & $\begin{array}{l}\text { Descriptive } \\
\text { terms }\end{array}$ & $\begin{array}{l}\text { Supporting ref- } \\
\text { erences }\end{array}$ \\
\hline $\begin{array}{l}\text { Rood } \\
\text { (neurophysiolog- } \\
\text { ical) }\end{array}$ & $\begin{array}{l}\text { Concerned with 'the interaction of } \\
\text { somatic, autonomic, and psychic } \\
\text { factors, and their role in regulations } \\
\text { of motor behaviour'. } \\
\text { Motor and sensory functions insepa- } \\
\text { rable } \\
\text { Focuses on the developmental se- } \\
\text { quence of recovery and the use of } \\
\text { peripheral input to facilitate move- } \\
\text { ment }\end{array}$ & $\begin{array}{l}\text { Activate/facilitate movement } \\
\text { and postural responses of pa- } \\
\text { tient in same automatic way as } \\
\text { they occur in the normal } \\
\text { Sequencing of movement from } \\
\text { basic to complex (supine lying; } \\
\text { rolling; prone lying; kneeling; } \\
\text { standing; walking) } \\
\text { Sensory stimulation (brush- } \\
\text { ing, icing, tapping, pounding, } \\
\text { stroking, slow stretch, joint } \\
\text { compression) to stimulate } \\
\text { movement at automatic level }\end{array}$ & $\begin{array}{l}\text { Ontogenetic se- } \\
\text { quences } \\
\text { Developmental } \\
\text { sequences } \\
\text { Postural stability } \\
\text { Normal patterns } \\
\text { of movement } \\
\text { Joint and cuta- } \\
\text { neous receptors } \\
\text { Golgi tendon or- } \\
\text { gans } \\
\text { Abnormal tone }\end{array}$ & $\begin{array}{l}\text { Goff 1969; Rood } \\
\text { 1954; Stockmey- } \\
\text { er } 1967\end{array}$ \\
\hline $\begin{array}{l}\text { Proprioceptive } \\
\text { neuromuscular } \\
\text { facilitation (PNF) } \\
\text { or Knott and } \\
\text { Voss }\end{array}$ & $\begin{array}{l}\text { Active muscle contractions intend- } \\
\text { ed to stimulate afferent proprio- } \\
\text { ceptive discharges into the CNS in- } \\
\text { creased excitation and recruitment } \\
\text { of additional motor units }\end{array}$ & $\begin{array}{l}\text { Diagonal and spiral patterns of } \\
\text { active and passive movement } \\
\text { Quick stretch at end of range to } \\
\text { promote contraction following } \\
\text { relaxation in antagonists }\end{array}$ & $\begin{array}{l}\text { Patterns of } \\
\text { movement } \\
\text { Stretch and pos- } \\
\text { tural reflexes } \\
\text { Manual pressure }\end{array}$ & $\begin{array}{l}\text { Kabat 1953; Voss } \\
1967\end{array}$ \\
\hline
\end{tabular}

Physical rehabilitation approaches for the recovery of function and mobility following stroke (Review) 
Table 1. Criteria for classification of neurophysiological and motor learning approaches (Continued)

(neurophysiolog- Assumes that central and peripheral ical)

stimulation are enhanced and facilitated in order to maximise the motor responses required

Cortex controls patterns of movement not singular muscular actions Necessary to return to normal developmental sequence for recovery
Brunnström (neurophysiological)
Uses primitive reflexes to initiate movement and encourages use of mass patterns in early stages of recovery

Aims to encourage return of voluntary movement through use of reflex activity and sensory stimulation Assumes recovery progresses from subcortical to cortical control of muscle function Stages of recovery: flaccidity; elicit major synergies at reflex level; establish voluntary control of synergies; break away from flexor and extensor synergies by mixing components from antagonist synergies; more difficult movement combinations mastered; individual joint movements become possible; voluntary movement is elicited
Maximal resistance is given by therapist to facilitate maximal activity in the range of the required movement.

Voluntary contraction of the targeted muscle(s)

Manual contact and therapist's tone of voice to encourage purposeful movement Isometric and isotonic contrac tions, traction and approximation of joint surfaces to stimulate postural reflexes
Isometric and isotonic contrac-

tion

Approximation

of joint surfaces

Afferent input

Use tasks that patient can master or almost master.

Sensory stimulation: from tonic neck or labyrinthine reflexes, or from stroking, tapping muscles

Bobath or neurodevelopmental approach (NDT) (neurophysiological)
Aim to control afferent input and facilitate normal postural reactions Aim to give patients the experience of normal movement and afferent input while inhibiting abnormal movement and afferent input To improve quality of movement on affected side, so that the 2 sides work together harmoniously Assumption that increased tone and increased reflex activity will emerge as a result of lack of inhibition from a damaged postural reflex mechanism. Movement will be abnormal if comes from a background of abnormal tone

Tone can be influenced by altering position or movement of proximal joints of the body
Facilitation of normal movement by a therapist, using direct handling of the body at key points such as head and spine, shoulders and pelvic girdle and, distally, feet and hands Volitional movement by patient is requested only against a background of automatic postural activity

NB. Techniques of treatment have changed over time; more recently they have become more active and functionally orientated

However, there is a lack of published material describing the current treatment principles of the Bobath approach

More recently (October 2000)

it has been emphasised that the concepts of the Bobath approach 'integrate with the main ideas of motor learning theory', and that advocated key
Normal movement

Abnormal postural reflex activity/tone Postural control Key points Reflex inhibitory patterns

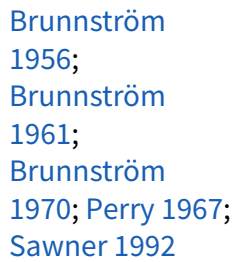

;


Table 1. Criteria for classification of neurophysiological and motor learning approaches (Continued)

treatment principles include

active participation, practice

and meaningful goals (Mayston

2000)

$\begin{array}{ll}\begin{array}{ll}\text { Johnstone } \\ \text { (neurophysiolog- }\end{array} & \begin{array}{l}\text { To control spasticity by inhibiting } \\ \text { ical) }\end{array} \\ & \text { tioning to influence tone } \\ & \text { Assumes that damaged postural re- } \\ & \text { flex mechanism can be controlled } \\ & \text { through positioning and splinting } \\ & \text { Based on hierarchical model that } \\ & \text { assumes recovery is from proximal } \\ & \text { to distal } \\ & \text { Aim to achieve central stability, with } \\ & \text { gross motor performance, before } \\ & \text { progressing to more skilled move- } \\ & \text { ments } \\ & \text { Inflatable air splints: apply even, } \\ & \text { deep pressure to address sensory } \\ & \text { dysfunction }\end{array}$
Use of inflatable splints
Emphasis on correct position and use of splints
Early stages: patient in side ly- ing, with splint on affected arm Treatment progresses through hierarchy of activities, pro- gressing from rolling through to crawling
Family involvement encouraged

$\begin{array}{ll}\text { Muscle tone } & \text { Johnstone } 1980 \\ \text { Air/pressure } & \text { Johnstone } 1989 \\ \text { splints } & \\ \text { Positioning } & \\ \text { Reflex inhibition } & \\ \text { Tonic neck reflex } & \\ \text { Anti-gravity pat- } & \\ \text { terns } & \end{array}$

terns

\begin{tabular}{|c|c|}
\hline $\begin{array}{l}\text { Carr and Shep- } \\
\text { herd or motor } \\
\text { learning or mo- } \\
\text { tor relearning or } \\
\text { movement sci- } \\
\text { ence } \\
\text { (motor learning) }\end{array}$ & $\begin{array}{l}\text { Assumes that neurologically im- } \\
\text { paired people learn in the same way } \\
\text { as healthy people. } \\
\text { Assumes that motor control of pos- } \\
\text { ture and movement are interrelat- } \\
\text { ed and that appropriate sensory in- } \\
\text { put will help modulate the motor re- } \\
\text { sponse to a task } \\
\text { Patient is an active learner } \\
\text { Uses biomechanical analysis of } \\
\text { movement } \\
\text { Training should be context-specific } \\
\text { Essential for motor learning: elimi- } \\
\text { nation of unnecessary muscle activi- } \\
\text { ty; feedback; practice } \\
\text { Focus is on cognitive learning }\end{array}$ \\
\hline
\end{tabular}

Carr and Shepherd or motor learning or moence Focus is on cognitive learning
(1) Analysis of task
(2) Practice of missing compo- nents
(3) Practice of task
(4) Transference of training
Biomechanical analysis with

Motor control
Motor relearning
Feedback
Practice
Problem solving
Training
Carr 1980; Carr 1982; Carr 1987a; Carr 1987b; Carr 1990; Carr 1998

movements compared to the normal

Instruction, explanation and feedback are essential parts of training

Training involves practice with guidance from therapist: guidance may be manual (but is used for support or demonstration, not for providing sensory input)

Identifiable and specific goals Appropriate environment

Conductive education or Peto (motor learning)
Aims to teach patient strategies for dealing with disabilities in order to encourage them to learn to live with or overcome disabilities Integrated approach emphasising continuity and consistency Assumes that feelings of failure can produce a dysfunctional attitude, which can prevent rehabilitation Teaches strategies for coping with disability

Active movements start with an intention and end with the goal Conductor assists patient to achieve movement control through task analysis and rhythmical intention or verbal reinforcement
Educational principles and repetition used as a method of rote learning

Highly structured day

Group work

Task analysis

Repetition and reinforcement of task through rhythmical intention or verbal chanting Activities broken down into components or steps Patient encouraged to guide movements bilaterally
Education

Rhythmical intention

Intention

Integrated system

Group work

Conductor Independence
Bower 1993; Cotton 1983; Kinsman 1988 
Table 1. Criteria for classification of neurophysiological and motor learning approaches (Continued)

Emphasis on learning rather than receiving treatments

Affolter
(motor learning)

Interaction between individual and environment fundamental part of learning

Perception seen as having an essential role in the cycle of learning Incoming information is compared with past experience ('assimilation'), which leads to anticipatory behaviour

Assimilation and anticipation seen as basic for planning and for performance of complex movements Feedback is important to learning process

\begin{abstract}
NB. This approach started from theory, rather than from clinical practice

Starting at an elementary level, there will be no anticipation The patient starts to initiate more steps

There is increased anticipation of the steps to be taken

As experience increases, the patient will start to search for missing objects

The patient is able plan more than 1 stage ahead and can perform new sequences if functional signals are familiar Not only can the patient think ahead but is able to check all the steps of the task in advance
\end{abstract}

\section{Sensory integra-} tion or Ayres (motor learning)

\section{Sensory feedback} Repetition

\author{
Perception \\ Assimilation \\ Anticipation \\ Complex human \\ performance
}

\section{Affolter 1980}

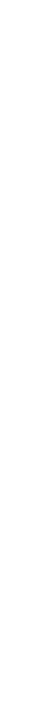

The criteria listed in this Table are those used in previous versions of this review. These criteria are not used in this updated version of the review. (See Table 2 for the criteria used for classification of interventions within this updated review).

Table 2. Classification of categories and individual treatment components

\begin{tabular}{lll}
\hline Categories & Treatment component & Description of individual treatment component \\
\hline Assistive devices (AD) & Walking aids & Devices to assist walking, including sticks and frames \\
\hline & Orthoses for walking & Externally applied orthoses to assist walking, including AFO, knee braces \\
\hline & Resting splints & Externally applied orthoses to maintain or improve limb alignment \\
\hline $\begin{array}{l}\text { Cardiopulmonary inter- } \\
\text { ventions }(\mathrm{Cl})\end{array}$ & $\begin{array}{l}\text { Aerobic/fitness/endurance } \\
\text { training }\end{array}$ & Activities to improve cardiopulmonary fitness \\
\hline $\begin{array}{l}\text { Functional task training } \\
\text { (FTT) }\end{array}$ & $\begin{array}{l}\text { ADL training } \\
\text { Sitting \&/or standing balance }\end{array}$ & $\begin{array}{l}\text { Various activities performed sitting \&/or standing with the aim of improv- } \\
\text { ing } \\
\text { training }\end{array}$ \\
\hline & $\begin{array}{l}\text { Sractice of tasks relevant to daily life, including both part and whole task } \\
\text { the ability to balance safely and independently }\end{array}$ \\
\hline
\end{tabular}


Table 2. Classification of categories and individual treatment components (Continued)

safely and independently

\begin{tabular}{|c|c|c|}
\hline & Transfer practice & $\begin{array}{l}\text { Practice of tasks aimed at improving ability to move from one position to } \\
\text { another }\end{array}$ \\
\hline & Walking & Practice of tasks aimed at improving ambulation \\
\hline & Stair climbing & Practice of tasks aimed at ability to go up and down stairs \\
\hline & Upper limb function training & $\begin{array}{l}\text { Practice of tasks aimed at improving the ability to move and use the arm, } \\
\text { such as reach, grasp, and hand-to-mouth activities }\end{array}$ \\
\hline & $\begin{array}{l}\text { Described as "MRP" (MRP } \\
\text { - Motor Relearning Pro- } \\
\text { gramme) }\end{array}$ & Described as MRP \\
\hline \multirow[t]{3}{*}{ Modality (Mo) } & Acupuncture & as an adjunct, delivered for either pain relief or movement therapy \\
\hline & $\begin{array}{l}\text { Physical agents (including } \\
\text { hot, cold, }\end{array}$ & as an adjunct, delivered for either pain relief or movement therapy \\
\hline & $\begin{array}{l}\text { TENS - Transcutaneous elec- } \\
\text { trical nerve stimulation) }\end{array}$ & \\
\hline \multirow[t]{2}{*}{$\begin{array}{l}\text { Musculoskeletal inter- } \\
\text { vention (active) }\end{array}$} & Muscle strengthening & $\begin{array}{l}\text { Practice of activities to progressively increase the ability to generate } \\
\text { muscle force, including using body weight and external resistance }\end{array}$ \\
\hline & $\begin{array}{l}\text { Active \& active-assisted } \\
\text { movement }\end{array}$ & $\begin{array}{l}\text { Moving a limb through its range of movement, under the patient's active } \\
\text { control with or without assistance }\end{array}$ \\
\hline \multirow[t]{5}{*}{$\begin{array}{l}\text { Musculoskeletal inter- } \\
\text { vention (passive) }\end{array}$} & $\begin{array}{l}\text { Increasing angle of upright } \\
\text { sitting }\end{array}$ & a form of positioning, to promote early sitting \\
\hline & Tilt table & To promote early lower limb loading \\
\hline & Passive movement & $\begin{array}{l}\text { Moving a limb through it's range of movement, whilst the patient is pas- } \\
\text { sive }\end{array}$ \\
\hline & Body \& limb positioning & $\begin{array}{l}\text { placing a limb or body part in a supported position, to maintain optimal } \\
\text { alignment }\end{array}$ \\
\hline & Massage & $\begin{array}{l}\text { Manipulation of soft tissue, using the hands or a tool designed for the } \\
\text { purpose }\end{array}$ \\
\hline \multirow[t]{4}{*}{$\begin{array}{l}\text { Neurophysiological in- } \\
\text { tervention }\end{array}$} & $\begin{array}{l}\text { Hands on facilitation of ('nor- } \\
\text { mal') movement (Bobath) }\end{array}$ & $\begin{array}{l}\text { Intervention which is described as facilitation of movement, referenced } \\
\text { to Bobath or Davies }\end{array}$ \\
\hline & $\begin{array}{l}\text { Inhibition of abnormal mus- } \\
\text { cle tone / normalising tone } \\
\text { (Bobath) }\end{array}$ & $\begin{array}{l}\text { Intervention which is described as inhibition of abnormal muscle tone or } \\
\text { as } \\
\text { normalising muscle tone, referenced to Bobath or Davies }\end{array}$ \\
\hline & Described as "Bobath" & Described as Bobath \\
\hline & $\begin{array}{l}\text { Trunk mobilisations / postur- } \\
\text { al reactions (Bobath) }\end{array}$ & $\begin{array}{l}\text { Intervention which is described as trunk mobilisations or postural reac- } \\
\text { tions to }\end{array}$ \\
\hline
\end{tabular}


Table 2. Classification of categories and individual treatment components (Continued)

\begin{tabular}{ll}
\hline $\begin{array}{l}\text { Proprioceptive Neuromuscu- } \\
\text { larfacilitation }\end{array}$ & Described as PNF \\
$\begin{array}{ll}\text { (PNF - proprioceptive neuro- } \\
\text { muscular facilitation) }\end{array}$ & $\begin{array}{l}\text { The use of excitatory techniques, such as brushing, striking, tapping, ic- } \\
\text { ing, }\end{array}$ \\
\hline Sensorimotor facilitation & to improve sensory awareness and promote muscle activity \\
\hline
\end{tabular}

AD: assistive devices; CPI: cardiopulmonary interventions; FTT: functional task training; MoD: Modality; Musc.(active): musculoskeletal intervention (active); Musc.(passive): musculoskeletal intervention (passive); NP: neurophysiological intervention. 


\begin{tabular}{|c|c|c|c|c|c|c|c|c|}
\hline Study & Group & Categoric & d treatment & omponents) & & & & \\
\hline & & $\begin{array}{l}\text { Assistive } \\
\text { Devices }\end{array}$ & $\begin{array}{l}\text { Cardiopul- } \\
\text { monary } \\
\text { intervention }\end{array}$ & $\begin{array}{l}\text { Functional } \\
\text { Task } \\
\text { Training }\end{array}$ & Modality & $\begin{array}{l}\text { Muscu- } \\
\text { loskeletal } \\
\text { intervention } \\
\text { (active) }\end{array}$ & $\begin{array}{l}\text { Musculoskele- } \\
\text { tal } \\
\text { intervention } \\
\text { (passive) }\end{array}$ & $\begin{array}{l}\text { Neurophysio- } \\
\text { logical } \\
\text { intervention }\end{array}$ \\
\hline Aksu 2001 & 'All groups' & - & - & - & - & - & - & $\begin{array}{l}\text { Described as } \\
\text { 'Bobath' }\end{array}$ \\
\hline Allison 2007 & 'Intervention' & - & - & $\begin{array}{l}\text { Sitting and/or standing balance } \\
\text { Sit-to-stand practice } \\
\text { Walking } \\
\text { UL function training }\end{array}$ & - & $\begin{array}{l}\text { Musc. } \\
\text { strengthen- } \\
\text { ing }\end{array}$ & - & - \\
\hline & 'Control' & - & - & $\begin{array}{l}\text { Walking } \\
\text { UL function training }\end{array}$ & - & $\begin{array}{l}\text { Musc. } \\
\text { strengthen- } \\
\text { ing }\end{array}$ & - & - \\
\hline Baer 2007 & 'Part practice' & - & - & Walking & - & - & - & - \\
\hline & 'Whole practice' & - & - & Walking & - & - & - & - \\
\hline Bai 2008 & $\begin{array}{l}\text { 'Early rehabili- } \\
\text { tation' }\end{array}$ & - & - & $\begin{array}{l}\text { ADL training } \\
\text { Sitting and/or standing balance } \\
\text { Walking }\end{array}$ & - & - & - & - \\
\hline Bale 2008 & $\begin{array}{l}\text { 'Functional } \\
\text { strength train- } \\
\text { ing' }\end{array}$ & - & - & $\begin{array}{l}\text { ADL training } \\
\text { Sitting and/or standing balance } \\
\text { Sit-to-stand practice } \\
\text { Walking } \\
\text { UL function training }\end{array}$ & - & $\begin{array}{l}\text { Musc. } \\
\text { strengthen- } \\
\text { ing }\end{array}$ & - & - \\
\hline & $\begin{array}{l}\text { 'Training as } \\
\text { usual' }\end{array}$ & - & - & ADL training & - & - & - & $\begin{array}{l}\text { Hands on fa- } \\
\text { cilitation }\end{array}$ \\
\hline
\end{tabular}




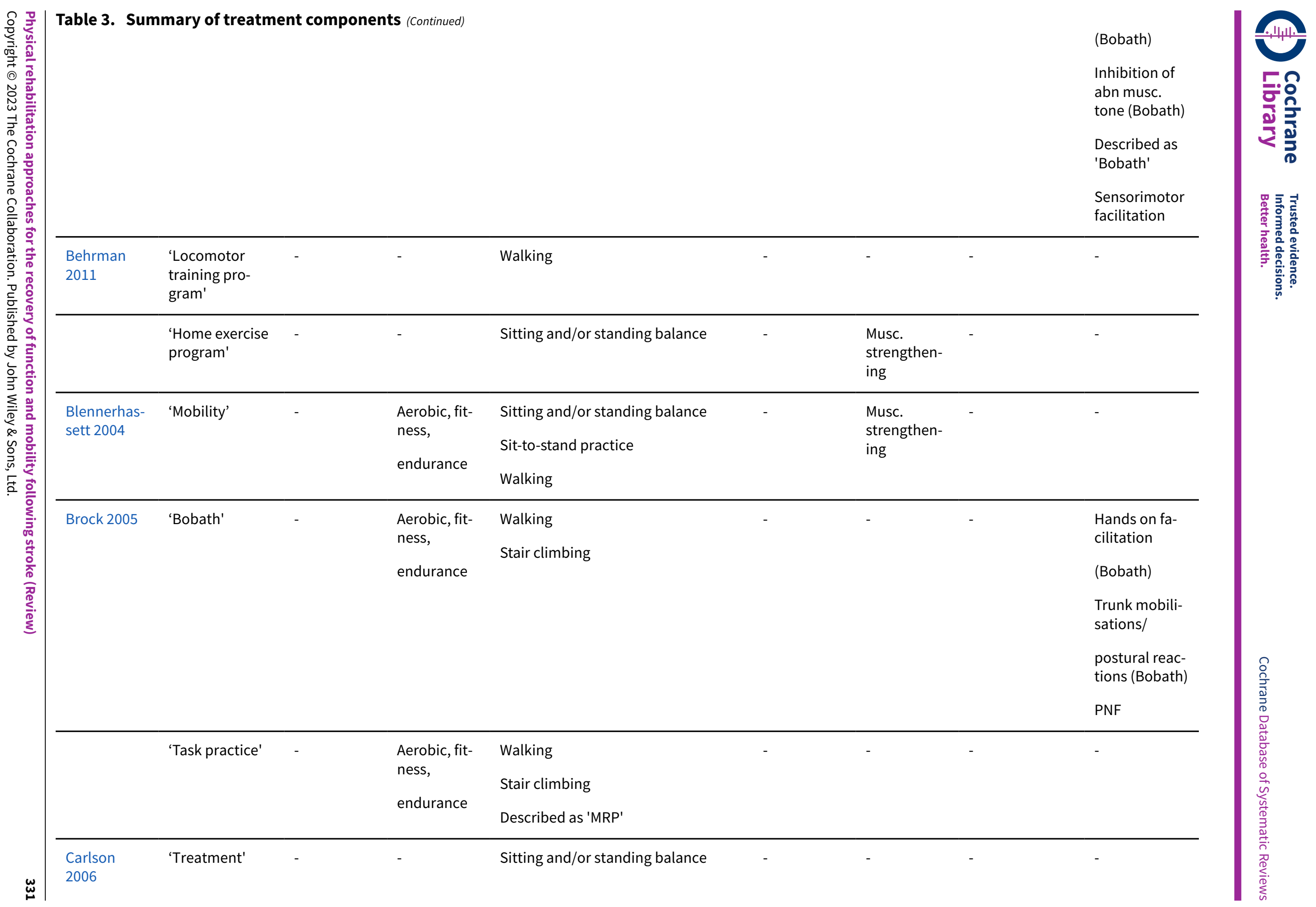




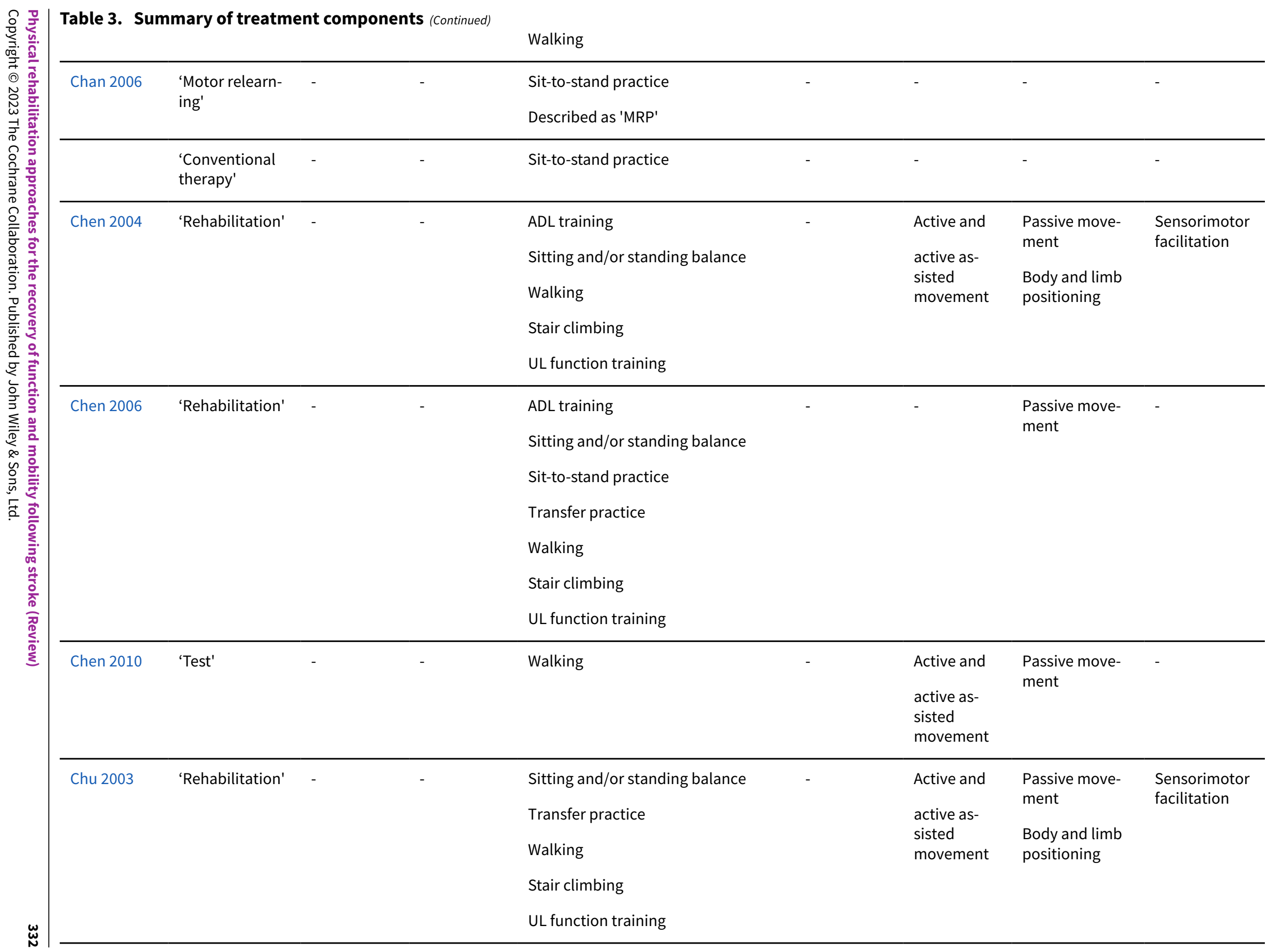




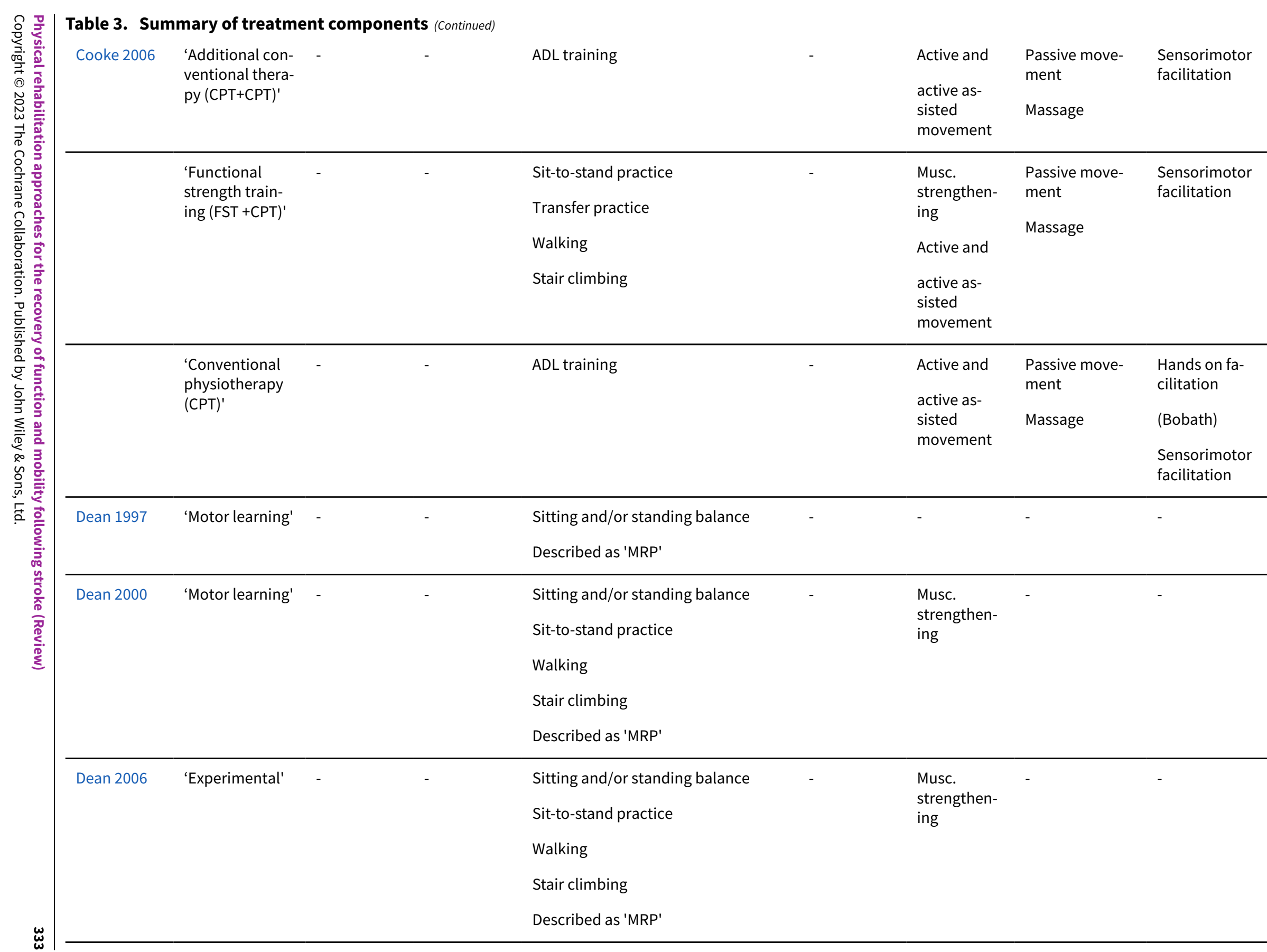




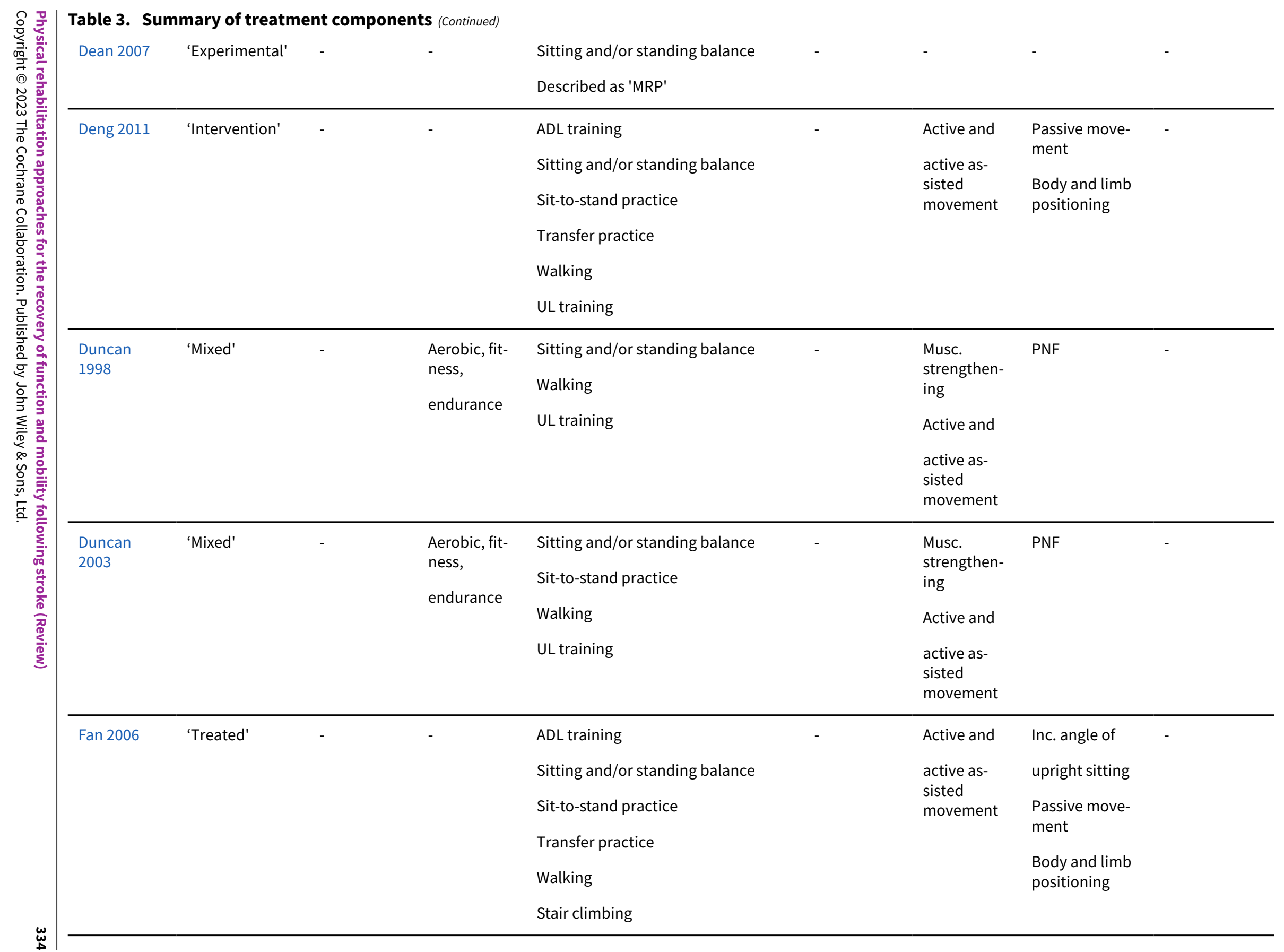




\begin{tabular}{|c|c|c|c|c|c|c|c|c|}
\hline Fang 2003 & $\begin{array}{l}\text { 'Additional ear- } \\
\text { ly physiothera- } \\
\text { py intervention' }\end{array}$ & - & - & - & - & - & $\begin{array}{l}\text { Passive move- } \\
\text { ment }\end{array}$ & $\begin{array}{l}\text { Described as } \\
\text { 'Bobath' }\end{array}$ \\
\hline $\begin{array}{l}\text { Fang } 2004 \\
\text { old }\end{array}$ & 'Rehabilitation' & - & - & - & - & - & $\begin{array}{l}\text { Passive move- } \\
\text { ment } \\
\text { Massage }\end{array}$ & - \\
\hline $\begin{array}{l}\text { Fang } 2004 \\
\text { young }\end{array}$ & 'Rehabilitation' & - & - & - & - & - & $\begin{array}{l}\text { Passive move- } \\
\text { ment } \\
\text { Massage }\end{array}$ & - \\
\hline Ge 2003 & 'Rehabilitation' & - & - & $\begin{array}{l}\text { Sitting and/or standing balance } \\
\text { Sit-to-stand practice } \\
\text { Transfer practice } \\
\text { Walking }\end{array}$ & $\begin{array}{l}\text { Acupunc- } \\
\text { ture } \\
\text { Physical } \\
\text { agents }\end{array}$ & $\begin{array}{l}\text { Active and } \\
\text { active as- } \\
\text { sisted } \\
\text { movement }\end{array}$ & Massage & $\begin{array}{l}\text { Described as } \\
\text { 'Bobath' }\end{array}$ \\
\hline Gelber 1995 & $\begin{array}{l}\text { 'Neurophysio- } \\
\text { logical (NDT)' }\end{array}$ & - & - & ADL training & - & - & - & $\begin{array}{l}\text { Hands on fa- } \\
\text { cilitation } \\
\text { (Bobath) } \\
\text { Inhibition of } \\
\text { abn musc. } \\
\text { tone (Bobath) }\end{array}$ \\
\hline & $\begin{array}{l}\text { 'Orthopaedic } \\
\text { (TFR)' }\end{array}$ & $\begin{array}{l}\text { Walking aids } \\
\text { Orthoses for } \\
\text { walking }\end{array}$ & - & ADL training & - & $\begin{array}{l}\text { Musc. } \\
\text { strengthen- } \\
\text { ing }\end{array}$ & $\begin{array}{l}\text { Passive move- } \\
\text { ment }\end{array}$ & - \\
\hline Green 2002 & 'Mixed' & & & $\begin{array}{l}\text { ADL training } \\
\text { Sitting and/or standing balance } \\
\text { Walking }\end{array}$ & & & & \\
\hline $\begin{array}{l}\text { Holmgren } \\
2006\end{array}$ & 'Intervention' & - & - & $\begin{array}{l}\text { Sitting and/or standing balance } \\
\text { Sit-to-stand practice }\end{array}$ & - & $\begin{array}{l}\text { Musc. } \\
\text { strengthen- } \\
\text { ing }\end{array}$ & - & - \\
\hline
\end{tabular}




\begin{tabular}{|c|c|c|c|c|c|c|c|c|c|}
\hline \multirow{8}{*}{ 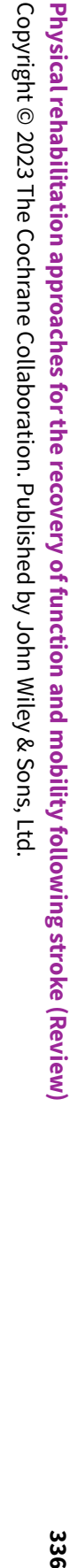 } & \multicolumn{4}{|c|}{ lable 3. Summary or treatment components (Continued) } & \multicolumn{5}{|l|}{ Walking } \\
\hline & Hou 2006 & 'Rehabilitation' & - & - & $\begin{array}{l}\text { ADL training } \\
\text { Sitting and/or standing balance } \\
\text { Sit-to-stand practice } \\
\text { Transfer practice } \\
\text { Walking } \\
\text { Stair climbing }\end{array}$ & $\begin{array}{l}\text { Physical } \\
\text { agents }\end{array}$ & $\begin{array}{l}\text { Active and } \\
\text { active as- } \\
\text { sisted } \\
\text { movement }\end{array}$ & $\begin{array}{l}\text { Passive move- } \\
\text { ment } \\
\text { Body and limb } \\
\text { positioning }\end{array}$ & $\begin{array}{l}\text { Described as } \\
\text { 'Bobath' } \\
\text { PNF }\end{array}$ \\
\hline & Howe 2005 & 'Mixed' & - & - & $\begin{array}{l}\text { Sitting and/or standing balance } \\
\text { Described as 'MRP' }\end{array}$ & - & - & - & - \\
\hline & $\begin{array}{l}\text { Hu } 2007 \\
\text { haem }\end{array}$ & 'Test' & & & $\begin{array}{l}\text { Details of individual components not } \\
\text { available }\end{array}$ & & & $\begin{array}{l}\text { Details of indi- } \\
\text { vidual compo- } \\
\text { nents not avail- } \\
\text { able }\end{array}$ & \\
\hline & Hu 2007 isch & 'Test' & & & $\begin{array}{l}\text { Details of individual components not } \\
\text { available }\end{array}$ & & & $\begin{array}{l}\text { Details of indi- } \\
\text { vidual compo- } \\
\text { nents not avail- } \\
\text { able }\end{array}$ & \\
\hline & Huang 2003 & 'Rehabilitation' & - & - & $\begin{array}{l}\text { Sitting and/or standing balance } \\
\text { Sit-to-stand practice } \\
\text { Transfer practice } \\
\text { Walking } \\
\text { Stair climbing } \\
\text { Described as 'MRP' }\end{array}$ & $\begin{array}{l}\text { Acupunc- } \\
\text { ture } \\
\text { Physical } \\
\text { agents }\end{array}$ & $\begin{array}{l}\text { Active and } \\
\text { active as- } \\
\text { sisted } \\
\text { movement }\end{array}$ & $\begin{array}{l}\text { Inc. angle of } \\
\text { upright sitting } \\
\text { Passive move- } \\
\text { ment } \\
\text { Body and limb } \\
\text { positioning }\end{array}$ & $\begin{array}{l}\text { Described as } \\
\text { 'Bobath' } \\
\text { PNF }\end{array}$ \\
\hline & $\begin{array}{l}\text { Hui-Chan } \\
2009\end{array}$ & 'PLBO-TRT' & - & - & $\begin{array}{l}\text { Sitting and/or standing balance } \\
\text { Sit-to-stand practice } \\
\text { Walking }\end{array}$ & - & - & - & - \\
\hline & Jiang 2006 & 'Treated' & - & - & ADL training & $\begin{array}{l}\text { Acupunc- } \\
\text { ture }\end{array}$ & - & $\begin{array}{l}\text { Passive move- } \\
\text { ment }\end{array}$ & - \\
\hline
\end{tabular}




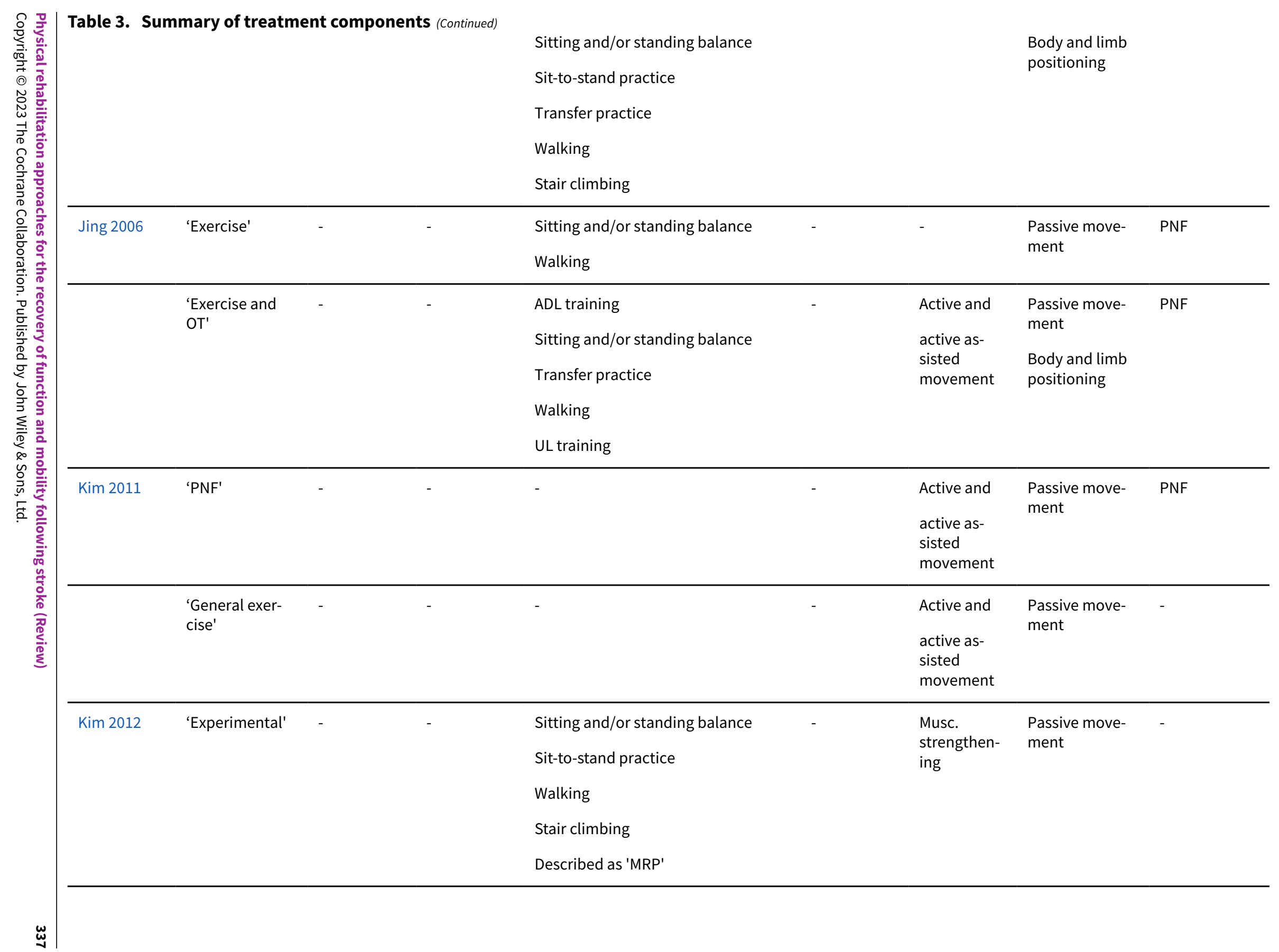




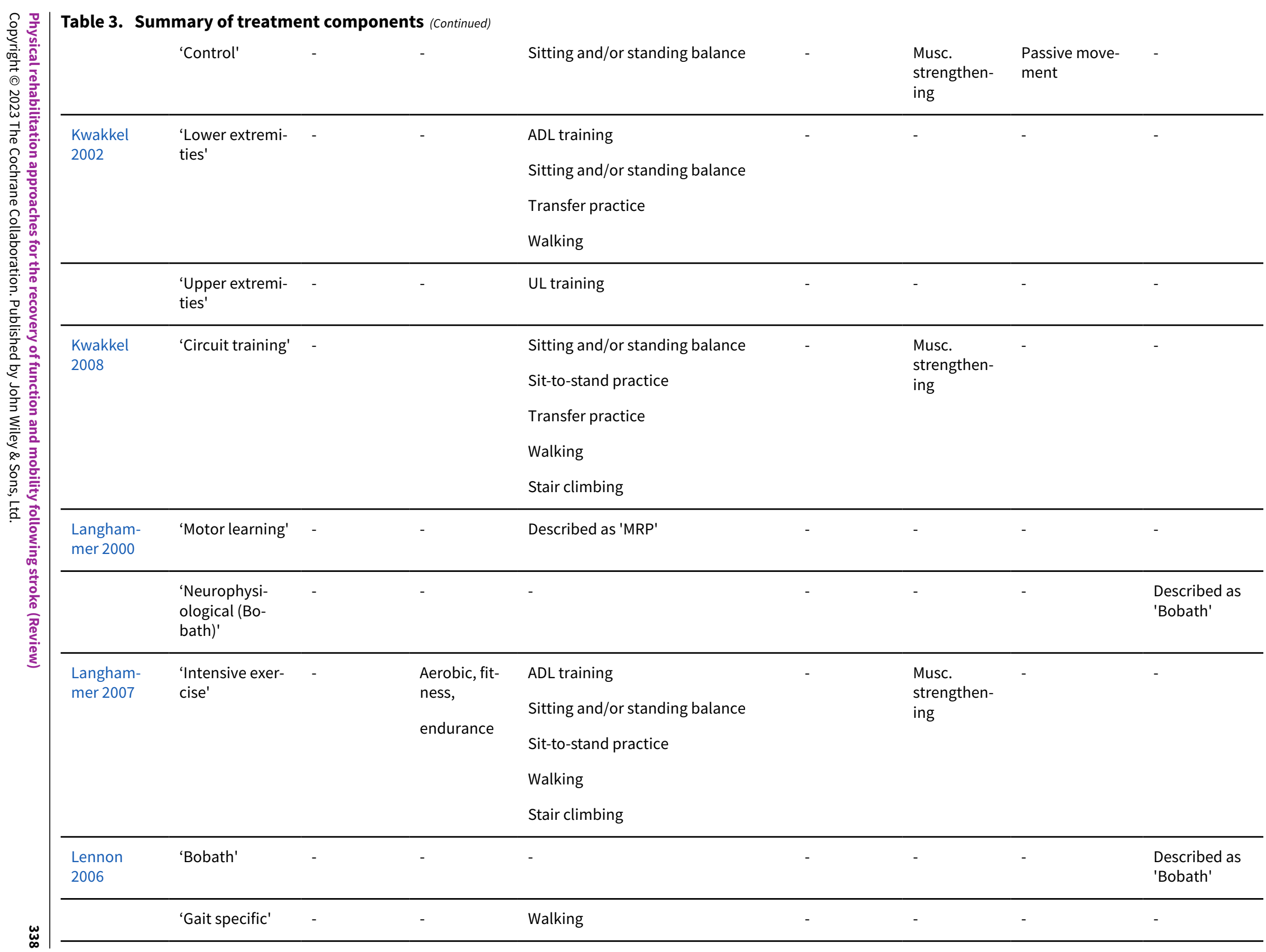




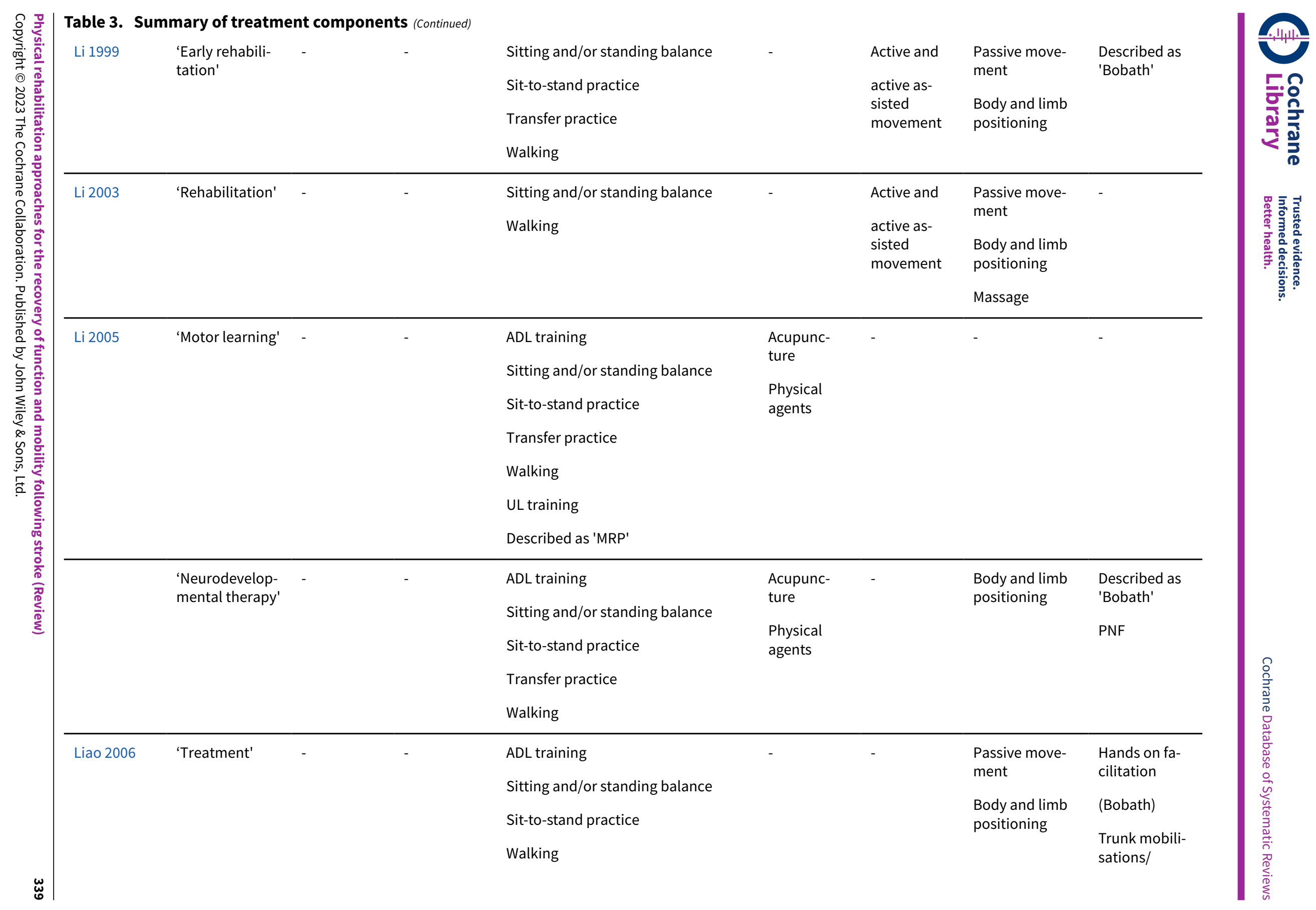




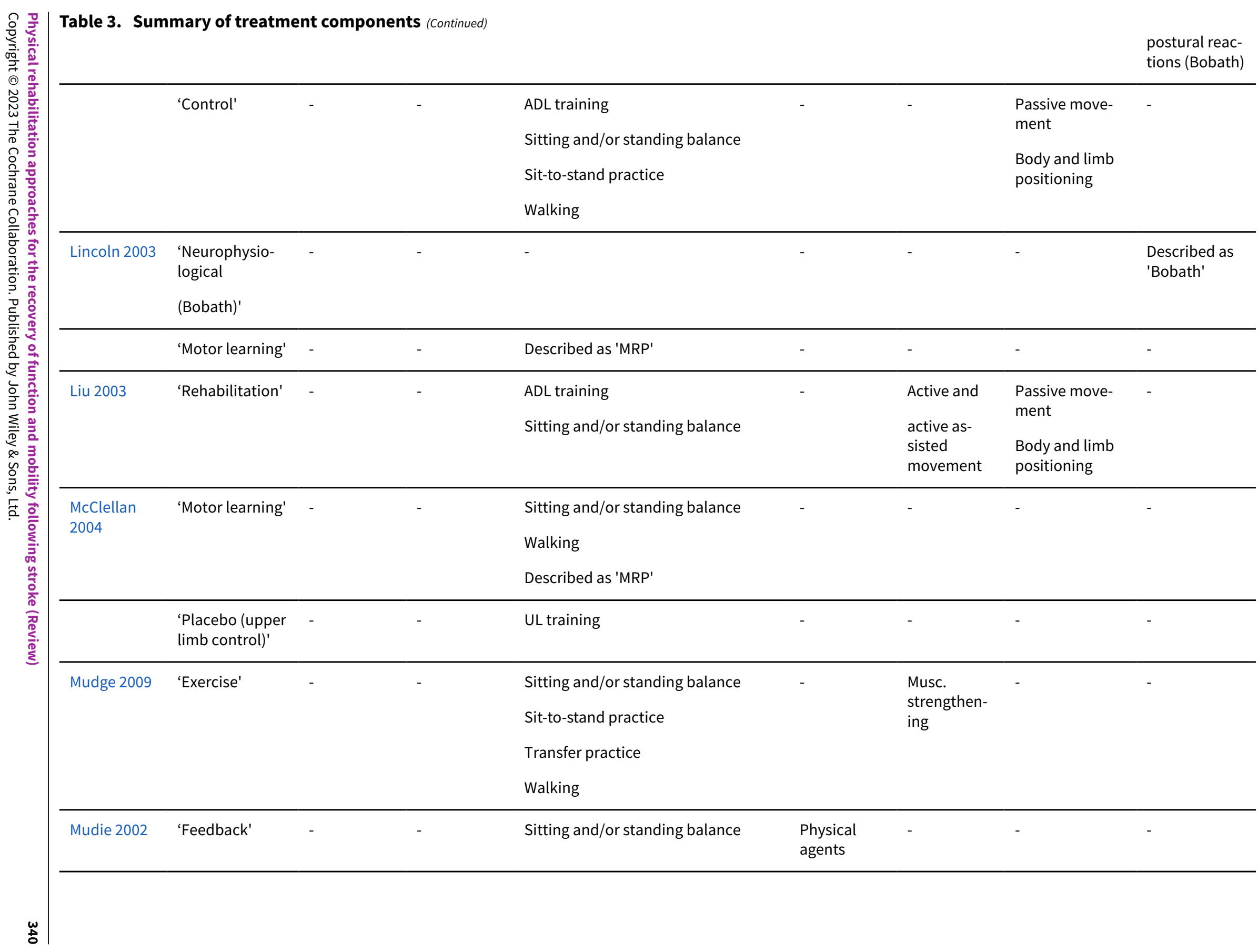




\begin{tabular}{|c|c|c|c|c|c|c|c|c|}
\hline & $\begin{array}{l}\text { 'Motor learning } \\
\text { (Task-related } \\
\text { training)' }\end{array}$ & - & - & $\begin{array}{l}\text { ADL training } \\
\text { Sitting and/or standing balance }\end{array}$ & - & - & - & - \\
\hline & $\begin{array}{l}\text { 'Neurophysio- } \\
\text { logical } \\
\text { (Bobath)' }\end{array}$ & - & - & 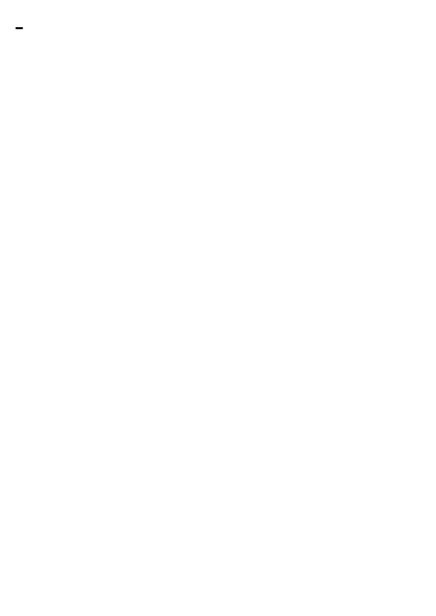 & $0^{2}$ & - & - & $\begin{array}{l}\text { Hands on fa- } \\
\text { cilitation } \\
\text { (Bobath) } \\
\text { Inhibition of } \\
\text { abn musc. } \\
\text { tone (Bobath) } \\
\text { Described as } \\
\text { 'Bobath' } \\
\text { Trunk mobili- } \\
\text { sations/ } \\
\text { postural reac- } \\
\text { tions (Bobath) }\end{array}$ \\
\hline Ni 1997 & $\begin{array}{l}\text { 'Comprehen- } \\
\text { sive rehabilita- } \\
\text { tion training' }\end{array}$ & $\begin{array}{l}\text { Orthoses for } \\
\text { walking }\end{array}$ & - & Sitting and/or standing balance & $\begin{array}{l}\text { Physical } \\
\text { agents }\end{array}$ & - & - & $\begin{array}{l}\text { Described as } \\
\text { 'Bobath' }\end{array}$ \\
\hline Pan 2004 & 'Rehabilitation' & - & - & $\begin{array}{l}\text { ADL training } \\
\text { Sitting and/or standing balance } \\
\text { Sit-to-stand practice } \\
\text { Transfer practice } \\
\text { Walking } \\
\text { Stair climbing }\end{array}$ & - & $\begin{array}{l}\text { Active and } \\
\text { active as- } \\
\text { sisted } \\
\text { movement }\end{array}$ & $\begin{array}{l}\text { Passive move- } \\
\text { ment } \\
\text { Body and limb } \\
\text { positioning }\end{array}$ & - \\
\hline Pang 2003 & 'Rehabilitation' & - & - & $\begin{array}{l}\text { ADL training } \\
\text { Sitting and/or standing balance } \\
\text { Transfer practice } \\
\text { UL training }\end{array}$ & - & - & $\begin{array}{l}\text { Passive move- } \\
\text { ment } \\
\text { Body and limb } \\
\text { positioning } \\
\text { Massage }\end{array}$ & - \\
\hline
\end{tabular}




\begin{tabular}{|c|c|c|c|c|c|c|c|c|}
\hline Pang 2006 & 'Treatment' & - & - & - & $\begin{array}{l}\text { Acupunc- } \\
\text { ture }\end{array}$ & - & - & $\begin{array}{l}\text { Described as } \\
\text { 'Bobath' }\end{array}$ \\
\hline $\begin{array}{l}\text { Pollock } \\
1998\end{array}$ & 'Mixed' & - & - & Sitting and/or standing balance & - & - & - & - \\
\hline & $\begin{array}{l}\text { 'Neurophysi- } \\
\text { ological (Bo- } \\
\text { bath)' }\end{array}$ & - & - & - & - & - & - & $\begin{array}{l}\text { Described as } \\
\text { 'Bobath' }\end{array}$ \\
\hline Qian 2004 & 'Treatment' & - & - & ADL training & $\begin{array}{l}\text { Acupunc- } \\
\text { ture } \\
\text { Physical } \\
\text { agents }\end{array}$ & - & $\begin{array}{l}\text { Body and limb } \\
\text { positioning }\end{array}$ & $\begin{array}{l}\text { Described as } \\
\text { 'Bobath' } \\
\text { PNF }\end{array}$ \\
\hline Qian 2005 & 'Treatment' & - & - & $\begin{array}{l}\text { ADL training } \\
\text { Walking }\end{array}$ & $\begin{array}{l}\text { Acupunc- } \\
\text { ture } \\
\text { Physical } \\
\text { agents }\end{array}$ & - & - & $\begin{array}{l}\text { Described as } \\
\text { 'Bobath' } \\
\text { PNF } \\
\text { Sensorimotor } \\
\text { facilitation }\end{array}$ \\
\hline $\begin{array}{l}\text { Richards } \\
1993\end{array}$ & 'Experimental' & - & - & Walking & $\begin{array}{l}\text { Physical } \\
\text { agents }\end{array}$ & $\begin{array}{l}\text { Musc. } \\
\text { strengthen- } \\
\text { ing }\end{array}$ & Tilt table & - \\
\hline & $\begin{array}{l}\text { 'Early conven- } \\
\text { tional' }\end{array}$ & - & - & - & - & - & - & $\begin{array}{l}\text { Described as } \\
\text { 'Bobath' }\end{array}$ \\
\hline & $\begin{array}{l}\text { 'Routine con- } \\
\text { ventional' }\end{array}$ & - & - & - & - & - & - & $\begin{array}{l}\text { Described as } \\
\text { 'Bobath' }\end{array}$ \\
\hline $\begin{array}{l}\text { Salbach } \\
2004\end{array}$ & 'Motor learning' & - & $\begin{array}{l}\text { Aerobic, fit- } \\
\text { ness, } \\
\text { endurance }\end{array}$ & $\begin{array}{l}\text { Sitting and/or standing balance } \\
\text { Sit-to-stand practice } \\
\text { Walking } \\
\text { Stair climbing } \\
\text { Described as 'MRP' }\end{array}$ & - & $\begin{array}{l}\text { Musc. } \\
\text { strengthen- } \\
\text { ing } \\
\text { Active and } \\
\text { active as- } \\
\text { sisted } \\
\text { movement }\end{array}$ & - & - \\
\hline
\end{tabular}




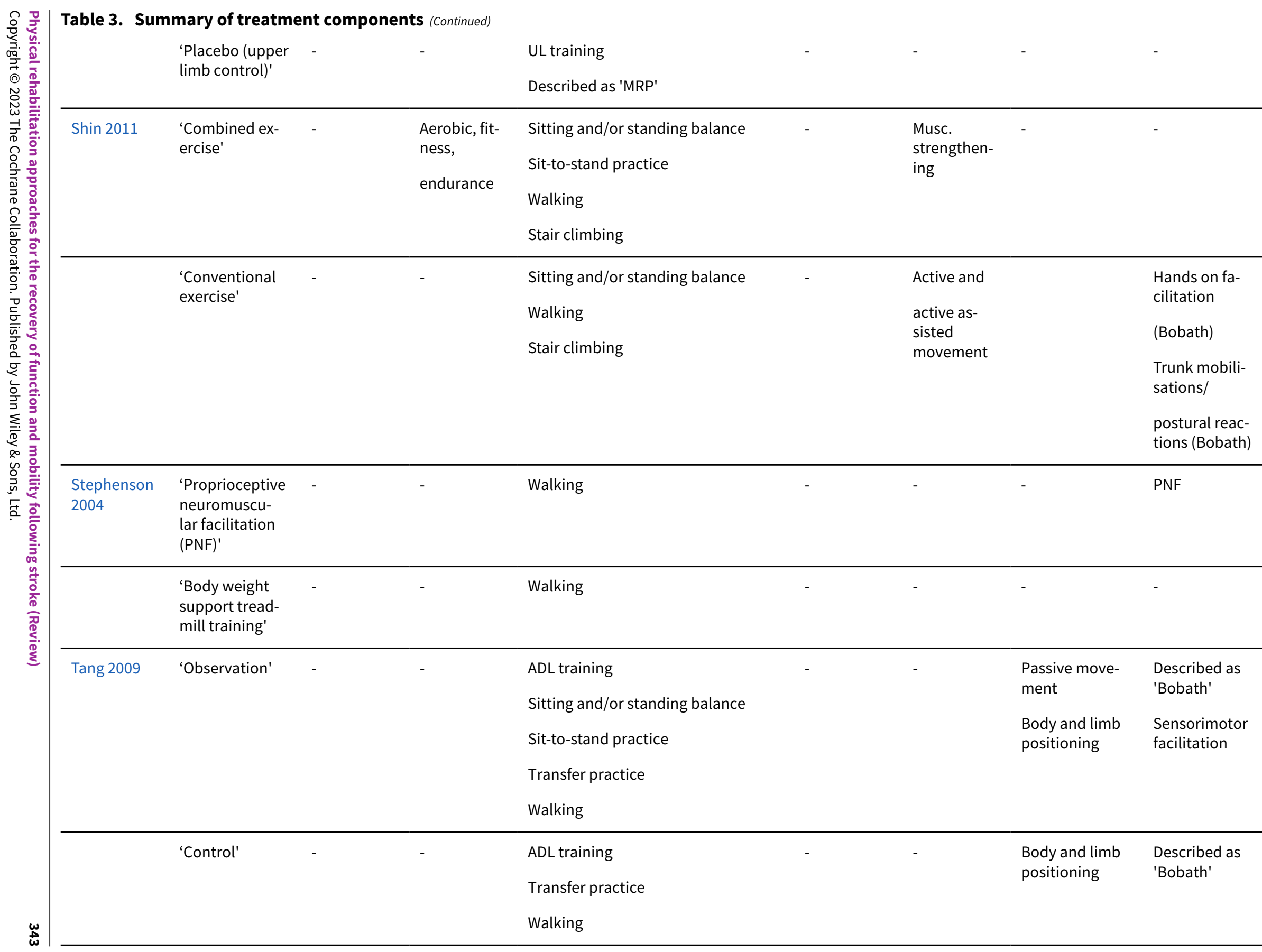




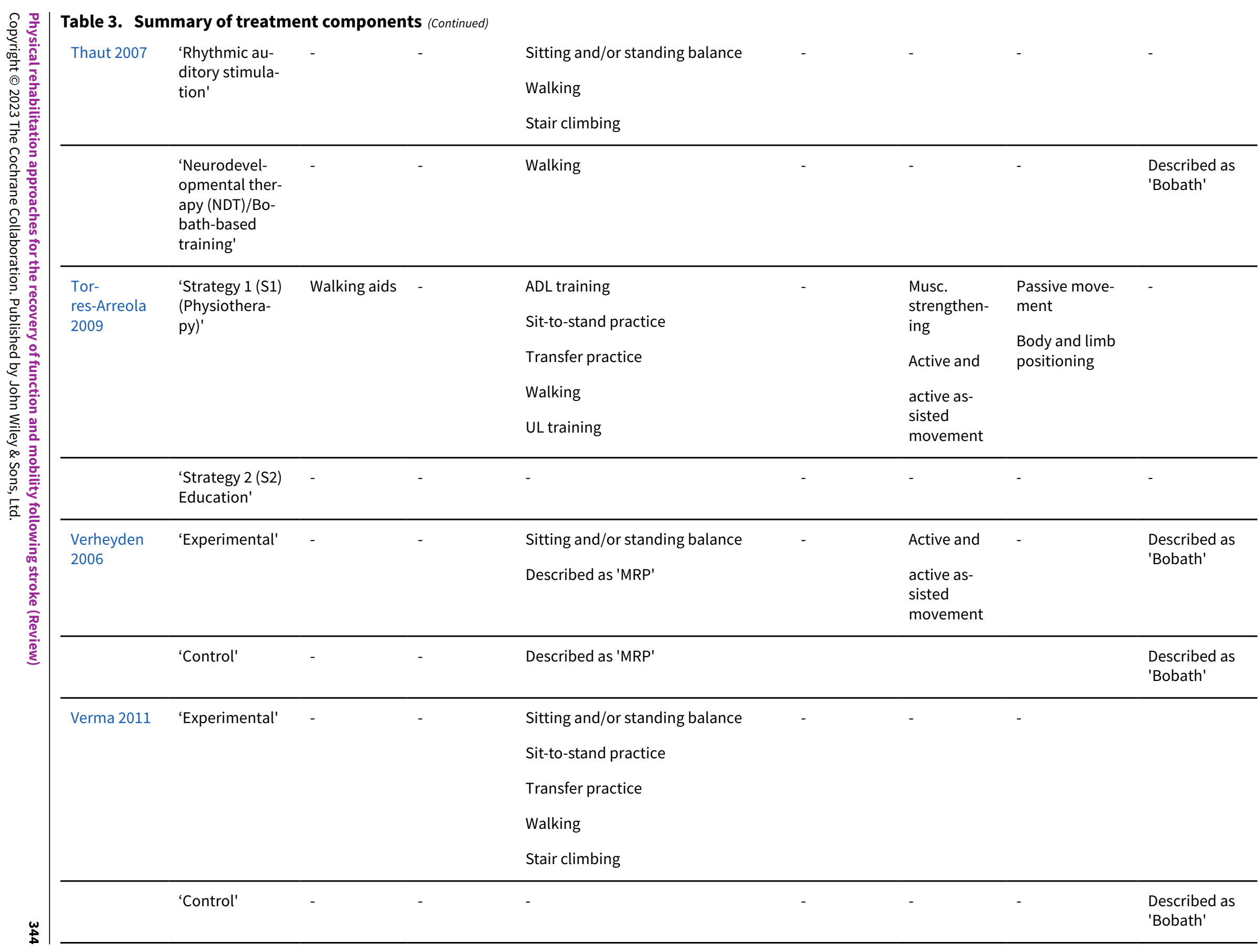




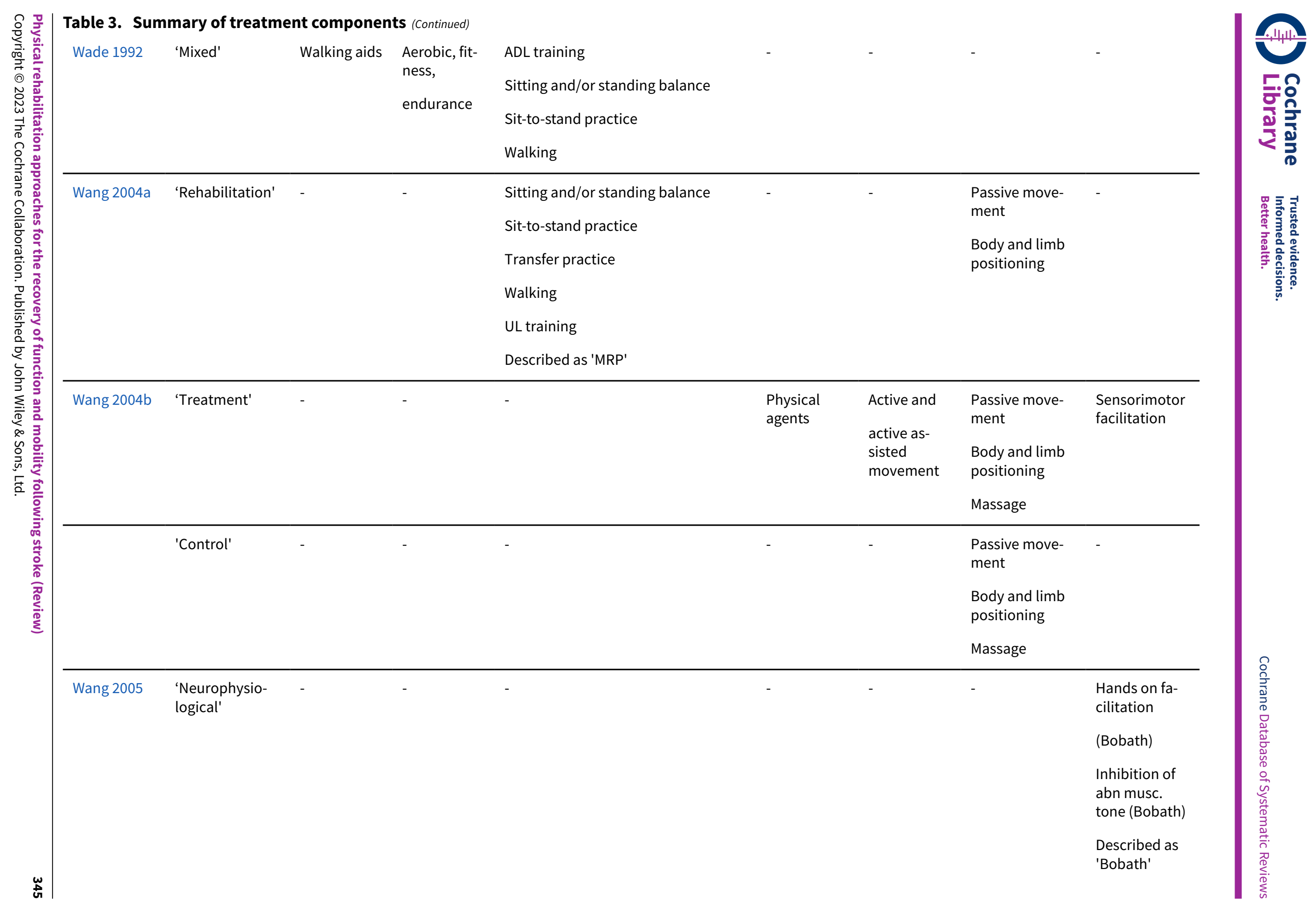




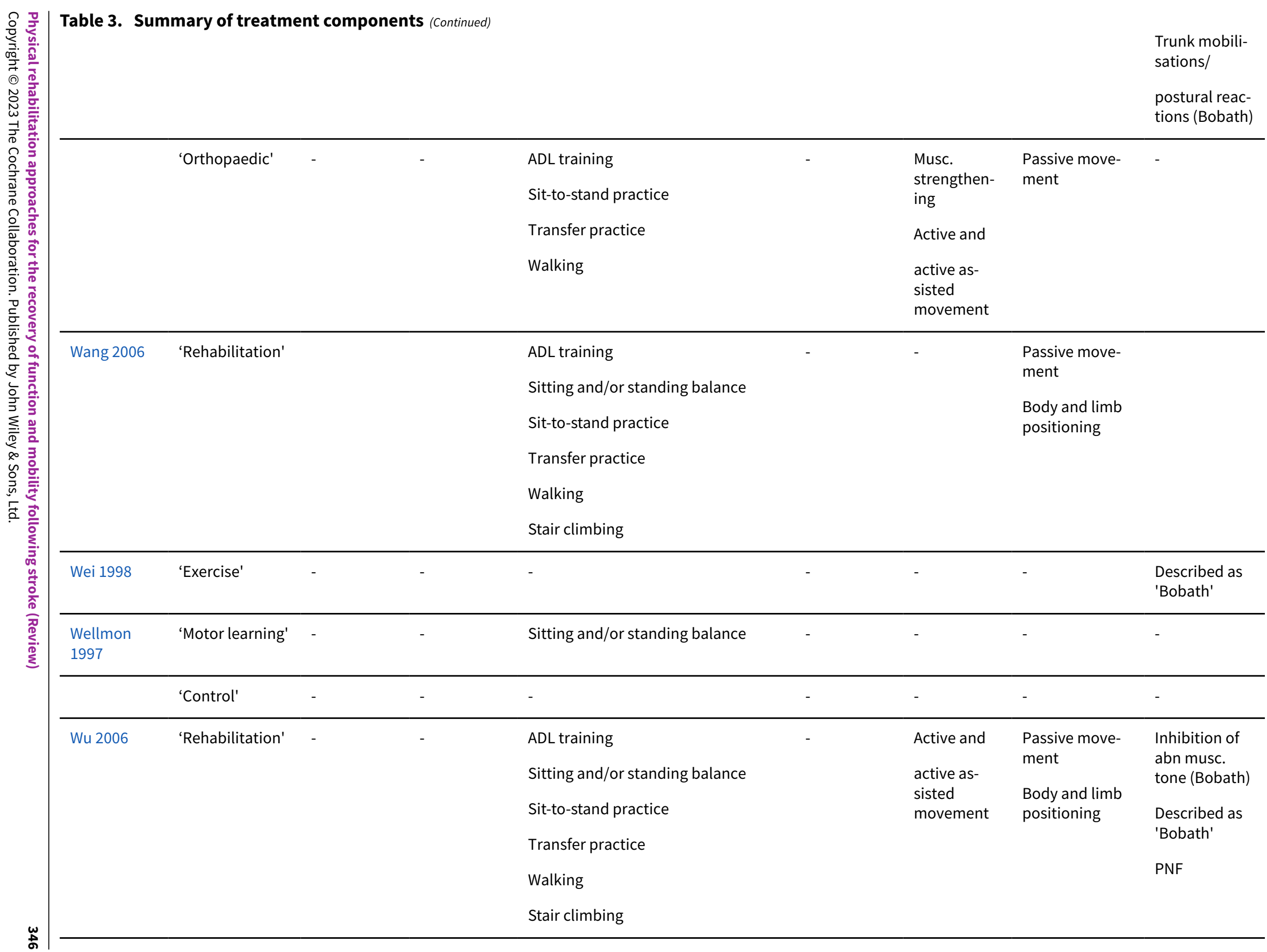




\begin{tabular}{|c|c|c|c|c|c|c|c|c|}
\hline Xiao 2003 & $\begin{array}{l}\text { 'Intensive reha- } \\
\text { bilitation' }\end{array}$ & - & - & - & $\begin{array}{l}\text { Physical } \\
\text { agents }\end{array}$ & - & - & $\begin{array}{l}\text { Described as } \\
\text { 'Bobath' } \\
\text { PNF }\end{array}$ \\
\hline & 'Conventional' & - & - & - & - & - & - & $\begin{array}{l}\text { Described as } \\
\text { 'Bobath' }\end{array}$ \\
\hline Xie 2003 & 'Rehabilitation' & - & - & $\begin{array}{l}\text { ADL training } \\
\text { Sitting and/or standing balance } \\
\text { Stair climbing } \\
\text { UL training }\end{array}$ & - & - & $\begin{array}{l}\text { Passive move- } \\
\text { ment } \\
\text { Body and limb } \\
\text { positioning } \\
\text { Massage }\end{array}$ & - \\
\hline Xie 2005 & 'Treatment' & - & - & $\begin{array}{l}\text { ADL training } \\
\text { Sitting and/or standing balance } \\
\text { Transfer practice } \\
\text { Walking }\end{array}$ & - & $\begin{array}{l}\text { Active and } \\
\text { active as- } \\
\text { sisted } \\
\text { movement }\end{array}$ & $\begin{array}{l}\text { Passive move- } \\
\text { ment } \\
\text { Body and limb } \\
\text { positioning }\end{array}$ & - \\
\hline Xu 1999 & 'Rehabilitation' & - & - & - & - & - & - & $\begin{array}{l}\text { Described as } \\
\text { 'Bobath' }\end{array}$ \\
\hline Xu 2003a & 'Rehabilitation' & - & - & $\begin{array}{l}\text { Sitting and/or standing balance } \\
\text { Walking }\end{array}$ & - & $\begin{array}{l}\text { Active and } \\
\text { active as- } \\
\text { sisted } \\
\text { movement }\end{array}$ & $\begin{array}{l}\text { Passive move- } \\
\text { ment } \\
\text { Body and limb } \\
\text { positioning } \\
\text { Massage }\end{array}$ & - \\
\hline Xu 2003b & 'Rehabilitation' & - & - & ADL training & - & - & $\begin{array}{l}\text { Body and limb } \\
\text { positioning }\end{array}$ & $\begin{array}{l}\text { Sensorimotor } \\
\text { facilitation }\end{array}$ \\
\hline Xu 2004 & 'Rehabilitation' & - & - & $\begin{array}{l}\text { ADL training } \\
\text { Sitting and/or standing balance } \\
\text { Transfer practice } \\
\text { Walking }\end{array}$ & - & - & $\begin{array}{l}\text { Passive move- } \\
\text { ment } \\
\text { Body and limb } \\
\text { positioning }\end{array}$ & $\begin{array}{l}\text { Described as } \\
\text { 'Bobath' }\end{array}$ \\
\hline
\end{tabular}




\begin{tabular}{|c|c|c|c|c|c|c|c|c|}
\hline Xue 2006 & ‘Training' & - & - & $\begin{array}{l}\text { Sit-to-stand practice } \\
\text { Transfer practice } \\
\text { Walking } \\
\text { Described as 'MRP' }\end{array}$ & - & $\begin{array}{l}\text { Active and } \\
\text { active as- } \\
\text { sisted } \\
\text { movement }\end{array}$ & $\begin{array}{l}\text { Passive move- } \\
\text { ment } \\
\text { Body and limb } \\
\text { positioning }\end{array}$ & $\begin{array}{l}\text { Described as } \\
\text { 'Bobath' }\end{array}$ \\
\hline Yan 2002 & 'Rehabilitation' & $\begin{array}{l}\text { Resting } \\
\text { splints }\end{array}$ & - & $\begin{array}{l}\text { Sitting and/or standing balance } \\
\text { Sit-to-stand practice } \\
\text { Transfer practice } \\
\text { Walking } \\
\text { Stair climbing }\end{array}$ & - & - & $\begin{array}{l}\text { Inc. angle of } \\
\text { upright sitting } \\
\text { Passive move- } \\
\text { ment } \\
\text { Body and limb } \\
\text { positioning }\end{array}$ & - \\
\hline Yelnik 2008 & $\begin{array}{l}\text { 'NDT-based } \\
\text { treatment' }\end{array}$ & - & - & $\begin{array}{l}\text { Sitting and/or standing balance } \\
\text { Sit-to-stand practice } \\
\text { Walking }\end{array}$ & - & - & - & $\begin{array}{l}\text { Hands on fa- } \\
\text { cilitation } \\
\text { (Bobath) } \\
\text { Inhibition of } \\
\text { abn musc. } \\
\text { tone (Bobath) } \\
\text { Described as } \\
\text { 'Bobath' }\end{array}$ \\
\hline & 'Multisensorial' & - & - & $\begin{array}{l}\text { Sitting and/or standing balance } \\
\text { Walking }\end{array}$ & - & - & - & - \\
\hline Yin 2003a & 'Rehabilitation' & $\begin{array}{l}\text { Resting } \\
\text { splints }\end{array}$ & - & Sitting and/or standing balance & - & - & $\begin{array}{l}\text { Body and limb } \\
\text { positioning }\end{array}$ & $\begin{array}{l}\text { Described as } \\
\text { 'Bobath' }\end{array}$ \\
\hline Zhang 1998 & $\begin{array}{l}\text { 'Early rehabili- } \\
\text { tation' }\end{array}$ & - & - & $\begin{array}{l}\text { ADL practice } \\
\text { Sitting and/or standing balance } \\
\text { Walking } \\
\text { Stair climbing }\end{array}$ & - & $\begin{array}{l}\text { Musc. } \\
\text { strengthen- } \\
\text { ing }\end{array}$ & $\begin{array}{l}\text { Passive move- } \\
\text { ment } \\
\text { Body and limb } \\
\text { positioning } \\
\text { Massage }\end{array}$ & $\begin{array}{l}\text { Sensorimotor } \\
\text { facilitation }\end{array}$ \\
\hline Zhang 2004 & 'Rehabilitation' & - & - & ADL practice & - & Active and & $\begin{array}{l}\text { Passive move- } \\
\text { ment }\end{array}$ & $\begin{array}{l}\text { Described as } \\
\text { 'Bobath' }\end{array}$ \\
\hline
\end{tabular}




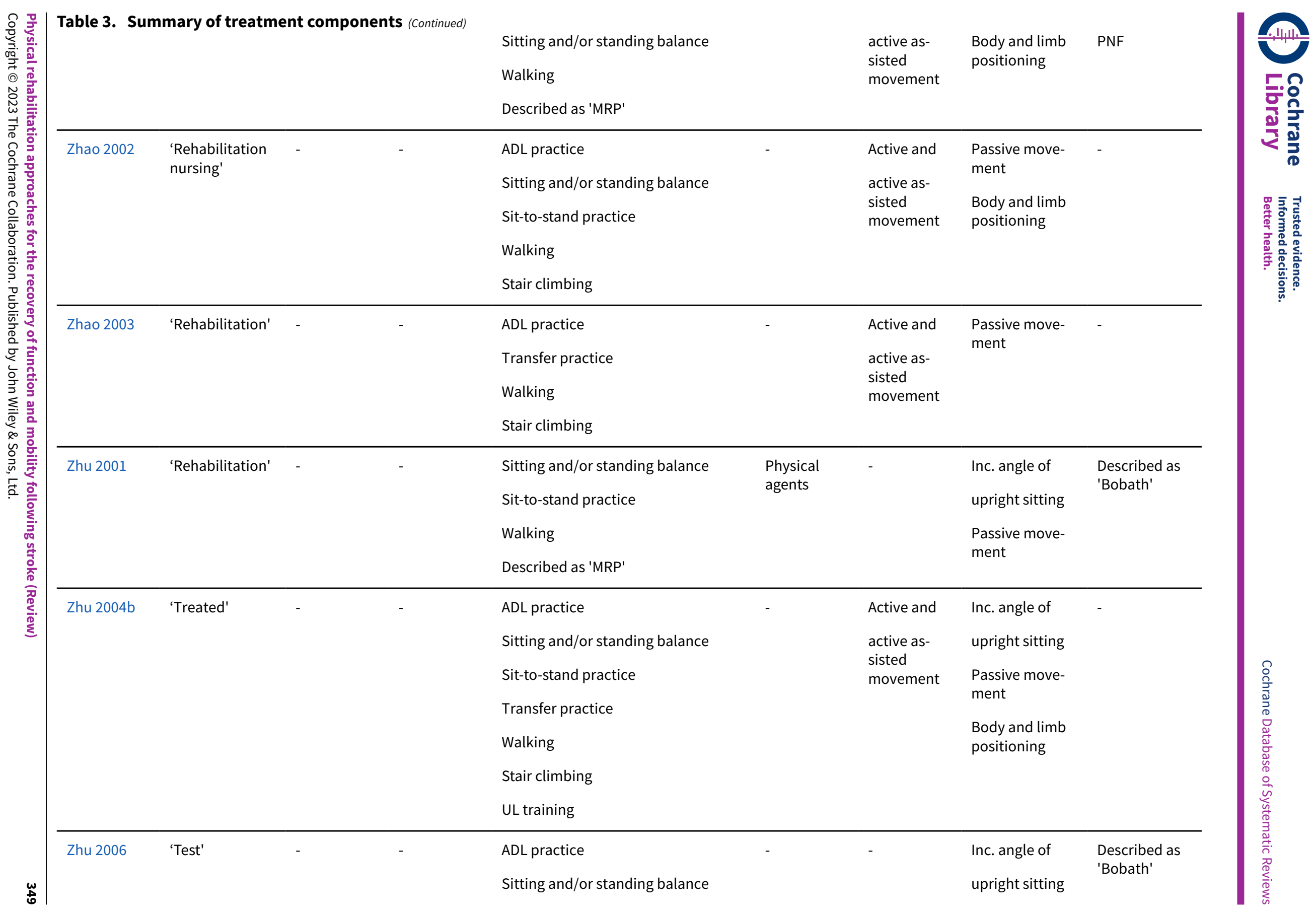




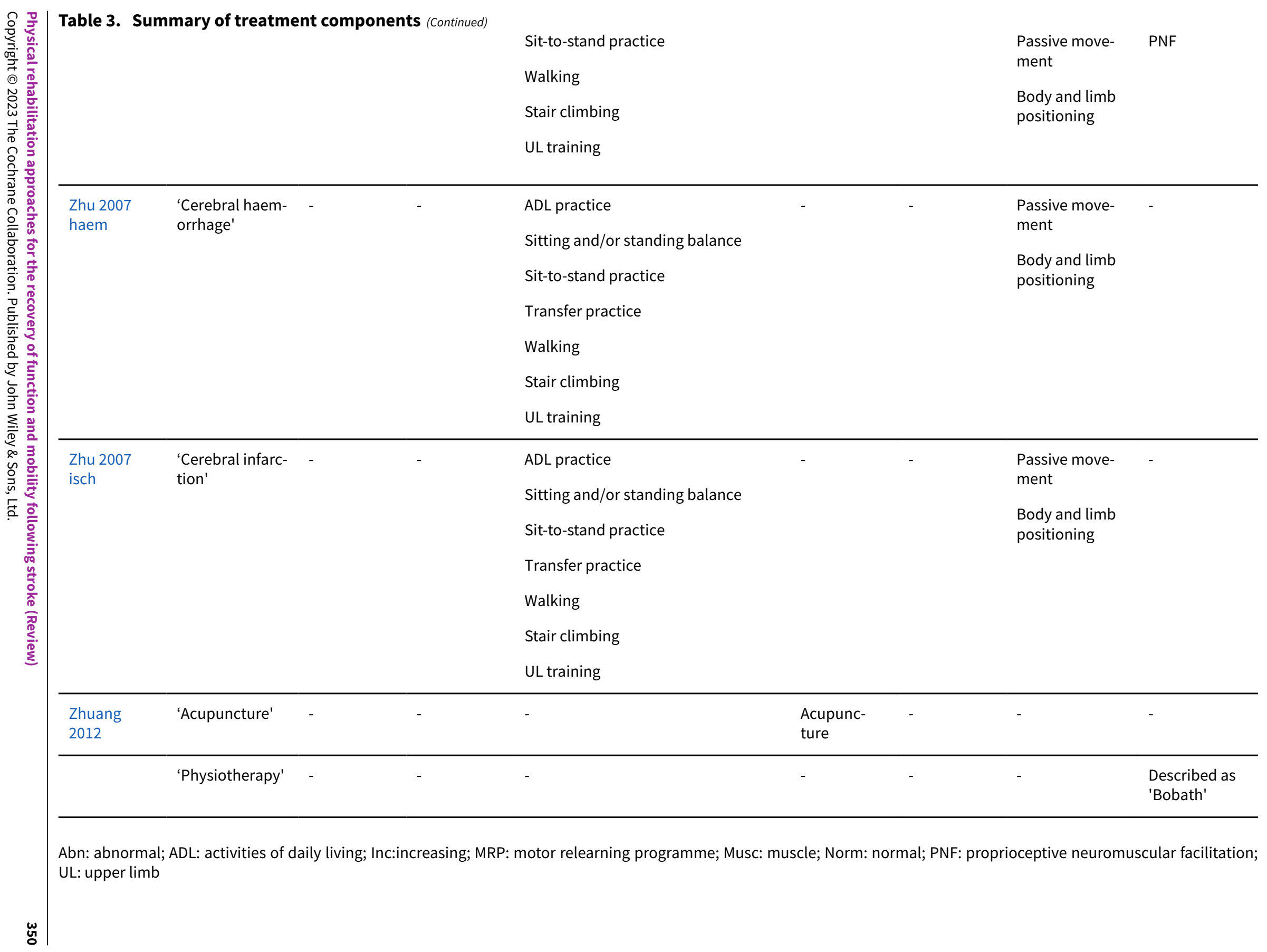




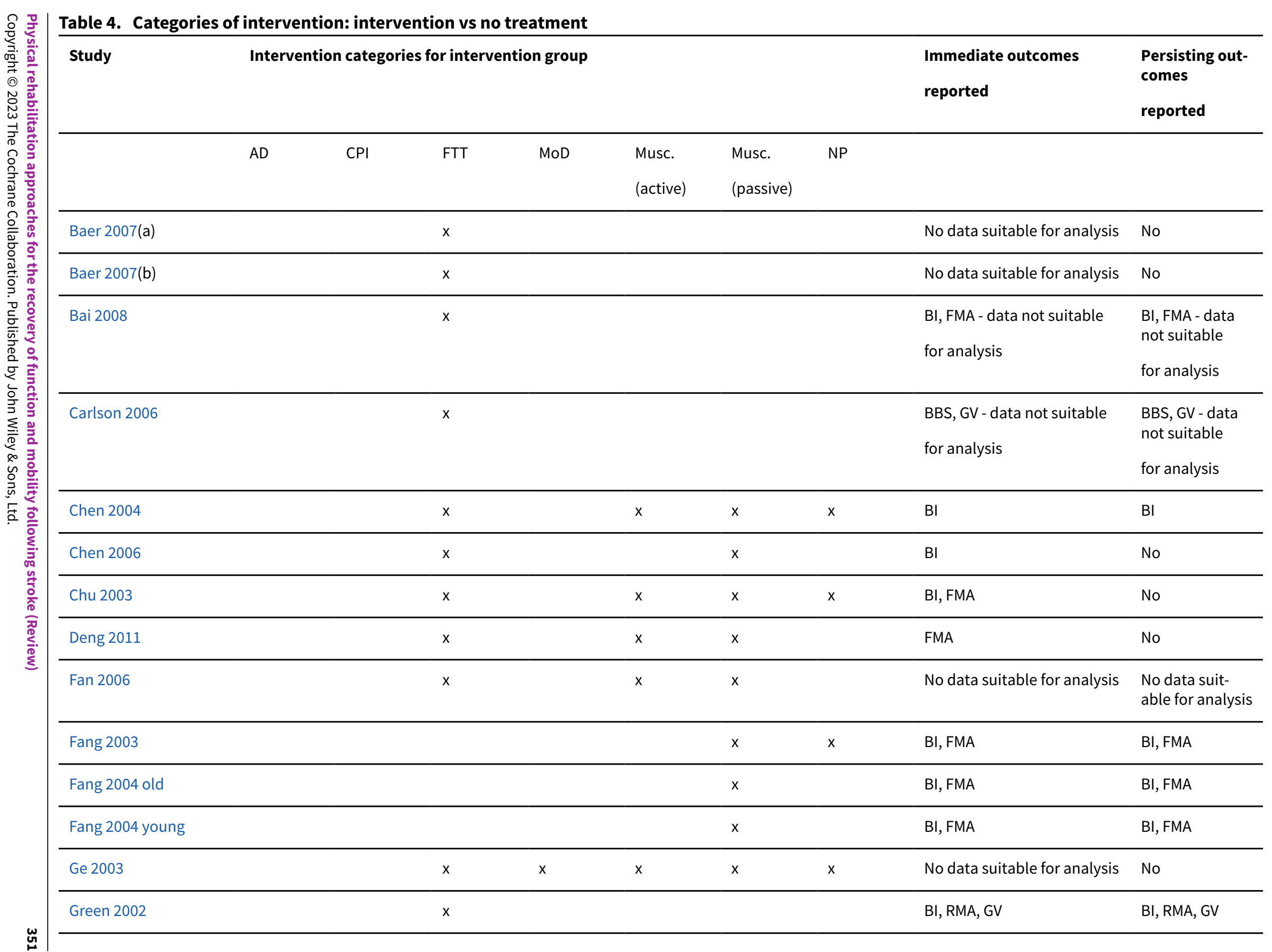




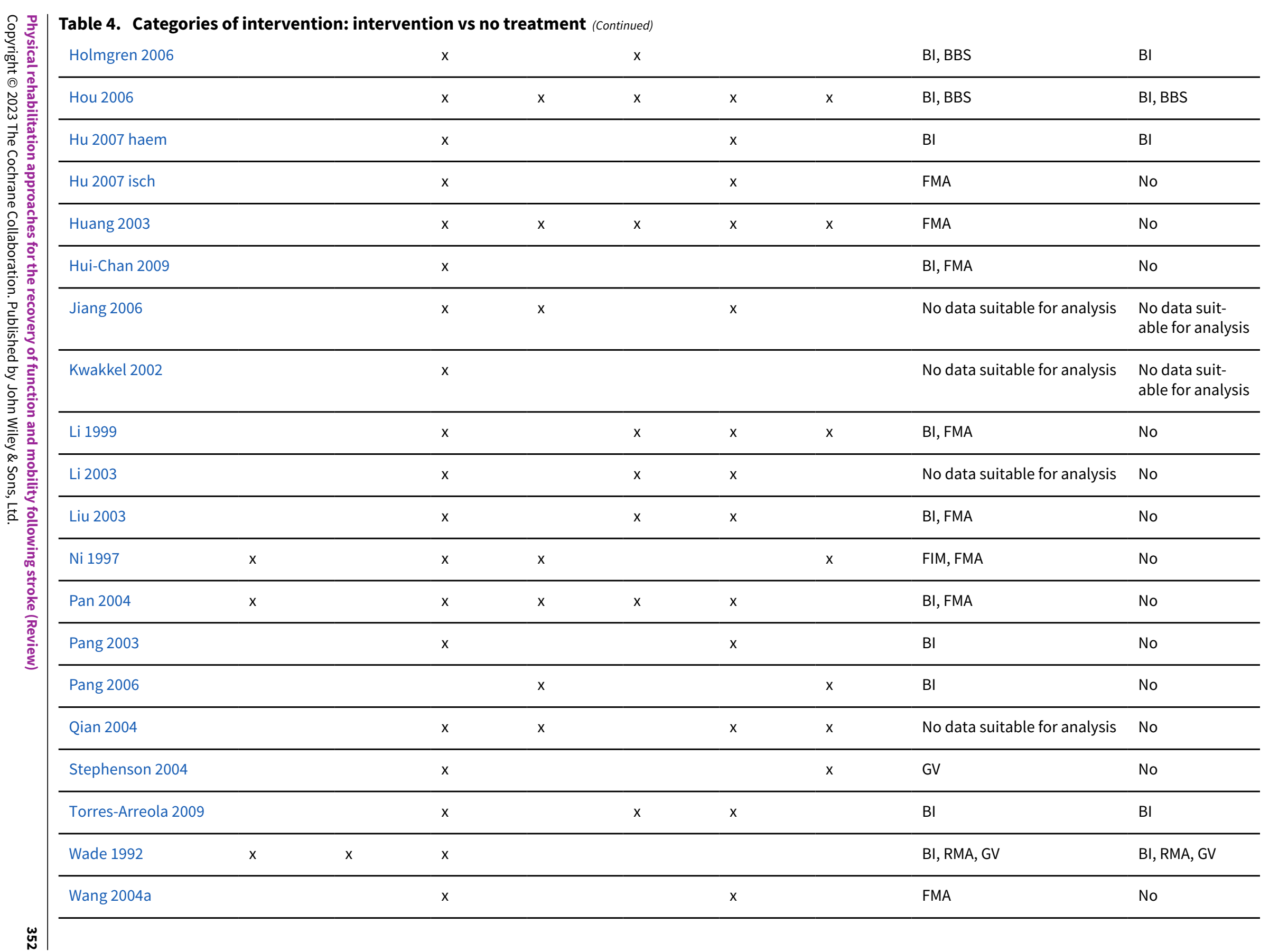




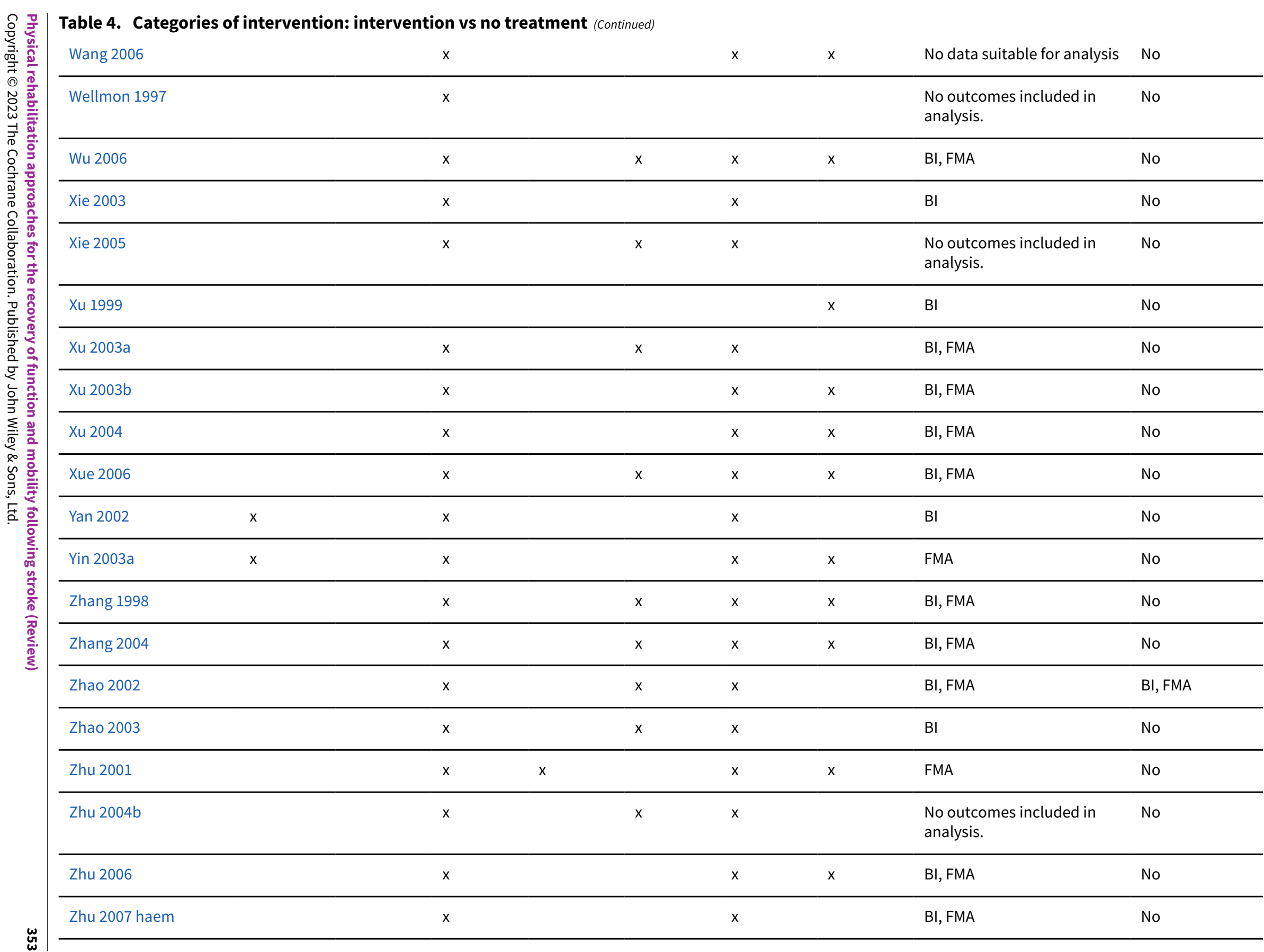




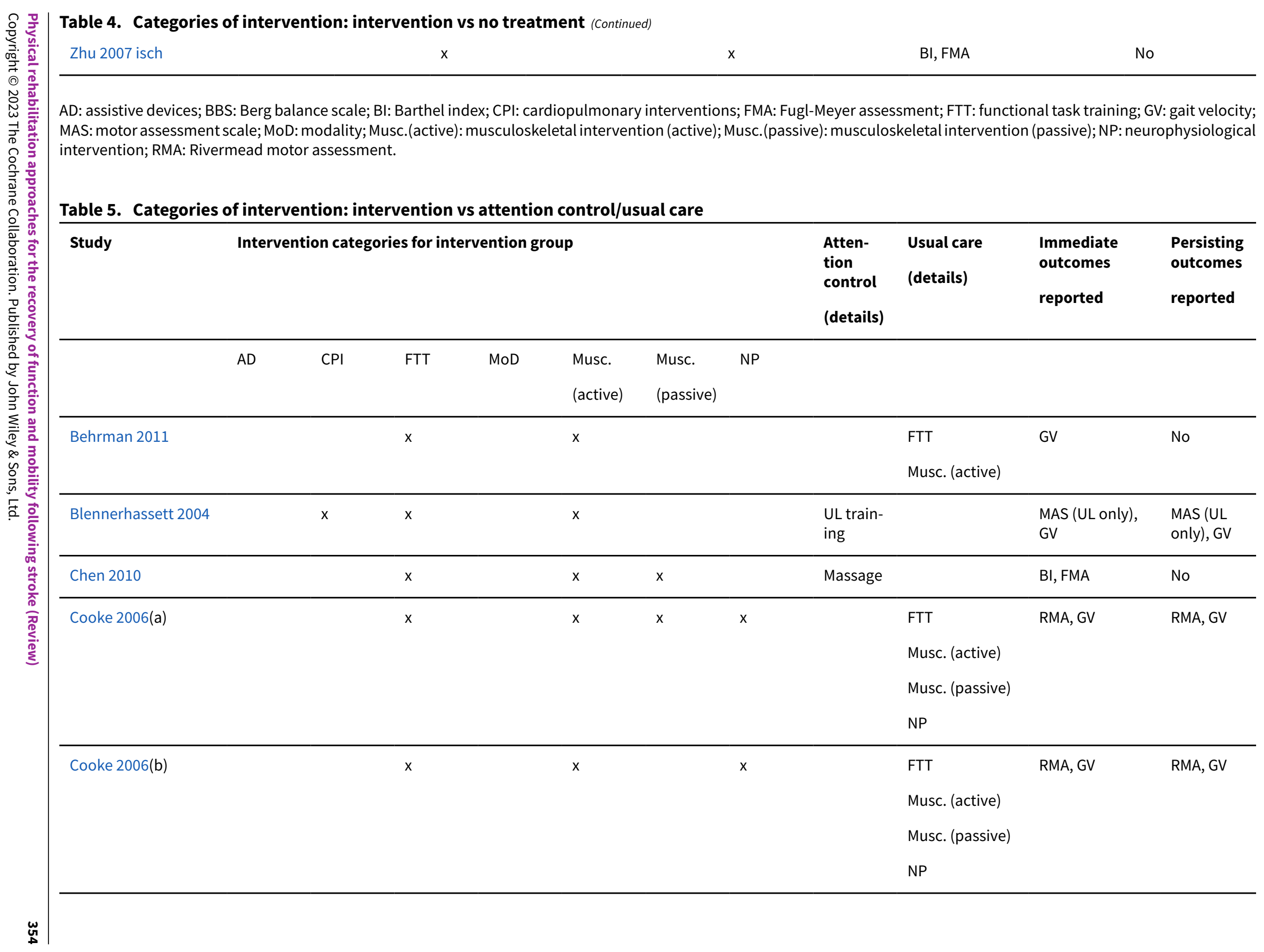

Table 5. Categories of intervention: intervention vs attention control/usual care 


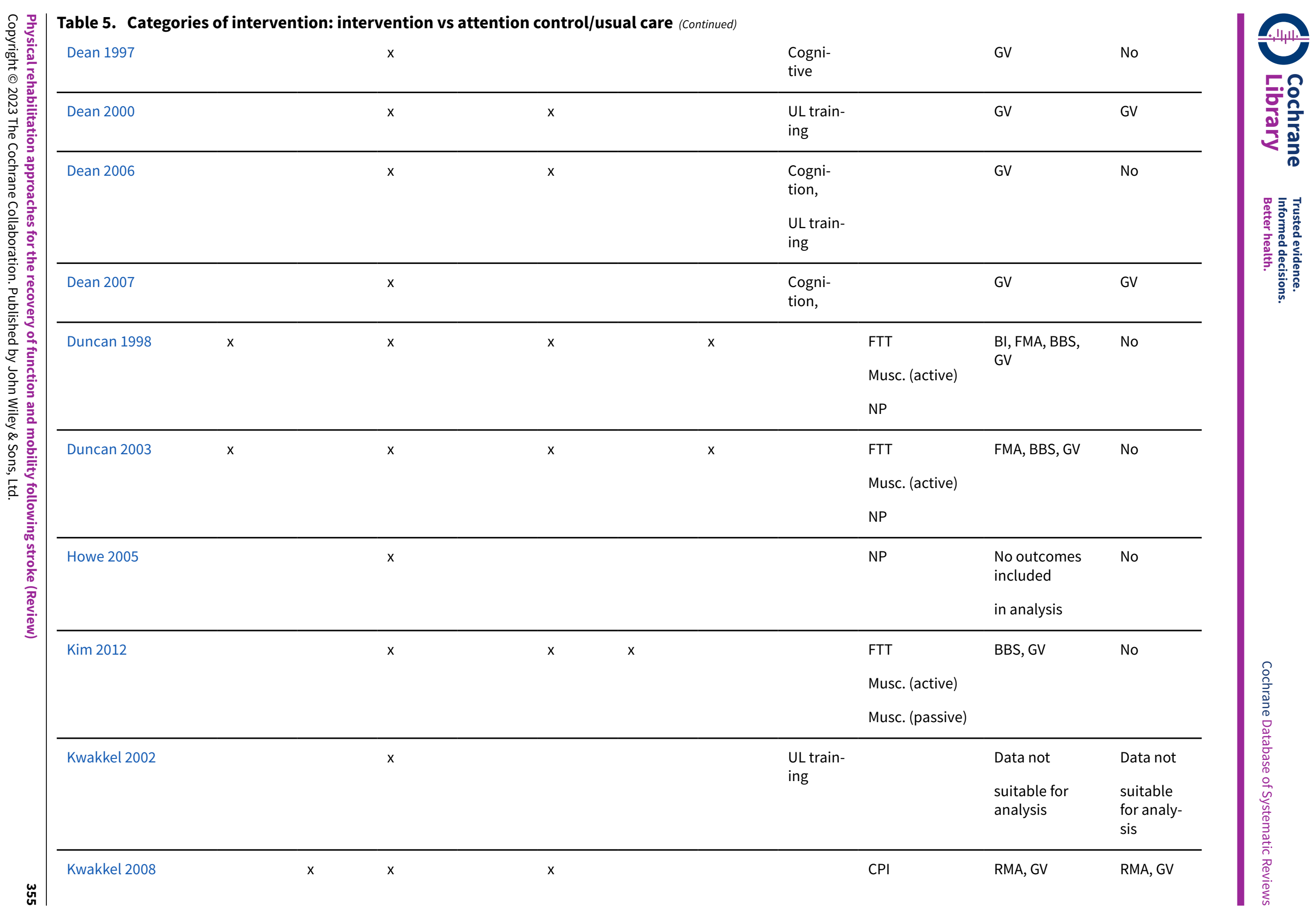




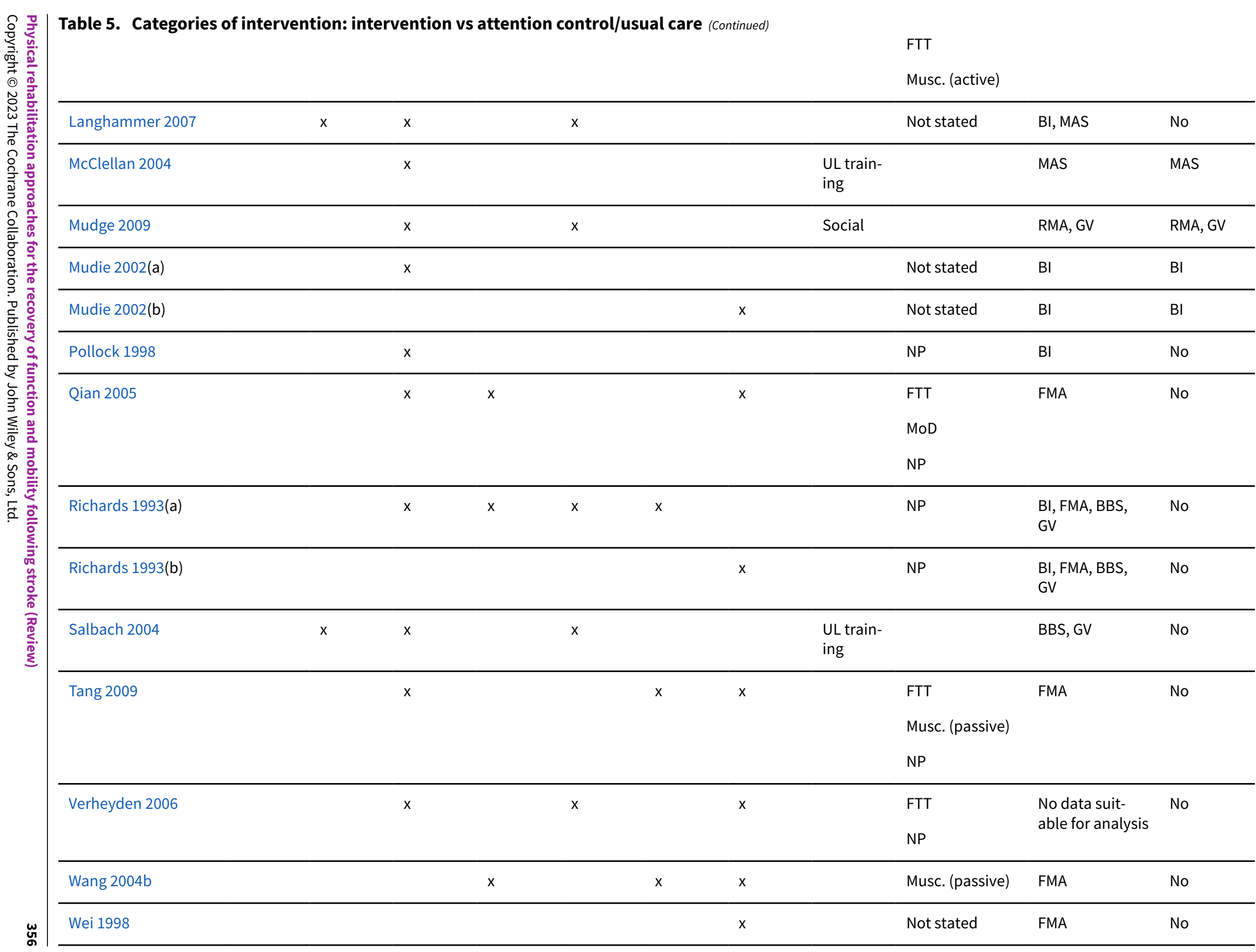




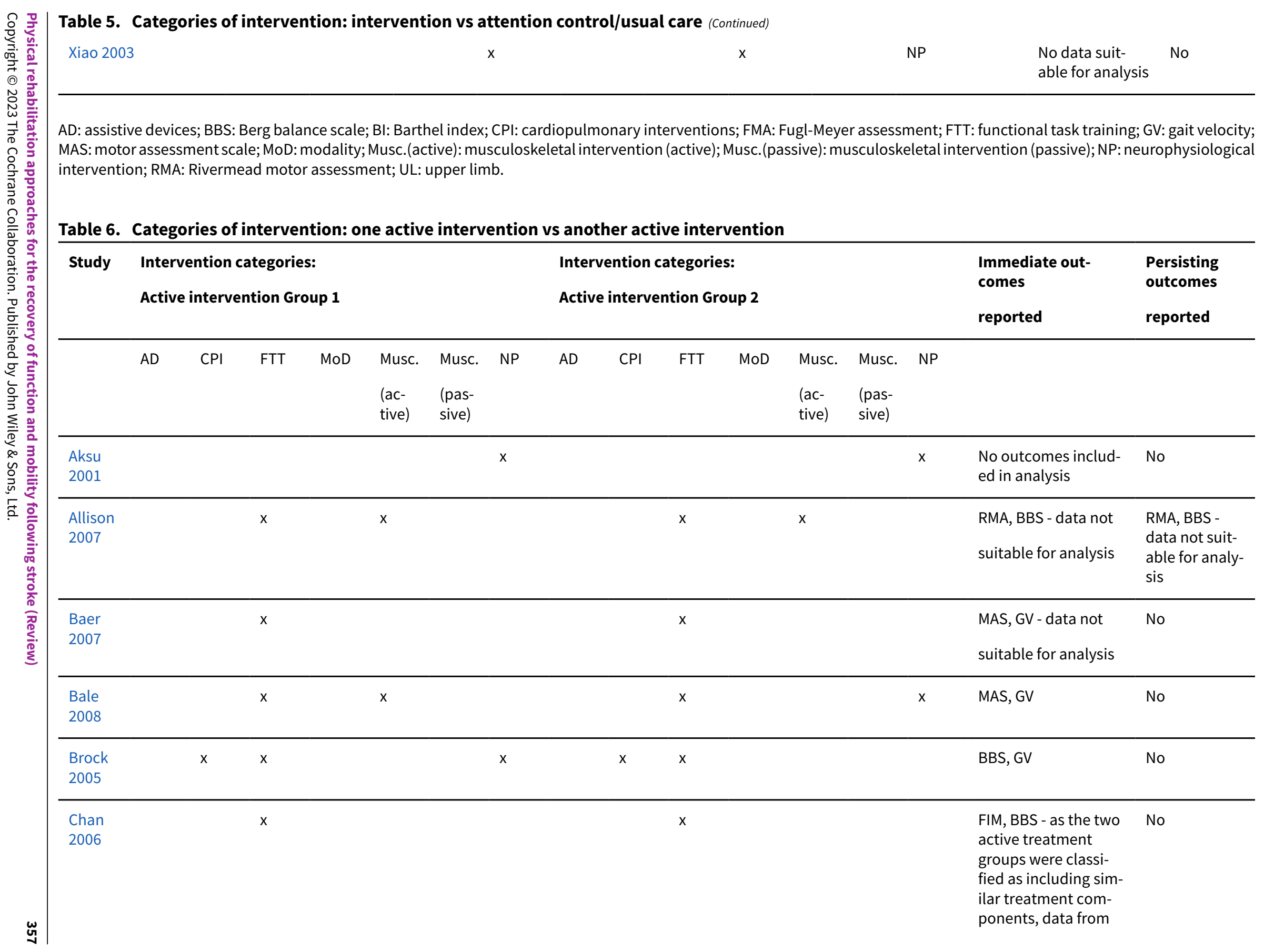




\begin{tabular}{|c|c|c|c|c|c|c|c|c|c|c|c|c|}
\hline & & & & & & & & & & $\begin{array}{l}\text { this study has not } \\
\text { been included within } \\
\text { the comparisons of } \\
\text { one active interven- } \\
\text { tion versus another } \\
\text { active intervention }\end{array}$ & & бٓㅇํㅇ \\
\hline $\begin{array}{l}\text { Cooke } \\
2006\end{array}$ & $x$ & $x$ & $x$ & $x$ & & $x$ & $x$ & & $x$ & $\begin{array}{l}\text { MAS, GV - as the two } \\
\text { active treatment } \\
\text { groups were classi- } \\
\text { fied as including sim- } \\
\text { ilar treatment com- } \\
\text { ponents, data from } \\
\text { this study has not } \\
\text { been included within } \\
\text { the comparisons of } \\
\text { one active interven- } \\
\text { tion versus another } \\
\text { active intervention }\end{array}$ & $\begin{array}{l}\text { MAS, GV - as } \\
\text { the two ac- } \\
\text { tive treatment } \\
\text { groups were } \\
\text { classified as } \\
\text { including sim- } \\
\text { ilar treatment } \\
\text { components, } \\
\text { data from this } \\
\text { study has not } \\
\text { been includ- } \\
\text { ed within the } \\
\text { comparisons } \\
\text { of one active } \\
\text { intervention } \\
\text { versus anoth- } \\
\text { er active in- } \\
\text { tervention }\end{array}$ & 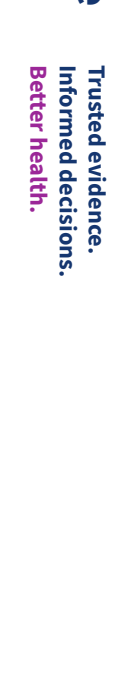 \\
\hline $\begin{array}{l}\text { Gelber } \\
1995\end{array}$ & $x$ & & & $x$ & $x$ & $x$ & $x$ & $x$ & & FIM, GV & FIM, GV & \\
\hline $\begin{array}{l}\text { Jing } \\
2006\end{array}$ & $x$ & $x$ & $x$ & $x$ & & $x$ & & $x$ & $x$ & $\begin{array}{l}\text { BI, FMA - as the two } \\
\text { active treatment } \\
\text { groups were classi- } \\
\text { fied as including sim- } \\
\text { ilar treatment com- } \\
\text { ponents, data from } \\
\text { this study has not } \\
\text { been included within } \\
\text { the comparisons of } \\
\text { one active interven- } \\
\text { tion versus another } \\
\text { active intervention. }\end{array}$ & $\begin{array}{l}\text { BI, FMA - as } \\
\text { the two ac- } \\
\text { tive treatment } \\
\text { groups were } \\
\text { classified as } \\
\text { including sim- } \\
\text { ilar treatment } \\
\text { components, } \\
\text { data from this } \\
\text { study has not } \\
\text { been includ- } \\
\text { ed within the } \\
\text { comparisons } \\
\text { of one active } \\
\text { intervention } \\
\text { versus anoth- }\end{array}$ & 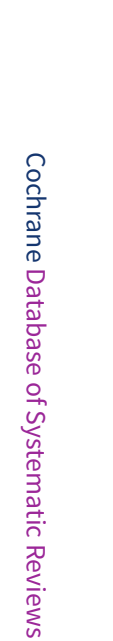 \\
\hline
\end{tabular}




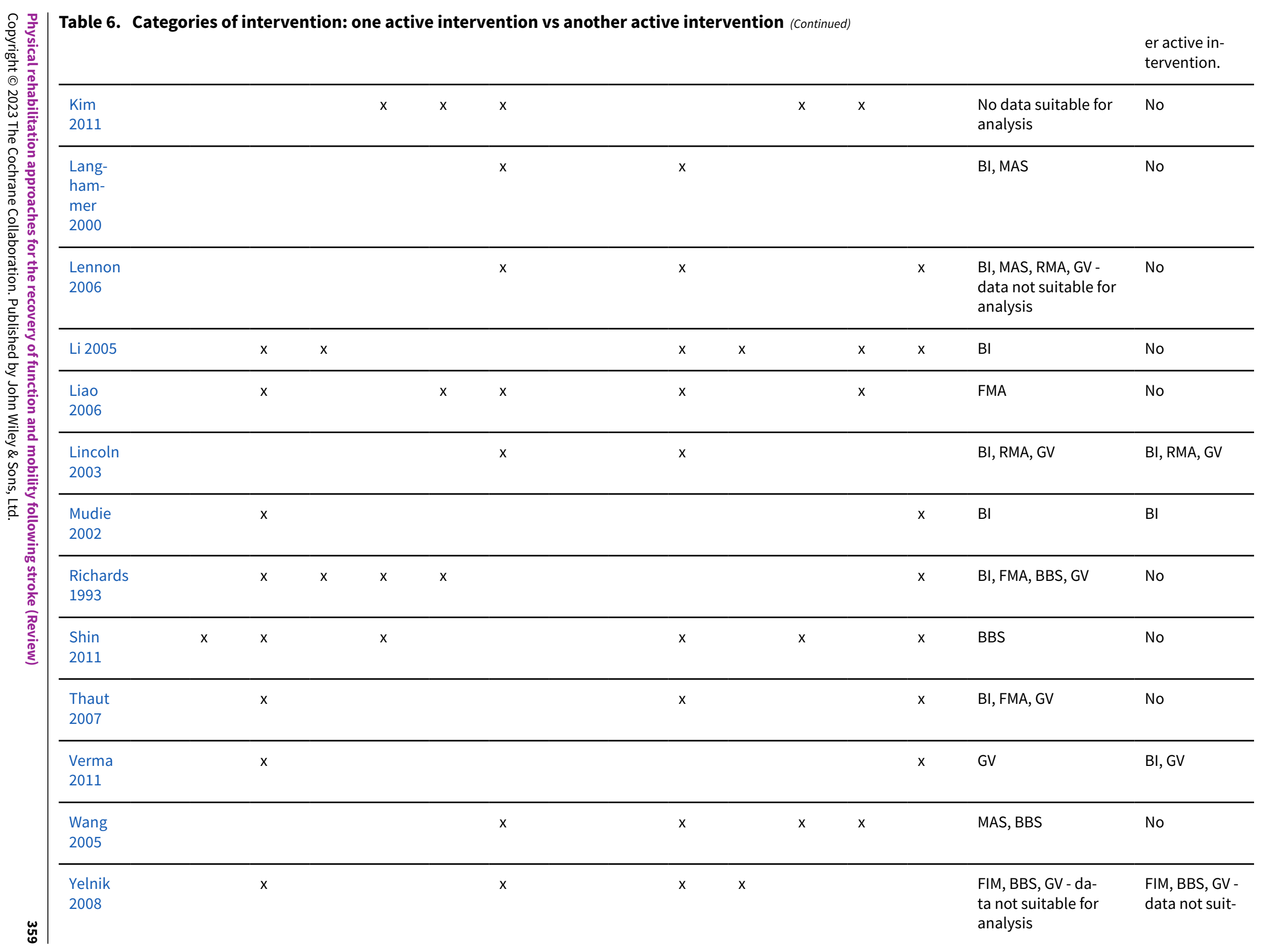




Zhuang $\quad x \quad$ No

AD: assistive devices; BBS: Berg balance scale; BI: Barthel index; CPI: cardiopulmonary interventions; FMA: Fugl-Meyer assessment; FTT: functional task training; GV: gait velocity; MAS: motor assessment scale; MoD: modality; Musc.(active): musculoskeletal intervention (active); Musc.(passive): musculoskeletal intervention (passive); NP: neurophysiological intervention; RMA: Rivermead motor assessment. 
Table 7. Summary of study setting

\begin{tabular}{|c|c|c|c|}
\hline Study & At recruitment & For intervention & Country \\
\hline Aksu 2001 & Not stated & Not stated & Turkey \\
\hline Allison 2007 & Inpatient stroke rehabilitation unit & Inpatient stroke rehabilitation unit & UK \\
\hline Baer 2007 & Not stated & Own homes & UK \\
\hline Bai 2008 & $\begin{array}{l}\text { Emergency department or Neurolo- } \\
\text { gy Department }\end{array}$ & $\begin{array}{l}\text { Dependent on stage of rehabilitation and could } \\
\text { include own homes, rehabilitation unit, } \\
\text { outpatient rehabilitation or community centre }\end{array}$ & China \\
\hline Bale 2008 & $\begin{array}{l}\text { Recruited from two rehabilitation } \\
\text { units, a hospital ward } \\
\text { and a rehabilitation centre }\end{array}$ & Not stated & Norway \\
\hline Behrman 2011 & $\begin{array}{l}\text { Recruited from multiple community } \\
\text { rehabilitation hospitals }\end{array}$ & Community & USA \\
\hline $\begin{array}{l}\text { Blennerhassett } \\
2004\end{array}$ & Rehabilitation Centre & Rehabilitation centre & Australia \\
\hline Brock 2005 & $\begin{array}{l}\text { Recruited from multiple rehabilita- } \\
\text { tion centres }\end{array}$ & Multiple rehabilitation centres & $\begin{array}{l}\text { Australia and Ger- } \\
\text { many }\end{array}$ \\
\hline Carlson 2006 & Not stated & Not stated & USA \\
\hline Chan 2006 & Outpatient rehabilitation centre & Outpatient rehabilitation centre & Hong Kong \\
\hline Chen 2004 & $\begin{array}{l}\text { Patients in neurological ward/ reha- } \\
\text { bilitation ward of } 4 \text { hospitals in Chi- } \\
\text { na }\end{array}$ & Not stated & China \\
\hline Chen 2006 & Inpatient University Hospital & Inpatient University Hospital & China \\
\hline Chen 2010 & Not stated & Not stated & China \\
\hline Chu 2003 & Inpatient, Hospital & Inpatient, Hospital & China \\
\hline Cooke 2006 & Multiple clinical centres (inpatient) & Multiple clinical centres (inpatient) & England \\
\hline Dean 1997 & $\begin{array}{l}\text { Own homes (recruited via stroke } \\
\text { clubs) }\end{array}$ & Own homes & Australia \\
\hline Dean 2000 & $\begin{array}{l}\text { Own homes (recruited from rehabil- } \\
\text { itation research group database) }\end{array}$ & Rehabilitation centre (outpatients) & Canada \\
\hline Dean 2006 & $\begin{array}{l}\text { Own homes (recruited via stroke } \\
\text { clubs) }\end{array}$ & Own homes & Australia \\
\hline Dean 2007 & Hospital rehabilitation unit & Hospital rehabilitation unit & Australia \\
\hline Deng 2011 & Hospital & $\begin{array}{l}\text { Before discharge: within hospital. After dis- } \\
\text { charge: home, }\end{array}$ & China \\
\hline
\end{tabular}


Table 7. Summary of study setting (Continued)

outpatient rehabilitation centres

\begin{tabular}{|c|c|c|c|}
\hline Duncan 1998 & $\begin{array}{l}\text { Previously inpatients, now dis- } \\
\text { charged? }\end{array}$ & Own homes & USA \\
\hline Duncan 2003 & Patients' own homes & Patients' own homes & USA \\
\hline Fan 2006 & In hospital & $\begin{array}{l}\text { Department of Neurology ward, rehabilitation } \\
\text { ward/ centre, } \\
\text { community or home setting, depending on } \\
\text { stage of rehabilitation }\end{array}$ & China \\
\hline Fang 2003 & Inpatient rehabilitation clinic & Inpatient rehabilitation clinic & China \\
\hline Fang 2004 old & Hospital & Not stated & China \\
\hline Fang 2004 young & Hospital & Not stated & China \\
\hline Ge 2003 & Rehabilitation department, Hospital & Not stated & China \\
\hline Gelber 1995 & Acute inpatient ward & Inpatient and outpatient rehabilitation centres & USA \\
\hline Green 2002 & $\begin{array}{l}\text { Recruited from hospital and com- } \\
\text { munity stroke registers }\end{array}$ & $\begin{array}{l}\text { Outpatient rehabilitation centre; patients' own } \\
\text { homes }\end{array}$ & England \\
\hline Holmgren 2006 & Rehabilitation unit (inpatients) & $\begin{array}{l}\text { Outpatient rehabilitation centre; patients' own } \\
\text { homes }\end{array}$ & Sweden \\
\hline Hou 2006 & Neurology ward & $\begin{array}{l}\text { Neurology ward, rehabilitation zone or rehabil- } \\
\text { itation centre, } \\
\text { own home or community depending on level of } \\
\text { rehabilitation }\end{array}$ & China \\
\hline Howe 2005 & Rehabilitation unit (inpatients) & Rehabilitation unit (inpatients) & England \\
\hline Hu 2007 haem & Not stated & Not stated & China \\
\hline Hu 2007 isch & Not stated & Not stated & China \\
\hline Huang 2003 & Not stated & Not stated & China \\
\hline Hui-Chan 2009 & Not stated & Own homes & Hong Kong \\
\hline Jiang 2006 & Not stated & $\begin{array}{l}\text { Neurology ward, rehabilitation ward/ centre, } \\
\text { community/home }\end{array}$ & China \\
\hline Jing 2006 & Not stated & Not stated & China \\
\hline Kim 2011 & Not stated & Not stated & Korea \\
\hline Kim 2012 & Inpatients, Hospital & Not stated & Korea \\
\hline Kwakkel 2002 & $\begin{array}{l}\text { Rehabilitation centres and nursing } \\
\text { homes }\end{array}$ & Rehabilitation centres and nursing homes & Netherlands \\
\hline Kwakkel 2008 & Rehabilitation centres (inpatient) & Multiple outpatient rehabilitation centres & Netherlands \\
\hline
\end{tabular}

Physical rehabilitation approaches for the recovery of function and mobility following stroke (Review)

Copyright $\odot 2023$ The Cochrane Collaboration. Published by John Wiley \& Sons, Ltd. 
Table 7. Summary of study setting (Continued)

\begin{tabular}{|c|c|c|c|}
\hline Langhammer 2000 & Acute inpatient ward & $\begin{array}{l}\text { Acute inpatient ward; rehabilitation units; out- } \\
\text { patients; own homes }\end{array}$ & Norway \\
\hline Langhammer 2007 & Acute inpatient ward & $\begin{array}{l}\text { Rehabilitation institutions, community, pa- } \\
\text { tients' homes and nursing homes. }\end{array}$ & Norway \\
\hline Lennon 2006 & Not stated & Not stated & Northern Ireland \\
\hline Li 1999 & Not stated & Not stated & China \\
\hline Li 2003 & Inpatient ward & Inpatient ward & China \\
\hline Li 2005 & Not stated & Not stated & China \\
\hline Liao 2006 & Not stated & Not stated & China \\
\hline Lincoln 2003 & Rehabilitation unit (inpatients) & Rehabilitation unit (inpatients); outpatients & England \\
\hline Liu 2003 & Hospital & 'All the trainings were done in the bed ward' & China \\
\hline McClellan 2004 & $\begin{array}{l}\text { Recruited on discharge from physio- } \\
\text { therapy services }\end{array}$ & Outpatients/patients' own homes & Australia \\
\hline Mudge 2009 & Private rehabilitation clinic & Private rehabilitation clinic & New Zealand \\
\hline Mudie 2002 & Rehabilitation unit (inpatients) & Rehabilitation unit (inpatients) & Australia \\
\hline Ni 1997 & Not stated & Not stated & China \\
\hline Pan 2004 & Not stated & Not stated & China \\
\hline Pang 2003 & Department of internal Neurology & Department of internal Neurology & China \\
\hline Pang 2006 & Not stated & Not stated & China \\
\hline Pollock 1998 & Stroke unit & Stroke unit & Scotland \\
\hline Qian 2004 & Not stated & Not stated & China \\
\hline Qian 2005 & Not stated & Not stated & China \\
\hline Richards 1993 & Acute inpatient ward & Acute inpatient ward & Canada \\
\hline Salbach 2004 & Patients' own homes (community) & $\begin{array}{l}\text { Outpatients/patients' own homes (self-prac- } \\
\text { tice) }\end{array}$ & Canada \\
\hline Shin 2011 & Outpatient rehabilitation centre & Outpatient rehabilitation centre & Korea \\
\hline Stephenson 2004 & Not stated & Not stated & USA \\
\hline Tang 2009 & Not stated & Not stated & China \\
\hline Thaut 2007 & Not stated & Not stated & Germany and USA \\
\hline Torres-Arreola 2009 & Hospital & Hospital and own homes (following discharge) & Mexico \\
\hline
\end{tabular}




\section{Table 7. Summary of study setting (Continued)}

\begin{tabular}{|c|c|c|c|}
\hline Verheyden 2006 & $\begin{array}{l}\text { Inpatient stroke rehabilitation cen- } \\
\text { tre }\end{array}$ & Inpatient stroke rehabilitation centre & Belgium \\
\hline Verma 2011 & Inpatient neurology ward & $\begin{array}{l}\text { Inpatient rehabilitation and/or outpatient re- } \\
\text { habilitation in day care units }\end{array}$ & India \\
\hline Wade 1992 & $\begin{array}{l}\text { Community (own homes and resi- } \\
\text { dential homes) }\end{array}$ & $\begin{array}{l}\text { Community (own homes and residential } \\
\text { homes) }\end{array}$ & England \\
\hline Wang 2004a & Not stated & Bedside and treatment room & China \\
\hline Wang 2004b & Not stated & Not stated & China \\
\hline Wang 2005 & Rehabilitation unit (inpatients) & Rehabilitation unit (inpatients) & Taiwan \\
\hline Wang 2006 & Not stated & $\begin{array}{l}\text { Neurology ward, rehabilitation zone, commu- } \\
\text { nity }\end{array}$ & China \\
\hline Wei 1998 & Hospital inpatients & Hospital inpatients & China \\
\hline Wellmon 1997 & Rehabilitation unit (inpatients) & Rehabilitation unit (inpatients) & USA \\
\hline Wu 2006 & Not stated & Ward, rehabilitation ward, community & China \\
\hline Xiao 2003 & Not stated & Not stated & China \\
\hline Xie 2003 & Hospital & Hospital & China \\
\hline Xie 2005 & Not stated & Hospital ward, home & China \\
\hline Xu 1999 & Not stated & Not stated & China \\
\hline Xu 2003a & Hospital & Neurology department & China \\
\hline Xu 2003b & $\begin{array}{l}\text { Inpatients, Department of Neurolo- } \\
\text { gy }\end{array}$ & Inpatients, Department of Neurology & China \\
\hline Xu 2004 & Not stated & Not stated & China \\
\hline Xue 2006 & $\begin{array}{l}\text { Department of Neurology, Yaan Peo- } \\
\text { ple's Hospital }\end{array}$ & Not stated & China \\
\hline Yan 2002 & Not stated & Hospital ward, rehabilitation centre & China \\
\hline Yelnik 2008 & Multi-center rehabilitation units & Multi-center rehabilitation units & France \\
\hline Yin 2003a & Neurology Department & Rehabilitation centre, Hospital & China \\
\hline Zhang 1998 & Not stated & Not stated & China \\
\hline Zhang 2004 & Not stated & $\begin{array}{l}\text { Department of Neurology- rehabilitation cen- } \\
\text { tres, Department of Rehabilitation, } \\
\text { community rehabilitation organisations, home }\end{array}$ & China \\
\hline Zhao 2002 & Not stated & Not stated & China \\
\hline
\end{tabular}


Table 7. Summary of study setting (Continued)

\begin{tabular}{llll} 
Zhao 2003 & Hospital & Neurology Department, Hospital & China \\
\hline Zhu 2001 & Not stated & Not stated & China \\
\hline Zhu 2004b & Not stated & Hospital, outpatient, community, home & China \\
\hline Zhu 2006 & Not stated & Not stated & China \\
\hline Zhu 2007 haem & Not stated & $\begin{array}{l}\text { Hospital, rehabilitation centre, home for inter- } \\
\text { vention groups. }\end{array}$ & China \\
\hline Zhu 2007 isch & Not stated & $\begin{array}{l}\text { Hospital, rehabilitation centre, home for inter- } \\
\text { vention groups. }\end{array}$ & China \\
\hline Zhuang 2012 & 'Stroke units in inpatient settings' & 'Stroke units in inpatient settings' & China \\
\hline
\end{tabular}




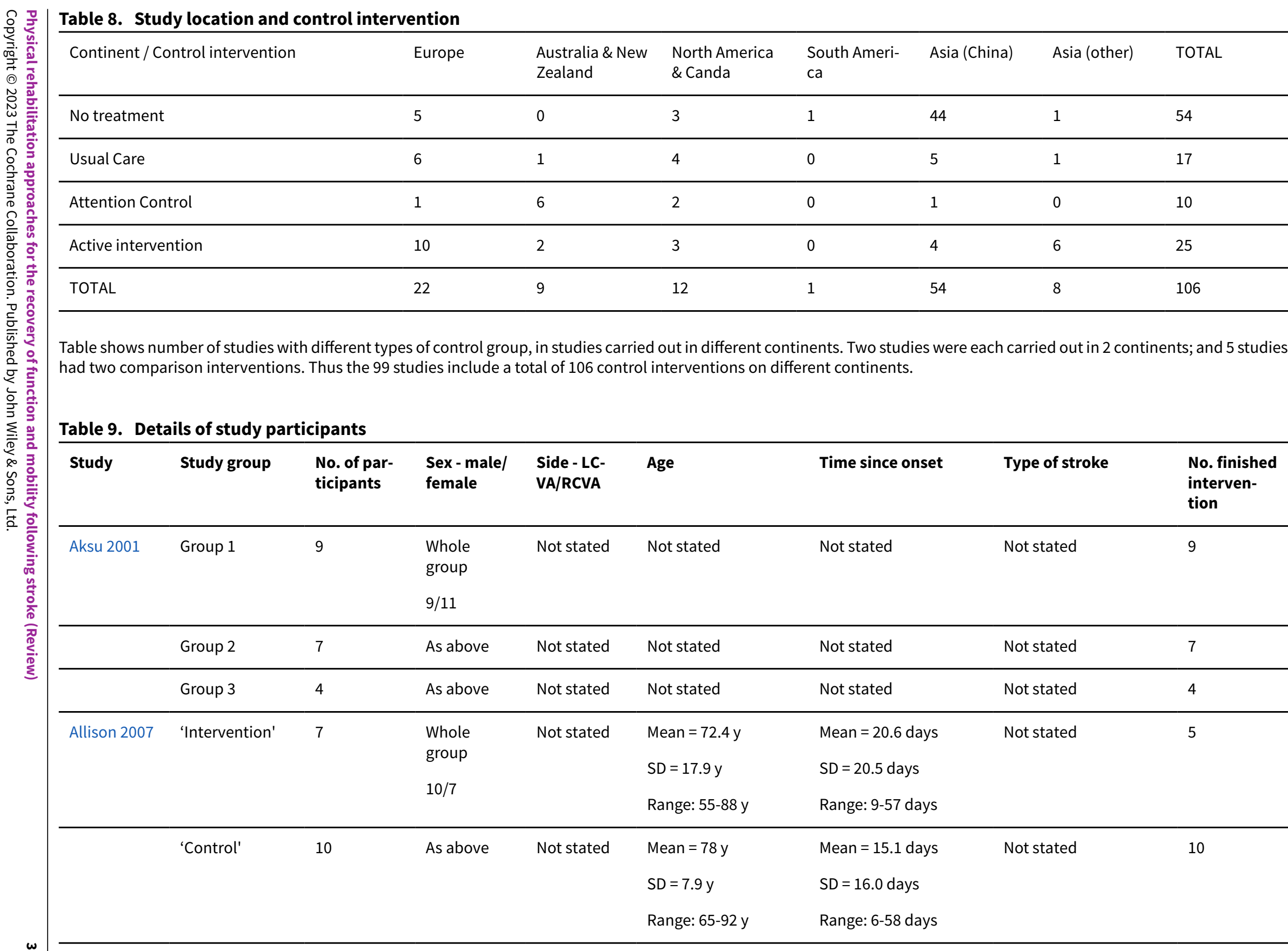




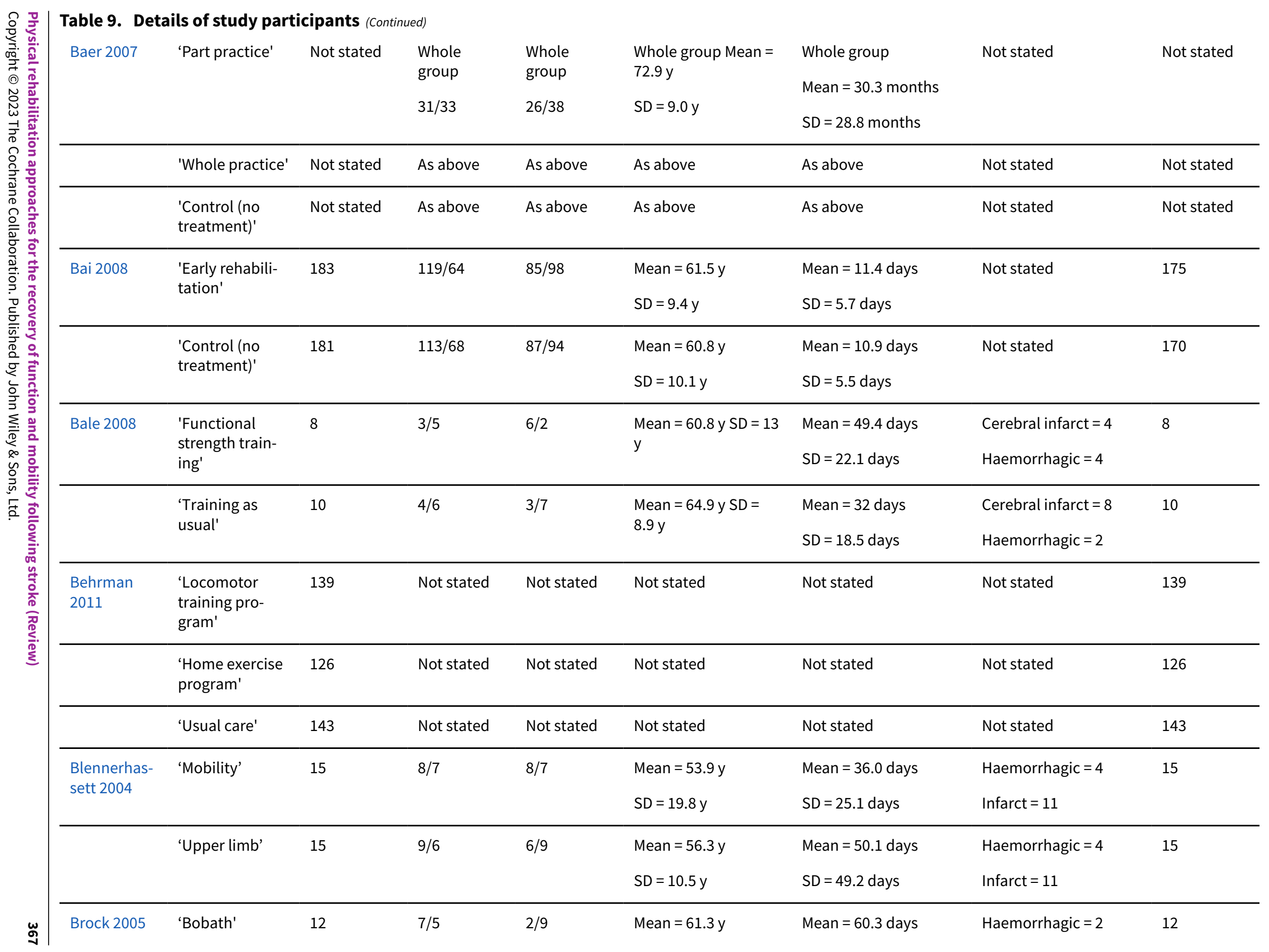




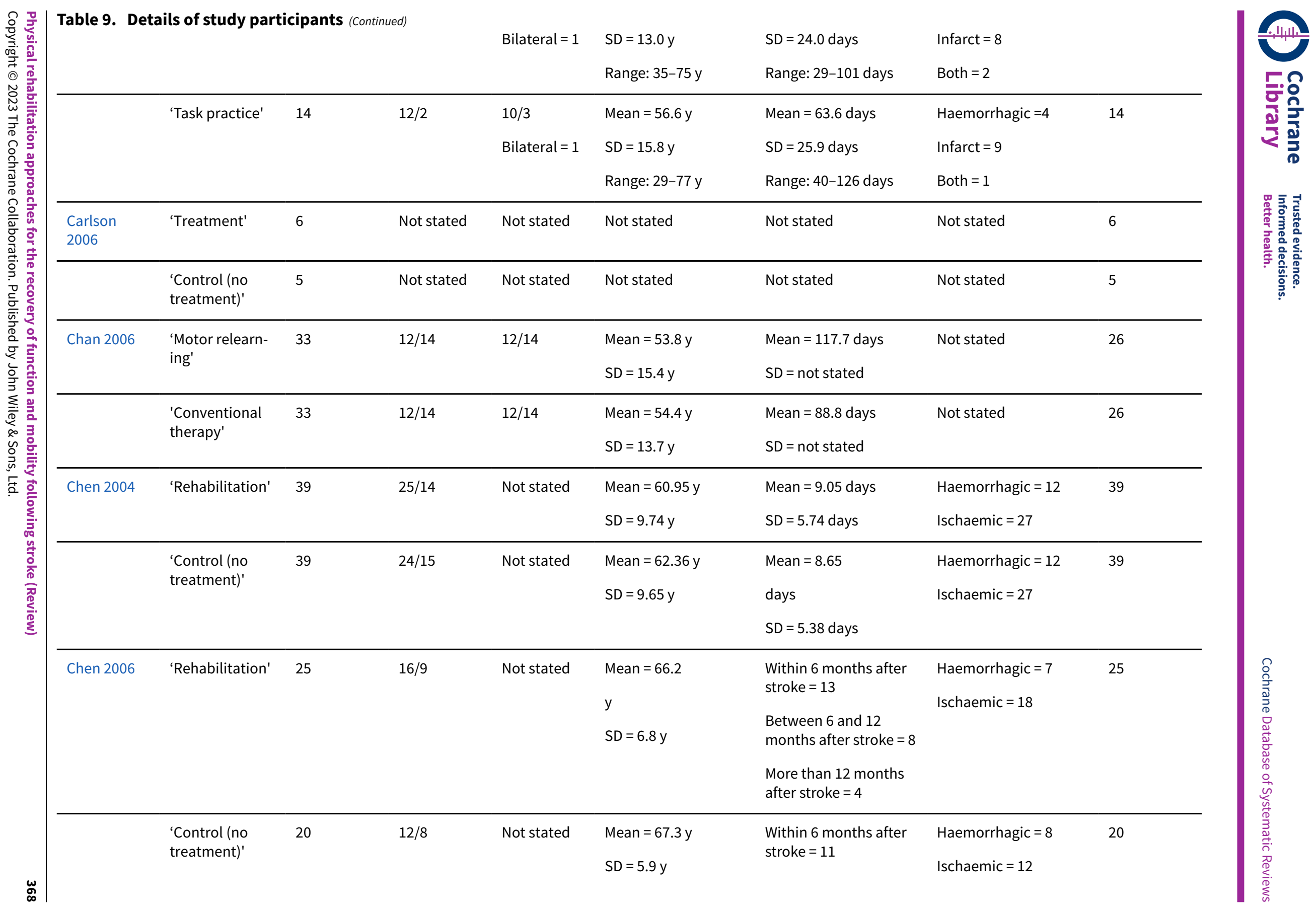




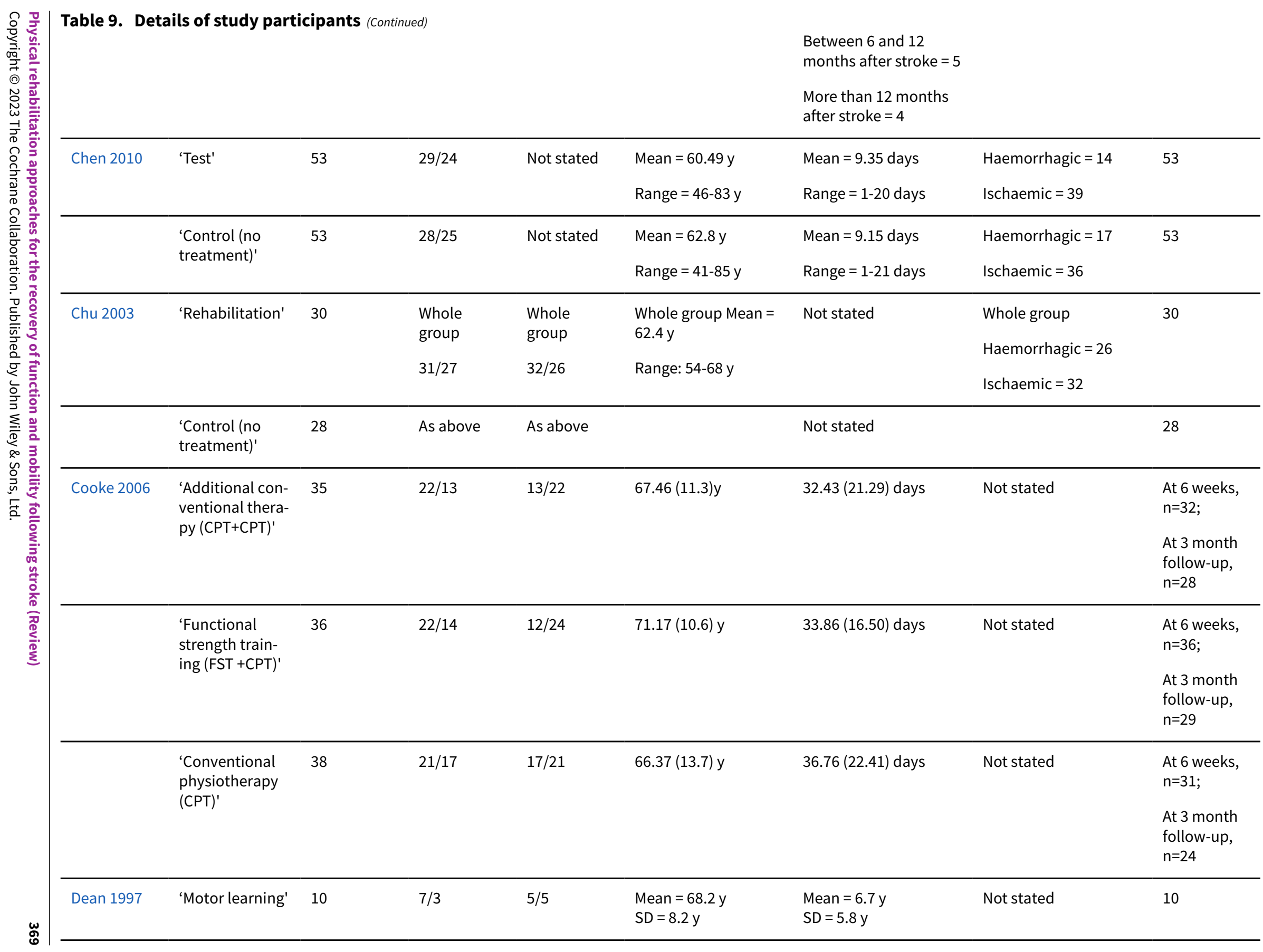




\begin{tabular}{|c|c|c|c|c|c|c|c|c|}
\hline & 'Placebo' & 10 & $7 / 3$ & $6 / 4$ & $\begin{array}{l}\text { Mean }=66.9 y \\
S D=8.2 y\end{array}$ & $\begin{array}{l}\text { Mean }=5.9 y \\
S D=2.9 y\end{array}$ & Not stated & 9 \\
\hline Dean 2000 & 'Motor learning' & 6 & $3 / 3$ & $3 / 3$ & $\begin{array}{l}\text { Mean }=66.2 \mathrm{y} \\
S D=7.7 \mathrm{y}\end{array}$ & $\begin{array}{l}\text { Mean }=2.3 y \\
S D=0.7 y\end{array}$ & Not stated & 5 \\
\hline & 'Placebo' & 6 & $4 / 2$ & $4 / 2$ & $\begin{array}{l}\text { Mean }=62.3 y \\
S D=6.6 y\end{array}$ & $\begin{array}{l}\text { Mean }=1.3 y \\
S D=0.9 y\end{array}$ & Not stated & 4 \\
\hline Dean 2006 & 'Experimental' & 76 & $38 / 38$ & $34 / 42$ & $\begin{array}{l}\text { Mean }=66.7 \mathrm{y} \\
\mathrm{SD}=14.3 \mathrm{y} \\
\text { Range: } 31-91 \mathrm{y}\end{array}$ & $\begin{array}{l}\text { Mean }=6.7 \mathrm{y} \\
\text { SD }=6.7 \mathrm{y} \\
\text { Range: } 0.1-24.8 \mathrm{y}\end{array}$ & Not stated & 65 \\
\hline & 'Control' & 75 & $40 / 35$ & $28 / 47$ & $\begin{array}{l}\text { Mean }=67.5 y \\
\text { SD }=10.2 y \\
\text { Range: } 40-85 y\end{array}$ & $\begin{array}{l}\text { Mean }=5.2 \mathrm{y} \\
\mathrm{SD}=5.4 \mathrm{y} \\
\text { Range: } 0.2-25.1 \mathrm{y}\end{array}$ & Not stated & 68 \\
\hline Dean 2007 & 'Experimental' & 6 & $5 / 1$ & $3 / 3$ & $\begin{array}{l}\text { Mean }=60 y \\
S D=7 y\end{array}$ & $\begin{array}{l}\text { Mean }=21 \text { days } \\
\text { SD }=8 \text { days Range: } 17 \text { - } \\
37 \text { days }\end{array}$ & Not stated & $\begin{array}{l}\text { At } 2 \text { weeks, } \\
n=6 ; \\
\text { At } 28 \text { week } \\
\text { follow-up, } \\
n=5\end{array}$ \\
\hline & 'Control' & 6 & $4 / 2$ & $1 / 5$ & $\begin{array}{l}\text { Mean }=74 y \\
S D=12 y\end{array}$ & $\begin{array}{l}\text { Mean }=37 \text { days } \\
\text { SD }=23 \text { days Range: } 13 \\
-75 \text { days }\end{array}$ & Not stated & $\begin{array}{l}\text { At } 2 \text { weeks, } \\
n=6 ; \\
\text { At } 28 \text { week } \\
\text { follow-up, } \\
n=4\end{array}$ \\
\hline Deng 2011 & 'Intervention' & 50 & $36 / 14$ & Not stated & $\begin{array}{l}\text { Mean }=57.08 y \\
S D=9.15 y\end{array}$ & $\begin{array}{l}\text { Stroke onset to admis- } \\
\text { sion to hospital: } \\
\leq 6 \text { hours: } n=31 \\
>6 \text { hours: } \mathrm{n}=19\end{array}$ & Not stated & 50 \\
\hline & $\begin{array}{l}\text { 'Control (no } \\
\text { treatment)' }\end{array}$ & 50 & $35 / 15$ & Not stated & $\begin{array}{l}\text { Mean }=56.98 y \\
S D=9.05 y\end{array}$ & $\begin{array}{l}\text { Stroke onset to admis- } \\
\text { sion to hospital: } \\
\leq 6 \text { hours: } n=30\end{array}$ & Not stated & 50 \\
\hline
\end{tabular}




\begin{tabular}{|c|c|c|c|c|c|c|c|c|}
\hline & & & & & & $>6$ hours: $n=20$ & & \\
\hline \multirow[t]{2}{*}{$\begin{array}{l}\text { Duncan } \\
1998\end{array}$} & 'Mixed' & 10 & Not stated & $4 / 6$ & $\begin{array}{l}\text { Mean }=67.3 y \\
S D=9.6 y\end{array}$ & Mean $=66$ days & Ischaemic $=10$ & 10 \\
\hline & 'Control' & 10 & Not stated & $\begin{array}{l}4 / 5+1 \\
\text { brainstem }\end{array}$ & $\begin{array}{l}\text { Mean }=67.8 \mathrm{y} \\
S D=7.2 \mathrm{y}\end{array}$ & Mean $=56$ days & $\begin{array}{l}\text { Haemorrhagic }=2 \\
\text { Ischaemic }=8\end{array}$ & 10 \\
\hline \multirow[t]{2}{*}{$\begin{array}{l}\text { Duncan } \\
2003\end{array}$} & 'Mixed' & $\begin{array}{l}50 \text { ( } 44 \text { com- } \\
\text { pleted inter- } \\
\text { vention) }\end{array}$ & $23 / 21$ & $\begin{array}{l}18 / 22 ; 4 \text { bi- } \\
\text { lateral }\end{array}$ & $\begin{array}{l}\text { Mean }=68.5 y \\
S D=9 y\end{array}$ & $\begin{array}{l}\text { Mean }=77.5 \text { days } \\
S D=28.7 \text { days }\end{array}$ & Ischaemic $=39$ & 44 \\
\hline & 'Control' & $\begin{array}{l}50 \text { ( } 48 \text { com- } \\
\text { pleted inter- } \\
\text { vention) }\end{array}$ & $27 / 21$ & $\begin{array}{l}22 / 22 ; 4 \text { bi- } \\
\text { lateral }\end{array}$ & $\begin{array}{l}\text { Mean }=70.2 \mathrm{y} \\
\mathrm{SD}=11.4 \mathrm{y}\end{array}$ & $\begin{array}{l}\text { Mean }=73.5 \text { days } \\
\text { SD }=27.1 \text { days }\end{array}$ & Ischaemic $=44$ & 48 \\
\hline \multirow[t]{2}{*}{ Fan 2006} & 'Treated' & 42 & $22 / 20$ & $21 / 21$ & $\begin{array}{l}\text { Mean }=64.53 \\
y \\
\text { SD }=10.77 y\end{array}$ & $\begin{array}{l}\text { Mean }=8.14 \text { days } \\
\mathrm{SD}=4.95 \text { days }\end{array}$ & $\begin{array}{l}\text { Haemorrhagic }=15 \\
\text { Ischaemic }=27\end{array}$ & 42 \\
\hline & $\begin{array}{l}\text { 'Control (no } \\
\text { treatment)' }\end{array}$ & 40 & $27 / 13$ & $\begin{array}{l}\text { (unable to } \\
\text { tell if data } \\
\text { pertains to } \\
\text { side of le- } \\
\text { sion } \\
\text { or side of } \\
\text { hemiplegia) }\end{array}$ & $\begin{array}{l}\text { Mean }=65.82 \mathrm{y} \\
S D=10.61 \mathrm{y}\end{array}$ & $\begin{array}{l}\text { Mean }=8.33 \text { days } \\
\mathrm{SD}=3.87 \text { days }\end{array}$ & $\begin{array}{l}\text { Haemorrhagic }=14 \\
\text { Ischaemic }=26\end{array}$ & 38 \\
\hline \multirow[t]{2}{*}{ Fang 2003} & $\begin{array}{l}\text { 'Additional ear- } \\
\text { ly physiothera- } \\
\text { py intervention' }\end{array}$ & 78 & $33 / 17$ & Not stated & $\begin{array}{l}\text { Mean }=65.49 y \\
S D=10.94 y\end{array}$ & Not stated & $\begin{array}{l}\text { Haemorrhagic }=13 \\
\text { Cerebral infarct }=37\end{array}$ & $\begin{array}{l}\text { At day } 30, n= \\
50 ; \\
\text { at } 6 \text { months, } \\
n=12\end{array}$ \\
\hline & $\begin{array}{l}\text { 'Routine thera- } \\
\text { py' }\end{array}$ & 78 & $44 / 34$ & Not stated & $\begin{array}{l}\text { Mean }=61.8 y \\
S D=10.94 y\end{array}$ & Not stated & $\begin{array}{l}\text { Haemorrhagic }=11 \\
\text { Cerebral infarct }=67\end{array}$ & $\begin{array}{l}\text { At day } 30, \mathrm{n}= \\
78 ; \\
\text { at } 6 \text { months, } \\
\mathrm{n}=12\end{array}$ \\
\hline
\end{tabular}




\begin{tabular}{|c|c|c|c|c|c|c|c|c|}
\hline $\begin{array}{l}\text { Fang } 2004 \\
\text { old }\end{array}$ & 'Rehabilitation' & 25 & $17 / 8$ & Not stated & $\begin{array}{l}\text { Whole group mean = } \\
65.49 \mathrm{y} \\
\mathrm{SD}=10.94 \mathrm{y}\end{array}$ & Not stated & $\begin{array}{l}\text { Whole group } \\
\text { Haemorrhagic = } 24 \\
\text { Ischaemic = } 102 \\
\text { Mixed = } 2\end{array}$ & $\begin{array}{l}\text { Whole } \\
\text { group: } \\
\text { At day } 30 \text {, } \\
n=45 ; \text { at } 6 \\
\text { months, } n= \\
14\end{array}$ \\
\hline & $\begin{array}{l}\text { 'Control (no } \\
\text { treatment)' }\end{array}$ & 45 & $26 / 19$ & Not stated & $\begin{array}{l}\text { Whole group mean }= \\
61.8 \mathrm{y} \\
\mathrm{SD}=10.9 \mathrm{y}\end{array}$ & Not stated & As above & $\begin{array}{l}\text { Whole } \\
\text { group: } \\
\text { At day } 30, \\
n=55 ; \text { at } \\
6 \text { months, } \\
n=12\end{array}$ \\
\hline $\begin{array}{l}\text { Fang } 2004 \\
\text { young }\end{array}$ & 'Rehabilitation' & 25 & $16 / 9$ & Not stated & As above & Not stated & As above & As above \\
\hline & $\begin{array}{l}\text { 'Control (no } \\
\text { treatment)' }\end{array}$ & 33 & $18 / 15$ & Not stated & As above & Not stated & As above & As above \\
\hline Ge 2003 & 'Rehabilitation' & 20 & $14 / 6$ & Not stated & $\begin{array}{l}\text { Mean }=61 \mathrm{y} \\
\mathrm{SD}=5 \mathrm{y}\end{array}$ & $\begin{array}{l}\text { Mean }=50 \text { days } \\
S D=22 \text { days }\end{array}$ & Not stated & $\begin{array}{l}\text { Unclear - } \\
\text { see notes in } \\
\text { character- } \\
\text { istics of in- } \\
\text { cluded stud- } \\
\text { ies }\end{array}$ \\
\hline & $\begin{array}{l}\text { 'Control (no } \\
\text { treatment)' }\end{array}$ & 28 & $20 / 8$ & Not stated & $\begin{array}{l}\text { Mean }=60 y \\
S D=5 y\end{array}$ & $\begin{array}{l}\text { Mean }=51 \text { days } \\
S D=26 \text { days }\end{array}$ & Not stated & $\begin{array}{l}\text { Unclear - } \\
\text { see notes in } \\
\text { character- } \\
\text { istics of in- } \\
\text { cluded stud- } \\
\text { ies }\end{array}$ \\
\hline Gelber 1995 & $\begin{array}{l}\text { 'Neurophysio- } \\
\text { logical (NDT)' }\end{array}$ & 15 & $9 / 6$ & $8 / 7$ & $\begin{array}{l}\text { Mean }=73.7 \mathrm{y} \\
\mathrm{SEM}=2.0 \mathrm{y}\end{array}$ & $\begin{array}{l}\text { Mean }=11.3 \text { days } \\
\mathrm{SEM}=1.1 \text { days }\end{array}$ & $\begin{array}{l}\text { Pure motor ischaemic } \\
=15\end{array}$ & 15 \\
\hline & $\begin{array}{l}\text { 'Orthopaedic } \\
\text { (TFR)' }\end{array}$ & 12 & $4 / 8$ & $5 / 7$ & $\begin{array}{l}\text { Mean }=69.8 \mathrm{y} \\
\mathrm{SEM}=2.9 \mathrm{y}\end{array}$ & $\begin{array}{l}\text { Mean }=13.8 \text { days } \\
\mathrm{SEM}=2.7 \text { days }\end{array}$ & $\begin{array}{l}\text { Pure motor ischaemic } \\
=12\end{array}$ & 12 \\
\hline Green 2002 & 'Mixed' & 85 & $49 / 36$ & $\begin{array}{l}56 / 26+3 \\
\text { 'other' }\end{array}$ & Mean $=71.5 \mathrm{y}$ & Not stated & Not stated & 81 \\
\hline
\end{tabular}




\begin{tabular}{|c|c|c|c|c|c|c|c|c|}
\hline & & & & & $\mathrm{SD}=8.7 \mathrm{y}$ & & & \\
\hline & $\begin{array}{l}\text { 'Control (no } \\
\text { treatment)' }\end{array}$ & 85 & $46 / 39$ & $\begin{array}{l}44 / 40+1 \\
\text { 'other' }\end{array}$ & $\begin{array}{l}\text { Mean }=73.5 y \\
S D=8.3 y\end{array}$ & Not stated & Not stated & 80 \\
\hline \multirow[t]{2}{*}{$\begin{array}{l}\text { Holmgren } \\
2006\end{array}$} & 'Intervention' & 15 & $9 / 6$ & Not stated & $\begin{array}{l}\text { Mean }=77.7 \mathrm{y} \\
\mathrm{SD}=7.6 \mathrm{y}\end{array}$ & $\begin{array}{l}\text { Mean }=139.7 \text { days } \\
S D=37.3 \text { days }\end{array}$ & $\begin{array}{l}\text { Cardioembolic stroke } \\
=4 \\
\text { Lacunar infarct = } 2 \\
\text { Other specified stroke } \\
=2 \\
\text { Unknown stroke = } 6 \\
\text { not applicable (be- } \\
\text { cause of } \\
\text { intracerebral haemor- } \\
\text { rhage) =1 }\end{array}$ & 15 \\
\hline & 'Control' & 19 & $12 / 7$ & Not stated & $\begin{array}{l}\text { Mean }=79.2 \mathrm{y} \\
\mathrm{SD}=7.5 \mathrm{y}\end{array}$ & $\begin{array}{l}\text { Mean }=126.8 \text { days } \\
S D=28.2 \text { days }\end{array}$ & $\begin{array}{l}\text { Large artery thrombo- } \\
\text { sis }=4 \\
\text { Cardioembolic stroke } \\
=5 \\
\text { Lacunar infarct }=8 \\
\text { Unknown stroke }=2\end{array}$ & 19 \\
\hline \multirow[t]{2}{*}{ Hou 2006} & 'Rehabilitation' & 40 & $25 / 15$ & Not stated & $\begin{array}{l}\text { Mean }=61.38 y \\
S D=9.99 y\end{array}$ & $\begin{array}{l}\text { Mean }=9.05 \text { days } \\
S D=5.74 \text { days }\end{array}$ & $\begin{array}{l}\text { Haemorrhagic }=12 \\
\text { Ischaemic }=28\end{array}$ & 40 \\
\hline & $\begin{array}{l}\text { 'Control (no } \\
\text { treatment)' }\end{array}$ & 40 & $24 / 16$ & Not stated & $\begin{array}{l}\text { Mean }=62.55 y \\
S D=9.60 y\end{array}$ & $\begin{array}{l}\text { Mean }=8.65 \text { days } \\
S D=5.38 \text { days }\end{array}$ & $\begin{array}{l}\text { Haemorrhagic }=12 \\
\text { Ischaemic }=28\end{array}$ & 40 \\
\hline \multirow[t]{2}{*}{ Howe 2005} & 'Mixed' & $\begin{array}{l}17 \text { (15 at } 4- \\
\text { week } \\
\text { follow up) }\end{array}$ & $9 / 8$ & $8 / 9$ & $\begin{array}{l}\text { Mean }=71.5 y \\
S D=10.9 y\end{array}$ & $\begin{array}{l}\text { Mean }=26.5 \text { days } \\
S D=15.7 \text { days }\end{array}$ & $\begin{array}{l}2 \text { TACS / } 7 \text { PACS / } \\
4 \text { LACS / } 1 \text { POCS / } 3 \\
\text { other }\end{array}$ & 15 \\
\hline & $\begin{array}{l}\text { 'Control (neuro- } \\
\text { physiological)' }\end{array}$ & $\begin{array}{l}18 \text { (18 at } 4- \\
\text { week }\end{array}$ & $9 / 9$ & $7 / 11$ & $\begin{array}{l}\text { Mean }=70.7 \mathrm{y} \\
S D=17.5 \mathrm{y}\end{array}$ & $\begin{array}{l}\text { Mean }=23.1 \text { days } \\
S D=17.5 \text { days }\end{array}$ & 3 TACS / 6 PACS / & 18 \\
\hline
\end{tabular}




\begin{tabular}{|c|c|c|c|c|c|c|c|c|}
\hline & & follc & & & & & 4 LACS / 3 POCS / 2 & \\
\hline $\begin{array}{l}\text { Hu } 2007 \\
\text { haem }\end{array}$ & $\begin{array}{l}\text { 'Test (haemor- } \\
\text { rhagic group)' }\end{array}$ & 178 & Not stated & Not stated & $\begin{array}{l}\text { Whole group } \\
\text { Mean = } \\
61 \mathrm{y} \\
\text { SD = 10y }\end{array}$ & $\begin{array}{l}\text { Whole group } \\
\text { Mean }=11 \text { days } \\
S D=6 \text { days }\end{array}$ & Not stated & $\begin{array}{l}\text { At } 1 \text { month } \\
\text { after stroke } \\
\mathrm{n}=178 ; \text { at } 3 \\
\text { months af- } \\
\text { ter stroke, } \\
\mathrm{n}=178 \text {; at } 6 \\
\text { months af- } \\
\text { ter stroke, } \\
\mathrm{n}=177\end{array}$ \\
\hline & $\begin{array}{l}\text { 'Control (no } \\
\text { treatment)' }\end{array}$ & 174 & Not stated & Not stated & As above & As above & Not stated & $\begin{array}{l}\text { At } 1 \text { month } \\
\text { after stroke } \\
\mathrm{n}=174 ; \text { at } 3 \\
\text { months af- } \\
\text { ter stroke, } \\
\mathrm{n}=168 \text {; at } 6 \\
\text { months af- } \\
\text { ter stroke, } \\
\mathrm{n}=168\end{array}$ \\
\hline Hu 2007 isch & $\begin{array}{l}\text { 'Test (ischaemic } \\
\text { group)' }\end{array}$ & 485 & Not stated & Not stated & $\begin{array}{l}\text { Whole group } \\
\text { Mean }=64 \mathrm{y} \\
\text { SD }=10 \mathrm{y}\end{array}$ & $\begin{array}{l}\text { Whole group } \\
\text { Mean }=10 \text { days } \\
\text { SD }=5 \text { days }\end{array}$ & Not stated & $\begin{array}{l}\text { At } 1 \text { month } \\
\text { after stroke } \\
\mathrm{n}=485 ; \text { at } 3 \\
\text { months af- } \\
\text { ter stroke, } \\
\mathrm{n}=478 \text {; at } 6 \\
\text { months af- } \\
\text { ter stroke, } \\
\mathrm{n}=471\end{array}$ \\
\hline & $\begin{array}{l}\text { 'Control (no } \\
\text { treatment)' }\end{array}$ & 480 & Not stated & Not stated & As above & As above & Not stated & $\begin{array}{l}\text { At } 1 \text { month } \\
\text { after stroke } \\
\mathrm{n}=480 \text {; at } 3 \\
\text { months af- } \\
\text { ter stroke, } \\
\mathrm{n}=473 \text {; at } 6 \\
\text { months af- } \\
\text { ter stroke, } \\
\mathrm{n}=469\end{array}$ \\
\hline Huang 2003 & 'Rehabilitation' & 25 & $17 / 8$ & $14 / 11$ & Mean $=64.61 \mathrm{y}$ & Mean $=6.45$ days & Haemorrhagic $=5$ & 25 \\
\hline
\end{tabular}




\begin{tabular}{|c|c|c|c|c|c|c|c|c|}
\hline & & & & & $\mathrm{SD}=12.37 \mathrm{y}$ & $\mathrm{SD}=3.70$ days & Ischaemic $=20$ & \\
\hline & $\begin{array}{l}\text { 'Control (no } \\
\text { treatment)' }\end{array}$ & 25 & $17 / 8$ & $12 / 13$ & $\begin{array}{l}\text { Mean }=65.351 \mathrm{y} \\
S D=11.71 \mathrm{y}\end{array}$ & $\begin{array}{l}\text { Mean }=6.89 \text { days } \\
\mathrm{SD}=3.20 \text { days }\end{array}$ & $\begin{array}{l}\text { Haemorrhagic = } 5 \\
\text { Ischaemic }=20\end{array}$ & 25 \\
\hline $\begin{array}{l}\text { Hui-Chan } \\
2009\end{array}$ & 'PLBO+TRT' & 25 & Not stated & Not stated & $\begin{array}{l}\text { Whole group mean }= \\
56.6 \mathrm{y} \\
\mathrm{SD}=7.9 \mathrm{y}\end{array}$ & $\begin{array}{l}\text { Whole group mean }= \\
4.7 \mathrm{y} \\
\mathrm{SD}=3.4 \mathrm{y}\end{array}$ & Not stated & 25 \\
\hline & $\begin{array}{l}\text { 'Control (no } \\
\text { treatment)' }\end{array}$ & 29 & Not stated & Not stated & As above & As above & Not stated & 29 \\
\hline Jiang 2006 & 'Treated' & 42 & $22 / 20$ & $\begin{array}{l}21 / 21 \\
\text { 'location of } \\
\text { disease' }\end{array}$ & $\begin{array}{l}\text { Mean }=64.53 y \\
S D=10.77 y\end{array}$ & $\begin{array}{l}\text { Mean }=8.14 \text { days } \\
\mathrm{SD}=4.95 \text { days }\end{array}$ & $\begin{array}{l}\text { Haemorrhagic }=15 \\
\text { Infarction }=27\end{array}$ & $\begin{array}{l}\text { Whole } \\
\text { group: } n=79 \\
\text { at } 6 \text { months }\end{array}$ \\
\hline & 'Control' & 40 & $27 / 13$ & $\begin{array}{l}15 / 25 \\
\text { 'location of } \\
\text { disease' }\end{array}$ & $\begin{array}{l}\text { Mean }=65.82 \mathrm{y} \\
S D=10.61 \mathrm{y}\end{array}$ & $\begin{array}{l}\text { Mean }=8.33 \text { days } \\
\mathrm{SD}=3.87 \text { days }\end{array}$ & $\begin{array}{l}\text { Haemorrhagic = } 14 \\
\text { Infarction }=26\end{array}$ & As above \\
\hline Jing 2006 & $\begin{array}{l}\text { 'Exercise and } \\
\text { occupational } \\
\text { therapy' }\end{array}$ & 120 & $69 / 51$ & $\begin{array}{l}\text { Whole } \\
\text { group } \\
73 / 87\end{array}$ & $\begin{array}{l}\text { Mean }=57.3 \mathrm{y} \\
\mathrm{SD}=12.5 \mathrm{y}\end{array}$ & $\begin{array}{l}\text { Mean }=5.2 \text { days } \\
\mathrm{SD}=4.2 \text { days }\end{array}$ & $\begin{array}{l}\text { Whole group } \\
\text { Haemorrhagic = } 66 \\
\text { Ischaemic = } 94\end{array}$ & 120 \\
\hline & $\begin{array}{l}\text { 'Exercise thera- } \\
\text { py' }\end{array}$ & 40 & $23 / 17$ & As above & $\begin{array}{l}\text { Mean }=54.5 y \\
S D=9.6 y\end{array}$ & $\begin{array}{l}\text { Mean }=4.6 \text { days } \\
\mathrm{SD}=3.7 \text { days }\end{array}$ & As above & 40 \\
\hline Kim 2011 & 'PNF' & 20 & $17 / 3$ & $12 / 8$ & $\begin{array}{l}\text { Mean }=51.4 \mathrm{y} \\
S D=5.7 \mathrm{y}\end{array}$ & $\begin{array}{l}\text { Mean }=22.9 \text { months } \\
\mathrm{SD}=12.2 \text { months }\end{array}$ & $\begin{array}{l}\text { Haemorrhagic }=8 \\
\text { Infarction }=12\end{array}$ & 20 \\
\hline & 'Control' & 20 & $14 / 6$ & $12 / 8$ & $\begin{array}{l}\text { Mean }=53.5 \mathrm{y} \\
S D=7.1 \mathrm{y}\end{array}$ & $\begin{array}{l}\text { Mean }=26.8 \text { months } \\
\mathrm{SD}=12.8 \text { months }\end{array}$ & $\begin{array}{l}\text { Haemorrhagic = } 9 \\
\text { Infarction }=11\end{array}$ & 20 \\
\hline Kim 2012 & 'Experimental' & 10 & Not stated & Not stated & $\begin{array}{l}\text { Mean }=52.5 y \\
S D=11.72 y\end{array}$ & $\begin{array}{l}\text { Mean }=7.7 \mathrm{y} \\
S D=6.11 \mathrm{y}\end{array}$ & Not stated & 10 \\
\hline
\end{tabular}




\begin{tabular}{|c|c|c|c|c|c|c|c|c|}
\hline & 'Control' & 10 & Not stated & Not stated & $\begin{array}{l}\text { Mean }=53.4 \mathrm{y} \\
S D=12.11 \mathrm{y}\end{array}$ & $\begin{array}{l}\text { Mean = } 13.1 \mathrm{y} \\
S D=10.62 \mathrm{y}\end{array}$ & Not stated & 10 \\
\hline $\begin{array}{l}\text { Kwakkel } \\
2002\end{array}$ & $\begin{array}{l}\text { 'Lower extremi- } \\
\text { ties' }\end{array}$ & 17 & $13 / 4$ & $7 / 10$ & $\begin{array}{l}\text { Mean }=60.8 \mathrm{y} \\
\text { SD }=10.6 \mathrm{y} \\
\text { Range: } 38-76 \mathrm{y}\end{array}$ & $\begin{array}{l}\text { Mean }=4.8 \text { weeks } \\
\text { SD }=3.1 \text { weeks } \\
\text { Range: } 2-9 \text { weeks }\end{array}$ & $\begin{array}{l}\mathrm{TACl}=6 \\
\mathrm{PACl}=10 \\
\mathrm{LACl}=1\end{array}$ & 17 \\
\hline & $\begin{array}{l}\text { 'Upper extremi- } \\
\text { ties' }\end{array}$ & 18 & $9 / 9$ & $8 / 10$ & $\begin{array}{l}\text { Mean }=64.3 y \\
S D=10.6 y \\
\text { Range: } 46-80 y\end{array}$ & $\begin{array}{l}\text { Mean }=5.9 \text { weeks } \\
\text { SD }=3 \text { weeks } \\
\text { Range: } 2-10 \text { weeks }\end{array}$ & $\begin{array}{l}\mathrm{TACl}=8 \\
\mathrm{PACl}=7 \\
\mathrm{LACl}=3\end{array}$ & 18 \\
\hline & 'Control' & 18 & $14 / 4$ & $7 / 11$ & $\begin{array}{l}\text { Mean }=62.1 \mathrm{y} \\
\text { SD }=10.6 \mathrm{y} \\
\text { Range: } 30-76 \mathrm{y}\end{array}$ & $\begin{array}{l}\text { Mean }=7.3 \text { weeks } \\
\text { SD }=3.6 \text { weeks } \\
\text { Range: } 2-10 \text { weeks }\end{array}$ & $\begin{array}{l}\mathrm{TACl}=9 \\
\mathrm{PACl}=6 \\
\mathrm{LACl}=3\end{array}$ & 18 \\
\hline $\begin{array}{l}\text { Kwakkel } \\
2008\end{array}$ & 'Circuit training' & 126 & $82 / 44$ & $\begin{array}{l}49 / 57 \\
\text { brainstem = } \\
6 \\
\text { cerebellum } \\
=14\end{array}$ & $\begin{array}{l}\text { Mean }=56 y \\
S D=10 y\end{array}$ & $\begin{array}{l}\text { Mean }=91 \text { days } \\
S D=42 \text { days }\end{array}$ & $\begin{array}{l}\text { Haemorrhagic }=23 \\
\text { Ischaemic }=103\end{array}$ & 125 \\
\hline & $\begin{array}{l}\text { 'Usual physio- } \\
\text { therapy' }\end{array}$ & 124 & $80 / 44$ & $\begin{array}{l}43 / 61 \\
\text { brainstem = } \\
14 \\
\text { cerebellum } \\
=6\end{array}$ & $\begin{array}{l}\text { Mean }=58 y \\
S D=10 y\end{array}$ & $\begin{array}{l}\text { Mean }=103 \text { days } \\
S D=51 \text { days }\end{array}$ & $\begin{array}{l}\text { Haemorrhagic }=24 \\
\text { Ischaemic }=100\end{array}$ & 117 \\
\hline $\begin{array}{l}\text { Langham- } \\
\text { mer } 2000\end{array}$ & $\begin{array}{l}\text { 'Neurophysi- } \\
\text { ological (Bo- } \\
\text { bath)' }\end{array}$ & 28 & $16 / 12$ & $17 / 11$ & $\begin{array}{l}\text { Whole group } \\
\text { Mean }=78 \mathrm{y} \\
\text { SD }=9 \mathrm{y} \\
\text { Range } 49 \text { to } 95 \mathrm{y}\end{array}$ & Not stated & Not stated & 24 \\
\hline & 'Motor learning' & 33 & $20 / 13$ & $17 / 16$ & See above & Not stated & Not stated & 29 \\
\hline
\end{tabular}




\begin{tabular}{|c|c|c|c|c|c|c|c|c|}
\hline $\begin{array}{l}\text { Langham- } \\
\text { mer } 2007\end{array}$ & $\begin{array}{l}\text { 'Intensive exer- } \\
\text { cise' }\end{array}$ & 35 & Not stated & $16 / 19$ & $\begin{array}{l}\text { Mean }=76 y \\
S D=12.7 y\end{array}$ & Not stated & $\begin{array}{l}\text { 'Cause of the stroke } \\
\text { was thrombosis or em- } \\
\text { bolism with } 29 \text { such } \\
\text { cases in the intensive } \\
\text { exercise group \& } 6 \text { be- } \\
\text { ing haemorrhages' }\end{array}$ & 32 \\
\hline & $\begin{array}{l}\text { 'Regular exer- } \\
\text { cise' }\end{array}$ & 40 & Not stated & $21 / 19$ & $\begin{array}{l}\text { Mean }=72 \mathrm{y} \\
S D=13.6 y\end{array}$ & Not stated & $\begin{array}{l}\text { 'Cause of the stroke } \\
\text { was thrombosis or em- } \\
\text { bolism with } 36 \text { such } \\
\text { cases in the regular } \\
\text { exercise group and } 4 \\
\text { being haemorrhages' }\end{array}$ & 32 \\
\hline $\begin{array}{l}\text { Lennon } \\
2006\end{array}$ & 'Bobath' & 30 & Not stated & Not stated & Not stated & Not stated & Not stated & 30 \\
\hline & $\begin{array}{l}\text { 'Gait specific } \\
\text { group' }\end{array}$ & 31 & Not stated & Not stated & Not stated & Not stated & Not stated & 31 \\
\hline Li 1999 & $\begin{array}{l}\text { 'Early rehabili- } \\
\text { tation' }\end{array}$ & 30 & Not stated & Not stated & $\begin{array}{l}\text { Mean }=58.1 \mathrm{y} \\
S D=11.9 y\end{array}$ & Not stated & $\begin{array}{l}\text { Haemorrhagic }=12 \\
\text { Ischaemic }=18\end{array}$ & 30 \\
\hline & $\begin{array}{l}\text { 'Control (no } \\
\text { treatment)' }\end{array}$ & 31 & Not stated & Not stated & $\begin{array}{l}\text { Mean }=59.20 \\
S D=10.2 y\end{array}$ & Not stated & $\begin{array}{l}\text { Haemorrhagic = } 12 \\
\text { Ischaemic }=19\end{array}$ & 31 \\
\hline Li 2003 & 'Rehabilitation' & 87 & $49 / 38$ & Not stated & $\begin{array}{l}\text { Mean }=63 y \\
S D=1 y\end{array}$ & Not stated & Not stated & 87 \\
\hline & $\begin{array}{l}\text { 'Control (no } \\
\text { treatment) } \\
\text { group' }\end{array}$ & 87 & $35 / 52$ & Not stated & Not stated & Not stated & Not stated & 87 \\
\hline Li 2005 & $\begin{array}{l}\text { 'Motor relearn- } \\
\text { ing' }\end{array}$ & 31 & Not stated & Not stated & $\begin{array}{l}\text { Mean }=51.4 \mathrm{y} \\
\mathrm{SD}=8.9 \mathrm{y}\end{array}$ & $\begin{array}{l}\text { Mean }=8.8 \text { days } \\
\mathrm{SD}=6.0 \text { days }\end{array}$ & Not stated & 31 \\
\hline & $\begin{array}{l}\text { 'Neurodevelop- } \\
\text { mental therapy' }\end{array}$ & 30 & Not stated & Not stated & $\begin{array}{l}\text { Mean }=54.6 \mathrm{y} \\
\mathrm{SD}=9.9 \mathrm{y}\end{array}$ & $\begin{array}{l}\text { Mean }=8.3 \text { days } \\
\mathrm{SD}=5.3 \text { days }\end{array}$ & Not stated & 30 \\
\hline
\end{tabular}




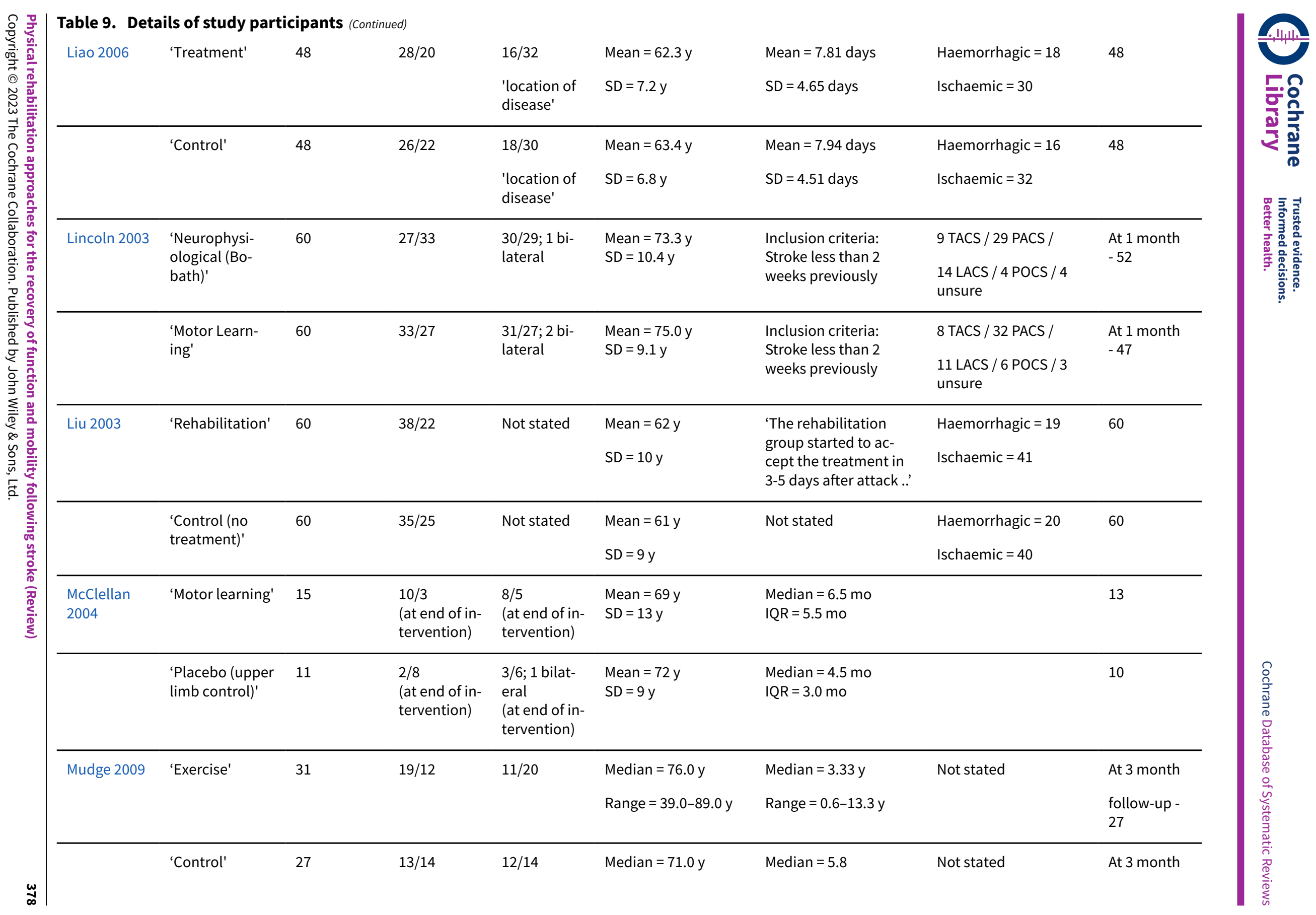




\begin{tabular}{|c|c|c|c|c|c|c|c|c|}
\hline & & & & 1 brainstem & Range $=44.0-86.0 \mathrm{y}$ & Range $=0.5-18.7 \mathrm{y}$ & & follow-up - \\
\hline \multirow[t]{3}{*}{ Mudie 2002} & 'Motor learning' & 10 & $\begin{array}{l}21 / 19 \text { for to- } \\
\text { tal of } 40 \text { re- } \\
\text { cruited }\end{array}$ & $\begin{array}{l}22 / 18 \text { for to- } \\
\text { tal of } 40 \text { re- } \\
\text { cruited }\end{array}$ & $\begin{array}{l}\text { Mean }=72.4 \mathrm{y} \\
\text { SD }=9.01 \mathrm{y} \\
\text { Range } 47 \text { to } 86 \mathrm{y} \\
\text { (for total of } 40 \text { re- } \\
\text { cruits) }\end{array}$ & $\begin{array}{l}\text { Range } 2 \text { to } 6 \text { weeks (for } \\
\text { total of } 40 \text { recruits) }\end{array}$ & $\begin{array}{l}\text { MCA infarct }=22 \\
\text { Haemorrhage }=11 \\
\text { Lacunar infarct }=4 \\
\text { Cerebellar infarct }=3 \\
\text { (for total of } 40 \text { recruits) }\end{array}$ & 10 \\
\hline & $\begin{array}{l}\text { 'Neurophysio- } \\
\text { logical' }\end{array}$ & 10 & $\begin{array}{l}21 / 19 \text { for to- } \\
\text { tal of } 40 \text { re- } \\
\text { cruited }\end{array}$ & $\begin{array}{l}22 / 18 \text { for to- } \\
\text { tal of } 40 \text { re- } \\
\text { cruited }\end{array}$ & $\begin{array}{l}\text { Mean }=72.4 \mathrm{y} \\
\text { SD }=9.01 \mathrm{y} \\
\text { Range }=47 \text { to } 86 \mathrm{y} \\
\text { (for total of } 40 \text { re- } \\
\text { cruits) }\end{array}$ & $\begin{array}{l}\text { Range }=2 \text { to } 6 \text { weeks } \\
\text { (for total of } 40 \text { recruits) }\end{array}$ & $\begin{array}{l}\text { MCA infarct }=22 \\
\text { Haemorrhage }=11 \\
\text { Lacunar infarct }=4 \\
\text { Cerebellar infarct }=3 \\
\text { (for total of } 40 \text { recruits) }\end{array}$ & 9 \\
\hline & $\begin{array}{l}\text { 'Control (no } \\
\text { treatment)' }\end{array}$ & 10 & $\begin{array}{l}21 / 19 \text { for to- } \\
\text { tal of } 40 \text { re- } \\
\text { cruited }\end{array}$ & $\begin{array}{l}22 / 18 \text { for to- } \\
\text { tal of } 40 \text { re- } \\
\text { cruited }\end{array}$ & $\begin{array}{l}\text { Mean }=72.4 \mathrm{y} \\
\text { SD }=9.01 \text { y } \\
\text { Range } 47 \text { to } 86 y \\
\text { (for total of } 40 \text { re- } \\
\text { cruits) }\end{array}$ & $\begin{array}{l}\text { Range } 2 \text { to } 6 \text { weeks (for } \\
\text { total of } 40 \text { recruits) }\end{array}$ & $\begin{array}{l}\text { MCA infarct }=22 \\
\text { Haemorrhage }=11 \\
\text { Lacunar infarct }=4 \\
\text { Cerebellar infarct }=3 \\
\text { (for total of } 40 \text { recruits) }\end{array}$ & 6 \\
\hline \multirow[t]{2}{*}{ Ni 1997} & $\begin{array}{l}\text { 'Comprehen- } \\
\text { sive rehabilita- } \\
\text { tion training' }\end{array}$ & 34 & $26 / 8$ & Not stated & $\begin{array}{l}\text { Mean }=55.56 y \\
S D=17.64 y\end{array}$ & $\begin{array}{l}\text { Mean }=19.21 \text { days } \\
\mathrm{SD}=7.59 \text { days }\end{array}$ & $\begin{array}{l}\text { Ischaemic = } 19 \\
\text { Haemorrhagic }=15\end{array}$ & 34 \\
\hline & $\begin{array}{l}\text { 'Control (no } \\
\text { treatment)' }\end{array}$ & 34 & $23 / 11$ & Not stated & $\begin{array}{l}\text { Mean }=53.25 y \\
S D=13.46 y\end{array}$ & $\begin{array}{l}\text { Mean }=18.31 \text { days } \\
\mathrm{SD}=9.64 \text { days }\end{array}$ & $\begin{array}{l}\text { Ischaemic }=20 \\
\text { Haemorrhagic }=14\end{array}$ & 34 \\
\hline \multirow[t]{2}{*}{ Pan 2004} & 'Rehabilitation' & 48 & $36 / 12$ & $26 / 22$ & $\begin{array}{l}\text { Mean }=64.2 \mathrm{y} \\
S D=11.5 \mathrm{y}\end{array}$ & Not stated & $\begin{array}{l}\text { Ischaemic }=30 \\
\text { Haemorrhagic }=18\end{array}$ & 48 \\
\hline & 'Control' & 48 & $32 / 16$ & $22 / 26$ & $\begin{array}{l}\text { Mean }=62.5 y \\
S D=13.7 y\end{array}$ & Not stated & $\begin{array}{l}\text { Ischaemic }=32 \\
\text { Haemorrhagic }=16\end{array}$ & 48 \\
\hline \multirow[t]{2}{*}{ Pang 2003} & 'Rehabilitation' & 50 & $32 / 18$ & Not stated & $\begin{array}{l}\text { Mean }=61.4 \mathrm{y} \\
\text { Range: } 37-76 \mathrm{y}\end{array}$ & Not stated & $\begin{array}{l}\text { Haemorrhagic }=21 \\
\text { Infarction }=29\end{array}$ & 50 \\
\hline & $\begin{array}{l}\text { 'Control (no } \\
\text { treatment)' }\end{array}$ & 36 & $25 / 11$ & Not stated & $\begin{array}{l}\text { Mean }=60 y \\
\text { Range: } 39-75 y\end{array}$ & Not stated & $\begin{array}{l}\text { Haemorrhagic = } 15 \\
\text { Infarction }=21\end{array}$ & 36 \\
\hline
\end{tabular}




\begin{tabular}{|c|c|c|c|c|c|c|c|c|}
\hline Pang 2006 & 'Treatment' & 41 & Not stated & Not stated & Not stated & Not stated & Not stated & 41 \\
\hline & $\begin{array}{l}\text { 'Control (no } \\
\text { treatment)' }\end{array}$ & 39 & Not stated & Not stated & Not stated & Not stated & Not stated & 37 \\
\hline $\begin{array}{l}\text { Pollock } \\
1998\end{array}$ & $\begin{array}{l}\text { 'Neurophysi- } \\
\text { ological (Bo- } \\
\text { bath)' }\end{array}$ & 19 & $12 / 7$ & $10 / 9$ & $\begin{array}{l}\text { Mean }=68.4 \mathrm{y} \\
S D=13.4 \mathrm{y}\end{array}$ & $\begin{array}{l}\text { Inclusion criteria: "less } \\
\text { than six weeks previ- } \\
\text { ously" }\end{array}$ & $\begin{array}{l}6 \text { TACS / } 3 \text { PACS / } \\
5 \text { LACS / } 2 \text { POCS / } 3 \\
\text { PICH }\end{array}$ & 11 \\
\hline & $\begin{array}{l}\text { 'Mixed (Neuro- } \\
\text { physiological } \\
+ \text { motor learn- } \\
\text { ing)' }\end{array}$ & 9 & $0 / 9$ & $7 / 2$ & $\begin{array}{l}\text { Mean }=73.1 \mathrm{y} \\
S D=10.3 \mathrm{y}\end{array}$ & $\begin{array}{l}\text { Inclusion criteria: "less } \\
\text { than six weeks previ- } \\
\text { ously" }\end{array}$ & $\begin{array}{l}2 \text { TACS / } 3 \text { PACS / } \\
4 \text { LACS / } 0 \text { POCS / } 0 \\
\text { PICH }\end{array}$ & 5 \\
\hline Qian 2004 & 'Treatment' & 23 & $11 / 12$ & Not stated & $\begin{array}{l}\text { Mean }=62.8 \mathrm{y} \\
\mathrm{SD}=14.3 \mathrm{y}\end{array}$ & $\begin{array}{l}\text { Mean }=13.9 \text { days } \\
\mathrm{SD}=8.5 \text { days }\end{array}$ & $\begin{array}{l}\text { Ischaemic }=15 \\
\text { Haemorrhagic }=8\end{array}$ & 23 \\
\hline & $\begin{array}{l}\text { 'Control (no } \\
\text { treatment)' }\end{array}$ & 19 & $9 / 10$ & Not stated & $\begin{array}{l}\text { Mean }=62.8 \mathrm{y} \\
\mathrm{SD}=17.2 \mathrm{y}\end{array}$ & $\begin{array}{l}\text { Mean }=12.1 \text { days } \\
\mathrm{SD}=9.7 \text { days }\end{array}$ & $\begin{array}{l}\text { Ischaemic = } 14 \\
\text { Haemorrhagic }=5\end{array}$ & 19 \\
\hline Qian 2005 & 'Treatment' & 20 & $11 / 9$ & Not stated & $\begin{array}{l}\text { Mean }=63.5 y \\
S D=15.5 y\end{array}$ & $\begin{array}{l}\text { Mean }=13.8 \text { days } \\
\mathrm{SD}=5.8 \text { days }\end{array}$ & $\begin{array}{l}\text { Ischaemic }=12 \\
\text { Haemorrhagic }=8\end{array}$ & 20 \\
\hline & 'Control' & 20 & $12 / 8$ & Not stated & $\begin{array}{l}\text { Mean }=63.7 y \\
S D=16.3 y\end{array}$ & $\begin{array}{l}\text { Mean }=13.5 \text { days } \\
\mathrm{SD}=7.3 \text { days }\end{array}$ & $\begin{array}{l}\text { Ischaemic }=14 \\
\text { Haemorrhagic }=6\end{array}$ & 20 \\
\hline $\begin{array}{l}\text { Richards } \\
1993\end{array}$ & 'Mixed (early)' & 10 & $5 / 5$ & $2 / 8$ & $\begin{array}{l}\text { Mean }=69.6 y \\
S D=7.4 y\end{array}$ & $\begin{array}{l}\text { Mean }=8.3 \text { days } \\
S D=1.4 \text { days }\end{array}$ & $\begin{array}{l}\text { Canadian Stroke Score } \\
\text { (maximum score }=15) \\
\text { Mean }=5.3 \\
\text { SD }=1.4\end{array}$ & 9 \\
\hline & $\begin{array}{l}\text { 'Neurophysio- } \\
\text { logical (early)' }\end{array}$ & 8 & $2 / 6$ & $6 / 2$ & $\begin{array}{l}\text { Mean }=67.3 \mathrm{y} \\
S D=11.2 \mathrm{y}\end{array}$ & $\begin{array}{l}\text { Mean }=8.8 \text { days } \\
\mathrm{SD}=1.5 \text { days }\end{array}$ & $\begin{array}{l}\text { Canadian Stroke Score } \\
\text { (maximum score }=15 \text { ) } \\
\text { Mean }=5.2 \\
\text { SD }=1.7\end{array}$ & 6 \\
\hline
\end{tabular}




\begin{tabular}{|c|c|c|c|c|c|c|c|c|}
\hline & $\begin{array}{l}\text { 'Neurophysio- } \\
\text { logical (conven- } \\
\text { tional)' }\end{array}$ & 9 & $6 / 3$ & $3 / 6$ & $\begin{array}{l}\text { Mean }=70.3 y \\
S D=7.3 y\end{array}$ & $\begin{array}{l}\text { Mean }=13.0 \text { days } \\
\mathrm{SD}=2.8 \text { days }\end{array}$ & $\begin{array}{l}\text { Canadian Stroke Score } \\
(\text { maximum score }=15) \\
\text { Mean }=6.0 \\
\text { SD }=1.8\end{array}$ & 8 \\
\hline $\begin{array}{l}\text { Salbach } \\
2004\end{array}$ & 'Motor learning' & 44 & $26 / 18$ & $27 / 17$ & $\begin{array}{l}\text { Mean }=71 y \\
S D=12 y\end{array}$ & $\begin{array}{l}\text { Mean }=239 \text { days } \\
\mathrm{SD}=83 \text { days }\end{array}$ & $\begin{array}{l}\text { Mild gait deficit }=19 \\
\text { Moderate }=17 \\
\text { Severe }=8\end{array}$ & 41 \\
\hline & $\begin{array}{l}\text { 'Placebo (upper } \\
\text { limb control)' }\end{array}$ & 47 & $30 / 17$ & $\begin{array}{l}24 / 22 ; 1 \text { bi- } \\
\text { lateral }\end{array}$ & $\begin{array}{l}\text { Mean }=73 y \\
S D=8 y\end{array}$ & $\begin{array}{l}\text { Mean }=217 \text { days } \\
S D=73 \text { days }\end{array}$ & $\begin{array}{l}\text { Mild gait deficit }=17 \\
\text { Moderate }=20 \\
\text { Severe }=10\end{array}$ & 43 \\
\hline Shin 2011 & $\begin{array}{l}\text { 'Combined Ex- } \\
\text { ercise' }\end{array}$ & 11 & $5 / 6$ & $8 / 3$ & $\begin{array}{l}\text { Mean }=58.1 \mathrm{y} \\
\mathrm{SD}=4.6 \mathrm{y}\end{array}$ & Not stated & Not stated & 11 \\
\hline & $\begin{array}{l}\text { 'Conventional } \\
\text { Exercise' }\end{array}$ & 10 & $3 / 7$ & $5 / 5$ & $\begin{array}{l}\text { Mean }=57.3 y \\
S D=4.4 y\end{array}$ & Not stated & Not stated & 10 \\
\hline $\begin{array}{l}\text { Stephenson } \\
2004\end{array}$ & $\begin{array}{l}\text { 'Body Weight } \\
\text { Support Tread- } \\
\text { mill Training' }\end{array}$ & 6 & Not stated & Not stated & $\begin{array}{l}\text { Whole group } \\
\text { Mean }=59.8 \mathrm{y} \\
\text { Range: } 42-80 \mathrm{y}\end{array}$ & Not stated & Not stated & 6 \\
\hline & $\begin{array}{l}\text { 'Proprioceptive } \\
\text { Neuromuscular } \\
\text { Facilitation-PNF } \\
\text { training' }\end{array}$ & 6 & Not stated & Not stated & See above & Not stated & Not stated & 6 \\
\hline & $\begin{array}{l}\text { 'Control (no } \\
\text { treatment)' }\end{array}$ & 6 & Not stated & Not stated & See above & Not stated & Not stated & 6 \\
\hline Tang 2009 & 'Observation' & 35 & $11 / 9$ & Not stated & $\begin{array}{l}\text { Whole group mean = } \\
61.98 \text { y Range: } \\
44-75 \text { y }\end{array}$ & Not stated & Not stated & 35 \\
\hline & 'Control' & 35 & $12 / 8$ & Not stated & See above & Not stated & Not stated & 35 \\
\hline
\end{tabular}




\begin{tabular}{|c|c|c|c|c|c|c|c|c|}
\hline Thaut 2007 & $\begin{array}{l}\text { 'Rhythmic au- } \\
\text { ditory stimula- } \\
\text { tion' }\end{array}$ & 43 & $22 / 21$ & $20 / 23$ & $\begin{array}{l}\text { Mean = 69.2 y } \\
S D=11 y\end{array}$ & $\begin{array}{l}\text { Mean }=21.3 \text { days } \\
S D=11 \text { days }\end{array}$ & $\begin{array}{l}\text { Location of stroke: } \\
\text { MCA = } 35 \\
\text { Internal capsule = } 4 \\
\text { Basal ganglia/thala- } \\
\text { mus = } 3 \\
\text { Subdural haematoma } \\
=1\end{array}$ & 43 \\
\hline & $\begin{array}{l}\text { 'Neurodevelop- } \\
\text { mental therapy } \\
\text { (NDT)/Bobath- } \\
\text { based training' }\end{array}$ & 35 & $19 / 16$ & $16 / 19$ & $\begin{array}{l}\text { Mean = 69.7 y } \\
S D=11 y\end{array}$ & $\begin{array}{l}\text { Mean }=22.2 \text { days } \\
\mathrm{SD}=12 \text { days }\end{array}$ & $\begin{array}{l}\text { Location of stroke: } \\
\text { MCA = } 30 \\
\text { Internal capsule = } 4 \\
\text { Basal ganglia/thala- } \\
\text { mus = } 1\end{array}$ & 35 \\
\hline $\begin{array}{l}\text { Tor- } \\
\text { res-Arreola } \\
2009\end{array}$ & 'Strategy 1' & 59 & $16 / 43$ & Not stated & $\begin{array}{l}\text { Mean }=69.4 \mathrm{y} \\
\mathrm{SD}=12 \mathrm{y}\end{array}$ & $\begin{array}{l}\text { Mean }=7.1 \text { days } \\
\mathrm{SD}=5.9 \text { days }\end{array}$ & Not stated & $\begin{array}{l}\text { At } 6 \text { month } \\
\text { follow-up = } \\
32\end{array}$ \\
\hline & 'Strategy 2' & 51 & $21 / 30$ & Not stated & $\begin{array}{l}\text { Mean }=69.8 \mathrm{y} \\
\mathrm{SD}=8.8 \mathrm{y}\end{array}$ & $\begin{array}{l}\text { Mean }=6.3 \text { days } \\
\mathrm{SD}=3.1 \text { days }\end{array}$ & Not stated & $\begin{array}{l}\text { At } 6 \text { month } \\
\text { follow-up = } \\
35\end{array}$ \\
\hline $\begin{array}{l}\text { Verheyden } \\
2006\end{array}$ & 'Experimental' & 17 & $11 / 6$ & $9 / 8$ & $\begin{array}{l}\text { Mean }=55 \mathrm{y} \\
\mathrm{SD}=11 \mathrm{y}\end{array}$ & $\begin{array}{l}\text { Mean }=53 \text { days } \\
\text { SD }=24 \text { days }\end{array}$ & $\begin{array}{l}\text { Haemorrhagic = } 2 \\
\text { Ischaemic }=15\end{array}$ & 17 \\
\hline & 'Control' & 16 & $9 / 7$ & $7 / 9$ & $\begin{array}{l}\text { Mean }=62 y \\
S D=14 y\end{array}$ & $\begin{array}{l}\text { Mean }=49 \text { days } \\
\mathrm{SD}=28 \text { days }\end{array}$ & $\begin{array}{l}\text { Haemorrhagic = } 3 \\
\text { Ischaemic }=13\end{array}$ & 16 \\
\hline Verma 2011 & 'Experimental' & 15 & $10 / 5$ & $8 / 7$ & $\begin{array}{l}\text { Mean }=53.27 \mathrm{y} \\
\mathrm{SD}=8.53 \mathrm{y}\end{array}$ & $\begin{array}{l}\text { Mean }=6.07 \text { weeks } \\
\text { SD }=3.30 \text { weeks }\end{array}$ & $\begin{array}{l}\text { Haemorrhagic = } 4 \\
\text { Ischaemic }=11\end{array}$ & 15 \\
\hline & 'Control' & 15 & $12 / 3$ & $7 / 8$ & $\begin{array}{l}\text { Mean }=55.07 y \\
S D=6.80 y\end{array}$ & $\begin{array}{l}\text { Mean }=6.60 \text { weeks } \\
\text { SD }=3.20 \text { weeks }\end{array}$ & $\begin{array}{l}\text { Haemorrhagic = } 3 \\
\text { Ischaemic }=12\end{array}$ & 15 \\
\hline
\end{tabular}




\begin{tabular}{|c|c|c|c|c|c|c|c|c|}
\hline Wade 1992 & 'Mixed' & 49 & $27 / 22$ & $\begin{array}{l}25 / 19 \\
5 \text { brainstem }\end{array}$ & $\begin{array}{l}\text { Mean }=72.3 \mathrm{y} \\
S D=9.7 \mathrm{y}\end{array}$ & $\begin{array}{l}\text { Mean }=53.1 \mathrm{mo} \\
\mathrm{SD}=29.5 \mathrm{mo}\end{array}$ & & 48 \\
\hline & $\begin{array}{l}\text { 'Control (no } \\
\text { treatment)' }\end{array}$ & 45 & $20 / 25$ & $\begin{array}{l}21 / 21 \\
3 \text { brainstem }\end{array}$ & $\begin{array}{l}\text { Mean }=72.0 y \\
S D=10.6 y\end{array}$ & $\begin{array}{l}\text { Mean }=59.6 \mathrm{mo} \\
\mathrm{SD}=35.3 \mathrm{mo}\end{array}$ & & 41 \\
\hline Wang 2004a & 'Rehabilitation' & 70 & $36 / 30$ & Not stated & $\begin{array}{l}\text { Mean }=63.1 \mathrm{y} \\
S D=9.8 \mathrm{y}\end{array}$ & Not stated & Not stated & 66 \\
\hline & $\begin{array}{l}\text { 'Control (no } \\
\text { treatment)' }\end{array}$ & 35 & $18 / 14$ & Not stated & $\begin{array}{l}\text { Mean }=65.2 \mathrm{y} \\
\mathrm{SD}=11.3 \mathrm{y}\end{array}$ & Not stated & Not stated & 32 \\
\hline Wang 2004b & 'Treatment' & 25 & $16 / 9$ & Not stated & $\begin{array}{l}\text { Mean }=62.1 \mathrm{y} \\
S D=10.2 \mathrm{y}\end{array}$ & $\begin{array}{l}\text { Mean }=54.2 \text { days } \\
S D=37.5 \text { days }\end{array}$ & $\begin{array}{l}\text { Haemorrhagic = } 11 \\
\text { Ischaemic }=14\end{array}$ & 25 \\
\hline & $\begin{array}{l}\text { 'Control (no } \\
\text { treatment)' }\end{array}$ & 25 & $15 / 10$ & Not stated & $\begin{array}{l}\text { Mean }=59.5 y \\
S D=11.4 y\end{array}$ & $\begin{array}{l}\text { Mean }=55.7 \text { days } \\
S D=35.3 \text { days }\end{array}$ & $\begin{array}{l}\text { Haemorrhagic }=9 \\
\text { Ischaemic }=16\end{array}$ & 25 \\
\hline Wang 2005 & $\begin{array}{l}\text { 'Neurophysio- } \\
\text { logical' }\end{array}$ & 21 & $14 / 7$ & $11 / 10$ & $\begin{array}{l}\text { Patients with spas- } \\
\text { ticity } \\
\text { Mean }=53.9 \mathrm{y} \\
\mathrm{SD}=11.8 \mathrm{y} \\
\text { Patients with relative } \\
\text { recovery } \\
\text { Mean }=62.4 \mathrm{y} \\
\mathrm{SD}=11.6 \mathrm{y}\end{array}$ & $\begin{array}{l}\text { Patients with spastici- } \\
\text { ty } \\
\text { Mean }=21.9 \text { days } S D= \\
7.4 \text { days } \\
\text { Patients with relative } \\
\text { recovery } \\
\text { Mean } 21.6 \text { days } \\
S D=9.3 \text { days }\end{array}$ & $\begin{array}{l}\text { Haemorrhagic }=7 \\
\text { Ischaemic }=14\end{array}$ & 21 \\
\hline & 'Orthopaedic' & 23 & $14 / 9$ & $9 / 14$ & $\begin{array}{l}\text { Patients with spas- } \\
\text { ticity } \\
\text { Mean }=59.3 \mathrm{y} \\
\mathrm{SD}=12.2 \mathrm{y} \\
\text { Patients with relative } \\
\text { recovery } \\
\text { Mean }=63.8 \mathrm{y} \\
\mathrm{SD}=13.1 \mathrm{y}\end{array}$ & $\begin{array}{l}\text { Patients with spastici- } \\
\text { ty } \\
\text { Mean }=20.7 \text { days SD = } \\
5.9 \text { days } \\
\text { Patients with relative } \\
\text { recovery } \\
\text { Mean }=19.6 \text { days SD = } \\
7.9 \text { days }\end{array}$ & $\begin{array}{l}\text { Haemorrhagic }=7 \\
\text { Ischaemic }=14\end{array}$ & 23 \\
\hline Wang 2006 & 'Rehabilitation' & 40 & $25 / 15$ & Not stated & $\begin{array}{l}\text { Mean }=61.38 y \\
S D=9.99 y\end{array}$ & $\begin{array}{l}\text { Mean }=9.05 \text { days } \\
S D=5.74 \text { days }\end{array}$ & $\begin{array}{l}\text { Ischaemic }=28 \\
\text { Haemorrhagic }=12\end{array}$ & 40 \\
\hline
\end{tabular}




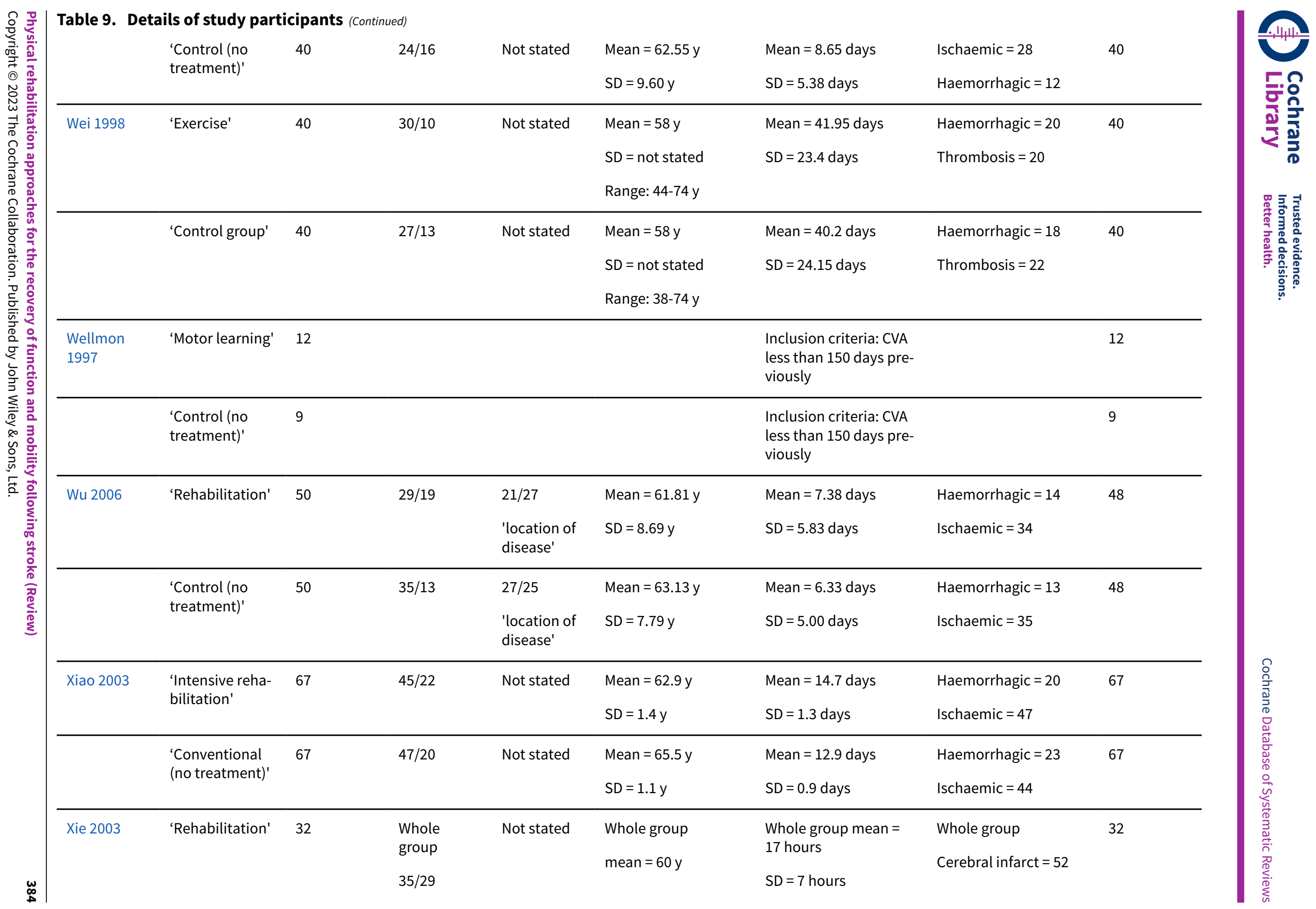




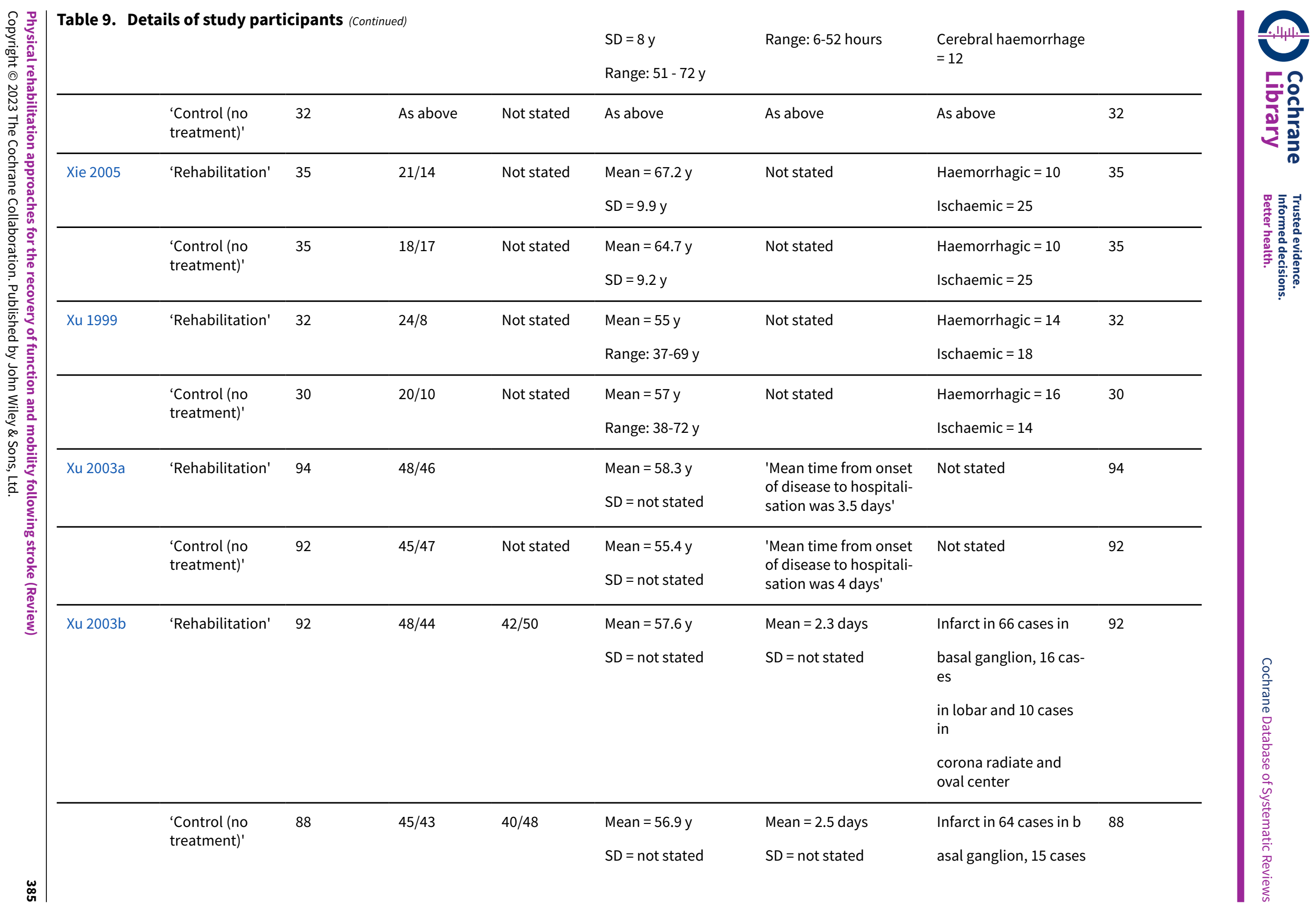




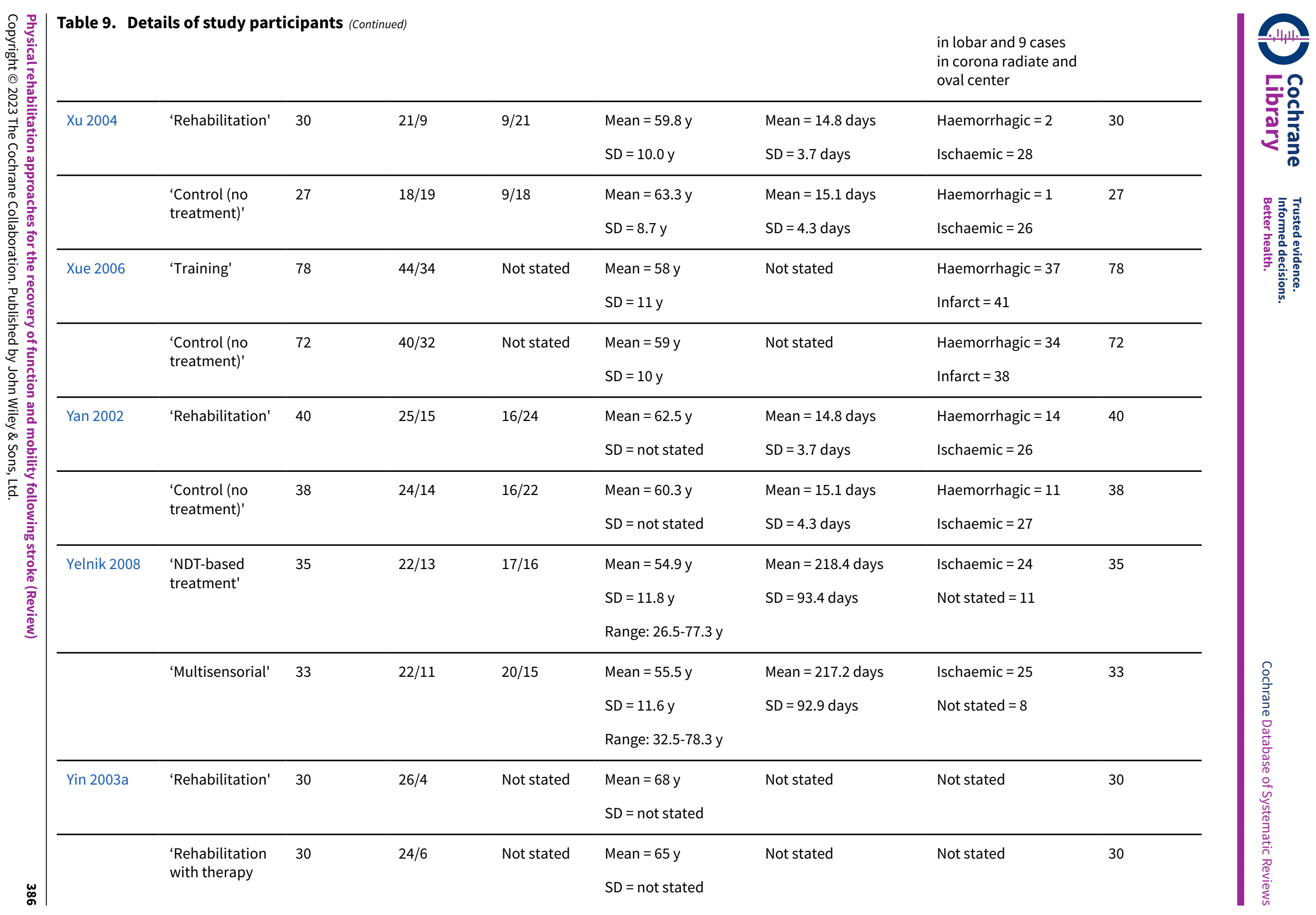




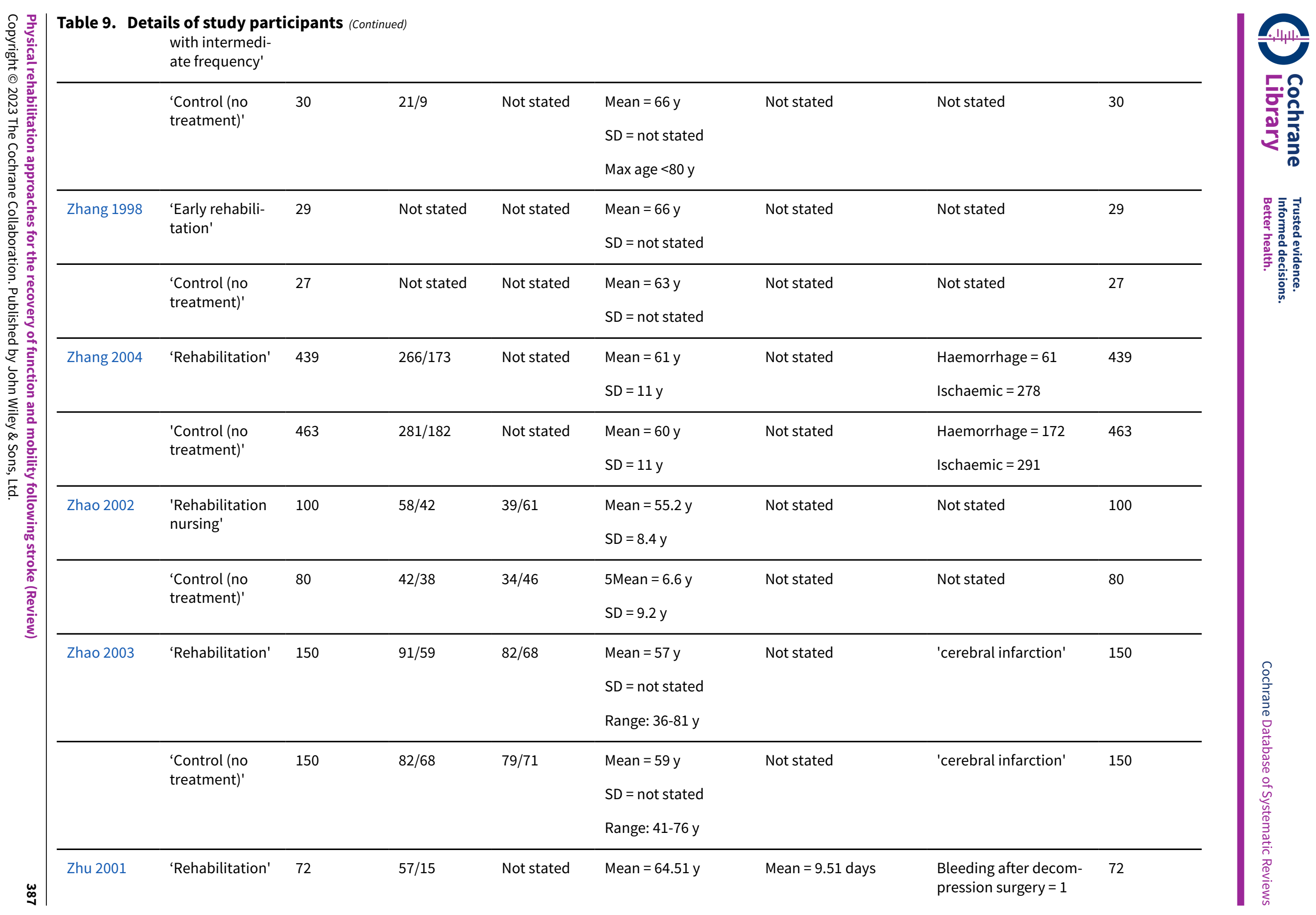




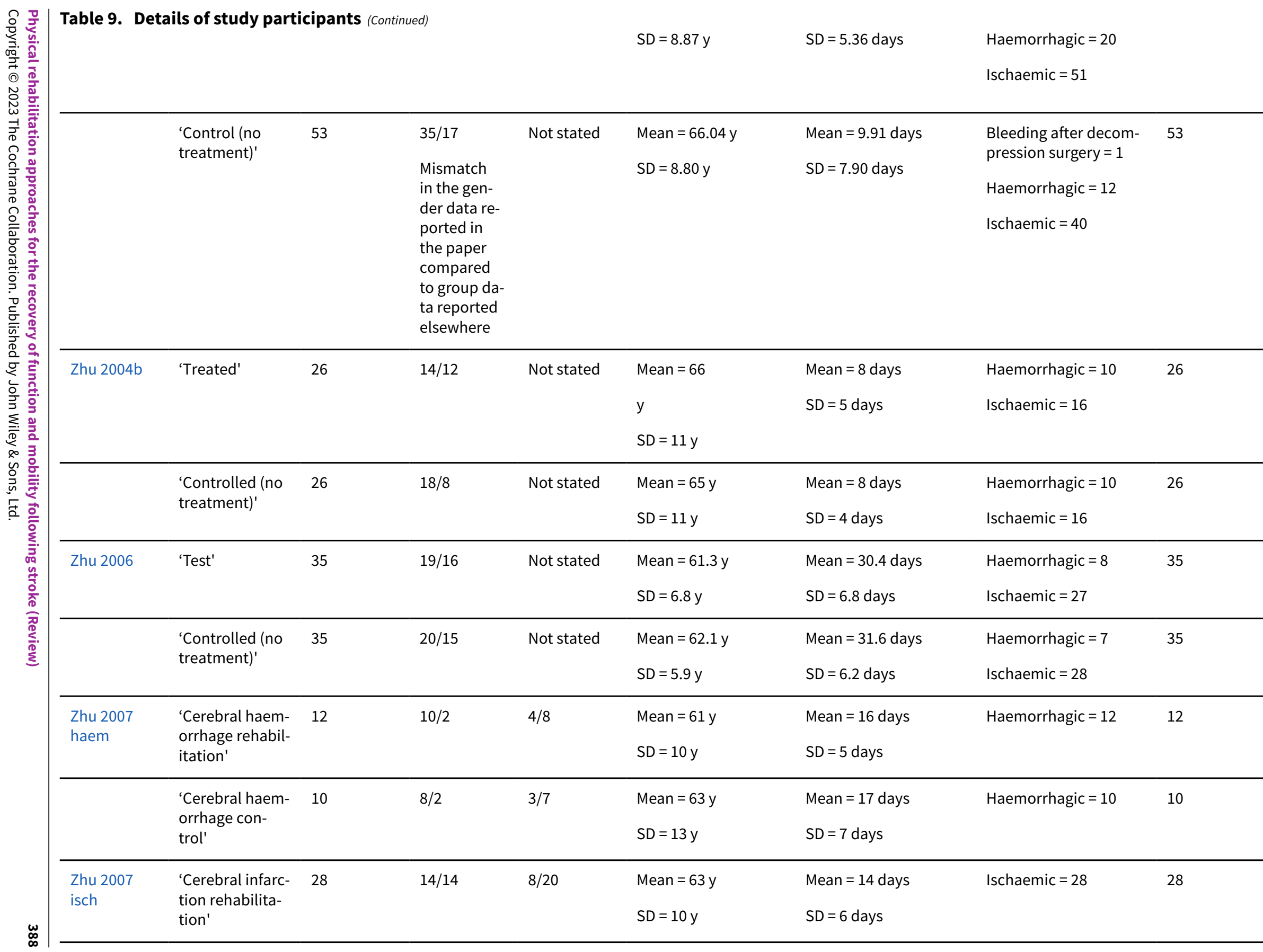




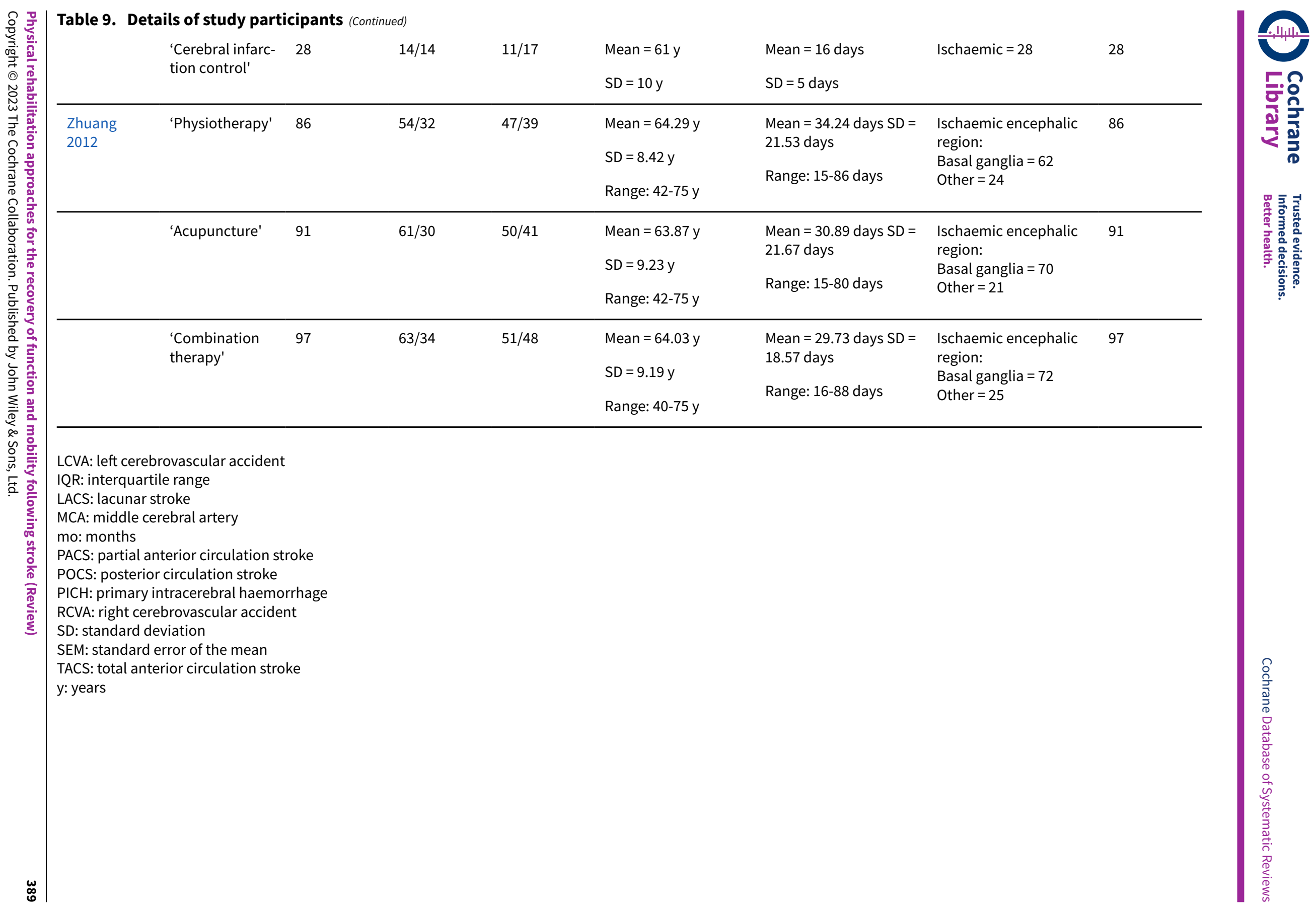


Table 10. Length and dose of intervention for those studies with Independence in ADL or Motor Function data in comparisons with no treatment.

\begin{tabular}{|c|c|c|c|}
\hline Study & $\begin{array}{l}\text { Length of intervention pe- } \\
\text { riod }\end{array}$ & Frequency of sessions & Length of sessions \\
\hline Chen 2004 & Not stated & Not stated & Not stated \\
\hline Chen 2006 & 3 months & 2/week & Not stated \\
\hline Chu 2003 & $\begin{array}{l}20 \text { days }-14 \text { months } \\
\text { (mean } 41.3 \text { days) }\end{array}$ & Daily & 40-60 minutes \\
\hline Deng 2011 & 6 weeks & 2/week & 60 minutes \\
\hline Fang 2003 & 3 months & 2/week & Not stated \\
\hline Fang 2004 old & 3 days & Daily & 45 minutes \\
\hline Fang 2004 young & 3 days & Daily & 45 minutes \\
\hline Green 2002 & Maximum 13 weeks & Minimum 3 contacts; & $\begin{array}{l}\text { Median number of treatments per patient } \\
\text { was three (IQR 2-7, range } 0-22 \text { ) and the mean } \\
\text { duration of every treatment was } 44 \text { min } \\
\text { (SD } 21 \text {, range } 10-90) \text {. }\end{array}$ \\
\hline Hou 2006 & 6 months & $\begin{array}{l}1-2 \text { times/day, 5/week; } \\
\text { increasing to } 2 / \text { day, 5-6/ } \\
\text { week }\end{array}$ & $30-40$ minutes \\
\hline Hu 2007 haem & Not stated & Not stated & Not stated \\
\hline Hu 2007 isch & Not stated & Not stated & Not stated \\
\hline Huang 2003 & 30 days & Daily & 45 minutes \\
\hline Li 1999 & 1 month & $2 /$ day & 30 minutes \\
\hline Liu 2003 & 15 days & 4/day & 30 minutes \\
\hline Ni 1997 & Average of 2 months & $2 /$ day & $30-45$ minutes \\
\hline Pan 2004 & Not stated & 3-4/day & 30 minutes \\
\hline Pang 2006 & 10 sessions & 5/week & 30 minutes \\
\hline Wade 1992 & $\begin{array}{l}\text { Mean visits }=4 \text { (range } 1-11) \\
73 \% \text { patients were seen one } \\
\text { to six times. }\end{array}$ & Not stated & $\begin{array}{l}\text { Ranged from } 1 \text { hour } 10 \text { minutes to } \\
3 \text { hours } 10 \text { minutes ( } m e a n=2 \text { hours } 4 \text { minutes) }\end{array}$ \\
\hline Wang 2004a & 30 days & 1-2/day & 45 minutes \\
\hline Wu 2006 & 6 months & Daily & Not stated \\
\hline
\end{tabular}


Table 10. Length and dose of intervention for those studies with Independence in ADL or Motor Function data in comparisons with no treatment. (Continued)

\begin{tabular}{|c|c|c|c|}
\hline Xu 1999 & 1 month & 2/day & 60 minutes \\
\hline Xie 2003 & Not stated & Massage 5-6/day; ADL 2/day & Massage $15-20$ minutes; ADL 30 minutes \\
\hline Xu 2003a & 21 days & Daily & Not stated \\
\hline Xu 2003b & 4 weeks & Daily & 60 minutes \\
\hline Xu 2004 & 1 month & 5/week & $40-50$ minutes \\
\hline Xue 2006 & 1 month & 3/day & 30 minutes \\
\hline Yan 2002 & 38 days & $\begin{array}{l}\text { Dependent on phase of re- } \\
\text { covery: } \\
\text { Early phase: } 2 / \text { day; } \\
\text { Rehabilitative treatment } \\
\text { (on bed): } 2 / \text { day, } \\
\text { increasing to } 3-4 \text { /day if par- } \\
\text { ticipants had no discomfort; } \\
\text { Rehabilitative treatment } \\
\text { (after leaving bed): } 2 \text { /day }\end{array}$ & $\begin{array}{l}\text { Dependent on phase of recovery: } \\
\text { Early phase: } 15 \mathrm{~min} / \text { session; } \\
\text { Rehabilitative treatment (on bed): } 30 \mathrm{~min} / \mathrm{ses} \text { - } \\
\text { sion; } \\
\text { Rehabilitative treatment (after leaving bed): } 60 \\
\text { minutes }\end{array}$ \\
\hline Yin 2003a & Not stated & Daily & 40 minutes \\
\hline Zhang 1998 & Not stated & Daily & 60 minutes \\
\hline Zhang 2004 & 6 months & Not stated & Not stated \\
\hline Zhao 2002 & $\begin{array}{l}\text { Mean } 31.6 \text { days (SD } 11.2 \\
\text { days) }\end{array}$ & 5/week & $30-45$ minutes \\
\hline Zhao 2003 & $\begin{array}{l}\text { РT and ОT: ' } 10 \text { days as a } \\
\text { treatment course, } \\
\text { persisting } 2 \text { courses' }\end{array}$ & Daily & $30-40$ minutes \\
\hline Zhu 2001 & Not stated & 5/week & 45 minutes (plus 20 minutes electrotherapy) \\
\hline Zhu 2006 & Not stated & 5/week & 60 minutes \\
\hline Zhu 2007 haem & Not stated & 5/week & 45 minutes \\
\hline Zhu 2007 isch & Not stated & 5/week & 45 minutes \\
\hline
\end{tabular}

OT: occupational therapy; PT = physical therapy

Table 11. Length and dose of intervention for those studies with Independence in ADL or Motor Function data in comparisons with usual care or attention control

\begin{tabular}{|c|c|c|c|}
\hline Study & Length of intervention period & $\begin{array}{l}\text { Frequency of ses- } \\
\text { sions }\end{array}$ & Length of sessions \\
\hline
\end{tabular}


Table 11. Length and dose of intervention for those studies with Independence in ADL or Motor Function data in comparisons with usual care or attention control (Continued)

\begin{tabular}{|c|c|c|c|}
\hline Chen 2010 & 4 weeks & Not stated & Not stated \\
\hline Cooke 2006 & 6 weeks & 4/week & 60 minutes \\
\hline Duncan 1998 & $\begin{array}{l}8 \text { weeks (then } 4 \text { weeks without ther- } \\
\text { apist) }\end{array}$ & 3/week & 90 minutes \\
\hline Duncan 2003 & 12-14 weeks & 36 sessions total & 90 minutes \\
\hline Kwakkel 2008 & 12 weeks & 2/week & 90 minutes \\
\hline Langhammer 2007 & Four 3 month sessions & 2-3/week & Minimum 20 hours total \\
\hline McClellan 2004 & 6 weeks & 2/week & Not stated \\
\hline Mudge 2009 & 4 weeks & 3/week & Not stated \\
\hline Mudie 2002 & 6 weeks & 5/week & 30 minutes \\
\hline Pollock 1998 & 4 weeks & 5/week & 60 minutes \\
\hline Qian 2005 & Not stated & Daily & 60 minutes \\
\hline Richards 1993 & Whilst in-patient & Not stated & Not stated \\
\hline Tang 2009 & 8 weeks & Daily & 45 minutes \\
\hline Wang 2004b & 4 weeks & 5/week & 30-45 minutes \\
\hline Wei 1998 & 12 weeks & 5/week & 45-60 minutes \\
\hline
\end{tabular}

Table 12. Summary of analyses performed

\begin{tabular}{|c|c|c|c|c|c|c|}
\hline \multirow{2}{*}{$\begin{array}{l}\text { Comparison } \\
\text { Outcome }\end{array}$} & \multicolumn{2}{|c|}{ Intervention vs no treatment } & \multicolumn{2}{|c|}{ Intervention vs usual care or control } & \multicolumn{2}{|c|}{ One active intervention vs another } \\
\hline & Immediate & Persisting & Immediate & Persisting & Immediate & Persisting \\
\hline $\begin{array}{l}\text { Independence in } \\
A D L\end{array}$ & Analysis 1.1 & Analysis 4.1 & Analysis 2.1 & Analysis 5.1 & Analysis 3.1 & Analysis 6.1 \\
\hline Motor Function & Analysis 1.2 & Analysis 4.2 & Analysis 2.2 & Analysis 5.2 & Analysis 3.2 & Analysis 6.2 \\
\hline Balance & Analysis 1.3 & Analysis 4.3 & Analysis 2.3 & Analysis 5.3 & Analysis 3.3 & Analysis 6.3 \\
\hline Gait velocity & Analysis 1.4 & Analysis 4.4 & Analysis 2.4 & Analysis 5.4 & Analysis 3.4 & Analysis 6.4 \\
\hline Length of stay & Analysis 1.5 & & Analysis 2.5 & & Analysis 3.5 & \\
\hline
\end{tabular}

Table 13. Summary of sub-group analyses performed

Comparison / Outcome Intervention vs no treatment Intervention vs usual care or control


Table 13. Summary of sub-group analyses performed (Continued)

\begin{tabular}{lllll} 
SUBGROUP & Independence in ADL & Motor Function & $\begin{array}{l}\text { Independence in } \\
\text { ADL }\end{array}$ & Motor Function \\
\hline Time after stroke & Analysis 7.1 & Analysis 9.1 & Analysis 8.1 & Analysis 10.1 \\
\hline Study geographical location & Analysis 7.2 & Analysis 9.2 & Analysis 8.2 & Analysis 10.2 \\
\hline Dose of intervention & Analysis 7.3 & Analysis 9.3 & Analysis 8.3 & Analysis 10.3 \\
\hline Provider of intervention & Analysis 7.4 & Analysis 9.4 & Analysis 8.4 & Analysis 10.4 \\
\hline Treatment components included & Analysis 7.5 & Analysis 9.5 & Analysis 8.5 & Analysis 10.5 \\
\hline
\end{tabular}

\begin{tabular}{lll}
\hline Comparison / Outcome & One active intervention vs another \\
\hline SUBGROUP & Independence in ADL & Motor Function \\
\hline Functional task training components & Analysis 11.1 & Analysis 12.1 \\
\hline Neurophysiological components & Analysis 11.2 & Analysis 12.2 \\
\hline Musculoskeletal components & Analysis 11.3 & Analysis 12.3 \\
\hline
\end{tabular}

\section{APPENDICES}

\section{Appendix 1. MEDLINE search strategy}

MEDLINE 2005 to 5 December $2012 \mathrm{~N}=2351$

1. cerebrovascular disorders/ or exp basal ganglia cerebrovascular disease/ or exp brain ischemia/ or exp carotid artery diseases/ or exp intracranial arterial diseases/ or exp "intracranial embolism and thrombosis"/ or exp intracranial hemorrhages/ or stroke/ or exp brain infarction/ or stroke, lacunar/ or vasospasm, intracranial/ or vertebral artery dissection/

2. (stroke or poststroke or post-stroke or cerebrovasc\$ or brain vasc\$ or cerebral vasc\$ or cva $\$$ or apoplex\$ or SAH).tw.

3. ((brain\$ or cerebr\$ or cerebell\$ or intracran\$ or intracerebral) adj5 (isch?emi\$ or infarct\$ or thrombo $\$$ or emboli\$ or occlus\$)).tw.

4. ((brain\$ or cerebr\$ or cerebell\$ or intracerebral or intracranial or subarachnoid) adj5 (haemorrhage\$ or hemorrhage\$ or haematoma\$ or hematoma\$ or bleed\$)).tw.

5. hemiplegia/ or exp paresis/

6. (hemipleg\$ or hemipar\$ or paresis or paretic).tw.

7. exp Gait Disorders, Neurologic/

8. 1 or 2 or 3 or 4 or 5 or 6 or 7

9. physical therapy modalities/ or exp exercise movement techniques/ or exp exercise therapy/ or rehabilitation/ or occupational therapy/

10. feedback/ or feedback, psychological/ or biofeedback, psychology/ or neurofeedback/ or feedback, sensory/

11. exercise/ or orthopedic procedures/

12. (physiotherapy or physical therapy or exercise therapy or rehabilitation).tw. 
13. (neurorehabilitation or feedback or biofeedback).tw.

14. (motor adj5 (train\$ or re?train\$ or learn\$ or re?learn\$)).tw.

15. neuromuscular facilitation.tw.

16. (movement adj5 (therap\$ or science)).tw.

17. ((neurodevelopmental or neurophysiological or orthop?edic) adj5 (therap\$ or treatment\$ or rehabilitation or principle\$ or approach \$or component\$ or concept\$)).tw.

18. (Bobath or Carr or Brunnstrom or Rood or Johnstone or NDT).tw.

19.9 or 10 or 11 or 12 or 13 or 14 or 15 or 16 or 17 or 18

\section{8 and 19}

21. cerebrovascular disorders/rh or exp basal ganglia cerebrovascular disease/rh or exp brain ischemia/rh or exp carotid artery diseases/ rh or exp intracranial arterial diseases/rh or exp "intracranial embolism and thrombosis"/rh or exp intracranial hemorrhages/rh or stroke/ rh or exp brain infarction/rh or vasospasm, intracranial/rh or vertebral artery dissection/rh

22. hemiplegia/rh or exp paresis/rh

23. 20 or 21 or 22

24. psychomotor performance/ or motor skills/ or "task performance and analysis"/

25. learning/ or "conditioning (psychology)"/ or problem-based learning/ or problem solving/

26. movement/ or locomotion/ or walking/ or dependent ambulation/ or gait/

27. motor activity/ or range of motion, articular/

28. postural balance/ or exp posture/ or supination/ or pronation/ or weight-bearing/

29. exp lower extremity/ or exp back/

30. (motor adj5 (skill\$ or activit\$ or function\$)).tw.

31. (learning or conditioning).tw.

32. (movement or gait or locomotion or walking or walk or mobility).tw.

33. (equilibrium or balance or postur\$ or supination or pronation).tw.

34. (body sway or stance or strength or weight?bearing or body weight support).tw.

35. (locomotor adj5 (recovery or training)).tw.

36. (weight adj5 (distribut\$ or transfer\$)).tw.

37. (sit or sitting or stand or standing or step or stepping or climb or climbing).tw.

38. (lower limb\$ or lower extremit\$ or ankle or leg or heel or calf or knee or hip or thigh or foot or trunk).tw.

39. or $/ 24-38$

40. 23 and 39

41. exp animals/ not humans.sh.

42. 40 not 41

43. Randomized Controlled Trials as Topic/

44. random allocation/

45. Controlled Clinical Trials as Topic/ 
46. control groups/

47. clinical trials as topic/

48. double-blind method/

49. single-blind method/

50. Placebos/

51. placebo effect/

52. cross-over studies/

53. Research Design/

54. randomized controlled trial.pt.

55. controlled clinical trial.pt.

56. clinical trial.pt.

57. (random\$ or RCT or RCTs).tw.

58. (controlled adj5 (trial\$ or stud\$)).tw.

59. (clinical\$ adj5 trial\$).tw.

60. ((control or treatment or experiment\$ or intervention) adj5 (group\$ or subject\$ or patient\$)).tw.

61. (quasi-random\$ or quasi random\$ or pseudo-random\$ or pseudo random\$).tw.

62. ((control or experiment\$ or conservative) adj5 (treatment or therapy or procedure or manage\$)).tw.

63. ((singl\$ or doubl\$ or tripl\$ or trebl\$) adj5 (blind\$ or mask\$)).tw.

64. (cross-over or cross over or crossover).tw.

65. placebo\$.tw.

66. sham.tw.

67. (assign\$ or allocat\$).tw.

68. controls.tw.

69. trial.ti.

70. or/43-69

71.42 and 70

72 . (2005\$ or $2006 \$$ or $2007 \$$ or $2008 \$$ or $2009 \$$ or $2010 \$$ or $2011 \$$ or $2012 \$)$.ed.

73. 71 and 72

MEDLINE search strategy (Original 2007 review)

1 exp cerebrovascular disorders/

2 stroke\$.tw.

3 cerebrovascular\$.tw.

4 (cerebral or cerebellar or brainstem or vertebrobasilar).tw.

5 (infarct\$ or isch?emi\$ or thrombo\$ or emboli\$).tw.

64 and 5

7 (cerebral or brain or subarachnoid).tw.

8 (haemorrhage or hemorrhage or haematoma or hematoma or bleeding).tw.

97 and 8

10 exp hemiplegia/ or "hemiplegi\$".mp.

Physical rehabilitation approaches for the recovery of function and mobility following stroke (Review) 
111 or 2 or 3 or 6 or 9 or 10

12 physical therapy/

13 exercise therapy/

14 rehabilitation/

15 occupational therapy/

16 exercise/

17 electric stimulation therapy/

18 "biofeedback (psychology)"/

19 feedback/

2012 or 13 or 14 or 15 or 16 or 17 or 18 or 19

21 (physiotherapy or physical therapy or exercise therapy or rehabilitation).tw.

22 (neurorehabilitation or feedback or biofeedback).tw.

23 (motor adj5 (train\$ or re?train\$ or learn\$ or re?learn\$)).tw.

24 neuromuscular facilitation.tw.

25 (movement adj5 (therap\$ or science)).tw.

26 (neurodevelopmental or neurophysiologic $\$$ or orthop?edic).tw.

27 (therap\$ or treatment\$ or rehabilitation or principle\$ or approach\$).tw.

2826 and 27

29 (bobath or carr or brunnstrom or rood or johnstone).tw.

3021 or 22 or 23 or 24 or 25 or 28 or 29

3120 or 30

3211 and 31

33 exp cerebrovascular disorders/rh

34 hemiplegia/rh

3532 or 33 or 34

36 motor skills/

37 exp psychomotor performance/

38 motor activity/

39 learning/

40 "conditioning (psychology)"/

41 movement/

42 locomotion/ or walking/

43 gait/

44 range of motion, articular/

45 activities of daily living/

46 exp posture/

47 equilibrium/

48 exp leg/

49 exp back/

50 weight-bearing/

5136 or 37 or 38 or 39 or 40 or 41 or 42 or 43 or 44 or 45 or 46 or 47 or 48 or 49 or 50

52 (motor adj5 (skill\$ or activit\$ or function\$)).tw.

53 (learning or conditioning).tw.

54 (movement or gait or locomotion or walk\$).tw.

55 (equilibrium or balance or postur\$).tw.

56 (body sway or stance or strength or weight? bearing or body weight support).tw.

57 (locomotor adj5 (recovery or training)).tw.

58 (ankle or leg or heel or calf or knee or hip or foot or trunk).tw.

59 lower limb.tw.

60 (weight adj5 (distribut\$ or transfer\$)).tw.

61 (sit or sitting or stand or standing or step or stepping or climb or climbing).tw.

6252 or 53 or 54 or 55 or 56 or 57 or 58 or 59 or 60 or 61

6351 or 62

6463 and 35

\section{Appendix 2. EMBASE search strategy}

EMBASE 2005 to 5 December $2012 \mathrm{~N}=4240$

1. cerebrovascular disease/ or basal ganglion hemorrhage/ or cerebral artery disease/ or cerebrovascular accident/ or stroke/ or exp carotid artery disease/ or exp brain hematoma/ or exp brain hemorrhage/ or exp brain ischemia/ or exp intracranial aneurysm/ or exp occlusive cerebrovascular disease/ 
2. stroke patient/ or stroke unit/

3. (stroke or poststroke or post-stroke or cerebrovasc\$ or brain vasc\$ or cerebral vasc\$ or cva\$ or apoplex\$ or SAH).tw.

4. ((brain\$ or cerebr\$ or cerebell\$ or intracran\$ or intracerebral) adj5 (isch?emi\$ or infarct\$ or thrombo $\$$ or emboli\$ or occlus\$)).tw.

5. ((brain\$ or cerebr\$ or cerebell\$ or intracerebral or intracranial or subarachnoid) adj5 (haemorrhage\$ or hemorrhage\$ or haematoma\$ or hematoma\$ or bleed\$)).tw.

6. hemiparesis/ or hemiplegia/ or paresis/

7. (hemipleg\$ or hemipar\$ or paresis or paretic).tw.

8. 1 or 2 or 3 or 4 or 5 or 6 or 7

9. physiotherapy/ or exp exercise/ or exp kinesiotherapy/ or rehabilitation/ or occupational therapy/ or exp feedback system/ or joint mobilization/

10. (physiotherapy or physical therapy or exercise therapy or rehabilitation).tw.

11. (neurorehabilitation or feedback or biofeedback).tw.

12. (motor adj5 (train\$ or re?train\$ or learn\$ or re?learn\$)).tw.

13. neuromuscular facilitation.tw.

14. (movement adj5 (therap\$ or science)).tw.

15. ((neurodevelopmental or neurophysiological or orthop?edic) adj5 (therap\$ or treatment\$ or rehabilitation or principle\$ or approach \$ or component\$ or concept\$)).tw.

16. (Bobath or Carr or Brunnstrom or Rood or Johnstone or NDT).tw.

\section{9 or 10 or 11 or 12 or 13 or 14 or 15 or 16}

\section{8 and 17}

19. cerebrovascular disease/rh or basal ganglion hemorrhage/rh or cerebral artery disease/rh or cerebrovascular accident/rh or stroke/ rh or exp carotid artery disease/rh or exp brain hematoma/rh or exp brain hemorrhage/rh or exp brain ischemia/rh or exp intracranial aneurysm/rh or exp occlusive cerebrovascular disease/rh

20. hemiparesis/rh or hemiplegia/rh or paresis/rh

\section{18 or 19 or 20}

22. physical performance/ or motor performance/ or object manipulation/

23. psychomotor performance/ or task performance/

24. learning/ or conditioning/ or problem based learning/

25. "movement (physiology)"/ or limb movement/ or locomotion/ or exp walking/ or leg movement/ or leg exercise/ or physical mobility/ or "range of motion"/

26. motor activity/ or psychomotor activity/

27. body equilibrium/ or body posture/ or sitting/ or standing/ or weight bearing/

28. exp leg/ or exp back/

29. (motor adj5 (skill\$ or activit\$ or function\$)).tw.

30. (learning or conditioning).tw.

31. (movement or gait or locomotion or walking or walk or mobility).tw.

32. (equilibrium or balance or postur\$ or supination or pronation).tw. 
33. (body sway or stance or strength or weight?bearing or body weight support).tw.

34. (locomotor adj5 (recovery or training)).tw.

35. (weight adj5 (distribut\$ or transfer\$)).tw.

36. (sit or sitting or stand or standing or step or stepping or climb or climbing).tw.

37. (lower limb\$ or lower extremit\$ or ankle or leg or heel or calf or knee or hip or thigh or foot or trunk).tw.

38. or $/ 22-37$

39. 21 and 38

40. (animal/ or nonhuman/ or animal experiment/) and human/

41. animal/ or nonhuman/ or animal experiment/

42. 41 not 40

43. 39 not 42

44. Randomized Controlled Trial/

45. Randomization/

46. Controlled Study/

47. control group/

48. clinical trial/

49. Crossover Procedure/

50. Double Blind Procedure/

51. Single Blind Procedure/ or triple blind procedure/

52. Parallel Design/

53. placebo/

54. "types of study"/

55. random\$.tw.

56. (controlled adj5 (trial\$ or stud\$)).tw.

57. (clinical\$ adj5 trial\$̣).tw.

58. ((control or treatment or experiment\$ or intervention) adj5 (group\$ or subject\$ or patient\$)).tw.

59. (quasi-random\$ or quasi random\$ or pseudo-random\$ or pseudo random\$).tw.

60. ((control or experiment\$ or conservative) adj5 (treatment or therapy or procedure or manage\$)).tw.

61. ((singl\$ or doubl\$ or tripl\$ or trebl\$) adj5 (blind\$ or mask\$)).tw.

62. (cross-over or cross over or crossover).tw.

63. (placebo\$ or sham).tw.

64. trial.ti.

65. (assign\$ or allocat\$).tw.

66. controls.tw.

67. or/44-66

Physical rehabilitation approaches for the recovery of function and mobility following stroke (Review) 
68.43 and 67

69 . (2005\$ or $2006 \$$ or $2007 \$$ or $2008 \$$ or $2009 \$$ or $2010 \$$ or $2011 \$$ or $2012 \$)$.em.

70.68 and 69

\section{Appendix 3. AMED search strategy}

AMED (Ovid) 1985 to 5 December $2012 \mathrm{~N}=1252$ (new search from scratch)

1. cerebrovascular disorders/or cerebral hemorrhage/ or cerebral infarction/or cerebral ischemia/ or cerebrovascular accident/ or stroke/

2. (stroke or poststroke or post-stroke or cerebrovasc\$ or brain vasc\$ or cerebral vasc\$ or cva\$ or apoplex\$ or SAH).tw.

3. ((brain\$ or cerebr\$ or cerebell\$ or intracran\$ or intracerebral) adj5 (isch?emi\$ or infarct\$ or thrombo $\$$ or emboli\$ or occlus\$)).tw.

4. ((brain\$ or cerebr\$ or cerebell\$ or intracerebral or intracranial or subarachnoid) adj5 (haemorrhage\$ or hemorrhage\$ or haematoma\$ or hematoma\$ or bleed\$)).tw.

5. hemiplegia/

6. (hemipleg\$ or hemipar\$ or paresis or paretic).tw.

7.1 or 2 or 3 or 4 or 5 or 6

8. physical therapy modalities/ or physiotherapists/ or occupational therapy modalities/ or occupational therapy techniques/ or occupational therapists/ or rehabilitation/ or rehabilitation modalities/or rehabilitation techniques/

9. exp exercise therapy/ or exercise/ or muscle stretching exercises/ or exp orthopedic equipment/

10. feedback/ or biofeedback/

11. exp neurodevelopmental therapy/

12. (physiotherapy or physical therapy or exercise therapy or rehabilitation).tw.

13. (neurorehabilitation or feedback or biofeedback).tw.

14. (motor adj5 (train\$ or re?train\$ or learn\$ or re?learn\$)).tw.

15. neuromuscular facilitation.tw.

16. (movement adj5 (therap\$ or science)).tw.

17. ((neurodevelopmental or neurophysiological or orthop?edic) adj5 (therap\$ or treatment\$ or rehabilitation or principle\$ or approach \$ or component\$ or concept\$)).tw.

18. (Bobath or Carr or Brunnstrom or Rood or Johnstone or NDT).tw.

19. or/8-18

20.7 and 19

21. psychomotor performance/ or exp motor skills/ or "task performance and analysis"/

22. learning/ or conditioning/ or problem solving/

23. balance/ or movement/ or exp gait/ or locomotion/ or walking/ or dependent ambulation/ or motor activity/ or pronation/ or "range of motion"/ or exp posture/ or sitting/ or weight bearing/

24. exp back/ or exp leg/

25. (motor adj5 (skill\$ or activit\$ or function\$)).tw.

26. (learning or conditioning).tw.

27. (movement or gait or locomotion or walking or walk or mobility).tw.

28. (equilibrium or balance or postur\$ or supination or pronation).tw.

Physical rehabilitation approaches for the recovery of function and mobility following stroke (Review) 
29. (body sway or stance or strength or weight?bearing or body weight support).tw.

30. (locomotor adj5 (recovery or training)).tw.

31. (weight adj5 (distribut\$ or transfer\$)).tw.

32. (sit or sitting or stand or standing or step or stepping or climb or climbing).tw.

33. (lower limb\$ or lower extremit\$ or ankle or leg or heel or calf or knee or hip or thigh or foot or trunk).tw.

34. or $/ 21-33$

35. 20 and 34

36. research design/

37. clinical trials/

38. randomized controlled trials/

39. comparative study/

40. double blind method/

41. meta analysis/

42. random allocation/

43. program evaluation/

44. placebos/

45. (clinical trial or clinical trial phase iii or meta analysis or clinical trialb or clinical trials or multicenter study or multicentre study or comparative studies or comparative study or randomised controlled trial or randomized controlled trial or controlled clinical trial or controlled trial).pt.

46. random\$.tw.

47. (controlled adj5 (trial\$ or stud\$)).tw.

48. (clinical\$ adj5 trial\$).tw.

49. ((control or treatment or experiment\$ or intervention) adj5 (group\$ or subject\$ or patient\$)).tw.

50. (quasi-random\$ or quasi random\$ or pseudo-random\$ or pseudo random\$).tw.

51. ((control or experiment\$ or conservative) adj5 (treatment or therapy or procedure or manage\$)).tw.

52. ((singl\$ or doubl\$ or tripl\$ or trebl\$) adj5 (blind\$ or mask\$)).tw.

53. (cross-over or cross over or crossover).tw.

54. placebo\$.tw.

55. sham.tw.

56. (assign\$ or allocat\$).tw.

57. controls.tw.

58. trial.ti. or (RCT or RCTs).tw.

59. or $/ 36-58$

60.35 and 59

\section{Appendix 4. CINAHL search strategy}

CINAHL (Ebsco) May 2005 to 5 December $2012 \mathrm{~N}=2098$

Physical rehabilitation approaches for the recovery of function and mobility following stroke (Review) 
S68.S46 AND S67

\section{S67.S47 OR S48 OR S49 OR S50 OR S51 OR S52 OR S53 OR S54 OR S55 OR S56 OR S59 OR S60 OR S63 OR S64 OR S65 OR S66}

\section{S66.PT meta analysis}

S65.TI ( meta analysis* or metaanalysis or meta-analysis or systematic review* ${ }^{\star}$ ) or AB ( meta analysis* or metaanalysis or meta-analysis or systematic review* ${ }^{\star}$ )

S64.TI ( counterbalance* or multiple baseline* or $A B A B$ design ) or $A B$ ( counterbalance* or multiple baseline ${ }^{\star}$ or $A B A B$ design )

S63.S61 and S62

S62.TI trial* or AB trial*

S61.TI ( clin* or intervention* or compar* or experiment* or preventive or therapeutic) or AB ( clin* or intervention* or compar ${ }^{\star}$ or experiment* or preventive or therapeutic)

S60.TI ( crossover or cross-over or placebo* or control* or factorial or sham ) or AB ( crossover or cross-over or placebo* or control* or factorial or sham )

S59.S57 and S58

S58.TI ( blind* or mask ${ }^{\star}$ ) or AB ( blind* or mask* ${ }^{\star}$

S57.TI ( $\operatorname{singl}{ }^{\star}$ or doubl ${ }^{\star}$ or tripl ${ }^{\star}$ or trebl ${ }^{\star}$ ) or AB ( $\operatorname{singl} l^{\star}$ or doubl ${ }^{\star}$ or tripl ${ }^{\star}$ or trebl $^{\star}$ )

S56.TI random* or AB random*

S55.PT systematic review

S54 .(MH "Community Trials") or (MH "Experimental Studies") or (MH "One-Shot Case Study") or (MH "Pretest-Posttest Design+") or (MH "Solomon Four-Group Design") or (MH "Static Group Comparison") or (MH "Study Design")

S53 .(MH "Clinical Research") or (MH "Clinical Nursing Research")

S52.(MH "Placebo Effect") or (MH "Placebos") or (MH "Meta Analysis")

S51 .(MH "Factorial Design") or (MH "Quasi-Experimental Studies") or (MH "Nonrandomized Trials")

S50.(MH "Control (Research)") or (MH "Control Group")

S49 .(MH "Crossover Design") or (MH "Clinical Trials+") or (MH "Comparative Studies")

S48.(MH "Random Assignment") or (MH "Random Sample+")

S47.PT randomized controlled trial or clinical trial

S46.S29 AND S45

S45.S30 OR S31 OR S32 OR S33 OR S34 OR S35 OR S36 OR S37 OR S38 OR S39 OR S40 OR S41 OR S42 OR S43 OR S44

S44.TI ( lower limb* or lower extremit* or ankle or leg or heel or calf or knee or hip or thigh or foot or trunk ) OR AB (lower limb* or lower extremit* or ankle or leg or heel or calf or knee or hip or thigh or foot or trunk )

S43.TI ( sit or sitting or stand or standing or step or stepping or climb or climbing ) OR AB ( sit or sitting or stand or standing or step or stepping or climb or climbing)

S42.TI ( weight N5 (distribut* or transfer ${ }^{\star}$ ) ) OR AB ( weight N5 (distribut ${ }^{\star}$ or transfer $^{\star}$ ))

S41.TI ( locomotor N5 (recovery or training) ) OR AB ( locomotor N5 (recovery or training) )

S40.TI ( body sway or stance or strength or weight\#bearing or body weight support) OR AB ( body sway or stance or strength or weight\#bearing or body weight support )

S39.TI ( equilibrium or balance or postur ${ }^{\star}$ or supination or pronation ) OR AB ( equilibrium or balance or postur ${ }^{\star}$ or supination or pronation ) 
S38.TI ( movement or gait or locomotion or walking or walk or mobility) OR AB ( movement or gait or locomotion or walking or walk or mobility)

S37.TI ( learning or conditioning ) OR AB ( learning or conditioning )

S36.TI ( motor N5 (skill* or activit* or function*) ) OR AB ( motor N5 (skill* or activit* or function*) )

S35.(MH "Back") OR (MH "Torso") OR (MH "Lower Extremity+")

S34 .(MH "Balance, Postural")

S33.(MH "Motor Activity")

S32 .(MH "Movement") OR (MH "Locomotion") OR (MH "Walking") OR (MH "Gait+") OR (MH "Pronation") OR (MH "Range of Motion") OR (MH "Rising") OR (MH "Sitting") OR (MH "Squatting") OR (MH "Stair Climbing") OR (MH "Standing+") OR (MH "Supination") OR (MH "WeightBearing") OR (MH "Weight Shifting")

S31.(MH "Learning") OR (MH "Conditioning (Psychology)") OR (MH "Problem Solving") OR (MH "Skill Retention")

S30.(MH "Psychomotor Performance") OR (MH "Motor Skills+") OR (MH "Task Performance and Analysis")

S29.S25 OR S26 OR S27 OR S28

S28.(MH "Gait Disorders, Neurologic/RH")

S27 .(MH "Hemiplegia/RH")

S26.(MH "Cerebrovascular Disorders+/RH")

S25.S12 AND S24

\section{S24.S13 OR S14 OR S15 OR S16 OR S17 OR S18 OR S19 OR S20 OR S21 OR S22 OR S23}

S23.TI ( Bobath or Carr or Brunnstrom or Rood or Johnstone or NDT ) OR AB ( Bobath or Carr or Brunnstrom or Rood or Johnstone or NDT )

S22.TI ( (neurodevelopmental or neurophysiological or orthop\#edic) N5 (therap* or treatment* or rehabilitation or principle* or approach* or component* or concept ${ }^{\star}$ ) ) OR AB ( (neurodevelopmental or neurophysiological or orthop\#edic) N5 (therap* or treatment* or rehabilitation or principle or approach $^{\star}$ or component $^{\star}$ or concept $\left.^{\star}\right)$ )

S21.TI ( movement N5 (therap* or science) ) OR AB ( movement N5 (therap* or science))

S20.TI neuromuscular facilitation OR AB neuromuscular facilitation

S19.TI ( motor N5 (train* or re\#train* or learn* or re\#learn*) ) OR AB ( motor N5 (train* or re\#train* or learn* or re\#learn*))

S18.TI ( neurorehabilitation or feedback or biofeedback) OR AB ( neurorehabilitation or feedback or biofeedback )

S17.TI ( physiotherapy or physical therapy or exercise therapy or rehabilitation ) OR AB ( physiotherapy or physical therapy or exercise therapy or rehabilitation )

S16.(MH "Orthopedic Equipment and Supplies+")

S15.(MH "Feedback") OR (MH "Biofeedback") OR (MH "Biofeedback (Iowa NIC)")

S14.(MH "Exercise+")

S13 .(MH "Physical Therapy") OR (MH "Rehabilitation") OR (MH "Home Rehabilitation+") OR (MH "Occupational Therapy") OR (MH "Therapeutic Exercise+") OR (MH "Neuromuscular Facilitation")

\section{$\mathrm{S} 12 . \mathrm{S} 1$ or $\mathrm{S} 2$ or $\mathrm{S} 5$ or $\mathrm{S} 8$ or $\mathrm{S} 9$ or $\mathrm{S} 10$ or $\mathrm{S} 11$}

S11.(MH "Gait Disorders, Neurologic+")

S10.TI ( hemipleg* or hemipar* or paresis or paretic ) or AB ( hemipleg* or hemipar* or paresis or paretic)

S9.(MH "Hemiplegia")

S8.S6 and S7

Physical rehabilitation approaches for the recovery of function and mobility following stroke (Review) 
S7.TI ( haemorrhage* or hemorrhage* or haematoma* or hematoma* or bleed ${ }^{\star}$ ) or AB ( haemorrhage* or hemorrhage* or haematoma* or hematoma* or bleed*

S6.TI ( brain* or cerebr* or cerebell* or intracerebral or intracranial or subarachnoid) or AB ( brain* or cerebr* or cerebell* or intracerebral or intracranial or subarachnoid)

S5.S3 and S4

S4.TI ( ischemi ${ }^{\star}$ or ischaemi ${ }^{\star}$ or infarct ${ }^{\star}$ or thrombo ${ }^{\star}$ or emboli* or occlus $^{\star}$ ) or AB ( ischemi ${ }^{\star}$ or ischaemi ${ }^{\star}$ or infarct $^{\star}$ or thrombo $^{\star}$ or emboli ${ }^{\star}$ or occlus* )

S3.TI ( brain* or cerebr ${ }^{\star}$ or cerebell ${ }^{\star}$ or intracran* or intracerebral ) or AB ( brain* or cerebr ${ }^{\star}$ or cerebell* or intracran* or intracerebral )

S2.TI ( stroke or poststroke or post-stroke or cerebrovasc* or brain vasc* or cerebral vasc or cva or apoplex or SAH ) or AB ( stroke or poststroke or post-stroke or cerebrovasc ${ }^{\star}$ or brain vasc* or cerebral vasc or cva or apoplex or SAH )

S1 .(MH "Cerebrovascular Disorders+") or (MH "stroke patients") or (MH "stroke units")

\section{WHAT'S NEW}

\begin{tabular}{lll}
\hline Date & Event & Description \\
\hline 17 April 2023 & Amended & $\begin{array}{l}\text { Small typographical error in Plain Language Summary and re- } \\
\text { sults section fixed. }\end{array}$ \\
\hline
\end{tabular}

\section{H I S T ORY}

Protocol first published: Issue 1, 2000

Review first published: Issue 2, 2003

\begin{tabular}{lll}
\hline Date & Event & Description \\
\hline 15 November 2013 & New search has been performed & $\begin{array}{l}\text { Title changed from "Physiotherapy treatment approaches for the } \\
\text { recovery of postural control and lower limb function" to "Phys- } \\
\text { ical rehabilitation approaches for the recovery of function and } \\
\text { mobility following stroke". We have updated the searches to De- } \\
\text { cember 2012. We included 79 new studies in this version: the re- } \\
\text { view now has } 96 \text { included studies involving 10,401 participants }\end{array}$ \\
\hline \multirow{2}{*}{15 November 2013} & $\begin{array}{l}\text { New citation required and conclusions } \\
\text { have changed }\end{array}$ & $\begin{array}{l}\text { A substantial amount of new information has been included in } \\
\text { this review. The conclusions of the review have changed since } \\
\text { the previous version; the comparisons and the method of cate- } \\
\text { gorising interventions have also changed. }\end{array}$ \\
\hline
\end{tabular}

\begin{tabular}{|c|c|c|}
\hline 30 September 2008 & Amended & Converted to new review format. \\
\hline 19 January 2006 & New search has been performed & $\begin{array}{l}2001 \text { Version } \\
\text { - } 4114 \text { trials from electronic searching } \\
\text { - } 167 \text { abstracts screened } \\
\text { - } 71 \text { full papers assessed } \\
\text { - } 11 \text { trials included (362 patients): Dean 1997; Dean 2000; Dun- } \\
\text { can 1998; Gelber 1995; Inaba 1973; Langhammer 2000; Pollock } \\
\text { 1998; Richards 1993; Stern 1970; Wagenaar 1990; Wellmon } 1997 \\
\text { Data for: } \\
\text { - four trials of neurophysiological versus other; }\end{array}$ \\
\hline
\end{tabular}




\begin{tabular}{|c|c|}
\hline Event & Description \\
\hline & $\begin{array}{l}\text { - } \text { four trials of motor learning versus other; } \\
\text { - } \text { four trials of mixed versus other; } \\
\text { - } 2005 \text { Update } \\
\text { - } 8408 \text { ( } 4294 \text { new) trials from electronic searching } \\
\text { - } 266 \text { ( } 99 \text { new) abstracts screened } \\
\text { - } 185 \text { (114 new) full papers assessed } \\
\text { - } 20 \text { (11 new) trials included (1087 patients; } 809 \text { new). New tri- } \\
\text { als: Duncan } 2003, \text { Green } 2002, \text { Hesse } 1998, \text { Howe } 2005, \text { Lincoln } \\
2003, \text { McClellan } 2004, \text { Mudie } 2002, \text { Ozdemir } 2002, \text { Salbach } 2004 \text {, } \\
\text { Wade } 1992, \text { Wang } 2005 a \\
\text { Trials comparing subgroups of the same approach were exclud- } \\
\text { ed (excluded Inaba } 1973 \text { and Wagenaar } 1990, \text { which were includ- } \\
\text { ed in original version). } \\
\text { - } \text { nine (five new) trials of mixed versus other. } \\
\text { Data for: } \\
\text { eight (four new) trials of neurophysiological (all Bobath) versus } \\
\text { other; }\end{array}$ \\
\hline
\end{tabular}

\section{CONTRIBUTIONS OF AUTHORS}

Alex Pollock (AP), for the 2007 and earlier versions: planned and co-ordinated all stages of the review; carried out searches, identified relevant studies and screened abstracts for relevant trials; co-ordinated and wrote the classification of rehabilitation approaches; wrote the 2007 amendment to the original protocol; classified the interventions administered in each trial; documented methodological quality of studies; contacted and communicated with trial authors; extracted data from included studies; planned the analyses and entered data into RevMan and wrote all drafts of the review. For this version: secured funding; planned and co-ordinated all stages of the review; screened abstracts; checked documented methodological quality of studies; planned analyses; checked data entry; carried out subgroup and sensitivity analyses; led stakeholder group meetings and wrote drafts of the review.

Pauline Campbell (PC), for this version: carried out searches, screened titles and abstracts; co-ordinated consensus decisions between review authors; entered descriptions of studies into RevMan; documented the methodological quality of studies; contacted and communicated with trial authors; extracted data from included studies and entered data into RevMan; created and entered data into tables summarising treatment components of included studies and contributed to writing and commented on draft versions of the written review.

Pei Ling Choo (PLC), for this version: screened abstracts in Chinese; translated necessary information for two review authors to reach consensus on inclusion of Chinese studies; translated written descriptions of interventions from Chinese into English; assessed methodological quality of Chinese studies; classified treatment components of all (Chinese and English) studies; contributed to stakeholder group meetings; contributed to conclusions drawn from the results and commented on draft versions of the written review.

Gill Baer (GB), for the 2007 and earlier versions: screened abstracts for relevant trials; contributed to the written criteria for classifying rehabilitation approaches; classified the interventions administered in each trial and discussed any discrepancies with AP to reach consensus; documented methodological quality of studies; extracted data from included trials and commented on draft versions of the written review. For this version: identified relevant studies for inclusion at full paper stage; discussed inclusion of studies at consensus meetings; contributed to stakeholder group meetings; contributed to conclusions drawn from the results and commented on draft versions of the written review.

Anne Forster (AF), for this version: contributed to the funding application that supported the stakeholder group and review update; contributed to stakeholder group meetings; provided methodological expertise in relation to identification of treatment components, including discussions with wider groups of physiotherapists and stroke survivors; contributed to conclusions drawn from the results and commented on draft versions of the written review. 
Jacqui Morris (JM), for this version: contributed to the funding application that supported the stakeholder group and review update; contributed to stakeholder group meetings; contributed to conclusions drawn from the results and commented on draft versions of the written review.

Valerie Pomeroy (VP), for the 2007 and earlier versions: contributed to the formation of the protocol; screened abstracts for relevant trials for the first version of the review; contributed to the written criteria for classifying rehabilitation approaches and commented on draft versions of the written review. For this version: contributed to conclusions drawn from the results and commented on draft versions of the written review.

Peter Langhorne (PL), for the 2007 and earlier versions: provided substantial input to the formation of the protocol and provided methodological support at all stages of the review; discussed disagreements between independent review authors (AP and GB) regarding inclusion and methodological quality of trials; supervised data analysis and commented on draft versions of the written review. For this version: contributed to the conclusions drawn from the results and commented on draft versions of the written review.

\section{DECLARATIONS OF INTEREST}

Authors Alex Pollock and Gillian Baer carried out trials included in this review (Baer 2007; Pollock 1998).

\section{SOURCES OF SUPPORT}

\section{Internal sources}

- No sources of support provided

\section{External sources}

- Chest Heart and Stroke, Scotland, UK

- The Big Lottery, UK

\section{DIFFERENCES BETWEEN PROTOCOLANDREVIEW}

\section{Title}

For the protocol and for versions of the review published in 2007 and earlier, the title of this review was 'Physiotherapy treatment approaches for the recovery of postural control and lower limb function following stroke.' For the 2013 version of this review, the title was changed to 'Physical rehabilitation approaches for the recovery of function and mobility following stroke.' We made this change to reflect the more international perspective of this review update and the fact that 'physiotherapy' is not a universally adopted term, and to more accurately reflect the primary and secondary outcomes of the review.

\section{Objectives}

The objectives within the protocol and versions published in 2007 and earlier were focused on "the recovery of postural control and lower limb function." In this update, this has been amended to read "the recovery of function and mobility." This difference relates only to the terminology used, not to the selection of primary or secondary outcomes within the review.

The reason for originally highlighting the focus on postural control and lower limb function was specifically to highlight that this review excluded studies related to upper limb interventions and recovery only. However, feedback from stakeholder group members indicated that the original objectives were perceived to be misleading, as they did not incorporate the primary outcomes, which were measures of disability, and prestated relevant measures of disability such as (1) independence in ADL scales*; or (2) motor function scales.* The stakeholder group reached consensus that the phrase "function and mobility" was more correct and appropriate to reflect the focus of this review within the stated objective (and title).

The original protocol stated a number of hypotheses. These have been removed from this update. (Original text: "Hypotheses to be tested: (1) physiotherapy treatment based on neurophysiological principles results in better recovery of postural control and lower limb function than treatment based on motor learning principles, orthopaedic principles or a mixture of treatment principles in patients with stroke; and (2) physiotherapy treatment based on motor learning principles results in better recovery of postural control and lower limb function than treatment based on orthopaedic principles, or on a mixture of treatment principles in patients with stroke.")

\section{* Names of measures of disability}

In the protocol and in previous versions of the review, the primary outcome of measures of disability was divided into two prestated subcategories of:

1. global dependency scales (including Barthel Activities of Daily Living Index, Functional Independence Measure (FIM), Modified Rankin Scale, Katz Index of Activities of Daily Living, Rehabilitation Activities Profile); and 
2. functional independence scales (including Motor Assessment Scale (MAS), Fugl-Meyer Assessment (lower limb section); Rivermead Mobility Index; Rivermead Motor Assessment).

For this update, we changed the names of each of these two subcategories (but not the content) because we considered that the original names were now potentially confusing, as other terms are more usually used in published research. The replacement names are (1) independence in ADL and (2) motor function.

\section{Outcomes}

The protocol defined secondary outcomes as measures of motor impairment, classifying them as measures of:

1. postural control and balance;

2. voluntary movement (including movement associated with gait);

3. tone or spasticity;

4. range of movement; and

5. strength.

The protocol also identified participation (handicap or quality of life) as an outcome of relevance to this review.

For the 2007 version of the review, the review authors documented and extracted descriptions and data from any outcomes falling into the groupings stated in the protocol. Based on the prestated groups of relevant outcomes and the availability of data from specific measures in the included trials, we discussed and reached consensus on which outcome measures should be included in the analysis. For this 2013 version of the review, the secondary outcomes remained the same as the outcomes analysed for the 2007 version (i.e. secondary outcomes).

1. Balance (Berg Balance Scale).

2. Gait velocity.

3. Length of stay.

In the protocol and in previous versions of this review, we carried out analysis only on outcomes reported immediately after the end of the intervention. In this 2013 version, we have carried out analyses on both outcomes reported immediately after the end of the intervention and on follow-up outcomes.

\section{N DEX TERMS}

\section{Medical Subject Headings (MeSH)}

Activities of Daily Living; Biofeedback, Psychology $\left[{ }^{*}\right.$ methods]; Leg [physiology]; Motor Skills; Physical Therapy Modalities; ${ }^{\star}$ Posture; Proprioception [physiology]; Randomized Controlled Trials as Topic; Recovery of Function; *Stroke Rehabilitation

\section{MeSH check words}

Adult; Humans 